\title{
An Investigation into New Zealand's \\ Climate during the Last Glacial Maximum: \\ A Climate Modelling Approach
}

\author{
by \\ Frank Drost
}

\author{
A thesis \\ submitted to the Victoria University of Wellington \\ in fulfilment of the \\ requirements for the degree of \\ Doctor of Philosophy \\ in Geophysics
}

Victoria University of Wellington

2006 
Menigmaal dwalen mijn gedachten

Zij proberen hun weg te vinden

Naar het niets

Niets zal hun helpen de weg te vinden

En mijn gedachten dwalen af

Frank Drost, 1985 


\section{Abstract}

New Zealand's climate during the Last Glacial Maximum has been investigated using the UKMO global and regional models HadAM3H (GCM) and HadRM3H (RCM). SSTs and sea-ice were supplied from a set of prior coupled model (HadCM3) runs and all models were set up according to the glacial conditions as specified by PMIP.

In the analysis of the global simulation, emphasis was placed on the climate of the Southern Hemisphere. Compared to the present day, the modelled climate of the LGM is mainly characterized by the different wind regimes, both in the zonal and meridional directions. In the zonal mean, the polar trough shifted equatorward, and the westerly wind increased slightly between approximately $30^{\circ} \mathrm{S}-50^{\circ} \mathrm{S}$, and decreased poleward of this zonal band. At the same time, there was an increase in the number of and/or strength of southerlies between $35^{\circ} \mathrm{S}$ $60^{\circ} \mathrm{S}$. This resulted in a reduction of the poleward zonal mean meridional heat transport, and an enhancement of the wave number 3 pattern in the mean zonal circulation. All these changes contributed to a weaker SAO during the LGM. Interannual variability was as today, dominated by the High Latitude Mode (HLM, or Antarctic Oscillation/Southern Annular Mode) and ENSO.

For the LGM, New Zealand was about $2.5^{\circ} \mathrm{C}-4^{\circ} \mathrm{C}$ cooler than in a preindustrial control simulation. The seasonal cooling was largest during winter. Excluding the Alpine region, the largest cooling geographically took place in the east of the South Island. Precipitation was in general reduced everywhere during the whole year, except for the east of the South Island. The westerly wind increased considerably over the North Island and the northern part of the South Island, but was weaker over the rest of the South Island. JJA was the exception 
with weaker westerly winds over all New Zealand, which was probably related to enhance blocking during that season. The stronger westerly wind accentuated the cooling over the North Island, except for the eastern region, where it mainly enhanced the dry conditions by preventing the moist easterly winds coming ashore. The weaker westerly wind in the south on the other hand encouraged enhanced penetration of moist winds. The most dramatic change in the modelled New Zealand climate was the large increase in the number of southerlies in each region, which were capable of bringing very cold polar air over most of the country. It was probably mainly the changes in the winds that lead to the harshness of New Zealand's climate during the LGM, increasing the seasonality in temperature and precipitation. It is suggested that they had therefore a controlling influence on the existence of some of the vegetation types in New Zealand. 


\section{Acknowledgements}

It was my general interest in earth sciences that had led me to undertake a $\mathrm{PhD}$ study in geophysics. But such a task was never possible without the very generous help and support I have received from many people and institutes.

For no apparent reason, it just happened that all my supervisors were called James. I would like to thank James McGregor (VicJim) for first initialising and then harbouring my interest in the atmospheric sciences, James Renwick (NIWAJim) for his enthusiasm, his guidance and for his open-door policy, which meant that I could at all times approach him with my many questions and problems, and James Shulmeister (Jamie) for contributing to my interest in paleoclimates. I couldn't have wished for a better team as my supervisors. In the beginning we joked about a probable situation in that I could defend myself by saying "But James said, ...", and that none of you would be the wiser who had said it, but such a situation never materialised. And that was simply the result of the good contacts we had with each other.

I would like to thank NIWA in general for supplying a fantastic research environment, in which I was able to fully participate. Interactions with atmospheric scientists, modellers, technicians and the occasional participation in fieldwork, all contributed to strengthen my interest in doing research. I guess that I have had contact with the bulk of the 200+ employees at NIWA, Greta Point, both academically as well as socially. The result was that I have been involved in organising international conferences, in modelling and climate discussions as well as have been involved in the social soccer games, netball and cricket matches. It is hard to single out people for their contribution to this study, but I would like to thank the following people at NIWA especially: Balakrishnan 
"Bhaski" Bhaskaran, who helped me with every aspect of my work; Hilary Oliver, who was instrumental in solving many programming problems; Phil Andrews, who helped me with the UM; Graham Rickard, with his expert knowledge of PV-WAVE; and of course James Renwick, whose contribution to this study can not be overestimated. Further, the conversations I had with Brett Mullan and Mike Revell have helped me in understanding several aspects of the atmosphere. The "Law of Murphy" was definitely applicable to many of my computer problems. Luckily the excellent IT support at NIWA was able to solve all the computer problems and they were often prepared to give personal assistance. Many thanks go out to Chris Edsall, Richard Rayner, Paul Radford and Colin Tinker in particular, but also to the rest of the IT group. Because of all these great people, I have thoroughly enjoyed my time at NIWA.

I would like to thank the New Zealand MetService for the introduction into the basics of atmospheric science and Clive Jones of the UK MetOffice for helping me with the vegetation and soil codes.

I would like to thank the Victoria University of Wellington for awarding me a PhD scholarship and the New Zealand Geophysical Society for awarding me the Jim Ansell Geophysics Scholarship for the year 2004. Further funding for my $\mathrm{PhD}$ study came from FRST contract C01X0202, "Adaptation to Climate Variability \& Change", and Marsden Grant Contract UOC301, "Orbital or Thermal Causes of Glaciation in New Zealand". I would like to thank the Meteorological Society of New Zealand and the Victoria University's Science Faculty for supporting me financially to attend conferences in New Zealand and abroad.

I have shared many great times with my fellow $\mathrm{PhD}$ students and friends based at NIWA. From the stench of rotten fish (giant squid), howling southerlies, leaking roofs to pleasant lunches on the grassy hill, seeing orcas and dolphins swimming past our offices till the dress-ups at the Winter Poultice: Neil and his mussels, Andy and his cricket, Susanne and her cakes, and most important of them all, Kelly and her love. Kelly, we started off as fellow students, we became friends, we got engaged, and we got married. My main achievement during my second period of study is having found you as my partner for life. I would like to 
thank you for having made this period the most enjoyable time of my life.

Finally, I would like to thank my parents for all that they have done for me. They as no other know how long the road has been. This study would never have happened without their continuous support throughout all those years. I am proud to say that I have the greatest parents that one could wish for, and I would like to dedicate this work to them. Thank you very much, Bea and Koos. 

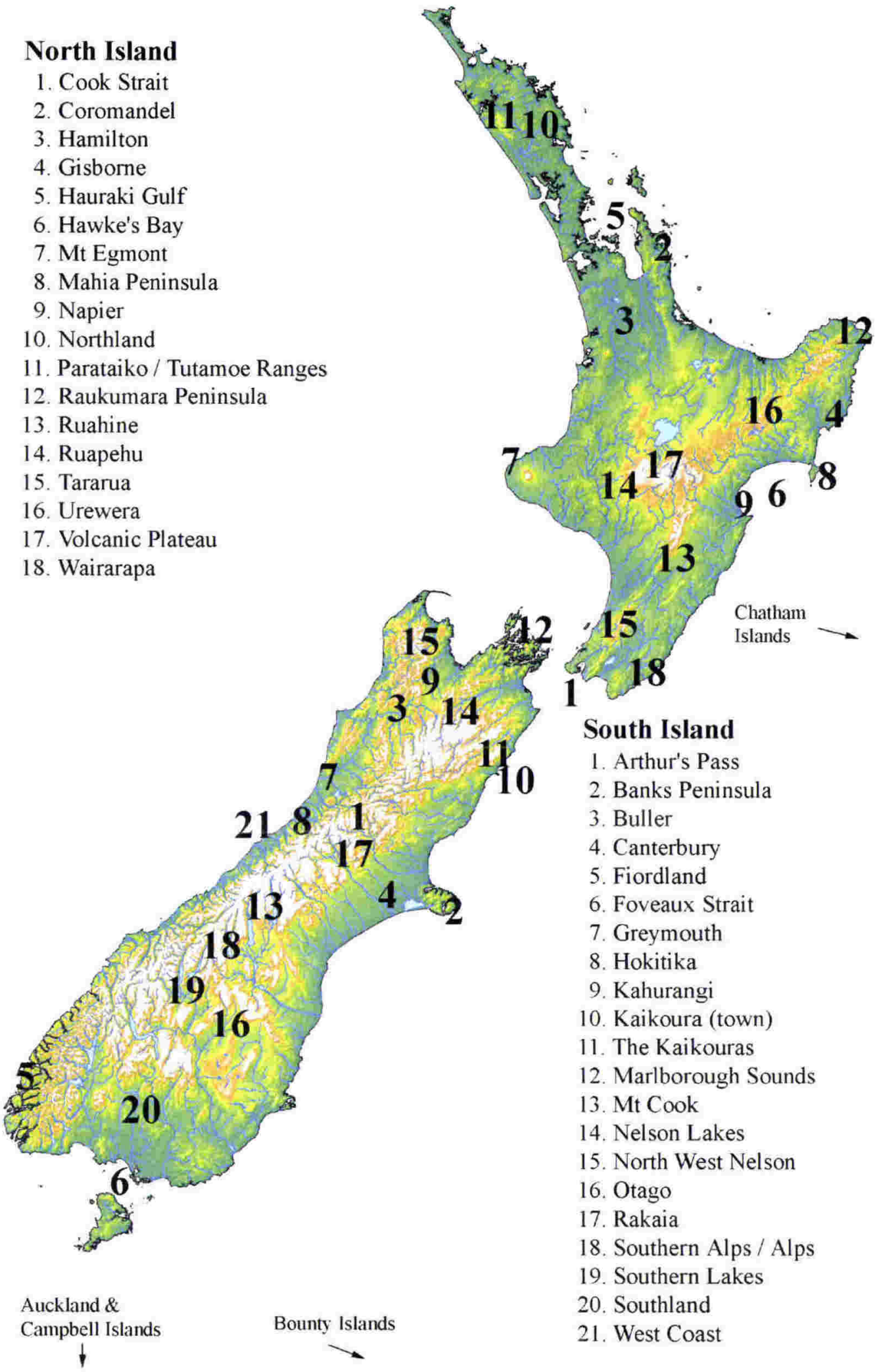

Figure I. Map of New Zealand with all the locations mentioned in this thesis. 


\section{List of Contents}

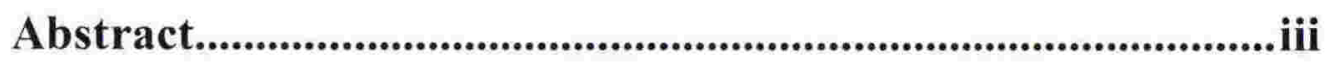

Acknowledgements .............................................................................

List of Contents ..............................................................................

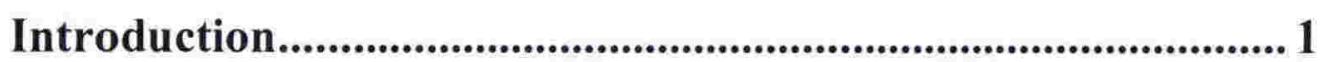

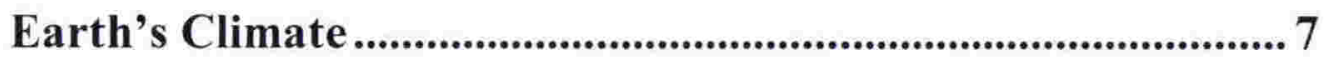

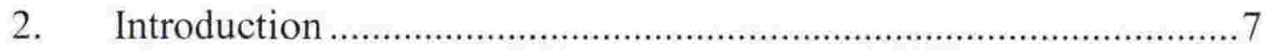

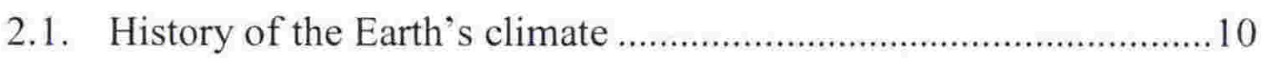

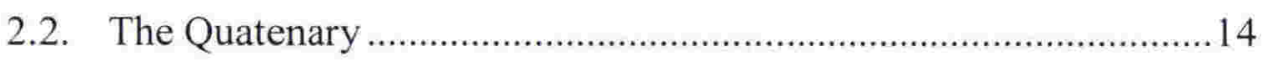

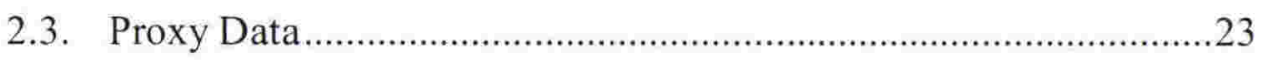

The Last Glacial Maximum ........................................................... 27

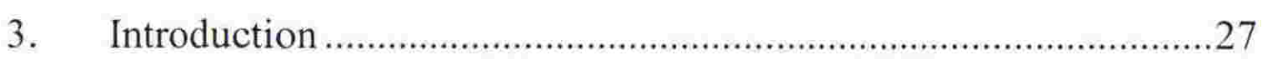

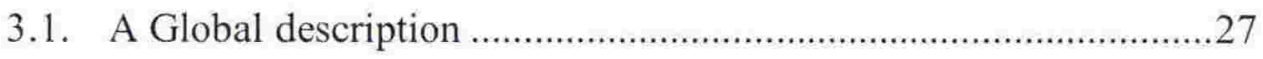

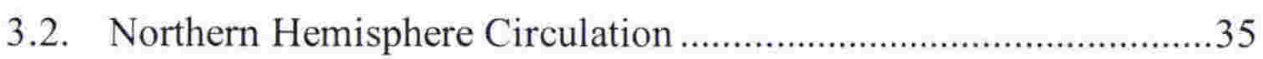

3.3. Southern Hemisphere Circulation ........................................................

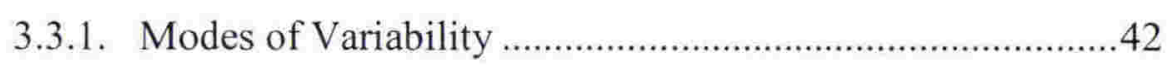

3.3.1.1. Semi-Annual Oscillation (SAO) ……………......42

3.3.1.2. Intraseasonal and Interannual Variations ...........44

3.3.2. New Zealand during the LGM ………………….................... 48

Climate Modelling .............................................................................53

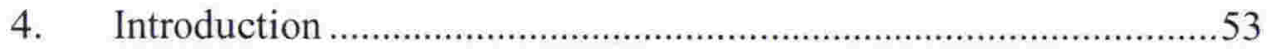

4.1. A Short History of Atmospheric Science .........................................54

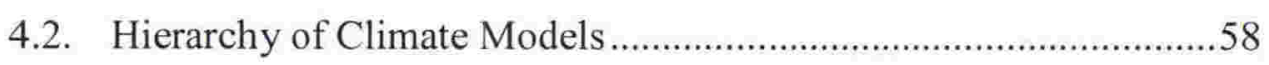


4.2.1. Zero Dimensional Models ..................................................59

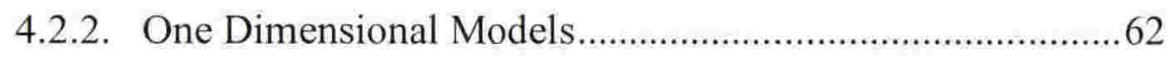

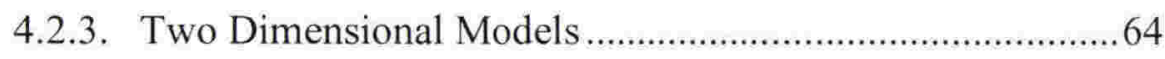

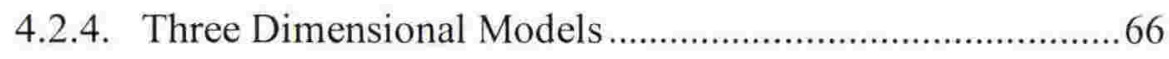

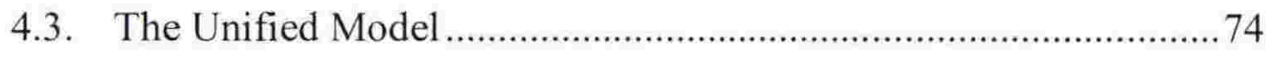

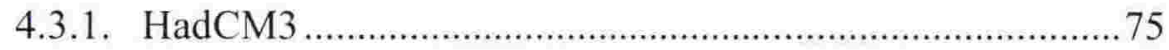

4.3.1.1. Atmospheric model: HadAM3 ........................ 76

4.3.1.2. Ocean model: HadOM3 …............................... 81

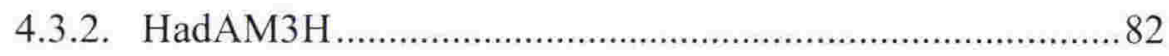

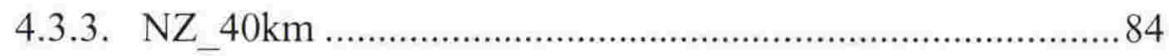

Pre-industrial simulation: Global ........................................ 88

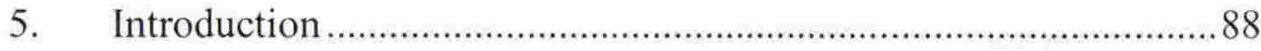

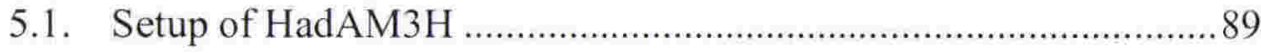

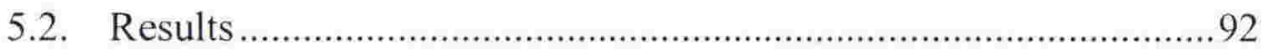

5.2.1. Sea Surface Temperature and Sea Ice............................. 92

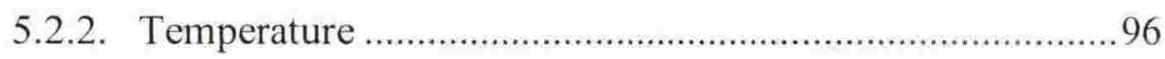

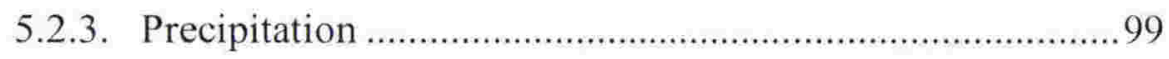

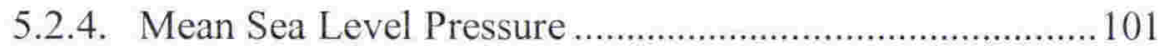

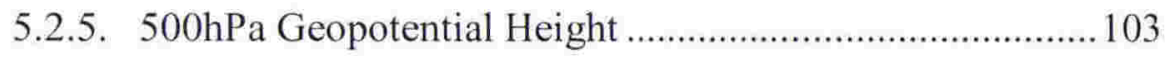

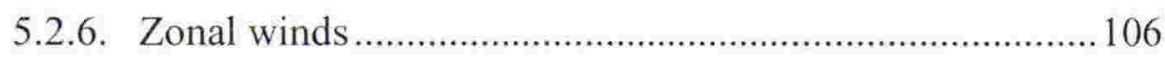

5.2.7. Meridional Fluxes .......................................................... 110

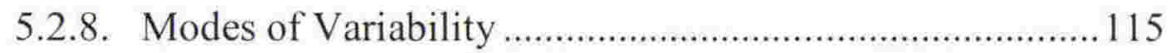

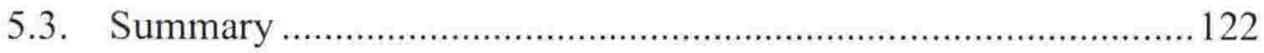

Pre-industrial simulation: New Zealand ................................. 125

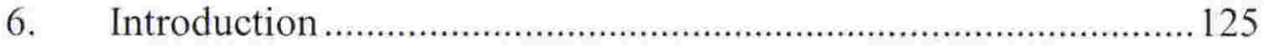

6.1. New Zealand Climate Database, CLIDB …................................. 126

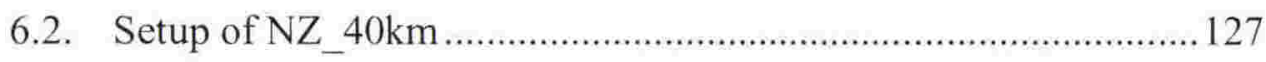

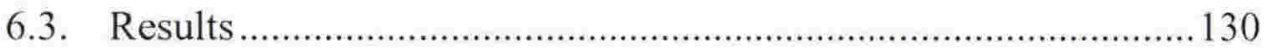

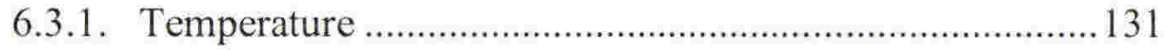

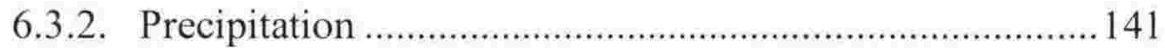

6.3.3. Mean Sea Level Pressure (MSLP) \& Winds..................... 151

6.3.3.1. Correlation MSLP-Temperature ................... 153 
6.3.3.2. Correlation MSLP-Precipitation .163

6.4. Summary 174

LGM simulation: Global........................................................178

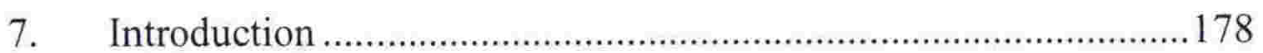

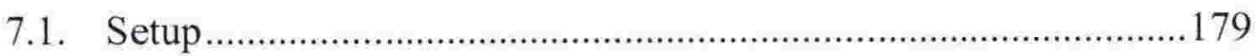

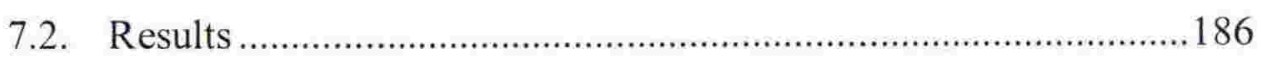

7.2.1. Sea Surface Temperature and Sea Ice .............................186

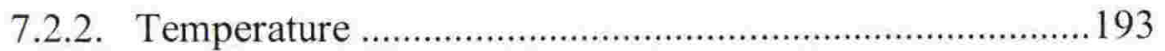

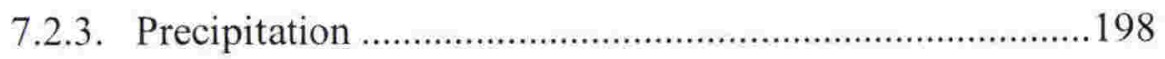

7.2.4. Mean Sea Level Pressure ............................................200

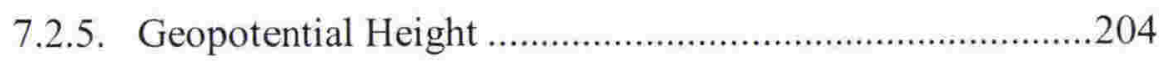

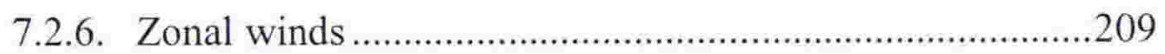

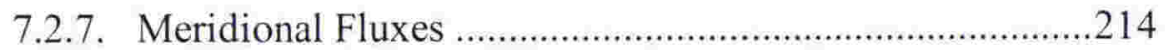

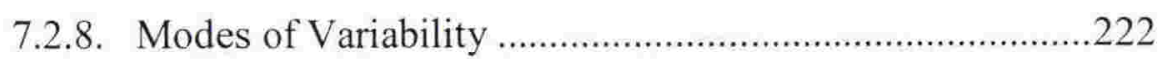

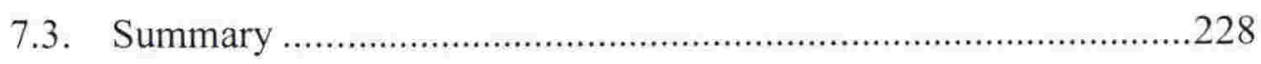

LGM simulation: New Zealand......................................... 234

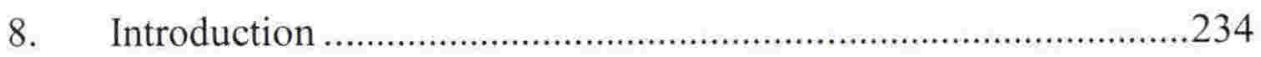

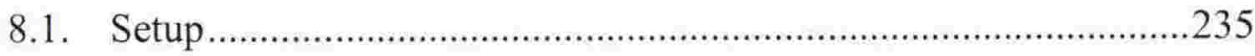

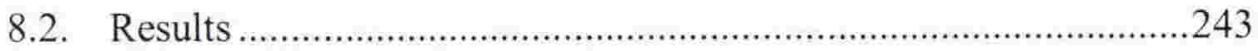

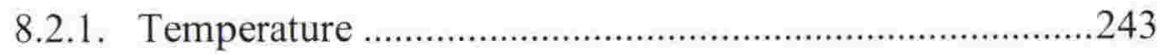

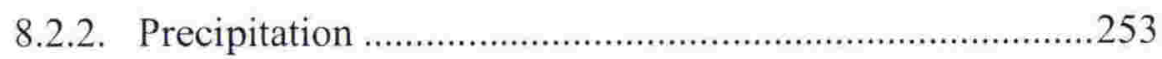

8.2.3. Mean Sea Level Pressure (MSLP) \& Winds.....................261

8.2.3.1. Correlation MSLP-Temperature ….................267

8.2.3.2. Correlation MSLP-Precipitation .....................273

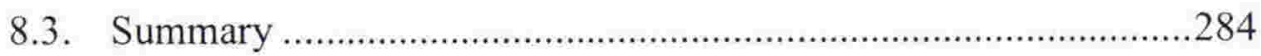

Summary and Conclusions ........................................................ 292

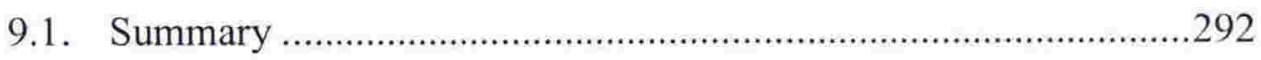

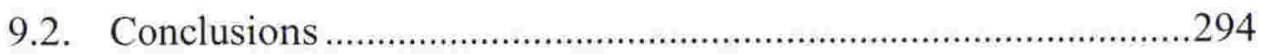

9.2.1. Global climate during the LGM ..................................2294

9.2.2. New Zealand's climate during the LGM.........................299

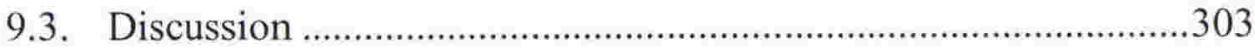


Chapter 1: Introduction

Abbreviations and Acronyms ............................................ 308

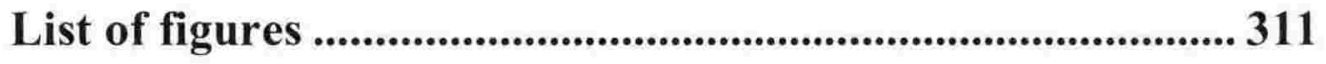

List of tables ................................................................. 322

References........................................................................ 324 


\section{Chapter 1}

\section{Introduction}

The latter part of the $20^{\text {th }}$ century saw our perception of Earth's climate change tremendously. If we previously thought that our climate was constant and would hardly ever change, we became aware that our climate had experienced, and most likely will experience, some enormous fluctuations. We had noticed of course how each year our climate slightly varied, which we usually referred to as for example, "We have a bad summer this year", or, "In my younger days, we had real winters". But only recently are we able to link up those experiences with climatic phenomena like ENSO and IPO. But those experiences did not prepare us for what we discovered in the last quarter of the $20^{\text {th }}$ century.

That our climate can experience much larger fluctuations was established by two independent disciplines. One was geology, from which we already knew that the climate was different in the past. The most well-known era would be the age of the dinosaurs, where the annual mean temperature was several degrees warmer than during the present day. But that was more than 65 million years ago. That hardly implied that there were extreme fluctuations in our climate, but merely an indication of a climate extreme. But analysis of many geological samples (proxy data) showed that our climate had undergone several stages of cooling and warming. One of the best known samples is the Vostok ice core, from which we were able to reconstruct the annual mean temperature up to 400.000 years in the past. And this reconstruction showed that there were several large temperature fluctuations in the past. Ice cores are only one kind of proxy data that can be used to reconstruct past climates. There is a multitude of geological proxy data that 
can help in getting a better picture of both the state of past climates and evolution from one state to another. Today, geological studies into our paleoclimates are significant and important areas of scientific research in their own right.

While geological proxy data provide direct proof that the climate of the Earth was not constant, the development of atmospheric models enabled us to study the physics and the chemistry factors that influenced the climate. The very first atmospheric models did not consider such a huge task as modelling the global climate state but were only capable of some very limited reconstructions. But with the progress of the science itself, and the development of the (super)computer, we were able to better simulate the state of the atmosphere. Those climate models showed us that if we changed some parameters in our equations, we could get a different climate state all together. And we were able to "tweak" our parameters appropriately by using values that the geologists had determined from their proxy data. Suddenly we did not only have proof of the possibility of the occurrence of different climates, we were also able to reconstruct those climates mathematically, and therefore understand them physically. Combining climate modelling studies with geology findings has enabled us to extrapolate the state of our current climate into the future. This has let to, among other results, the subject of global warming, which is a very important ecological, economical and political topic in present times.

But no matter how much we have learned from those studies, there is still no comprehensive climate model that can correctly reconstruct past climates, simulate the present day climate, or make completely accurate predictions of the state of the climate in the future. Verifying climate forecasts can only happen after the forecasting period has past, and the length of such a period is at least several decades. Climate forecasters will be challenged when stating that their prediction is most correct. The argument is that if that same model fails to correctly simulate the present day climate or a climate from the past, it is doubtful it can make fully accurate predictions for the future. A lot of current effort is spent on improving these climate models. The ultimate verifiable climate model would be one that is capable of simulating the present day climate. Parallel to such a task, our capabilities in modelling climates from the past will 
improve, which will help to create better climate models. Some climates from the past are in general easier to reconstruct. Rather than try to reconstruct a transitional climate, often a climate extreme is simulated. A very common climate to simulate is an ice age climate, and in particular, the Last Glacial Maximum, or LGM. This period was only about 20.000 years ago. Since this is so recent, there is a lot of geological data available to us that can help us to reconstruct the climate of that era and thereby verify, or find errors in the outcome of the models.

Geological proxy data can only give us an indication of what the climate was like. They can not give us an absolute picture. Difficulties in the dating of the geological samples (due to the large range of timescales involved, inaccuracies, "contaminated" samples, and so on), regional variations in geography, the time and spatial range of the possible climate indicator, and so on, results in that proxy data can only give us a band of possible climates. If the available proxy data shows that the Earth in the annual mean was colder during the LGM, then that does not exclude the possibility that some regions on Earth were warmer than today. One should always distinguish between global and regional aspects of the climate.

The aim of this study is to reconstruct the climate of New Zealand during the Last Glacial Maximum. Both a global and a regional climate model will be set up under LGM conditions. The output of the LGM simulations will be compared mainly against the output of a pre-industrial control simulation. The climate of the pre-industrial era is the most recent equilibrium climate, since the last hundred or more years have seen increasing greenhouse gas forcing and a nonequilibrium global climate. However, it is assumed that the pre-industrial climate was not that much different than the present day climate and that therefore comparing the result of the LGM simulation with the pre-industrial climate is similar to comparing it to the present day climate. In order to understand the local climate of a region as small as New Zealand, first one needs to understand the general circulation in which it is embedded. Most of the climate aspects of New Zealand will be governed by the state of the general circulation. For that 
reason the global climate during the LGM, and then in particular the climate of the Southern Hemisphere, is investigated. Subsequently, the regional model will investigate local climate aspects in New Zealand. In short, the two most important questions are:

1) What was the general state of the LGM climate, and in particular the general circulation in the Southern Hemisphere? What were the main anomalies on the annual mean circulation in the Southern Hemisphere compared to a recent "control" climate? And how did the different general circulation affect the climate in the South-west Pacific (New Zealand)?

2) How was New Zealand's climate affected by the different general circulation during the LGM, and how was New Zealand's climate during the LGM different than to that of the present day (or during pre-industrial times)?

Although this study is mainly about modelling the climate of the LGM, the structure of this thesis is set up similar to this introduction. One can not separate geology and climate modelling in the field of paleoclimate studies. An overview is given of the LGM climate and climate modelling, and both topics have an introduction that places them in a historical context: The history of Earth's climate and the development of atmospheric science. Both topics come together in the core of the thesis. The structure of this thesis is therefore as follows:

- Chapter 1: An introduction and an overview of the thesis.

- Chapter 2: An overview of Earth's climatic history.

It starts at the very beginning with Earth's first climate, and describes its general evolution towards today's climate. This chapter indicates that our climate is variable, and has had quite a few different configurations in time.

- Chapter 3: The climate of the LGM.

It describes the possible cause of the ice age (Milankovitch cycles, thermohaline circulation) and details some aspects of the climate of the LGM as determined from geological proxy data and from climate simulations. It 
describes global climatic features, features of the climate of the Northern Hemisphere $(\mathrm{NH})$, and particularly the climate of the Southern Hemisphere (SH) and New Zealand during the LGM as reconstructed by the aforementioned studies. The topic of climate modelling is discussed in further detail in the next chapter.

- Chapter 4: Climate modelling.

This chapter has a similar structure as chapter 2 and 3 . It begins with a historic overview (like chapter 2), and then goes into detail (like chapter 3) into one aspect of atmospheric science, climate modelling. Just as one can not understand one climate, like an ice age, without understanding the larger time frame of the evolution of climates, it is important to understand how the climate models used in this study fit in. What are their main achievements and what are their limitations? This chapter discusses various climate models, and indicates which one is most suitable for this study. It discusses the General Circulation Models (the GCMs: HadCM3, HadAM3, HadAM3H) and Regional Climate Model (RCM: NZ_40km) ${ }^{1}$ used in this study in detail. Before simulating the climate of the LGM, the model's performance needs to be investigated under a control simulation. This is done in chapter 5 and 6 .

- Chapter 5: Pre-industrial simulation: Global.

This chapter describes the capability of $\mathrm{HadAM} 3 \mathrm{H}$ in simulating the preindustrial climate. Some global fields are described and compared against data from the National Centers for Environmental Prediction (NCEP). The differences between the datasets are investigated. The reasons for the differences can be grouped in roughly three categories: the data are related to two different climates, the differences are related to the initial conditions of the simulation, or the differences are related to the model used. Only those

\footnotetext{
' These models are all versions of the United Kingdom MetOffice (UKMO) Unified Model (the UM) and are the $\underline{H a d l e y}$ Center $\underline{C}$ Coupled model version $\underline{3}$ (HadCM3), $\underline{A}$ tmosphere model version $\underline{3}$ (HadAM3) and the $\underline{A}$ tmosphere $\underline{H}$ igh resolution version $\underline{3}(\mathrm{HadAM} 3 \mathrm{H})$ for the GCMs and the $\underline{N}$ ew $\underline{Z}$ ealand RCM configuration with a $\underline{40 \mathrm{~km}}$ resolution (NZ_40km).
} 
fields that will be described in the LGM simulation will be investigated.

- Chapter 6: Pre-industrial simulation: New Zealand.

Chapter 6 extends modelling into regional studies. New Zealand's climate is simulated by an RCM that is embedded within the GCM. New Zealand's climate is discussed across six regions and seasonal aspects are investigated.

- Chapter 7: LGM simulation: Global.

Chapter 7 discusses the output of the global LGM simulation. The output is mainly compared to the output of the control (pre-industrial) simulation. Accounting for the inherent biases in the model and the effect of the initial and boundary conditions on the simulations, the differences between the simulations can then be related to aspects of the LGM climate.

- Chapter 8: LGM simulation: New Zealand.

This chapter details what the climate of New Zealand was like during the LGM. Comparisons with the control simulation and with proxy data are made. Primarily it investigates temperature, precipitation and characteristics of the wind during the LGM.

- Chapter 9: Summary and conclusions. 


\section{Earth's Climate}

\section{Introduction}

In the global and time mean, the climatic state of the Earth can be seen as the balance between incoming (shortwave) radiation and outgoing (longwave) radiation. In an equilibrium climate, the incoming and outgoing energy balance each other. Any deviation from the mean state of the climate is then solely due to changes in the internal energy balance. Fig. 2-1 gives a general overview of the various energy flows within the atmosphere. These flows are far from constant and vary continually. The atmosphere and oceans continually try to balance out

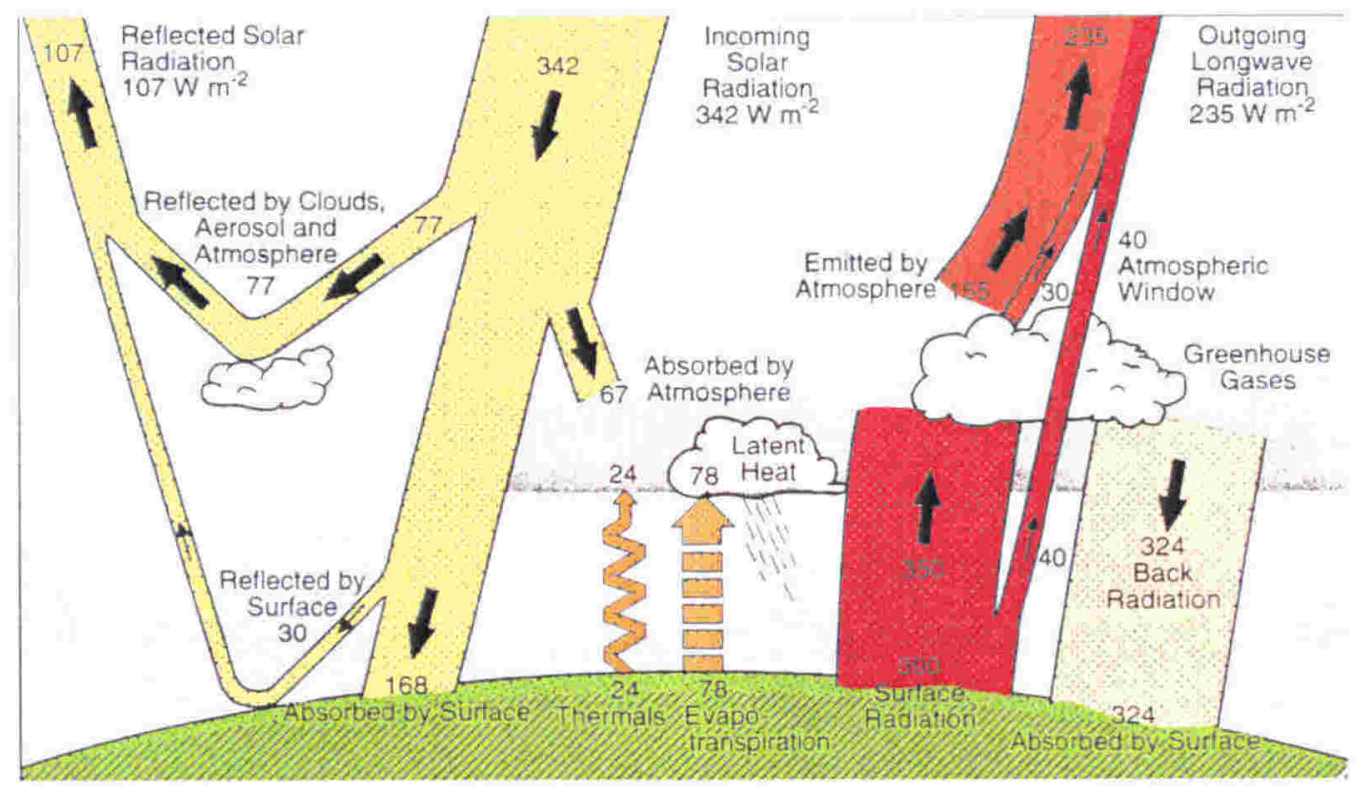

Fig. 2-1. Schematic diagram representing the Earth's annual global mean energy budget (Kiehl and Trenberth, 1997). 
any discrepancies from the mean steady state. Such efforts at time scales of a week or less is what we call weather. Averaging weather patterns over a longer period is then referred to as a climate. Although climates are an averaged state of our atmosphere, they are far from constant. If one looks solely at a short-time period (within the lifespan of a human being) there are intra and interannual variations. The main interannual variation is the one of course associated with the seasons. But when you filter the seasonal mean out of the data, there are other, less obvious interannual climatic signals. For instance, the Madden-Julian Oscillation (MJO) (e.g. Madden and Julian, 1971) is a 30 to 60 days oscillation in the zonal wind and convection in the tropical Pacific. The MJO affects the entire tropical troposphere, but is most evident in the Indian and Western Pacific oceans. After the march of the seasons, the biggest interannual climate signal is the El Niño/Southern Oscillation (ENSO) cycle.

Interannual climate changes associated with El Niño and La Niña not only have major impacts on regional climates but they affect the climate of the whole world. The occurrence of droughts and floods over a number of regions of the world can often be linked to an El Niño or a La Niña. The easterly trade winds weaken during an El Niño, reducing the upwelling of cold deep water in the Eastern Pacific This results in warming of the ocean surface, which in turn reduces the wind even more. The warmer waters shift from the west to the east and in doing so displaces the main convective systems along the equator eastward as well. This again leads to changes in the jetstreams which in turn affect the underlying climates. During an El Niña this process is reversed with a stronger than average temperature gradient along the equatorial Pacific.

The Southern Oscillation Index (SOI) is an atmospheric index used to help define whether an El Niño or a La Niña occurs. The SOI is the normalized monthly mean sea level pressure (MSLP) difference between Tahiti and Darwin. When there is a significantly negative SOI, where the pressure in the West Pacific is higher than in the East Pacific, you have an El Niño event. Plotting the SOI against time, one sees that El Niños occur every three to seven years (Fig. 2-2). The impact of El Niños is in turn modulated by the decadal variation of our climate. The impact of El Niños and La Niñas in some Pacific regions can be 


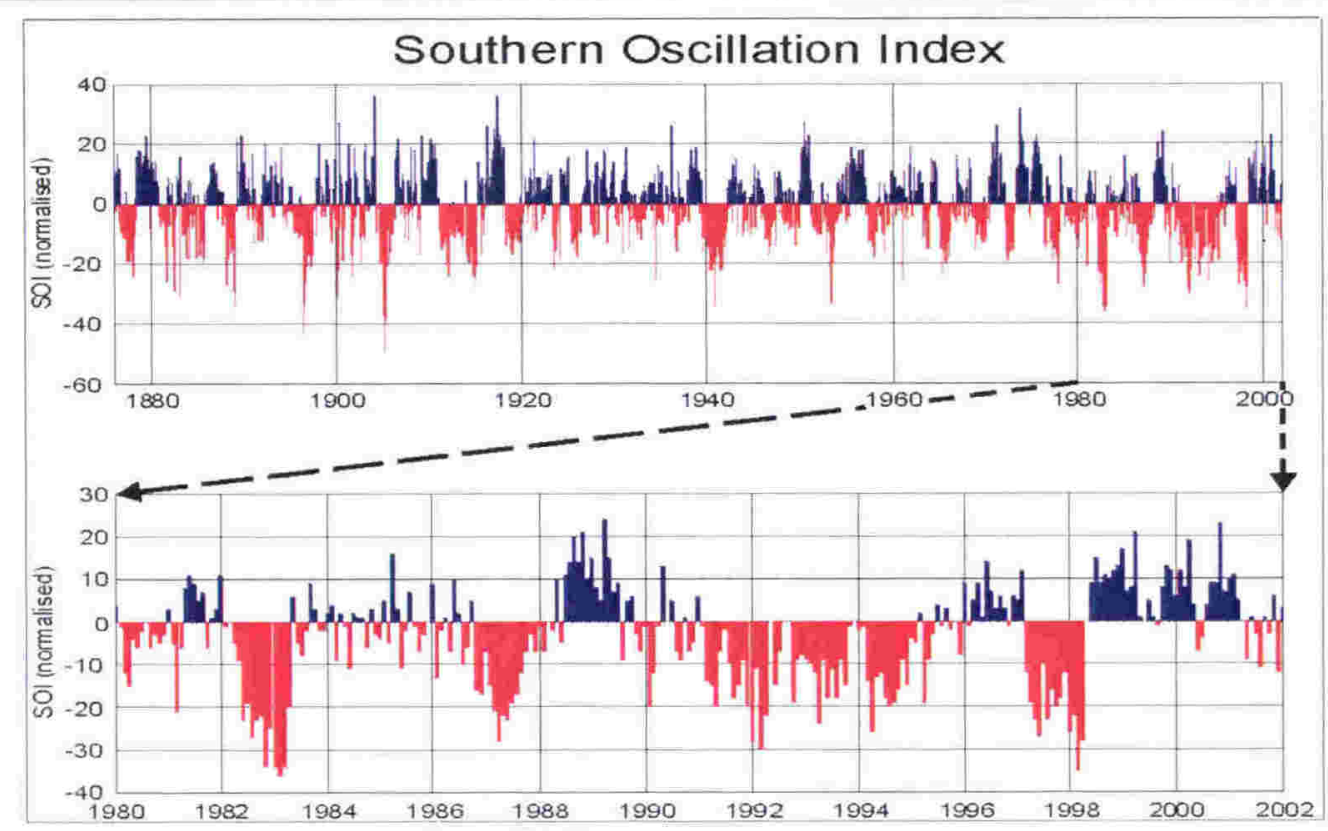

Fig. 2-2 . Southern Oscillation Index (SOI). The data is normalized over the period 19411980 and then multiplied with 10. Negative values are coloured red and are El Niño years. Positive (blue) values are La Niña years. Original monthly mean pressure data over Darwin and Tahiti were obtained from the Bureau of Meteorological Research Centre (BMRC).

either enhanced or diminished depending on whether they occur during the positive or negative phase of the Interdecadal Pacific Oscillation (IPO). For instance, Salinger et al. (2001) describe the effects of the interaction between El Niño and the different phases of the IPO in the New Zealand region. They find that ENSO-IPO teleconnections strengthen during the positive phase of the IPO.

All of these short-term climate fluctuations are relatively minor compared to fluctuations that have taken place over much longer time scales. There are several different causes for these larger events. Their origins can be found extraterrestrial (solar output, Earth's orbit), on the surface of the Earth (oceans, ice distribution, vegetation) and in the Earth's interior (tectonics drives landmass distribution and mountain building). Saltzman (1990) describes these fluctuations by comparing the response time of the different forcings. To understand these fluctuations on very long time scales, we have to understand the evolution of our past climates. The following section gives a brief overview of the history of the climate of the Earth. 


\subsection{History of the Earth's climate}

The Earth is nearly five billion years old (see Table 2-1). The first rocks probably were formed after a few hundred million years after the forming of the Earth. Not much is known about the climate of those days, because none of these

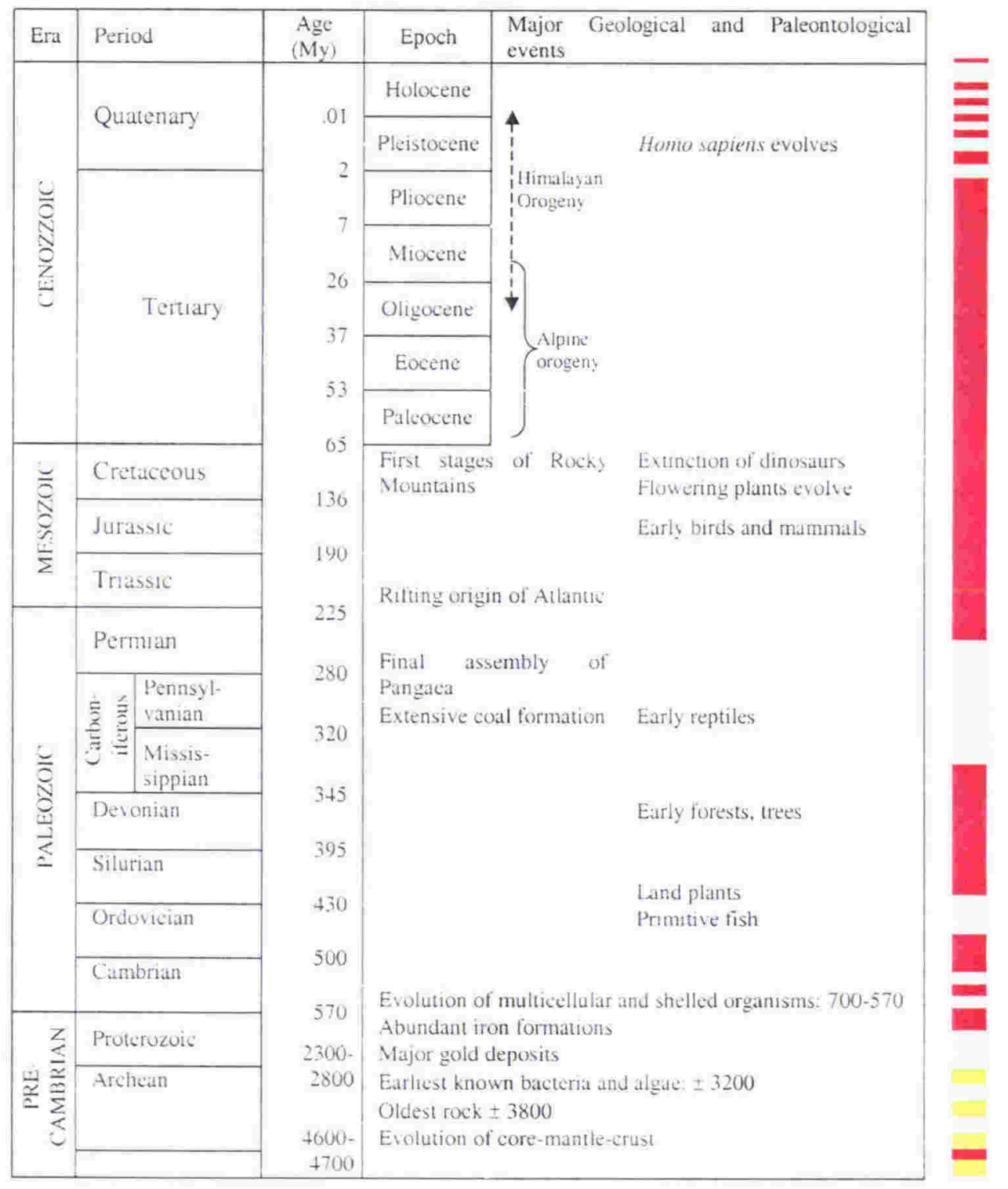

Table 2-1. The Geological Time Scale (after Press and Siever, 1982). The colour bar on the right indicates very schematically how the average global temperature has varied in time compared to today's average global temperature (Constructed using information from Frakes et al., 1994). Blue indicates a cooler climate, red a warmer climate, and yellow means that not much is known about that period. Note the irregular time scale. 
rocks were preserved. The oldest preserved rocks date back to about 3.8 billions of years ago (Press and Siever, 1982). These rocks indicate that the climate was relatively warm at that time. As the result of outgassing from volcanoes, the atmosphere was probably thicker than in the present day and would have trapped more heat. The exact composition of the earliest atmosphere is not known, but it probably did not have much oxygen. This can be deduced from the abundant iron deposits that were deposited in the following billions of years. These iron deposits are indicative of an oxygen-poor environment.

A change in the environment took place at about two billion years ago. Certain iron deposits disappeared and were replaced by red sandstone. The reddish colour would have been the result of iron reacting with oxygen, forming iron-oxide. This is the first time there is an indication of the atmosphere containing oxygen. The oxygen would have been the result of the development of oxygen forming organisms.

There are indications that ice ages might have occurred in the Precambrian, 900-600 millions of years (My) ago, but the Earth was apparently ice-free for most of that era (e.g. Frakes et al., 1994). The early Paleozoic, about 500My ago, had significantly higher levels of atmospheric $\mathrm{CO}_{2}$ than the earlier Precambrian, which would have resulted in a warmer climate. There was an abrupt change during the middle Ordovician, about 475-490My ago, when tectonic forces moved the then existing continents to form the supercontinent Pangaea. Geological records indicate that the climate varied due to these tectonic shifts. The main continent, Gondwanaland, went across the South Pole. An ice age existed from the middle Ordovician to the early Silurian (about 430My ago). There were massive coal formations during the Carboniferous (300-350My ago) indicating a warm climate. And from the Permian we find glacial deposits in widely separated areas, indicating another ice age. It's not known how much this "wandering" of the continents influenced the climate, but there is a remarkable correspondence between the occurrences of glaciation during the Paleozoic and the position of the paleo south pole (Fig. 2-3). Crowley et al. (1987) found that the position of the pole is crucial in finding whether the continent was ice-free or 


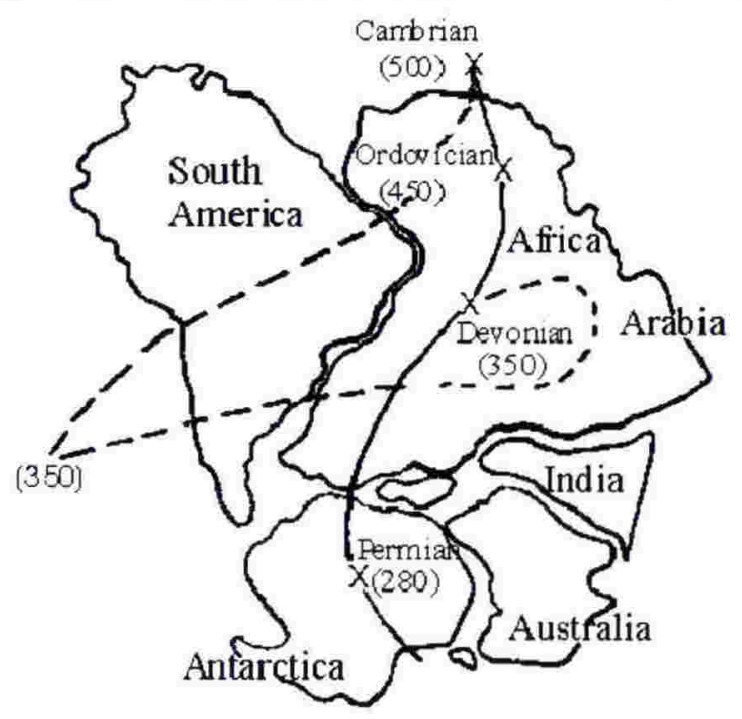

Fig. 2-3. Reconstruction of the "wandering of the South Pole" during the Paleozoic. The position of the pole in time (in My ago) is indicated by the crosses. Two possible "wander" paths, solid and dashed line, are presented (Crowley et al., 1987).

not. Crowley et al. (1993) hypothesize that pole-edged supercontinents could have permanent snow cover, where as pole-centred supercontinents could be snow-free in summer.

Another dramatic effect influencing the climate during the Paleozoic was the development of first plants, and later on, trees. The occurrence of vegetation would have reduced the surface albedo and would have affected the hydrological cycle. Probably even more important, it would have affected the chemistry of the atmosphere in that the plants converted $\mathrm{CO}_{2}$ into $\mathrm{O}_{2}$, and thereby reduced an important greenhouse gas, probably inciting a cooler climate as well.

Little is known about the climate during the period of Pangaea's existence (Permian - Jurassic, 150-250My ago). A likely climatic feature of the supercontinent is that it was probably dry and cold with seasonal extremes, common to a continental climate. Pangaea was formed during the Paleozoic, but it broke up again during the Mesozoic. Opening of the oceans, rifting and mountain-forming had their effects on the climate (Barron and Washington, 1984). Climates of the Mesozoic, and in particular during the Cretaceous, were generally warm and stable. Tropical plants grew across the Arctic circle. The late 
Mesozoic and the early Cenozoic (about 100My ago) saw widely varying warm climates with extremes of global mean temperatures $10^{\circ} \mathrm{C}$ warmer than today's. These extremes can not be solely explained by the different geographical distribution of the continents. The tectonic changes did influence the $\mathrm{CO}_{2}$ cycle. Volcanism increased as a result of an increase of subduction of carbon-rich oceanic crust. This process, together with the increase of rifting, increased the atmospheric amount of $\mathrm{CO}_{2}$ several fold. Gerard and Dols (1990) found that the increase of $\mathrm{CO}_{2}$ in the atmosphere was the main factor explaining the magnitude of the warming during the Cretaceous.

The climates of the Tertiary can be described as a general trend going from warm (the Mesozoic) to cold climates (the Quatenary). Superimposed on this trend are significant fluctuations (Crowley and North, 1991). With the northward movement of the continents of Australia and South America, detaching themselves from Antarctica, the Southern Ocean circulation became circumpolar. The effect of this was that there was no longer meridional transport from the equator to the south pole by the ocean. This resulted in the temperature of the surface and deep waters to cool off more than $10^{\circ} \mathrm{C}$ and the isolation caused by the circumpolar current helped to keep it cold. Ice sheets were formed over East Antarctica.

Continental drift closed the Panama Isthmus about 3.0My ago. This resulted in a different circulation in the North Atlantic. This particular tectonic event might very well have been the initiation of the last glaciation, which is apparent presently. There is evidence of glaciation in the North Atlantic around 2.5My ago (Shackleton et al., 1984).

Following the Tertiary is the Quatenary, which is the current period. The Quatenary is in general classified as an ice age although warm periods did occur. More is known about the climate of the Quatenary than any other climate from the past, and the next section will deal with the climate of the Quatenary in more detail. 


\subsection{The Quatenary}

The Quatenary is a very well-studied period. Mainly because it is so recent, we can still find a lot of evidence of what the climate was like during this period. A characteristic of the climate of the Quatenary is that it underwent some remarkable fluctuations (Fig. 2-4). There were various periods where both seaice and land ice expanded considerably. These glaciations (stadials) were accompanied by changes in sea level and by changes both in the dynamics and the chemistry of ocean and atmosphere. These glaciations alternated with warmer periods (interstadials), where these changes in oceans and atmosphere were reversed. The general theory that is most accepted in explaining these climatic variations comes from Milankovitch (see for instance Berger, 1978: Berger and Loutre, 1994: Imbrie, 1994). Milankovitch noticed that there was a relationship between the Earth's position with respect to the sun, and the climate fluctuations.

Milankovitch found that the Earth "wobbles" in its orbit. The angle of the Earth's tilt varies between $22.2^{\circ}$ and $24.5^{\circ}$ and is currently $23.5^{\circ}$ (Fig. 2-5A). The tilt of the Earth (obliquity) causes the seasons, and the seasonal contrasts vary as this tilt changes. A larger tilt means that the summer hemisphere will receive more solar radiation while the winter hemisphere will receive less, with the opposite occurring for a smaller tilt. Secondly, the Earth's orbit is not circular and the distance Sun-Earth varies through the year. Currently the Earth is nearest to the sun in January (perihelion), and furthest away in July (aphelion). Earth's orbit around the sun is described by its eccentricity (Fig. 2-5B), which is a relationship between the major and minor axes of its orbit. Changes in eccentricity are very small and affect incoming solar radiation only slightly (less that $0.2 \%$ annually). However, eccentricity modulates the amplitude of the precession cycle. The precession of the Earth is the change in position of the solstices and equinoxes. Fig. 2-5C illustrates two different situations. The Southern Hemisphere tilts towards the sun at aphelion in the diagram on the left, where as in the diagram on the right it is the Northern Hemisphere that tilts towards the sun. All these variations in the Earth's orbit have their effect on the climate of the Earth. 


\section{Chapter 2: Earth's Climate}
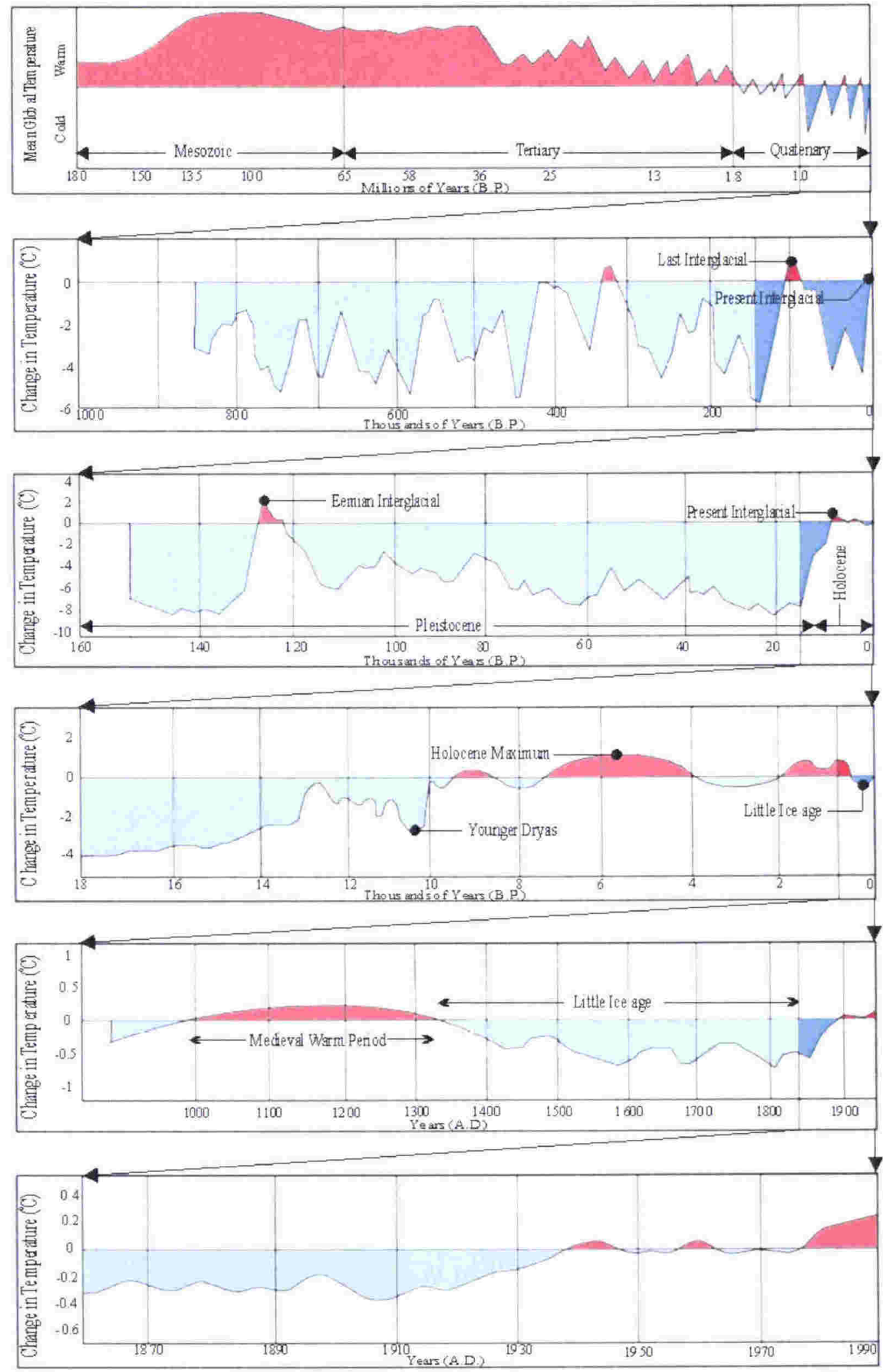

Fig. 2-4. The changes in mean global temperature during the Quatenary. Notice the increasing temporal resolution and changes in the temperature scale (reconstructed from Eddy and Bradley, 1991). 


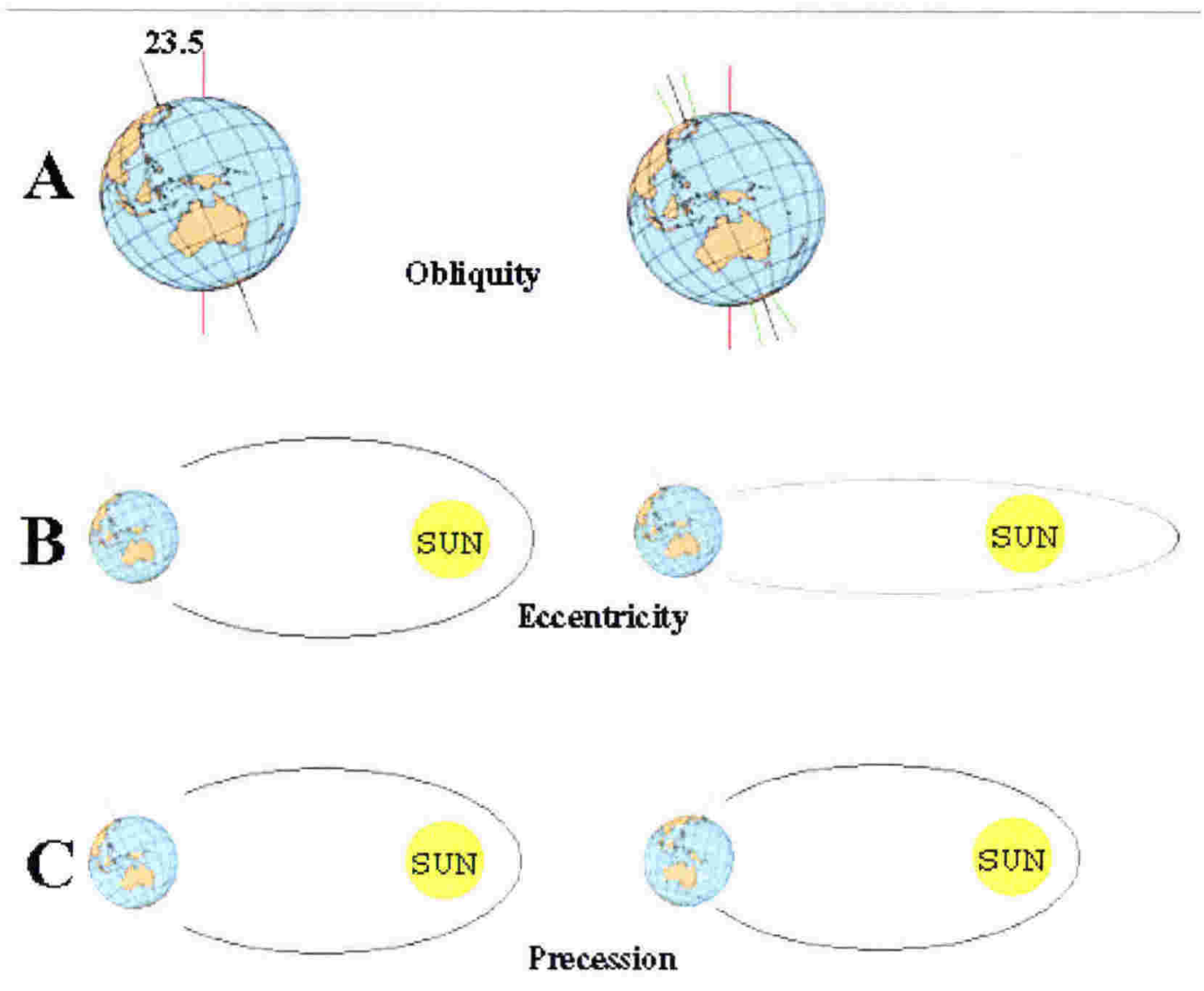

Fig. 2-5. Schematic diagram illustrating the three main orbital changes: A. Obliquity. The current tilt of the axis of the Earth is $23.5^{\circ}$. The green lines represent the minimum and maximum tilt. $\mathbf{B}$. Eccentricity. The eccentricity is highly exaggerated. C. Precession. The solstices and equinoxes move around Earth's orbit.

It is easy to determine that there are several superimposed cycles of climate fluctuations, 19 and 23,41 and $100 \mathrm{Ky}^{1}$, with another less well determined cycle of about 400Ky (Berger, 1978: Barnola et al., 1987: Genthon et al., 1987: Crowley and North, 1991: Imbrie, 1994: Bradley, 1999). Fig. 2-6 shows each individual cycle with its frequency spectrum. Variations in the precession of the Earth cause the 19 and $23 \mathrm{Ky}$ cycles, variations in the obliquity the $41 \mathrm{Ky}$ cycle, and variations in the eccentricity the $100 \mathrm{Ky}$ and the $400 \mathrm{Ky}$ cycles.

The eccentricity is actually the only variable of those three that affects the annual amount of solar radiation reaching the Earth. It has been a major task to

\footnotetext{
${ }^{1} \mathrm{Ky}=1000$ years.
} 


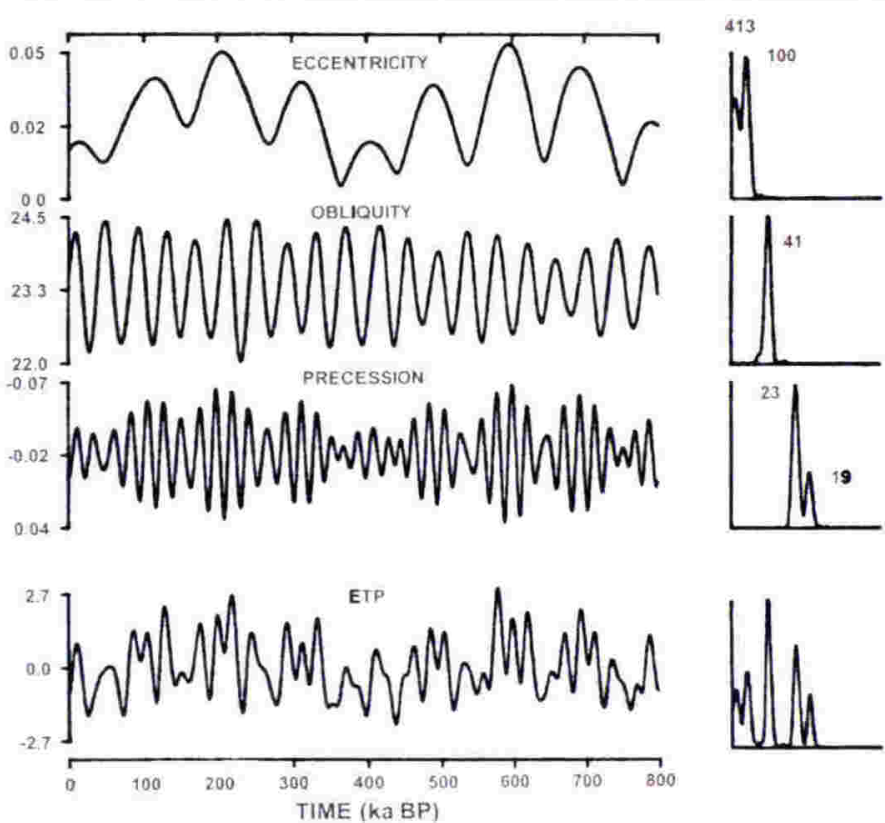

Fig. 2-6. Graphical representation of the variations in eccentricity, obliquity, precession and the combination of these three factors (ETP) over the last $800 \mathrm{Ky}$ with their corresponding frequency spectrum. Note the different vertical scales (Crowley and North, 1991).

actually link the small change in solar radiation to the cyclic occurrence of ice ages. Solar output could have varied by a larger degree, masking any variations due to the eccentricity. The question still remains how the weak signal of eccentricity is amplified and leads to the major climatic shifts that have taken place. The most obvious candidates for amplifying the eccentricity signal are icealbedo feedbacks and variations in atmospheric carbon dioxide. Lin et al. (1992) used a one-level nonlinear seasonal energy balance climate model to show how variations in Earth's orbit can cause ice ages, but that abrupt climate changes can also take place as the result of changes in $\mathrm{CO}_{2}$. Another explanation is given by Paillard (1998) who proposes multiple equilibriums for the climate, and that the various different climates are merely climates at a different equilibrium.

Obliquity and precession variations affect geographical and seasonal insolation. They cause no net annual decrease or increase of incoming solar radiation, but rather lead to amplitude variations in the geographical distribution of solar radiation. Their effects are modulated by the interaction with some of the physical properties of the Earth, like the albedo (particular land-sea distribution, 
ice sheets, clouds and vegetation) and chemistry of the atmosphere (e.g. carbon dioxide and trace gases).

Imbrie et al. (1984) found a very high correlation between the oxygen isotope record and orbital forcing. Up to $85 \%$ of the isotopic variance could be explained by the Milankovitch cycles. Berger et al. (1989) found that the Milankovitch cycles were traceable back to about 500My ago, albeit that their periods have changed in time. The orbital forcing is the most significant factor affecting our climate for long periods and changes in the orbital geometry are the fundamental cause of the succession of Quaternary ice ages (Hays et al., 1976).

Other factors affect climate variability on shorter periods. As mentioned earlier, those factors have a considerable, sometimes even a dominant, influence on the climate of the Quatenary. Variations in ice extent, both sea-ice as well as land ice, changes in ocean circulation, vegetation, the chemistry of the atmosphere, all modulated the climate of the Quatenary. These variations can amplify the effects of orbital forcing (e.g. Genthon et al., 1987), but can individually lead to considerable fluctuations in the climate as well. Many of these factors are linked to each other. A change in one factor is most likely followed by a change in the others. It is therefore not always clear which factor initiated a climate change.

During the Quatenary, onset of periods of glaciation can often be related to changes in oceanic circulation. This is in particular the case for changes in the thermohaline circulation (THC) of the North Atlantic Ocean. The principle of the North Atlantic conveyor belt is well known. Driven by the meridional overturning gyre, warm, salty surface water from the subtropics is transported to the higher latitudes in the North Atlantic via the Gulfstream. At high latitudes, this water cools, it becomes denser than its surroundings, and sinks to the bottom of the ocean. This North Atlantic Deep Water (NADW) then flows along the ocean floor to lower latitudes and to the South Atlantic as a deep western boundary current. Closure of the gyre takes place through upwelling processes (e.g. differences in diffusivity, forcing by the winds). The oceanic circulation is a major transporter of energy (heat) from low latitudes to the higher latitudes in the Northern Hemisphere. If this circulation is interrupted, it will have major 
consequences for meridional heat transport in the North Atlantic in particular, but also for the global heat distribution in general.

It is now clear that this circulation is not constant and in fact might even have stopped at times (the "off" mode). During certain times there were massive influxes of fresh water in the North Atlantic ocean which was the result of ice calving (other possible causes of influx of fresh water are melting of the ice sheets and jökulhlaups). This resulted in the North Atlantic water at high latitudes to become less salty, and therefore less dense. This decrease of density prevented the water from sinking to the ocean floor, and therefore the production of NADW stopped. The Atlantic circulation was now interrupted. The direct effect of this was a reduction of the Atlantic circulation with the warm Gulfstream not flowing deep into the high latitudes anymore. Less energy was transported to the higher latitudes, resulting in a cooler pole. Colder temperatures resulted in an increase in the polar ice sheets. Ganopolski and Rahmstorf (2001) indicated that a geographical shift in the location of the NADW formation rather than switching the NADW "on" and "off", could simulate these events more easily. Their ultimate result was the same however, in that the North Atlantic conveyor belt could exist in more than one mode. The cessation or reduction of the North Atlantic Thermohaline Circulation started a cumulative effect of cooling. The reduction in temperature increased the size and thickness of the ice sheets. This in turn caused the atmosphere to cool even more, partly because of its direct contact with the ice sheets, but also because the ice sheets increased the global albedo. A change in the THC affected the climate therefore globally (e.g. Seidov and Haupt, 1997).

Heinrich (1988) discovered some curious deposits in the eastern North Atlantic. He linked the "dropstones" deposits to ice-rafted debris. Heinrich suggested that with an increase of freshwater influx in the North Atlantic, either as the result of an increase of summer meltwater or an increase of iceberg production, polar water was able to penetrate further south. With polar water penetrating further south, icebergs, and the debris they were transporting (Fig. 2-7A), were also transported further south. Eventually the icebergs melted and 

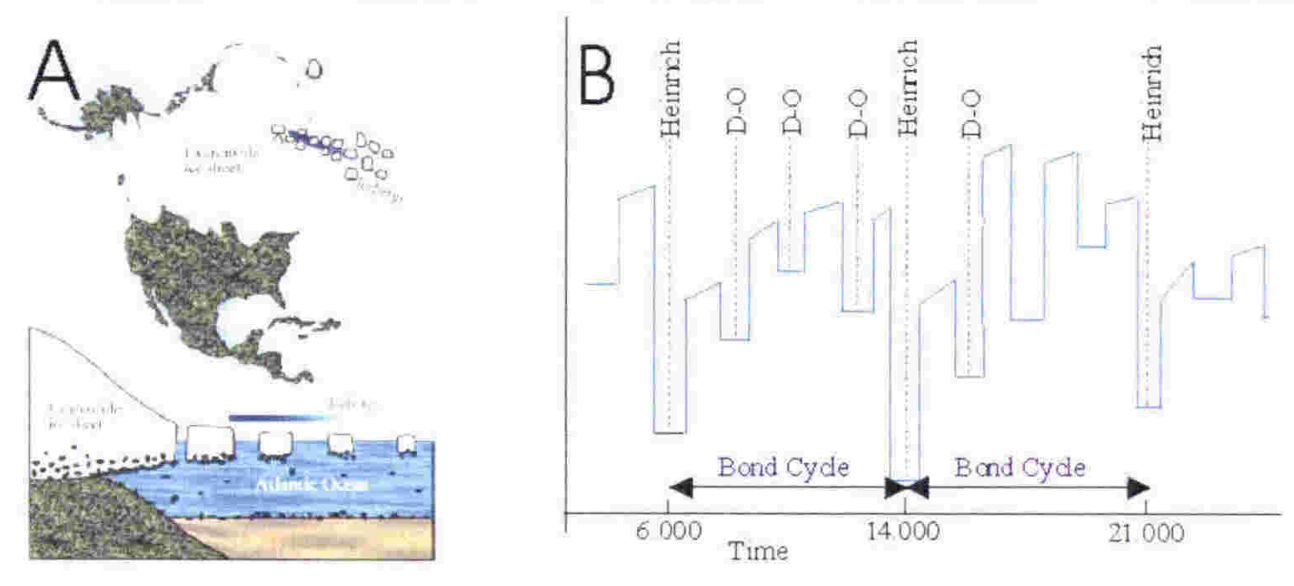

Fig. 2-7. (A) Icebergs break off of the ice sheet, carrying debris with it, which is subsequently deposited in the deep North Atlantic (Bard, 2002). (B) A very schematic time series indicating the cyclicity of Heinrich and Dansgaard-Oeschger (D-O) cycles. Vertical scale can be e.g. proxy data or temperature.

the debris they carried was deposited. These deposits can be seen clearly in sediment cores. The quantity and quality of the ice-rafted debris not only indicates what the climate was like at the time of deposition, but geological analysis of the debris can also establish the source of the sediment. During the colder periods (the so-called Heinrich events), the source of the debris was from the Laurentide ice sheet and the Hudson Bay area.

Each Heinrich event was followed abruptly by a change to warmer climates. A plausible explanation could be that due to a net increase of evaporation at the high latitudes ${ }^{1}$, the ocean became saltier, and therefore denser. The denser ocean would sink, and this would have triggered the conveyor motion again. Dokken and Jansen (1999) also suggested that the conveyor belt would start up due to an increase in salinity of the North Atlantic ocean, but they suggested that that was due to brine release during the cold phase. With the initiation of the circulation, heat was transported to the higher latitudes again, resulting in warming of the pole, and melting of the ice. This in turn would have reduced the albedo again, resulting in a global warming. This warming trend caused a chain of minor cooling events (Dansgaard-Oeschger cycles) and eventually another Heinrich

\footnotetext{
' Evaporation would become more dominant when the decrease in precipitation outweighted the decrease in evaporation.
} 
event with the next massive discharge of icebergs into the North Atlantic (e.g. Broecker, 1994: Bond and Lotti, 1995). The sequence of a chain of DansgaardOeschger cycles within the Heinrich cycles is called a Bond Cycle. These cycles are quite periodical, with Dansgaard-Oeschger cycles every 2.000-3.000 years, and the Heinrich events every 7.000-10.000 years (Fig. 2-7B). Variations in Earth's orbit (precession, obliquity and eccentricity) and Heinrich - DansgaardOeschger cycles, are the most recognizable causes for periodical variations in the climate of the Quatenary, but are surely not the only ones. As can be seen in Fig. $2-4$, there are many more fluctuations at many more different time scales. What causes these variations in the climate is still an area of major research.

Now that it is understood that the climate of the Quatenary was subject to major fluctuations, we can have a look at the last "extreme" climate of the Quatenary. As mentioned earlier, the whole Quatenary can be regarded as an ice age, but there were short times that the climate was quite a bit warmer (the interglacials). The previous interglacial, the Eemian, about 125.000 years ago, ended a very long glaciation period. There are indications that that happened very rapidly. Duplessy and Shackleton (1985) suggested that the change was due to a major influx of fresh water in the North Atlantic as the result of the melting of the continental ice sheets. They suggested that NADW formation stopped completely in the northern Atlantic Ocean (the "off" mode). The NADW started up again at the inception of the next glaciation in response to the increasing salinity of the world oceans. The Eemian lasted about 10.000 years.

The Eemian was followed by the last period of glaciation, the one that lasted till about 10.000 years ago. This period of glaciation featured similar climate fluctuations, culminating in a glacial maximum about 20.000 years ago, the Last Glacial Maximum (LGM). The Last Glacial Maximum was abruptly followed by a warming period, which culminated in today's interglacial. The change from glacial to interglacial was not a smooth event, but was also characterized by several fluctuations (see Fig. 2-4). Alley et al. (1999) hypothesized that these millennium scale fluctuations were also due to Heinrich and DansgaardOeschger oscillations. Most noticeable was a temporarily cooling event during 
the younger Dryas (about 11.000 years ago). Manabe and Stouffer (1999) suggested that it was due, again, to the melting of the continental ice sheets. The effect of this cooling was probably not strong enough to be felt worldwide (e.g. Markgraf, 1991: McGlone, 1995: Bennett et al., 2000). The glaciation of the younger Dryas appeared mostly in the Northern Hemisphere.

After this period of cooling, the temperature rose sharply again and entered the present Interglacial. A temperature maximum occurred during the midHolocene, around 6.000 BP. This maximum was mainly due to an increase in summer temperature, rather than an increase in the year round temperature (e.g. Crowley and North, 1991). The main continental ice sheets had disappeared by this stage. The increase of temperature also resulted in global changes in precipitation patterns, with an increase of the monsoonal circulation. The latter part of the Holocene was slightly cooler and drier again.

The last 1000 years of the Holocene first had a relatively warmer period during the Middle Ages (1000-1300), followed by what is called "The Little Ice Age" (1350-1850). Besides geological proxy data, historical data in the form of writings and drawings, do illustrate these particular climate periods. The mean global temperature increased dramatically during the last 150 years. Although slowly at first, the mean global temperature increased during the latter part of the twentieth century at a rate not seen for many millions of years. This increase has been attributed to an ever-increasing amount of carbon dioxide being released into the atmosphere as a result of the rapid industrialisation. Besides carbon dioxide, many new, man-made gases have been dumped in the atmosphere. Even in relatively small amounts, these "trace gases" have a considerable effect on our climate.

In the previous paragraphs, geological proxy data were mentioned several times as the basis of our knowledge of what our past climates were like. Since there is really no other way to find actual data about the state of past climates, it is important to extract the correct information from these data. The following section will give a short overview of what proxy data is available, and what climatic information can be extracted from it. 


\subsection{Proxy Data}

There are various disciplines that use a variety of methods to gather information about recent paleoclimates (Quatenary). Table 2-2 presents an overview of the principal sources of proxy data for paleoclimate reconstructions

(1) Glaciological (ice cores)

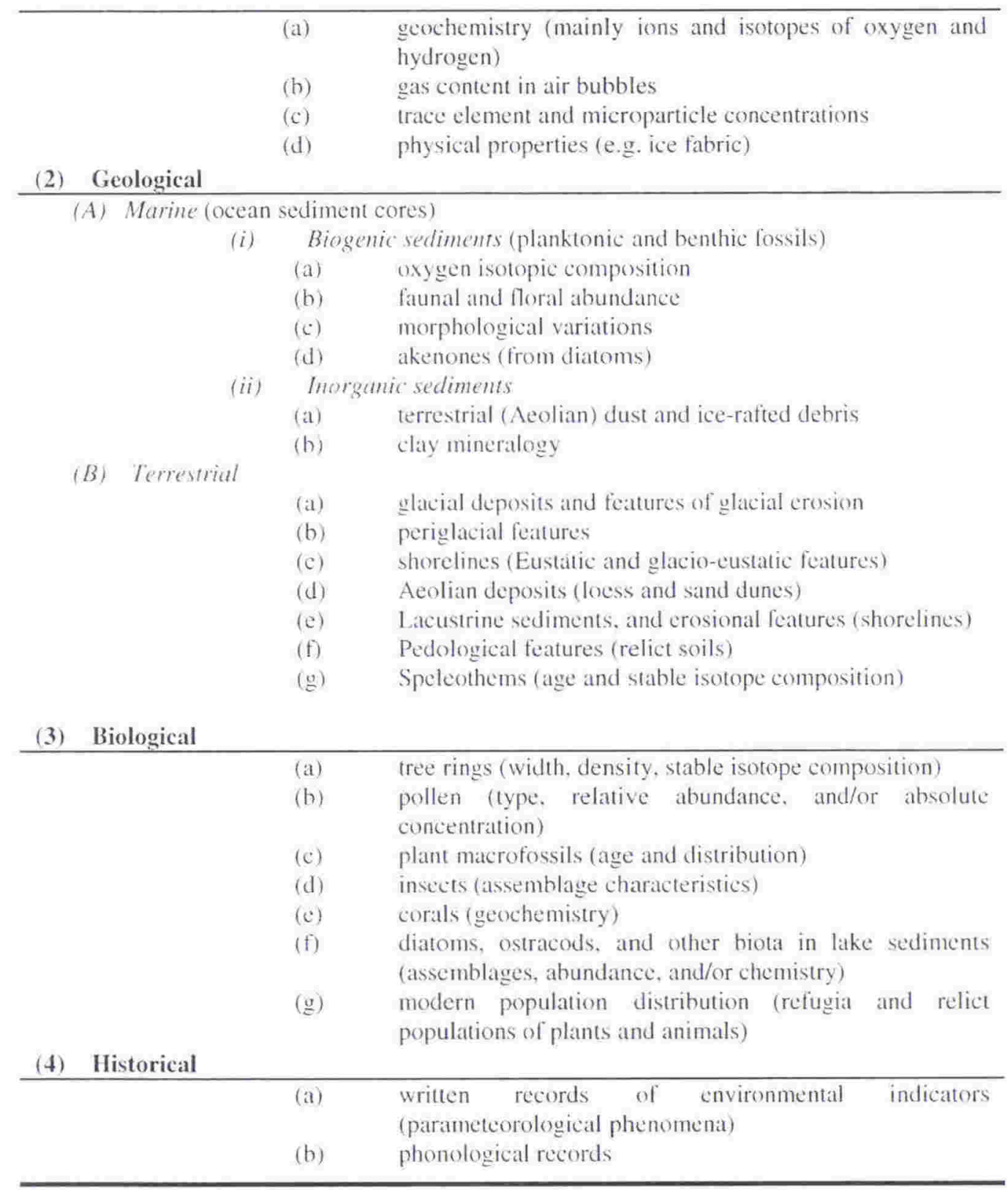

Table 2-2. Principal sources of proxy data for paleoclimate reconstructions (after Bradley, 1999). 


\begin{tabular}{|c|c|c|c|}
\hline Archive & $\begin{array}{l}\text { Minimum sampling } \\
\text { interval }\end{array}$ & $\begin{array}{l}\text { Temporal range } \\
\text { (order: yr) }\end{array}$ & $\begin{array}{l}\text { Potential } \\
\text { information derived }\end{array}$ \\
\hline Historical records & day/hr & $\sim 10^{\circ}$ & T.P.B.V.M.L.S \\
\hline Tree rings & $\mathrm{yr} /$ season & $\sim 10^{4}$ & T.P.B.V.M.S \\
\hline Lake sediments & $\mathrm{yr}$ (varves) to $20 \mathrm{yr}$ & $-10^{4}-10^{6}$ & T.B.M,P.V.C \\
\hline Corals & $\mathrm{Yr}$ & $-10^{4}$ & $\mathrm{C}_{\mathrm{w}}, \mathrm{L}, \mathrm{T}, \mathrm{P}$ \\
\hline Ice cores & Yr & $\sim 5 \times 10^{5}$ & T.P.C. B. V.M.S \\
\hline Pollen & $20 \mathrm{yr}$ & $\sim 10^{5}$ & T.P.B \\
\hline Speleothems & 100 & $-5 \times 10^{5}$ & $\mathrm{C}_{\mathrm{w}}, \mathrm{T}, \mathrm{P}$ \\
\hline Paleosols & $100 \mathrm{yr}$ & $-10^{6}$ & T.P.B \\
\hline Loess & $100 \mathrm{yr}$ & $-10^{6}$ & P.B.M \\
\hline Geomorphic features & $100 \mathrm{yr}$ & $-10^{6}$ & T,P.V.L \\
\hline Marine Sediments & $500 \mathrm{yr}$ & $\sim 10^{6}$ & T, C, B., I,L.P \\
\hline \multicolumn{4}{|c|}{$T=$ temperature } \\
\hline \multicolumn{4}{|c|}{$\mathrm{P}=$ precipitation, humidity, or water balance (P-E) } \\
\hline \multicolumn{4}{|c|}{$C=$ chemical composition of air $\left(C_{n}\right)$ or water $\left(C_{n}\right)$} \\
\hline \multicolumn{4}{|c|}{$\mathrm{B}=$ information on biomass and vegetation patterns } \\
\hline \multicolumn{4}{|c|}{$\mathrm{V}=$ voluanic eruptions } \\
\hline \multicolumn{4}{|c|}{$\mathrm{M}=$ geomagnetic field variations } \\
\hline \multicolumn{4}{|c|}{ I. = sea lesel } \\
\hline \multicolumn{4}{|l|}{$S=$ solar attivity } \\
\hline in rare rivelumsams & (1arred iediments) s & () $r$ & \\
\hline
\end{tabular}

Table 2-3. Characteristics of natural archives (after Bradley, 1999).

and Table 2-3 gives an overview what timescale they detail. Extracting climatic information from these proxy data is often a difficult task. The sample needs to be calibrated properly. Calibration is done by investigating features of a similar sample under present day climatic conditions. It is assumed that the relationships between past climatic conditions and the sample are the same as in the present day. Once these relationships are known under present day conditions, the paleoclimate record of the sample can be obtained by interpolating/extrapolating these relationships for the sample.

An example of this calibration technique is the relationship between $\delta^{18} \mathrm{O}^{1}$ and temperature. When evaporation occurs, the lighter compounds will evaporate first. The compounds that include ${ }^{16} \mathrm{O}$ are lighter than the same compounds that

$$
\begin{aligned}
& { }^{1} \delta^{18} \mathrm{O} \text { is the ratio between the isotopes }{ }^{18} \mathrm{O} \text { and }{ }^{16} \mathrm{O} \text { in a sample. } \\
& \delta^{18} \mathrm{O}=\left\{\frac{\left({ }^{18} \mathrm{O} /{ }^{16} \mathrm{O}\right)_{\text {sample }}-\left({ }^{18} \mathrm{O} /{ }^{16} \mathrm{O}\right)_{\text {standard }}}{\left({ }^{18} \mathrm{O} /{ }^{16} \mathrm{O}\right)_{\text {standard }}}\right\} \times 1000
\end{aligned}
$$


include ${ }^{18} \mathrm{O}$. This means that ${ }^{16} \mathrm{O}$ will evaporate more easily, leaving a relatively higher concentration of ${ }^{18} \mathrm{O}$ in the oceans. When atmospheric water precipitates, some of it will end up in the continental ice sheets. The water compounds of these ice sheets are then relatively depleted of ${ }^{18} \mathrm{O}$ compared to the ocean. The variation between the $\delta^{18} \mathrm{O}$ of the ice sheets and the $\delta^{18} \mathrm{O}$ of the oceans provides information about the temperature of the climate in the time the ice sheets were formed. Therefore $\delta^{18} \mathrm{O}$ is an indicator of whether a past climate was relatively warm or cold. In general, the larger the ratio of $\delta^{18} \mathrm{O}$, the colder the climate is. However, care should be taken in the interpretation of the outcomes. The $\delta^{18} \mathrm{O}$ method does not apply universally, and the link between surface temperature and $\delta^{18} \mathrm{O}$ seems to vary in time and space. Several modelling studies have indicated that regional and seasonal aspects can have a profound effect on the surface temperature- $\delta^{18} \mathrm{O}$ relationship (e.g. Jouzel et al., 1997: Delaygue et al., 2000: Noone and Simmonds, 2002), making it difficult to determine uniquely the exact relationship between those parameters. In fact, Noone and Simmonds (2002) found that daily temperature correlations for Antarctica were opposite to those of the annual mean and Brown and Simmonds (2004) found that the correlations were reversed when moisture transport took place from cooler to warmer latitudes in their experiment with idealized continents located around the equator.

To increase the time resolution of the climatic record, it is important to understand the relationships between climatic conditions and proxy data from more than one discipline. As Table 2-3 shows, the various records cover different time periods. The period that is covered by most, if not all records, will be a more accurate determination of the age of a climatic signal than if the age was determined by a single kind of proxy data. Combining the information from all these proxy data will give a better and more complete database. For reliable paleoclimate reconstructions it is important to have an as complete and accurate database as possible. The most complete and longest recording of the climate in the last several 100.000 years, comes from the Vostok ice core ${ }^{1}$ (e.g. Barnola et

\footnotetext{
I A recently analysed sample, the EPICA Dome C core, records the climate to about 740.000 years ago (Augustin et al., 2004).
} 


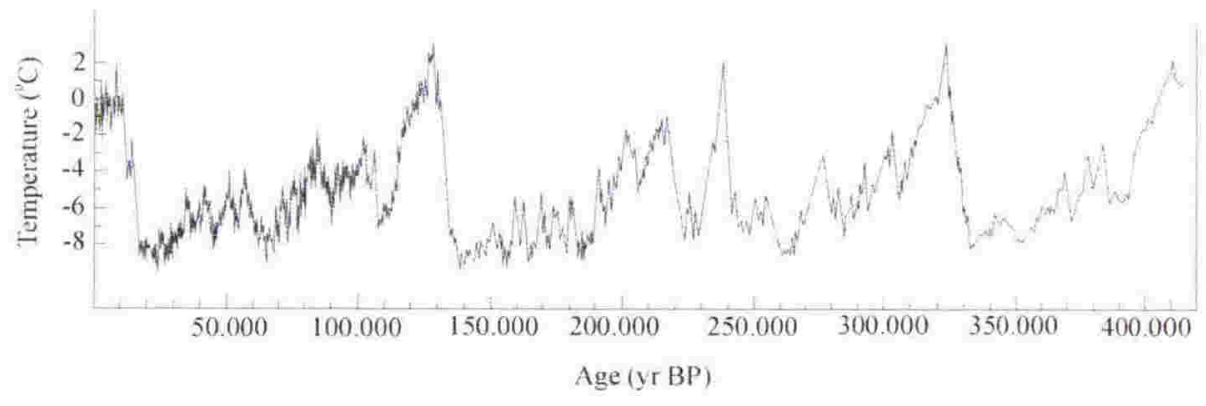

Fig. 2-8. Temperature fluctuations in the last 400.000 years relative to today's global mean temperature (Petit et al., 1999. This figure comes from their Figure 3. For illustrative reasons, only temperature has been plotted here).

al., 1987: Genthon et al., 1987: Petit et al., 1999). The ice core gave information about a variety of climatic indicators.

Besides $\delta^{18} \mathrm{O}$, other climatic indicators that could be studied include e.g. the quality and quantity of sedimented dust, the amount of sodium, the deuterium profile, and trace gases (e.g. $\mathrm{CO}_{2}$ and $\mathrm{CH}_{4}$ ). All of these indicators have their own specific link to climatic variables like temperature, wind, and humidity. Combining the information of all these different climate indicators gives us an astonishing insight into the climate of the Quatenary. As an example, Fig. 2-8 gives the general temperature trend as determined by the proxy data from the Vostok ice core.

It must be clear that detailed geological proxy data are only available for more recent geological times. The further back in time, the harder it is to find appropriate proxy data, and the more difficult they are to date. The time resolution is coarser for older samples. One period that is very well sampled, and of which we can make a reasonable reconstruction, is the LGM. Past climates can be referred to as "warm" or "cold", or "windier", "drier", "dustier" or as more or less humid then the present day climate. The next chapter will look the most recent "cold" climate in detail; the Last Glacial Maximum. 


\section{The Last Glacial Maximum}

\section{Introduction}

For successful climate model validation and interpretation of output, it is necessary to be able to reconstruct past climates. One of the main eras often modelled is the Last Glacial Maximum (LGM). The Last Glacial Maximum occurred about 20.000 years ago (see Fig. 2-4). Although the purpose of this study is to reconstruct New Zealand's climate during the Last Glacial Maximum, it is essential that we understand the global climate of the LGM first. After all, regional climates are influenced by the global circulation. This chapter will discuss the characteristics of the Last Glacial Maximum and its climate. Reconstructions of the climate during the LGM are done by both direct interpretation of geological data, and by modelling, using the geological record as proxy data, driving and forcing the climate models. Some aspects of the climate during the LGM will be speculated on, in order to lay the framework for the analysis of the data that will be discussed in chapters $5,6,7$ and 8 . The theory and the use of climate models are discussed in chapter 4 .

\subsection{A Global description}

Many studies have been dedicated to reconstructing the climate during the Last Glacial Maximum. An abundance of geological data is found around the world. Studies of marine organisms (like forams and diatoms), glaciers and ice sheets, rainfall, sedimentation records, and many other biological and physical 
studies provide us with a view on what the state of the climate was at the LGM (see section 2.3). The first international project that set out to combine the various data was the CLIMAP project (Climate: Long-range Investigation Mapping and Prediction: CLIMAP, 1976: 1981). Some of CLIMAP's major findings for the LGM were:

\section{1) Albedo:}

An increase in albedo occurred as the result of the extensive areas covered in ice, a vegetation change from forests to grassland, steppes and deserts, and the lowering of the sea level.

\section{2) Oceans:}

The average cooling over the whole ocean surface was about $2.3^{\circ} \mathrm{C}$ and the global average surface temperature decreased by about $5.3^{\circ} \mathrm{C}$. The main reasons for the cooling of the oceans were the equatorward shift of the polar fronts and an increase in upwelling (and therefore cooling) in the eastern equatorial regions. This was particularly the case in the Eastern Pacific. Areas of major cooling are found at high latitudes, particularly in the Northern Hemisphere.

3) Ice sheets:

The existence of huge continental ice sheets, up to $3 \mathrm{~km}$ thick over the Northern Hemisphere continents, and a dramatic increase in the extent of sea-ice for both hemispheres. The increase of the ice sheets reduced the sea level by about 85 meters.

Many models which have described the climate during the Last Glacial Maximum have used boundary conditions as reconstructed by the CLIMAP project. However the simulated Last Glacial Maximum climate is subject to significant uncertainties related to these boundary conditions. SSTs (Sea Surface Temperatures), sea-ice distribution and concentration, and ice-sheet topography are not perfectly known. Different values for these parameters can give quite 
different results. Over the years some doubt has arisen about the accuracy of the reconstructed tropical SSTs (e.g. Webb et al., 1997: Bush and Philander, 1998) and the extent of sea-ice (e.g. Burckle and Mortlock, 1998: Crosta et al., 1998b) as described in CLIMAP. Therefore several studies have used coupled oceanatmosphere models to compute SSTs instead of using prescribed SSTs in atmosphere-only models (e.g. Manabe and Broccoli, 1985: Dong and Valdes, 1998: Ganopolski et al., 1998: Delaygue et al., 2000), while others have used, for instance, Peltier's (1994) reconstruction of the ice sheets (e.g. Dong and Valdes, 2000: Kageyama and Valdes, 2000) or have simply "modified" some of the CLIMAP boundary conditions (e.g. Toracinta et al., 2004). Crowley (2000) gives a review of CLIMAP's tropical SST findings and of others who favour a larger decrease of tropical SSTs, and suggests that the "truth" is probably somewhere in between. The Paleoclimate Modelling Intercomparison Project (PMIP, e.g. World Climate Research Programme-111, 2000) was set up in the 1990's to facilitate model to model comparisons under the same paleoclimate forcing conditions in order to better understand past climate changes. The two time periods investigated were the Holocene Maximum (6kBP, see Fig. 2-4) and the LGM. The simulations for the LGM were performed either in a coupled version with a slab model (computed SSTs), or have used SSTs from CLIMAP. There were several distinct differences between the computed SSTs and CLIMAP SSTs (e.g. Dong and Valdes, 1998). PMIP's findings are often quoted as being more correct than CLIMAP's, particularly PMIP's reconstruction of SSTs and sea-ice fields. For that reason, PMIP's findings are often used in climate simulations of the LGM. The modelling study done here has used the boundary conditions specified by PMIP.

The cause of the colder climate during the LGM was due to a combination of the factors mentioned earlier in this section, none of which were by themselves the dominant cause of the ice age. A reduction in atmospheric $\mathrm{CO}_{2}$, an increase in the extent of the polar ice sheet and continental glaciation, and a shallower, and possibly less vigorously, North Atlantic Thermohaline Circulation (NATC), are all suggested as causes (e.g. Broccoli and Manabe, 1987: Bintanja and 
Oerlemans, 1996: Weaver et al., 1998: Broccoli, 2000) for the colder climate. Other studies have shown that other variables, such as the number of airborne dust particles (e.g. Harvey, 1988: Lunt and Valdes, 2001), vegetation changes (e.g. Levis et al., 1999) and cloud cover (e.g. Hewitt and Mitchell, 1997) all had an effect in cooling the climate as well.

\section{- Albedo:}

During the LGM, the orbital parameters of the Earth were similar to their current values so that the incoming solar radiation during the LGM was not significantly different than today's. Orbital forcing therefore is unlikely to be a major cause of the colder climate. But the combination of a higher albedo, resulting in more radiation being reflected back, with a reduction of atmospheric $\mathrm{CO}_{2}$, resulting in less long-wave radiation being trapped, had a major effect (Broccoli, 2000). Their combined effects would have altered the effect of the incoming solar radiation. Changes in the height and amount of clouds, as well as in their composition would also have affected the radiation balance (e.g. Hewitt and Mitchell, 1997), but their total effect would be hard to determine (Stephens and Webster, 1981).

\section{- Oceans:}

A lot of effort has gone in to understanding the role of the oceans in explaining the climate of the LGM. Any cooling of the surface temperature in the tropics will inevitably influence the atmosphere globally. Liu et al. (2000) point out the importance of accurately determining the east/west SST gradient across the equatorial Pacific during the LGM. Any change in this gradient could imply that the climate was more like an El Niño or a La Niña climate. Yin and Battisti (2001) came to a similar conclusion. They found that the absolute temperature difference between the present and the LGM tropical SSTs is of less importance in explaining the differences between the two climates than the differences between their tropical SSTs patterns. Using a coupled ocean-atmosphere GCM, Bush and Philander (1998) found that the western tropical Pacific also had a strong cooling (CLIMAP indicates that the main tropical cooling was in the 
Eastern Pacific), that there was a strengthening of the trade winds, and an enhancement of the tilt of the thermocline. The latter point was also found by Andreasen et al. (2001) and Hewitt et al. (2002). These findings indicate that the climate during the LGM might have been more similar to a La Niña climate. Other studies however, find indications that the climate during the LGM might have been more like an El Niño climate (e.g. Koutavas et al., 2002).

Using an atmospheric GCM under LGM conditions, Webb et al. (1997) found that if the ocean during the LGM was transporting heat at present day rates, that would lead to enhanced cooling, particularly in the tropics. The cause of the latter was mainly subscribed to an increase in low clouds (increase of albedo), where as the enhanced global cooling was due to reductions in atmospheric water vapour and high clouds which decreased greenhouse forcing. Weaver et al. (1998) however found that the ocean heat transport during the LGM was different than today's. Ocean models under glacial conditions indicate that there were significant changes in circulation patterns (e.g. Lautenschlager et al., 1992: Fichefet et al., 1994: Hovine and Fichefet, 1994: Hewitt et al., 2002). Most notably, there was a change in deep water formation in the North Atlantic. The production of the North Atlantic Deep Water (NADW) became much shallower (e.g. Fichefet et al., 1994: Weaver et al., 1998), to a depth of about 2500m, and ceased to exist north of about $55^{\circ} \mathrm{N}$. Intermediate waters in the Pacific and in the Atlantic ocean were more vigorously ventilated. The Atlantic conveyor belt was weaker and the circulation closed itself in the North Atlantic. Antarctic Bottom Water $(\mathrm{AABW})$ was able to penetrate to higher northern latitudes, replacing NADW. The effects of a reduced or southwards shift of the NATC, has been discussed in chapter 2. The LGM sees indeed a cooling of the pole and an expansion of the polar ice sheets as the result of a changed NATC. There are some indications that this was more the result of a southward movement than a reduction in the NATC. Indeed, Lautenschlager et al. (1992), Hewitt et al. (2001), and Hewitt et al. (2002), using an ocean model or a coupled oceanatmosphere model, all find that the NATC might actually have increased in the northeast of the Atlantic. The latter two authors even indicate that a possible strengthening of the NATC during the LGM might have resulted in warming in 
parts of the Northern Atlantic, though this warming is at odds with paleoclimate proxy data (e.g. CLIMAP, 1976). Kitoh et al. (2001) and Bjornsson and Mysak (2001), also using coupled ocean-atmosphere models, find the southerly shift and the strengthening of the NATC during the LGM as well, but subscribe their latter finding to a shortcoming in their model, and declare it contradicts paleoclimate findings.

Another possible important change in the ocean circulation occurred as the result of the near-closure of the Indo-Pacific throughflow. Due to the lowering of the sea level, Australia was connected to Papua New Guinea and the South-East Asian Archipelago had become one landmass. The increase in landmass had a blocking effect on the exchange of water between the Indian and Pacific Ocean. The wind-driven South Pacific gyre now had two of its outlets, the Indo-Pacific throughflow and the flow through the Baring Strait, altered. If the forcing were the same, it would mean that the mass balance of the Pacific Ocean circulation was altered, resulting in either a change of direction or a change in the strength of one or more of its currents. Hirst and Godfrey (1993) for instance showed that a change in the Indo-Pacific throughflow could considerably alter the East Australian Current (EAC) ${ }^{1}$. Instead of turning east at about $32^{\circ} \mathrm{S}$, the current would go south all the way to Tasmania. Lautenschlager et al. (1992) however found a reduction in the East Australian current. However, both scenarios would result in lowering the SSTs around New Zealand, since the waters around New Zealand would have been deprived of their main heat-bearing current. Colder SSTs around New Zealand during the LGM have been documented by several authors (e.g. Barrows et al., 2000: Carter et al., 2002).

\section{- Ice sheets:}

The increase in the volume of the cryosphere is particularly prominent in the Northern Hemisphere, where the polar ice cap extended southwards and covered Northeast America (the Laurentide ice sheet) and North-western America (Cordilleran ice sheet), Greenland and parts of northern Eurasia (CLIMAP, 1976:

\footnotetext{
I The EAC is a western boundary current, and together with the local eddies, transports heat to higher latitudes.
} 
Peltier, 1994) and reached heights possibly over $2 \mathrm{~km}^{1}$. The enlarged ice sheets affected the climate of the LGM in several ways. The radiative balance was different as the result of a higher albedo. The high albedo was the result of the high reflectivity of the ice sheets and the increase of the landmass at the expense of the oceans (due to lowering of the sea level). Due to its direct contact with the atmosphere, the enlarged ice sheets lowered the high latitude surface temperature and changed the locations of maximum temperature gradients. The latter would have had affected the locations of storm tracks and thereby regional climates. The large atmospheric circulations changed due to the large increase in orography. The latter two effects resulted in major changes in the climate of the midlatitudes in the Northern Hemisphere. Manabe and Broccoli (1985) and Broccoli and Manabe (1987) speculated that the ice-sheet was the main contributor to the cooling of the Northern Hemisphere, where as the reduction in $\mathrm{CO}_{2}$ content in the atmosphere was the primary contributor to lower temperatures in the Southern Hemisphere. A similar result was found by Mitchell (1993), although Bintanja and Oerlemans (1996) suggest that the $\mathrm{CO}_{2}$ effect was more pronounced for the Northern Hemisphere.

It is thought that the effect of the change in the amount of sea-ice in the Southern Hemisphere was not as dramatic as in the Northern Hemisphere. There are major differences between the CLIMAP (1976: 1981) and Peltier (1994) reconstructions of the extent of the sea-ice around Antarctica, but unlike in the Northern Hemisphere high latitudes, there was virtually no additional land covered by ice ${ }^{2}$. Modelling studies by Huybrechts (1990) and Budd et al. (1998) give support to the hypothesis that the Antarctic ice sheet basically followed glacial periods in the Northern Hemisphere by means of sea-level teleconnections. The lowering of the sea level during the LGM resulted in an advance of the grounding line of the Antarctic sea-ice (Huybrechts, 1990: Budd

\footnotetext{
Although the extent of the ice sheets is well constrained through geological data (e.g. sedimentation records of diatoms and forams), the possible height of the ice sheets is determined by modelling the visco-elastic response of the Earth to different loading of the ice sheets. By tracking the relative sea level back in time to the LGM, the amount of loading can be determined approximately.

${ }^{2}$ Exemptions were for some midlatitude land masses with high orography that protrude far enough south to be covered by glaciers, like e.g. South America and the Southern Alps of New Zealand.
} 
et al., 1998: Krinner and Genthon, 1998). Studies by Crosta et al. (1998a: 1998b), Burckle and Mortlock (1998) and Delaygue et al. (2000) indicate possible seasonal changes in sea-ice extent, which would imply seasonal variations in local wind patterns and therefore variations in precipitation. Overall, due to the lower temperature, precipitation over Antarctica was reduced (Krinner and Genthon). The reduction of precipitation meant that the thickness of the ice sheet in the interior of Antarctica was similar, or slightly thinner, than in the present day (e.g. Budd et al., 1998: Krinner and Genthon, 1998: Delaygue et al., 2000: Krinner et al., 2000).

These different surface conditions during the LGM influenced the main atmospheric circulation patterns. With the poles cooling down more than the tropics, the zonal mean meridional temperature gradient steepened. The meridional temperature gradient, together with the geostrophic wind equation ${ }^{1}$ and the thermal wind equation ${ }^{2}$, explains the main large-scale atmospheric flow at midlatitudes, that is, the predominantly westerly winds. Rind (1998) describes how the climate during the LGM changed as a result of a steeper meridional temperature gradient. Through the thermal wind equation, an increasing meridional temperature gradient will result in an increasing westerly flow at the mid-latitudes. In order to maintain this stronger westerly flow, the baroclinicity should increase as well. Hall et al. (1996a), Dong and Valdes (1998), and Kageyama and Valdes (2000) all found that there were large local increases in baroclinicity during the LGM, especially in the Atlantic storm track. They also noted that there were considerable differences in the responses between the Northern and Southern Hemisphere to this different climate. The following section describes the differences between the Northern and Southern Hemispheres.

\footnotetext{
The geostrophic wind equation describes the horizontal flow which is the result of the balance between the horizontal pressure gradient force and the Coriolis acceleration.

${ }^{2}$ The thermal wind equation relates the vertical gradient of the horizontal geostrophic wind to the horizontal temperature gradient and is a consequence of geostrophic and hydrostatic balance.
} 


\subsection{Northern Hemisphere Circulation}

The most dramatic changes occurred in the Northern Hemisphere. As a result of the expanded polar ice sheet, not only did the zonal mean meridional temperature gradient become steeper, resulting in stronger westerly winds, but the positions of the maximum temperature gradients shifted too. In today's climate, the position of the maximum temperature gradient can be found at the land-sea boundary. This is particularly true in the winter, when the land cools off considerably more than the surrounding seas. The land-sea temperature contrasts are the most important variations on the mean temperature field for the development of transient eddies. During the LGM however, the locations of the maximum temperature gradients shifted from the land-sea to the ice-sea boundary. As a result of this shift, zonal changes in the temperature gradient produced changes in the stationary waves and storm track activity. These in turn altered the large-scale precipitation distribution and the pattern of latent heat release. Changes in storm track activity and diabatic heating in turn affected the stationary waves. This was in particular true for the North Atlantic storm track, since the polar ice sheet expansion was more prominent in North America.

Modelling studies have indicated that the two main Northern Hemisphere storm tracks both had a strong eastward shift at the LGM (e.g. Valdes and Hall, 1994: Dong and Valdes, 1998). The primary sites for the generation of midlatitude systems moved eastward in both storm tracks, onto the sea-ice edge where the strongest temperature gradient resided. Both storm tracks were much more zonally confined, following closely the sea-ice edge, resulting in a change in precipitation patterns as well. Kageyama et al. (1999) found that these results were consistent for a range of models. The models were not conclusive however in determining whether a higher baroclinicity necessarily implied stronger transients. Because of differences in meridional heat transport, some models favoured energy transport by the transient eddies, where as others found that the planetary waves were more important. Rind (1987) found that the different responses to the ice age forcings depended on the different boundary conditions used to drive the model. For instance, variations in land-sea contrast and orography altered the types of eddies (transient versus stationary), their 
generation (barotropic versus baroclinic), their wave number distribution and the location of the storm tracks.

Another interesting aspect of the midlatitude storm tracks in the Northern Hemisphere during the LGM was their possible shift towards longer wavelengths (e.g. Kageyama and Valdes, 2000). The effect of the baroclinic eddies is to limit the meridional temperature gradient from becoming supercritical (Stone, 1978, the "baroclinic adjustment"). When the meridional temperature gradient exceeds a critical value (becomes supercritical), the most unstable wave will be excited and baroclinic instability will occur. The most unstable wave grows and transports heat until it reaches its threshold where upon excess heat will be transported by the next longer wave. So through internal interactions of the unstable waves, baroclinic waves will shift to longer wavelengths. (Hayashi and Golder, 1987: Cehelsky and Tung, 1991, the "nonlinear baroclinic adjustment": Rind, 1998: Welch and Tung, 1998a: Welch and Tung, 1998b). Longer wavelengths are more efficient at carrying heat, since they saturate less easily. A change of wavelength would have changed the regional climates by affecting the advection of weather patterns.

Perturbations in temperature are much more confined to the lower levels during the LGM (Hall et al., 1996a: Dong and Valdes, 1998: Kageyama et al., 1999: Kageyama and Valdes, 2000). The tropopause was lower as a result of the cooler temperatures. The heat transport at the lower levels was larger than during the present day, but integrated vertically through the whole atmosphere, it was less. The increase of heat transport at lower levels is consistent with an increase in transient eddies. Garcia (1991) shows that with an increase in the forcing, locations of wave breaking extend to lower altitudes. The stronger vertical decrease of horizontal heat transport is consistent with a more stable atmosphere during the LGM, where the extent of vertical movement was reduced. Although these deductions of what the climate was like during the LGM come from climate models, there are various factors that influence the outcome of a simulation. Resolutions and parameterizations play an important part in each simulation. Dong and Valdes (2000) found that low resolution models give poor simulations of planetary waves and storm tracks. Rind (1988) suggested that the 
variations between low and high resolutions in the model he used are due to the way they model energy transfers (for details on parameterization issues, see chapter 4).

From geological proxy data (section 2.3) and from climate models it is clear that the climate during the LGM was quite different in the Northern Hemisphere. It was not just colder, but the interactions of the cryosphere, the atmosphere and the ocean, altered the main circulation patterns, which in turn affected regional climates as well. In order to interpret regional climates during the LGM, it is therefore important to understand the main circulation patterns. For understanding New Zealand's climate during the LGM, we need to understand the climate of the midlatitudes in the Southern Hemisphere during the LGM.

\subsection{Southern Hemisphere Circulation}

In today's climate, the Southern Hemisphere's atmosphere is characterized by a pronounced zonal circulation. Variability on the zonal circulation is characterized mainly by the lower wave numbers, although their amplitudes are in general smaller than their counterparts in the Northern Hemisphere (e.g. van Loon and Jenne, 1972: Trenberth, 1980). Trenberth (1980) found that both the mean fields as well as the individual wave patterns show remarkable consistency with only very small seasonal variations. It seems that the Southern Hemisphere stationary waves are caused mainly by the off-centre positioning of the main orography in Antarctica and asymmetries in the sea-surface temperatures (e.g. van Loon and Jenne, 1972: Karoly, 1983: James, 1988), although remote forcing from lower latitudes also plays a role (e.g. Quintanar and Mechoso, 1995).

In the Southern Hemisphere, the planetary waves are very weak. It are the transient eddies which the whole year round are the main drivers of the meridional heat transport (e.g. van Loon, 1979: van Loon and Kidson, 1993). If a change in the mean planetary wavelength occurred in the Southern Hemisphere during the LGM then there needs to have been a very strong increase in the meridional temperature gradient. But as mentioned, although the meridional 
temperature gradient had increased, it most likely was not as steep as first described by the CLIMAP group. There have been no indications of significant changes in the frequency and amplitude of the Southern Hemisphere's stationary waves during the LGM. Dong and Valdes (1998) found that the Southern Hemisphere planetary waves remained weak and hardly changed in their LGM simulation. But even if they had changed, the effect would have been less dramatic than in the Northern Hemisphere. With the general circulation being nearly barotropic, storm tracks in the Southern Hemisphere are much more zonally aligned than their northern counterparts. Any variations in the mean field, as a result of any possible variations in the planetary waves, would have had minimal consequences for any 'preferred' positions of troughs and ridges, and therefore for specific climate patterns at mid latitudes.

The changes in the general circulation pattern of the Southern Hemisphere during the LGM were therefore probably less profound than they were in the Northern Hemisphere. With no large landmasses in the Southern Hemisphere, the zonal temperature contrasts during the LGM were not much different than in today's climate. But what might be of more relevance for the Southern Hemisphere is how the transient eddies might have changed under LGM conditions. The strength of the eddies is closely related to changes in meridional temperature gradient which are associated with changes in sea-ice (Hall et al., 1996a). Rind (1987) noticed an increase in baroclinic energy generation due to the expanded sea-ice extent. The main discussion point for the Southern Hemisphere therefore could be: "What was the sea-ice distribution during the LGM?" The strongest temperature gradients are found between regions with the largest difference in sea-ice concentration. The maximum extent ${ }^{1}$ and seasonal variability of sea-ice could influence the meridional temperature gradients and therefore could influence the preferred positions of the storm tracks.

CLIMAP (1976: 1981) saw a huge increase in the extent of the ice sheets, and in particular the mainly marine Western Antarctic ice sheet which expanded up

\footnotetext{
In many discussions, as well as in this thesis, references to sea ice extent often refer to the distribution and the concentration of sea ice, rather than to maximum extension only. Excemptions to this assumption will be indicated clearly in this thesis.
} 
to its continental edge. But CLIMAP's findings in this are questioned. Because the sea-ice extent is important for determining the mid-latitudinal meridional temperature gradient, and therefore for understanding the main westerly flow and its perturbations, a whole range of studies have been dedicated to improve our knowledge about how sea-ice might have changed during the LGM. For instance, Crosta and Pichon (1998b) and Burckle and Mortlock (1998) used the dependence of certain diatoms on open sea, as opposed to sea covered by ice, to estimate the extent of the LGM sea-ice. Others used modelling studies (e.g. Huybrechts, 1990: Budd et al., 1998) together with geochemical records (e.g. Morse et al., 1998). Although these studies were not always conclusive, the main point they had in common was that the sea-ice extent in the Southern Hemisphere as described by CLIMAP was probably too large. This meant that CLIMAP's SSTs were too low, particularly during JJA. Fig. 3-1 clearly shows the differences in prescribed (CLIMAP) and computed SSTs in the Southern Hemisphere's midlatitudes. In general, studies that used CLIMAP's sea-ice extent have a stronger westerly flow than studies that used a less extensive seaice cover, since the meridional temperature gradient in the latter is less steep. Dong and Valdes (1998) simulated the LGM climate with both prescribed and computed SSTs and investigated their different climatic responses. They found an increase in the westerlies around $60^{\circ} \mathrm{S}$ in their simulation with CLIMAP SSTs (and therefore a large sea-ice cover), where as they hardly noticed any change in the westerlies in a similar simulation with computed SSTs (with less extensive sea-ice).

Together with the static stability of the atmosphere, which determines the ability of the atmosphere to become turbulent as the result of buoyancy effects, the horizontal temperature gradient largely determines the occurrence of baroclinicity. Therefore, changes in the meridional temperature gradient as the result of changes in the location of the sea-ice edge could result possibly in different locations of origin for the storm tracks. Analysing data from only one year, Cavalieri and Parkinson (1981) found a relationship between the areas of rapid ice growth and areas of cyclonicity. However Simmonds and Wu (1993) determined that the location of storm tracks was insensitive to a reduction, and 

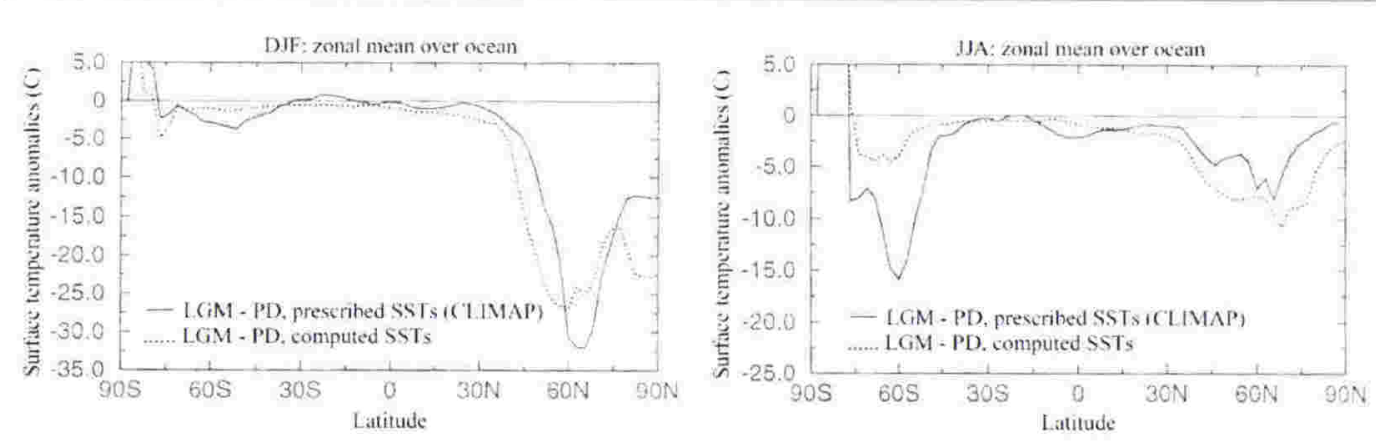

Fig. 3-1. Zonal mean sea surface temperature anomalies between the LGM and Present Day (PD) simulations for DJF and JJA. Note the large anomalies at $60^{\circ} \mathrm{S}$ during JJA which are the result of the difference in sea-ice extent between prescribed and computed sea-ice (after Dong and Valdes, 1998).

even a full removal of the sea-ice. They found that a change in the extent of the sea-ice affected the number of cyclones and cyclogenesis mainly regionally. The sensitivity of the location of the storm tracks to the sea-ice extent was discussed further by Simmonds (1996), who noted that there was only a subtle connection on interannual time scales. Although no strong relationship is found between the location of storm tracks and the sea-ice extent, cyclone behaviour seems to be influenced by changes in sea-ice concentration.

Various authors have developed different schemes determining the main areas of cyclogenesis. Taljaard (1967) found that the Southern Hemisphere storm tracks originate and develop in the midlatitudes and travel eastward and poleward. Similar findings came from Trenberth (1991) and Sinclair (1995) who both found a strong relationship between the storm track and the major upper tropospheric jetstream. Sinclair and Revell (2000) studied extratropical cyclogenesis in the Southwest Pacific. They used cyclonic vorticity advection as indication of cyclogenesis. Since areas of strong cyclonic vorticity advection are commonly associated with jet streaks, they were in fact linking cyclogenesis to the upper air jetstream. During the austral winter, cyclogenesis often moves south of the subtropical jetstream in the Pacific region, to areas of strongest SST gradients. Other areas of preferred cyclogenesis are baroclinic zones along the east coasts of Australia and South America in winter (Sinclair, 1995). Jones and Simmonds (1993) and Simmonds and Keay (2000a: 2000b) found high cyclone 
density and cyclogenesis at higher latitudes, at latitudes associated more with the polar trough. The differences between the studies are ascribed to the use of different storm tracking systems. In general, the region between $30^{\circ} \mathrm{S}$ and $60^{\circ} \mathrm{S}$ shows the largest transient eddy activity and is therefore often called the area of the storm tracks (Karoly et al., 1998).

Using the UGAMP (United Kingdom Global Atmospheric Modelling Programme) model under GCM conditions, Dong and Valdes (1998) found that the Southern Hemisphere sea-ice extent was less than that described by CLIMAP and that the mean sea level pressure and the winds over the high latitudes hardly differed from the present day. Also using the UGAMP model, Wyrwoll et al. (2000) investigated how the westerly flow and the baroclinicity could have varied in the Southern Hemisphere during the LGM under CLIMAP boundary conditions. Besides finding that the westerly flow had increased, they found that there was a general poleward displacement of the zonal maximum of the westerly winds, but with significant regional differences. Over the Indian Ocean-Australia sector, the poleward displacement was nearly non-existent during the summer. The associated poleward displacement of baroclinicity is in contrast with the findings of the shift in westerly flow in the Northern Hemisphere. In the Northern Hemisphere, the shift was determined by the change of the position of the maximum temperature gradient from the land-sea edge to the ice-sea edge. If the Southern Hemisphere storm tracks would depend similarly on the extent of the sea-ice, then they would have moved equatorward during the LGM as well, following the expanded sea-ice margin. This in fact was found by Morse et al. (1998), who suggested that the storm tracks had indeed moved equatorward during the LGM. However, Wyrwoll et al.'s (2000) study showed that the changes in the position of the westerly flow during the LGM were more similar to the variations in the present day circulation during winter: an increase and poleward displacement of the westerlies and having a more zonal character. With respect to the precipitation during the LGM, their reconstructions suggested that there was a reduction in precipitation in south Australia, consistent with some paleoclimatic evidence (Colhoun et al., 1996: Hunt and Barrows, 1999). They suggested that this was caused by the fact that the main band of baroclinicity had 
moved poleward together with the general colder conditions during the LGM. Similar interpretations were made by Hope and Simmonds (2003).

Although the Southern Hemisphere's mean zonal circulation might not have changed as much as it did in the Northern Hemisphere, the characteristics of its seasonal cycle and its variability on the mean flow might have been different. The next section discusses the Southern Hemisphere's climate variability and presents some speculations on what the variability might have been during the LGM.

\subsection{1.}

\section{Modes of Variability}

\subsubsection{Semi-Annual Oscillation (SAO)}

The SAO is the main seasonal variation of the zonal flow in the middle-high latitudes of the Southern Hemisphere. It is characterized by a twice yearly variation of both the strength and the position of the polar trough of low pressure surrounding Antarctica. From March to June, and from September to December, the circumpolar zone of low pressure expands equatorward and weakens at the same time, where as from June to September, and from December to March it contracts and intensifies (van Loon, 1967: Meehl, 1991: Mullan and McAvaney, 1995). As the result of the different heat capacities between the mid $\left(50^{\circ} \mathrm{S}\right)$ and high $\left(65^{\circ} \mathrm{S}\right)$ latitudes (ocean versus polar continent), warming and cooling have different lag times. Because of these different lag times, the temperature contrast between the two latitudinal bands has two maxima; one during the austral autumn (March/April) and one during spring (September/October. See Fig. 3-2). Differences between the cooling and heating rates between these times ${ }^{\prime}$ give rise to the maximum being larger during autumn. The variations in temperature gradient give rise to variations in both the strength of the westerlies and to changes in cyclonic activity. The strongest westerly winds occur during March and September south of about $50^{\circ} \mathrm{S}$ and during June and December north of about $50^{\circ} \mathrm{S}$.

\footnotetext{
${ }^{1}$ Autumn cooling at $65^{\circ} \mathrm{S}$ takes place more rapidly than the spring warming at $50^{\circ} \mathrm{S}$.
} 


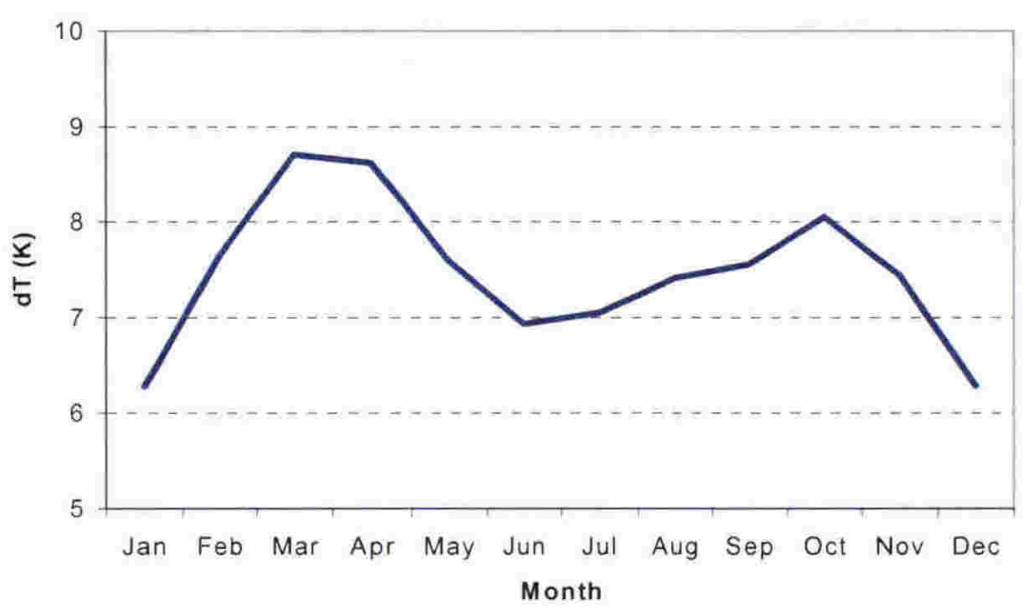

Fig. 3-2. The meridional temperature difference between latitudes $50^{\circ} \mathrm{S}$ and $65^{\circ} \mathrm{S}$ for climatological $500 \mathrm{hPa}$ temperature. Computed from 30 years (1971-2000) of NCEP reanalysis data.

The SAO is not a constant and varies in time. Meehl (1991) found that an altered seasonal cycle of SSTs and ocean heat storage at $50^{\circ} \mathrm{S}$ can result in a change in the SAO signal. Meehl et al. (1998) showed that the SAO was stronger before and weaker after 1979. They described this to an anomalous change in the temperature gradient between $50^{\circ} \mathrm{S}$ and $65^{\circ} \mathrm{S}$, which was the result of a warming trend. This resulted in a "flattening of the seasonal cycle of baroclinicity".

Walland and Simmonds (1999) point out that the variations in the meridional temperature gradient by themselves however do not fully explain the variations in baroclinicity. They found that although the temperature gradient is larger in March than in October, when studying variations in baroclinicity as the result of the different states of the SAO, it is important to include the static stability of the atmosphere as well. The static stability in the Southern Hemisphere's early spring is lower than in early autumn. The effect of this is that although the temperature gradient maximum in autumn might be larger than the one in spring, the lower static stability in spring amplifies the effect of the temperature gradient more, resulting in greater baroclinicity in spring than in autumn. An interesting climatic feature happens during winter when the average pressure over Australia increases. This increase in pressure is in contrast with the decrease in pressure at 
other longitudes in the same latitudinal band. Van Loon (1967) pointed out that during this period the pressure increases eastward of Australia, unlike during other seasons. This phenomenon could possibly support the birth of "blocking highs" around New Zealand.

The question arises whether the SAO was much different during the LGM. From the previous description one can assume that a different meridional temperature gradient during the LGM could indeed have affected the SAO. Stammerjohn et al. (2003) found that the SAO responds to the meridional temperature gradient as modulated, among others, by regional/global warming. Would a colder climate therefore also affect the SAO?

\subsubsection{Intraseasonal and Interannual Variations}

The Southern Hemisphere circulation exhibits a range of climatic variations after the mean seasonal cycle is filtered out. Kidson (1999) gives an overview of what signals can be detected within these variations, their timescales, and their proportion to the total variance (Fig. 3-3). Although climatic variations on

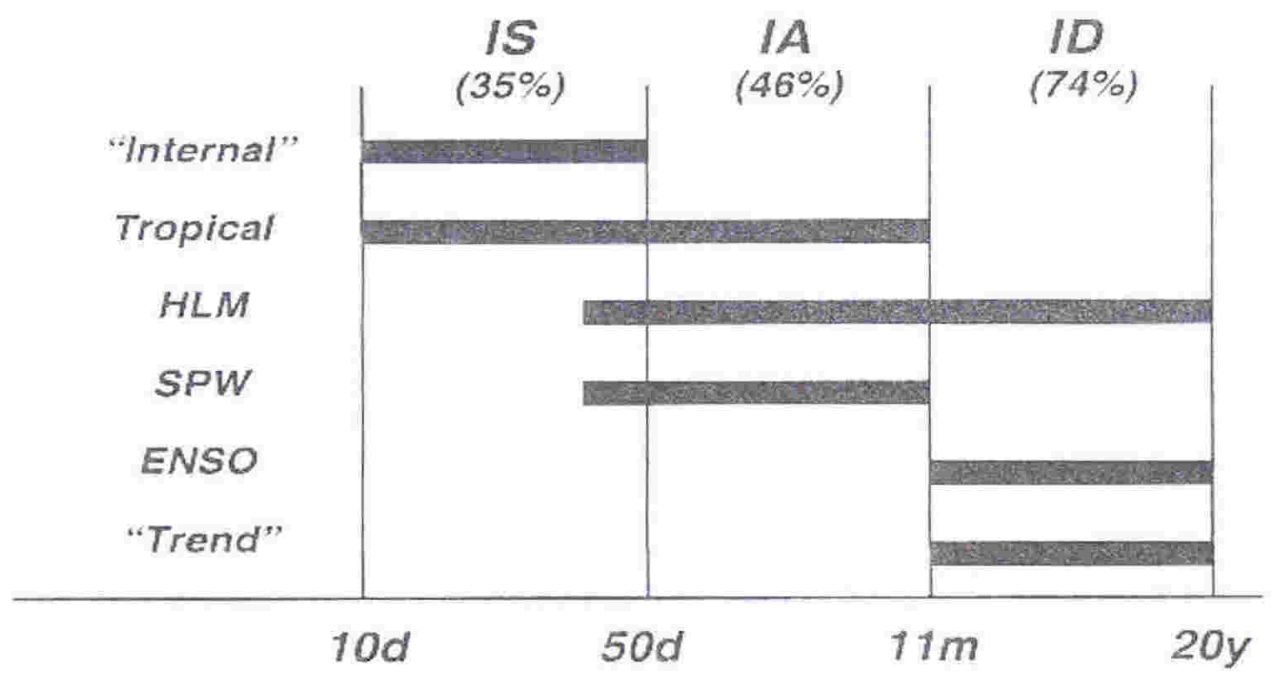

Fig. 3-3. Timescales associated with modes identified by EOF analysis (IS = intraseasonal, IA $=$ interannual, $\mathrm{ID}=$ intra-decadal, $\mathrm{d}=$ days, $\mathrm{m}=$ months, $\mathrm{y}=$ years $)$. The percentages indicate the proportion of variance in each band accounted for by the leading EOFs (after Kidson, 1999). 
timescales over several years are of interest, this study is not investigating these "long term" variations, but examines only the intraseasonal and interannual variations within defined time slices.

Kidson (1988a) looked at the time and spatial variation of the Southern Hemisphere $500 \mathrm{hPa}$ zonal wind and found that there are several signals in the variations. These variations can be understood by examining the $500 \mathrm{hPa}$ geopotential height fields, and are well described through EOF analysis ${ }^{1}$ (e.g. Kidson, 1988b: Karoly, 1990: Ghil and Mo, 1991: Kidson, 1991). Fig. 3-4 shows the four leading modes of variations of the 30day low-pass $500 \mathrm{hPa}$ geopotential fields presented as rotated EOFs (REOF) ${ }^{2}$. These figures are from Renwick and Revell (1999: The fourth panel was provided by the authors) and are representative of the leading EOFs of the intraseasonal variations on the mean Southern Hemisphere circulation. The leading EOFs explain around $40 \%$ of the monthly mean variance of the Southern Hemisphere circulation at the midlatitudes.

The main signal, or the first EOF, is primarily zonally-symmetric with opposing peaks at $40^{\circ} \mathrm{S}$ and $60^{\circ} \mathrm{S}$ with a wave number 3 pattern embedded in it (Wave number 3 is largely rotated out in Fig. 3-4. Unrotated EOF contains a stronger wave number 3 pattern). Kidson (1988a) shows that this pattern varies during the year, and is more of a wave number 4 pattern during summer. Variations in geopotential height at the latitudes of $40^{\circ} \mathrm{S}$ and $60^{\circ} \mathrm{S}$ give rise to variable gradients in geopotential height between these latitudes. These intraseasonal modulations on the mean field are often called the "High Latitude Mode" (HLM), the "Antarctic Oscillation" (AAO) or the "Southern Annual Mode" (SAM). Since the Southern Hemisphere circulation is virtually barotropic on the monthly timescale, the modulation on the mean field is done through momentum adjustment. A geopotential height gradient that is steeper than the long-term mean results therefore in increasing westerly winds and baroclinicity

\footnotetext{
${ }^{1}$ EOF is a mathematical approach to examine the temporal and spatial variance in a dataset.

${ }^{2}$ Interpretation of EOFs might be difficult if adjacent modes are not well-resolved in terms of their eigenvalues. Rotation of their basis can improve the relationship between the spatial and temporal patterns and known physical mechanisms, while keeping the eigenvectors orthogonal.
} 


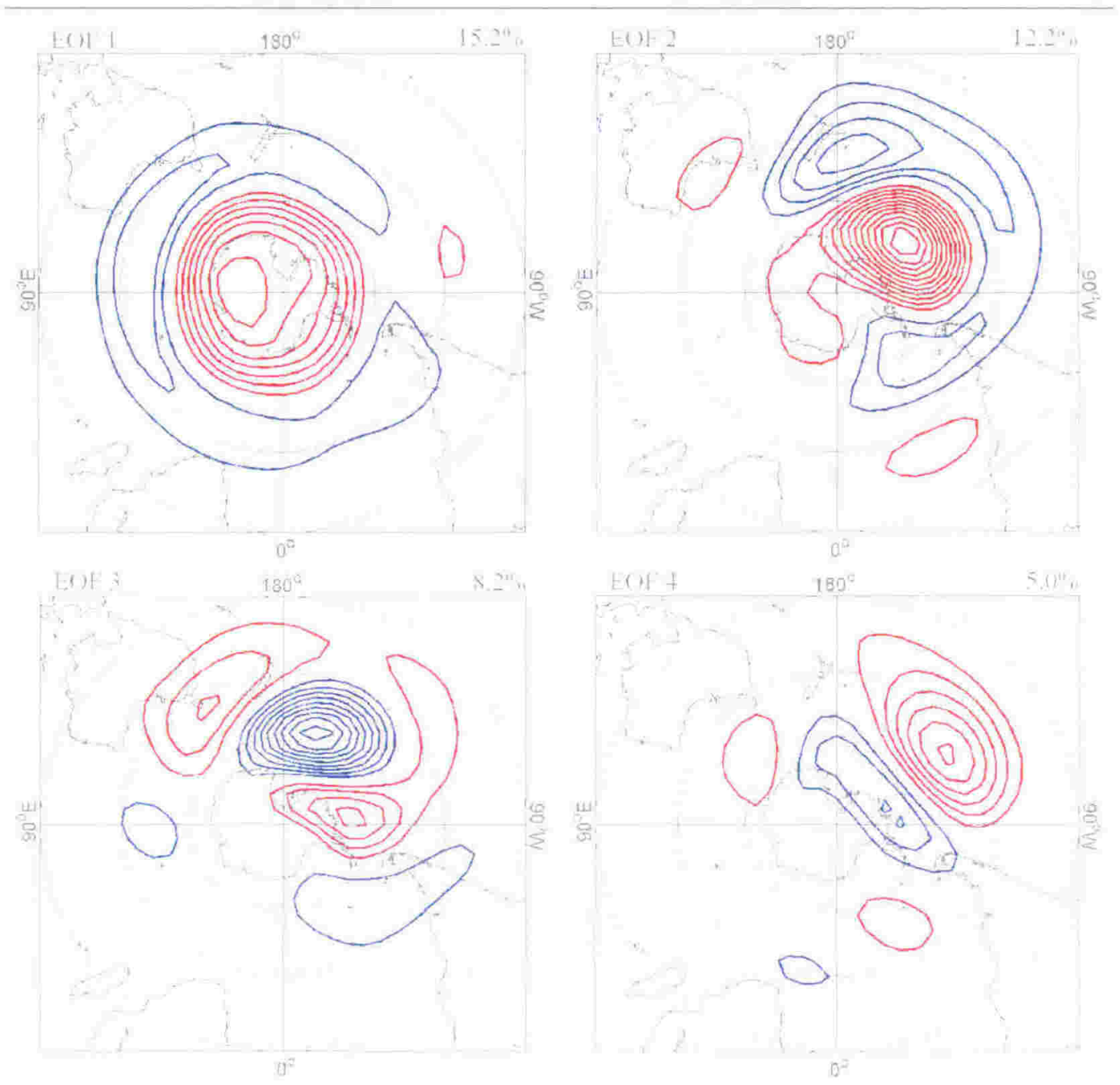

Fig. 3-4. The four leading rotated EOFs of 30-day low-pass filtered 500hPa geopotential heights, shown as covariance maps. The contour interval is $10 \mathrm{~m}$, with negative contours in blue, positive contours in red. The figures at the top of each panel indicate the fraction of low-pass filtered variance accounted for by each REOF (from Renwick and Revell, 1999).

at higher latitudes (Karoly, 1990). This phase of the HLM is referred to as the "positive" mode of the HLM. Hartmann (1995) details how the two different modes (positive vs. negative) have different characteristics and shows in particular the difference in their baroclinicity.

Studies have shown that the HLM may change its behaviour under global warming (e.g. Kushner et al., 2001). The question therefore arises whether it might have varied during the LGM as well. During the LGM, the pole's temperature had decreased much more than the temperature at the midlatitudes. This would have resulted in a larger decrease of the tropopause height at the pole 
than at the midlatitudes, giving rise to an increase in the geopotential height gradient between the mid and high latitudes. If the amplitude of the HLM on the meridional gradient of the geopotential height during the LGM was the same as present, then it would have been relatively weaker than today, since the mean westerly flow was stronger during the LGM and the gradient would therefore have been steeper. However, with changes in the HLM intimately linked to changes in the transients (e.g. Hartmann, 1995: Limpasuvan and Hartmann, 1999), if the HLM had changed during the LGM, then it could have altered baroclinicity and precipitation patterns. This could have resulted in changes in regional climates, for instance in the climate of New Zealand.

The second and third leading modes of the low-frequency variations in the Southern Hemisphere (EOF $2+3$ ) move in quadrature and resemble a wavetrain pattern, which moves from Australia to South America. These signals are associated with ENSO (e.g. Karoly, 1989: Renwick, 1998: Mo, 2000) and have been shown to influence the number of blocking events in the midlatitudes, particularly in the southeast of the Pacific (Renwick, 1998: Renwick and Revell, 1999) ${ }^{1}$. The fourth EOF is also associated with ENSO, and is indicative of the effect of El Niño during the Southern Hemisphere summer months (or in other words, during its mature stage). It represents a "dipole" between the middle and high latitudes, with positive anomalies at middle latitudes and negative anomalies at high latitudes (e.g. Karoly, 1989: Kidson et al., 2002).

As mentioned in section 3.1, for studying the climate at the midlatitudes during the LGM, it is not sufficient to determine only the amount of cooling of the tropical SSTs. With climate variability in the midlatitudes related to ENSO patterns, it is just as important to get the east-west SST-gradient in the equatorial tropics correctly (Liu et al., 2000: Yin and Battisti, 2001). This gradient will indicate whether the LGM climate had characteristics associated more with an El Niño or a La Niña climate. Analysis of the second and third EOFs of the Southern Hemisphere climate during the LGM could further indicate whether the

\footnotetext{
${ }^{1}$ However, ENSO is not the only cause of interannual variability over the southeast Pacific Ocean. Renwick (2002) found that interannual variability over that region was also related to Antarctic sea-ice variability.
} 
climate during the LGM was indeed more like either one of these climatic patterns. Both patterns would have had consequences for the climate at the midlatitudes.

\subsubsection{New Zealand during the LGM}

New Zealand is located in the midlatitudes of the Southern Hemisphere, about $166^{\circ} \mathrm{E}-179^{\circ} \mathrm{E}, 34^{\circ} \mathrm{S}-48^{\circ} \mathrm{S}$, with its main axes, about $1600 \mathrm{~km}$ long, oriented roughly SW - NE. It consists of two main islands, the North and South Island, and numerous smaller islands. Only about a fifth of its continental shelf is above water. New Zealand is very mountainous, and a mountain chain runs virtually through the whole country from north to south. The mountain range is particularly high in the South Island (the Southern Alps) with several peaks well over $3000 \mathrm{~m}$ high. New Zealand's climate can in general be regarded as temperate, which is a reflection of the major influence that the surrounding oceans have.

New Zealand occupies a unique position in the general circulation of the Southern Hemisphere. Its continental shelf sits in the path of the main ocean circulation and forces its Southern Hemisphere branch (the Antarctic Circumpolar Current) to dip southwards in order to get around this obstruction. Its landmass sits in the prevailing westerly winds and influences its passage. For an air parcel to get pass this obstruction, it has to ascend and cross the mountains. The air rises in the west, cools off, condensation occurs, and eventually the moisture is removed through precipitation. When the air has passed over the mountains, its moisture content has been lowered considerably. At the same time, due to its descent to lower altitudes and release of latent heat, the air warms up, also resulting in lowering the relative humidity. The climatic effect of the mountains therefore is a wet west coast and a dry east coast with often föhn-like winds. This is particularly the case for the South Island, where the mountains are more continuous and are higher than in the North Island. At the same time, the northern regions can be affected by (sub)tropical cyclones coming from the north, and the (far) south regularly sees intrusions of polar air. In a nutshell, New 
Zealand's climate is dominated by the ocean and the main regional differences are induced by the topography.

With the SSTs being lower during the LGM, the question arises how New Zealand's climate during the Last Glacial Maximum was different to today's climate. When describing New Zealand's climate during the LGM it is necessary to understand the global circulation patterns during the LGM, and in particularly the Southern Hemisphere circulation. The global circulation and the circulation of the Southern Hemisphere during the LGM have been discussed in the first part of chapter 3 .

\section{- Oceans}

Due to the significant increase of the ice sheets and polar icecaps during the LGM, the sea level was lowered. The result was that the main islands of New Zealand were connected into one big landmass. The closure of Foveaux Strait and Cook Strait would have had only a minimal impact on the ocean currents flowing around New Zealand, since the mass transport through these passages is relatively small compared to the other currents circulating around New Zealand. The main effect of the enlarged landmass on the coastal currents was that they shifted further offshore.

There are indications however that the main currents around New Zealand during the LGM did change, but more as the result of changes in the driving forces from somewhere else. The strengthened westerly flow would have accelerated the Antarctic Circumpolar Current (ACC), and it could also have moved slightly equatorward. New Zealand's continental shelf interrupts the predominantly zonal flow of the ACC and forces it to take a diversion to the south to get around it. Any changes in the strength and direction of the ACC would have affected the currents around New Zealand. A change in the currents around New Zealand implies an alteration in the advection of ocean heat to New Zealand, and has therefore a direct impact on the climate of New Zealand. Carter (2001) gives a good overview of the possible changes to the currents around New Zealand (Fig. 3-5). With the ACC moving slightly northward, it brings colder waters to New Zealand's continental shelf. There are indications of iceberg 


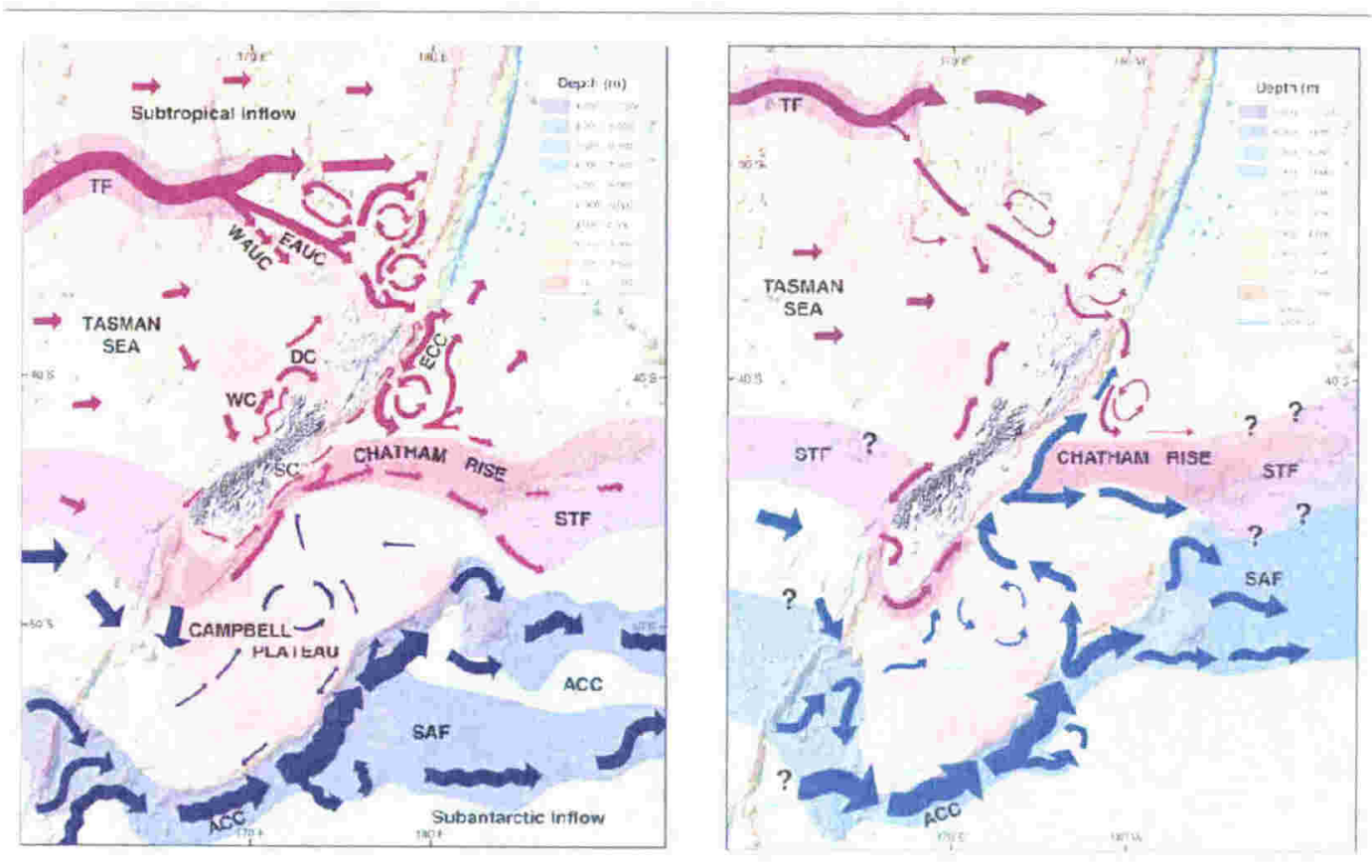

Fig. 3-5. Currents around New Zealand, Present Day (left) and Last Glacial Maximum (right). Abbreviations for fronts are: $\mathrm{TF}=$ Tasman, $\mathrm{STF}=$ SubTropical, $\mathrm{SAF}=$ SubAntarctic. Abbreviations for currents are: WAUC $=$ West Auckland, EAUC $=$ East Auckland, ECC $=$ East Cape, $\mathrm{DC}=$ D'Urville, $\mathrm{WC}=$ Westland, $\mathrm{SC}=$ Southland, $\mathrm{ACC}=$ Antarctic Circumpolar (from Carter, 2001).

excursions onto the Campbell Plateau during the LGM (Carter et al., 2002). The Tasman Front probably moved equatorward (e.g. Barrows et al., 2000) and was weaker during the LGM, resulting in a less developed East Auckland Current (EAUC) and East Cape Current (ECC). This enabled the Southland Current (SC) to penetrate further northwards, across the Chatham Rise, replacing the warm waters from the ECC with colder waters (e.g. Nelson et al., 1993: Nelson et al., 2000). However the Subtropical Front (STF) directly to the east of New Zealand had probably stayed in place. It is suggested that the waters to the east of New Zealand saw enhanced upwelling, possibly as the result of stronger winds during the LGM. This resulted in extra cooling of the eastern coastal waters during the LGM.

\section{- Cryosphere}

An interesting feature of New Zealand during the LGM was the extensive glaciers covering most of the Southern Alps. These glaciers extended all the way 
to the central west coast. The glaciers would have filled in most of the present day valleys, up to $2000-2500 \mathrm{~m}$ thick, and could have been as high as $3000 \mathrm{~m}$ near Mt Cook (J. Shulmeister, personal communication). The conclusion to be drawn from this extensive glaciation is that accumulation of ice and snow (through precipitation) had to be higher than ablation (melting, evaporation). Although precipitation was probably lower overall than what it is today, the colder temperatures prevented any rapid decline of the glaciers. The filling-in of the valleys by the glaciers would have raised and widened the barrier to the westerly flow, further accentuating the differences in the climates on either side of the main divide. The mountains of the North Island were probably free from large glaciers, but some peaks of the Central Plateau and the southern North Island might have been covered in permanent ice.

In order to reconstruct the past climates of New Zealand, geological studies have either investigated in detail certain areas of New Zealand, or have studied a particular climatic indicator across New Zealand. Combining the results of all these studies enables us to determine what New Zealand's climate during the LGM might have been like. This is done in several papers, such as Markgraf et al. (1992), McGlone et al. (1993), and in a more recent paper by Shulmeister et al. (2004).

Vegetation proxy data gives a good indication of what New Zealand's climate was like during the LGM. The common vegetation in New Zealand during the LGM was mostly grasslands and shrubs. Forests are thought to have only existed in isolated, sheltered areas. Patches of forest were more common in the North Island than in the South Island, with the Far North being the only area actually covered by forests. The vegetation data of New Zealand implies a harsh climate, where strong winds, droughts and frosts were not uncommon. The average annual temperature was probably about $4.5^{\circ} \mathrm{C}-5^{\circ} \mathrm{C}$ cooler than today. The precipitation was reduced compared to today, giving rise to drier conditions overall during the LGM. Only the Far North may have received precipitation comparable to present day levels. Another indicator of drier conditions in New Zealand during the LGM is the amount of löss that can be found in the 
sedimentation records. Drier conditions lead to an increase in erosion and consequently to an increase of windblown sediment. An increase in aeolian sediment requires either stronger winds or longer periods of windy conditions, or simply an increase in exposure of the original bedrock. It is thought that that was particularly the case for southern and central New Zealand. In short, one can sum up New Zealand's climate during the LGM as one that shows more characteristics of a continental climate than its present day climate.

Our knowledge of the characteristics of a climate from the past comes mainly from geological proxy data. But recently, researchers have placed more reliance on computer models for simulating past climates. Paleoclimate modellers and geologists now work together in reconstructing past climates. Chapter 4 will discuss the topic of climate modelling. 


\section{Chapter 4}

\section{Climate Modelling}

\section{Introduction}

The last 30 years have seen a remarkable increase in studies of our climate. We have become more aware of the variability of our climate system and its impact on our society. Natural and human induced climatic phenomena like El Niño, the ozone hole, and "global warming", to name a few, are not only scientific issues, but are also important social, political, and economic topics. For those reasons, climate studies have become prominent research programs. Many national and international groups have been established to monitor and study our climate. Building on the knowledge of the atmosphere developed over the last century, we are now capable of describing the dynamics of the atmosphere and its interactions with the surrounding spheres ${ }^{1}$ in mathematical form. The workings of the climate system can now be simulated through computer models. These climate models in turn help us to understand our past, current and possible future climates (Simmonds, 1998).

This chapter will deal with the topic of climate models. It will give a brief overview of the history of atmospheric research, followed by a general overview of some climate models, and a more detailed description of the model used in this study.

\footnotetext{
${ }^{1}$ These spheres are for instance the biosphere and cryosphere.
} 


\subsection{A Short History of Atmospheric Science}

At the start of the twentieth century, people were trying for the first time to use physics to describe the atmosphere. The Norwegian scientist Vilhelm Bjerknes in 1904 (Charney, 1950) was the first to suggest that weather forecasting should be possible by using the laws of physics. He believed that both atmospheric physics as well as the necessary meteorological observations techniques had developed to such a point that together they should enable us to give a complete characterization of the atmosphere at a given place and time. $\mathrm{He}$ suggested that only seven quantities were necessary for solving the equations describing the state and motions of the atmosphere. Those quantities were the three components of the velocity of air, $u, v$, and $w$ (zonal, meridional and vertical), the pressure $p$, the temperature $\theta$, the mass density $\rho$, and the water content per unit volume $\mu$. Bjerknes did not believe that the equations describing the atmosphere could be solved analytically ${ }^{1}$. He therefore developed a "graphical calculus", a technique that enabled him to determine atmospheric values using graphs. One of the well known graphical methods is the tephigram (Devised by W.N. Shaw in 1925), and is still in common use today.

Bjerknes founded the Bergen School of Meteorology in 1917. This school made a large contribution to the knowledge of the workings of the atmosphere and they introduced a whole range of new concepts to meteorology. Their formulations of air mass, warm fronts, cold fronts, and occluded fronts, enabled them to formulate a working model for the main atmospheric movements. They established the theory that small disturbances in the atmosphere can lead to cyclogenesis.

In 1922 Lewis Fry Richardson was the first to solve a simplified version of the seven equations describing the state and motions of the atmosphere. He presented the idea that future weather can be predicted by solving these equations with the present weather as the initial conditions. While correct in principle, Richardson was not successful in his own numerical experiment, which was

\footnotetext{
${ }^{1}$ These equations are the continuity equation, the humidity and thermodynamic equations, the equation of motion (three-dimensional) and the gas law.
} 
mainly attributed to the lack of sufficient and accurate initial data, as well as having integration steps that were too large (hours) which caused numerical errors (Fleming, 1996). It was Carl-Gustaf Rossby who eventually laid the foundation by combining weather forecasting with dynamical meteorology. In his early years he had worked at Bjerknes' institute in Bergen, but he became most famous for his work during his time in the United States. Rossby's main contribution was that he was able to include large-scale planetary features in the meteorological models. His equations fitted the observations well and could be solved. In 1940, Rossby and his fellow workers were able to make numerical predictions for a one-layer atmosphere. "Rossby's great achievement was to devise a theory that was based on physics, that described atmospheric phenomena that were of importance to forecasters, and whose predictions could be calculated" (Nebeker, 1995).

The time between the two World Wars saw an explosion of meteorological data gathering. The arrival of the punch-card machine in the meteorological world allowed meteorologists to do much more with much more data than ever before. The most dramatic impact that World War II had on meteorology was the introduction of the electronic computer, which developed out of wartime technology. It was von Neumann who integrated meteorology and the electronic computer. In 1944, von Neumann had become involved with the building of the first electronic computer, the ENIAC, and by 1946 he identified that meteorology was a prime application to test on an electronic computer (Cressman, 1972). His drive to solve the highly non-linear equations by means of the computer got many people interested. Rossby invited von Neumann to submit a proposal for a meteorology project. In July 1946, the Meteorology Project at the Institute for Advanced Study (IAS) in Princeton was founded. Jule Charney, who would become a pivotal person in the development of atmospheric science, joined this project in 1948 (Thompson, 1990). One of the key early breakthroughs by Charney was the quasi-geostrophic approximation, where a near balance between the pressure gradient force and the Coriolis force is assumed. The approximation therefore assumed that the gravitational acceleration is balanced by the vertical 
pressure gradient ${ }^{1}$. This is a very accurate approximation except at very small spatial scales $(<10 \mathrm{~km})$ and outside regions of strong vertical motion, e.g. vigorous convection or flow over steep orography. The quasi-geostrophic approximation simplified the equations of motion into equations with pressure as the only variable. At the same time the problem of fulfilling the CourantFriedrichs-Lewy ${ }^{2}$ stability criterion was reduced since this approximation filters out the fast travelling atmospheric sound and gravity waves and allows for a larger integration time step. In the early 1950's, Charney's group made a lot of progress in numerical weather prediction. Starting with a simple model, they increased the complexity by addressing the shortcomings of the model (Smagorinsky, 1983). This was a time where many developments took place in atmospheric modelling. The idea of "long-range forecasting", or in other words, incorporating features of the general circulation, was first conceptualize.

Starr (1948) had outlined the concept of the general circulation in his analysis of the transfer of absolute angular momentum within the atmosphere. Rossby and Starr (1949) had concluded that the advection of relative angular momentum through cyclones and anti cyclones plays an integral part in the mechanics of the general circulation. These "perturbations" on the mean zonal flow can cause baroclinic instability. Barotropic models were replaced by baroclinic models ${ }^{3}$ in order to incorporate the advection of angular momentum in the general circulation. Charney (1947) and Eady (1949) developed the first simple models of baroclinic instability. Emerging from these early models was the simple 2layer model, which became a common model to describe features of baroclinic instability (e.g. Phillips, 1954: Smagorinsky, 1963: Moura and Stone, 1976: Held, 1978: Stone, 1978). Although these models had a lot of shortcomings, they were able to describe the main features of the atmospheric circulation quite well and so they are still regularly being used.

\footnotetext{
${ }^{1}$ This is the hydrostatic balance where $d p / d z=-\rho g$

${ }^{2}$ The Courant-Friedrichs-Levy criterion states that the horizontal resolution of the model must be larger than the product of the integration timestep times the phase speed of the fastest traveling atmospheric wave, $\Delta x \geq \Delta t \cdot c$ ${ }^{3}$ Barotropic models, where isobars and isentropes are parallel to each other, do not allow vertical exchanges,
unlike baroclinic models where the geostrophic wind varies with height.
} 
What had become clear in the 1950's was that the computer was an essential tool for operational forecasting. There were spectacular improvements in the speed, memory capacity and reliability of the electronic computers in the 1950's and 1960's. In 1976 the first of the Cray computers, the first of the so-called supercomputers, became available. The increase of computer power allowed for the development of more advanced models. In the mid 1960's the primitiveequation models, based on Bjerknes' theories and integrated by Richardson, replaced the baroclinic models for daily forecasting (Cressman, 1972). Gravity waves were included in these models, since the very fast computers were capable of solving the equations with very short integration steps.

The development of the forecasting models, their algorithms, and the parameterization of difficult to define atmospheric variables, are closely related to the development of general circulation models. Von Neumann was the first to suggest the "infinite forecast" whereby he looked at the larger, planetary weather systems. In 1955, Norman Phillips was the first to successfully model the general motions of the Earth's atmosphere (A description of Phillips' work is given by Lewis, 1998). Smagorinsky's paper (1963) about a primitive equation' model for the general circulation did much to increase the interest in general circulation modelling. This could be seen as the start of global modelling, and therefore the start of modelling the state of the atmosphere on longer timescales. In fact, weather modelling moved into climate modelling.

The following decades saw a significant development in the field of atmospheric science. The tremendous development of numerical analysis techniques, the introduction of the electronic computer, massive increases in conventional and new data (e.g. from satellites), all improved our understanding of the workings of the atmosphere. New studies (e.g. chaos theory, paleo studies, atmospheric chemistry) and improved interactions with sources affecting our atmosphere (e.g. oceans, bio- and cryosphere, human impacts) all led to a more complete picture of our atmosphere. One of the most complete atmospheric

\footnotetext{
The primitive equations are the simplified versions of the equations describing the conservation of momentum, mass and energy. It assumes that the hydrostatic and shallow atmosphere approximations are correct (see e.g. section 4.2.4).
} 

As mentioned in the previous section, climate models have evolved in time as more and more became known about atmospheric circulations and as the computer power increased. The earliest models were very much simplifications of the real circulation. Today the most comprehensive climate models are the socalled "coupled General Circulation Models" (coupled GCMs). These models solve the set of equations describing the circulation for both the atmosphere and the ocean (the "primitive equations", see section 4.2.4). Included with these models can be standalone models such as ice-sheet models and models describing the biosphere. But other, simpler models are still very useful for getting a general picture of some aspects of the atmosphere. This section will discuss some of the more common climate models.

\subsubsection{Zero Dimensional Models}

The simplest climate model is the zero order model. This is also called the energy-radiation model. This model only looks at the balance of incoming and outgoing radiation. The incoming radiation is summed and that value can be presented as the total radiative energy that the Earth receives from the sun. If it is assumed that the Earth radiates like a black body, then radiation and temperature are related to each other through the Stefan-Boltzmann equation:

$$
R_{E}=\sigma T^{4}
$$

where $R_{E}$ is the Earth's radiation, $T$ is the equivalent black body temperature, and $\sigma$ is the Stefan-Boltzmann constant. The difference in incoming $R_{i}$ and outgoing radiation $R_{o}$ therefore indicates whether the Earth is warming up, or whether it is cooling down.

$$
\Delta R=R_{i}-R_{o}
$$


If the incoming radiation balances the outgoing radiation, $\Delta R=0$, then the average temperature in time and over the whole Earth does not change ${ }^{1}$.

The chemical composition of the atmosphere plays a very important part in trapping outgoing longwave radiation. Some of the atmospheric gases, especially water vapour and $\mathrm{CO}_{2}$ are particularly effective in trapping Earth's infrared radiation. Their spectrum of absorption bands lies well within the spectrum of terrestrially-emitted radiation. Since we know that the composition of the atmosphere is not constant (increase of "greenhouse" gases by man, emissions by volcanoes, and absorption of carbon by forests and oceans, to name a few), this simple model can not be applied directly to the Earth's surface, as it does not include the crucial feedbacks that influence the radiation balance. Feedbacks are interactions within the system that either enhance (positive) or counteract (negative) the effect of any disturbance. The increase of greenhouse gases in the atmosphere is directly linked to the phenomenon of "global warming".

Other important feedbacks in the radiation scheme are related to the albedo of the Earth and to the distribution and specifications of clouds. Over a third of the incoming radiation is reflected back to space, mainly by the Earth's surface and by clouds (Fig. 2-1). A simple feedback mechanism can easily be demonstrated in the case of the effect of ice on the incoming solar radiation. A change of albedo of the Earth by increasing/decreasing the polar ice sheets, will have a measurable result on the incoming amount of solar radiation. An increase in the size of the icecap will result in a higher proportion of incoming radiation being reflected back to space. This is a positive feedback, since if less solar radiation hits the Earth, the Earth will cool off, and ice sheets can grow. This in turn will increase the albedo of the Earth, so more radiation is reflected back to space, which will result in the Earth to cool off, and so on.

Clouds however have a more complicated effect. Clouds can both reflect incoming solar radiation and trap outgoing radiation. These effects depend on the albedo, the type and the heights of the clouds. Clouds at lower altitudes have a cooling effect (they radiate more outwards than that they trap heat), where as

\footnotetext{
${ }^{1}$ Both $R_{i}$ and $R_{\theta}$ vary in time (on all time scales) and in space.
} 
clouds at higher altitudes do just the opposite and tend to warm up the atmosphere (Stephens and Webster, 1981). Under a global warming scenario, an increase of clouds at low altitude would be a negative feedback, where as an increase of clouds at higher altitude would be a positive feedback. Including the effect of feedbacks on the radiation scheme, equation 4.2 should then read:

$$
\Delta R=R_{i}-R_{0} \pm \widetilde{E}_{t}
$$

where $\widetilde{E}_{t}$ is a variable heating term that depends on time. The sign of this variable depends on whether it has a positive or negative feedback on the radiation balance.

One of the remarkable aspects of this simple model is that if we only vary the albedo by varying the amount of ice cover on Earth, it shows that the climate can have three different stable states. A stable state is where incoming solar radiation is balanced by the outgoing longwave radiation. Fig. 4-1 shows the three states as

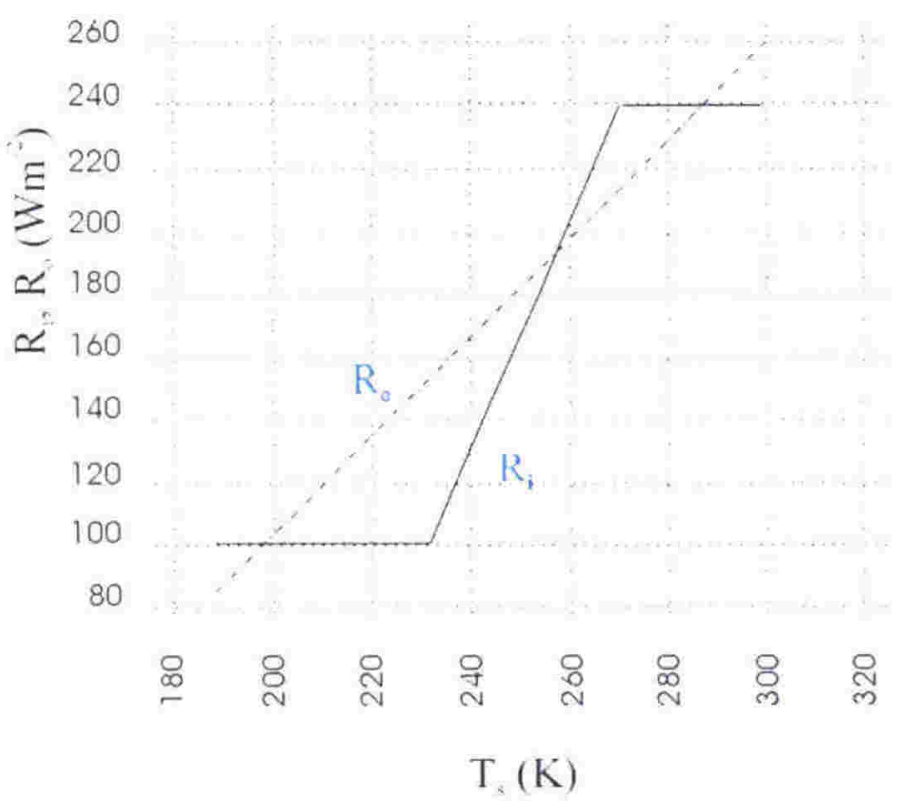

Fig. 4-1. Zero dimensional climate model with ice albedo feedback. The outgoing longwave radiation $R_{0}$ and the incoming shortwave radiation $R_{i}$ are plotted against the globally averaged surface temperature $T_{s}(K)$. There are three climate states where the incoming radiation equals the outgoing radiation (after Trenberth, 1992). 
a "warm" state, which can be seen as the current climate, an intermediate climate, and a cold climate, where the whole Earth is covered in ice. This model is therefore useful in estimating in a preliminary way whether the climate is in a stable state or not. From it one can derive the "climate sensitivity" parameter which determines the magnitude of the temperature change as the result of a perturbation (North et al., 1981: Trenberth, 1992).

\subsubsection{One Dimensional Models}

There are two different kinds of one-dimensional models, but both have a space coordinate as a variable.

\section{A) Energy balance model}

This model is a direct extension of the zero-dimension model. It assumes a latitudinal distribution of solar radiation. Because of the uneven distribution of solar radiation on the Earth, heat is transported from the equator to the pole. Therefore this model needs a latitudinal heat transport term in the equation, $\widetilde{E}_{\text {lat }}$ :

$$
\Delta R=R_{j}-R_{0} \pm \widetilde{E}_{x, t}+\widetilde{E}_{l a t}
$$

Without this latitudinal heat transport, the equator would be too hot, and the poles too cold. Fig. 4-2 gives a simplification of the effect of the heat transport on the latitudinal temperature gradient. The latitudinal heat transport term reduces the latitudinal temperature gradient. The time-dependent heating term

now also has a latitudinal dependency, $\widetilde{E}_{x, t}$. The energy balance model is an effective model in studies examining mechanisms of poleward heat transport (North et al., 1981).

B) Radiative-convective model

This type of model simulates the vertical profile of atmospheric temperature under the assumption of radiative-convective equilibrium. The radiative 


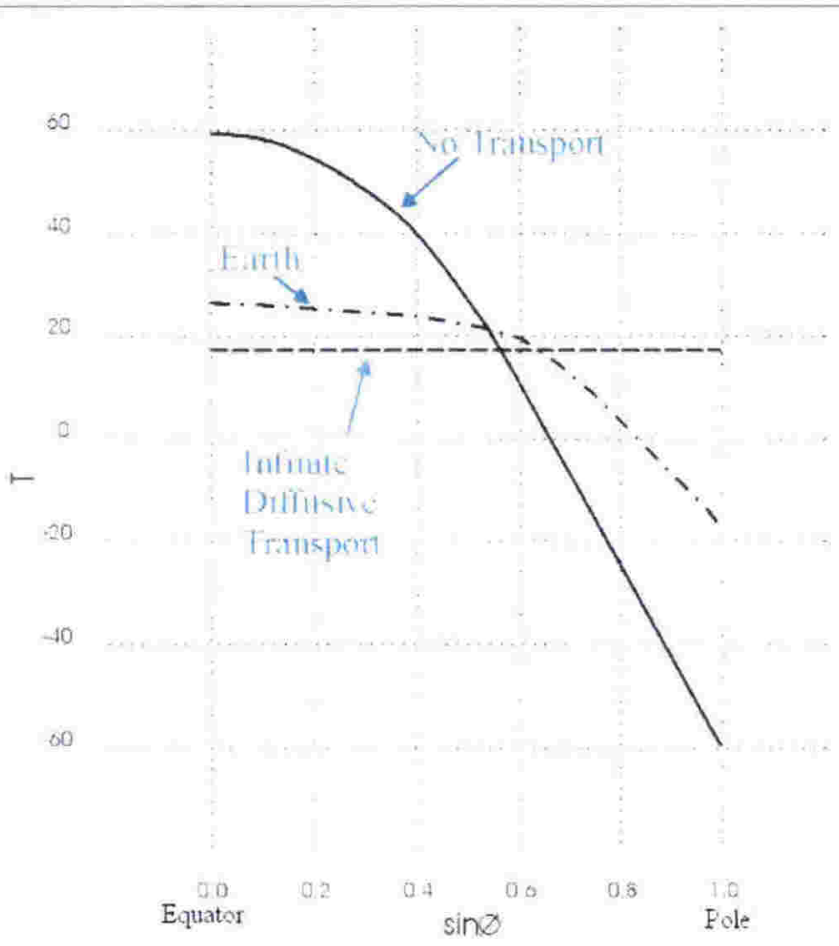

Fig. 4-2. Temperature (in ${ }^{\circ} \mathrm{C}$ ) versus the sine of latitude (Ø). The effect of heat transport for the cases of no heat transport, infinite heat transport, and a schematic effect of heat transport for the real Earth are presented (after North et al., 1981).

equilibrium temperature profile for the atmosphere is determined on the assumption that the temperature decreases with height according to the moist adiabatic lapse rate ${ }^{l}$. However, the troposphere is never exactly in radiative equilibrium. There is continuous vertical movement of air parcels that are either slightly warmer or colder than their surroundings. This convective adjustment acts to smooth out radiative and other heating imbalances. The equilibrium temperature profile therefore depends on both radiation and convection. Radiative-convective models have several vertical layers to calculate the contribution at each level of the radiation and diabatic heating and convection to the thermal equilibrium.

There are several variables that need to be incorporated in radiation balance schemes. Clouds, water vapour, $\mathrm{CO}_{2}, \mathrm{O}_{3}$, surface albedo, aerosols and trace gases all have their influence on the radiation balance. Stephens and Webster (1981)

\footnotetext{
${ }^{1}$ The radiative equilibrium temperature profile is unstable with respect to the dry adiabatic lapse rate since the effect of moisture (i.e. latent heat) is not taken into account.
} 


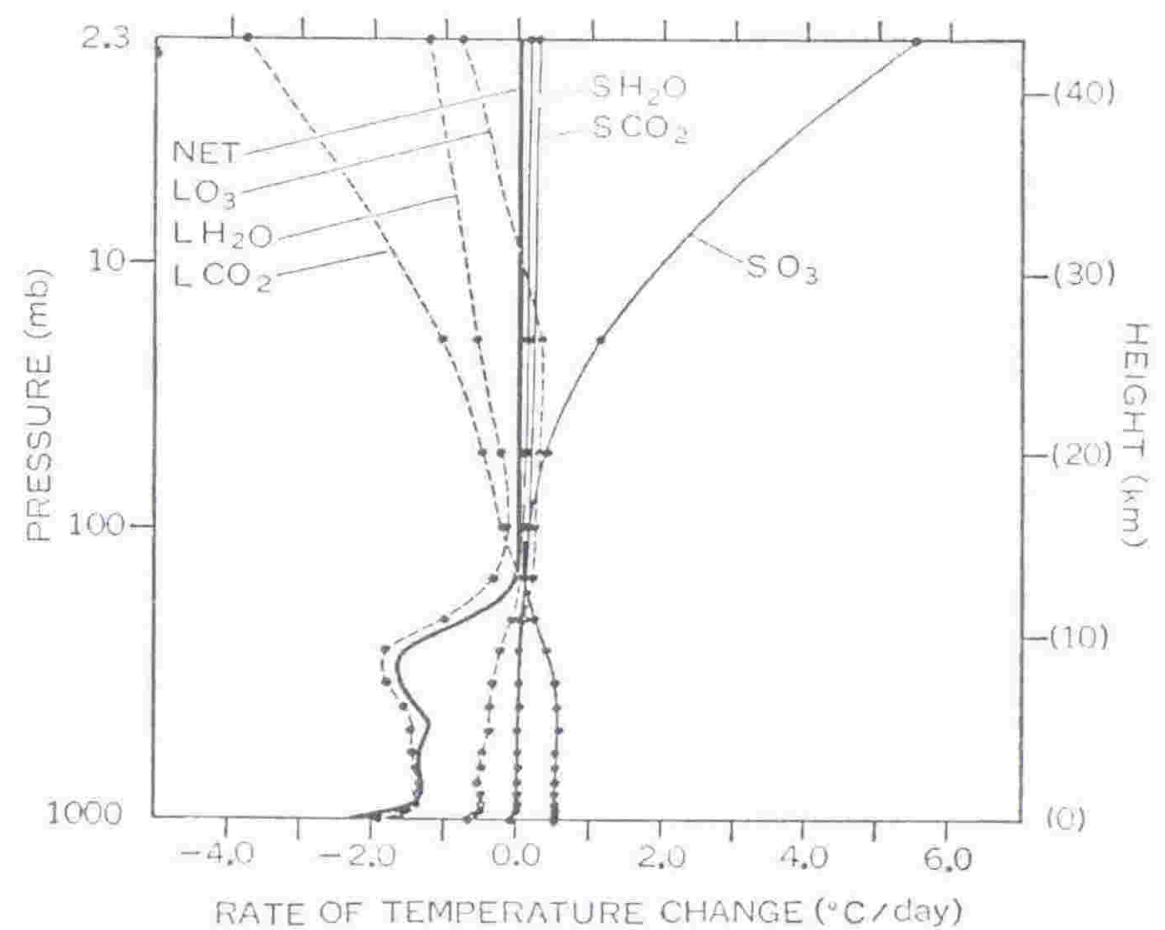

Fig. 4-3. Vertical distribution of the components of radiative heat balance for the thermal equilibrium of a clear atmosphere. $\mathrm{LH}_{2} \mathrm{O}, \mathrm{LCO}_{2}$, and $\mathrm{LO}_{3}$ show the effect of the longwave radiation of water vapour, $\mathrm{CO}_{2}$ and $\mathrm{O}_{3}$ respectively. $\mathrm{SH}_{2} \mathrm{O}, \mathrm{SCO}_{2}$, and $\mathrm{SO}_{3}$ show the rate of temperature change due to the absorption of solar radiation by those gases (Manabe and Strickler, 1964).

use a one-dimensional radiative convective model to gauge the influence of clouds on simple climate systems. Fig. 4-3 gives an indication of the effect of the major atmospheric gases to the radiative heat balance as a function of height. It is not possible to give precise values for their contribution to the radiation balance, so often their influence needs to be parameterized.

\subsubsection{Two Dimensional Models}

Two-dimensional models are either vertically or zonally integrated. The former presents a two-dimensional domain by longitude and latitude, the latter by latitude and altitude. 


\section{A) Energy balance model}

The energy balance model (EBM) as a two dimensional model has the space variables of latitude and longitude. The advantage of a two-dimensional EBM over a one-dimensional EBM is that it has a realistic orography, and therefore includes the different aspects of heating and cooling of the continents and the oceans. This kind of model can be used to study local variations in radiation balance, and it is often used in paleoclimate studies. The variations in radiation can be due to changes both in insolation (orbital forcing) as well as in the outgoing radiation (e.g. change in albedo due to change in ice sheets, land-sea distribution). Crowley et al. (1987) used a two-dimensional energy balance model to look at the seasonal variations in the temperature of a super-continent as it might have been during the Paleozoic. North et al. (1983) used this simple model to reconstruct the Last Glacial Maximum. They found that the climate sensitivity to small changes in the solar constant were similar to those of other accepted models. Lin et al. (1992) used such a model to study the astronomical theory (Milankovitch) of ice ages. Saltzman (1990) gives a brief overview of general concepts that could help in formulating models for paleoclimate studies, including the energy balance model.

\section{B) Zonally averaged model}

Instead of looking at horizontal fields and average over altitude, this model takes the zonal average and retains the altitude. Because of this simplification, this model is easy on the computational demand. This kind of model is often coupled to the ocean and land surface. It is useful in examining climate changes as a result of changes in these fields, particularly their albedo. The changes in albedo would primarily occur as the result of changes in land and sea-ice coverage as well as the occurrence of snow. In contrast with the EBM, orography effects are not fully incorporated. This is partly remedied by splitting the zonal direction up in segments which would allow separate treatment of the land and ocean.

This model can be particularly useful for paleoclimate studies. Farrell (1990) used this kind of model to investigate the Hadley Circulation during equable 
climates, like the Eocene and the Cretaceous. Harvey (1988) studied the possible positive feedback of the increase of aerosols in the atmosphere during the Last Glacial Maximum, and Harvey (1989) evaluated the climatic effects of global changes in land glacier ice, seasonal land snow cover, vegetation and sea-ice during the Last Glacial Maximum. Bintanja and Oerlemans (1996) and Bjornsson and Mysak (2001) investigated the Last Glacial Maximum by coupling the model to a two-dimensional zonal averaged ocean model, and Tarasov and Peltier (1997) also included an ice-sheet model to such a coupled model.

\subsubsection{Three Dimensional Models}

A) General Circulation Model (Global Climate Model, GCM)

The most elaborate of all climate models is the GCM. It integrates the equations describing the motion of a fluid on a rotating sphere and includes the interactions between the atmosphere, ocean and land and the internal feedbacks of the system. The atmospheric equations of motion are the Navier-Stokes equations of motion for viscous compressible fluids. Usually the atmosphere is assumed to be in hydrostatic balance, which is a good approximation at the horizontal scales modelled in typical GCMs. Another simplification often made is the shallow-water approximation. The Earth is assumed to be spherical, resulting in only the horizontal component of the Coriolis force being retained, and any curvature components are removed ${ }^{\prime}$. Applying these simplifications on the general atmospheric equations, result in the "primitive" equations. These equations in pressure coordinates are:

Equation of Motion (Conservation of Momentum)

$$
\frac{D \mathbf{V}}{D t}+\nabla_{p} \Phi+f \mathbf{k} \times \mathbf{V}=0
$$

\footnotetext{
'With the vertical movements being much smaller than the radius, the radius is set to the mean radius of the Earth.
} 
Continuity Equation

(Conservation of Mass)

Thermodynamic Equation

(Conservation of Energy)

$$
\begin{aligned}
& \nabla_{p} \cdot \mathbf{V}+\frac{\partial \omega}{\partial p}=0 \\
& c_{p} \frac{D T}{D t}-\alpha \omega=Q
\end{aligned}
$$

Where $\mathbf{V}$ is the vector velocity, $f$ is the Coriolis parameter, $\mathbf{k}$ is the vertical unit vector, $\nabla_{p}$ is the two-dimensional gradient operator on a surface of constant pressure, $\Phi$ is the geopotential field (height times gravity), $c_{p}$ is the specific heat of dry air, $\omega$ is the vertical velocity, and $Q$ is the heating term. $\alpha=1 / \rho$ and $\omega=D p / D t . D / D t$ is the time derivative, which in pressure coordinates can be written:

$$
\frac{D}{D t}=\frac{\partial}{\partial t}+\mathrm{V} \cdot \nabla+\frac{\partial p}{\partial t} \frac{\partial}{\partial p}
$$

Together with the equations of conservation of water vapour and the hydrostatic equation ( $\partial \Phi / \partial p=\alpha)$, these equations are integrated, from a given initial state, for a rotating spherical Earth. Since these equations are prognostic (they have a time derivative), future states of the atmosphere can be calculated from its current state. There are principally two ways of integrating these equations:

- The spectral method assumes that the horizontal representation of the main variables, velocity, temperature and pressure, can be represented by truncated series of spherical harmonic functions. After solving the primitive equations for these variables, their values may be converted onto a grid on the Earth.

- The finite difference method solves the primitive equations for each grid box of a regular grid. Often these grids are defined in terms of regular latitude and longitude steps, or according to some standard map projection.

It is not common to solve the equations with the height as the vertical coordinate. For computational reasons, the solutions are often expressed on pressure, sigma or hybrid levels, or on levels of constant potential temperature. 
The vertical levels are therefore not equally spaced, but depending on the coordinate used, are concentrated around areas of most importance (e.g. planetary boundary layer, tropopause). Sadourny (1994) gives a mathematical summary of what is involved with global atmospheric modelling.

Of crucial importance is the specification of the grid on which the model will map its parameters (or the wave number cut off, in the case of a spectral model). The atmospheric features that are investigated determine the resolution of the grid. Furthermore, the size of the grid depends on the computational power and the time period over which the model is integrated. The horizontal resolution of the grid must be larger than the product of the integration time step times the phase speed of the fastest travelling wave (the Courant-Friedrichs-Levy criterion). High resolution global climate models can have a horizontal resolution varying from $100-200 \mathrm{~km}$, and have $15-30$ layers in the vertical. With the Earth Simulator coming online in March 2002, horizontal resolutions of $10 \mathrm{~km}$ (or less) are now also possible for multi-year integrations. The resolution determines which parameters can be dealt with explicitly and which need to be parameterized (see below) in a model. And given the non-linear nature of many feedbacks and parameterization schemes, it is important that the resolution should be chosen so that these schemes are most appropriate for the model. Largely because of these parameterizations, it is not true that the model necessarily performs better with increasing resolution. Various studies have investigated the relation between resolution and model accuracy (e.g. Rind, 1988: Dong and Valdes, 2000: Davies and Brown, 2001).

Although GCMs are state of the art computer climate models, they are discrete and do not mimic the continuous nature of the atmosphere and oceans. There are small-scale processes that can not be modelled, since they occur at scales well below the resolution of the model. Processes that take place on these small scales need to be "parameterized", i.e. described in terms of variables at the resolved scales. Cloud dynamics, rainfall and evaporation, sea-ice, interactions between atmosphere and ocean and interactions between atmosphere and biosphere, the effect of aerosols and trace gases, all need to be included in the model. Parameterization of these processes means that their weighted effect is 
calculated within a grid box around a model point. A lot of research is particularly aimed at improving our understanding of these small-scale processes and improving their parameterization in climate models (an overview of the dynamics of some of these small-scale processes can be found in e.g. Kantha and Clayson, 2000). The largest errors and model uncertainties are mainly associated with the incorrect inclusion of these small-scale processes in the parameterization schemes.

In atmospheric science, GCMs are often configured to model only the atmosphere (and are called atmosphere GCMs or AGCMs). AGCMs only determine the state and circulation of the atmosphere. Important variables external to the atmosphere like SSTs and sea-ice are given as boundary forcings. However, the equations of motion can also be solved for determining the state of the oceans. GCMs that model the state of the oceans are called ocean General Circulation Models (OGCM). Although the equations of motion for AGCMs and OGCMs are virtually the same, there are some distinct differences between an atmospheric and an ocean model. The most obvious difference is of course that OGCMs deal with water, not air. Where as the atmosphere is driven thermally, the ocean is driven both thermally and mechanically. The driving forces for the ocean circulation are the wind stress at the surface, heat fluxes through the surface and density variations caused by salinity variations. The hydrostatic assumption is also valid for the oceans, but now density variations are also ignored (the Boussinesq approximation ${ }^{1}$ ). Delecluse (1994) gives a good overview of what is involved with ocean modelling.

Ocean GCMs have their own mathematical difficulties. Because the ocean's higher density, relevant scales of motion in the oceans are much smaller than in the atmosphere ${ }^{2}$. Important global circulations like the Gulfstream and the Kuroshio Current take place at much smaller scales compared to the mean

\footnotetext{
${ }^{1}$ The Boussinesq approximation assumes that density is constant except in its product with the gravitational acceleration (the buoyancy term).

${ }^{2}$ The Rossby wave number, $\mathrm{R}_{0} \equiv \mathrm{U} /\left(\mathrm{f}_{0} \mathrm{~L}\right.$ ) ( $\mathrm{U}$ is the velocity scale, $\mathrm{f}_{0}$ the Coriolis parameter and $\mathrm{L}$ is the horizontal length scale), is a measure of the validity of the geostrophic approximation, and is in the ocean about $1 / 10^{\text {th }}$ of that in the atmosphere.
} 
atmospheric circulation patterns. Therefore OGCMs require a higher resolution than AGCMs in order to properly model the mean oceanic circulation, the largescale overturning circulation. Furthermore, the mean meridional oceanic transport mechanisms, the mesoscale eddies, also take place at a very small scale. It is important to include the effect of these eddies on the general overturning circulation, since the ocean eddies are the main means of transporting heat meridionally. Just like their atmospheric counterpart, these small-scale processes that take place below the grid resolution of the model need to be parameterized. Processes that need to be parameterized in OGCMs are these eddy fluxes, as well as turbulent mixing in the surface layer, and processes of diffusion and convection.

Ocean processes also take place on much larger timescales than atmosphere processes. Atmospheric motions have timespans from minutes to years. Ocean motions can vary from minutes to hundreds of years. Thus in oceanic modelling, a model needs to be "spun up" for a longer time to get to its "equilibrium" state. On the other hand, much larger timesteps are allowed than in atmospheric modelling, since the flows are much slower. Other complications for oceanic modelling are that oceans have a complex bathymetry and that there are relatively fewer data from the ocean to verify simulation output against. Sampling is very sparse and non-uniform over the oceans.

Although OGCMs are the most elaborate ocean models, simpler models are often used in studying the oceanic circulation. Examples of simple ocean models are single-basin models, solely wind-driven circulation models, box models, models describing solely the thermohaline circulation and two-dimensional models. For instance, Marotzke (1989) uses several simplified models to look at the steady-states of the thermohaline circulation in the Atlantic Ocean.

\section{B) Coupled General Circulation Model (CGCM)}

A coupled GCM includes both an atmospheric and an oceanic component. There are various scenarios in coupling an atmospheric circulation model to an oceanic model. The most complete model would be one that combines an AGCM and an OGCM. The description for each individual model is the same as for their 
stand alone cases. The CGCM has then the difficulty to combine two models that are defined for processes that have different time scales and different resolutions, and to incorporate the fluxes between them. As mentioned before, modelling the general ocean circulation requires a much longer integration time than modelling the atmosphere. Separate schemes need to be set up to pass on information, specifically heat fluxes, from the ocean to the atmosphere and vice versa at discrete intervals. Other exchanges that take place between ocean and atmosphere are the exchanges of gases (like the main atmospheric gases $\mathrm{O}_{2}$ and $\mathrm{N}_{2}$, and trace gases like $\mathrm{CO}_{2}$ and $\left.\left(\mathrm{CH}_{3}\right)_{2} \mathrm{~S}\right)$ and exchanges of fresh water (precipitation and evaporation).

For the case of atmospheric studies, simpler versions of coupled GCMs usually take a simpler ocean format. A common simplification is not to model the whole ocean circulation, but restrict the model only to the top layer, above the thermocline. This simplification still requires all the schemes describing the exchanges between the atmosphere and the ocean, but it does not require a long integration anymore, since the deep ocean does not need to be modelled. Another simplification would be to model the ocean by what is called a "slab ocean". This kind of ocean only exchanges heat fluxes with the atmosphere. No ocean circulation is modelled, so this form of "coupled" modelling would therefore not be called a CGCM.

\section{C) Regional Climate Model (RCM)}

The enormous amount of computation that is required to model the atmosphere of the whole Earth restricts the model's resolution, both in the horizontal as well as in the vertical. The higher the resolution, the more calculations are required. A common resolution for global climate models these days is about $250-300 \mathrm{~km}$ in the horizontal with $15-20$ levels in the vertical. Of course with this kind of resolution, it is not possible to obtain a detailed output for any specific area. Topography is smoothed, and any local orographic effects are not taken into account. It is often desirable to have a more detailed output for a smaller area. Regional Climate Models do just that. Regional Climate Models are essentially the same models as GCMs, but configured to run over a limited 
(non-global) domain. The initial and boundary conditions at the edge of the RCM domain are given by the GCM, and the RCM simulates the circulation in its domain. Nesting a RCM within a GCM in grid point space is the most common approach (Giorgi, 1990: Fenessy and Shukla, 2000), but Kida et al. (1991) point out that it is also possible to link global and regional models with spectral boundaries. This process of having a higher resolution model sitting within a lower resolution model can be repeated again, so that consecutively higher resolution models can sit within each other (an example is in McGregor and Walsh, 1994). This is referred to as "multiple nesting".

The main reason to downscale a GCM to an RCM region is to improve the realism of the circulation on a smaller scale. The use of a RCM does not automatically mean that it provides a better capability in simulating regional climates, since often improvements in physical parameterizations of the GCM can be more important. A RCM should add detail that is the result of its own structure, and which could not have been obtained by simply interpolating the GCM to a higher resolution. It should not just enhance geographical detail, but should be capable of determining the internal variability of the regional domain.

Regional modelling allows us to examine the local effects of global variations. The importance of regional modelling has been underlined by the IPCC in that regional issues have received prominent attention in their reports (IPCC, 2001). This thesis uses a regional climate model (chapter 6 and 8) to investigate the climate in New Zealand during the LGM.

D) Earth System Model (ESM) and Earth System Model of Intermediate Complexity (EMIC)

The latest generation of three dimensional climate models are the so-called "Earth System Models". Building on GCMs, which are based on describing the full dynamics of atmospheric and oceanic motion, ESMs also describe a relatively complete set of interactions between climate system components. Interactions between the atmosphere, ocean, cryosphere and biosphere are modelled as a different set of systems. Various modules can be linked to the model, like ice-sheet, vegetation, and chemistry modules. The number of 
interactions within and between each system and module, and the resulting feedbacks are enormous ${ }^{1}$. Hence, ESMs are computationally much more demanding than GCMs. Simpler versions of the ESMs are the "Earth System Models of Intermediate Complexity" (EMICs). EMICs can be characterized by the number of interacting components they use in describing the Earth (Claussen et al., 2002). EMICs include most of the processes used in GCMs, but they are now more in parameterized form. This simplification enables EMICs to either simulate feedback processes between as many components of the climate system as possible and are very well-suited to very long term climate simulations. Ganopolski et al. (1998) used an EMIC to simulate the Last Glacial Maximum, and integrated the system for 5000 model years to compute an equilibrium climate.

Although it might be obvious that higher order climate models might be more complete, there are several issues one has to think about before deciding on a climate model. As mentioned before, the computational costs can be enormous and therefore often due to financial constraints, simpler climate models are chosen. Other ways to reduce computational costs is to reduce the length of integration or the number of feedbacks in the climate system.

At the same time, a climate modeller needs to know exactly what is wanted as output. He needs to decide on which climate model is most suitable. But no matter what the constraints are, any climate model needs to be tested upon its capability to model the climate correctly. A climate model should be tested against a known climate from the past. If the model can successfully reconstruct past climates, you can be more confident that the model is capable of reconstructing today's climate or predict future climate states.

The subject of this study is to investigate the climate of New Zealand during the Last Glacial Maximum. For this, a Regional Climate Model had to be defined. This RCM is nested within a GCM. The following sections will describe the model used for this study and its different configurations.

\footnotetext{
' An example of this can be found in the overview that Randall, Khairoutdinov et al. (2003) gave on cloudsystem resolving models and their affect on the running of a GCM (they call them super-GCMs).
} 


\subsection{The Unified Model}

The model used in this study is based on the Hadley Centre's (United Kingdom Met Office, UKMO) Unified Model (Cullen, 1993), or in short, the UM. The UM is used for numerical weather prediction, oceanographic forecasting, climate prediction and middle atmosphere research. It supports global as well as regional models. It can run in atmosphere or ocean only modes as well as in coupled versions of them. It can be linked to other models, like cryosphere and biosphere models. It can also include a whole range of "external" data (ancillary data), such as land surface data (e.g. vegetation, soil moisture, root depth), ocean data (e.g. SSTs, sea-ice) and other atmospheric variables (e.g. ozone, chemistry, aerosols).

The UM integrates the main atmospheric equations (conservation of momentum, mass and energy. See section 4.2.4.). The equations are integrated using a hybrid vertical coordinate (Simmons and Burridge, 1981). The hybrid coordinates are terrain-following sigma coordinates (normalized by the surface pressure) in the bottom layers, pressure coordinates in the top layers (where they are almost horizontal), and a linear combination for the layers in between. The advantage of having terrain-following coordinates is that it is much more convenient in the lower layers of the atmosphere, while pressure coordinates are more likely to give accurate results in the upper layers. Unlike many other models, the UM does not make the shallow atmosphere approximation (see section 4.2.4) and retains all three components of the Coriolis force. The UM uses a split-explicit finite difference integration scheme (Cullen and Davies, 1991). Also unlike some other models, the UM does include the fast travelling gravity waves. The splitting now refers to the separation of the fast moving vertical advection term (a basic state temperature) and the terms associated with the slower moving horizontal advection by the winds (e.g. pressure, temperature and surface winds). The UM regularly uses three adjustment steps per advection step. The average wind fields can then be used to determine the total advection. The UM uses an Arakawa-B grid, where pressure and thermodynamic variables are located at the centre of a grid box, and velocity components on the corners. 


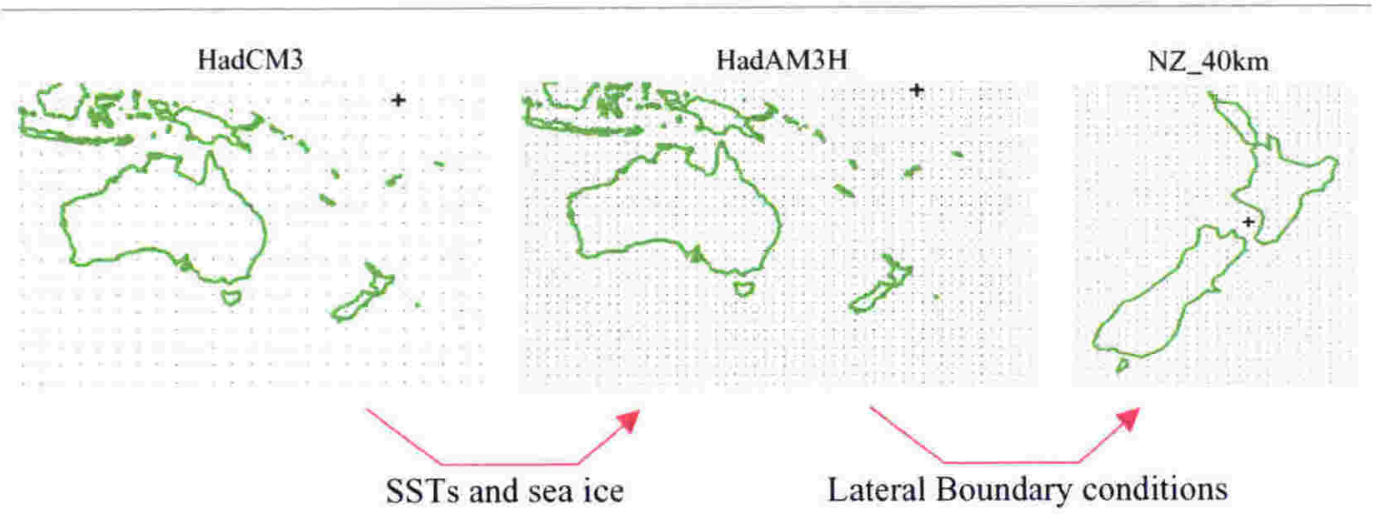

Fig. 4-4. The sequence of the models. The GCMs only show the area of Australasia and the RCM is greatly zoomed in as well. Each dot is a grid point of that particular model. The coupled model HadCM3 supplies the SSTs and sea-ice for the atmospheric model HadAM3H, which in turn supplies the lateral boundary conditions for the regional model NZ_40km.

This thesis is for a large part built on the output of HadCM3 (Hadley Centre

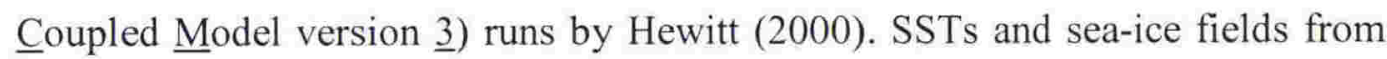
these simulations were used as forcings in this study. Many atmospheric parameter settings were set at the same values as in Hewitt's (2000) simulations. Differences in atmospheric settings are specified in section 4.3.2. A higher

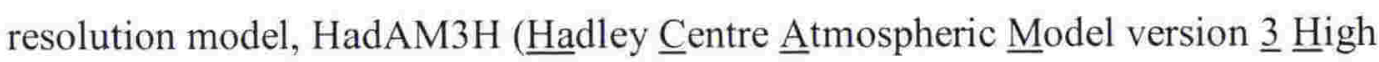
Resolution), capable of simulating the climate in the Northern Hemisphere more realistically was made available at the start of the study, and it was decided to use that model. Several different RCM configurations were tested but only the RCM with a $40 \mathrm{~km}$ resolution (NZ_40km) was used in this study. The lateral boundary conditions were supplied by HadAM3H. The place of the three models in this study is schematically presented in Fig. 4-4. A description of the models follows.

\subsubsection{HadCM3}

The main model was HadCM3, which is a tested and proven model (Gordon et al., 2000). All other models used in this study were merely different configurations of HadCM3. HadCM3 is a coupled atmosphere-ocean GCM. The coupled OAGCM (Ocean-Atmosphere GCM) couples the atmospheric part (HadAM3) to the ocean part (HadOM3) and a sea-ice model, once a day, every 
day. The atmospheric model runs first for one day, during which all the atmospheric-ocean fluxes are calculated. These are then passed on to the ocean and sea-ice model. These models are then integrated and the ocean surface fluxes are passed back to the atmospheric model, after which the atmospheric part integrates the next day. This cycle repeats itself for the duration required.

This study did not run the coupled model, but used the SSTs and sea-ice values of a coupled model simulation with similar settings as in this study, to force a higher resolution atmospheric model (A detailed description of this simulation is presented in Hewitt, 2000). Of more importance to this study is the atmospheric component (HadAM3) of the coupled model since the highresolution atmospheric model used in this study $(\operatorname{HadAM} 3 \mathrm{H})$ is basically the same. HadAM3 is discussed in section 4.3.1.1, and is followed by only a short description of the ocean model (section 4.3.1.2). Section 4.3.2 deals with HadAM3H but discusses only those settings that are different than HadAM3.

\subsubsection{Atmospheric model: HadAM3}

The atmospheric model has a horizontal resolution of $2.5^{\circ} \times 3.75^{\circ}$. This is the equivalent of a resolution of about $278 \mathrm{~km}$ in the meridional direction and a zonal resolution of about $417 \mathrm{~km}$ at the equator, increasing to a resolution of about $295 \mathrm{~km}$ at $45^{\circ}$. This produces a global grid of $73 \times 96$ points. There are 19 levels in the vertical in hybrid coordinates. The levels are spaced unevenly with regions of more detailed vertical structure covered by more levels (e.g. the boundary layer is covered by 5 levels: 1000, 994, 956, 905, and $835 \mathrm{hPa}$ ), with the highest level set at $5 \mathrm{hPa}$. The integration step is 30 minutes and the model uses a 360 day calendar. A full description of HadAM3 is given in Pope et al. (2000). Only the basic features of the model are summarized below.

\section{- Radiation:}

The radiation scheme used is the one by Edwards and Slingo (1996) and modified by Cusack and Edwards (1999). This scheme uses the two-stream approximation in both the long-wave and short-wave radiation. Instead of 
looking at the whole radiative spectrum, the radiation scheme splits the spectrum in discrete intervals. These bands correspond to the main radiative bands of the main atmospheric gases. In the long-wave, absorption is due to $\mathrm{H}_{2} \mathrm{O}, \mathrm{O}_{3}, \mathrm{CO}_{2}$, $\mathrm{CH}_{4}, \mathrm{~N}_{2} \mathrm{O}, \mathrm{CFC} 11$ and $\mathrm{CFC} 12$, and in the shortwave, absorption is due to $\mathrm{H}_{2} \mathrm{O}$, $\mathrm{O}_{3}, \mathrm{CO}_{2}$, and $\mathrm{O}_{2}$. There are eight long-wave bands and six short-wave bands. Where there is more than one gas in a band, gas overlap is treated in the manner similar to that described by Edwards (1996). The effect of clouds on the radiation balance is modelled using the method of Slingo (1989). Furthermore, the effect of aerosols on the radiation balance is included through the scheme of Cusack et al. (1998) which incorporates water-soluble, dust, soot, and oceanic and stratospheric sulphates. Inclusion of this scheme improves the model's global and annual mean solar radiation budget at the surface.

\section{- Clouds:}

Although clouds have a considerable influence on the radiation balance of the atmosphere, their representation in GCMs is still being investigated. The radiative properties of clouds (transmissivity, reflectivity, and absorptivity) depend on their water content, the phase of the water (liquid or solid) and the size distribution of water droplets or ice particles. Incorporating the effect of clouds in HadAM3 is achieved through a scheme that separates the dependence of the cloud radiative properties on the (liquid) water content and equivalent radius of the drop size distribution (Slingo, 1989). Smith (1990) turned this into a prognostic cloud scheme where the cloud radiative properties are calculated interactively. Cloud layers consisting of water droplets, ice particles, or a combination of them (mixed phase clouds), are treated separately. Cloud formation depends on a probability distribution of a saturation variable in a grid box. The advantage of this scheme is that large-scale clouds can form before mean saturation takes place in the grid box. Gregory and Morris (1996) updated this scheme to let full glaciation occur at a higher temperature $\left(\right.$ at $-9^{\circ} \mathrm{C}$ instead of $-15^{\circ} \mathrm{C}$ ). This resulted in an increase of potential ice particles in the cloud layer. Consequently, depletion of cloud water within the region of mixed phase cloud happens faster, since fallout of ice particles is unconstrained by a critical value of 
cloud ice content as it is in the rain parameterization.

\section{- Precipitation/evaporation:}

Models that do not include a prognostic cloud water variable precipitate all condensed water as soon as it forms. In Smith's (1990) prognostic cloud water scheme, the rate of conversion of cloud liquid water to precipitation is parameterized. The precipitation scheme treats liquid and ice clouds differently, since the amount of cloud condensation nuclei $(\mathrm{CCN})$ to make up water droplets and ice nuclei differ. Since only a few CCNs will act as ice nuclei, cloud water in ice clouds will attach over fewer particles, and therefore grow bigger faster, resulting in them to detrain quicker and easier. The model distinguishes between the possible occurrence of precipitation over land or over sea, reflecting the differences in cloud condensation nuclei over the different areas. Evaporation of rain and snow is described by the scheme developed by Gregory (1995), which deals with precipitation both on grid-scale (stratiform clouds) as well as on subgrid-scale (convective clouds).

\section{- Convection:}

Convective processes are important phenomena in weather and climate studies since they affect the large-scale atmosphere through subsidence in the cloud environment and detrainment of heat, moisture and liquid water from convective towers. The convection scheme used is the mass flux convection scheme as described by Gregory and Rowntree (1990). Because clouds come in a large variety, rather than try to model the convective effect of each cloud type, this scheme uses a single cloud model. This single cloud model is set up so that it represents the effects of an ensemble of clouds where the clouds' varying characteristics and depths are taken into account by allowing a proportion of the ensemble to undergo complete detrainment before the maximum cloud height has been reached. The convection scheme is applicable to moist convection of all types (shallow, deep, and midlevel) as well as to dry convection. The model is updated to include downdraughts as well as described by Gregory and Allen (1991). 
Convection also affects the vertical transport of horizontal momentum in the atmosphere. The scheme used to model convective momentum transport is the one from Gregory et al. (1997). Their scheme is based on the mass flux convection scheme mentioned before. It includes a simple parameterization of the effects of pressure gradients within a cloud to the flow within a cloud. This result in the cloud characteristics being better defined.

\section{- Gravity-wave drag:}

Orography acts as an obstacle to air movement. When the atmospheric flow moves over mountains, stress is exerted. This stress is proportional to the subgridscale variance of orography and the wind speed. In stable conditions, the stress on the flow will excite waves. These gravity waves travel upwards, thereby reducing the static stability and increasing the vertical wind shear. The wave will break when the Richardson Number ${ }^{1}$ falls below a critical value. When the wave breaks, it exerts a drag which leads to instability of the atmospheric flow. HadAM3 uses the scheme developed by Gregory et al. (1998), which incorporates low-level wave-breaking associated with lee waves and anisotropic orography. Inclusion of this scheme reflects mainly the seasonal change to the flow as the result of the orographically induced drag. Just like deep convection, orographically forced gravity waves play an important role in the momentum budget in the global circulation.

\section{- Boundary layer:}

There are many processes taking place within the boundary layer that influence the dynamics of the atmosphere. They transport momentum, heat, moisture, aerosols and pollutants mainly in the vertical direction. The influence of the boundary layer processes is parameterized using the scheme of Smith (1990). Defining the predicted cloud water variable enables the model to formulate the model's boundary layer mixing in terms of variables that are conserved during the phase changes of water, rather than to formulate some physical processes

\footnotetext{
${ }^{1}$ The Richardson number is a dimensionless ratio of buoyant suppression of turbulence to shear generation of turbulence.
} 
independently or with no explicit regard to cloud at all. The scheme has two parts: the surface exchange scheme which determines the turbulent transport in the bottom layer (from the surface to the atmosphere), and the boundary layer turbulent mixing scheme which determines the turbulent transports above the surface layer.

\section{- Land Surface Scheme:}

The scheme used is MOSES (Met Office Surface Exchange Scheme: Cox et al., 1999), which is a 4-layer hydrology and thermodynamics model. It simulates the freezing and melting of soil moisture and the soil thermal characteristics are realistic functions of soil moisture content (e.g. specific heat capacity and volumetric concentrations of water and ice, soil thermal conductivity). The land surface albedo depends on the vegetation type and snow amount. The total evaporation depends also on the amount of evapotranspiration, which in turn depends on the vegetation type. Evapotranspiration depends on the stomatal resistance to temperature, $\mathrm{CO}_{2}$ concentration and vapour pressure. Vegetation type and vegetation cover are included in the model through an external data file which contain parameterized values for the vegetation characteristics. Land Surface Schemes are fairly new and the biological cycle's influence on climate has only recently become a component in climate models.

\section{- Ozone:}

Ozone is found predominantly in the stratosphere, but is also an important tropospheric gas. Mainly known for its blocking effect on ultraviolet radiation, it is also a good absorber of solar radiation in the near infra-red. Absorption of solar radiation by ozone heats the middle atmosphere and causes the temperature increase with height that defines the stratosphere and tropopause. HadAM3 uses the 4-dimensional ozone climatology of Li and Shine (1995), which is based on both satellite measurements of the stratosphere, and some ground based measurements of the troposphere. 
Other inputs that are required for the model are the landmask and the orography file, which define respectively which gridpoints are defined as land or water in the model, and the orography used in the model. Many parameters, like diffusion, damping coefficients, critical humidity, trace gases (particularly $\mathrm{CH}_{4}$ and $\mathrm{CO}_{2}$ ), are variables that need to be defined in the model setup itself. Some of these fields will be discussed in more detail in the sections dealing with the settings of each simulation.

\subsubsection{Ocean model: HadOM3}

The Navier-Stokes equations on a rotating sphere are solved with the Boussinesq approximation (see section 4.2.4), the hydrostatic approximation and the turbulent viscosity hypothesis (approximate all the stresses generated at subgrid scales that are not resolved as an enhanced molecular mixing). The "primitive equations" describe the large-scale ocean circulation. The rigid lid approximation is used, which means that fresh water fluxes at the surface do not lead to changes in the volume of the ocean. The circulation is solved for the barotropic and baroclinic parts separately. The model has a horizontal resolution of $1.25^{\circ} \times 1.25^{\circ}(144 \times 290$ grid points which corresponds to about $140 \times 140 \mathrm{~km})$ and there are 20 levels in the vertical. The layers are stacked so to give the highest resolution in the top part of the ocean where most of the dynamics take place.

Driving forces are again radiation and momentum variations, but now also salinity variations in the ocean. The main transport mechanisms are diffusion and advection. Energy (heat) and salinity are transported along isopycnal (constant density) surfaces. Since these surfaces do not coincide with the model's grid, the model runs a "along isopycnal diffusion scheme". Just as with the atmospheric model, the ocean model needs particular schemes and parameterizations to simulate subgrid dynamics, e.g. vertical mixing of momentum and tracers, eddy mixing, and overflow schemes. Sub-grid parameterizations are even more important for ocean modelling than that they are for atmospheric modelling, because most of the thermodynamic processes as well as all the energetic 
mesoscale variability, take place at sub grid resolution. For a detailed description of HadOM3, and the coupling to HadAM3, see Gordon et al. (2000).

\subsection{2. $\quad$ HadAM3H}

HadAM3H is an implementation of HadAM3 at double the horizontal resolution. A description of HadAM3H is given by Williams et al. (2001) and Hudson and Jones (2002a). The differences with HadAM3 are discussed below.

\section{- Resolution:}

Pope and Stratton (2002) describe how the output from UM models (including HadAM3) vary with resolution. It was clear that systematic errors decreased when increasing the resolution from HadAM3. Basic model features, like horizontal winds, temperatures, moisture, vertical velocities and transients all improved, although precipitation biases increased. For this thesis it was decided that the higher resolution model was suitable. HadAM3H's horizontal resolution is double the resolution of HadAM3 in both latitudinal and longitudinal direction $\left(1.25^{\circ} \times 1.875^{\circ}\right)$. This gives a resolution of $139 \mathrm{~km}$ in the latitudinal direction and a longitudinal resolution varying from around $200 \mathrm{~km}$ at the equator to about $150 \mathrm{~km}$ at $45^{\circ}$. This produces a global grid of $145 \times 192$ points. There is no change in the vertical resolution. To stay within the constraints of the CFL criterion, the integration step was halved compared to HadAM3, to 15 minutes.

\section{- Radiation:}

Several changes made in HadAM3H affect the radiation schemes. Included in HadAM $3 \mathrm{H}$ is a scheme that treats the radiative effects of convective anvil cirrus (Gregory, 1999). The scheme increases both long-wave and short-wave forcings in the tropics and causes a warming of the upper tropical troposphere. Included is also a full radiative coupling of the vegetation canopy to the soil surface. And in order to generate a more realistic radiation balance, some of the cloud parameters have been altered. The time constant for conversion of cloud liquid water to precipitation has been reduced, the ice-fall speed has increased, and the threshold 
for conversion of layer cloud water to precipitation has been doubled over the ocean and increased by a factor of 2.5 over land.

\section{Clouds:}

Included in HadAM3H is a scheme representing anvil clouds (Gregory, 1999). HadAM3H allows vertical variation of cloud amount. In the case of deep convection (where the top of the clouds extend above the freezing level), cloud fraction is increased linearly with model level from the freezing level to the cloud top level, representing the anvil cloud, and decreased to a constant value below the freezing level, to represent the convective tower. The effect of the scheme is that it reduces some of the cloud water, resulting in less thick clouds. Anvil clouds show an increase in warming at the base of the cloud, and an increase in cooling at the top of the cloud. These radiative effects are dealt with in the radiation scheme (see above: radiation).

For cloud formation to occur within a gridbox, a critical relative humidity value has to be exceeded. Rather than to have one static value (grid cell averaged cloud amount), or using a cloud overlapping scheme (e.g. Ridout et al., 1994), HadAM3H includes a scheme that parameterizes the horizontal variance of saturation within a gridbox (Cusack et al., 1999b). This parameterization predicts the subgrid variability from the resolved variability on the climate scale. The critical relative humidity now depends on the spatial and temporal variability of relative humidity.

Furthermore, HadAM3H includes a parameterization that adjusts the cloud fraction to 0.6 rather than to 0.5 when the gridbox specific humidity reaches saturation, and the values of some cloud parameters have been adjusted (see above: radiation).

\section{- Chemistry:}

One of the main optional additions is the inclusion of a simple sulphur cycle with representation of the effect of the sulphate aerosols (mentioned in the internal technical note of Williams et al., 2001). However, as will be mentioned in chapter 5 , this feature was switched off in this study. 


\section{- Precipitation:}

It is known that the UM has difficulties in modelling the precipitation in areas with strong uplift, like in the Indonesian Archipelago. As indicated before, although the higher resolution models show some improvement in climate modelling, the errors in the precipitation increased. To counter this effect, as mentioned under radiation, the threshold of cloud liquid water for the formation of precipitation over sea was doubled and over land multiplied by two and a half. The fraction of a grid box on which precipitation falls was adjusted.

\section{- Diffusion:}

The diffusion parameter has been increased (to increase damping) to prevent the accumulation of energy at the smaller grid scale.

\subsubsection{NZ_40km}

$\mathrm{NZ} 440 \mathrm{~km}$ is the name given to the RCM configuration that is used in this study for regional simulations over New Zealand. It is nested within HadAM3H, as discussed before (see section 4.2.4 C). The dynamics and physics of the RCM NZ_40 km are the same as in the GCM HadAM3H, except for certain parameterization constants that had to be set differently to account for the higher resolution of the RCM (Hudson and Jones, 2002a: 2002b).

When constructing a regional domain for the regional climate model by following longitude and latitude, it is inevitable that the area is distorted away from a perfect rectangular shape towards a pyramid shaped area the nearer you get to the pole, where there is a "convergence" of the meridians. This can cause problems in calculating the atmospheric circulation in the domain. These problems are mainly related to the CFL-criterion. The UM already uses Fourier filtering at high latitudes in the global models to avoid the need for a very short time step. The need for filtering in regional models is avoided by an appropriate choice of the coordinate pole, so that no part of the region lies at latitude greater than $45^{\circ}$. The coordinate pole is displaced away from the Earth's North Pole in such a way that the equator of the rotated grid passes through the centre of the 


\section{Chapter 4: Climate Modelling}

\begin{tabular}{|l|c|}
\hline Latitude Rotated Pole & 49.5 \\
\hline \hline Longitude Rotated Pole & 175 \\
\hline \hline Latitude top left corner of region in relation to the rotated pole & 12 \\
\hline \hline Longitude top left corner of region in relation to the rotated pole & 164.5 \\
\hline \hline Number of grid points East-West & 95 \\
\hline \hline Number of grid points North-South & 68 \\
\hline \hline Horizontal grid point spacing (in degrees) & 19 \\
\hline \hline Number of vertical levels & 19.36 \\
\hline
\end{tabular}

Table 4-1. Coordinates of the regional domain NZ 40km.

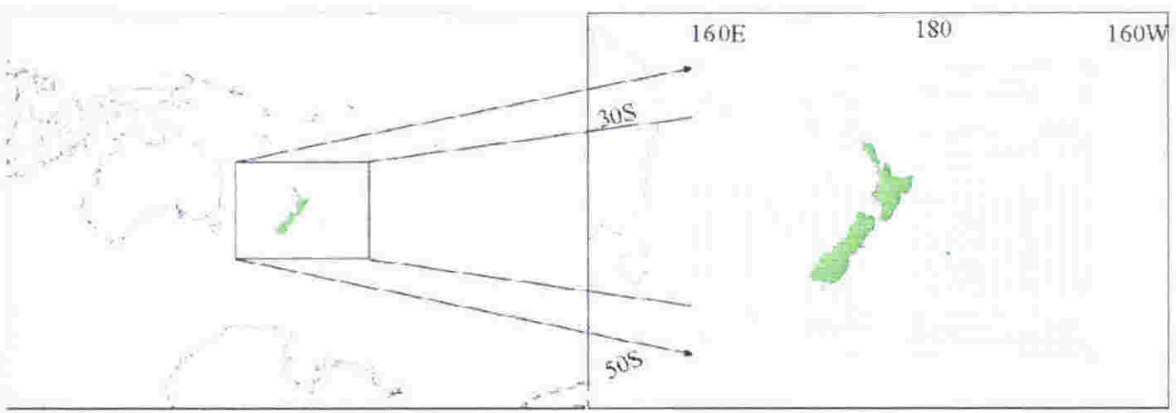

Fig. 4-5. New Zealand in the South-West Pacific and the Regional Domain NZ_40km with all grid points. The rectangular grid is the shape of the grid after rotation, and this rotated grid is presented here at its coordinates of origin.

regional domain. Coordinates for the new pole are then selected so to give a near uniform grid spacing over the regional domain. The result is that in the rotated coordinates, the regional domain remains virtually a rectangular shape. After the integrations, the grid can be rotated back to its original position. The regional model has 95 grid points in the East-West direction, and 68 in the North-South. The horizontal resolution is $0.36^{\circ}$, which is equivalent to $40 \mathrm{~km}$. New Zealand's landmass (the North and South Islands and Stewart Island) is modelled by 180 points ${ }^{1}$. There are 19 levels in the vertical. Table 4-1 gives an overview of the coordinates of the regional domain of NZ_40km, and Fig. 4-5 shows New Zealand's position in the South-West Pacific and the regional domain with all the grid points of the model.

1 The GCM HadCM3 covered New Zealand only by three points, and HadAM3H covered New Zealand by nine points (see Fig. 4-4). 
The global model supplies the lateral boundary conditions for the regional model. The prognostic variables that are passed on to the regional model are the surface pressure, the horizontal wind components ( $u$ and $v$ ), the specific total water content (summation of the specific humidity, specific cloud water and cloud ice content), and the liquid water potential temperature (liquid water temperature normalized by the Exner function). These variables are passed on to the regional model at regular intervals by linearly interpolating their values to grid points at the rim of the regional model. Within this "rim" zone, the values are averaged, based on their values within the regional and global grid. The effect of weighing the lateral boundary conditions assures a smooth transition from global to regional grid, and reduces any spurious effects "extreme" boundary conditions can have on the regional model. The model has a rim of four grid points.

The regional model uses "external" data representing New Zealand, such as the orography, landmask, vegetation and soil surface parameters. Sea surface temperature and sea-ice are interpolated from the global simulation. New Zealand is a tectonically active country, which results in very steep mountains and numerous volcanoes. There are several mountain peaks higher than $3000 \mathrm{~m}$ with Mt Cook the highest mountain in Australasia with its height of $3754 \mathrm{~m}$. However, with a resolution of 40 kilometres, many features of the New Zealand landscape are smoothed or averaged out. At the modelled resolution, the highest point in the model is just less than $1800 \mathrm{~m}$ and the mountains of the Southern Alps vary in height between 1100-1500m. New Zealand also has many reasonable sized lakes (up to $600 \mathrm{~km}^{2}$ ), but they too disappear at the resolution used. This has consequences for other input variables like soil moisture and vegetation. Both of these variables and the parameters derived from them are simplified compared to reality.

Although the orography at the modelled resolution is less steep than in reality, there is still a significant gradient from sea to mountain in the model. It is known that problems can occur in the calculations of the vertical fields as the result of rapid uplift, as occurs when the atmosphere moves over a mountain range. For that reason, often a very small integration time step is used, in order to 
stay within the CFL criterion. The integration time step used for the regional model is 4 minutes.

In section 8.1 we will see that the orography during the LGM was quite different than it is today. Due to the extensive coverage of the glaciers in the South Island, which were several kilometres thick at some places, the averaged height of the orography at the resolution modelled goes up to well over 2 kilometres, with the Mt Cook area 3 kilometres high. This results in an even steeper gradient in the orography, and which could be of concern when calculating the vertical fields. 


\section{Pre-industrial simulation:}

\section{Global}

\section{Introduction}

This study is to investigate the climate during the Last Glacial Maximum, and then in particular New Zealand's climate during the LGM. Since regional climates are influenced by the mean state and the fluctuations of the general circulation they are embedded in, it is essential to understand the general circulation first before studying regional features. HadAM3H is used to reconstruct the general circulation and HadRM3H $\left(\mathrm{NZ} \_40 \mathrm{~km}\right)$ is used for the regional model. At the same time, when using a climate model to make statements about past and future climates, it is important to understand the behaviour of the model. What are the model's limitations and biases? The performance of the basic model HadAM3 (Pope et al., 2000) and the impact of new physical parameterizations in the model, including on the higher resolution configuration of HadAM3H (Pope and Stratton, 2002: Hudson and Jones, 2002a), have been well documented. This study makes use of the findings of those studies, and the reader is referred to those papers for a detailed discussion on the relevancy of the models.

In order to be able to ascribe findings of the LGM simulations (chapter 7 and 8) to either the climate of the LGM or to a bias in the models, following Hewitt (2000), the global and regional model are first described under a "control" 
simulation. Together with the analysis on the behaviour of the models described in the papers mentioned above, features of the simulated climate of the LGM can be better qualified. Chapter 5 discusses the GCM and chapter 6 deals with the RCM. Although HadAM3H is a global model, most of the emphasis in this study is on its ability in modelling the Southern Hemisphere circulation. Some particular climatic features are discussed in more detail.

Instead of running $\mathrm{HadAM} 3 \mathrm{H}$ under present day conditions, it was tested in a pre-industrial setting. The advantage of running the model under a pre-industrial setting is that you can assume that the tropospheric chemistry can be kept constant. "New" aspects of the climate that have featured over the last century, for instance the ozone hole and global warming, are therefore not included. The assumption however is that the general circulation during the pre-industrial period was not that much different than today and that therefore direct comparisons can be made between the model's output and today's climate, at least qualitatively. For this study, the 1971-2000 National Centers for Environmental Prediction's (NCEP) reanalysis (Kalnay et al., 1996) climatology was used to compare the model's output. Although the NCEP climatology is based on a very comprehensive reanalysis, it is still not well constrained poleward of $40^{\circ} \mathrm{S}$, with the largest differences close to the surface and decreasing rapidly with height (Kistler et al., 2001).

\subsection{Setup of HadAM3H}

The initial conditions came from HadCM3 under a pre-industrial setup. HadCM3 was run for 500 years, after which it was determined that the model had run to an equilibrium state (Hewitt, 2000). Most of the fields required for HadAM3H were directly taken from HadCM3. Global fields from HadCM3 were interpolated onto the $\operatorname{HadAM} 3 \mathrm{H}$ grid using bilinear interpolation. Some parameters and fields needed for HadAM3H are mentioned below. They get a special mention here since most of these fields will be different for the LGM simulation. 


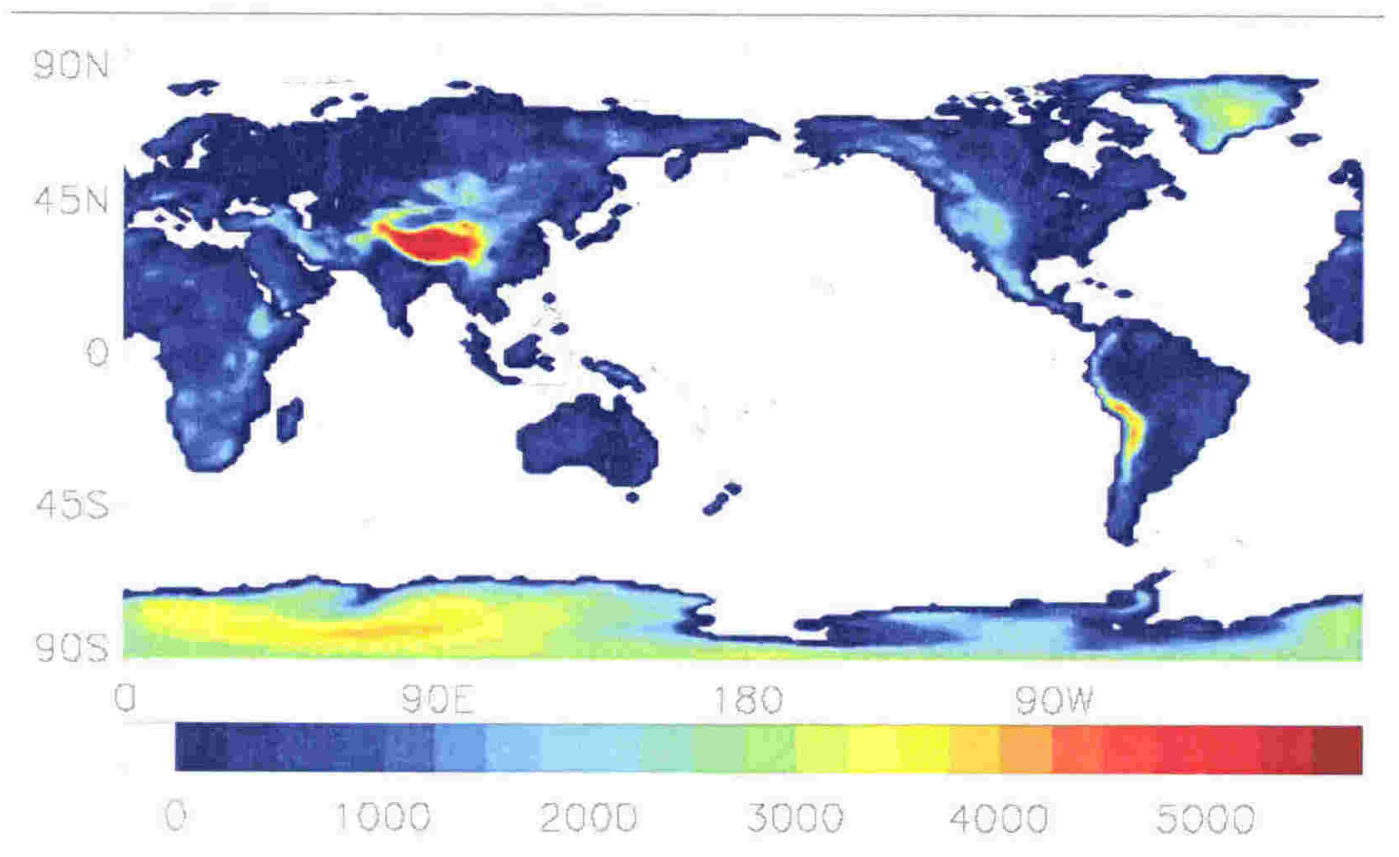

Fig. 5-1. The global orography in the pre-industrial simulation (elevation in m.).

\section{- Orography:}

HadAM3H's orography differs from HadCM3's as the result of it being at a higher horizontal resolution. Simply interpolating HadCM3's orography onto HadAM3H would not give all the new landpoints correctly. The original dataset, the US Navy 10" resolution dataset (Joseph, 1980), was interpolated onto the HadAM3H grid.

As was pointed out by Palmer et al. (1986), increasing the model resolution induces a bias in the westerly wind, which enhances the instability of the atmosphere. This instability can be countered by a reduction of the surface stress, which will reduce the surface drag of the atmosphere. Consequently, this will reduce the vertical shear and therefore will inhibit baroclinicity, The gravity wave drag coefficients were therefore reduced to account for the higher resolution of orography (Pope and Stratton, 2002).

\section{- SST and sea-ice:}

SSTs and sea-ice distribution and concentration for the pre-industrial simulation were updated monthly and were provided by HadCM3. To determine these fields for the higher resolution grid of $\operatorname{HadAM} 3 \mathrm{H}$, the fields were first interpolated 
onto a resolution grid equal to HadCM3's resolution, but without any landmasses. After determining the SSTs and the sea-ice fields for this "ocean" world, these fields were interpolated onto the grid of $\operatorname{HadAM} 3 \mathrm{H}$. HadAM3H landmass distribution was then placed on top of these fields. Since no dataset exist for SSTs in pre-industrial times, no proper validation can be made about the accuracy of these values. The AGCM (HadAM3H) is of course very sensitive to SST and sea-ice forcings, hence any biases in HadCM3 fields will be reflected in HadAM3H. Some impacts of these SSTs and sea-ice fields on the atmosphere will be discussed in section 5.2.1.

\section{- Chemistry and ozone:}

As mentioned before, the advantage of running the model under a pre-industrial setup, is that it simplifies the model setup considerably. $\mathrm{CO}_{2}$ was kept constant at $280 \mathrm{ppm}, \mathrm{CH}_{4}$ at $792 \mathrm{ppb}, \mathrm{N}_{2} \mathrm{O}$ at $285 \mathrm{ppb}$, and the interactive sulphur cycle was excluded. As the result of this, any introduced trends in the atmospheric fields are solely due to trends in SSTs. The ozone fields are defined for 19 levels, and are given in monthly zonal means.

\section{- Vegetation and soil:}

The assumption is that the vegetation during the pre-industrial period was not that much different than today, and that in this simulation the present day global vegetation can be used. For the vegetation data, the Wilson/Henderson-Sellers (1985, WHS) data was used. The $1^{\circ} \times 1^{\circ}$ dataset was interpolated onto the HadAM3H grid.

The model was run for 30 model years. The model stabilized within a few months, but it was decided arbitrarily to analyse only the last 21 years of the simulation. In section 5.2 the results are discussed, with the emphasis on the Southern Hemisphere circulation, and in particular on aspects affecting New Zealand's climate. 


\subsection{Results}

HadAM3H's output is compared to the NCEP reanalysis data from the period 1971-2000. The procedure used was to interpolate the NCEP reanalysis data (resolution of $2.5^{\circ} \times 2.5^{\circ}$ ) onto the HadAM3H grid (resolution of $1.875^{\circ} \times 1.25^{\circ}$ ) in order to be able to make direct comparisons between the various fields. A perfect agreement between the two datasets is not expected, since HadAM3H was set up for a pre-industrial climate, and the monthly updated SSTs and sea-ice fields were derived from HadCM3, and not from observational data. Nevertheless, general circulation patterns are expected to be similar. In this chapter, some of the main fields are analysed. Where there are major differences between the model output and the reanalysis dataset, a more detailed analysis will discuss possible causes of the anomaly.

\subsubsection{Sea Surface Temperature and Sea Ice}

The computed SSTs for the pre-industrial simulation are for each season very similar to the NCEP climatology. The main differences between the two datasets are in the high latitudes and in the North Pacific (Fig. 5-2). In the North Pacific, HadCM3 shows substantially lower SSTs than the NCEP reanalysis. Gordon et al. (2000) discussed the cool SSTs in HadCM3 in the North Pacific, which they ascribed to the poor simulation of surface heat fluxes in that region. The differences in the high latitudes SSTs can be related to the differences between the sea-ice extent of HadCM3 and the NCEP climatology. The differences in SST and sea-ice between $45^{\circ} \mathrm{S}$ and $75^{\circ} \mathrm{S}$ show a correlation of -0.93 for austral winter and spring, and -0.73 for the austral summer and autumn, for a difference in sea-ice fields of 0.3 and higher ${ }^{1}$. For the Northern Hemisphere between $60^{\circ} \mathrm{N}$ and $89^{\circ} \mathrm{N}$, these values are -0.80 and -0.41 . This implies that lower SST values in HadCM3 are the result of greater sea-ice extent in that area, and vice versa.

\footnotetext{
I The amount of sea ice within a gridbox is given as a percentage of the total gridbox area: 0 means that there is no sea ice present within the gridbox, 1 means the gridbox area is fully covered by sea ice.
} 


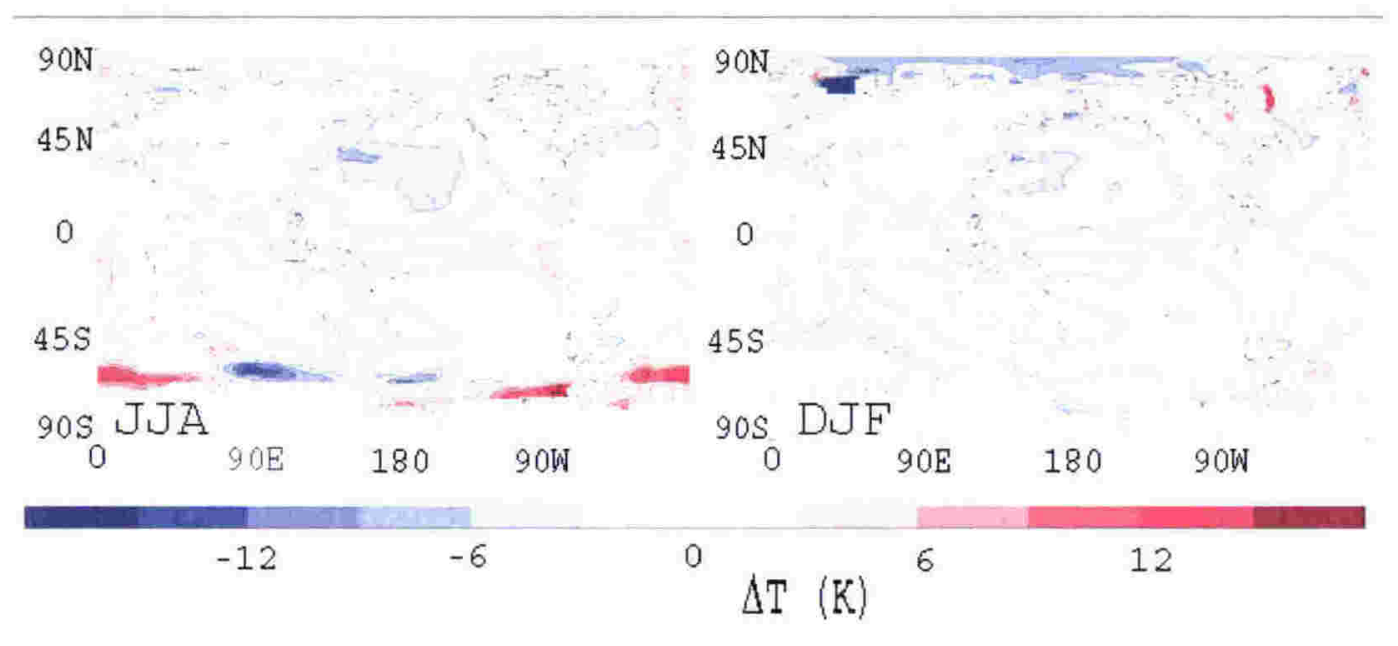

Fig. 5-2. SST HadCM3 - SST $_{\text {NCEP }}$ for the seasonal averaged JJA and DJF.

Gordon et al. (2000) noticed that the SSTs in the Southern Hemisphere in HadCM3 are too high. They found that the peak SST errors in the Southern Ocean are in regions of strong SST gradients and therefore could possibly be associated with the incorrect positioning of the ACC. This overall warming of the Southern Hemisphere is not obvious in this simulation, but the effect of a possible error in the location of the ACC could also partially explain the warmer temperatures in the higher southern latitudes (see section 5.2.2). The reason that the SSTs in the Southern Hemisphere in this simulation are not higher than in NCEP might be related to the fact that the computed SSTs were determined in a simulation under pre-industrial settings. Of concern is that higher SSTs at the high latitudes in the Southern Hemisphere could result in a different SAO signal than observed today. This will be discussed later, but these discrepancies should be kept in mind when comparing atmospheric fields in these regions with the NCEP climatology. Also, errors in the temperature gradients in the Indian Ocean will significantly influence cyclogenesis in that region, which will have consequences for New Zealand. However, this will not be investigated further, since no comparison can be made with the climate of the LGM. Other discrepancies in the SSTs which were also noted by Gordon et al. (2000) are the cooling in the central equatorial Pacific, the warming in the east of the Pacific and the Atlantic south of the equator, and the regional cooling in the North Atlantic, which they ascribed to some large local errors associated with shifts in 


\begin{tabular}{|l|r|r|r|r|}
\hline & DJF & MAM & JJA & SON \\
\hline NCEP & 5.09 & 2.81 & 1.84 & 3.93 \\
\hline HadAM3H $_{\text {pre-industrial }}$ & 5.74 & 2.56 & 2.58 & 5.01 \\
\hline
\end{tabular}

Table 5-1. Southern Oscillation Index (non-normalised) for the four seasons for HadAM $3 \mathrm{H}$ and NCEP climatology.

the North Atlantic Current.

During the last few decades we have gained a lot of insight into the phenomena of El Niño and La Niña, the El Niño - Southern Oscillation, ENSO. The different states of ENSO affect the climate in many places around the world. In climate modelling it is therefore important to correctly incorporate the effects of ENSO. Table 5-1 gives the seasonally averaged, non-normalised, mean sea level pressure difference between Tahiti and Darwin for HadAM3H and the NCEP climatology. We can see from this table that compared to the NCEP climatology, HadAM3H has a stronger pressure gradient across the Pacific, which would result in stronger tradewinds. Since the NCEP reanalysis of 19712000 contains some strong El Niños, it would seem that HadAM3H underestimates the strength and/or occurrence of El Niños in this simulation. This is a direct result of the inability of HadCM3 to calculate the SSTs in the eastern Pacific correctly, as was mentioned earlier.

A clearer picture on the capability of HadCM3 to simulate ENSO is formed when comparing the standard deviation in SST between the NCEP climatology and HadCM3. Fig. 5-3 shows the standard deviation in annual mean SSTs for the NCEP climatology and HadCM3 for the latitude bands of $50^{\circ} \mathrm{N}-50^{\circ} \mathrm{S}$. Ocean areas that at any time are covered in sea-ice are coloured white. NCEP clearly shows a large variability in the Pacific, indicating the 'classic' ENSO pattern. HadAM3H however shows much lower variability in the Pacific, implying a weaker ENSO signal (refer to section 5.2.8: Modes of Variability). The fact that ENSO is less prominent in HadAM3H than that it is in the NCEP climatology has an impact on the interannual variability in $\mathrm{HadAM} 3 \mathrm{H}$, as discussed later. 

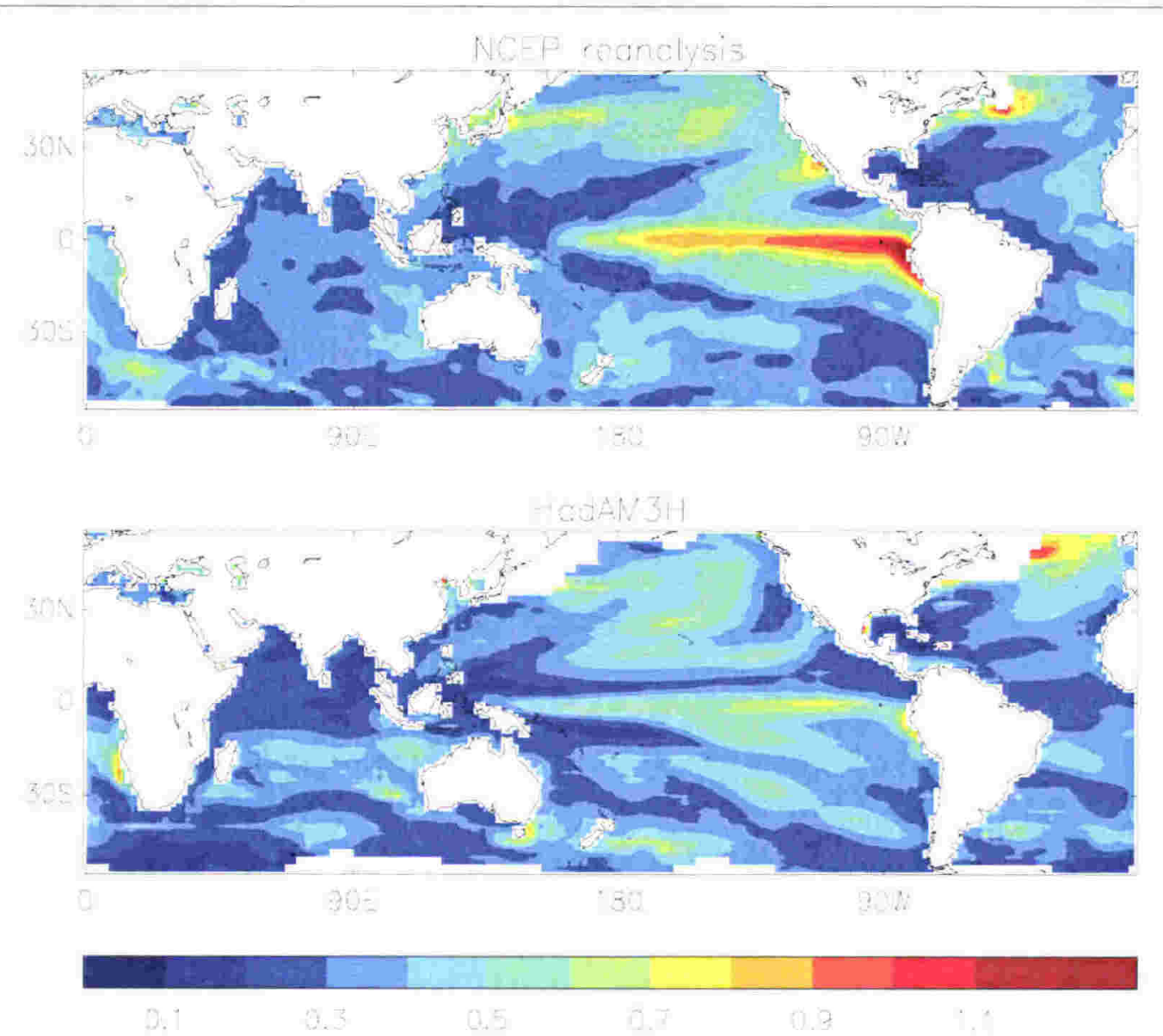

Fig. 5-3. Standard deviation of the mean annual SST for the NCEP reanalysis and HadCM3 (in $\left.{ }^{\circ} \mathrm{C}\right)$.

When comparing the HadCM3 and NCEP reanalysis sea-ice fields, the seaice in the Northern Hemisphere winter extends too far south in the North Pacific and the Barents Sea remains covered in ice the whole year round (Fig. 5-4). But of more importance to this study is the difference in sea-ice extent in the Southern Hemisphere. The difference is particularly large in the austral winter (JJA), where there is clearly a zonal wave number one pattern in the difference in sea-ice extent between the simulation and the reanalysis data, with a maximum south of Australia, which extends into the Western Pacific, and a minimum mainly in the Atlantic region. This wave one pattern in the difference in the seaice extent is also present during DJF, albeit of a smaller magnitude. It is also rotated slightly, with the extremes now clearly in the eastern and western hemisphere. This difference in the sea-ice pattern between HadCM3 and the 


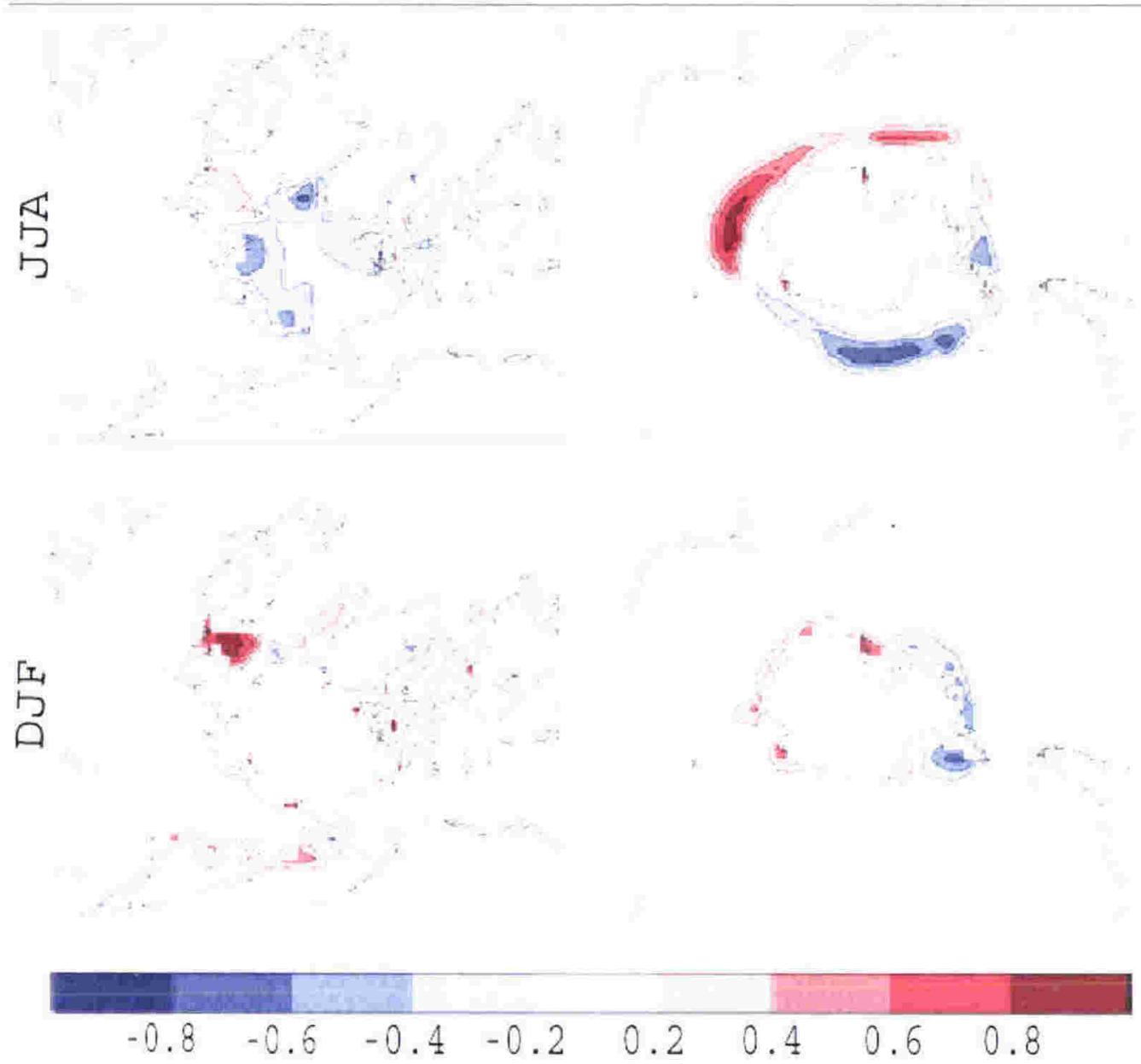

Fig. 5-4. Polar plots of the Northern Hemisphere (left) and Southern Hemisphere (right) of seaice $_{\text {HadAM3H }}$ - sea-ice NCEP, seasonally averaged for JJA and DJF. Units refer to the extent of sea-ice cover within the grid box: 0 is no sea-ice, \pm 1 is fully covered by sea-ice..

NCEP reanalysis, as well as the related difference in their SST pattern, obviously result in related atmospheric patterns, as will be shown later.

\subsubsection{Temperature}

Differences between HadAM3H and NCEP reanalysis surface temperatures over the oceans are directly related to the use of SSTs from HadCM3. This is in particular true for the high latitudes, where differences in modelling the orography also gives rise to substantial surface anomalies (Fig. 5-5). The surface temperatures of $\mathrm{HadAM} 3 \mathrm{H}$ over the continents are in general warmer at low latitudes, and the Northern Hemisphere is cooler at middle and high latitudes 


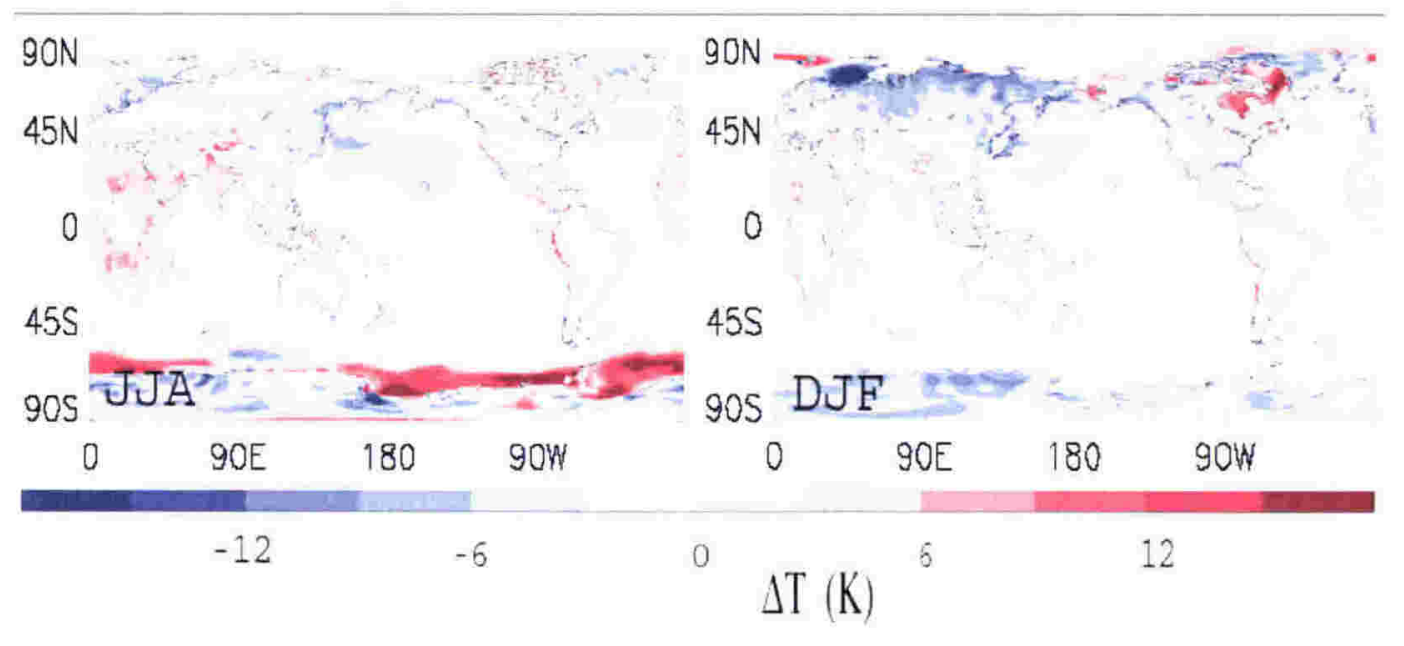

Fig. 5-5. The difference in surface temperature between the pre-industrial HadAM3H and NCEP reanalysis for the seasonal averaged JJA and DJF.

than in the reanalysis. These cooler temperatures also showed up in HadAM3 (Pope et al., 2000). The cooler temperatures continue upward into the atmosphere, except over Antarctica. The comparison of the vertical zonal averaged temperature structure between the two datasets reveals some clear differences (Fig. 5-6). The warmer temperatures in HadAM3H in Antarctica can be attributed to differences in definition of temperature at height for HadAM3H and NCEP (NCEP data was vertical interpolated onto the 13 levels of HadAM3H) and to the effect of different resolutions. Pope et al. (2000) indicate that the inclusion of the radiation scheme of Edwards and Slingo (1996) resulted in an increase of the surface temperature over Antarctica.

Also, as mentioned in section 5.2.1, HadCM3 has higher SSTs at high latitudes, resulting in warmer surface temperatures in HadAM3H near the Antarctic continent. This can be seen clearly in Fig. 5-6 and this warming effect continues with height. From today's observations we know that the increase in greenhouse gases has resulted in lower temperatures in the stratosphere. The lower temperatures near the tropopause in HadAM3H over Antarctica could be the result of the higher modelled surface temperatures in Antarctica. Although this feature should be much stronger in the NCEP reanalysis data, and should therefore show a positive anomaly over Antarctica, it is one more indication that HadAM3H has difficulties modelling the climate over Antarctica. The Southern 


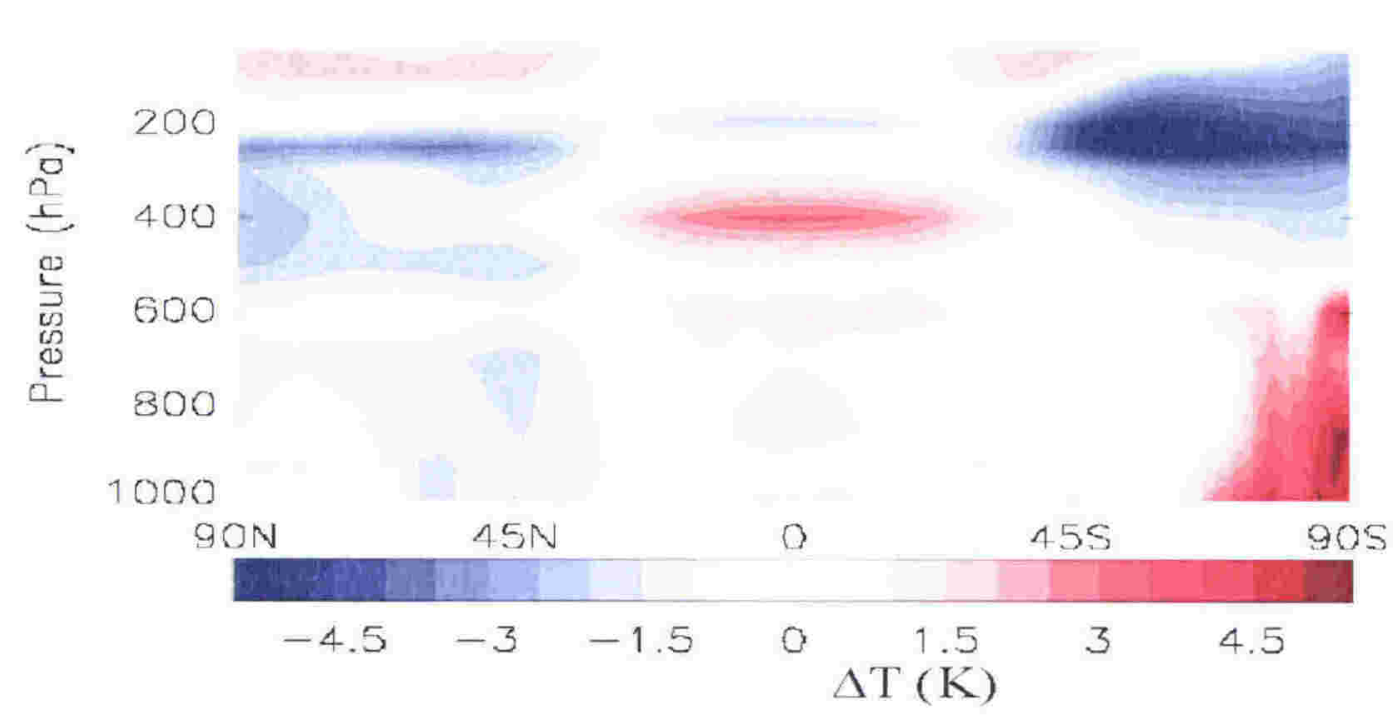

Fig. 5-6. Zonally averaged annual temperature difference between HadAM3H and NCEP climatology.

Hemisphere high latitudes are also an area that has undergone some major changes since the industrial revolution, referring in particular to the "ozone hole" feature. Direct comparison between the two datasets in this region is therefore difficult.

The pre-industrial HadAM3H atmosphere can in general be regarded as cooler than the NCEP reanalysis atmosphere, as one would expect. However, HadAM3H shows substantial warmer temperatures in the tropics, which are related to the larger amount of precipitation (see Fig. 5-8A), which gives rise to a larger release of latent heat. As a consequence of convection taking place over a larger area in the tropics, there is a larger amount of cloud cover in the tropics, resulting in a lower amount of outgoing long wave radiation compared to the NCEP data (Fig. 5-8B). The enhanced cloud cover in the tropics is also a positive feedback on the warming of the tropical atmosphere. With the warming in the tropics, and cooler temperatures in the mid latitudes, the meridional temperature gradient is steeper for the pre-industrial HadAM3H simulation. This has consequences for the westerly flow and the meridional transport of heat and momentum, which will be discussed later. 


\subsubsection{Precipitation}

Fig. 5-7 shows the modelled average daily total precipitation for the four seasons. The major precipitation bands of the ITCZ (Intertropical Convergence Zone) and the SPCZ (South Pacific Convergence Zone), as well as the monsoonal patterns are clearly identifiable. The North Pacific and North Atlantic show enhanced precipitation along their storm tracks. Major dry areas are in North Africa, the Middle East, Antarctica, and over the subtropical oceans, with dry summer conditions existing in Australia, Brazil, Southern Africa, Canada and Central Asia. Precipitation is one of the most difficult parameters to model, since many of the variables that are needed to determine precipitation take place at subgrid-scale level (refer to section 4.3). It is therefore not surprising that HadAM $3 \mathrm{H}$ has difficulties in getting the extremes in precipitation right over the Tropical regions.

Reasons for the larger amount of precipitation in the tropics in $\operatorname{HadAM} 3 \mathrm{H}$ (Fig. 5-8A) are not straight forward. It occurs mainly in the ITCZ and in particular over the Western Pacific warm pool. The cooler SSTs in the equatorial Pacific and the warmer SSTs in the Western Pacific in HadCM3 increase the

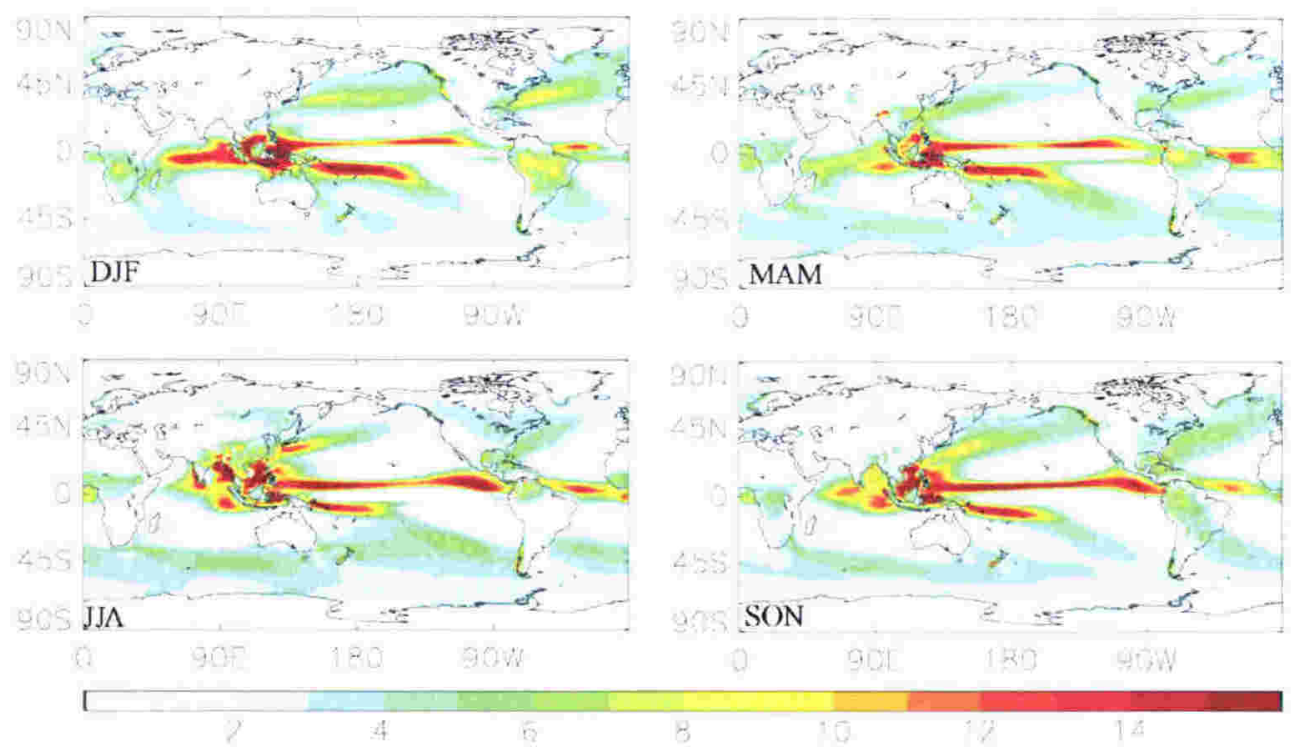

Fig. 5-7. Average daily total precipitation ( $\mathrm{mm} /$ day) for the four seasons as modelled by HadAM3H. 


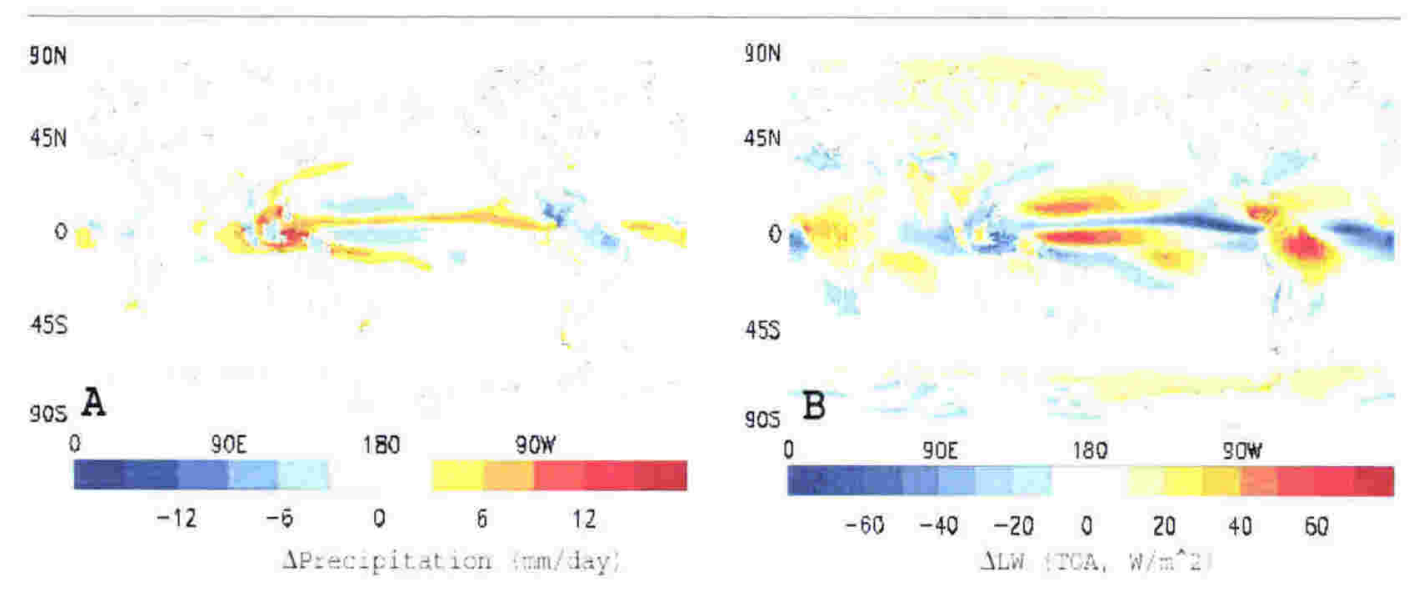

Fig. 5-8. A: Difference in annual averaged precipitation between the pre-industrial HadAM3H and NCEP climatology. B: Difference in annual averaged outgoing LW radiation (TOA) between the pre-industrial HadAM3H and the NCEP climatology.

east-west gradient in SST, resulting in stronger easterly winds over the central equatorial Pacific and enhanced precipitation over the western equatorial Pacific. The larger amount of precipitation over Indonesia is also partly the result of the inclusion of the radiation scheme of Edwards and Slingo (1996). Pope et al. (2000) describe the effect of the radiation scheme on the global precipitation pattern in more detail. A feature that stands out is that the landmasses in the Indonesian region, and other landmasses in the tropics, show a reduction in precipitation. Such a pattern was also noticed by Kumar et al. (2004). They noticed that since 1976, as the result of a warming trend in tropical SSTs, that there was a positive feedback in precipitation over the oceans (increasing precipitation), but a negative feedback in precipitation over land (decreasing precipitation) with warmer SSTs. The increase in precipitation in the ITCZ was also documented by Hudson and Jones (2002a). Owing to the mass continuity principle, an increase in convection in one place (resulting in an increase in precipitation) will result in an increase in descending motion in another place (increase of dry conditions). Positive and negative precipitation anomalies therefore could cancel each other out. Hudson and Jones (2002a) were also able to find these patterns in ensembles of simulations of two different AGCMs. These models indicated that a change in the tropical circulation has a major effect on the precipitation trends. 
What also needs to be considered is that the difference in precipitation over land and sea could be a reflection of the different thresholds for cloud liquid water for the formation of precipitation over land and ocean. As discussed in section 4.3.2, several of HadAM3H's precipitation related parameters were adjusted to improve the modelling of the global precipitation. It could be that these adjustments were not appropriate or sufficient enough for the tropical regions. No sensitivity studies were done to examine this.

A further comment that can be made about the precipitation anomaly in the Pacific between HadAM3H and the NCEP reanalysis is that there were some particularly strong El Niños during the 1971-2000 period. That resulted in less precipitation in the Western Pacific and enhanced precipitation in the equatorial Pacific. The positive anomaly in the Western Pacific and the negative anomaly over the tropical Pacific Ocean can therefore also partially be explained by the choice of the climatology data. Since HadCM3 and HadAM3H were not set up to model ENSO in particular, the precipitation anomalies in the Pacific are probably smaller if the data was compared with climatology data that includes less and/or less severe El Niño events. However, this was not further investigated.

Regions that experience a relatively smaller increase in precipitation in HadAM3H are also found along the warm ocean currents, like the Gulfstream and the Kuroshio flow, as well as in the SPCZ. Although only of minor significance since the resolution is too coarse to make any absolute comparisons, but of interest to this study nevertheless, is that $\mathrm{HadAM} 3 \mathrm{H}$ also shows larger amount of precipitation in southern New Zealand. This could be related to the steep orography of the South Island of New Zealand, but as will be pointed out later, it is also related to the enhanced wave number 3 pattern of the mean zonal circulation.

\subsubsection{Mean Sea Level Pressure}

The global mean sea level pressure pattern is very similar to the NCEP reanalysis in space, but varies in magnitude (Fig. 5-9). Most of the spatial variations can be explained by the variations in temperature. The cooler SSTs in 
the North Pacific and in the North Atlantic in HadCM3 during JJA result in their low pressure zones being less deep in $\operatorname{HadAM} 3 \mathrm{H}$ than in the reanalysis. The warmer landmasses at the lower latitudes in HadAM3H show lower mean sea level pressures. This is in particularly true for Africa, which sees an increase in direct warming, and for South-East Asia, which sees an increase in warming as the result of a more vigorous Hadley circulation. The warmer temperatures in the Western Pacific are the result of the warmer SSTs in that region (Fig. 5-2) and an increase in latent heat. The warmer temperatures lead to HadAM3H's lower mean sea level pressures in this region. The cooler landmasses at high latitudes, and in particular the Eurasian continent, show higher MSLP in HadAM3H. This is similar to HadAM3, which main systematic error is the high pressure at high latitudes for most of the year (Pope et al., 2000).

The reduced MSLP in the North Atlantic during DJF could be associated with the equatorward shift of the Atlantic jetstream during the boreal winter months (this will be discussed in section 5.2.6). However, the reduction in MSLP is much less over the North Pacific though the jetstream has varied similarly in this region. This might be the result of the cooler SSTs in the North Pacific, which effect would be to increase MSLP. The North Pacific shows higher MSLP than the NCEP reanalysis in all other seasons (not shown).

The differences in mean sea level pressure over areas of high orography, like the Himalayas, the Andes and the Rockies, are the result of differences in describing pressure with height, as well as the result of the different resolutions between the two datasets. HadAM3H consistently shows lower MSLP in areas at high altitudes. Furthermore, the difference in mean sea level pressure at the poles is also due to differences in sea-ice cover. Similar to surface temperature, mean sea level pressure is difficult to model correctly at the poles. Mean sea level pressure over the poles was therefore not presented in Fig. 5-9.

The largest anomaly is to the south of Australia during JJA, where the mean sea level pressure in HadAM3H is much lower than in NCEP. Other zones of lower pressure are found in the South Pacific and in the South Atlantic. The location of the anomaly south of Australia corresponds with the area of enhanced sea-ice cover in HadAM3H. The SSTs and sea-ice have a significant influence 

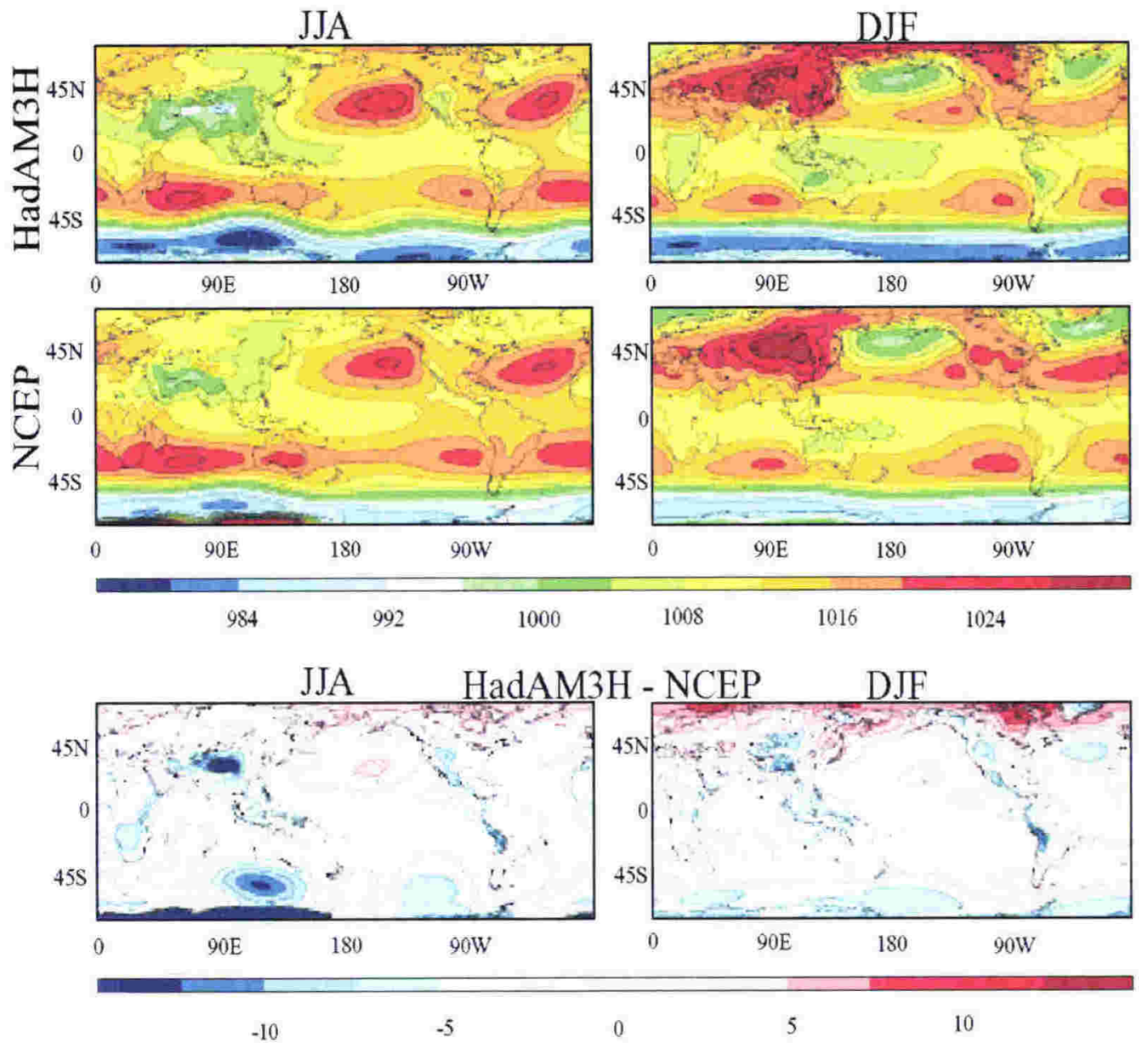

Fig. 5-9. Mean Sea Level Pressure (hPa) for JJA and DJF from HadAM3H and the NCEP reanalysis, and the difference between them. Note the different scales. Only latitudes $75^{\circ} \mathrm{S}$ $75^{\circ} \mathrm{N}$ are given, since the values and the associated errors at the poles are model and resolution dependent.

on MSLP. Since the Southern Hemisphere's atmosphere can be regarded as barotropic in the mean, these zones of low mean sea level pressure are also present in the 500hPa geopotential height field (section 5.2.5).

\subsection{5. $\quad 500 \mathrm{hPa}$ Geopotential Height}

The modelled $500 \mathrm{hPa}$ geopotential heights are lower than in the NCEP reanalysis in the North Pacific, North Atlantic, Northern Asia, and in a more complicated pattern, all around the southern midlatitudes, the whole year round 


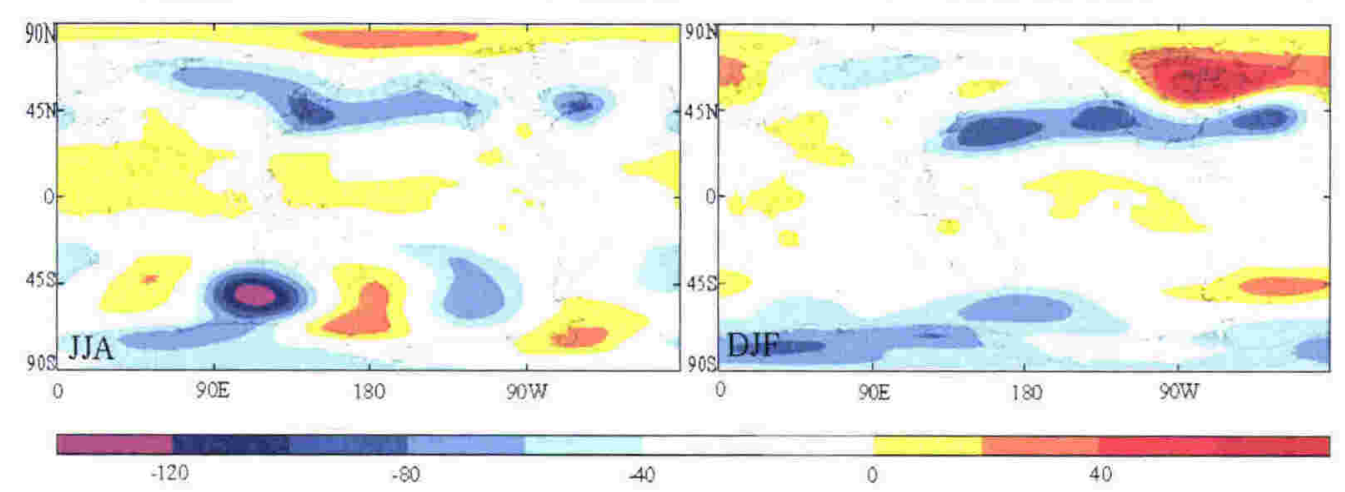

Fig. 5-10. The difference in 500hPa geopotential height $(\mathrm{m})$ between HadAM3H and the NCEP climatology for JJA (left) and DJF (right).

(Fig. 5-10). This result is not surprising as the UM is known to overestimate the westerly flow in midlatitudes (Pope et al., 2000) which would require a steeper meridional gradient of geopotential. Since the differences in geopotential height between HadAM3H and the NCEP climatology at low latitudes are small, the steepening of the meridional gradient has to be the result of lower geopotential heights at higher latitudes.

Of particular interest to this study is the behaviour of the model in the Southern Hemisphere. Unlike the $500 \mathrm{hPa}$ geopotential height anomalies in the Northern Hemisphere, which move equatorward (poleward) during the northern winter (summer) and are relatively consistent the whole year round, the Southern Hemisphere anomalies show different seasonal patterns and vary in strength considerably. Polar projections of the anomalies show clearly lower geopotential heights over Antarctica, particularly over east Antarctica, surrounded with various patterns in the high and middle latitudes (Fig. 5-11). The anomalies during JJA and SON are particularly clear since they show the characteristic wave number 1 and wave number 3 patterns. The circulation associated with the wave number 3 pattern is particularly strong during the austral winter months and it is obvious that $\mathrm{HadAM} 3 \mathrm{H}$ accentuates this pattern. The maximum anomaly of over $120 \mathrm{~m}$ during JJA is plausible in the short-term but for it to show up in the climatology indicates a systematic bias in HadAM3H. The anomalies during DJF and MAM show a more complicated and generally weaker pattern, where DJF resembles an inverted wave number 1 and MAM a wave number 4 pattern. The 

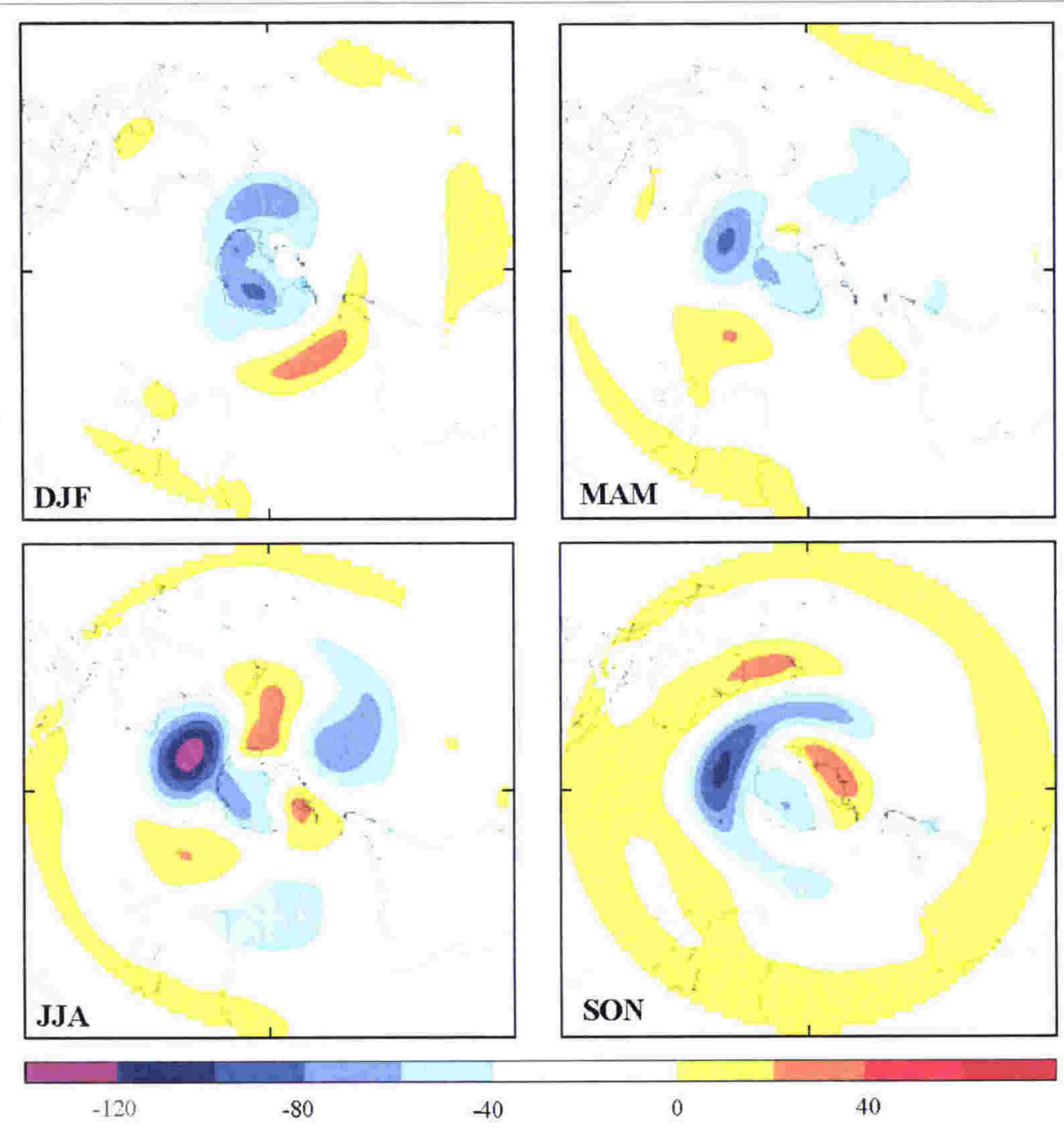

Fig. 5-11. Polar projections of the seasonal differences in $500 \mathrm{hPa}$ geopotential heights between HadAM3H and the NCEP climatology (m).

generally lower geopotential heights at the polar and high latitudes during these seasons again indicate the stronger westerly flow in the midlatitudes in the model.

Meridional transport in the Southern Hemisphere takes place mainly through the eddies since the planetary waves are relatively weak. Since HadAM3H enhances the wave number 3 pattern of the mean zonal circulation, especially during the austral winter and spring, it is expected that meridional transport in HadAM3H will also be larger than in the NCEP climatology. This particular point will come back in later discussions. The enhancement of the wave number 
3 pattern of the Southern Hemisphere circulation affects other features of the general circulation of the Southern Hemisphere as well, and will be referred to regularly in the chapters to come. Changing the characteristics of the mean zonal circulation can also change aspects of regional climates. This will be of importance when investigating the regional climate model for New Zealand in chapter 6.

\subsubsection{Zonal winds}

It was mentioned in the previous section that the UM overestimates the westerly flow of the midlatitudes. Fig. 5-12 indicates that the westerly winds are indeed too strong in $\operatorname{HadAM} 3 \mathrm{H}$, but there are also some interesting patterns in the differences with the NCEP climatology. During the austral winter months (JJA), HadAM3H's subtropical jetstream in the Southern Hemisphere is not only much stronger over the Indian Ocean, but is also broader in the meridional direction. The zonal extent of the maximum of the subtropical jet east of Australia is larger than in the reanalysis. The main differences in the subtropical jetstream in the Southern Hemisphere between HadAM3H and NCEP (Fig. 5-12, bottom) are all found on the eastern side of the three oceans, and resemble the wave number 3 pattern which was mentioned in section 5.2.5. These regions show stronger westerly winds in HadAM3H than in NCEP. Poleward of these positive anomalies, there is a similar pattern of negative anomalies, indicating that HadAM3H underestimates the strength of the zonal flow there.

These patterns can be explained by the enhanced wave number 3 pattern of the mean zonal circulation in the Southern Hemisphere. The larger amplitude of the wave number 3 pattern in HadAM3H shows up mainly at low latitudes. However, there are small local minima in the anomaly patterns at latitudes south of $75^{\circ} \mathrm{S}$ during JJA, indicating that the enhanced wave number 3 pattern does penetrate slightly more southwards as well. The overall effect would be that the mean zonal flow has moved slightly equatorward. Where the amplitude of the wave number 3 pattern in HadAM3H is larger than in NCEP, it has stronger mean zonal winds at low latitudes and weaker mean zonal winds at high latitudes 

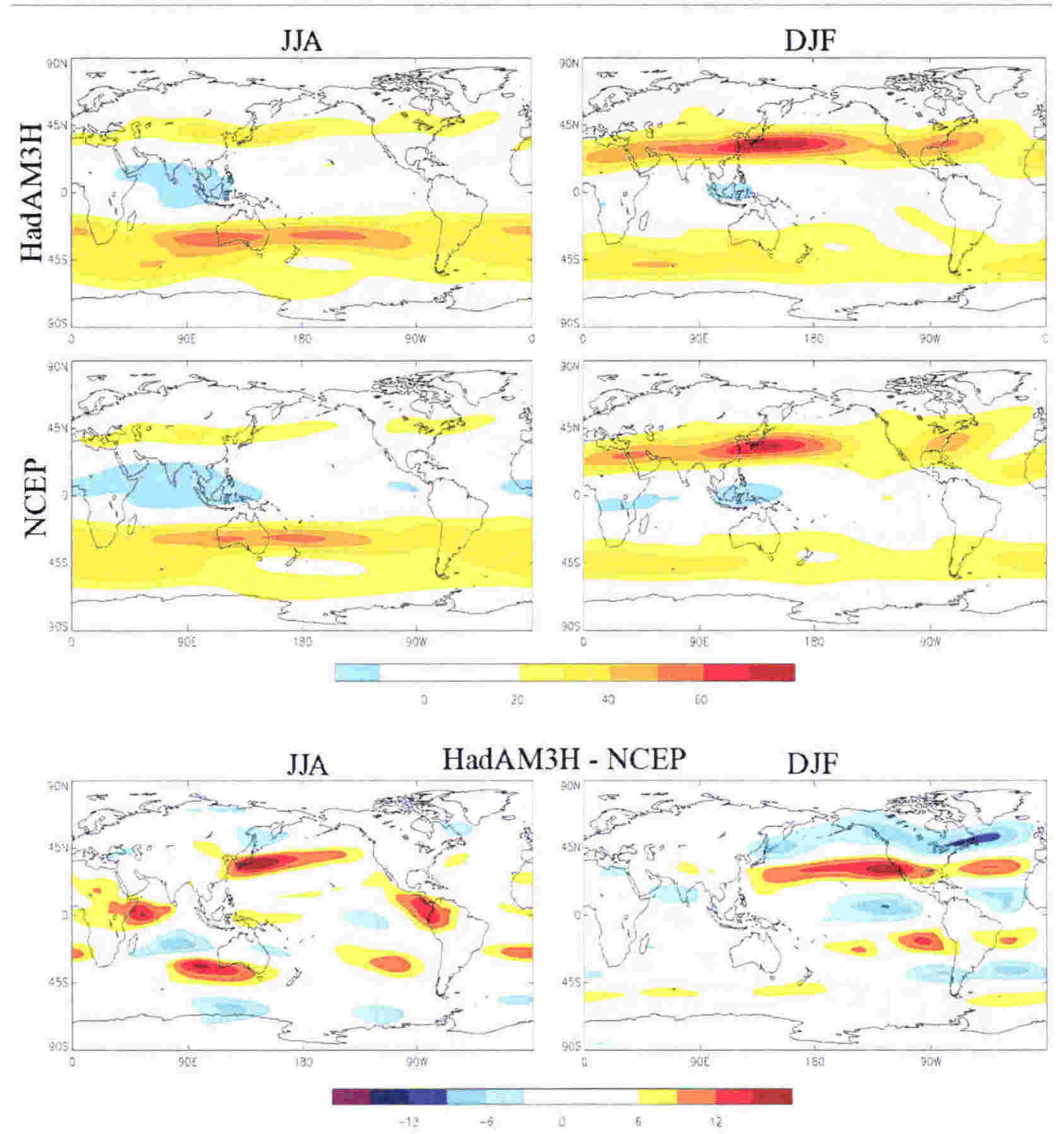

Fig. 5-12. 200hPa wind speed (in m/s) for HadAM3H (top), the NCEP climatology (middle), and the difference between them, HadAM3H-NCEP (bottom) for JJA (left) and DJF (right). Note the different scales.

than the NCEP climatology. The more wavelike character of the general circulation therefore explains the local maxima in the anomalies within the zonal bands of $25^{\circ} \mathrm{S}-45^{\circ} \mathrm{S}$, and the local minima in the anomalies within the zonal bands of $55^{\circ} \mathrm{S}-70^{\circ} \mathrm{S}$ (see Fig. 5-13).

Another distinctive feature of the Southern Hemisphere, especially during JJA, is the splitting of the jetstream east of Australia into a sub-polar jet (SPJ) and a sub-tropical jet (STJ). Splitting of the jetstream enhances the occurrence of 


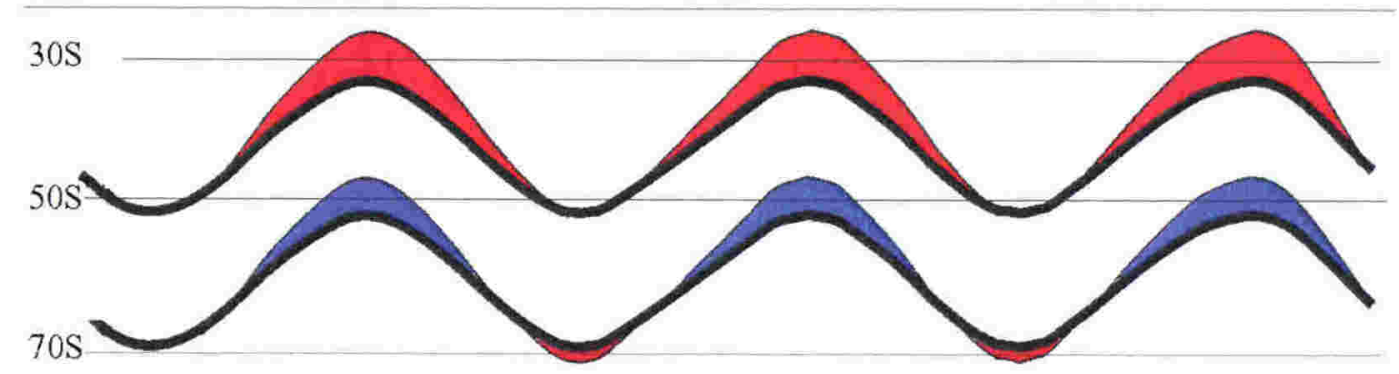

Fig. 5-13. A schematic diagram illustrating the $200 \mathrm{hPa}$ anomalies in the Southern Hemisphere. The thick wavy lines mark the mean flow of the NCEP climatology, and the thin wavy lines mark the mean flow modelled by HadAM3H. The wave structure is greatly enlarged for clarity. Due to the enhanced wave structure of HadAM3H, the winds are stronger in HadAM3H than NCEP at low latitudes (areas coloured red), and are weaker at high latitudes (areas coloured blue) in the areas associated with the peaks in the wave pattern.

"blocking highs" over the New Zealand region (e.g. van Loon, 1956: Trenberth, 1980: Renwick and Revell, 1999). HadAM3H does simulate this splitting albeit less pronounced than in the NCEP climatology. This might be the result of the effective equatorward movement of the main jet as the result of the enhanced wave number 3 pattern. Trenberth and Mo (1985) and Trenberth (1986) found that blocking is associated with an enhanced wave number 3 pattern, although a more recent study (Renwick, 2005) has shown that the occurrence of blocking in the Southern Hemisphere is dominated by a wave number 1 pattern. The Southern Hemisphere $200 \mathrm{hPa}$ winds at high latitudes are stronger than in the NCEP climatology around the whole globe during DJF. There is a weak wave number 3 pattern within this band of stronger winds, with one of its maxima lying over New Zealand.

There is a similar pattern in the westerly winds in the Northern Hemisphere, particularly during DJF. HadAM3H clearly overestimates the strength of the Northern Hemisphere $200 \mathrm{hPa}$ winds as well, and also has the jet streams much more elongated. But similar to their Southern Hemisphere counterparts, the anomaly fields show a "dipole" pattern with stronger winds at low latitudes and weaker winds at middle/high latitudes, implying a meridional displacement of jet location. But the anomaly patterns of the $200 \mathrm{hPa}$ zonal winds show a strong wave number 1 pattern instead of a wave number 3 pattern. The equatorward displacement of the strongest winds takes place mainly over the North Pacific, 

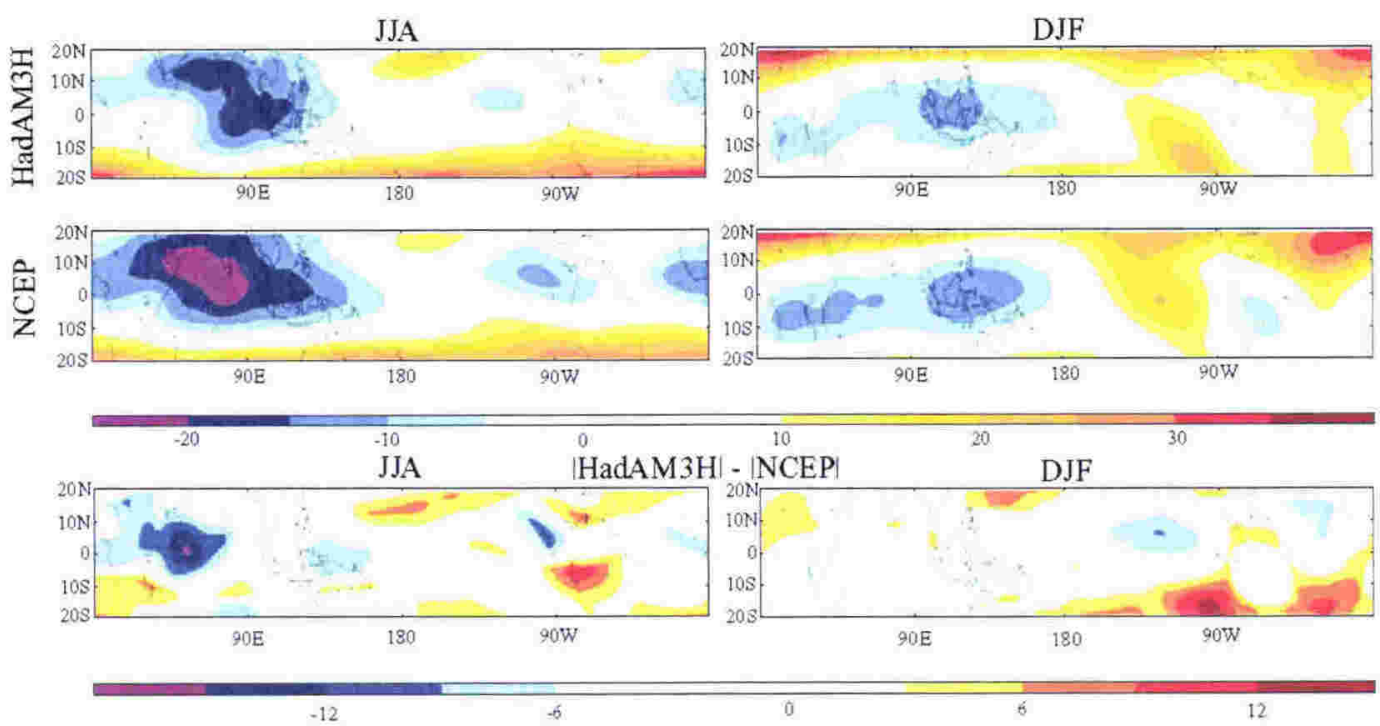

Fig. 5-14. 200hPa mean zonal wind ( $\mathrm{u}$, in $\mathrm{m} / \mathrm{s}$ ) for HadAM3H (top), NCEP (middle) and $\mid$ HadAM $3 \mathrm{H}|-| \mathrm{NCEP} \mid$ (bottom) for the zonal band $20^{\circ} \mathrm{S}-20^{\circ} \mathrm{N}$ for JJA (left) and DJF (right). Note the different scales.

North America and the North Atlantic. The equatorward movement of these winds is consistent with the description in HadAM3 (Pope et al., 2000).

HadAM3H shows relatively weak $200 \mathrm{hPa}$ easterly winds during JJA over the equatorial Pacific and Indian Ocean, implying a strengthened Walker circulation. The enhanced convection over Indonesia leads to enhanced divergence above. The difference patterns of the absolute zonal wind at 200hPa (Fig. 5-14) show negative values over Indonesia. However, the NCEP climatology is taken over the period 1971-2000, which included several strong El Niños (refer to Table 5-1). When comparing the data with the ECMWF dataset (1979-1993) used by Hurrell et al. (1998), which excludes the strong El Niño year of 1998, HadAM3H seems to simulate the $200 \mathrm{hPa}$ winds in the tropics fairly well. HadAM $3 \mathrm{H}$ has also a weaker monsoonal circulation in the Indian Ocean than the NCEP climatology. The reduction in the monsoonal circulation leads to a reduction in precipitation over India and East Africa (Fig. 5-8A). The associated increase in long-wave radiation (Fig. 5-8B) results in warming of the Indian continent and of equatorial Africa (Fig. 5-5). The decrease in the monsoonal circulation was also described in HadAM3 (Pope et al., 2000). 


\subsubsection{Meridional Fluxes}

The total time-mean and zonal-mean meridional transport can be split up in two terms; one representing the contribution by the mean meridional circulation and one representing the deviations from that mean, or in other words, the eddies. The eddy component is then again split up in a transient and stationary component $^{1}$. In the midlatitudes, the mean meridional circulation is thermally indirect (Ferrel cell), and it are therefore mainly the eddies that drive the meridional circulation at those latitudes. The most prominent eddies are the transient eddies. The contribution of the stationary eddies to the meridional circulation is only similar to the contribution of the transient eddies in the Northern Hemisphere during DJF (e.g. Hartmann, 1994).

Since HadAM3H overestimates the strength of the westerly flow in the midlatitudes, the meridional momentum fluxes in $\mathrm{HadAM} 3 \mathrm{H}$ are also stronger than in the NCEP climatology (Fig. 5-15). The maxima of the momentum fluxes occur at heights where the westerly winds are strongest, which is at the tropopause at about $200 \mathrm{hPa}$. Analysing the differences between the two datasets reveals a slight equatorward shift of the maximum momentum flux in the Northern Hemisphere in HadAM3H, which follows from the equatorward shift of the zonal wind mentioned in section 5.2.6. Such a shift is less obvious in the Southern Hemisphere. The contribution of the stationary and transient eddy momentum fluxes to the total eddy flux, and the differences between the NCEP climatology and HadAM3H can be identified from Fig. 5-16 and Fig. 5-17. Fig. 5-16 shows that the contribution of the stationary eddies to the total covariance is indeed only significant in the Northern Hemisphere during DJF. The stationary eddies are only weakly developed in the Southern Hemisphere, which is indicated by their very low contribution to the total covariance there. HadAM3H has reconstructed the position of the stationary eddies reasonably well, but their maxima seem to be more similar to the ECMWF analysis for 1979-1993 (Karoly

$[\overline{v \zeta}\rfloor=[\bar{v}\rfloor \bar{\zeta}]+\left[\overline{v^{\prime} \zeta^{\prime}}\right]+\left[\overline{v^{*} \zeta *}\right]$, where $v$ is meridional wind, $\zeta$ is a climatic variable. The square brackets refer to the zonal average, the overbar denotes the mean value, ' is transient eddy component and * is stationary eddy component. 

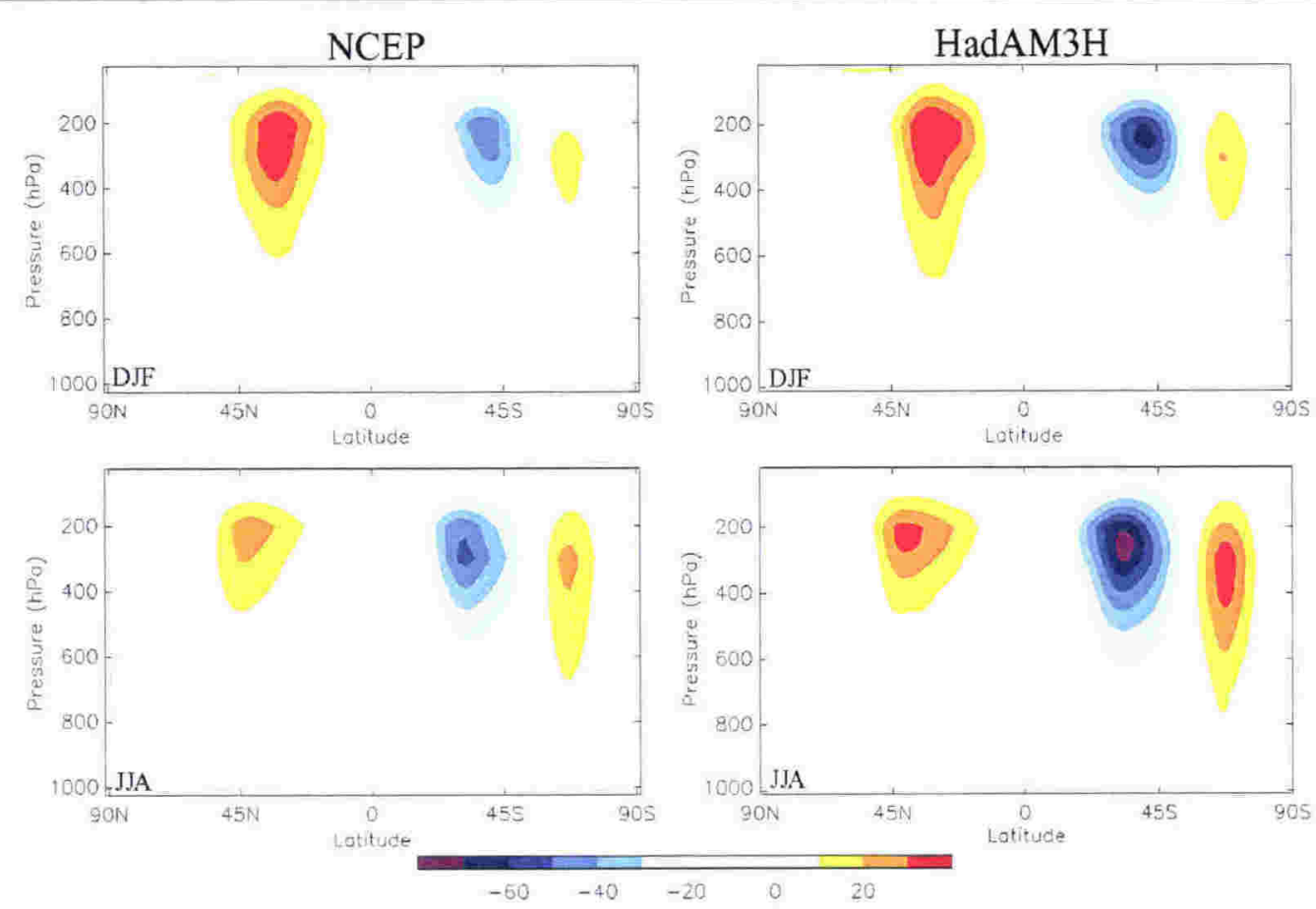

Fig. 5-15. Covariance of $\mathrm{u}$ and $\mathrm{v}\left(\mathrm{m}^{2} / \mathrm{s}^{2}\right)$ for NCEP (left) and HadAM3H (right) for DJF (top) and JJA (bottom). Northwards and eastwards are positive.

et al., 1998) than to NCEP for 1971-2000.

In general, the poleward stationary momentum flux in the Northern Hemisphere lower mid latitudes $\left(30^{\circ} \mathrm{N}\right)$ as modelled in $\mathrm{HadAM} 3 \mathrm{H}$ is slightly weaker than in the NCEP climatology, where as the equatorward stationary momentum flux in the higher mid latitudes $\left(60^{\circ} \mathrm{N}\right)$ in $\mathrm{HadAM} 3 \mathrm{H}$ is slightly stronger than in the NCEP climatology. This trend is opposite to the general equatorward movement of the strongest westerly winds, and it can therefore be concluded that it is primarily the transient component that gives rise to an increase in the eddy meridional momentum flux. This is in particular the case for the transitional seasons of MAM and SON (not shown). However, Held and Phillips (1992) found that an increase in modelled eddy meridional momentum flux was due to an increase in the meridional resolution. Meridional momentum transport takes place mainly by the transients in the Southern Hemisphere and they are distinctively stronger in HadAM3H than in NCEP (Fig. 5-17). This is the result of the combined effects of the stronger westerly flow and the accentuated wave number 3 pattern of the mean zonal circulation in $\operatorname{HadAM} 3 \mathrm{H}$. 

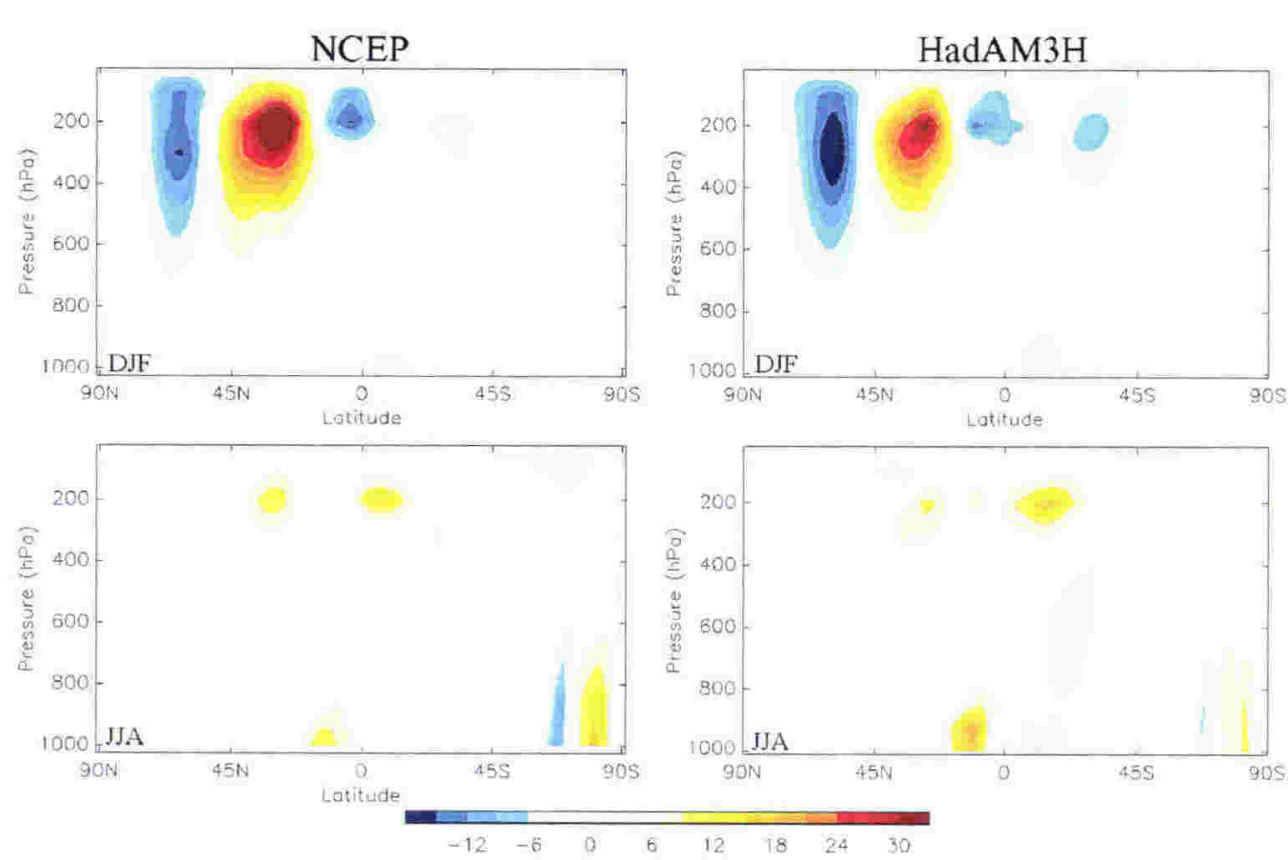

Fig. 5-16. Stationary eddy momentum flux $\left(\mathrm{m}^{2} / \mathrm{s}^{2}\right)$ for NCEP (left) and HadAM3H (right) for DJF (top) and JJA (bottom). Northwards and eastwards are positive.
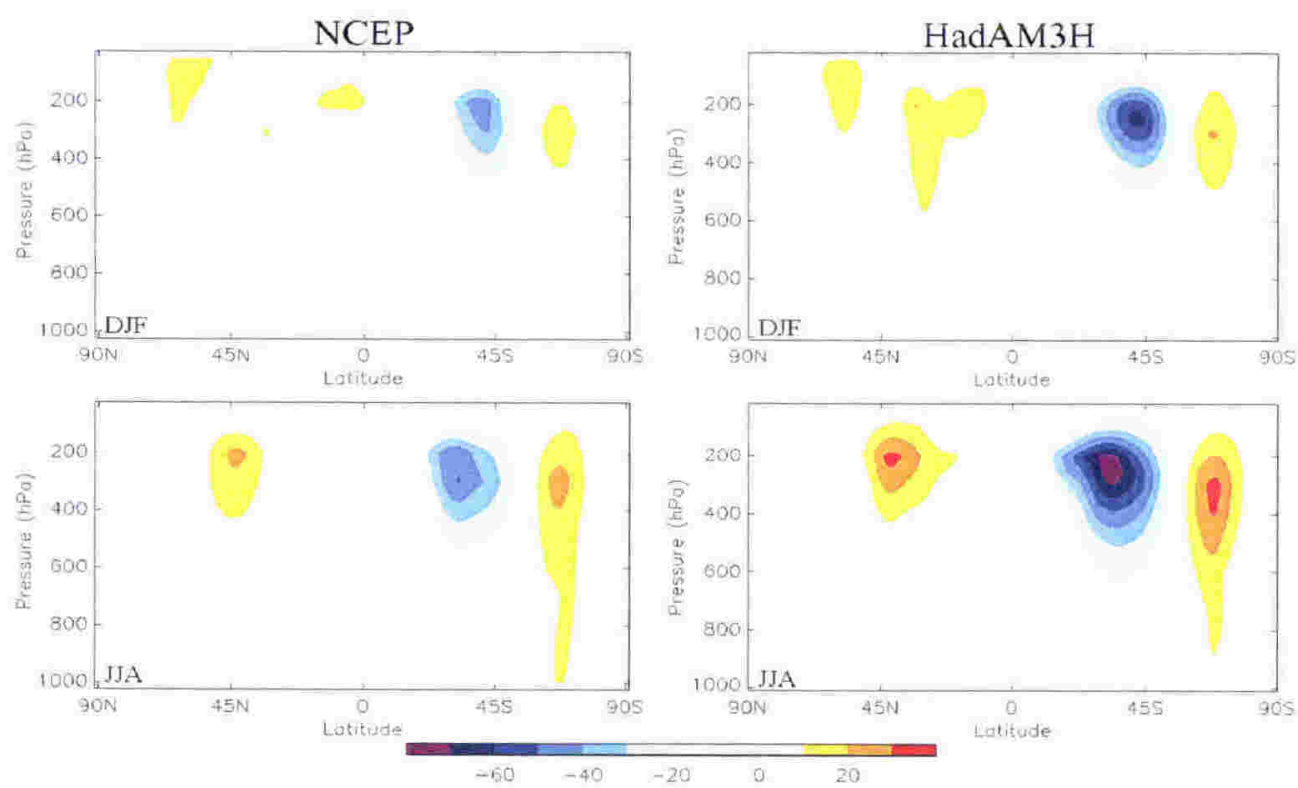

Fig. 5-17. Transient eddy momentum flux $\left(\mathrm{m}^{2} / \mathrm{s}^{2}\right)$ for NCEP (left) and HadAM3H (right) for DJF (top) and JJA (bottom). Northwards and eastwards are positive.

The transient eddy momentum fluxes are particularly stronger than in NCEP during winter and spring when the wave number 3 pattern is most prominent. 


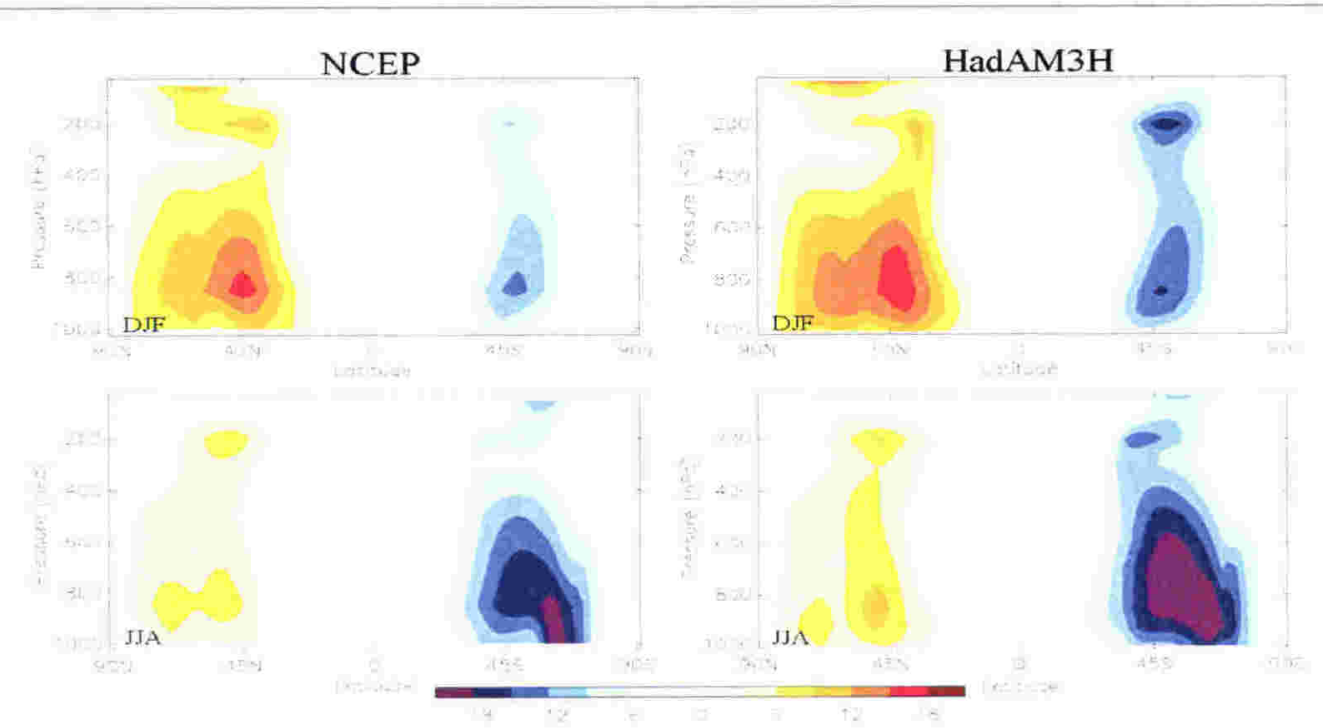

Fig. 5-18. Covariance of $\mathrm{v}$ and $\mathrm{T}(\mathrm{mK} / \mathrm{s})$ for NCEP (left) and HadAM3H (right) for DJF (top) and JJA (bottom). Northwards is positive.

The meridional heat flux shows similar patterns as the meridional momentum flux. The mean meridional heat flux has a stronger signature in the tropics than the mean meridional momentum flux. The mean meridional heat flux in HadAM3H indicates a slightly more vigorous Hadley Cell than in the NCEP climatology (not shown). It is known that the Hadley circulation in HadAM3 is too strong (Gregory et al., 1997: Pope et al., 2000). Fig. 5-18 shows the total covariance of $\mathrm{v}$ and $\mathrm{T}$ for HadAM3H and the NCEP climatology for DJF and JJA. HadAM3H overestimates the meridional heat flux in both hemispheres during the entire year. Fig. 5-19 and Fig. 5-20 present the different contributions of the eddies to the meridional heat flux. HadAM3H has simulated the stationary eddy heat flux quite well and the largest differences between the NCEP climatology and HadAM3H are found in the transients. The transients are stronger in both hemispheres in HadAM3H. Maxima are at 700-925hPa, they are largest in the winter months and are more prominent in the Southern Hemisphere. The largest difference occurs during JJA in the Southern Hemisphere between $35^{\circ} \mathrm{S}-65^{\circ} \mathrm{S}$ and is again related to the stronger wave number 3 pattern in the mean zonal circulation. An increase of the transient eddy heat fluxes in the Southern Hemisphere should result in an increase in cyclonic activity. Chen and Yen (1997) indeed find that the enhancement of the wave 

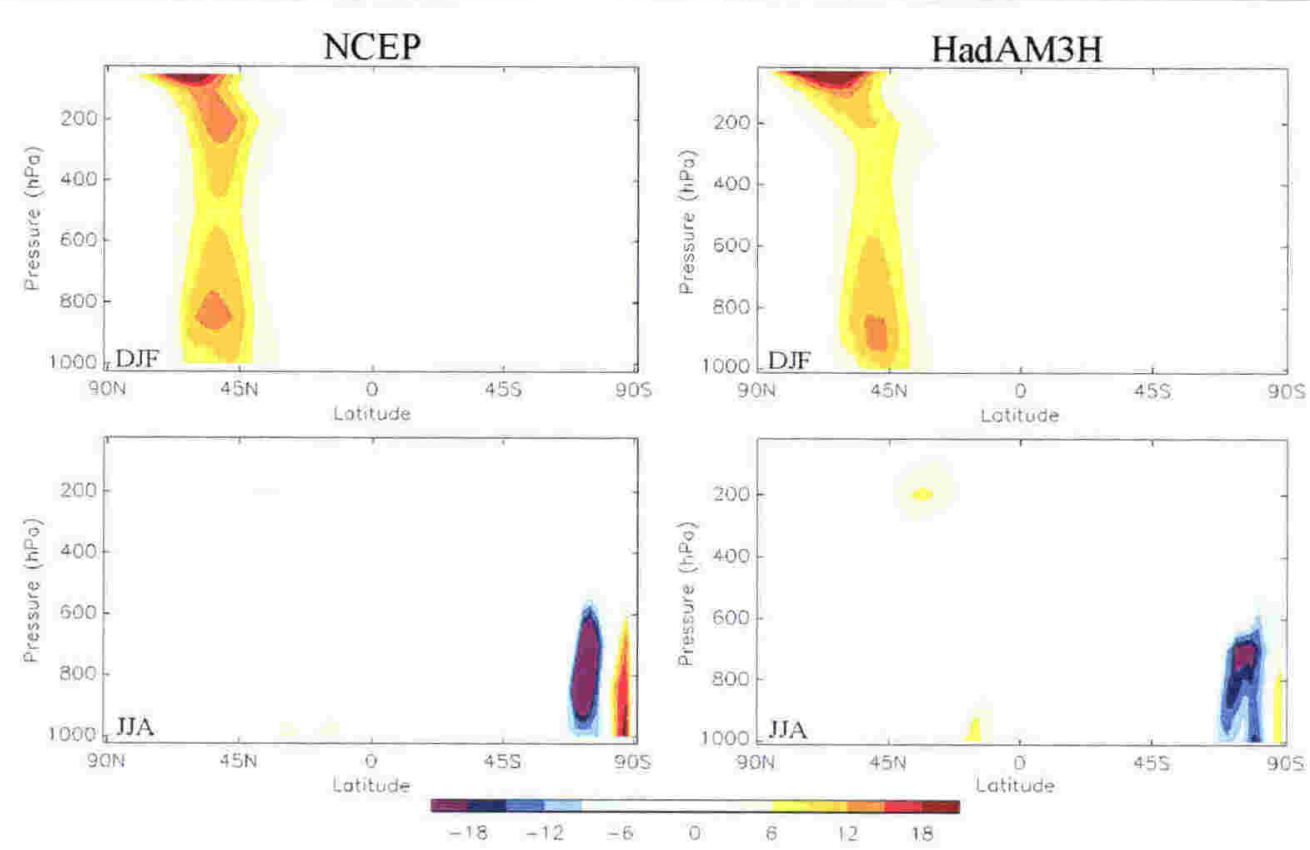

Fig. 5-19. Stationary eddy heat flux (mK/s) for NCEP (left) and HadAM3H (right) for DJF (top) and JJA (bottom). Northwards is positive.

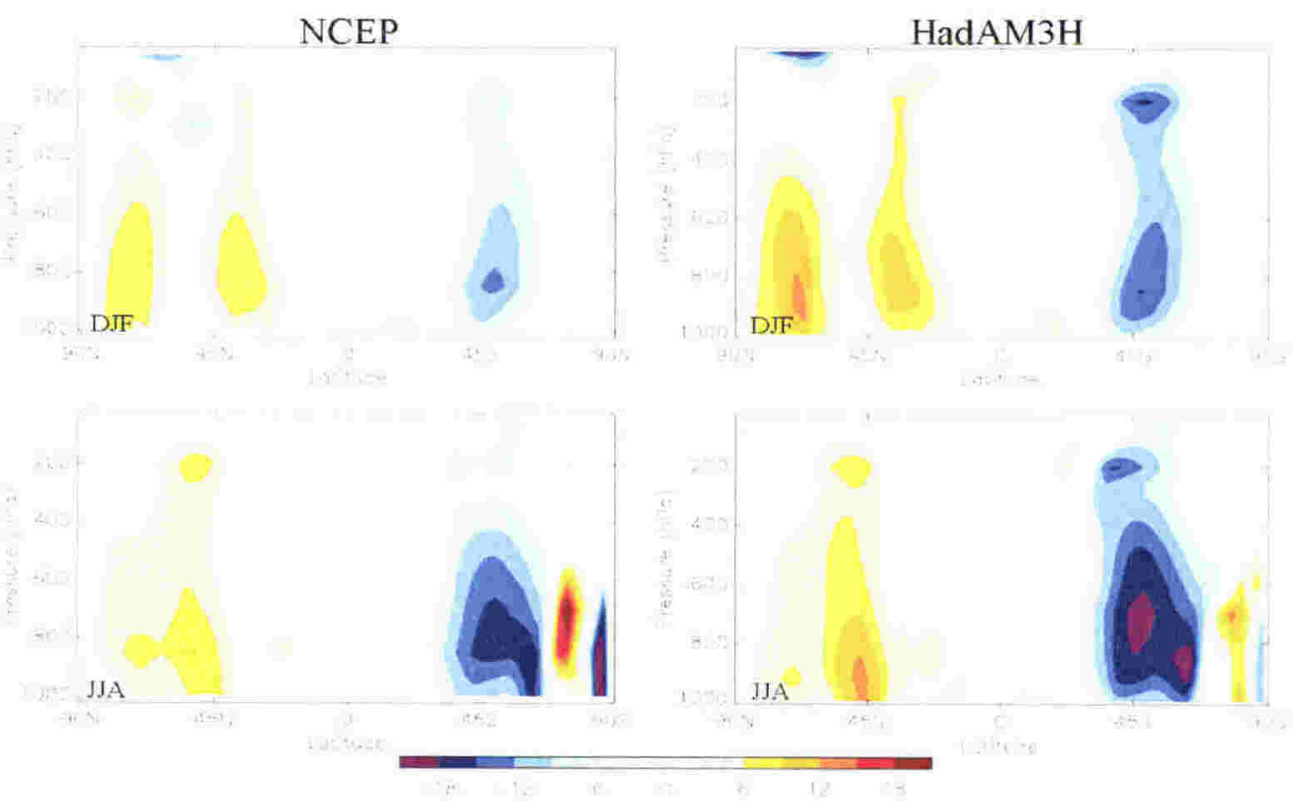

Fig. 5-20. Transient eddy heat flux (mK/s) for NCEP (left) and HadAM3H (right) for DJF (top) and JJA (bottom). Northwards is positive.

number 3 pattern together with the deepening and equatorward movement of the polar trough results in an enhancement of cyclonic activity. 


\subsubsection{Modes of Variability}

In the previous sections, features of the mean zonal circulation as simulated by HadAM3H have been discussed. The model's output agrees with the main features of the Southern Hemisphere, and the anomalies were related to a stronger westerly flow together with an enhanced wave number 3 pattern of the mean zonal circulation. In this section the variability of the mean flow in the Southern Hemisphere as modelled by HadAM3H will be investigated.

\section{Semi-Annual Oscillation (SAO)}

Fig. 5-21 shows the SAO of the NCEP climatology and HadAM3H. The $\mathrm{SAO}$ is computed from the $500 \mathrm{hPa}$ temperatures and the gradient is determined between $50^{\circ} \mathrm{S}$ and $65^{\circ} \mathrm{S}$. The NCEP data shows the normal SAO pattern, with peaks during late summer/early autumn and during spring. HadAM3H does model the meridional temperature gradient quite well, both in intensity as well as in time. However, HadAM3H fails to model the second peak during the austral spring. Although $\mathrm{HadAM} 3 \mathrm{H}$ was set up for the pre-industrial period, it is not likely that the meridional temperature gradient was so much different during that time. The fact that HadAM3H is not able to reconstruct the peak in the SAO

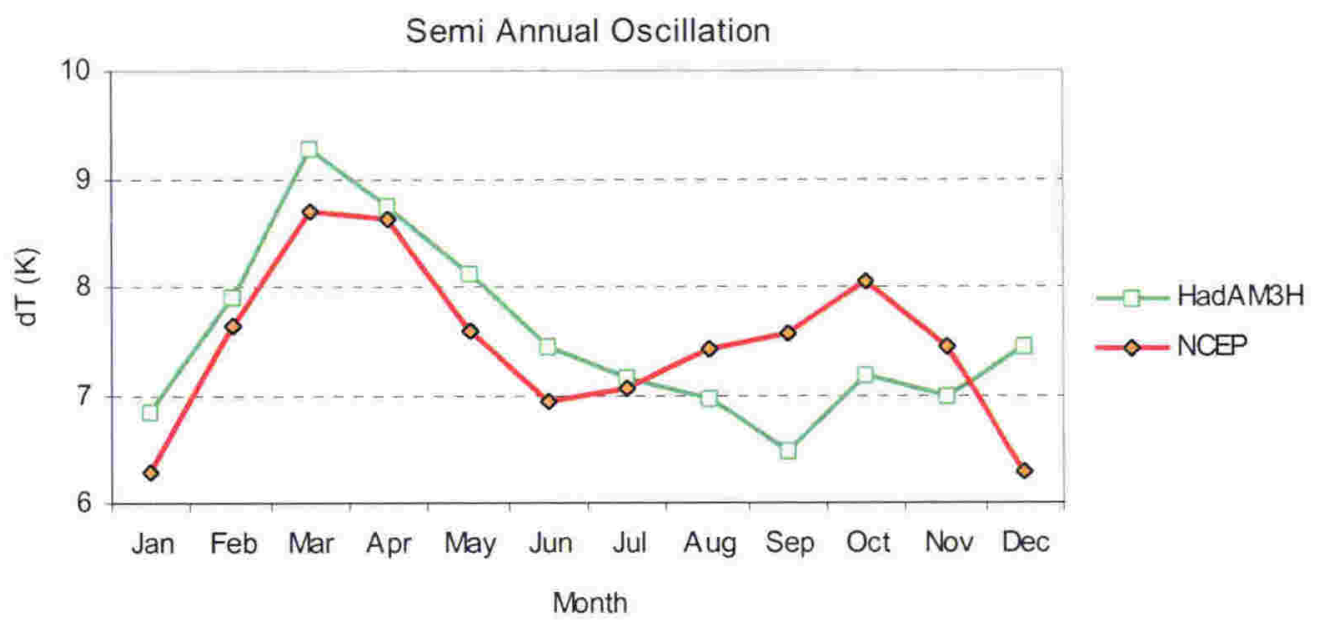

Fig. 5-21. Meridional temperature differences between latitude $50^{\circ} \mathrm{S}$ and $65^{\circ} \mathrm{S}$ for climatological $500 \mathrm{hPa}$ temperatures for HadAM3H and NCEP. 


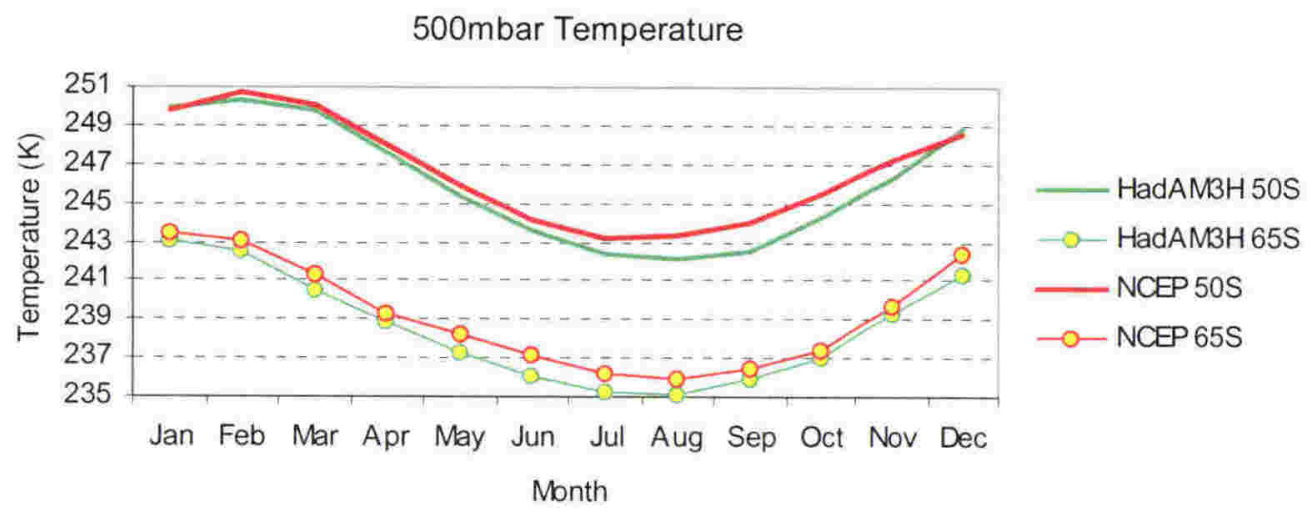

Fig. 5-22. Zonal mean $500 \mathrm{hPa}$ temperature for HadAM3H and NCEP for $50^{\circ} \mathrm{S}$ and $65^{\circ} \mathrm{S}$.

signal during the austral spring is because HadCM3 fails to produce accurately the annual cycle of SSTs at $40^{\circ} \mathrm{S}-60^{\circ} \mathrm{S}$. Fig. $5-22$ presents the $500 \mathrm{hPa}$ temperature profiles at $50^{\circ} \mathrm{S}$ and $65^{\circ} \mathrm{S}$ and gives a clearer picture on why the SAO in HadAM3H is different. During the austral summer months, the amplitude of the $\mathrm{SAO}$ in HadAM3H is slightly larger than the amplitude of the SAO in NCEP. This is primarily the result of $\mathrm{HadAM} 3 \mathrm{H}$ having cooler temperatures at $65^{\circ} \mathrm{S}$. During autumn, the temperature at $65^{\circ} \mathrm{S}$ in HadAM3H drops slightly faster than in NCEP, causing the first peak of the SAO in HadAM3H to decrease slower than in NCEP. It can be expected therefore that HadAM3H has slightly stronger winds and higher baroclinicity than NCEP during MAM. But the main difference between the NCEP SAO and HadAM3H's SAO is during the austral winter and spring, and is related to the warming at $50^{\circ} \mathrm{S}$ in $\mathrm{HadAM} 3 \mathrm{H}$ which lags the warming in the NCEP climatology. This has been documented by Gordon et al. (2000) who showed that the SSTs in HadCM3 at $40^{\circ}-60^{\circ} \mathrm{S}$ were too low. In this way, it is clear that the ocean heat storage plays an important role on the atmospheric circulation patterns (Meehl, 1991).

Simmonds and Jones (1998) have pointed out that periods of a weak SAO can be quite extensive. For that reason, the period of 21 years over which the SAO was determined could have been biased towards a weaker SAO. Analysis of the monthly variability of the SAO can indicate whether the weak second peak in the 
SAO was common during that period. The standard deviation of the interannual variability of the SAO is weakest during SON $\left(0.5-0.7^{\circ} \mathrm{C}\right)$ and strongest during DJF and MAM $\left(0.7-0.9^{\circ} \mathrm{C}\right)$. Their equivalent values in the NCEP climatology are $0.85-1.0^{\circ} \mathrm{C}$ and $1.0-1.2^{\circ} \mathrm{C}$ (DJF). This indicates that the weak SAO during SON was quite robust in the simulation.

Several studies have shown that the strength of the SAO is related to the amplitude of the wave number 3 pattern of the general circulation (e.g. Trenberth and Mo, 1985: Trenberth, 1986). It was noticed that there was a weakening in the SAO in the late 1970s, which was associated with an intensification of this wave number 3 pattern, a deepening and equatorward movement of the polar trough, an anomalous southward and intensified subtropical ridge which resulted in an enhancement of cyclone activity, and an increase of the strength of the westerlies (e.g. van Loon et al., 1993: Hurrell and van Loon, 1994: Chen and Yen, 1997: Meehl et al., 1998: Burnett and McNicoll, 2000). Some of these features show up in $\mathrm{HadAM} 3 \mathrm{H}$ during winter and spring, but less so during summer and autumn. Nevertheless, the SAO as modelled by HadAM3H seems to be consistent with those findings, even though its reduction during winter and spring is more extreme than described in those studies.

\section{Intraseasonal and Interannual Variations}

After removing the annual cycle in the mean sea level pressure data, intraseasonal and interannual variations can be determined using EOF analysis. Only the variability in the Southern Hemisphere is analysed. Fig. 5-23 shows the 3 leading EOFs of the monthly averaged mean sea level pressure in the NCEP climatology (top row) and HadAM3H (bottom row) for the zonal bands of $20^{\circ} \mathrm{S}$ $80^{\circ} \mathrm{S}$. For the NCEP climatology, the first EOF accounts for nearly $25 \%$ of the variations and is the characteristic High Latitude Mode (HLM), which describes the "see-sawing" variation of the difference in mean sea level pressure between the middle and high latitudes. It has a distinctive wave number 3 pattern with maxima virtually at equal distance from each other over the Indian Ocean, the Western Pacific and the Western Atlantic. In this configuration it is presented in its positive phase. The second and third EOF of the NCEP climatology are the 


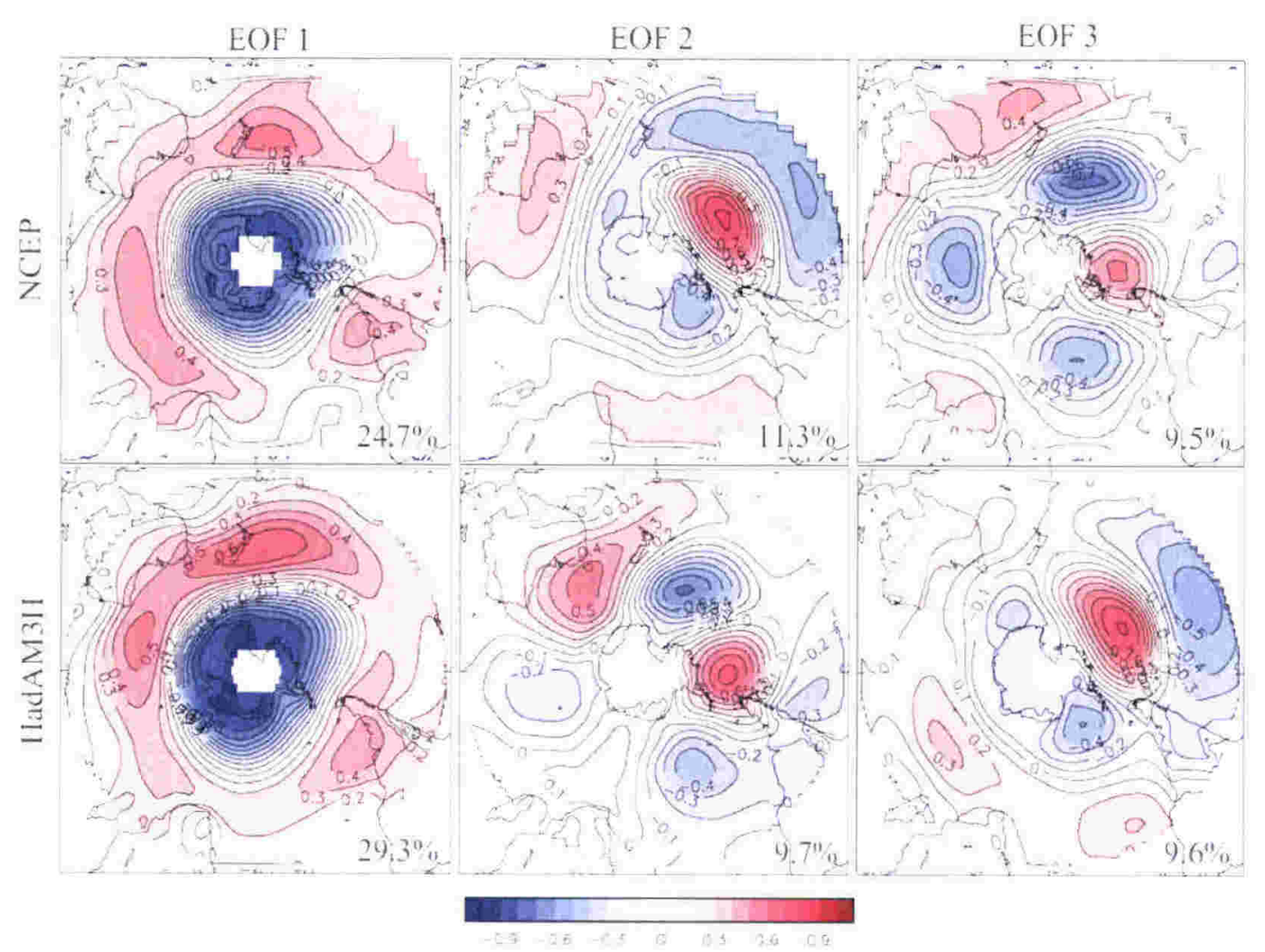

Fig. 5-23. Intraseasonal variation of the Southern Hemisphere $\left(20^{\circ} \mathrm{S}-80^{\circ} \mathrm{S}\right)$ circulation as presented by EOF analysis. Top NCEP, bottom HadAM3H. The bottom right hand corner of each EOF indicates the amount of variation it explains.

variations associated with ENSO, and show the Pacific dipole and the PacificSouth America wavetrain. They account for $11.3 \%$ and $9.5 \%$ of the variations respectively. The Pacific dipole pattern is more prominent in summer/autumn, and the wavetrain pattern is more dominant in winter/spring (e.g. Karoly, 1989). Fig. 5-24 shows the characteristic correlation patterns of the timeseries of the second and third EOF with SSTs. The three leading EOFs account for nearly half of the monthly variations with the next three EOFs for about $5 \%$ each again (not shown).

HadAM3H has essentially the same three leading EOFs although there are some differences (Fig. 5-23). The first EOF accounts for nearly 30\% of the variance, indicating that the HLM in HadAM3H is more prominent than in the NCEP climatology. The maximum over the Indian Ocean has moved eastwards, 


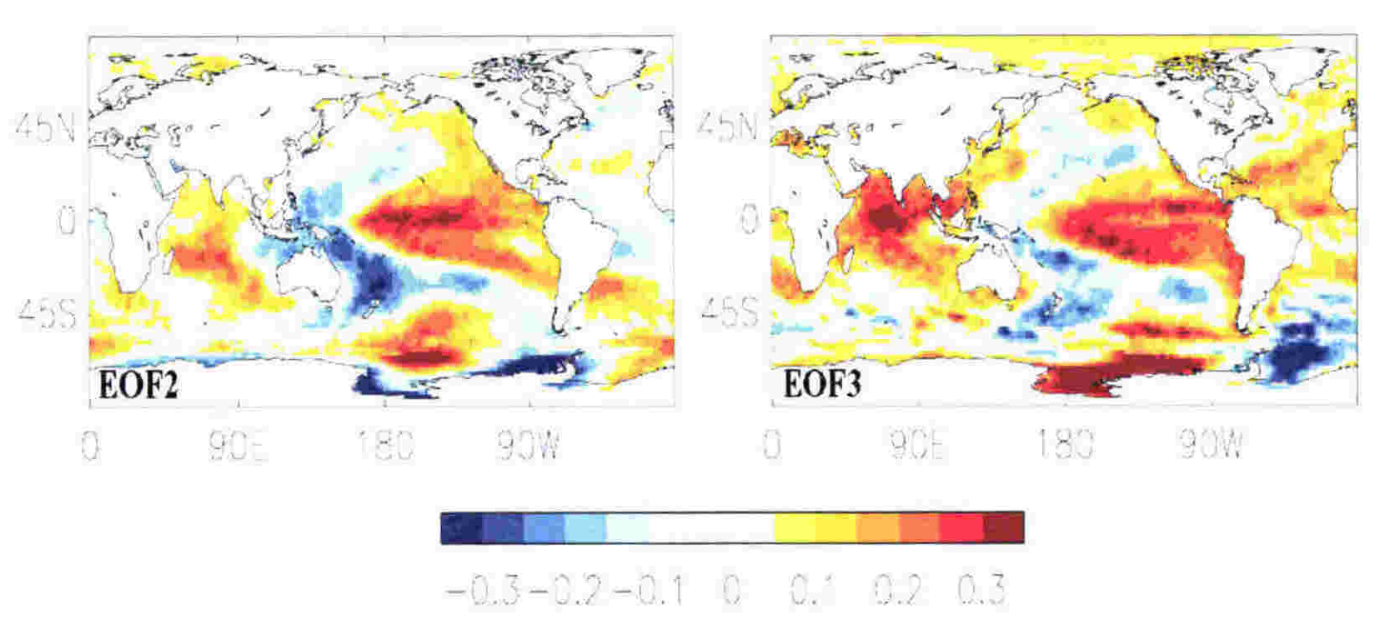

Fig. 5-24. Correlation patterns of the timeseries of EOF2 and EOF3 (NCEP) with SST.

and is much closer to the maximum over the Western Pacific. Both maxima have become stronger. The trough in the East Pacific is now farther to the west. The HLM mode in HadAM3H gives rise to stronger westerly winds, especially over the Western Pacific. It is clear that interannual variability as the result of the HLM mode is enhanced over New Zealand in HadAM3H.

Fig. 5-3 had shown that HadCM3 failed to properly reconstruct the extent and strength of the interannual variability in the Pacific SSTs. It is therefore expected that analysis of the ENSO variability in the NCEP and HadAM3H data will show some differences between the datasets. However, the second and third EOF pattern of HadAM3H are quite similar to the EOF patterns of the NCEP climatology, albeit that the wavetrain pattern now accounts for a similar amount of the variability as the Pacific dipole pattern (both about $10 \%$ ). The wave number 3 pattern in the second EOF of HadAM3H (the "wavetrain") is somewhat weaker than in the NCEP climatology and shows larger maxima over the Pacific region. The wavetrain seems to start more from the south of Australia, rather then from the east of Australia. Such a configuration enhances more the southerly winds than the south-westerly winds over New Zealand. However, applying the 'North test' (North et al., 1982), the second and third EOF overlap each other considerably, implying mixing between their patterns. Linear combinations of EOFs 2 and 3 could be configured to more closely resemble those from the NCEP data. The SST differences between NCEP and HadCM3 


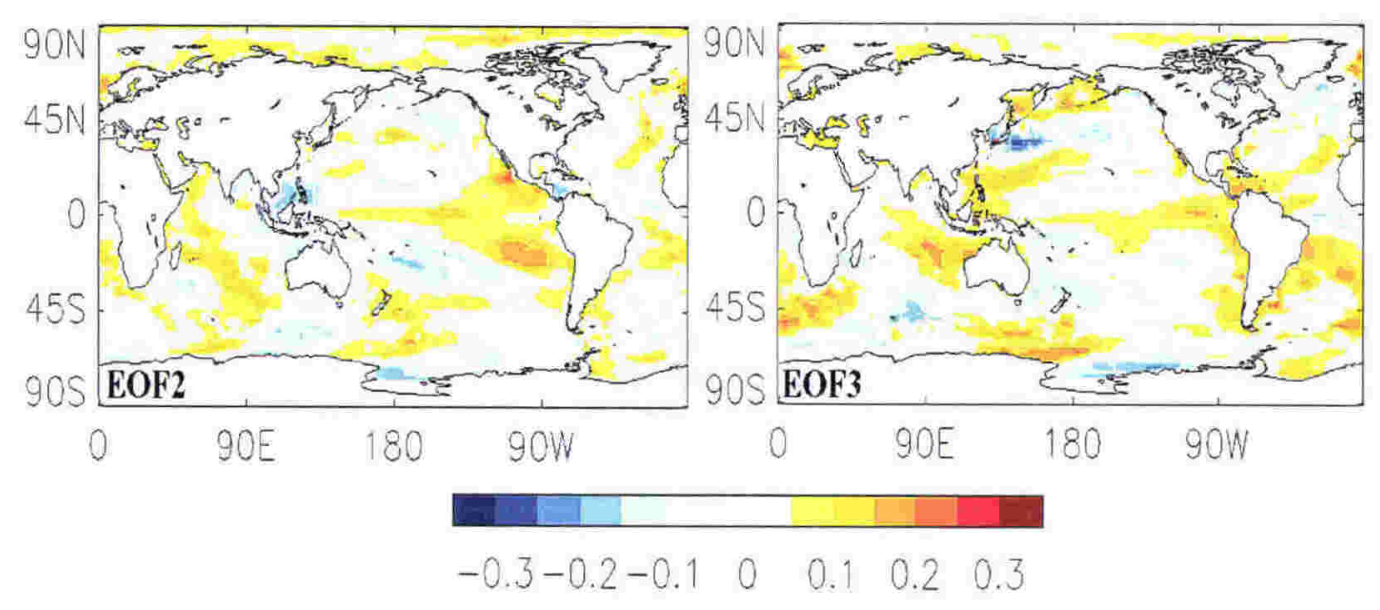

Fig. 5-25. Correlation patterns of the timeseries of EOF2 and EOF3 (HadAM3H) with SSTs.

are directly responsible for the differences in the interannual variations in mean sea level pressure. The correlation patterns of the timeseries of the second and third EOF from HadAM3H with the computed SSTs only indicate weakly the ENSO variability in the tropical Pacific (Fig. 5-25). Both correlation patterns show a maximum in the (eastern) Pacific, but both their amplitude and extent are anomalous. Although the second and third EOF are not related to the classical ENSO patterns, they are referred to as ENSO signals throughout this thesis, because of their similarity to the NCEP-based ENSO-related EOFs.

Further analysis of the timeseries of the EOFs shows more differences. Fig. 5-26 shows the distribution of the time series ${ }^{1}$ for the first three EOFs in standard deviation units. The second EOF of $\operatorname{HadAM} 3 \mathrm{H}$, the "wavetrain", is compared to its counterpart in NCEP where it is the third EOF (see Fig. 5-23). The HLM (EOF1), which is commonly characterized by a negative and positive phase, shows up quite distinctively in the NCEP climatology. It indicates that the HLM in NCEP is most often in either one of its phases, and less often in the "transition" zone between the phases (bimodal distribution). HadAM3H however does not show the two peaks and is much flatter across the whole distribution (unimodal distribution). HadAM3H has a bias towards the positive phase but shows a few more months in a strong negative phase.

\footnotetext{
'These are the EOF coefficients, or principal components.
} 

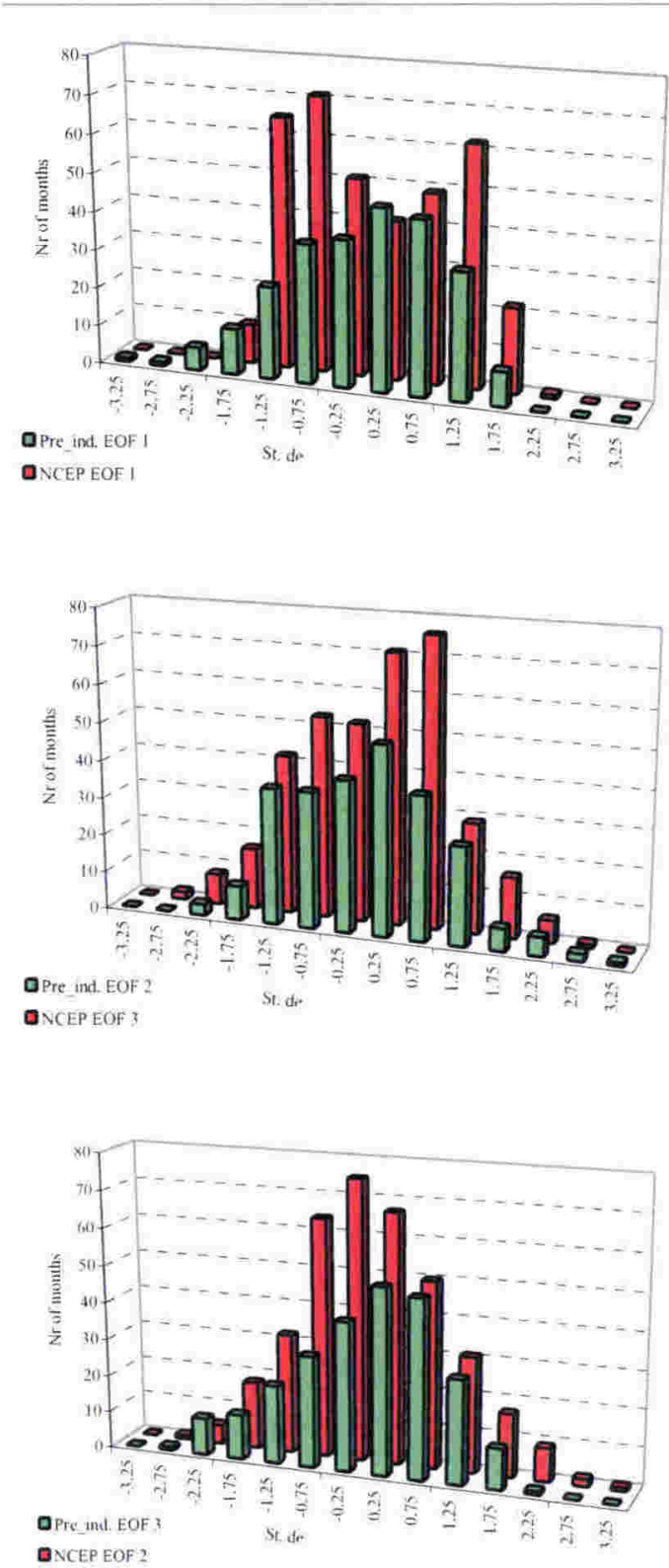

Fig. 5-26. Distribution of the EOF coefficients for the first three EOFs for NCEP and HadAM3H. X-axis is standard deviation and $y$ axis is number of months. NCEP is over the period of 1971-2000 and HadAM3H is over 21 years, hence the difference in the total numbers.
The wavetrain pattern (NCEP EOF 3 versus HadAM3H EOF 2, the middle graph in Fig. 5-26) sees a distinctive positive peak and a weak negative one in the NCEP climatology. The positive phase is associated with El Niño and the negative with La Niña. It is evident that the 1971-2000 climatology was influenced more by El Niños than by La Niñas, which was already commented upon earlier. In general, the distribution in time of the positive and negative phase of EOF2 in $\operatorname{HadAM} 3 \mathrm{H}$ is quite evenly spread. It does show a small positive peak (El Niño) but fails to model the negative peak (La Niña), although there is a small bias towards negative values.

The time series belonging to the "dipole" pattern (NCEP EOF 2 versus $\operatorname{HadAM} 3 \mathrm{H}$ EOF 3, the bottom graph in Fig. 5-26) shows a very symmetrical, Gaussian-shaped distribution for NCEP with a small bias towards a negative distribution. HadAM3H shows a similar distribution but with a shift more towards the positive values. From the time series analysis it can be concluded that although the three leading EOFs of the interannual variability as modelled by HadAM3H account for a similar percentage of the total variability 
as they do in the NCEP climatology, the distribution of their amplitude differ noticeably.

\subsection{Summary}

The performance of the Unified Model HadAM3H has been investigated under a pre-industrial configuration. By simulating the climate during preindustrial times it is possible to simplify the tropospheric chemistry by omitting the interactive sulphur cycle and keep the atmospheric gases $\mathrm{CO}_{2}, \mathrm{CH}_{4}$ and $\mathrm{N}_{2} \mathrm{O}$ constant at $280 \mathrm{ppm}, 729 \mathrm{ppb}$ and $285 \mathrm{ppb}$ respectively. The output of the simulation was compared to the NCEP 1971-2000 climatology and it is assumed that the climate of the pre-industrial times was qualitatively not different than today's climate and that such a comparison is therefore valid. Of the NCEP climatology it is known that the largest uncertainties appear poleward of $40^{\circ} \mathrm{S}$, and although the emphasis in this simulation is on the reconstruction of the circulation in the Southern Hemisphere, the dataset was deemed to be appropriate for this study.

HadAM3H is an atmospheric model that is virtually the same as its basic model of a lower resolution, HadAM3. It uses the SSTs and sea-ice fields determined by the coupled model HadCM3 under the same configuration. The biases in these models have been described by Pope et al. (2000: HadAM3) and Gordon, Cooper et al. (2000: HadCM3). Relevant to this study are that the westerly winds are too strong, HadCM3's computed SSTs in the Pacific show only weak variability, cooler SSTs in the North Pacific and warmer SSTs in the Southern Hemisphere's high latitudes. The peak SST errors in the Southern Ocean are in regions of steep SST gradients and therefore could possibly be associated with the incorrect positioning of the ACC. The considerable differences between the NCEP climatology and HadCM3 SSTs at high latitudes are highly correlated to the differences between their sea-ice fields. NCEP shows larger interannual variability in the central Pacific (ENSO) than HadAM3H.

The climate of the pre-industrial is globally cooler than the NCEP climatology. The main differences in the surface temperature between the two 
climatologies over the oceans are related to their differences in SSTs, and over land are often related to how well the orography has been incorporated. The latter is especially relevant for Antarctica, which climate is particularly difficult to reconstruct. $\mathrm{Had} \mathrm{AM} 3 \mathrm{H}$ shows considerable warmer temperatures in the tropics, which are the result of an increase in latent heating, associated with an increase in precipitation there. With differences in Pacific SSTs, trade winds, convection and cloud amount, the amount of precipitation in the tropics in NCEP and HadAM3H shows large variations. Precipitation is one of the hardest climatic variables to model. HadAM3H at least correctly identifies the ITCZ, SPCZ and the monsoonal precipitation patterns.

HadAM3H not only overestimates the mean westerly winds, it also shows an enhanced wave number 3 pattern in the Southern Hemisphere midlatitudes, particularly in winter. This results in an overestimation of the meridional momentum and heat fluxes, and then in particular their transient eddy component. The eddy momentum flux in the Northern Hemisphere shifts slightly equatorward, following the equatorward movement of the strongest $200 \mathrm{hPa}$ winds. An equatorward shift of the strongest mean winds also occurs in the Southern Hemisphere, although this shift was not apparent in the transient eddy momentum flux of the Southern Hemisphere. The transient component of the meridional heat transport is particularly enhanced in the Southern Hemisphere, indicating stronger cyclonicity.

Several modes of variability in the Southern Hemisphere were investigated. The SAO showed the characteristic peak in the meridional difference between the $50^{\circ} \mathrm{S}$ and $60^{\circ} \mathrm{S} 500 \mathrm{hPa}$ temperature during autumn, but failed to show a distinct peak during spring. This was primarily due to the lag in warming at $50^{\circ} \mathrm{S}$ during spring, which was related to the cooler temperatures in the SSTs at $40^{\circ} \mathrm{S}$ $60^{\circ} \mathrm{S}$. A weakening of the SAO is associated with an intensification of the wave number 3 pattern in the mean zonal circulation, a deepening and equatorward movement of the polar trough and an increase in the strength of the westerlies. These characteristics have all been found in this simulation to a certain degree as well. In the analysis of many climatic parameters, it showed that HadAM3H varied from NCEP the most during SON. The anomalous SAO signal in 
HadAM3H during SON is therefore a direct indication that HadAM3H has its largest errors during this period.

An investigation into interannual variability through EOF analysis shows that the first three EOFs in HadAM3H account for nearly $50 \%$ of all the variations on monthly and longer timescales. This is only slightly higher than in the NCEP climatology, but there were some notable differences. The HLM in HadAM3H explains nearly $30 \%$ of the variability against "only" $25 \%$ in NCEP, its maximum in the Indian Ocean has intensified and has shifted eastwards, the Pacific maximum has also intensified and is geographically enlarged, and the East Pacific trough has shifted westwards. The time series of the HLM does not show the distinct peaks of the positive and negative phase seen in the time series in NCEP, and shows much more a Gaussian distribution. Although there is only a weak correlation between the timeseries of the second and third EOF with the SSTs in the tropical Pacific, it is assumed that they are associated with ENSO. The ENSO signal in the tropical Pacific is quite weak in HadCM3, but both EOF patterns still explain nearly $10 \%$ of the interannual variation in the Southern Hemisphere. The pacific dipole pattern accounts for $11.3 \%$ of the variability in $\operatorname{NCEP}\left(2^{\text {nd }} E O F\right)$, and accounts "only" for $9.6 \%$ in HadAM3H $\left(3^{\text {rd }} E O F\right)$. The wave train pattern, which is more dominant during winter/spring, does not start from the east coast of Australia in HadAM3H. The largest variability is south of Australia, from where the wave train starts. This could possibly be associated with the anomalous low SSTs south of Australia that are found during winter and spring, resulting in a larger range of SSTs during the year. The effect is that the positive phase of the $2^{\text {nd }}$ EOF in HadAM $3 \mathrm{H}$ (the wave train pattern) brings more southerlies, rather than south-westerlies, over the New Zealand region. Time series analysis shows that the wave train pattern of $\mathrm{HadAM} 3 \mathrm{H}$ captures the distribution between El Niño and La Niña quite well, but that the dipole pattern shows a preference towards the positive phase in HadAM3H. Since the second and third EOF are degenerate, an improved reconstruction of tropical SST variability could possibly lead to EOF patterns more similar to those of NCEP. That would result in more realistic simulations of ENSO variability in the Southwest Pacific. 


\section{Chapter 6}

\section{Pre-industrial simulation:}

\section{New Zealand}

\section{Introduction}

In this chapter the performance of the RCM for New Zealand is discussed. In the only other regional climate simulation similar to this study, Renwick et al. (1998) showed that the skill of specifying New Zealand's climate did increase noticeably when nesting a high-resolution RCM inside a GCM. The climate of New Zealand will be mainly described by the temperature and precipitation patterns of several regions during the various seasons. Although analysis of the extremes would be very useful in accessing the performance of the RCM, only monthly and seasonal means were stored during the runs. Temperature and precipitation data can easily be compared against the observed data and proxy data for those climatic indicators available from the LGM. New Zealand's temperature and precipitation patterns have the additional value of responding significantly to changes in the general circulation (e.g. Salinger and Mullan, 1999). Comparing the data against the observations will show to what degree the model is capable in simulating the climate of New Zealand. Once that is established, the output of the LSM simulations can then he analvsed and 
extensively by Hudson and Jones (2002b) and Frei et al. (2003) and the reader is referred to these papers for an analysis of the model's behaviour.

Local climate variability is a sensitive indicator of changes in the large-scale circulation. In chapter 5 it was determined that the modelled general circulation in the Southern Hemisphere had an enhanced wave number 3 pattern and the modelled westerlies were too strong. The regional model's output will be affected by these anomalous patterns and therefore discrepancies with today's climate are expected.

The description of this RCM, NZ_40km, was given in section 4.3 .3 and its specifications are given in Table 4-1. As with the GCM HadAM3H, the simulation is done under pre-industrial conditions, that is, the tropospheric chemistry has been kept constant and put at levels present during pre-industrial times. The output of the regional model is verified against the New Zealand Climate Database (CLIDB: e.g. Penney, 2001). After a short description of CLIDB, the setup and the results of the regional climate model of New Zealand are discussed in this chapter. Geographical locations that are being referred to are all marked on the map on page 1 .

\subsection{New Zealand Climate Database, CLIDB}

The CLIDB database is a comprehensive set of atmospheric data of New Zealand, which is maintained by the National Institute of Water and Atmospheric Research (NIWA). Its data comes for instance from automatic weather stations, radio sondes and synoptic data from the MetService (Meteorological Service of New Zealand). This dataset is highly detailed and has a very good coverage of New Zealand, although the Southern Alps and Fiordland have a relatively low coverage, due to the difficult terrain. As an example, Fig. 6-1 shows two presentations of data of CLIDB, the New Zealand Daily Average Temperature and the New Zealand Mean Annual Rainfall for the period 1971-2000. The data to create these maps included stations that were not operational over the full period. Their data was therefore "weighted" according to the length of time they 


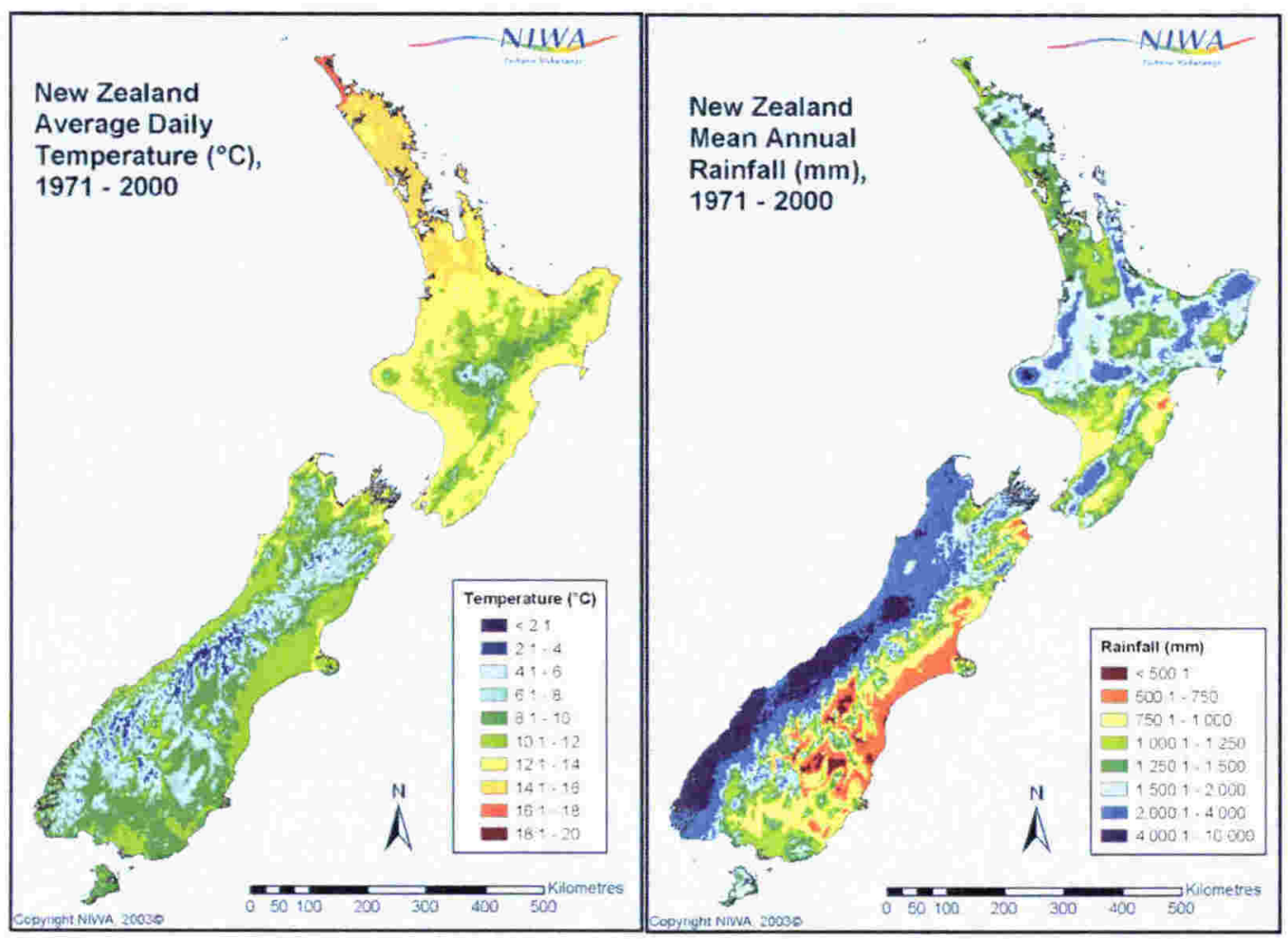

Fig. 6-1. New Zealand Average Daily Temperature $\left({ }^{\circ} \mathrm{C}\right)$ and New Zealand Mean Annual Rainfall (mm) for the period of 1971-2000. Source Climate Database of New Zealand, CLIDB. Printed by permission of NIWA.

were operational. There were in total about 2000 stations.

The CLIDB data was interpolated onto a $0.05^{\circ}$ resolution grid covering New Zealand. The CLIDB was subsequently interpolated onto the lower resolution grid of the RCM to enable comparison between RCM output and CLIDB data.

\subsection{Setup of $\mathrm{NZ}_{-} 40 \mathrm{~km}$}

The initial conditions come from HadAM3H. The RCM was started 10 years into the GCM simulation and ran nested inside the GCM for the next 20 years. The boundary conditions were updated every 6 hours. For the analysis of the RCM data, only the last 15 years of the simulation have been used. Some of the fields required for running the RCM were taken directly from the GCM and were interpolated onto the higher resolution grid, e.g. SST, sea-ice and ozone. 


\section{- Orography:}

As in the determination of the global orography for $\mathrm{HadAM} 3 \mathrm{H}$, the original orography dataset used was the US Navy 10" resolution dataset. This dataset was interpolated onto the regional domain (Fig. 6-2). The gravity wave drag coefficients were reduced again to account for the higher resolution of orography (refer to Orography: section 5.1). There are 180 land points in the RCM.

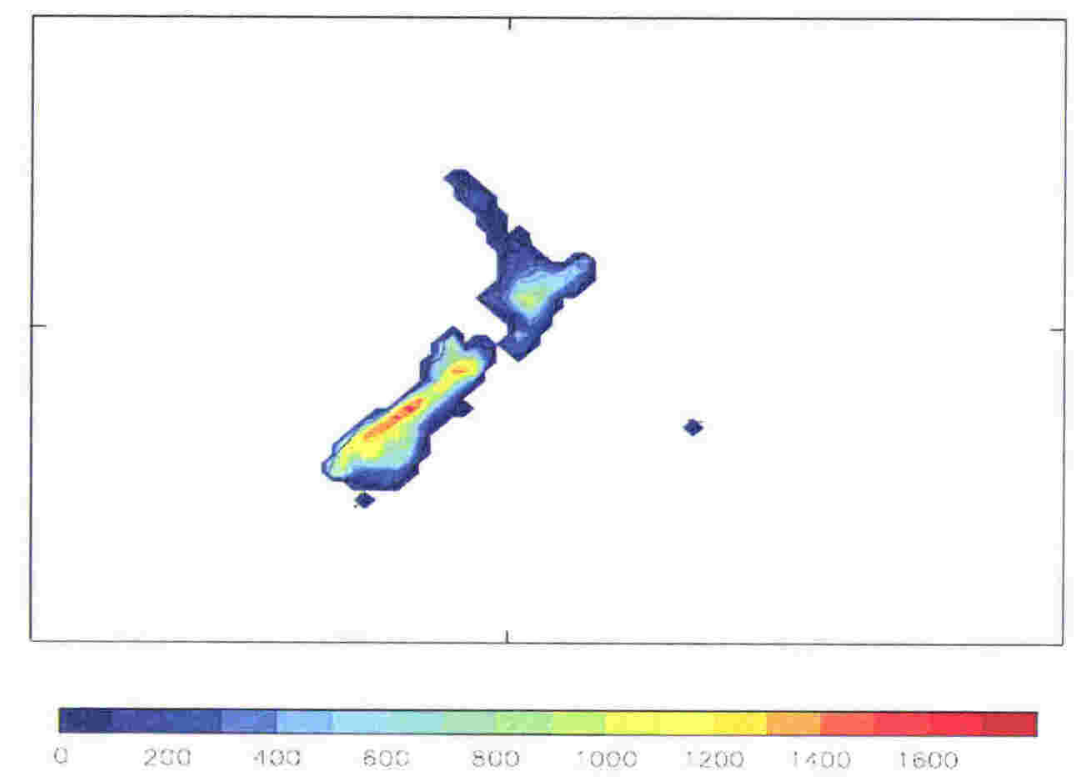

Fig. 6-2. The orography (in $\mathrm{m}$ ) and the domain of the regional model NZ_40km in its rotated position.

\section{- SST and sea-ice:}

The SSTs and sea-ice fields were interpolated from the GCM. As with the global simulation, they were updated every month. The averaged SSTs for DJF and JJA are presented in Fig. 6-3. There is a gradient of about $10^{\circ} \mathrm{C}$ between south and north New Zealand. The Tasman Front flows from west to east at about $35^{\circ} \mathrm{S}$, separating the warmer subtropical waters from the colder polar waters (Fig. 3-5) It flows around the north of New Zealand as the East Auckland Current (EAUC), and continues to the east of New Zealand in the East Coast Current (ECC). This water can easily be identified in the SSTs of the regional model as the tongue of warm water curling around the north of New Zealand. There is a sharp 

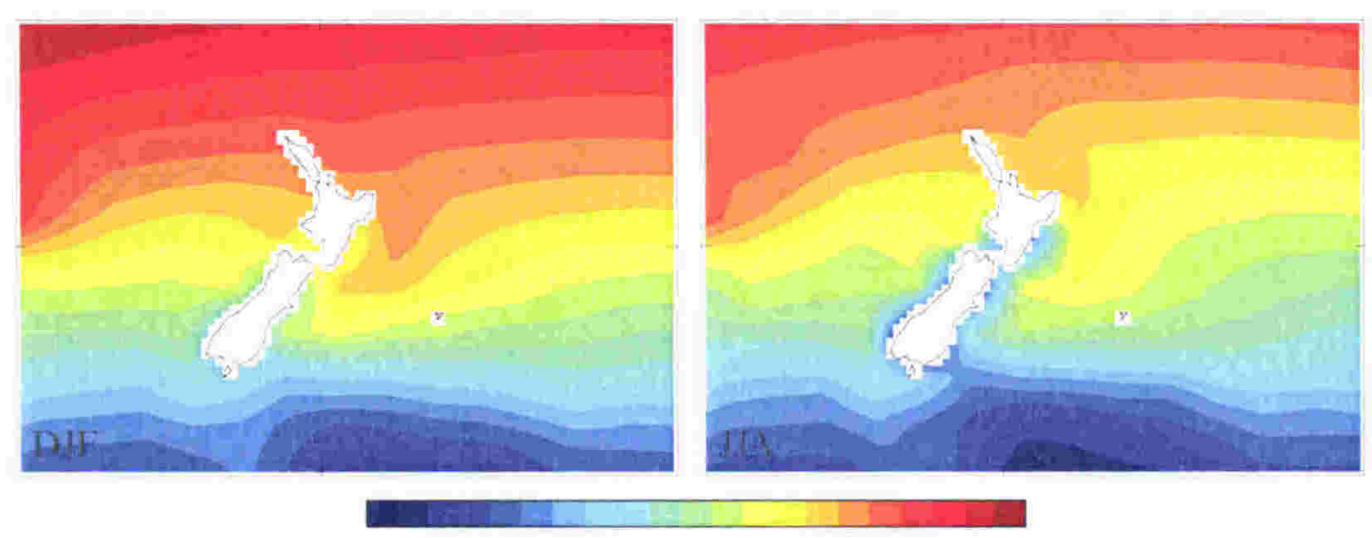

Fig. 6-3. Average SSTs (K) for DJF and JJA for NZ_40km.

temperature gradient east of New Zealand at $43^{\circ} \mathrm{S}$ where the Chatham Rise separates the warmer ECC from the Antarctic Circumpolar Current (ACC). This gradient is slightly too large compared to the present day climatology as was shown by Gordon et al. (2000), with both the southern waters too cool and the northern waters too warm.

At no time were there any incursions of sea-ice in this regional domain. There are therefore no climatic effects related to regional sea-ice in this simulation.

\section{- Vegetation and soil:}

The global vegetation data set (WHS, Wilson and Henderson-Sellers, 1985) with its resolution of $1^{\circ} \times 1^{\circ}$ covered New Zealand only with 33 points. That number of points is not sufficient to describe the large variety in vegetation in New Zealand. The global vegetation dataset described New Zealand mainly as grazing and pasture (in the east and north of the country), with forests in the west and with some dwarf shrubs in Fiordland. Although there are probably better high resolution vegetation databases for New Zealand available, there is none however in a format that can easily be converted in a format that can be used by the UM. It was therefore decided to just interpolate the global vegetation dataset onto the regional domain. The soil type used was also obtained from the WHS dataset, and was also interpolated onto the RCM domain. 
Some parameters in the UM have been adjusted as the result of the higher resolution. For instance, the diffusion parameters have been reduced and the grid box fraction over which convective precipitation is assumed to fall is increased and the grid box fraction over which large scale precipitation is assumed to fall is reduced. These adjustments are in line with usual practice in running the RCM at $40 \mathrm{~km}$ resolution at the MetOffice.

\subsection{Results}

As discussed in section 4.3.3, for computational reasons it was convenient to move the coordinate pole away from the geographic pole so that the rotated equator passes through the centre of the New Zealand domain. For the analysis of the data and for the presentation of the results, the regional domain with its data was rotated back to its real location. This would give a slight distortion in the position of the grid points, and therefore the location of a data point. This is illustrated as an example in Fig. 6-4. This figure shows the modelled mean sea level pressure for DJF in the domain used for the simulation (A), and when rotated back to its original position (B). Comparing $\mathrm{A}$ with $\mathrm{B}$ shows the amount of distortion that occurs in the return rotation. Often the analysis is done on data that are on or over land and the presentation will show then only the land area! In Fig. 6-4, C and D are the land areas of the output of the simulation and the domain used for the analysis respectively. The distortion shows up mainly in the more "jagged" contour lines.

New Zealand's National Institute of Water and Atmospheric Research (NIWA) presents its seasonal climate outlooks for six defined regions. These regions were determined by an REOF analysis, and are characterized by having similar behaviour in their covariances (Mullan, 1998). Some of the data here, as well as in chapter 7 will also be analysed using these regions. Fig. $6-5$ shows the six different regions. It is acknowledged here that by restricting some of the analysis to regional means, the statistics are calculated over a smaller number of

\footnotetext{
' The Chatham Islands are present but were not included in any analysis, hence the size of the area.
} 


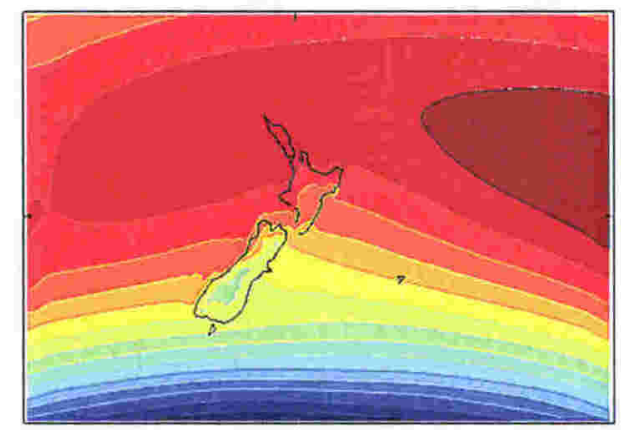

A

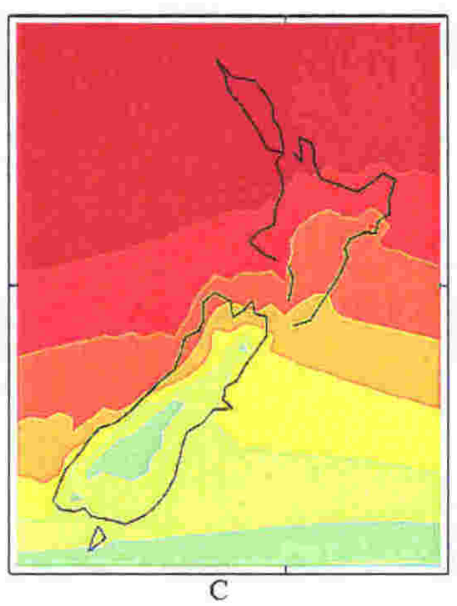

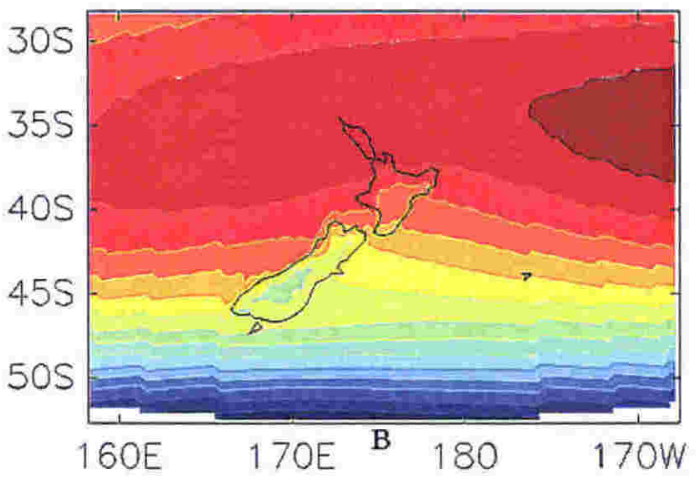

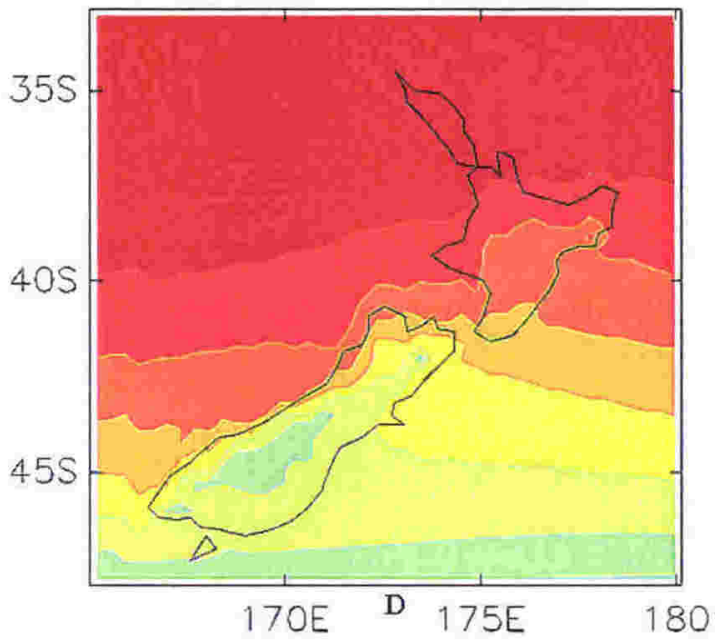

1002100410061008101010121014

Fig. 6-4. The modelled DJF mean sea level pressure (in $\mathrm{hPa}$ ) in the domain of the simulation $(\mathrm{A})$, rotated back to its original position (B), and their zoomed in versions of the land areas (C and D respectively).

points, possibly reducing the significance of some of the regional findings. However, the regional analysis may be appropriate to the smallest scales properly represented in a model with $40 \mathrm{~km}$ grid spacing.

\subsubsection{Temperature}

One of the few parameters that can be compared directly with the CLIDB database is the surface temperature. Fig. 6-6 shows the mean annual surface temperature from the RCM and CLIDB. Only the grid points corresponding to 


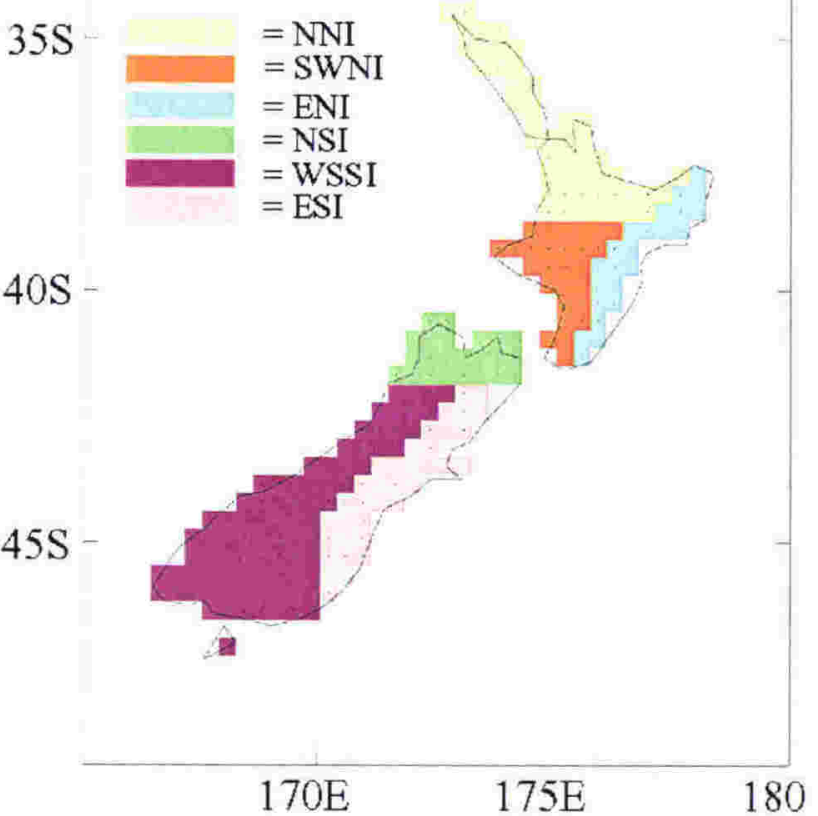

Fig. 6-5. The grid points and the different regional areas of the New Zealand landmass. NNI $=$ North North Island, SWNI $=$ South-West North Island, ENI $=$ East North Island, NSI $=$ North South Island, WSSI $=$ West and South South Island, ESI $=$ East South Island.

land in the RCM are presented since no comparison can be made with CLIDB in points over sea'. Although the Chatham Islands are present in the RCM and climate data for the Islands are available, since the Chatham Islands occupy only one grid point far away from the main landmass of New Zealand, they were omitted from the discussions in this study. The CLIDB temperature data was interpolated onto the RCM grid to allow for direct comparisons (Fig. 6-6) ${ }^{2}$.

The modelled and recorded (CLIDB) surface temperatures are similar in both their magnitude and patterns over New Zealand. The greater area of the Southern Alps, extending from Fiordland to the Nelson Lakes region and including the Inland Kaikouras, stands out the region with the lowest mean annual surface temperatures. The narrow coastal strip of relatively low land along the West

\footnotetext{
' In order to cover the country completely, grid points that are over the sea but whose grid box is partially land are included as well. That explains the coastal contours of warm temperatures around Northland, and Bay of Plenty, which are in fact sea points. There are no land grid points in the Cook Strait.

${ }^{2}$ After interpolation onto the lower resolution grid of $\mathrm{NZ}_{-} 40 \mathrm{~km}$, there were no data points covering the North Cape, the eastern tip of the East Coast, the southwestern tip of Fiordland and the south of Stewart Island.
} 


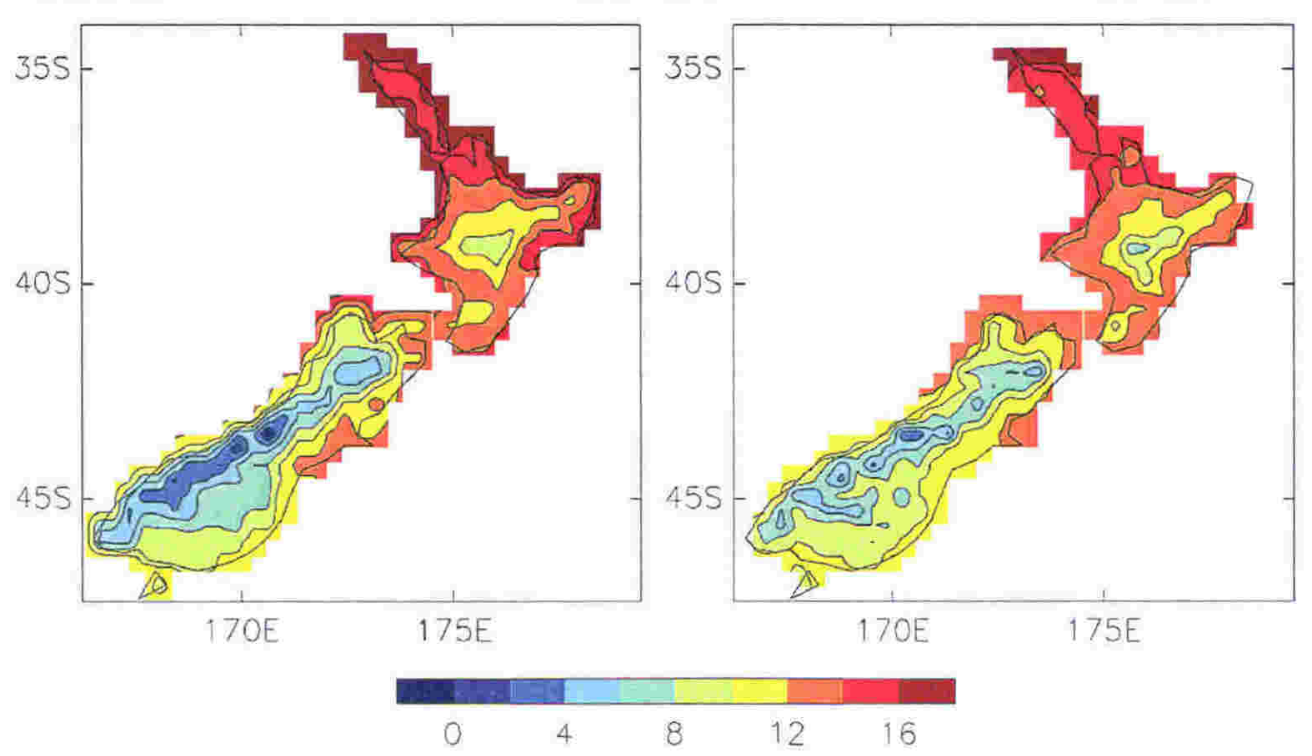

Fig. 6-6. The mean annual surface temperatures $\left({ }^{\circ} \mathrm{C}\right.$ ) in the RCM (left) and CLIDB (right).

Coast north of about Hokitika/Greymouth shows up as it has higher mean annual surface temperatures than the inland mountains. The area of relatively low orography continues into the northern region of the South Island, including Kahurangi, North West Nelson and the Marlborough Sounds. The Marlborough Sounds have the highest mean annual surface temperatures in the South Island. The mean annual surface temperature of Southland is higher than in inland Otago, indicating the influence of the sea. However the cooler temperatures of inland Otago extend too far to the east. The east coast of the South Island, and in particularly Canterbury, shows the higher mean annual temperatures found there, although they are slightly higher than in CLIDB.

The regional variation in temperature in the North Island is less than in the South Island, primarily as a result of the North Island being less mountainous. The elevated region of the Volcanic Plateau in the centre of the North Island has lower mean annual surface temperatures than the surrounding areas. The warmest areas are found north of about Hamilton, in the Hawke' Bay and around Gisborne. As it is in the South Island, the mean annual surface temperatures in the east are slightly too high. Table 6-1 gives the mean annual surface temperatures for the different regions of Fig. 6-5 as well as for the entire country. This table clearly shows that the largest differences in mean annual surface 
Chapter 6: Pre-industrial simulation: New Zealand

\begin{tabular}{|c|c|c|c|}
\hline \multicolumn{2}{|l|}{ Region } & CLIDB & $\mathrm{RCM}$ \\
\hline North North Island & $-\quad$ NNI & 14.07 & 14.00 \\
\hline South-West North Island & - SWNI & 11.64 & 11.48 \\
\hline East North Island & - ENI & 11.68 & 12.81 \\
\hline North South Island & - NSI & 11.44 & 9.80 \\
\hline West and South South Island & - WSSI & 7.88 & 6.35 \\
\hline East South Island & - ESI & 9.50 & 9.99 \\
\hline New Zealand & & 10.49 & 9.95 \\
\hline
\end{tabular}

Table 6-1. The mean annual surface temperature $\left({ }^{\circ} \mathrm{C}\right)$ for each region of Fig. 6-5 and for the whole of New Zealand as determined from CLIDB and NZ $40 \mathrm{~km}$.

temperature between the RCM and CLIDB occur in regions with high orography, and in the east of New Zealand. In the former regions, the mean annual surface temperatures in the RCM are too low, and in the latter regions the mean annual surface temperatures are too high.

A more detailed discussion of the difference in the mean surface temperatures between the RCM and the CLIDB is given in the analysis of their mean seasonal temperatures. Table 6-2 and Fig. 6-7 show the differences in mean seasonal surface temperatures between the RCM and CLIDB. The graphs are indicative of the difficulty of comparing data from a relative low-resolution climate model with a high-resolution climate dataset. New Zealand is very mountainous and so it is important to compare data not only from the same longitude and latitude, but

\begin{tabular}{||r|r|r|r|r|r|r|r|r||}
\hline \multicolumn{1}{|c|}{ Region } & \multicolumn{4}{|c|}{ CLIDB } & \multicolumn{4}{|c||}{ RCM } \\
\hline \hline & DJF & MAM & JJA & SON & DJF & MAM & JJA & SON \\
\hline \hline NNI & 18.02 & 14.94 & 10.02 & 13.31 & 18.54 & 14.39 & 9.67 & 13.40 \\
\hline \hline SWNI & 15.93 & 12.24 & 7.36 & 11.02 & 16.06 & 11.75 & 7.13 & 10.99 \\
\hline \hline ENI & 16.09 & 12.27 & 7.24 & 11.10 & 18.29 & 12.79 & 7.66 & 12.49 \\
\hline \hline NSI & 15.53 & 12.23 & 7.11 & 10.89 & 14.50 & 9.93 & 5.25 & 9.52 \\
\hline \hline WSSI & 12.34 & 8.47 & 3.10 & 7.60 & 10.74 & 6.82 & 2.15 & 5.70 \\
\hline \hline ESI & 14.16 & 10.06 & 4.52 & 9.24 & 15.60 & 9.88 & 4.38 & 10.09 \\
\hline \hline NZ & 14.82 & 11.15 & 5.96 & 10.02 & 14.71 & 10.22 & 5.38 & 9.51 \\
\hline \hline
\end{tabular}

Table 6-2. Mean seasonal surface temperatures (in ${ }^{\circ} \mathrm{C}$ ) in CLIDB and NZ_40km by region. 


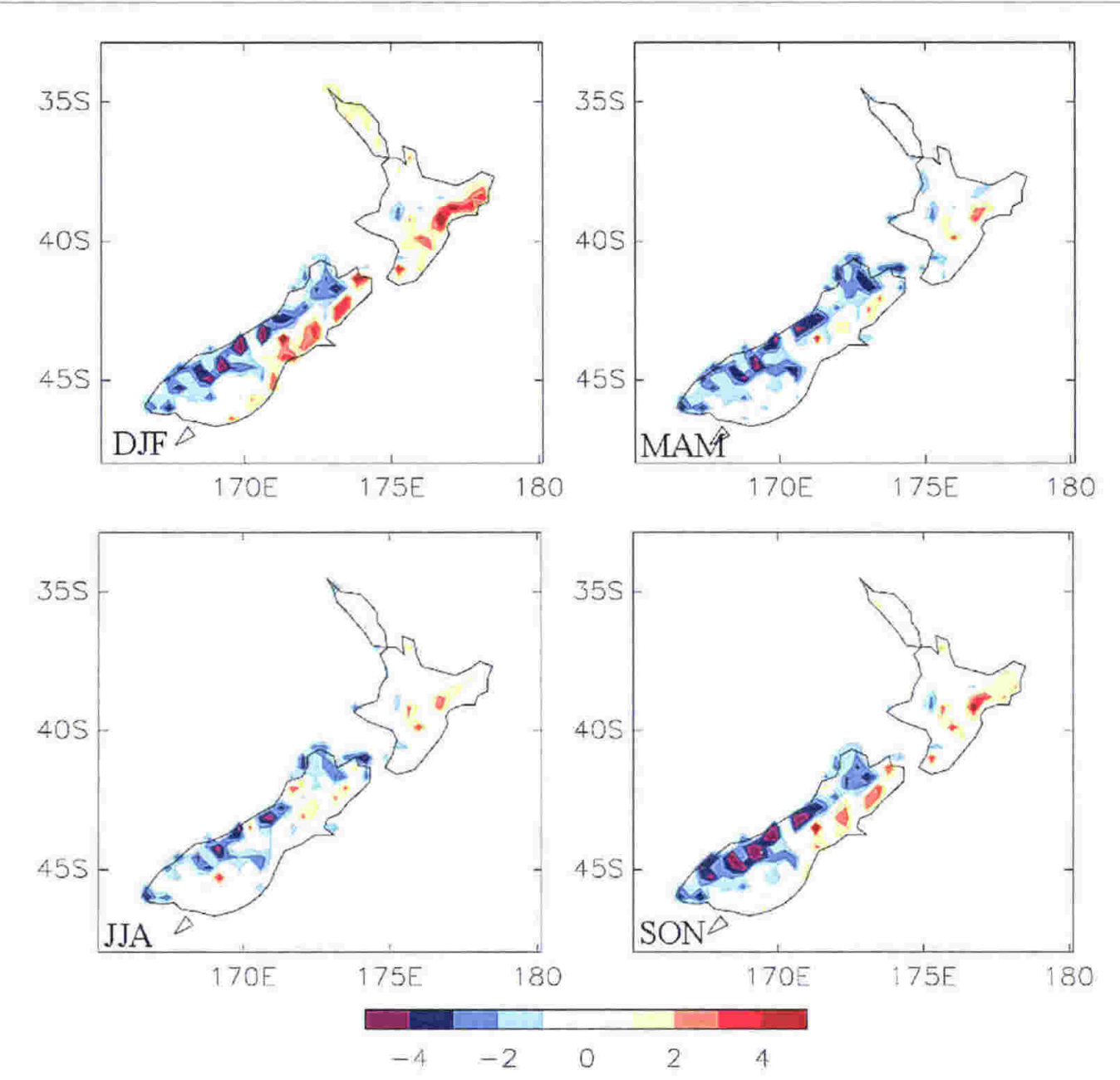

Fig. 6-7. The difference in mean seasonal surface temperature between NZ_40km and CLIDB ( $\mathrm{T}_{\mathrm{NZ} \_40 \mathrm{~km}}-\mathrm{T}_{\mathrm{CLIDB}}$ in $\left.{ }^{\circ} \mathrm{C}\right)$.

also at the same altitude. Altitude is an important spatial component, especially for parameters such as temperature and precipitation (see section 6.3.2). The modelled orography in each grid point shows the average altitude of an area of roughly $1600 \mathrm{~km}^{2}$. If this average altitude is different than the altitude of the equivalent point in CLIDB by as much as $150 \mathrm{~m}$, there would be a difference in surface temperature of $1^{\circ} \mathrm{C}$ simply because of orographic reasons.

The effect of the different orography between CLIDB and NZ_40km on surface temperature can partially be countered by placing the RCM surface temperatures at the same altitude as in the CLIDB dataset, by adjusting their values according to the lapse rate. Such an adjustment was done for instance in Moberg and Jones (2004). Such an approach was not applicable in this study for 
several reasons. It was not possible to link the RCM surface temperatures to one uniquely determined altitude since there were many CLIDB stations within a gridbox. No algorithm that would weigh the importance of the stations within each gridbox was known to the author. Secondly, averaging out their different heights would only result in reducing the magnitude of the differences at best, but would not change their patterns. And thirdly, the simulated climate of New Zealand during the LGM (chapter 8) will be compared to the climate of the preindustrial. It is not known how to adjust data points at different altitude during the LGM. As will be discussed in chapter 8, differences in altitude between preindustrial and LGM values are very difficult to compare with each other.

The RCM temperatures are considerably cooler than observed in the mountains. This is in particularly true for the Southern Alps, but single points of high orography, like Mt. Egmont, the Volcanic Plateau and the Northern Tararuas, do consistently stand out too. The general warmer temperatures out east in the RCM are probably caused by an enhanced "föhn" effect of the model; that is dry winds coming off the mountains, compressing and thereby warming the air. This would particularly have been significant during SON. The eastern regions would have experienced stronger westerly winds than observed in the spring seasons, since the GCM had modelled the westerly winds too strong. Another cause for the high surface temperatures in the east could be that the hydrological cycle was affected by the very dry conditions of the soils. It was found that the variability in maximum daily surface temperatures was too high, particularly during DJF, which probably was related to the soils drying out to quickly (Drost et al., 2005). The small variations in the seasonal differences indicate that the simulation gets the seasonal trend in general quite right.

Cross sections can give a good indication on how well the RCM has picked up regional features. Fig. 6-8 shows the cross sections superimposed on the orography of the RCM, and Fig. 6-9 shows the temperature profiles of those cross sections $^{1}$. The criterion for choosing the locations of the cross sections was that they had to give the best coverage of the various different climate patterns in

\footnotetext{
' Only 5 cross sections will be discussed. The sixth, the Southern Fiordland-Southland cross section, will only be discussed in chapter 8.2 .2 since it serves a different purpose.
} 


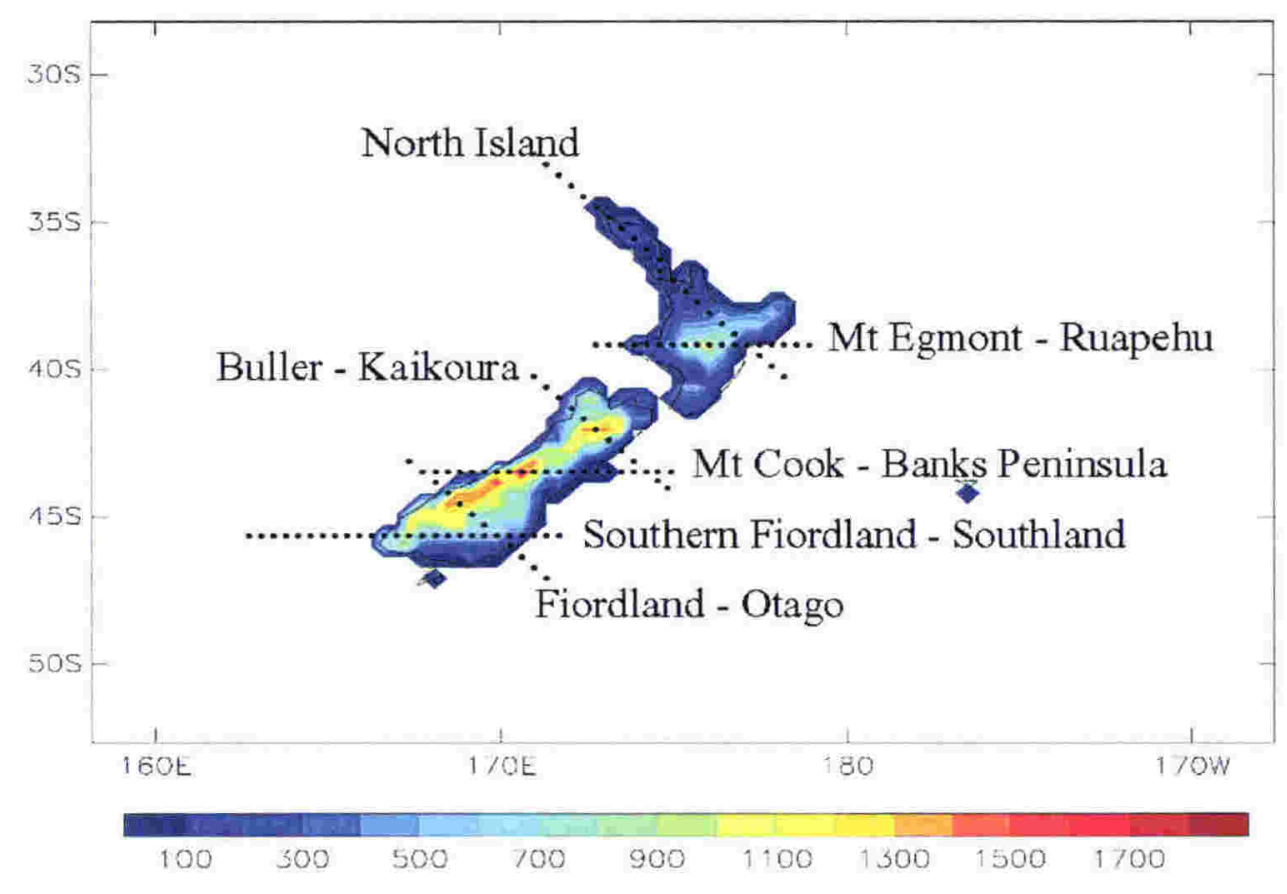

Fig. 6-8. The cross sections for the temperature profiles of Fig. 6-9 and the precipitation profiles of Fig. 6-12.

New Zealand (North versus South, West versus East and mountain areas versus low lands). The temperature profiles are presented against the high-resolution orography of CLIDB (black line) and against the orography of NZ_40km (brown line). The CLIDB temperature data (purple line) is presented in its highresolution format, and not in the low-resolution format of the RCM as it was done for Fig. 6-6 and Fig. 6-7. The CLIDB temperature profile is very strongly inversely related to its orography, indicating the warm valleys and the cold mountain peaks. The resolution of the RCM is too low to pick up the sharp changes in the real orography and its temperature profiles should be compared against its low-resolution orography. Regional trends that are not picked up by the regional model are then identifiable. At the same time the RCM temperature profile can be compared to the temperature profile of the GCM (green line). The RCM picks up clearly regional patterns that are not seen by the GCM.

The cross sections of the North Island (North Island and Mt EgmontRuapehu) have the lowest and most gently sloped orography. The impact of orography on temperature is less in the North Island than in the South Island. The modelled temperature follows the CLIDB temperature very well, and during 


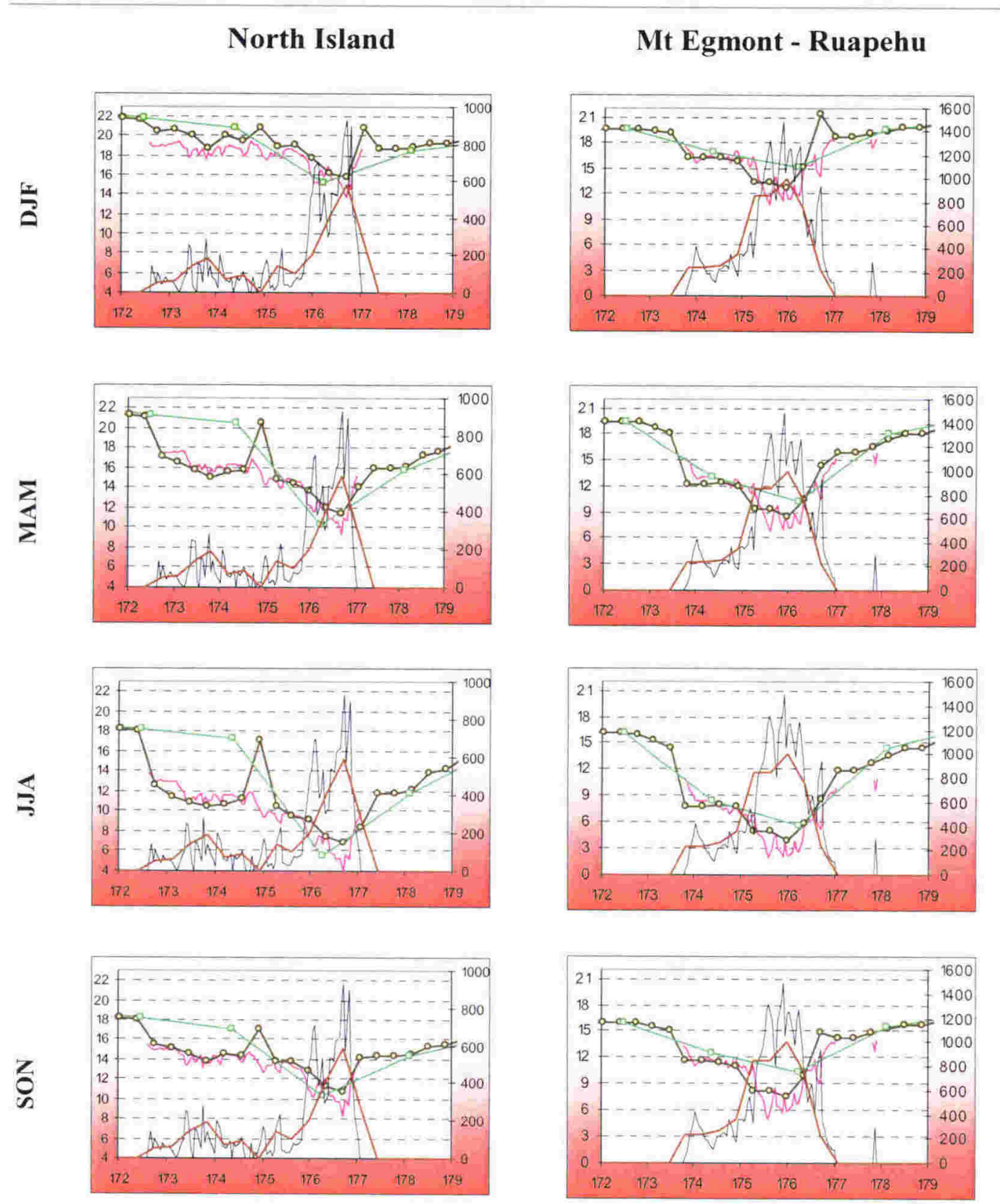

Fig. 6-9. Temperature profiles of the cross sections of Fig. 6-8. X-axis: longitude, Y-axis: left Temperature $\left({ }^{\circ} \mathrm{C}\right)$, right - Height $(\mathrm{m})$. Y-axis scale is different for each cross section. Legend: Thin black - CLIDB orography, Brown - NZ_40km orography, Dark green with - GCM temperature, Olive green with - NZ_40km temperature, Purple - CLIDB temperature.

none of the seasons differs by more than $2^{\circ} \mathrm{C}$. The largest differences between the modelled and CLIDB temperatures are found in the mountainous areas. The modelled temperatures are also consistently higher east of the ranges, and at times indicate that the atmosphere over land is warmer than over the sea. 


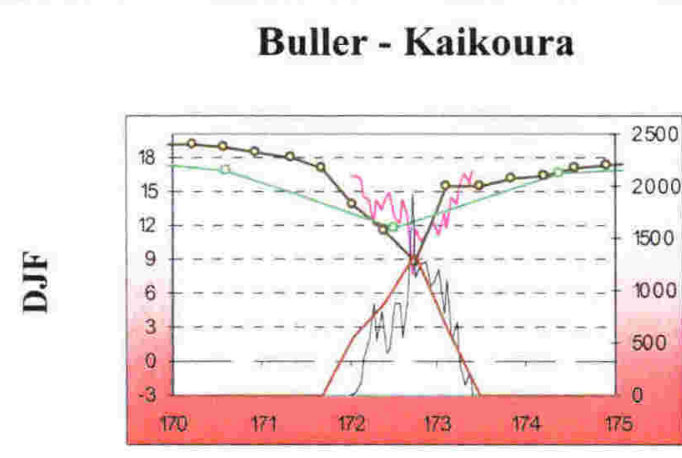

\section{Mt Cook - Banks Peninsula}
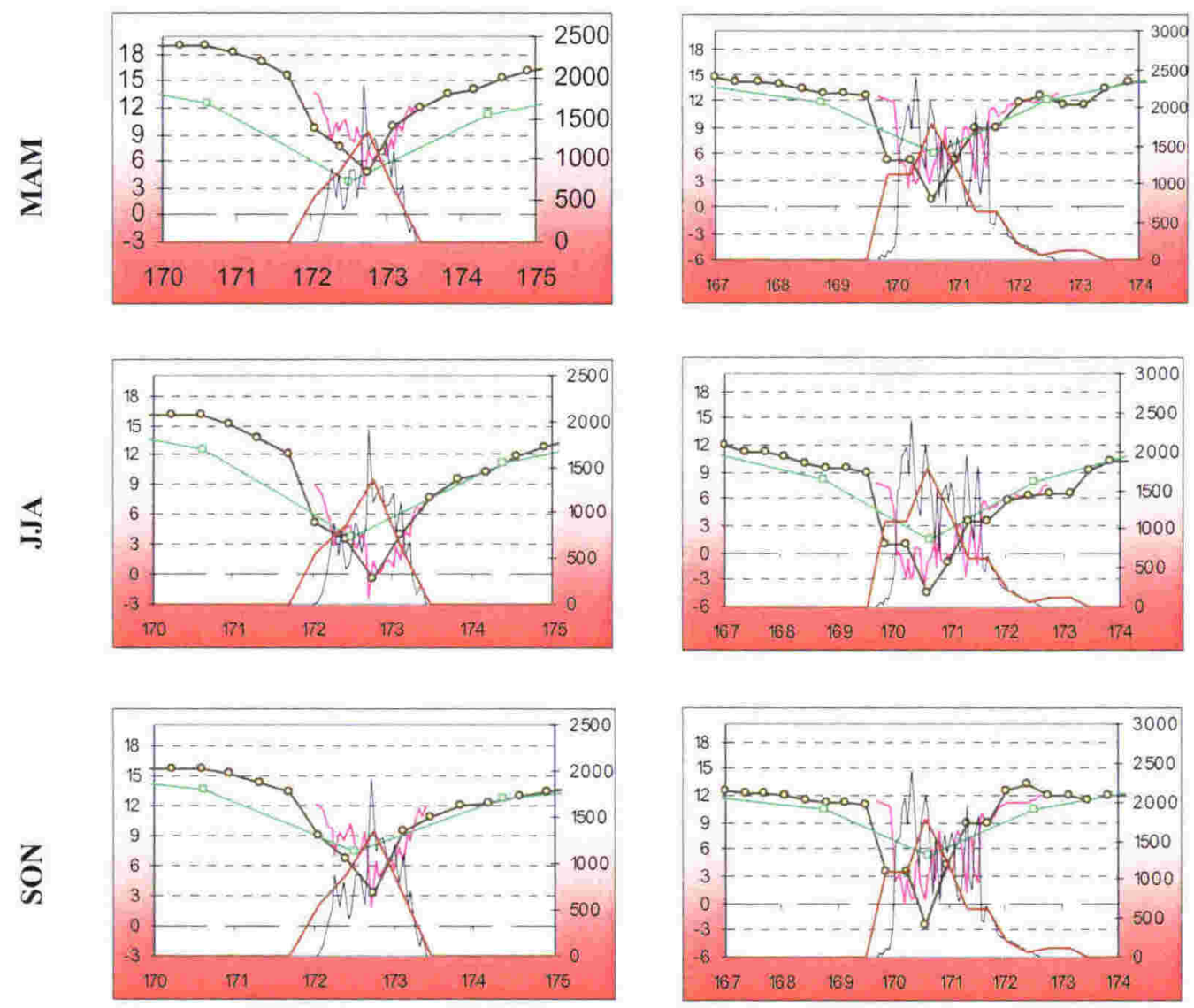

Fig. 6-9. continuation.

The landmass at $177.9^{\circ} \mathrm{E}$ in the Mt Egmont-Ruapehu cross section is the Mahia Peninsula. The Mahia Peninsula did not have its own grid point in the model, hence the model does not show orography at $177.9^{\circ} \mathrm{E}$. Only the CLIDB temperature is presented at the Mahia Peninsula.

Because of the very mountainous terrain in the South Island, the modelled temperatures do not accurately reflect the local temperatures down south. However, the RCM captures the regional trends correctly. The Buller - Kaikoura 

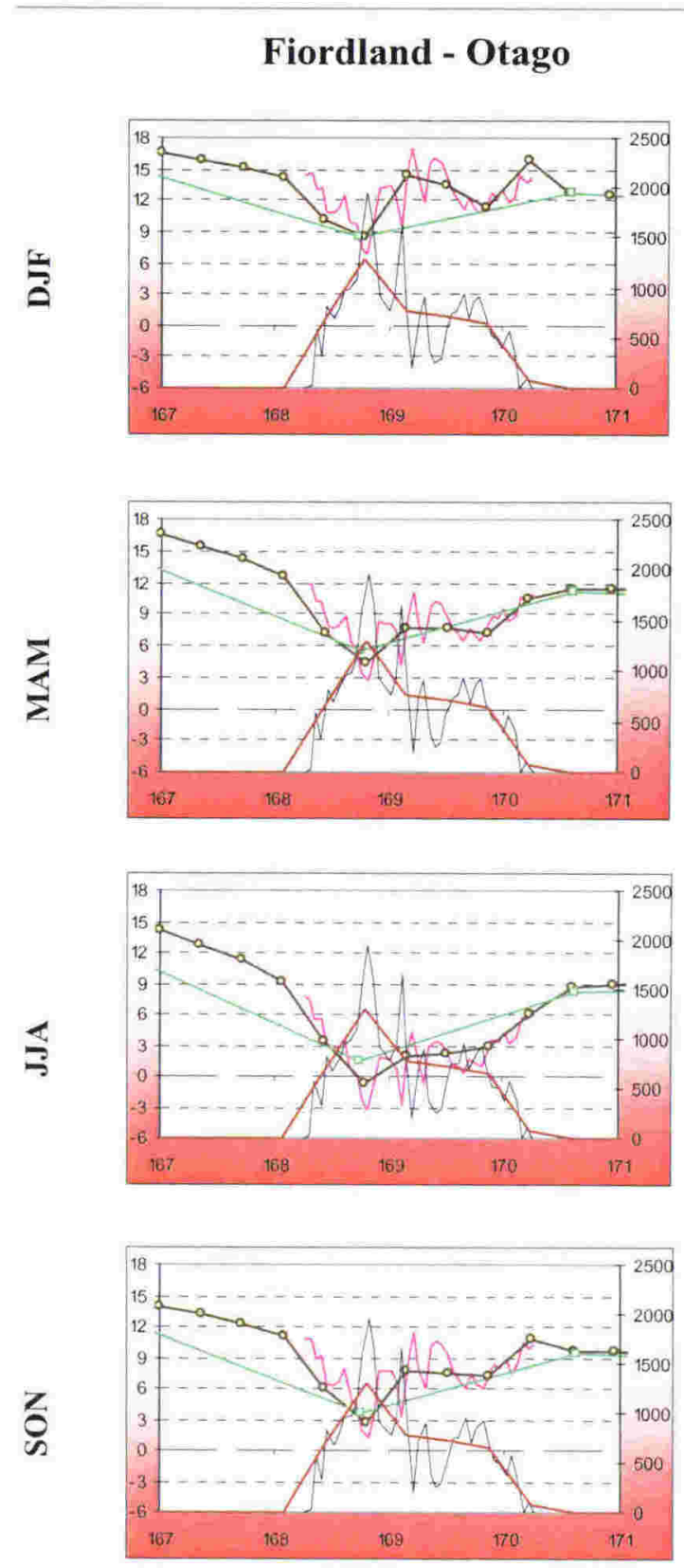

Fig. 6-9. continuation. cross section in particular shows clearly that the misrepresentation of local temperatures is due to the low resolution. The temperature profile is inversely related to the height of the orography, but the resolution of the modelled orography is too low to capture the strong local variation in orography. The modelled temperature in the mountains is generally lower than the CLIDB temperature. This is partially caused by the difference in their orography. The simulation captures the warmer temperatures in the east, but they are often too high.

These graphs indicate that the enhanced regional detail gained by using a RCM could possibly have been obtained by simply interpolating/downscaling the GCM profile once it has incorporated the higher resolution

orography. It is obvious that orography has a strong impact on temperature. However, simple interpolation can not account for the enhanced warming in the east of both islands. Interpolation can not account for the different affects of compressional warming, reduced cloud cover, and enhanced solar warming that all occur on the lee-side of the mountains.

The largest difference in surface temperature between the GCM and RCM is to the west in the North Island cross section. This is because the nearest GCM 
grid point to the cross section is still a sea point (at $174.3^{\circ} \mathrm{E}$ ). The large anomaly in the RCM temperature profile at $175^{\circ} \mathrm{E}$ is because that grid point is just out at sea (Hauraki Gulf) as well. The main difference in the temperature profiles between the RCM and GCM in the South Island, and in particular for the Buller Kaikoura and Fiordland - Otago cross sections, are related to the opposite. The GCM grid boxes at New Zealand's latitude are about $150 \times 150 \mathrm{~km}$ across. A land point in the GCM extends therefore much further to the west when compared to a land point of the RCM. The low surface temperature of the GCM off shore, west of the South Island, is because the GCM grid point is a land point, hence the large difference with the RCM temperature.

\subsubsection{Precipitation}

Precipitation is also a climate variable that is well covered by the CLIDB database and can therefore be used to verify the RCM's output. Fig. 6-10 shows the mean annual precipitation for the RCM and CLIDB. The presentation of the data is similar as in Fig. 6-6 in that it focuses on the land area and that the highresolution CLIDB data is projected onto the lower resolution of the RCM. The low resolution version of the CLIDB data still shows the characteristics of the precipitation pattern in New Zealand. It shows that the maximum rainfall is in the Southern Alps and can be more than $6 \mathrm{~m}$ per year (compare with the figure on the right in Fig. 6-1). The effect of the Southern Alps on precipitation is very strong with the highest amount of precipitation to the west of the main divide, and the lowest amount of precipitation on the eastern side. The driest areas are in South Canterbury and Otago. Precipitation patterns in the North Island are defined less sharply. But even in this low resolution, the effect of the orography is still apparent with the highest rainfall over the Tararuas, Mt Egmont, the Volcanic Plateau and the Ureweras. Even the minor regional maxima in precipitation over the Ruahines, the Coromandel and Northland are identifiable. The mean annual precipitation for the six regions and for the whole of New Zealand is presented in Table 6-3. 


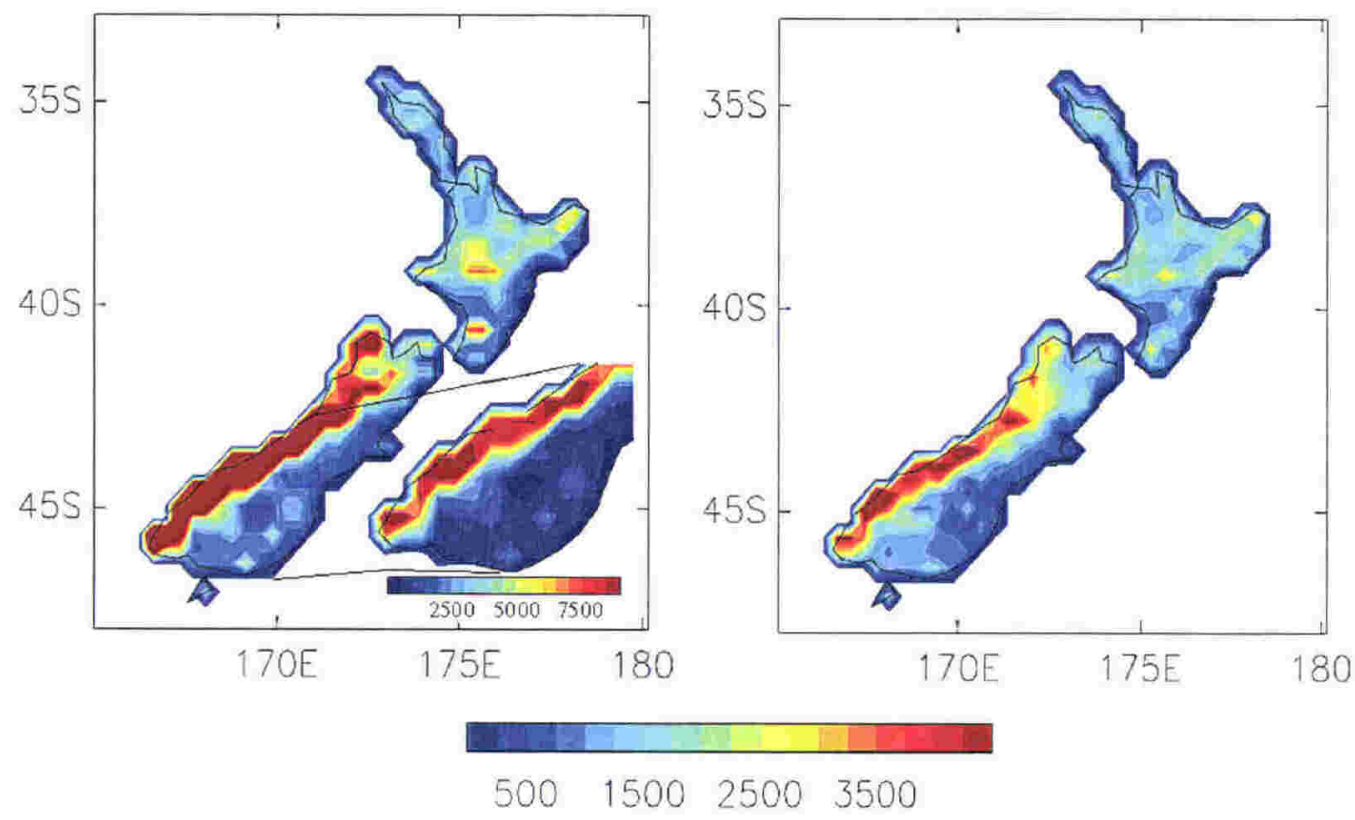

Fig. 6-10. The mean annual precipitation $(\mathrm{mm})$ for the RCM (left) and CLIDB (right). Precipitation in the southern part of the South Island in the RCM is plotted separately again with its own legend.

\begin{tabular}{|l|c|c|}
\hline \multicolumn{1}{|c|}{ Region } & CLIDB & RCM \\
\hline \hline North North Island & 1446.6 & 1556.3 \\
\hline \hline South-West North Island & 1563.5 & 1924.0 \\
\hline \hline East North Island & 1473.9 & 1201.5 \\
\hline \hline North South Island & 1834.4 & 2680.8 \\
\hline \hline West and South South Island & 2394.7 & 3864.7 \\
\hline \hline East South Island & 922.8 & 844.9 \\
\hline \hline New Zealand & 1748.8 & 2363.0 \\
\hline
\end{tabular}

Table 6-3. The mean annual precipitation (in $\mathrm{mm}$ ) for each region of Fig. 6-5 and for the whole of New Zealand as determined from CLIDB and NZ_40km.

The regional model does a good job in simulating the precipitation patterns of the North Island. However, the amount of precipitation is in general too high. The amount of precipitation is particularly large over the Southern Alps. This is again associated with the anomalously strong westerly winds in the GCM. The computed and observed mean annual precipitation patterns in Fig. 6-10 are 
presented on the same scale to enable direct comparisons between the two datasets. The maximum corresponds to the maximum amount of precipitation in the CLIDB data. The disadvantage of such a presentation is that the maximum amount of precipitation over the Southern Alps in the RCM can not be determined from the figure. For that reason is the southern part of the South Island taken apart and presented again with a legend that is more suitable for that region (inset in Fig. 6-10). The modelled precipitation shows clearly the impact of the Southern Alps with high precipitation in the west, and dry regions in the east. But as mentioned before, the amount of precipitation is too high, and over the Southern Alps can be as twice as high as the observed precipitation'.

As discussed earlier, the CLIDB and RCM orography are not the same and a large part of the difference in precipitation can be attributed to the different grid point heights in the two datasets. Similar as for surface temperature, it is therefore difficult to compare the CLIDB precipitation directly with the RCM precipitation since recordings and simulated data are at different heights. It is known that precipitation is difficult to simulate over steep orography and for high resolution (e.g. Giorgi and Marinucci, 1996: Pope and Stratton, 2002). Although some precipitation parameters were adjusted (sections 4.3.2 and 6.2), it remained difficult to simulate the precipitation correctly. The enhanced precipitation in the regional model is not solely caused by the dynamics of the model. Precipitation was already enhanced in the GCM and the conditions suitable for precipitation were passed on to the regional domain by the initial and boundary conditions. Therefore precipitation was likely to be enhanced in the RCM as well. Using the same high resolution model for the region of southern Africa, Hudson and Jones (2002b) described similar findings in their climate simulation of South Africa.

The seasonal differences between the NZ_40km and CLIDB precipitation patterns (Fig. 6-11 and Table 6-4) show similarities with the differences in their surface temperatures. The largest anomalies are found in the areas of high orography, and then in particular in the Southern Alps. The modelled precipitation is especially too high during spring. This is a direct indication of the

\footnotetext{
${ }^{1}$ These values might still be realistic for some local areas. Fig. 6-1 shows highest precipitation bracket for 4$10 \mathrm{~m} /$ year.
} 

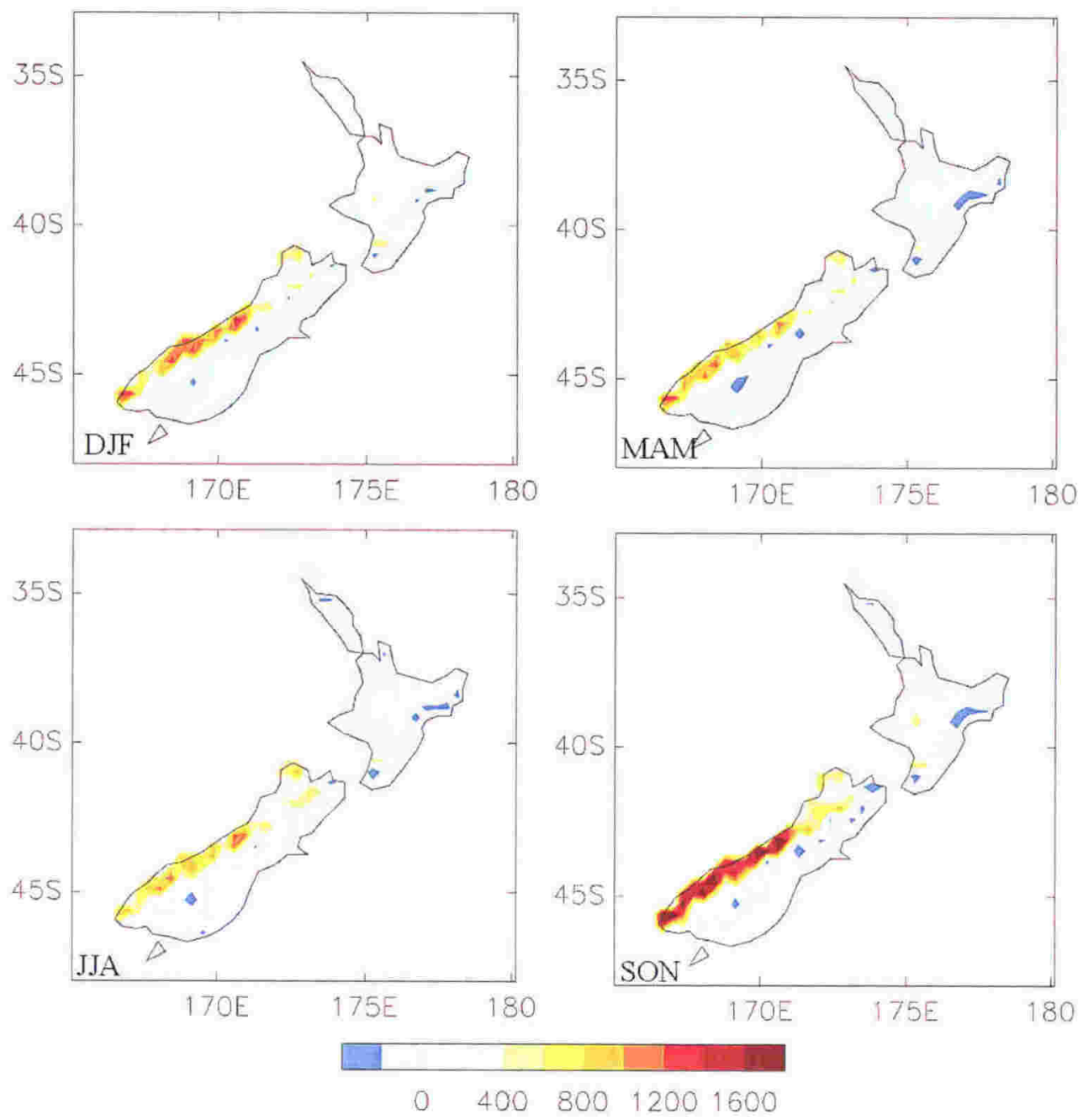

Fig. 6-11. The difference in mean seasonal precipitation between the NZ $40 \mathrm{~km}$ and CLIDB (in $\mathrm{mm}$ ).

impact of the GCM on the simulation of the regional climate. The largest anomalies in the mean zonal circulation occurred during SON and through the boundary conditions have affected the climate of the regional domain. The eastwest gradient in precipitation across the South Island is further accentuated since the RCM overestimates the general drier conditions east of the main divide. This is in particular the case for the driest areas, the Gisborne - Hawke's Bay region in the North Island and Central Otago in the South Island. Other areas that are modelled often too dry are the Wairarapa, Canterbury and Southland. Precipitation in the North Island is in general slightly too low except for the ranges and during JJA. 


\begin{tabular}{|l|r|r|r|r|r|r|r|r||}
\hline \hline Region & \multicolumn{4}{|c|}{ CLIDB } & \multicolumn{4}{|c|}{ RCM } \\
\hline \hline & DJF & MAM & JJA & SON & DJF & MAM & JJA & SON \\
\hline \hline NNI & 273.7 & 346.0 & 471.7 & 355.2 & 429.2 & 350.6 & 451.0 & 325.4 \\
\hline \hline SWNI & 324.7 & 373.1 & 460.1 & 405.5 & 490.1 & 395.6 & 489.5 & 548.8 \\
\hline \hline ENI & 287.4 & 392.6 & 456.1 & 337.8 & 292.4 & 299.6 & 383.5 & 226.1 \\
\hline \hline NSI & 382.1 & 450.0 & 499.5 & 502.8 & 604.3 & 611.2 & 777.8 & 687.5 \\
\hline \hline WSSI & 587.6 & 602.4 & 565.9 & 638.6 & 914.1 & 871.1 & 855.6 & 1223.8 \\
\hline \hline ESI & 216.0 & 228.6 & 242.6 & 235.6 & 213.3 & 188.4 & 262.1 & 181.0 \\
\hline \hline NZ & 391.0 & 434.6 & 469.8 & 453.4 & 575.0 & 531.4 & 591.8 & 664.7 \\
\hline \hline
\end{tabular}

Table 6-4. Mean seasonal precipitation (in $\mathrm{mm}$ ) in CLIDB and NZ_40km by region.

It was suggested that the regional features of the surface temperature could to a degree have been obtained by simple interpolation of the GCM values. Since precipitation and temperature are to a certain extent related to each other, it could be that regional precipitation might also be determined by simply interpolating/downscaling the amount of precipitation determined by the GCM. This can be further investigated by examining the precipitation patterns along the cross sections of Fig. 6-8. Fig. 6-12 shows the five precipitation profiles along the cross sections. The setup of these graphs is identical to Fig. 6-9, except that the variable is precipitation.

The Northland part of the North Island cross section shows higher precipitation and larger spatial variation in the amount of precipitation than the recorded amount of precipitation (CLIDB) during DJF. Although this region experiences its lowest rainfall during this season in the present day, the computed amount of precipitation is only slightly less than during the wettest season, JJA (see Table 6-4). A possible explanation for the reduction in seasonality of precipitation in NNI could be that the enhanced wave number 3 pattern of the simulated Southern Hemisphere circulation allows more warm moist northerlies into this region. An increase in the number of northerlies would explain the enhanced warming that is found in this region as well (Fig. 6-7). Furthermore, the high variability in precipitation is also an indication of enhanced convective precipitation (not shown). 


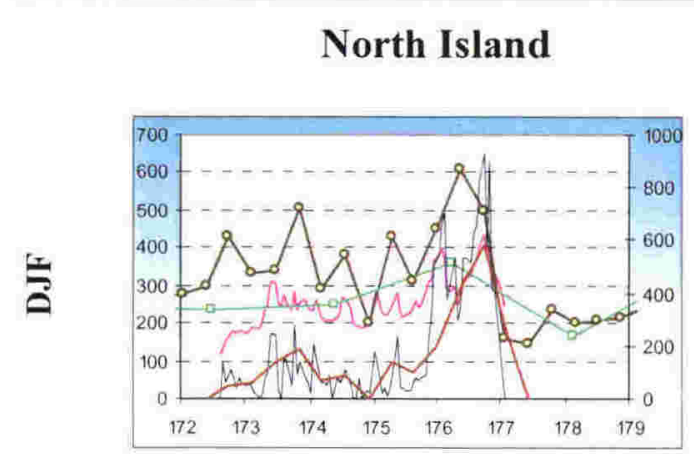

\section{Mt Egmont - Ruapehu}
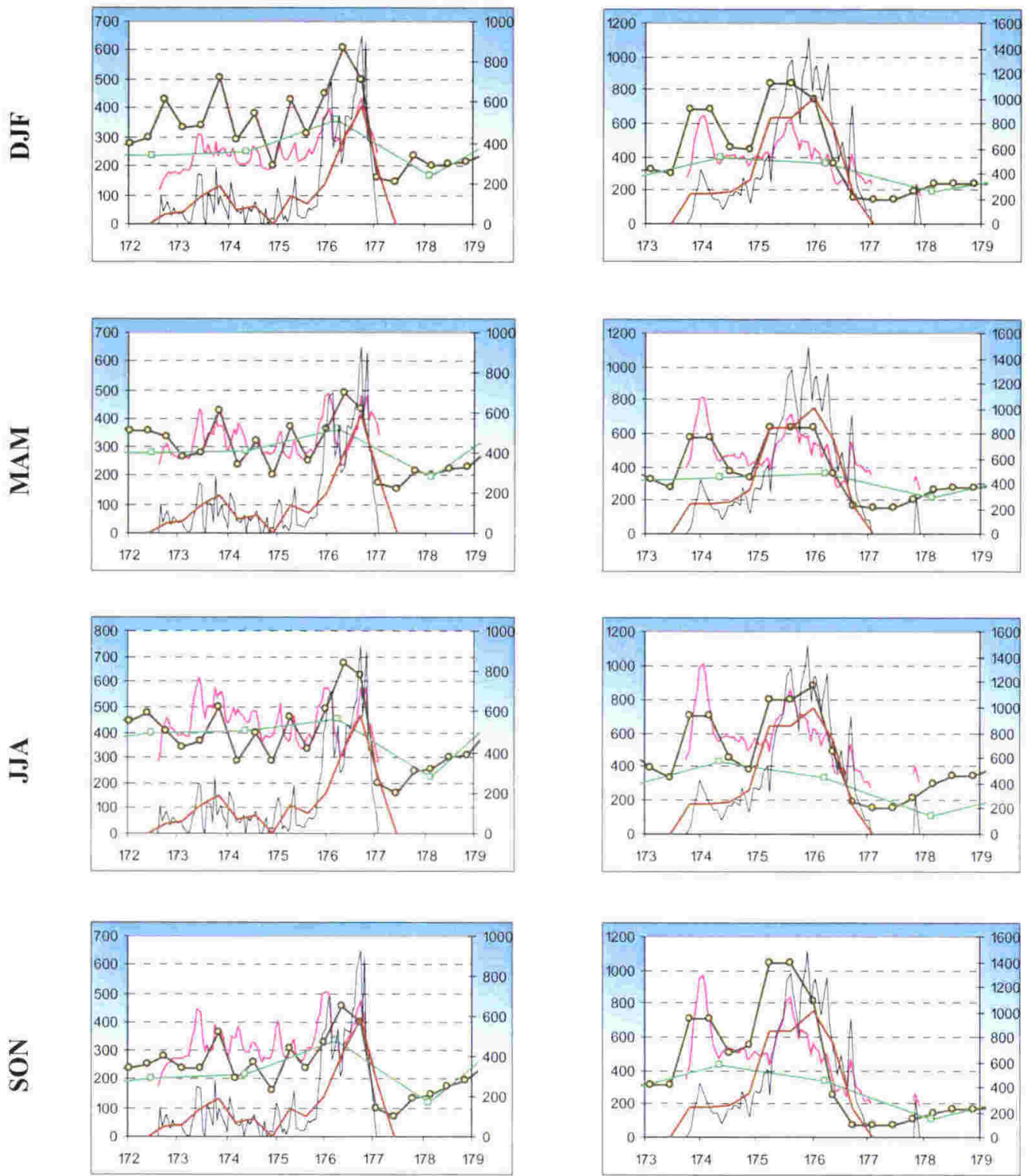

Fig. 6-12. Precipitation profiles of the cross sections in Fig. 6-8. X-axis: longitude, Y-axis: left Precipitation (mm), right - Height $(\mathrm{m})$. Both y-axis scales change for each cross section. Legend: Thin black - CLIDB orography, Brown - NZ_40km orography, Dark green with - GCM precipitation, Olive green with $-\mathrm{NZ}$ - $40 \mathrm{~km}$ precipitation, Purple - CLIDB precipitation.

The maximum in precipitation is clearly linked to the orography, with the maximum in precipitation generally one grid point west of the maximum in modelled height. The profile of the modelled precipitation along the North Island cross section is similar to the precipitation profile of CLIDB during the rest of the year. There is a distinct peak in the precipitation profile linked to the 


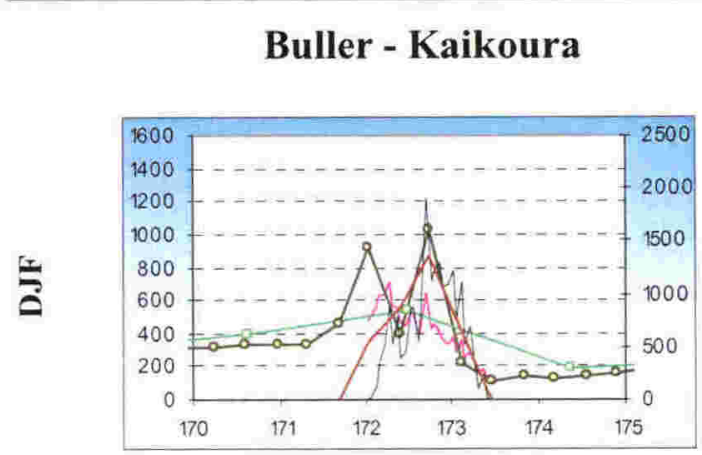

\section{Mt Cook - Banks Peninsula}
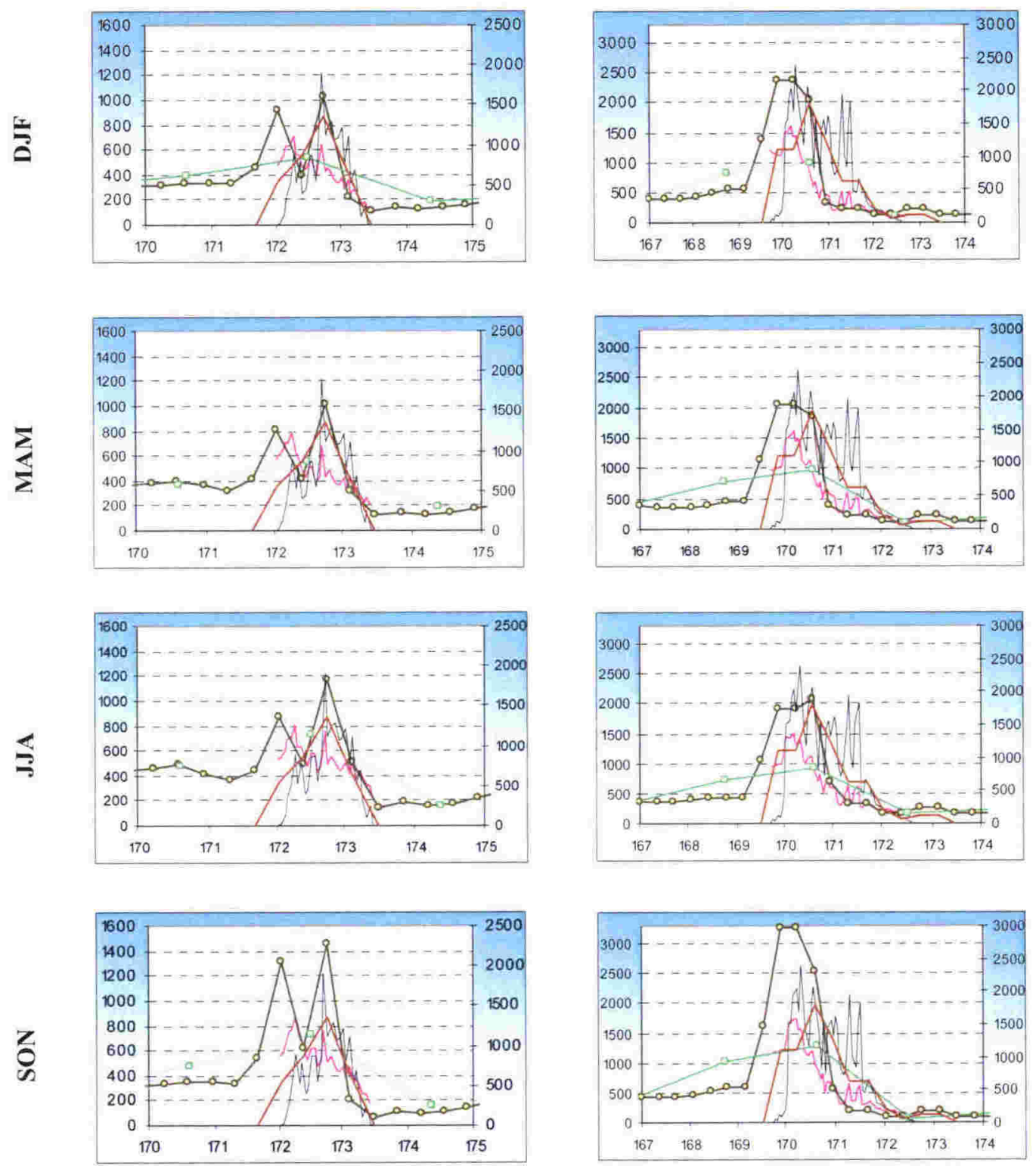

Fig. 6-12. continuation.

modelled Parataiko/Tutamoe Ranges, which is similar to the peak in the CLIDB pattern. The regional model does not simulate correctly the precipitation pattern over the other ranges in Northland due to its low resolution. But the amount of precipitation increases every time where there is an increase in the height of the modelled orography. The precipitation pattern over the Urewera ranges only show one peak, which is in accordance with its modelled orography.

One of the pleasing aspects of the precipitation patterns of the RCM is that it 

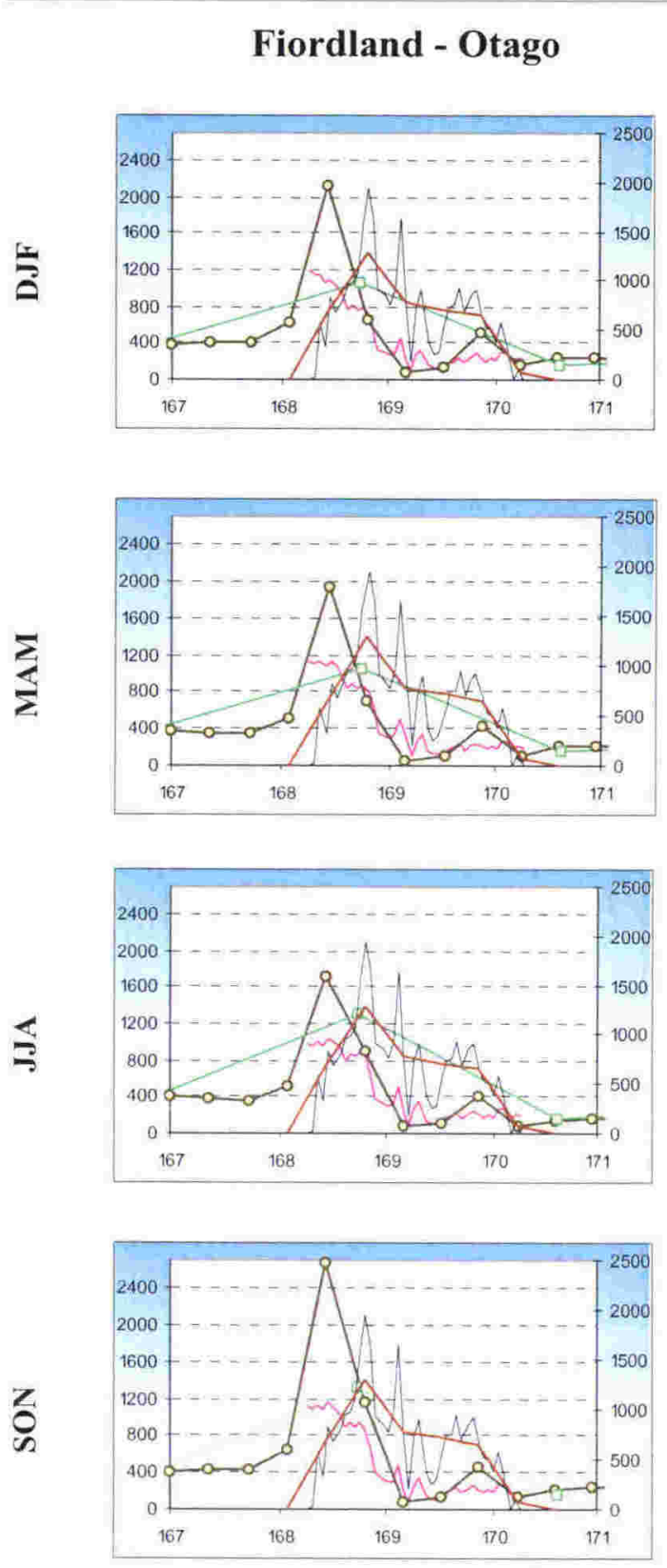

Fig. 6-12. continuation. showed that the regional model was able to simulate the enhanced precipitation as the result of a single mountain. Although Mt Egmont was only modelled by two grid points, the $\mathrm{RCM}$ has done quite a reasonable job in reproducing the enhanced precipitation in that area. The precipitation over the Volcanic Plateau follows the modelled orography quite well, and shows a similar pattern as the cross sections for the CLIDB data, with the maximum precipitation west of the Plateau and drier conditions to the east. The seasonal variation is also similar to the seasonal variation in CLIDB, albeit that the modelled precipitation is again slightly too high, especially during spring. The lone peak in the CLIDB orography in the east is the Mahia Peninsula, which landmass does

not show up in the RCM.

The main differences in precipitation patterns between the RCM and CLIDB appear in the South Island. They are related to both the different impacts of their different orography as to the anomalous general circulation modelled by the GCM. The latter showed up particularly during spring when the amplitude of the SAO was weak, indicating stronger westerly winds and an increase in baroclinicity at midlatitudes. The impact on the regional domain is that 
precipitation is nearly $200 \%$ of that of CLIDB at some grid points during SON. All South Island cross sections show a very large amount of precipitation directly west of the highest peaks in the modelled orography. Those grid points correspond to the first land points at the resolution used. Clearly the model fails to properly simulate precipitation under extreme uplift situations. Although some parameterizations related to precipitation were adjusted for the increase in horizontal resolution (section 4.3.2), it is apparent that some other adjustments need to be made as well in situations of extreme uplift. Giorgi and Marinucci (1996) discussed how an increase in horizontal resolution does not necessarily result in an improvement in the simulation of some aspects of precipitation without a proper tuning of the appropriate parameterizations. In simulating an extreme precipitation event along the West Coast of the South Island, Katzfey (1995) found that an increase in horizontal resolution together with a more accurate representation of the orography, captured the observed distribution of precipitation better.

The Buller-Kaikoura cross section shows two peaks in the modelled precipitation, but their modelled orography has only one peak. This seems to be at odds with precipitation following the orography in the model. However, the virtual non-alignment of precipitation with orography is the result of the direction of the cross section. The model calculates the amount of precipitation using the history and amount of precipitation of each neighbouring grid point. But this is done only in the zonal and meridional direction, and not in a northwest - southeast direction. The "diagonal" Buller - Kaikoura cross section therefore connects precipitation with orography at different latitudes. The two peaks in the precipitation do correspond to two different locations where orography increases steeply (at $172.04^{\circ} \mathrm{E}, 41.32^{\circ} \mathrm{S}$ and $172.76^{\circ} \mathrm{E}, 42.04^{\circ} \mathrm{S}$ respectively), but which do not show up on the northwest - southeast aligned cross section. The orography at $172.40^{\circ} \mathrm{E}, 41.68^{\circ} \mathrm{S}$ is higher than at $172.04^{\circ} \mathrm{E}$, $41.32^{\circ} \mathrm{S}(866.29 \mathrm{~m}$ versus $556.22 \mathrm{~m})$ but it is in the "rain shadow" of already two land points immediately to its west $\left(171.68^{\circ} \mathrm{E}, 41.68^{\circ} \mathrm{S}\right.$ and $172.04^{\circ} \mathrm{E}, 41.68^{\circ} \mathrm{S}$ at a height of $304.91 \mathrm{~m}$ and $615.74 \mathrm{~m}$ respectively), where as $172.04^{\circ} \mathrm{E}, 41.32^{\circ} \mathrm{S}$ is only the first land grid point at that latitude. Although this was of consequence in 
the analysis of the North Island cross section as well, since the zonal and meridional variations were relatively minor, it was not of much significance for that cross section. Although to a much lesser extent, a similar pattern was apparent in the temperature profile of the Buller - Kaikoura cross section.

The precipitation profile of the Mt Cook - Banks Peninsula cross section shows high amount of precipitation to the west of the main divide and tapers of quickly to dry conditions in the east. Although the modelled amount of precipitation in the west is much higher than observed, the amount of precipitation in the east is relatively similar to the recorded amount of precipitation (see Fig. 6-11 and Table 6-4). Since most of the precipitation in the east of the South Island comes from southerlies and north-easterlies, and only a very small percentage from the west (less than 10\%), the reduction in any spillover from the west does not have a large impact on the amount of precipitation in the east. No daily data was available to further support this by correlating precipitation with wind direction. The main impact of the dry westerly wind on the climate of the east of the South Island would be to enhance warming over land (see Fig. 6-6, Fig. 6-7 and Fig. 6-9) and drying of the soils.

The Fiordland - Otago cross section shows the same pattern as the other South Island cross sections, with too much precipitation at landfall in the west, and dry conditions in the east. The highlands of Otago show a slight increase in precipitation that does not show up in the CLIDB data. This is again most likely related to the difference between modelled height and the actual height of the data point.

Compared to the simulation of the GCM, it would seem that there is no major improvement in simulating precipitation over New Zealand by an RCM. However, it is hard to correctly compare data points at different heights. Clearly precipitation is orography dependent, but the RCM does not seem to simulate precipitation in areas with steep orography very well. Simply interpolating the GCM values and adjust them for height could give similar amount of precipitation as the RCM. The GCM gives reasonable values over low-lying areas and shows the west-east gradient in precipitation. 


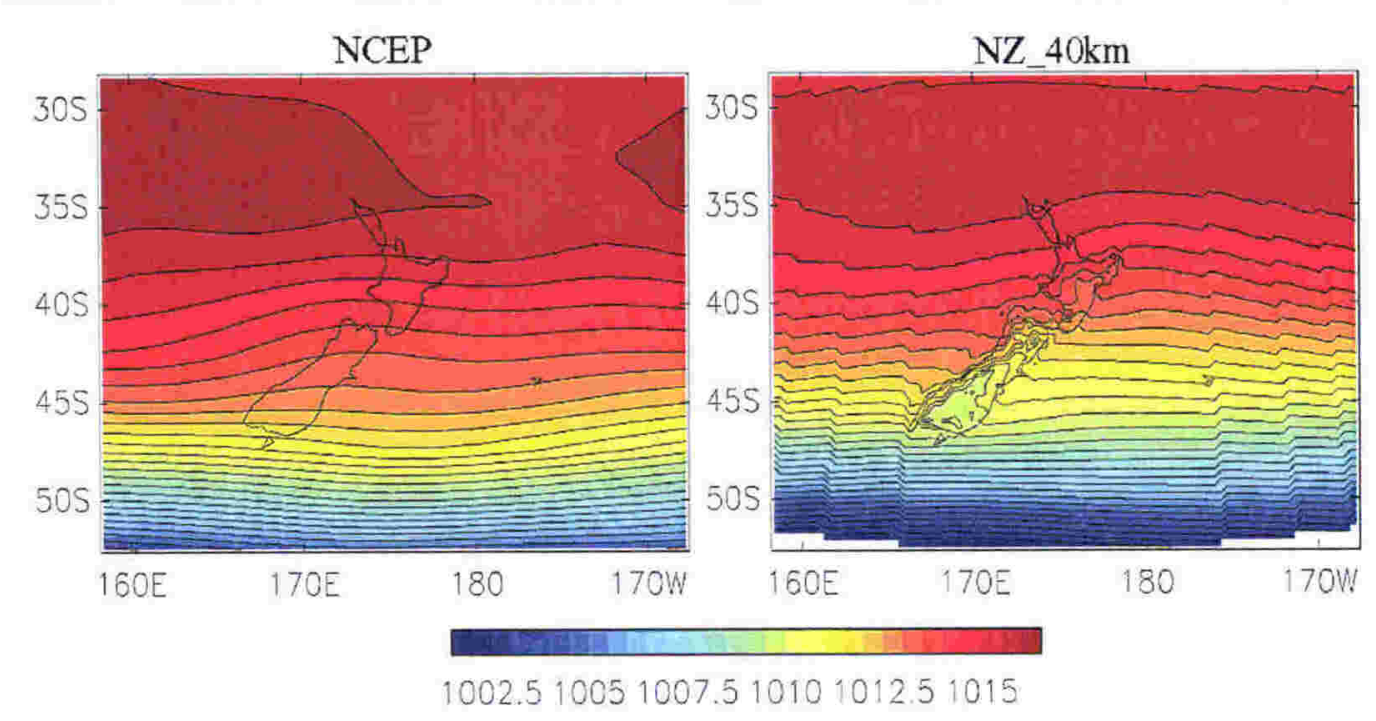

Fig. 6-13. Annual mean sea level pressure for NCEP and the RCM (in $\mathrm{hPa}$ ).

\subsubsection{Mean Sea Level Pressure \& Winds}

At the time of this study, no seasonal or annual CLIDB mean sea level pressure and wind data was available that could easily be converted into a format compatible with the model output. Therefore no comparisons could be made between the CLIDB dataset and the RCM output for these variables. It is however worthwhile to analyse the RCM's wind in this section, so that comparisons can be made later with the wind regimes during the LGM (chapter 8). The winds are either analysed directly by looking at their zonal and meridional components, or indirectly by looking at the mean sea level pressure. The aim in this section is to relate precipitation and temperature patterns as described in the previous sections to mean sea level pressure patterns and therefore to wind direction. For which regions do temperature and precipitation correlate positively/negatively with the winds? And how do these patterns change during the year?

Fig. 6-13 gives the annual mean sea level pressure from the NCEP climatology (interpolated onto the RCM grid) and from the RCM. In the annual mean the wind is slightly more southwest over the North Island and more northwest over the West Coast in the RCM than in the NCEP climatology. There is more detail in the mean sea level pressure pattern east of the main divide in the 


\begin{tabular}{||c|c|c|}
\hline \multirow{2}{*}{} & \multicolumn{2}{|c|}{ RCM } \\
\cline { 2 - 3 } & $\mathrm{u}$ wind & $\mathrm{v}$ wind \\
\hline \hline North North Island & 1.483 & 0.356 \\
\hline \hline South-West North Island & 1.794 & -0.693 \\
\hline \hline East North Island & 2.553 & -0.647 \\
\hline \hline North South Island & 1.713 & -0.054 \\
\hline \hline West and South South Island & 1.946 & -0.908 \\
\hline \hline East South Island & 2.217 & -0.890 \\
\hline \hline New Zealand & 1.913 & -0.521 \\
\hline
\end{tabular}

Table 6-5. The mean annual zonal (u) and meridional wind (v) in $\mathrm{m} / \mathrm{s}$ for each region. Westerly and southerly winds are positive.

RCM than in the NCEP data since the RCM is of a higher resolution. Fig. 6-13 shows that the RCM has lower mean sea level pressure to the east of the mountain ranges in both the North and South Island, indicating that the RCM has probably modelled the local wind regimes in those areas correctly. Table 6-5 shows the strength of the annual mean wind in its components for each region. The positive values for the zonal wind are westerly winds in the annual mean, and positive values for the meridional wind are southerlies in the annual mean. This table clearly indicates that the RCM has predominantly south-westerly winds over Northland where as the rest of New Zealand is influenced more by the north-westerly winds.

Trenberth (1976) described the Southern Hemisphere circulation in the Australasian region through indices for the zonal and meridional wind and through indices based on EOF analysis in his study of atmospheric trends in the New Zealand region. This approach was used by Salinger (1980a: 1980b), who described the precipitation and temperature patterns in New Zealand with respect to the dominant wind directions ${ }^{\prime}$. Correlation indices and principal component analysis led him to describe the patterns for up to eight different regions. Although New Zealand was divided up into six regions in the previous section,

\footnotetext{
${ }^{1}$ The approach by indices was also used in a study on future climate scenarios in New Zealand (Mullan et al., 200I)
} 
the slightly different defined regions have several boundaries in common and characteristics of these regions can therefore be compared with each other.

Annual mean correlation maps of the time series of the regional monthly mean temperature ${ }^{\prime}$ against the mean sea level pressure at each grid point in the RCM over the whole period only show weak correlations $(-0.3 /+0.3)$ for each region (not shown). Correlation maps of precipitation against the mean sea level pressure also show weak correlations $(-0.2 /+0.2)$. But for both variables, their regional correlation maps look all very similar. The temperature correlation maps all show negative correlation values in the north, and positive values in the south. Such a configuration means that of all wind directions, in the annual mean, it is the westerly wind that has the strongest influence on the temperature of each reg. The precipitation correlation patterns all show a positive value roughly to the north-west and a negative value roughly to the south/south-east of New Zealand. Such a configuration indicates that it are the north-easterlies that are the dominant moisture-bearing winds. The low correlation for both annual patterns is due to seasonal effects, and more can be learned by investigating the seasons separately.

\subsubsection{Correlation MSLP-Temperature}

The regional mean seasonal temperature is correlated against the mean sea level pressure at each grid point. Details of the correlations and wind implications are described. The correlation patterns are also compared against the patterns obtained by correlating the NCEP seasonal mean sea level pressure with the observed regional temperatures (in the seasonal mean; from CLIDB). A similar analysis will be done for the LGM simulation in chapter 8 and correlation patterns between the two simulations will be compared to each other. As was discussed in the previous chapter and in other studies (e.g. Pope et al., 2000) the model overestimates the strength of the westerly winds. It is therefore expected

\footnotetext{
${ }^{1}$ The overall mean monthly value of temperature, precipitation and mean sea level pressure were removed from each month prior to the calculation in order to remove any seasonal effects.
} 
that some of the correlation patterns of the simulation will differ from the correlation patterns of the observed data.

Seasonal correlation patterns show clearly much higher correlations than the annual correlation patterns. Also examined, but not always presented, are the correlations of the meridional and zonal winds with temperature. These correlations can give more detail than just the correlations with mean sea level pressure, and are often referred to. A discussion of the correlation patterns of each season follows.

\section{- DJF}

(NCEP) The correlation patterns of NCEP mean sea level pressure with the observed regional temperature for DJF are presented in Fig. 6-14. It is clear that there is a strong positive correlation with warmer temperatures over the country with a zone of high pressure to the east of the country. The resulting northeasterlies will advect warm air onto the country. The opposite configuration, a low in mean sea level pressure to the east of the country, will result in cooling under south-westerly winds.

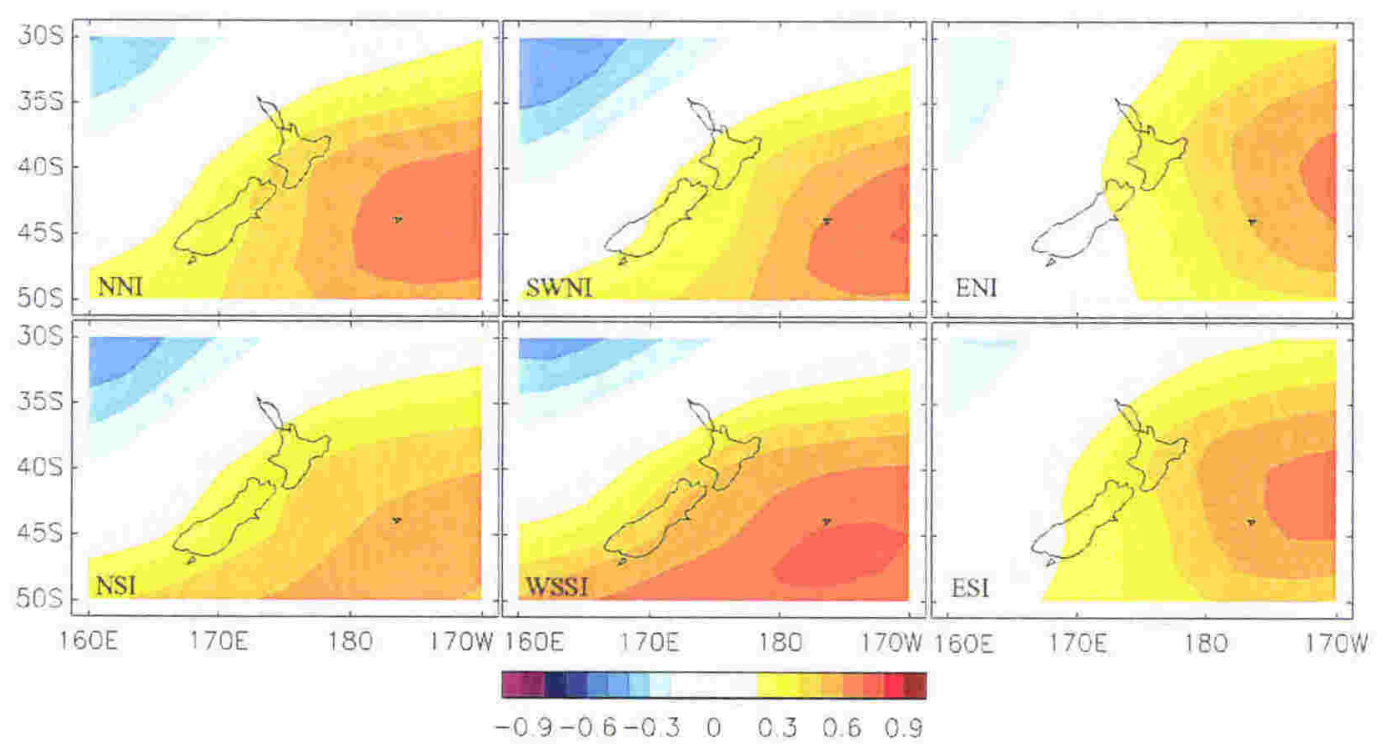

Fig. 6-14. Correlation maps of mean sea level pressure (NCEP) versus observed temperature (CLIDB) for the six different regions during DJF. 


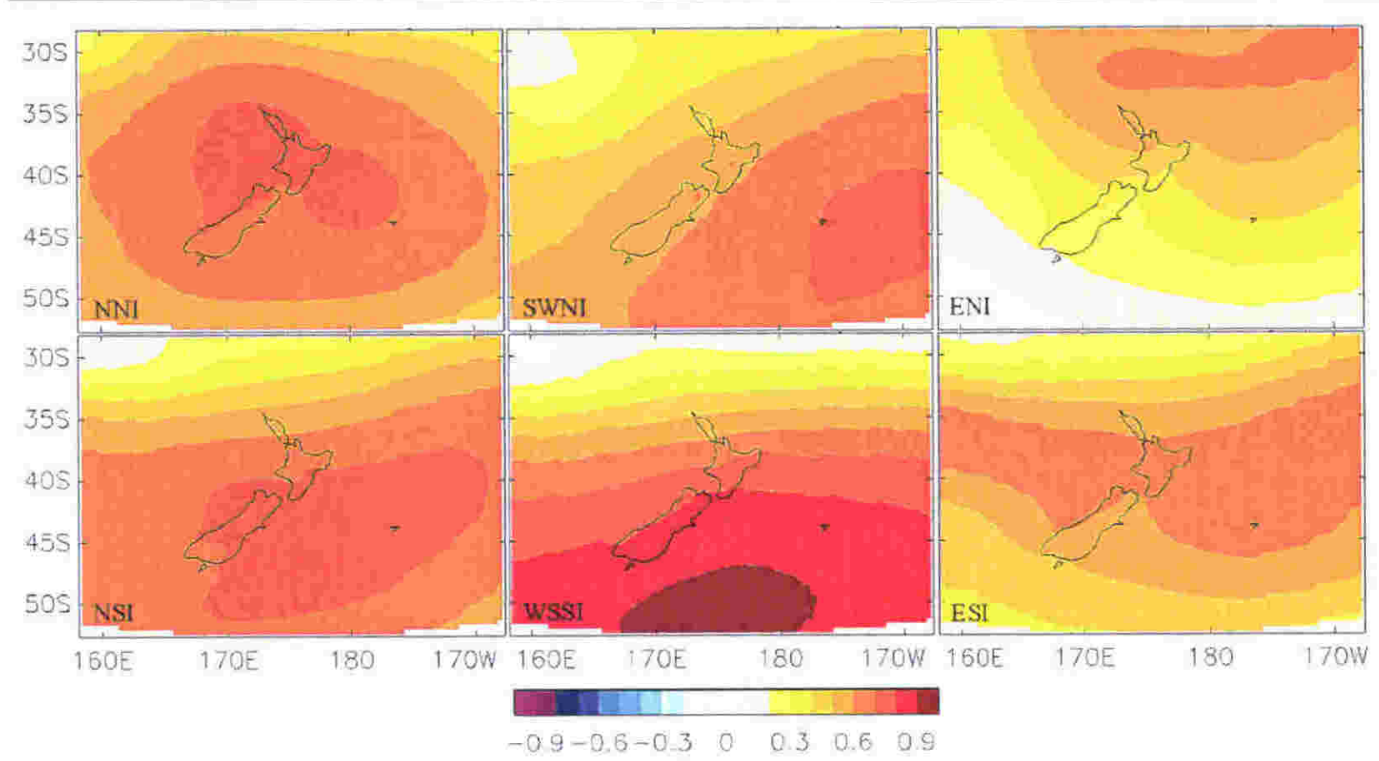

Fig. 6-15. Correlation maps of mean sea level pressure versus temperature for the six different regions during DJF (RCM).

(RCM, Fig. 6-15) The correlation patterns of the modelled mean sea level pressure and temperature for DJF are completely dominated by the positive correlations ranging from +0.4 to +0.8 . For the regions NNI and NSI the maximum correlation sits virtually directly on top of the region. This indicates that clear skies during this season results in warming since the incoming solar radiation is unimpeded. At the same time, cooler temperatures are due to unsettled, dull weather with impeded solar radiation. The temperature response in SWNI and WSSI is also influenced by a "high", but its position is more to the east and south respectively. Those areas are then sheltered from the prevailing north-easterly winds by the Volcanic Plateau and the Alps and experience leeside warming effects (compressional warming, reduced cloudiness, increase incoming solar radiation) as well ${ }^{1}$. This particular pattern is consistent with the wind regimes of the NCEP/CLIDB correlation patterns. The effect of lee-side warming is even stronger for ENI and ESI when the zone of high pressure is in the north or over the North Island, creating a westerly flow over the country. In section 6.3.1 (Fig. 6-7 and Fig. 6-8, cross sections of North Island, Mt Egmont -

\footnotetext{
${ }^{1}$ With the winds predominantly westerly, such a correlation pattern is in general interpreted as the westerly wind having a cooling effect on the western regions.
} 
Ruapehu and Mt Cook-Banks Peninsula) it was evident that the model overestimates the warmer temperatures in the east. It was suggested that as the result of a stronger westerly flow, the amount of compressional warming was too high in the east of both islands.

The correlation patterns for the RCM during DJF differ considerably from the NCEP patterns. Only some regions (SWNI, NSI and WSSI) indicate the link of an easterly flow with warmer temperatures, but even in these regions is the full correlation not well captured. The differences in the NCEP and RCM correlation patterns are due to the anomalous westerly flow (and therefore the anomalous mean sea level pressure) as modelled by the GCM.

- MAM

(NCEP) The correlation patterns of NCEP mean sea level pressure with the observed regional temperature for MAM are presented in Fig. 6-16. The patterns are very similar for each region with a positive correlation between mean sea level pressure and temperature to the east of the country, and a negative correlation to the west. This indicates that the temperature response of the regions is related to advection of warm/cold air from the north/south.

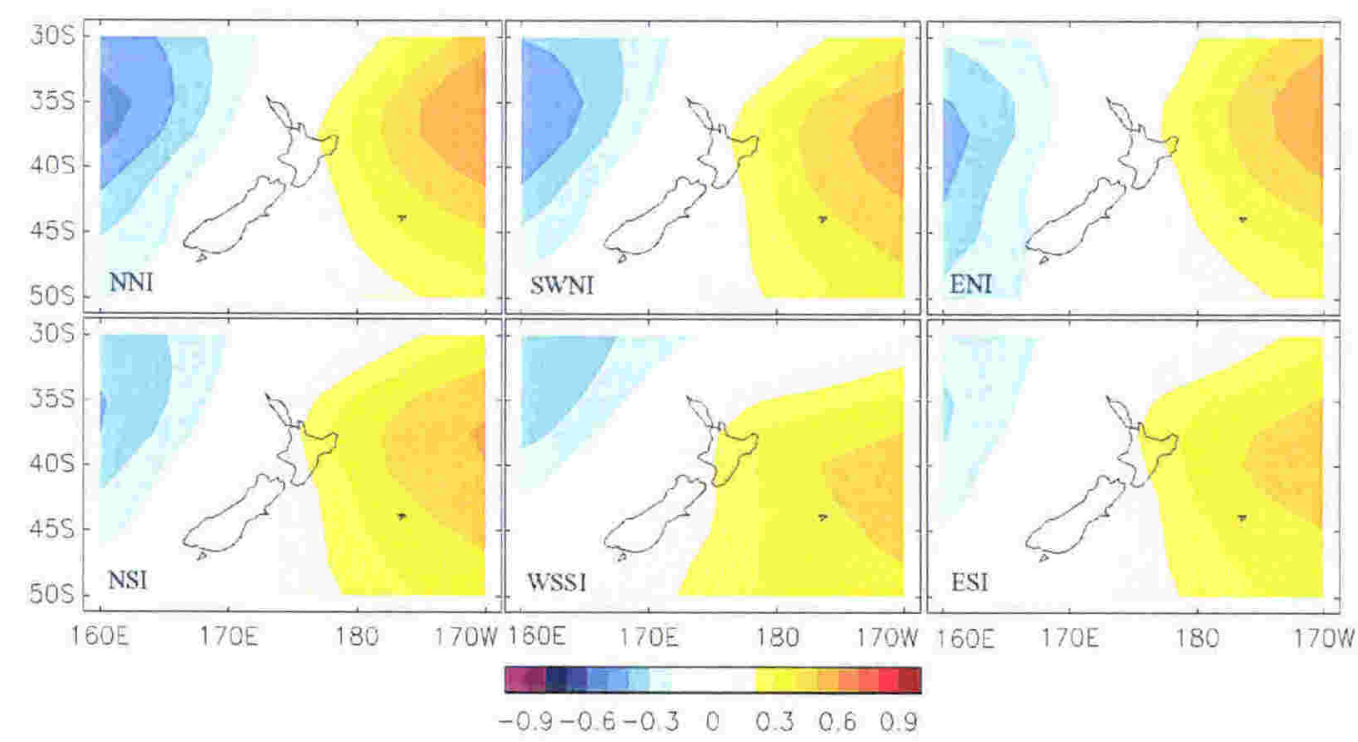

Fig. 6-16. Correlation maps of mean sea level pressure (NCEP) versus temperature (CLIDB) for the six different regions during MAM. 


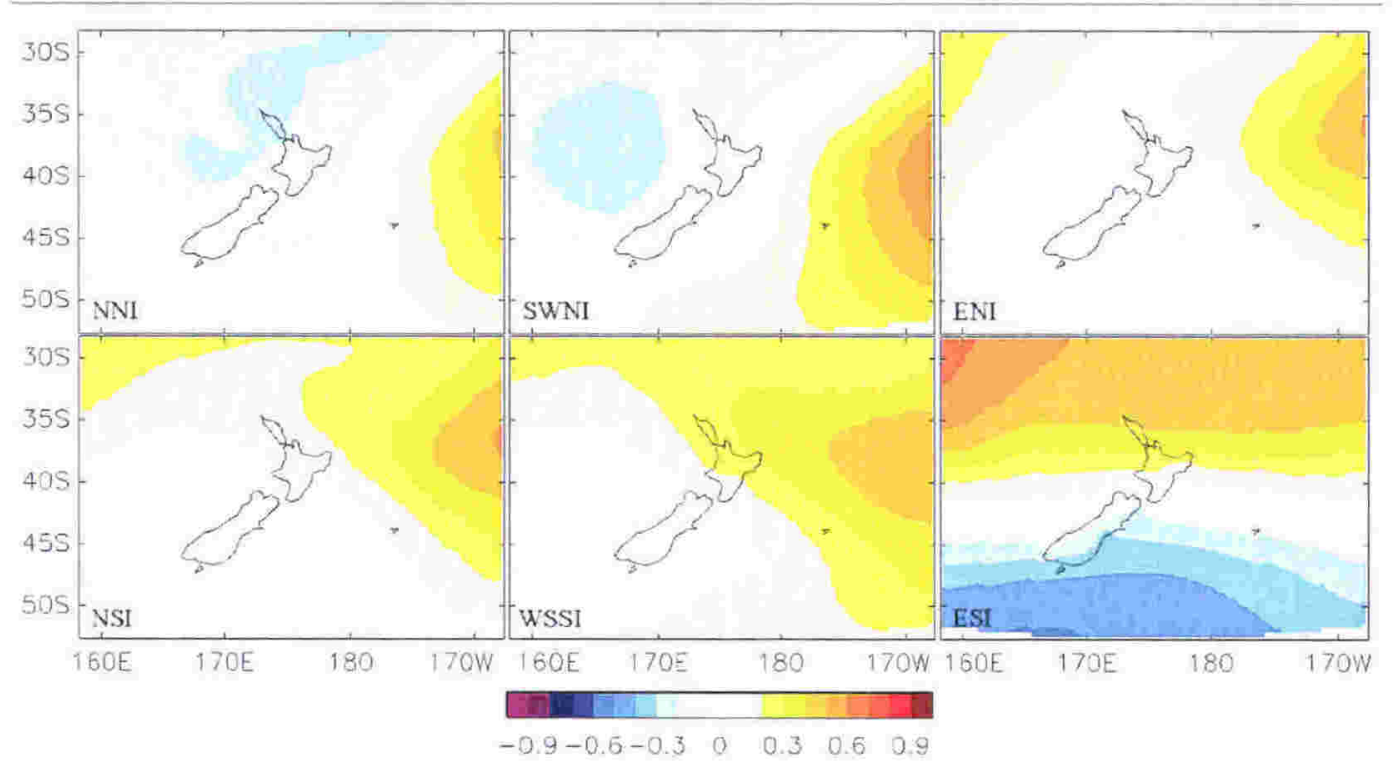

Fig. 6-17. Correlation maps of mean sea level pressure versus temperature for the six different regions during MAM (RCM).

(RCM, Fig. 6-17) Correlation patterns of the modelled data during MAM are quite different than during DJF. The patterns show that it is the advection of warm and cold air that determines the temperature response. The correlation values are lower for each region (ranging from -0.4 to +0.5 ). Similar as during DJF, the correlation patterns of mean sea level pressure with temperature are different from the correlation patterns of the observed data (Fig. 6-16). To a certain extent, there is a positive correlation of temperature with a northerly flow in NNI and SWNI, but it is quite weak. Temperature in both NSI and WSSI is positively correlated to a zone of high pressure in the east. The eastern regions, and then in particular ESI, show a positive correlation with a westerly flow (Fig. 6-18). The westerly winds cause lee-side warming in the east.

- JJA

(NCEP) The correlation patterns of the observed data during JJA are distinctively different for the North and South Island regions (Fig. 6-19). Although all regions have a positive correlation pattern with a zone of high pressure in the east, the largest positive correlations are found in the South Island and lie virtually over the country. 


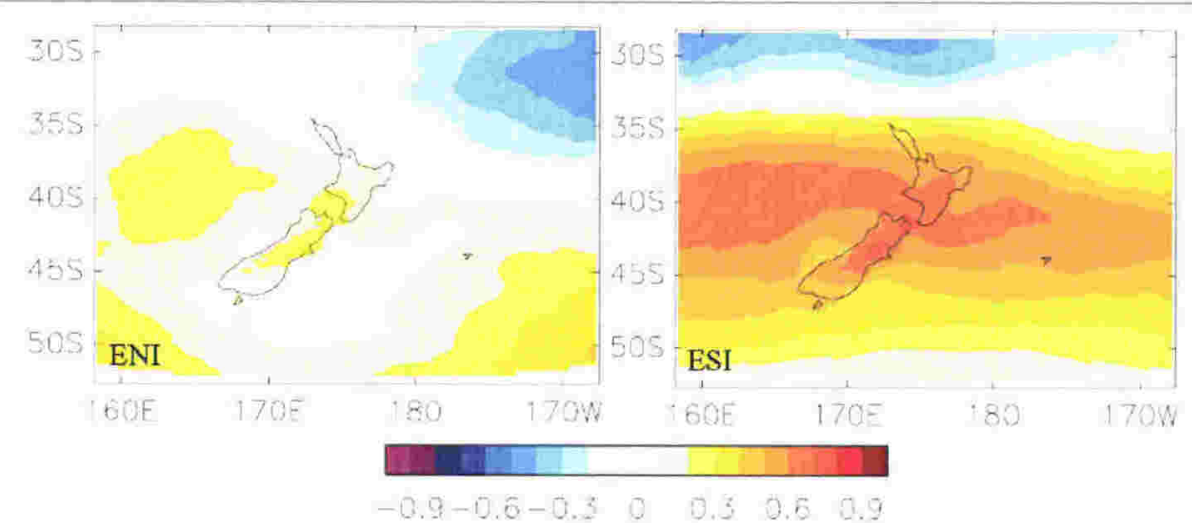

Fig. 6-18. Correlation maps of the zonal wind (at $1000 \mathrm{hPa}$ ) versus temperature for ENI (left) and ESI (right) during MAM. Westerly wind is positive.

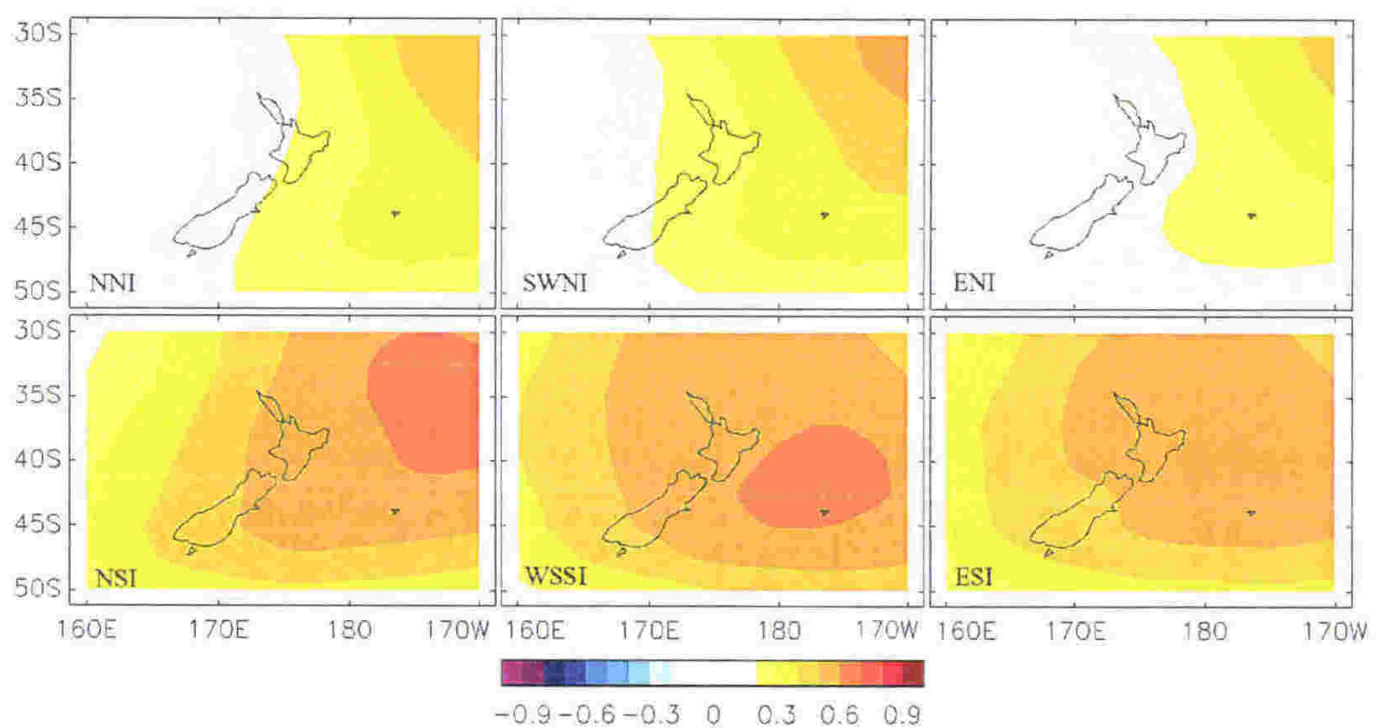

Fig. 6-19. Correlation maps of mean sea level pressure (NCEP) versus temperature (CLIDB) for the six different regions during JJA.

(RCM, Fig. 6-20) The correlation patterns of the modelled mean sea level pressure with temperature during JJA shows a very strong dipole pattern with large negative correlations (up to -0.9) in the west and large positive correlations (up to +0.7 ) in the east. This is the case for each region, with only small deviations in the locations and amplitude of the maximum correlations. All correlation maps show a strong relationship between temperature and northeasterly/south-westerly winds. The temperature in all regions is highly correlated 


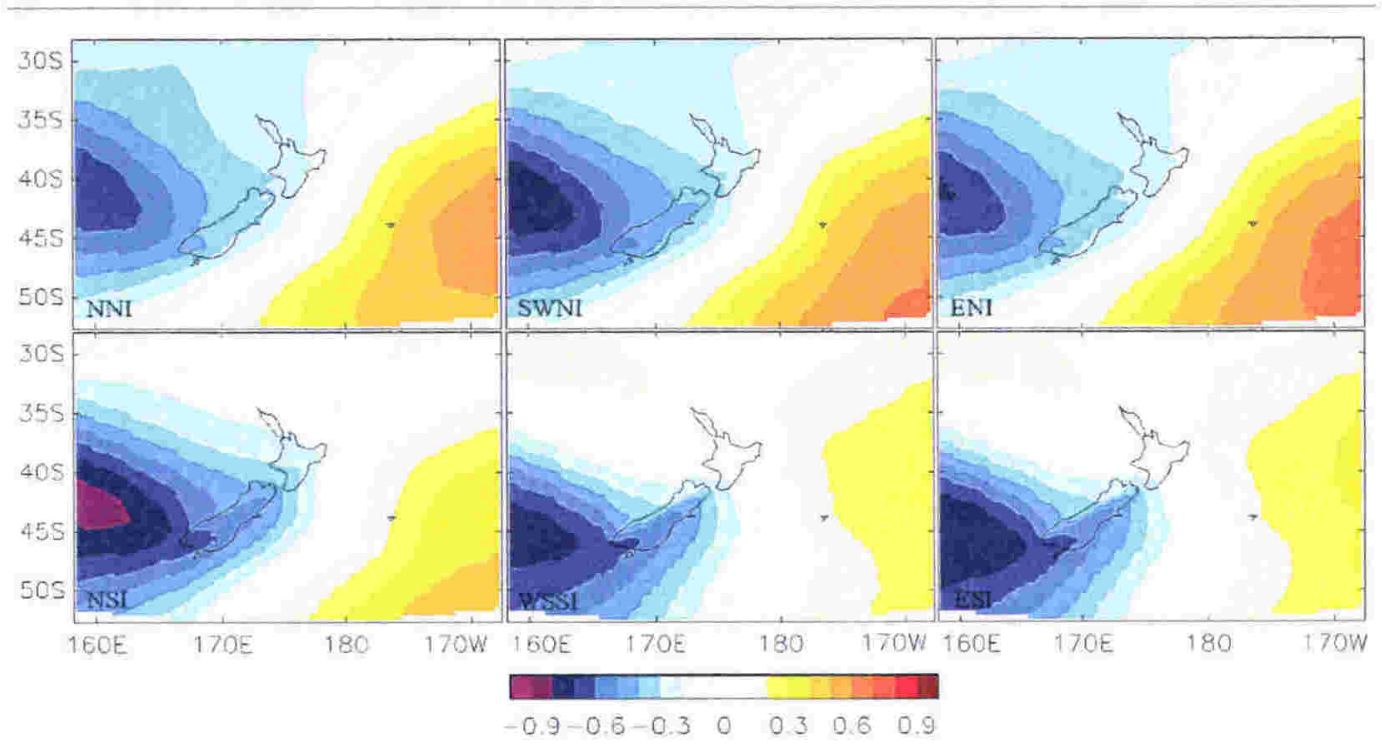

Fig. 6-20. Correlation maps of mean sea level pressure versus temperature for the six different regions during JJA (RCM).

(up to -0.9 ) to a meridional wind just to the east of New Zealand (this is shown for SWNI in Fig. 6-21). Warmer temperatures in the North Island are also strongly related to an easterly flow. Opposite to its cooling effect during DJF, WSSI experiences a warming influence from the westerly wind. This is understandable since the westerly wind advects relatively warm ocean air onto

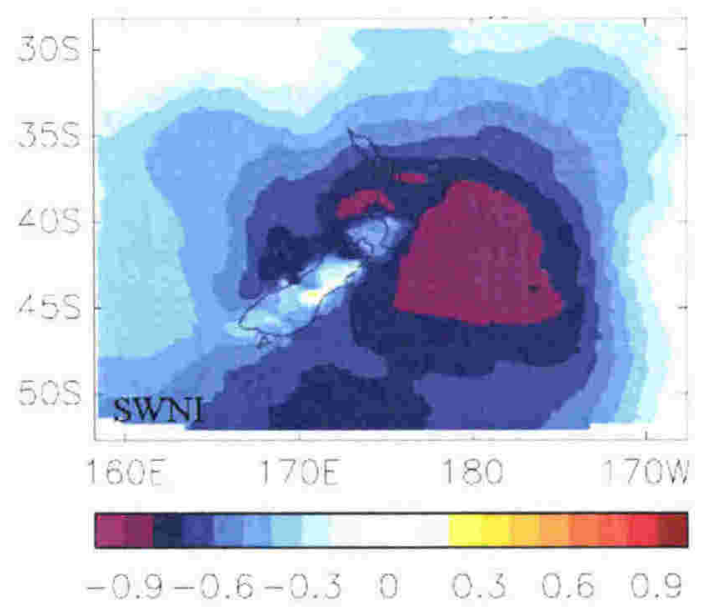

Fig. 6-21. Correlation map of the meridional wind (at $1000 \mathrm{hPa}$ ) versus temperature for SWNI during JJA. Northerly wind is negative. the region which temperatures are relatively cold during the winter. Warming in ESI is still related to a strong westerly flow over New Zealand, but the strongest correlations with the westerly wind (up to +0.7 ) have shifted equatorward $\left(35^{\circ} \mathrm{S}\right)$ compared to their maximum in MAM $\left(40^{\circ} \mathrm{S}\right.$, see Fig. 6-22).

The correlation patterns of the modelled parameters differ from the NCEP correlation patterns 


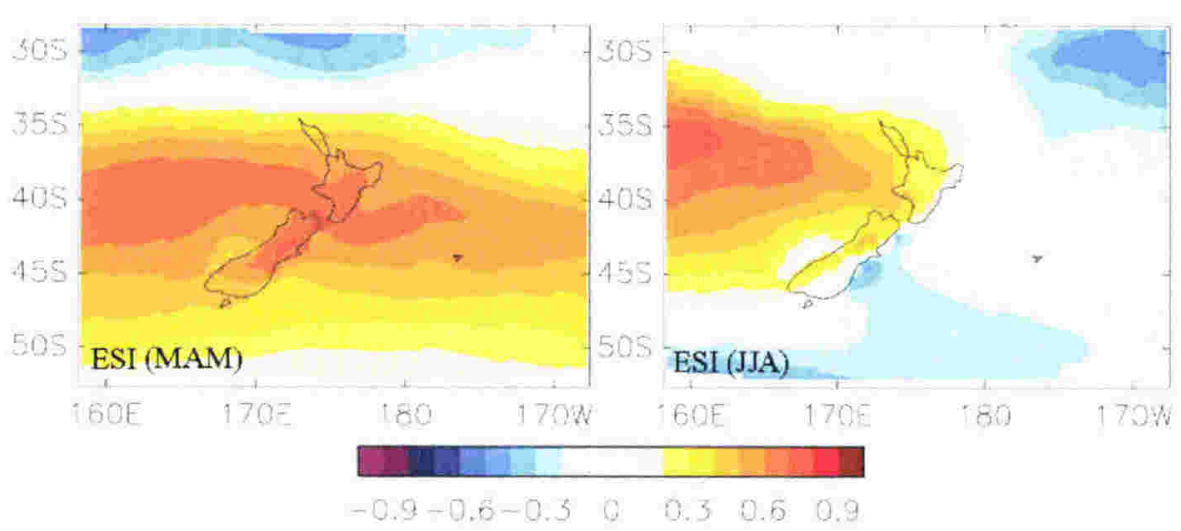

Fig. 6-22. Correlation maps of the zonal wind (at $1000 \mathrm{hPa}$ ) versus temperature for ESI during MAM (left) and for ESI during JJA (right). Westerly wind is positive.

significantly during JJA. Trenberth and Mo (1985) and Trenberth (1986) had found a positive connection between the amplitude of the wave number 3 pattern of the mean zonal circulation and blocking. Since the mean zonal circulation in the global simulation had an enhanced wave number 3 pattern, it could be expected that a feature as blocking might show up in the correlation patterns of MSLP with temperature. However, opposite to the correlation patterns of the NCEP/CLIDB data, the correlation patterns for the modelled parameters do not indicate any influence of blocking over New Zealand during JJA. As for the previous two seasons, these different patterns are the result of the anomalously strong westerlies.

\section{$-S O N$}

(NCEP) The correlation patterns of NCEP mean sea level pressure with the observed regional temperature for SON are presented in Fig. 6-23. All regions have a positive correlation with a "high" to the east. The temperature response in NNI, SWNI and NSI is correlated to a northerly/southerly flow. WSSI experiences warming under a north-easterly wind direction, where as warming in the eastern regions ENI and ESI is correlated to a north-westerly. The latter two regions in particular are affected by lee-side warming. 


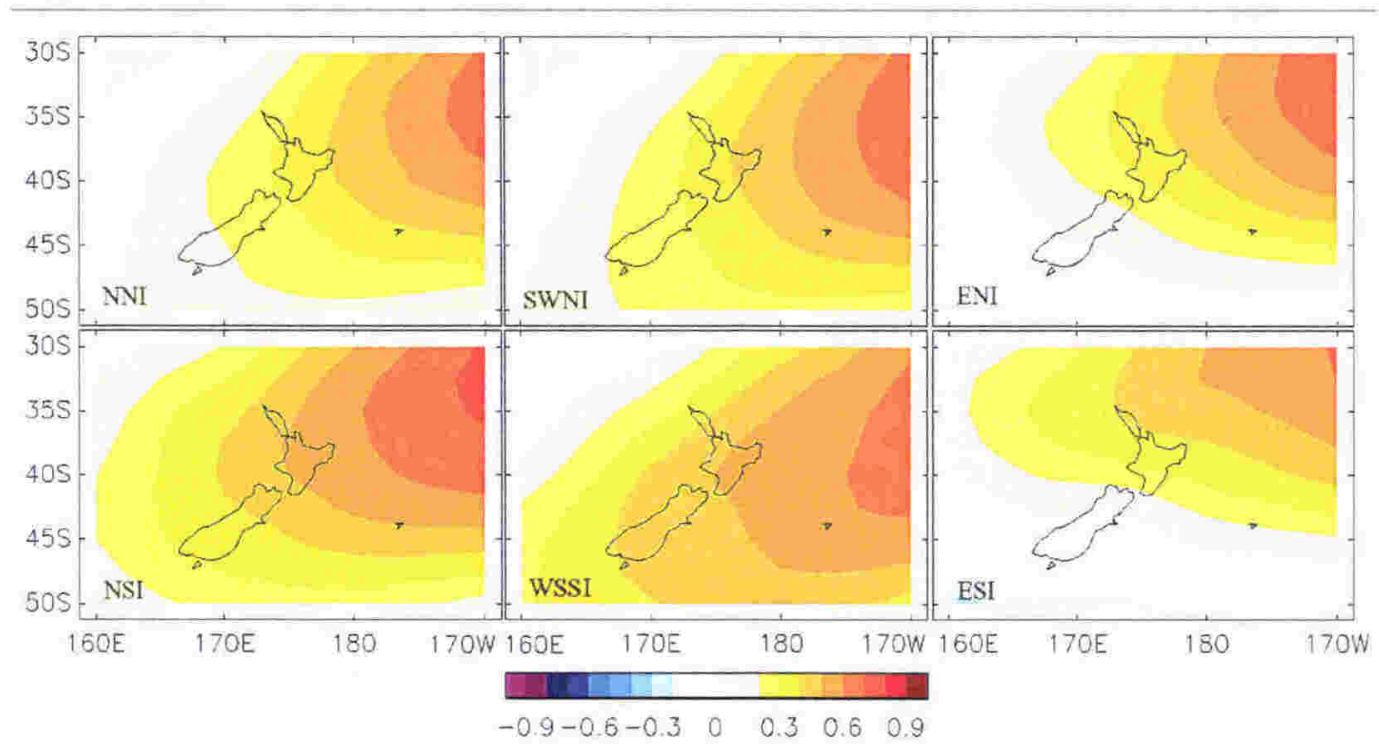

Fig. 6-23. Correlation maps of mean sea level pressure (NCEP) versus temperature (CLIDB) for the six different regions during SON.

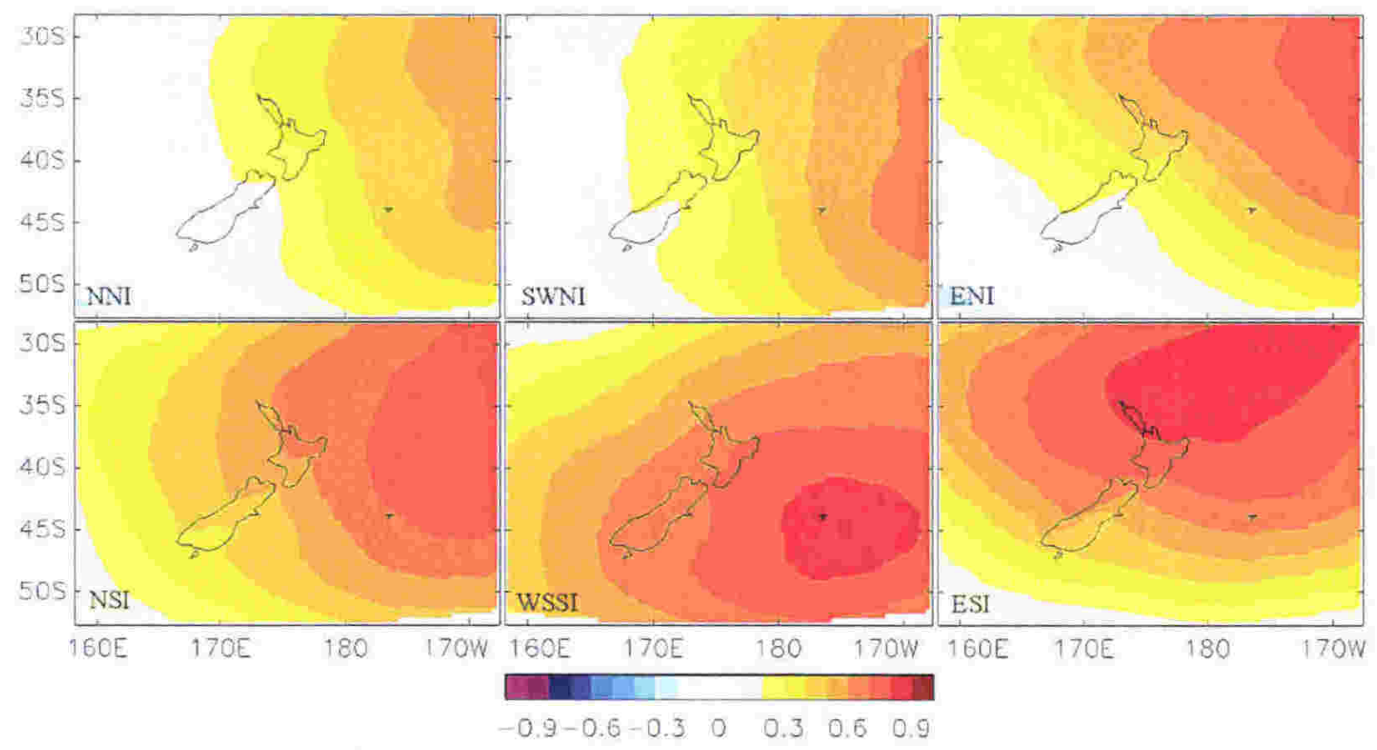

Fig. 6-24. Correlation maps of mean sea level pressure versus temperature for the six different regions during SON (RCM).

(RCM, Fig. 6-24) The correlation patterns for SON are dominated by large positive correlations in the east (up to +0.8 ). The resulting northerly/southerly flow warms/cools every region. Temperature is indeed stronger correlated to the meridional wind (up to -0.9$)$ than to the zonal wind $(-0.3 /+0.3)$ for the regions in the North Island (this is shown for NNI in Fig. 6-25). The zone of high pressure 


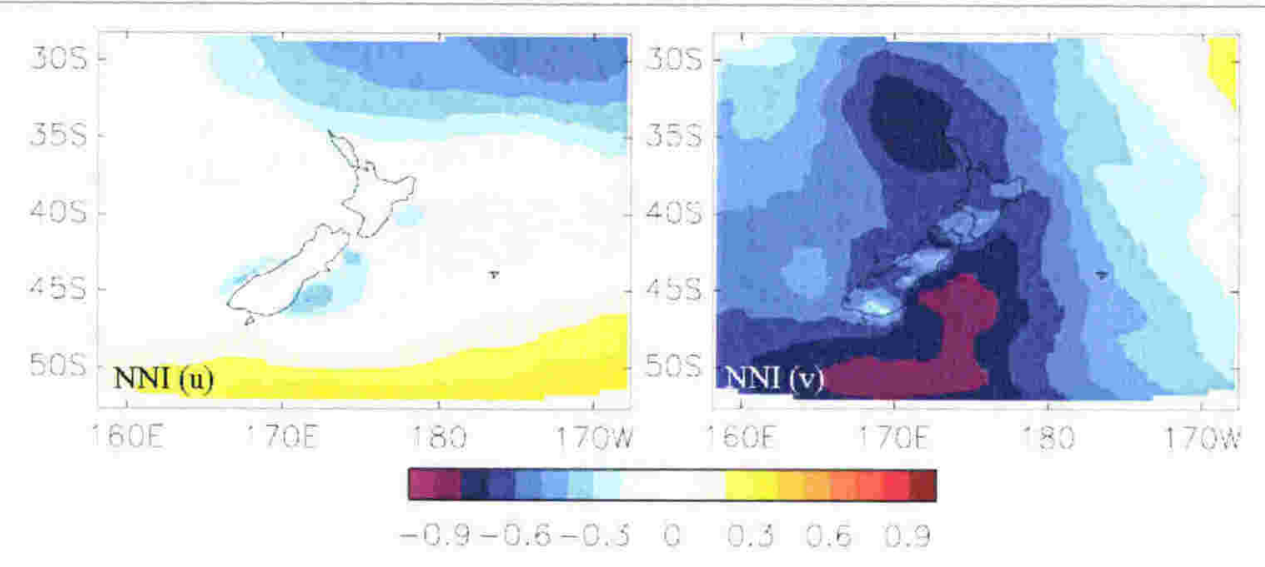

Fig. 6-25. The correlation map of the zonal wind (at $1000 \mathrm{hPa}$ ) versus temperature (left) and the correlation map of the meridional wind (at $1000 \mathrm{hPa}$ ) versus temperature (right) for NNI during SON. Westerly wind is positive, northerly wind is negative.

in the correlation patterns of ENI and ESI is more to the northeast. The associated wind would give rise to lee-side warming. This has a strong impact on the temperature in ESI. This lee-side warming is partially responsible for the modelled temperatures being too high in those regions (see Fig. 6-7, Fig. 6-8). Warming in WSSI is again strongly correlated to a north-easterly flow, which would result in lee-side warming to the west of the mountains.

Of all the seasonal correlation maps, the correlation patterns of SON show the most similarity with the correlation patterns of the NCEP reanalysis with CLIDB data. At first this seems to be at odds with earlier statements made in chapter 5 where it was determined that the largest differences between the modelled circulation patterns and the reanalysis data were during SON. One of the main anomalies was that the westerly winds were too strong. However, the westerly wind in the present day has its most dominant influence on the climate of New Zealand during SON. It is therefore not surprising to see that the correlation patterns of the simulated and observed data are similar during that season. The conclusion remains that the largest errors in the regional domain are introduced by the GCM.

Except for DJF, the pre-industrial simulation indicates that the meridional wind has a stronger influence on the regional temperature in New Zealand than 
the zonal wind. This is in general agreement with the NCEP climatology. However, this does not imply that the (meridional) wind is the dominant factor determining the temperature response in a region. The correlations for NNI and SWNI are the most consistent during the year and show a positive correlation of north-easterlies with temperature. The largest seasonal variations occur in the two most southern regions, where the temperature response to the zonal wind is dominated by westerlies at one time and by easterlies at other times. The relationships between temperature and mean sea level pressure in this simulation are consistent with the main findings of Salinger (1980b): warm/cold temperatures are related to northerlies/southerlies as the result of a high/low to the east (southeast) of the country. The orography modulates the impact of the zonal wind on temperature. Lee-side warming occurs in the east under westerly winds, and to a lesser degree, occurs in the west under easterly winds.

\subsubsection{Correlation MSLP-Precipitation}

The regional mean seasonal precipitation is correlated against the seasonal mean sea level pressure at each grid point. Negative values mean a lowering of the mean sea level pressure implies enhanced precipitation, or an increase in the mean sea level results in reduced precipitation. The correlations do not imply that mean sea level pressure is the sole, or even the strongest determinant of the occurrence of precipitation, but just as with the MSLP-temperature correlations are discussed here in order to investigate later (chapter 8) the differences in their relationships under a different climate. Correlations of the winds with precipitation are also examined. The seasonal patterns show again much higher correlations than the annual patterns. The correlation patterns of each season are discussed below.

\section{- DJF}

(NCEP) The correlation patterns of NCEP mean sea level pressure with the observed regional precipitation (CLIDB) for DJF are presented in Fig. 6-26. All regions show a strong correlation with a "low" to the west of the country. 


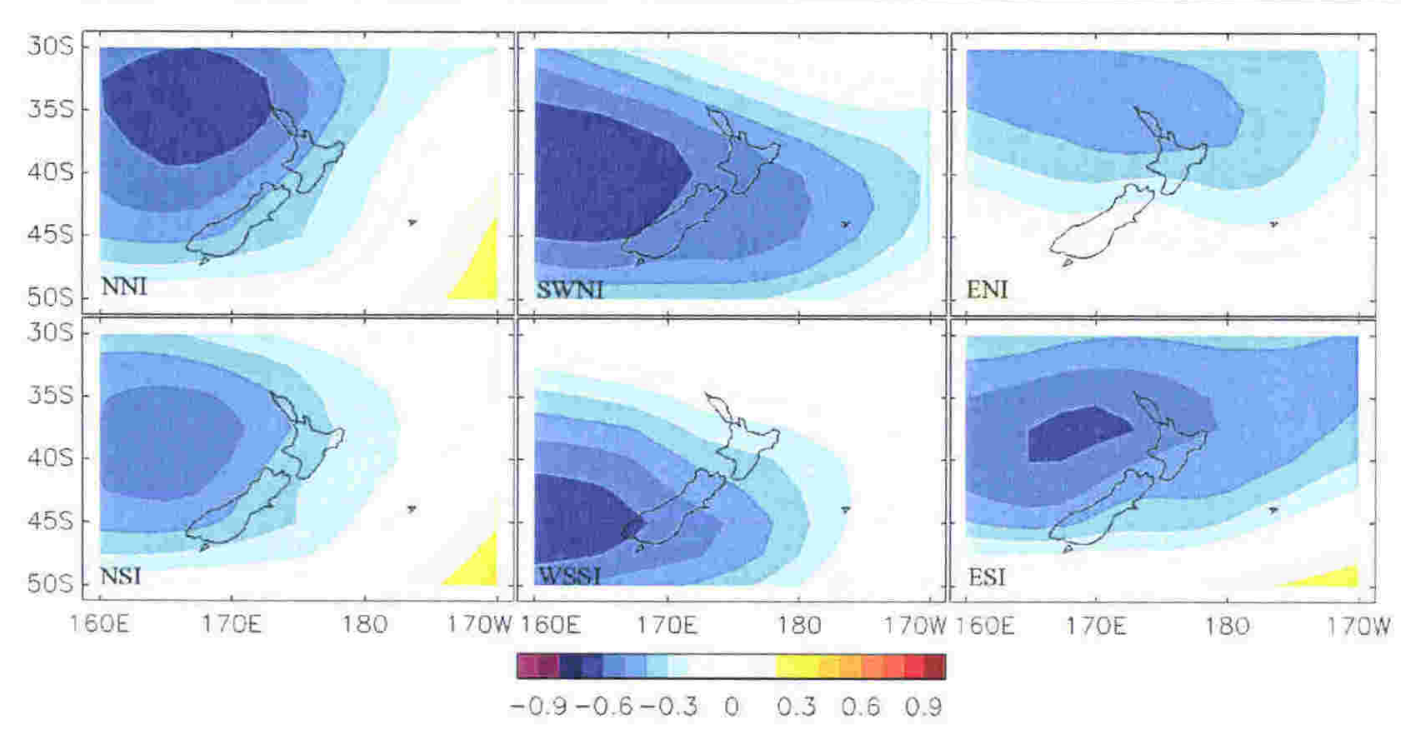

Fig. 6-26. Correlation maps of mean sea level pressure (NCEP) versus precipitation (CLIDB) for the six different regions during DJF.

Precipitation in NNI and NSI has the strongest correlation with a northerly wind, and precipitation in SWNI and WSSI has a strong correlation with a northwesterly. The region of low MSLP for the eastern regions sits more to the northwest of the country and ENI and ESI get their moisture from an easterly direction.

(RCM, Fig. 6-27) Correlation values for DJF vary in general between $-0.4 /+0.4$, except for precipitation in WSSI, which has a stronger correlation $(-0.6)$ with a low to the southwest of the country. The correlation maps for the regions in the North Island indicate that precipitation is mainly coming from the north. The occurrence of precipitation in SWNI has a more westerly component and precipitation in ENI has a more easterly component of the northerly wind. The correlation patterns for NNI in particular can be associated with (tropical) lows coming down from the north. Correlating precipitation in NNI with the zonal and meridional wind gives a correlation of over -0.9 with a northerly wind and -0.2 for an easterly wind (Fig. 6-28). Precipitation in both NSI and ESI is correlated to a region of low MSLP to the west of the North Island. Precipitation in NSI is particularly correlated to northerly winds (up to -0.8) where as precipitation in ESI shows correlation values of 0.5 with easterly and southerly winds. WSSI gets 


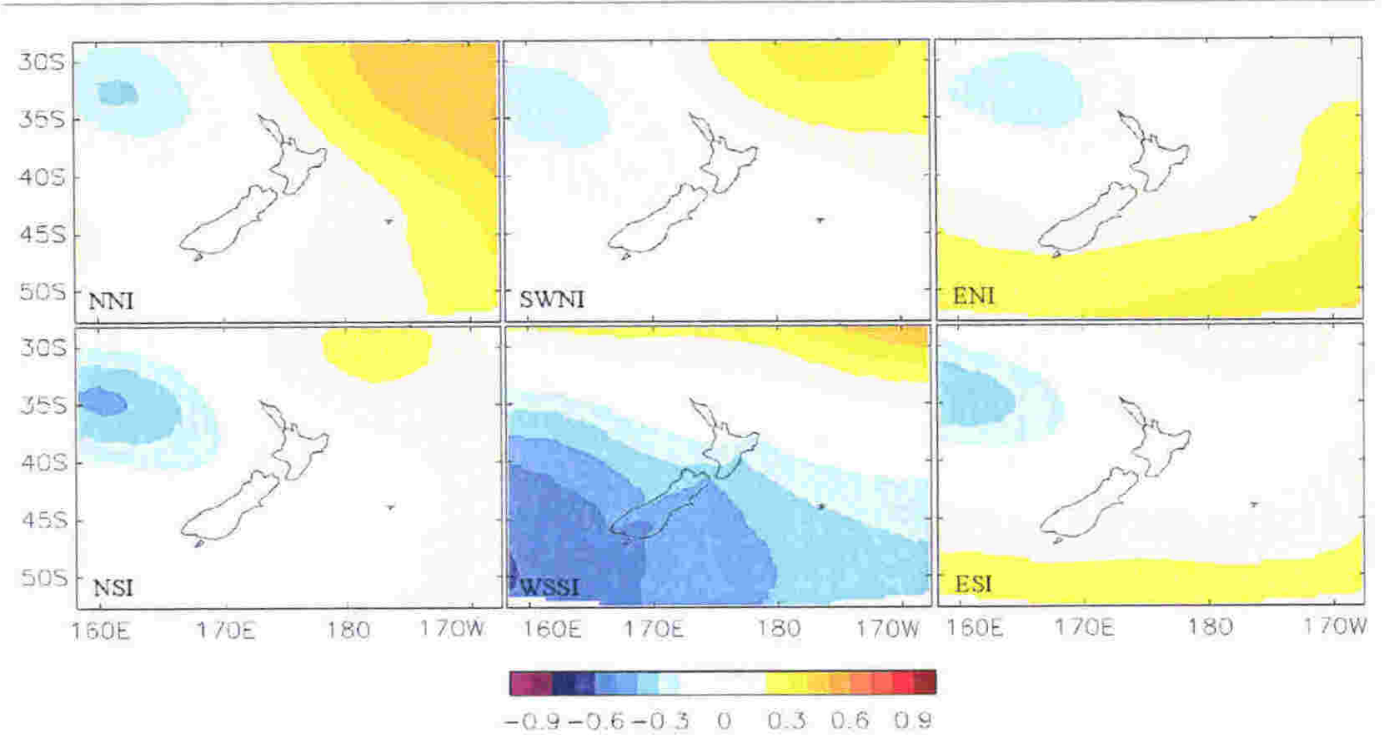

Fig. 6-27. Correlation maps of mean sea level pressure versus precipitation for the six different regions during DJF (RCM).

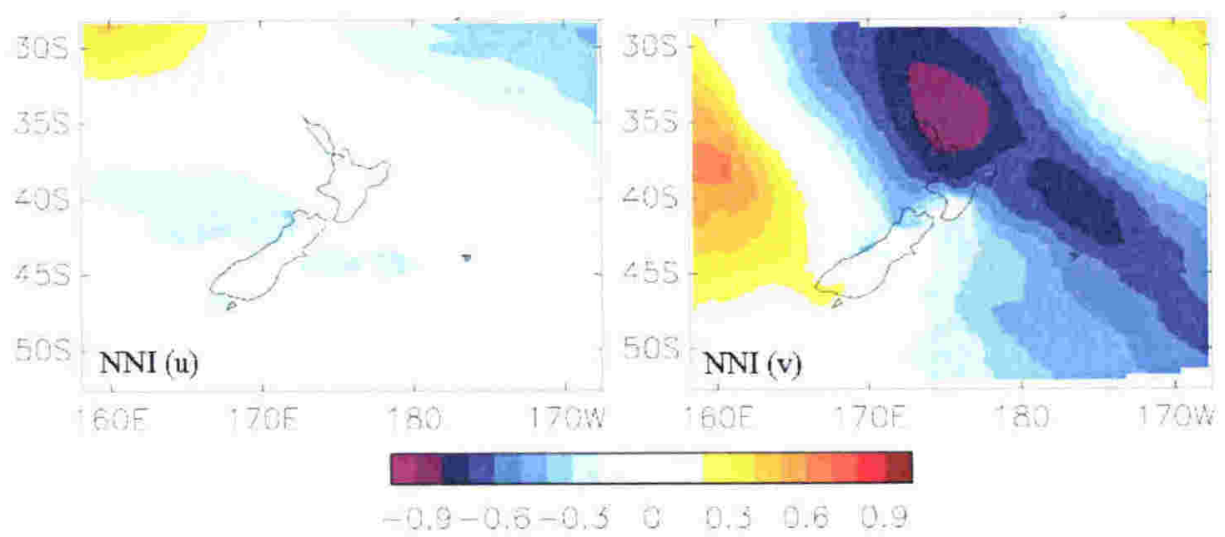

Fig. 6-28. The correlation map of the zonal wind (at $1000 \mathrm{hPa}$ ) versus precipitation (left) and the correlation map of the meridional wind (at $1000 \mathrm{hPa}$ ) versus precipitation (right) for NNI during DJF. Westerly wind is positive, northerly wind is negative.

its moisture predominantly from the northwest, with precipitation in this area having a correlation up to +0.7 with a westerly and -0.5 with a northerly wind.

Although the correlation patterns for the observed and computed MSLP and precipitation look quite different from each other, the correlation patterns of the RCM data does capture the essence of the link between precipitation and MSLP for each region. Precipitation in NNI and NSI is correlated predominantly to a northerly wind component, precipitation in WSSI has a strong correlation with a 


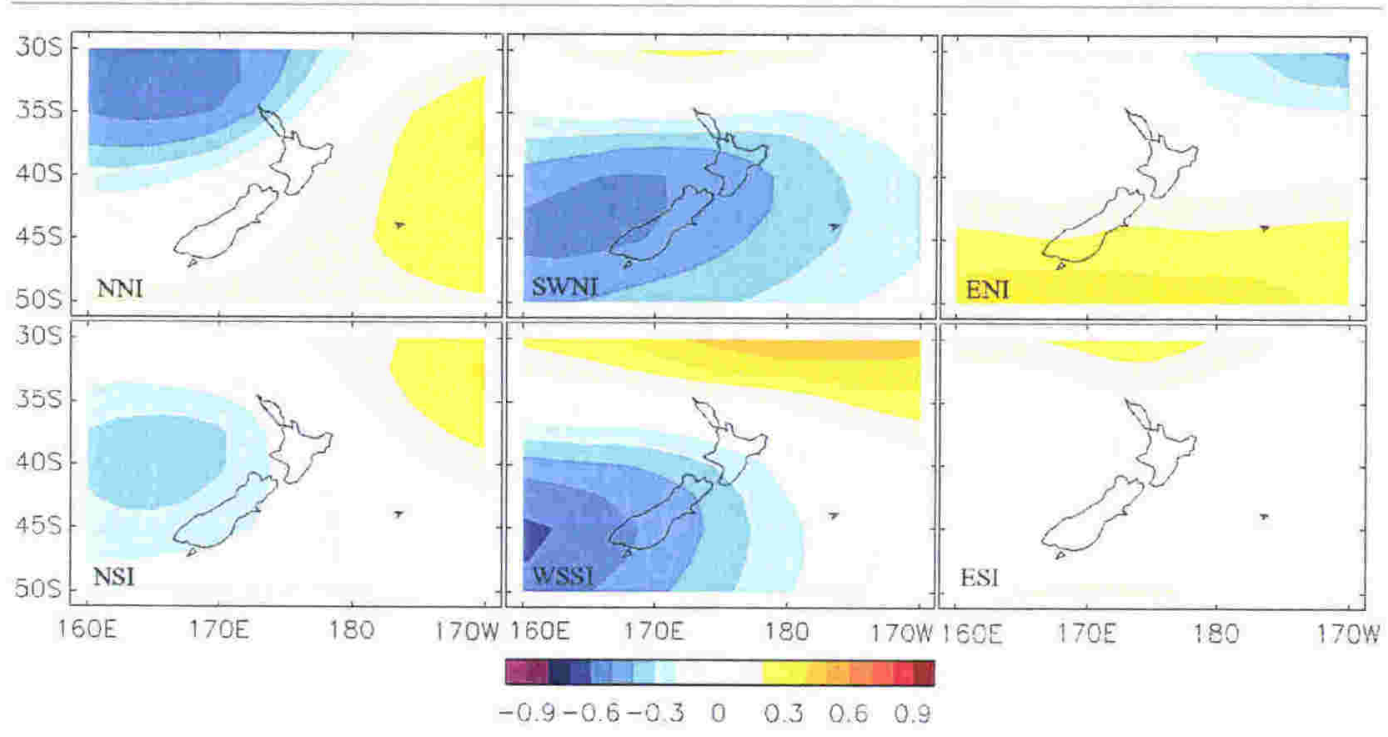

Fig. 6-29. Correlation maps of mean sea level pressure (NCEP) versus precipitation (CLIDB) for the six different regions during MAM.

westerly wind, and precipitation in the eastern regions is correlated to an easterly wind. All correlations for the RCM are quite weaker than the NCEP/CLIDB correlations, but the range is quite similar (NCEP/CLIDB:-0.7- +0.2 versus RCM: -0.4 - +0.4) indicating similar windstrength.

\section{- MAM}

(NCEP) The correlations of NCEP mean sea level pressure with the observed regional precipitation for MAM (Fig. 6-29) are in general weaker than during DJF, but show a similar pattern. The largest differences between the two seasons are found in the east where precipitation in ENI is now correlated to a "high" in the south and a "low" to the north, and precipitation in ESI is only weakly correlated to any windpattern.

(RCM, Fig. 6-30) Correlations during MAM are higher than during DJF and the maximum correlation is up to -0.9 . Precipitation in NNI has a very high correlation with a large low to the northwest, which also covers New Zealand's northern tip. Precipitation in NNI is highly correlated to (tropical) lows coming from the north (correlation with a northerly wind up to -0.8), similar as during DJF. Precipitation in SWNI has a high correlation with a low sitting right over 


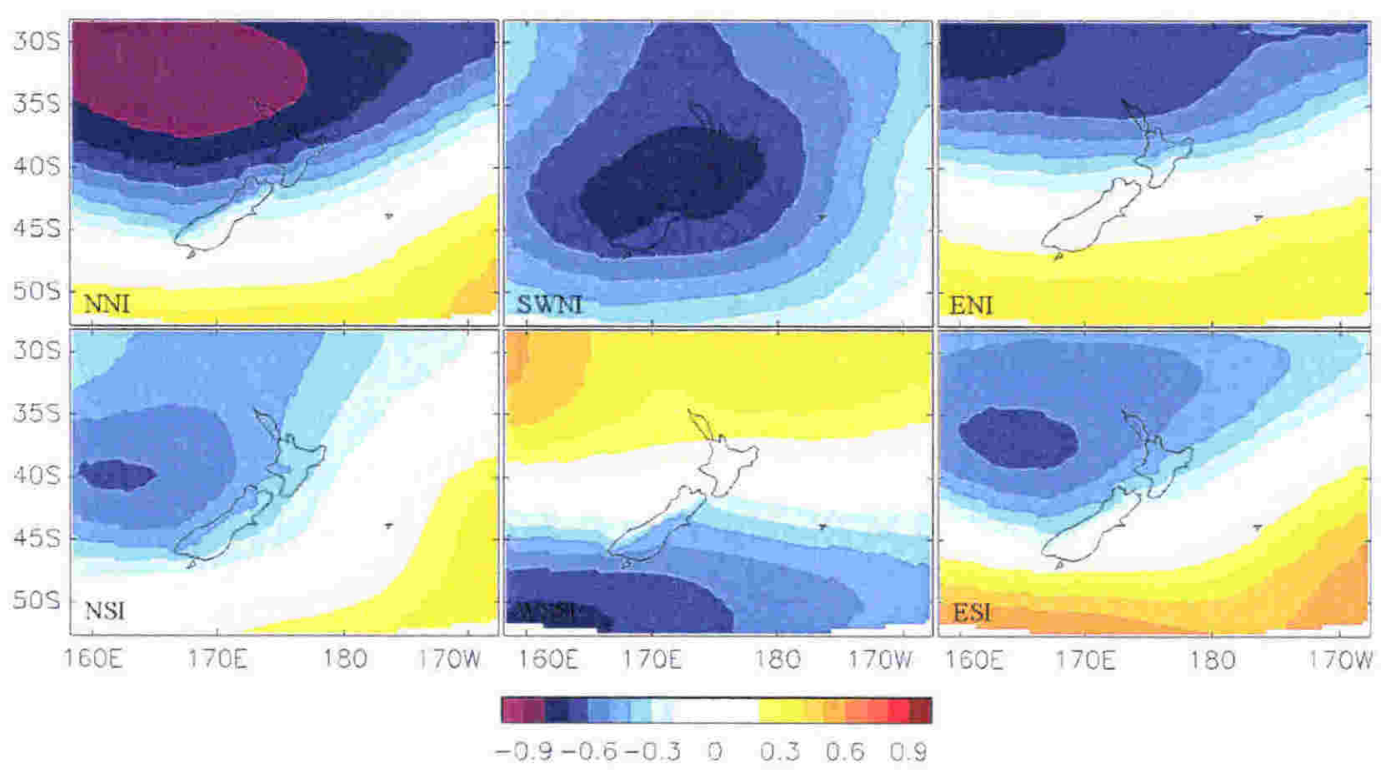

Fig. 6-30. Correlation maps of mean sea level pressure versus precipitation for the six different regions during MAM (RCM).

New Zealand. It has high correlations (up to 0.8 ) with all winds at the regional domain's outer edge, but only very weak correlations with any wind direction in its larger region (Fig. 6-31). The correlation patterns for precipitation in NNI and SWNI are similar to their correlation patterns for temperature. This indicates that wind patterns that would tend to warm up the region also enhance the occurrence of precipitation during this season (temperature and precipitation are correlated up to +0.6 in these regions).

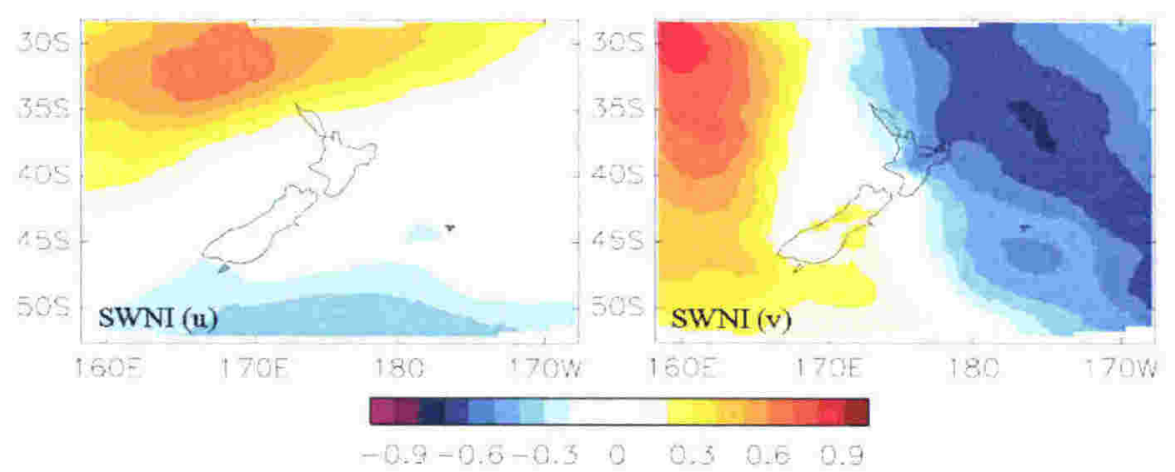

Fig. 6-31. The correlation map of the zonal wind (at 1000hPa) versus precipitation (left) and the correlation map of the meridional wind (at $1000 \mathrm{hPa}$ ) versus precipitation (right) for SWNI during MAM. Westerly wind is positive, northerly wind is negative. 
Precipitation in the eastern regions is strongly correlated to a zone of low pressure to the west/northwest of the country, which is positioned in such a way to give these regions maximum exposure to easterly winds. The difference between the eastern regions is that the contribution of precipitation coming from the south to the precipitation in ENI is relatively much smaller than in ESI. The correlation patterns of precipitation with temperature for ENI and ESI differ from the patterns for NNI and SWNI since the correlations in their equivalent zonal and meridional winds are the opposite of each other. The effect is that warming (as the result of a strengthening of the westerly wind) goes together with a decrease in precipitation, and cooling (as the result of a weakening of the westerly wind) with an increase in precipitation. Precipitation in WSSI is stronger correlated to a westerly wind than during DJF, indicating that fronts embedded in the mean circulation are the systems that bring the most precipitation to this area.

The correlations for the RCM data are quite higher than the NCEP/CLIDB correlations. Most patterns show a similarity to the NCEP/CLIDB correlation patterns albeit that the precipitation in NSI in the RCM comes more from a north-easterly direction where as the observed data shows it comes from the northwest.

\section{- JJA}

(NCEP) The correlation patterns of NCEP mean sea level pressure with the observed regional precipitation for JJA are presented in Fig. 6-32. Although the correlation patterns with MSLP is slightly different than in the previous two seasons, the dominant direction of the moisture-bearing winds remains nearly the same for each region. Precipitation in SWNI is strongly correlated with a "low" right over the country, implying dull, unsettled conditions overhead correlates highly to precipitation in this region. Precipitation in WSSI is correlated to a very zonal westerly flow. Precipitation in both eastern regions is strongly correlated to easterly winds. 


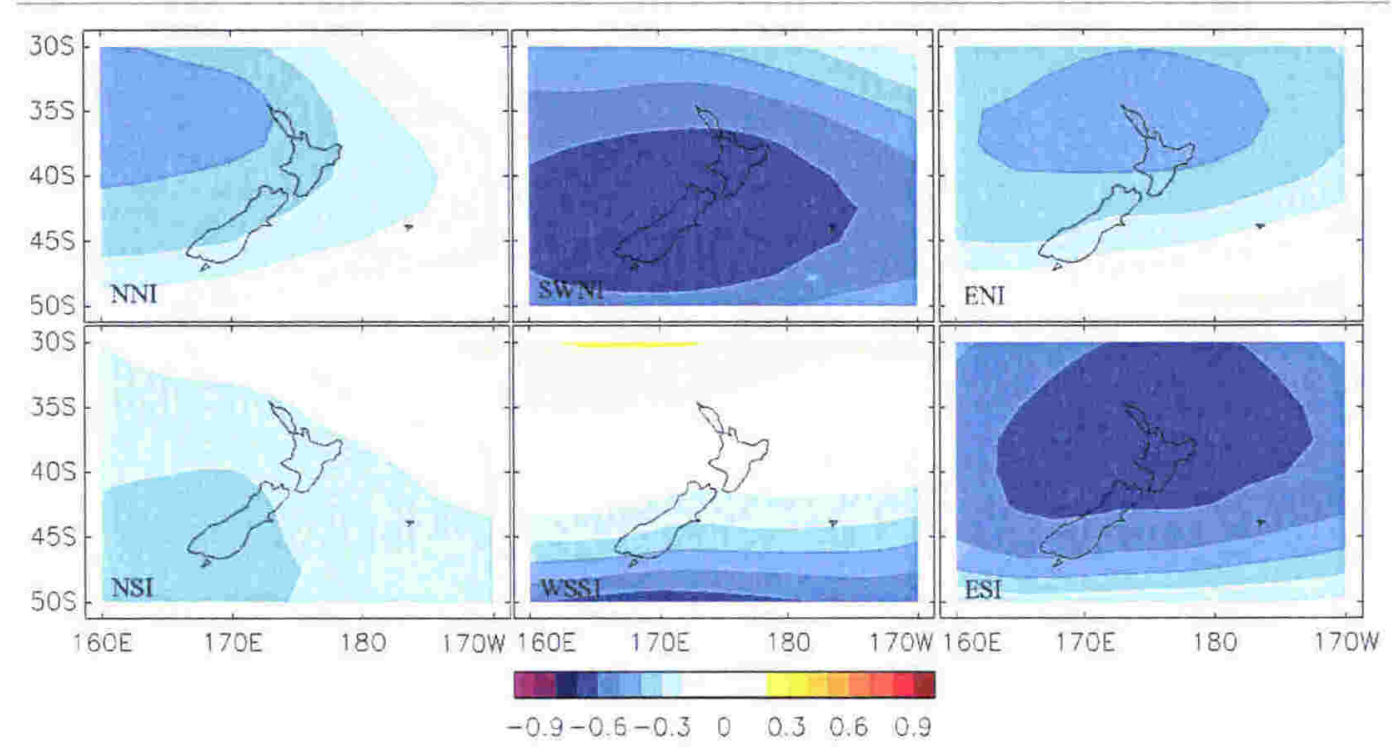

Fig. 6-32. Correlation maps of mean sea level pressure (NCEP) versus precipitation (CLIDB) for the six different regions during JJA.

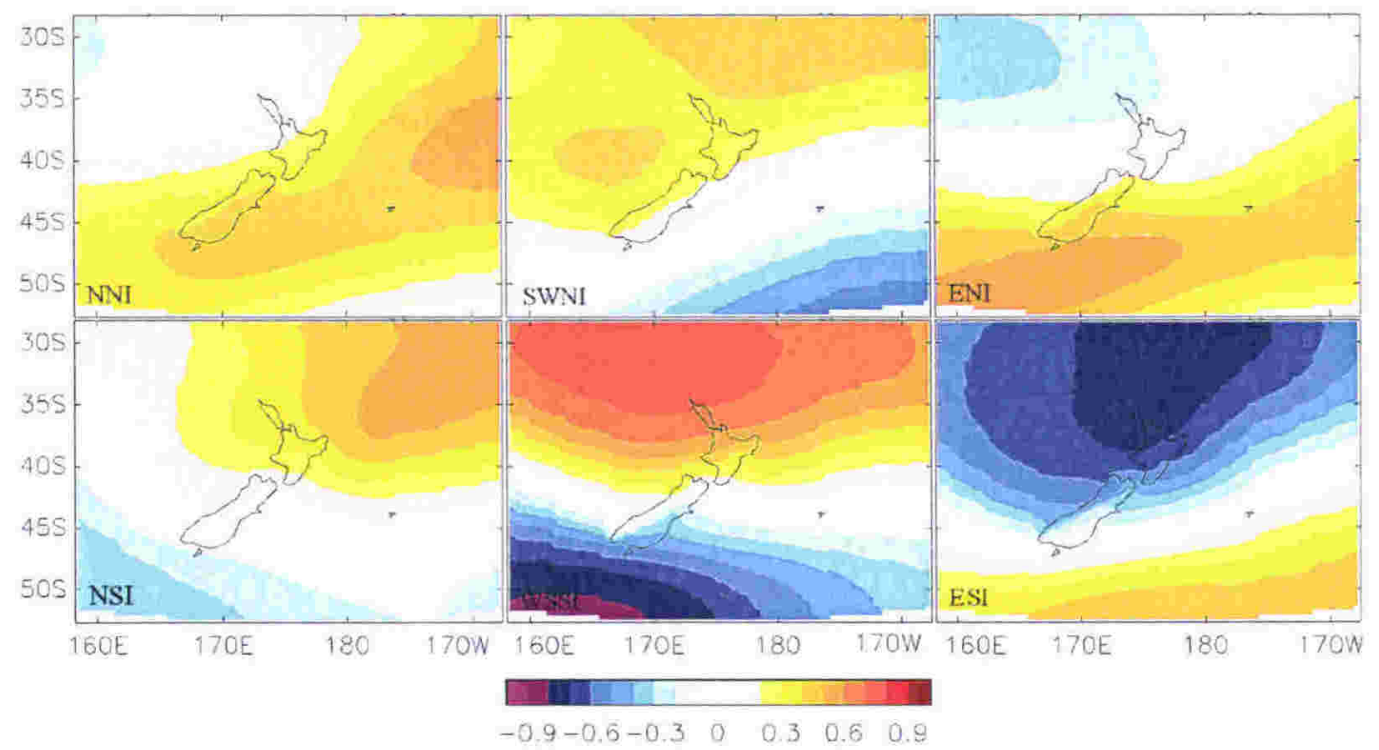

Fig. 6-33. Correlation maps of mean sea level pressure versus precipitation for the six different regions during JJA (RCM).

(RCM, Fig. 6-33) The correlation patterns for NNI and ENI during JJA are similar to those of DJF. Precipitation in both regions is correlated to a low to the northwest of the country. Precipitation in NNI comes mainly from the northeast, and precipitation in ENI comes mainly from the (south)east. The other regions show different and uniquely winter patterns. The correlation maps are dominated 


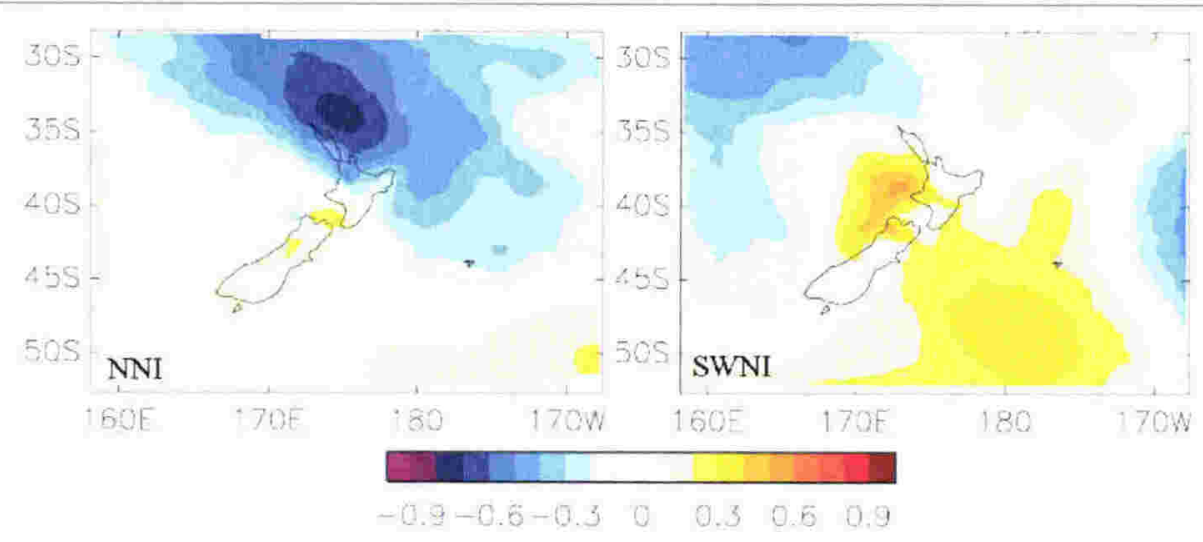

Fig. 6-34. Correlation maps of the meridional wind (at 1000hPa) versus precipitation for NNI and SWNI during JJA. Northerly wind is negative.

by linkages to high rather than to low mean sea level pressure zones, which is perhaps indicative of the fact that this season experiences a larger occurrence of blocking highs. The exception is precipitation in ESI, which correlation pattern is similar as during MAM. Correlations for the four most northern regions are only up to 0.5. The two southern regions, WSSI and ESI, have much higher correlations, and are as high as 0.9 . This indicates the dominance of the northwesterlies and south-easterlies respectively on the occurrence of precipitation in those regions.

For most regions the correlation maps of precipitation and temperature with the mean sea level pressure are to some degree similar, but the responses for each region vary. The northerly flow correlates positively with precipitation in NNI, but correlates negatively for SWNI as a result of the sheltering effect of the Volcanic Plateau (see Fig. 6-34). This means that northerly winds result in warmer temperatures and dry conditions in this region. Temperature and precipitation are positively correlated in the Raukumara Peninsula and NSI. The largest positive correlations of temperature with precipitation are found for WSSI, where the dominant northwester both warms the land as well as enhances the occurrence of precipitation'. Temperature and precipitation are not correlated

\footnotetext{
${ }^{I}$ A cautionary note needs to be placed here. WSSI is a large area and includes regions with different climatic characteristics, as there are the wet West Coast, the cold Southern Alps and dry inland Otago. The northwesterly winds will not cause warmer temperatures in the coastal region of the West Coast, but could
} 
to each other in inland ESI, but are positively correlated along the South Canterbury Coast (up to 0.6). It is difficult to make absolute statements about this large local contrast in the temperature-precipitation correlation pattern. A possible interpretation might be that the night-time cooling away from the coast during JJA can be very large and can offset the daytime warming, where as the coast would be constantly under the influence of the "warm" SSTs and would not experience such a strong cooling.

Although the correlation patterns for the RCM are quite different again than the patterns for the observed data, the implied dependency on the dominant moisture-bearing wind direction is practically the same. The region for which this does not count is SWNI. The anomalous correlation pattern of SWNI was not investigated further.

- SON

(NCEP) The eastern regions have the most consistent mean sea level pressureprecipitation correlation patterns. Both eastern regions show that precipitation also comes predominantly from the east during SON as well (Fig. 6-35). The correlation pattern of the zonal wind with precipitation for SWNI and WSSI are similar and show that precipitation in these regions is strongly correlated to a westerly flow (not shown). Precipitation in NNI is correlated to a north-easterly and in NSI to a north-westerly wind direction.

(RCM, Fig. 6-36) In section 6.3.2 it was established that the amount of precipitation was not very well simulated during SON. This was mainly ascribed to the erroneous westerly circulation in the Southern Hemisphere. However, it was found that the correlation patterns of modelled MSLP and temperature were most similar to the correlation patterns of the observed MSLP and temperature during SON. To a certain extent, this is true for the correlation patterns of MSLP with precipitation as well.

very well have a warming effect on the Southern Alps and Otago. Averaging over all these regions result in the northwesters having a warming effect on WSSI. 


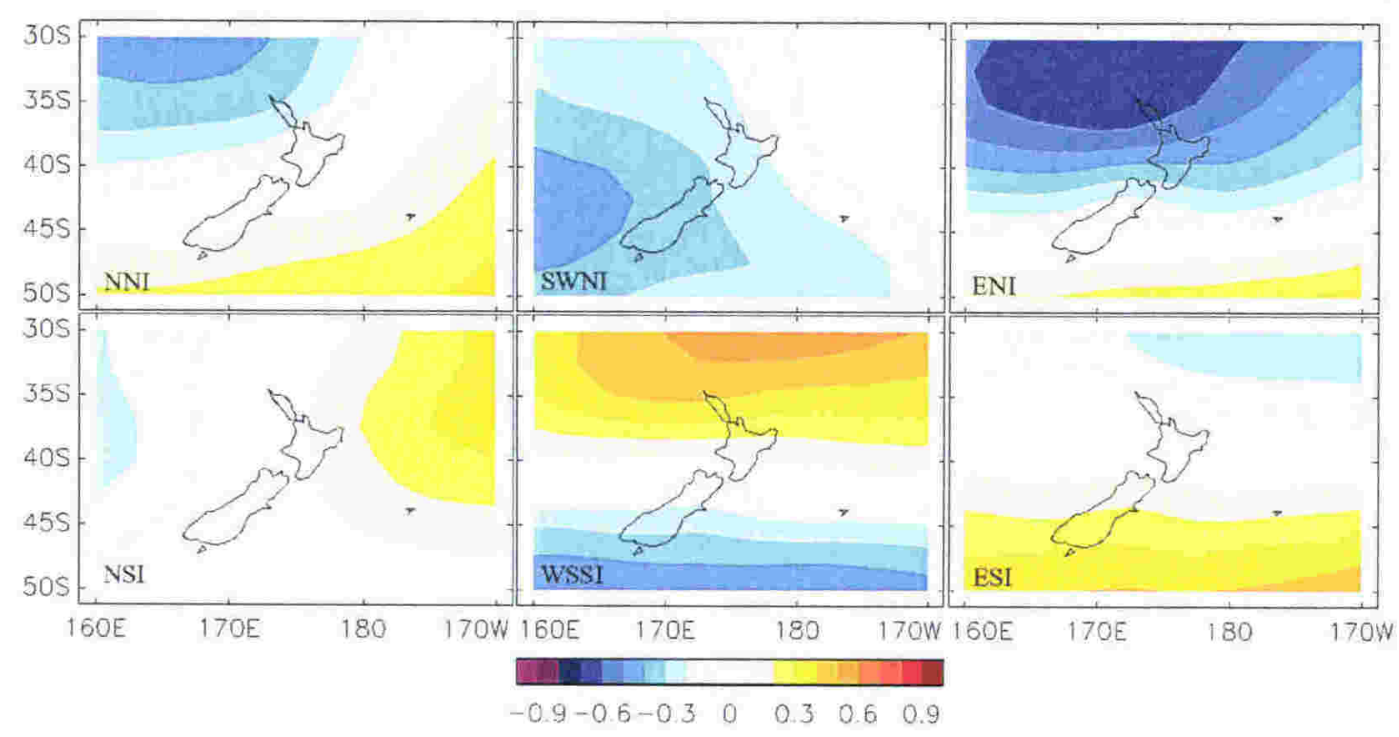

Fig. 6-35. Correlation maps of mean sea level pressure (NCEP) versus precipitation (CLIDB) for the six different regions during SON.

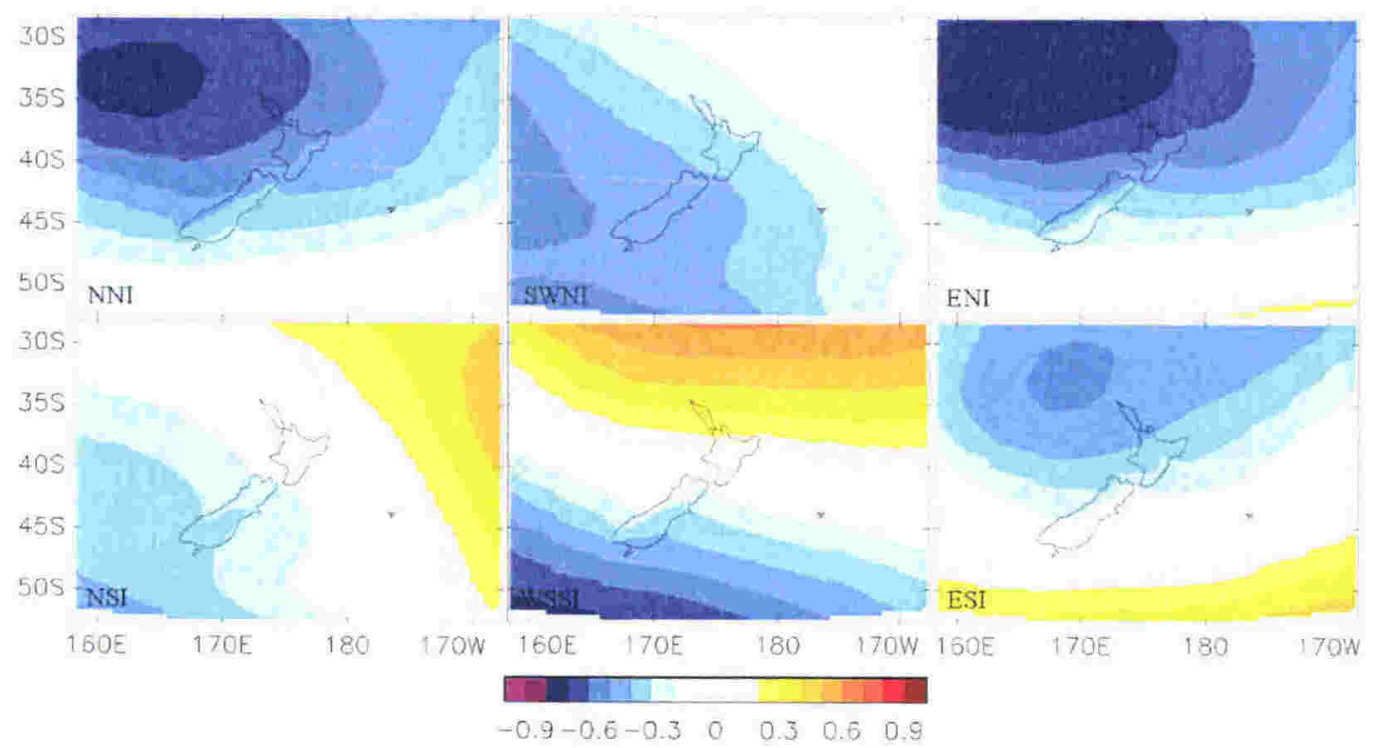

Fig. 6-36. Correlation maps of mean sea level pressure versus precipitation for the six different regions during SON $(\mathrm{RCM})$.

As during the other seasons, precipitation in NNI and ENI show their highest correlations with a low to the northwest of New Zealand. The strongest correlations are found during the transitional seasons, with SON also showing a maximum correlation of -0.8 . The pattern of WSSI is also consistent with the correlation patterns during other seasons, and again shows the dominance of the 
northwester on the occurrence of precipitation in this region. The patterns of SWNI and NSI differ slightly from their MAM pattern, and both seem to have a westward shift of their negative maximum in their correlation patterns. Although precipitation in those areas is still very highly correlated to a northerly flow (up to -0.9 ), the correlation with the zonal wind during SON is to a westerly wind $(+0.5)$ instead of an easterly as during MAM (-0.3, see Fig. 6-37).

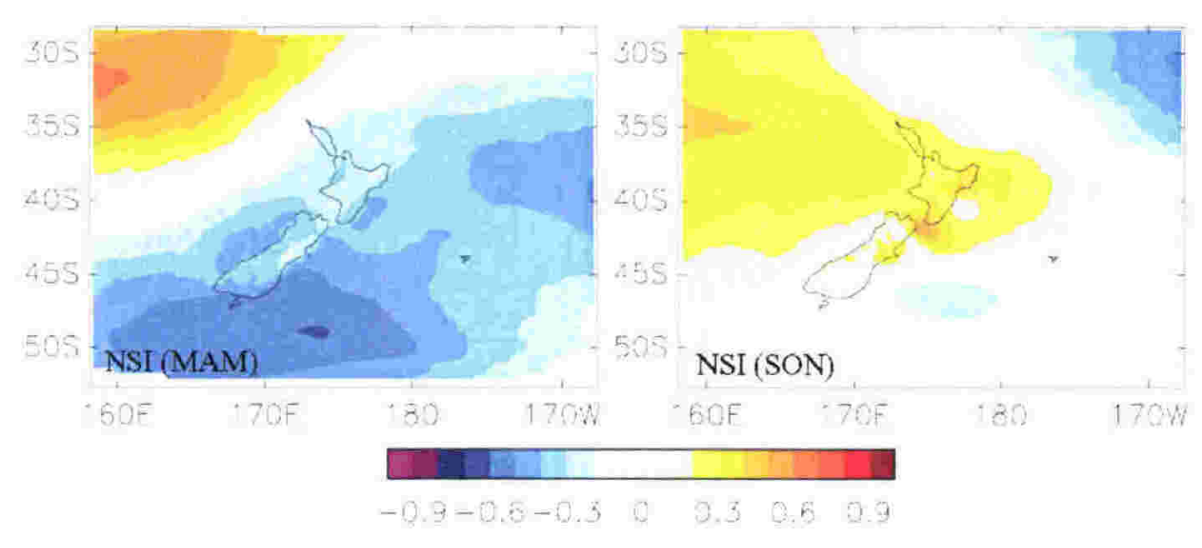

Fig. 6-37. Correlation maps of the zonal wind (at $1000 \mathrm{hPa}$ ) versus precipitation for NSI during MAM (left) and for NSI during SON (right). Westerly wind is positive.

The strongest correlations between MSLP and precipitation are during MAM and SON. There are some very strong and consistent correlations of precipitation to particular wind patterns during all seasons. Precipitation in WSSI always has a very strong correlation to westerly winds, where as easterlies are the main moisture-bearing winds for ENI and ESI. Zonal winds have a relatively stronger impact on the occurrence of precipitation than meridional winds. Precipitation in NNI, NSI and WSSI are correlated to northerlies the whole year round. This is the same for SWNI, except during JJA when there is quite an anomalous pattern. Southerlies are the dominant meridional winds for bringing precipitation to ENI and ESI the whole year round. In general, the relationships found between precipitation and mean sea level pressure here are consistent with the findings of Salinger (1980a).

An indication of the relationship between surface temperature and precipitation was given at various times. The extent of such a relationship is 
difficult to qualify since the physics and dynamics of the processes leading to precipitation in the model are linked to temperature and vice versa. Furthermore, it is the temperature of the air column that indicates how much moisture an air parcel can contain, and not the surface temperature. And precipitation results in cooling of the atmosphere. Therefore, the suggestions made about a possible correlation between surface temperature and precipitation needs to be understood in the context of the prevailing winds in each region.

\subsection{Summary}

An RCM (NZ_40km) was embedded within the GCM HadAM3H and its output was analysed. An RCM gives more geographical detail in the region of interest. The RCM was largely successful in simulating New Zealand's climate. Spatial and seasonal variations were by and large picked up correctly and were consistent with observed data. Due to the nature of New Zealand's steep and strongly varying orography, the main limitation in interpreting the RCM's output was related to the difficulty in representing New Zealand's orography appropriately in the model. The current resolution of $40 \mathrm{~km}$ was sufficient to analyse New Zealand's mean climate through cross sections and by regions. The climatic variations within a gridbox of $1600 \mathrm{~km}^{2}$ are still quite large.

Temperature and precipitation were the two climatic variables that were verified against the observed data. These variables were chosen since they have a long record and good geographical distribution in the database, and they are excellent local indicators of changes in the mean circulation. The latter is of importance when examining the different climate of the LGM in chapter 8.

The coldest regions in the simulation were associated with areas of high orography. The mountain range of the Southern Alps stood out as the region with lowest temperatures. The resolution was sufficiently fine that the model was also able to pick up the cooler temperatures of the smaller ranges like the Tararuas, Ruahines, Ureweras and the Volcanic Plateau. Even lone mountains as Mt Egmont were picked up. In general, the simulated temperatures in the mountain 
ranges were colder than the observed temperature. This was mainly due to the differences in altitude of the data, which in turn was the result of the differences between the modelled and real orography. The grid points in the model were often at higher altitudes than the climate stations. The eastern regions in both the North and South Island were consistently warmer than observed. This was attributed to the enhanced lee-side warming in the simulation which was partly caused by the stronger westerly flow of the GCM. The seasonal variations were entirely consistent with the climate database data.

The correlation patterns of MSLP with temperature show quite different configurations than the correlation patterns of NCEP MSLP with observed temperature. This was ascribed to the anomalous westerly flow of the GCM. However, most patterns did indicate to a certain extent the correct correlation between wind and temperature. The interpretation of these correlation maps resulted in similar findings as found by Salinger (1980b). In general, westerly winds give cooling in the west, with the exception during JJA, when they advect relatively warm air onto the coast. The same westerlies cause warming in the east (föhn winds), except during DJF, where the wind has a slight cooling effect. The temperature response is generally stronger correlated to the meridional wind than to the zonal wind, in particular during JJA and SON. Southerlies during those seasons can bring very cold conditions to eastern regions. In the simulation, DJF is characterized by having the strongest correlations between temperature and a high in MSLP centred nearly over the country. This implies that "clear skies" have a substantial influence on regional temperatures. This is less the case for the other seasons when it is mainly the advection of warm/cold air that affects the regional temperatures.

The feature that stands out the most in the precipitation analysis is the precipitation's very strong relationship with orography. Orographic precipitation is prominent all along the Southern Alps, but shows up also over the lesser mountain ranges. Precipitation is relatively well-simulated over areas with low and relatively smooth orography, but the amount is too much, up to $100 \%$ of the recorded precipitation, along the highest alpine regions. The anomalous amount 
of precipitation is the result of three processes. The largest errors occur as the result of the difficulty in modelling the orography correctly. Similar to the anomalies in temperature in the alpine regions, observed and simulated precipitation are not determined at the same height. The highest amount of annual precipitation takes place along the West Coast of the South Island, and can be as much as $6 \mathrm{~m}$. The annual precipitation in the simulation is up to $10 \mathrm{~m}$. Although this amount of precipitation is possibly in the highest nevees (see Fig. 6-1), this is deemed to be excessive. Secondly, it is known that the UM has difficulties in simulating precipitation properly over steep terrain. Some parameterizations had to be adjusted for the high-resolution regional model. However, these adjustments were not tested for the extreme steep orography that exists in New Zealand, and it can therefore very well be that these adjustments were still not adequate. Thirdly, the GCM's westerly flow was inherently too strong and had an enhanced wave number 3 pattern. Both features induce enhanced precipitation. The latter reason was particularly obvious during SON, when both the westerly circulation and the regional precipitation had their largest anomalies. Analysis of precipitation through surface plots and along cross sections showed that the geographical variation was modelled correctly with the highest rainfall in the west and the driest regions in the east. However, precipitation in the west was generally too high. The seasonality in precipitation was reconstructed relatively accurate, except again during SON, when the modelled precipitation was in general too high in the west and too low in the east. DJF also experienced enhanced precipitation, particularly in NNI, SWNI and NSI, which was probably caused by increased incursions of (tropical) lows. Precipitation in those regions shows a very strong correlation with the meridional wind.

Precipitation was in general very strongly correlated to MSLP. Particularly consistent correlated were lows to the south and highs to the north with precipitation in WSSI, and the opposite configuration with precipitation in the eastern regions. Although precipitation in NNI, NSI and WSSI is strongly correlated to northerly winds, and is correlated to southerlies in ENI and ESI, the influence of the meridional wind on precipitation patterns is weaker than for 
temperature. The correlation patterns of MSLP with precipitation for the RCM are often different than the correlation patterns of NCEP MSLP with observed precipitation. But similar to the correlation patterns of temperature, the general correlation between windpatterns and precipitation in each region is by and large correctly simulated. The relationships found between precipitation and mean sea level pressure were consistent with the findings of Salinger (1980a).

To a certain extent, some of the increased geographical detail gained by using a RCM could have been obtained by simply interpolating the GCM values and correct them for the increased resolution in orography or by using a simple statistical downscaling scheme. Several regional responses are similar to the GCM's response (e.g. lower temperatures with height, increased precipitation on the western side of the Alps). The climate on the lee-side of the mountain ranges was most affected by the regional dynamics and physics. The strength of the use of a RCM lays in its capability in analysing local variability as a result of regional effects. Investigating this capability in this RCM was not the purpose of this work however ${ }^{1}$. The output of the RCM under pre-industrial conditions will be compared against the same RCM under LGM conditions in chapter 8 . Differences in their responses will be related to both the different general circulation patterns as well as to the different local conditions.

\footnotetext{
I The internal variability of a regional climate model for New Zealand was investigated in an internal report for NIWA (Drost et al., 2005).
} 


\section{LGM simulation:}

\section{Global}

\section{Introduction}

In chapter 5 the performance of the model HadAM3H was investigated under pre-industrial settings. In this chapter, HadAM3H's reconstruction of the climate during the LGM will be discussed. The climate of the LGM was quite different from the pre-industrial, as was discussed in chapter 3 . The equations governing the general circulation remain the same, but initial and boundary conditions for the simulation are quite different. These conditions are constrained by geological proxy data. Since the biases in the model are known (chapter 5, Pope et al., 2000: Pope and Stratton, 2002: Hudson and Jones, 2002a), it is possible, to a certain extent, to separate climate characteristics of the LGM from those introduced by model biases. Section 7.1 discusses the different settings for this simulation, and section 7.2 presents the results.

At times, absolute values of a climate parameter is given, but most of the time the simulated climate of the LGM is compared against the simulated climate of the pre-industrial in order to emphasize the differences between the two climatic states. The emphasis is on the Southern Hemisphere, but comparisons are also made for the Northern Hemisphere and the tropics in order to examine the global circulation. Especially since not many modelling studies have focused on the climate of the Southern Hemisphere during the LGM, it is for validity reasons 
prudent to examine the simulated circulations in those regions. If the simulated climate in those areas has similar features as found in other studies, it would give more credit to the findings for the Southern Hemisphere.

\subsection{Setup}

The model is initialised from the end of a 500year HadCM3 LGM simulation which was described by Hewitt (2000). The boundary conditions for that simulation were set up as much as possible according to the specifications of PMIP. There are several important fields for the LGM simulation that are different than in the pre-industrial simulation. These are discussed below.

\section{- Orbital parameters:}

The orbital parameters during the LGM were slightly different from the present day and pre-industrial times (Table 7-1). The small changes in eccentricity, obliquity and precession of the Earth did not contribute greatly to any change in radiative forcing and therefore had only a minor impact on the climate of the LGM (e.g. Hewitt and Mitchell, 1997: Weaver et al., 1998: Broccoli, 2000). The value for these parameters in the LGM simulation (Hewitt and Mitchell, 1997) were based on the calculations of Berger (1978).

\begin{tabular}{|l|r|r|}
\hline & $0 \mathrm{~K} \mathrm{BP}$ & $21 \mathrm{~K} \mathrm{BP}$ \\
\hline \hline Longitude of perihelion (relative to the vernal equinox) & $102.5^{\circ}$ & $114.4^{\circ}$ \\
\hline \hline Obliquity & $23.44^{\circ}$ & $22.95^{\circ}$ \\
\hline \hline Eccentricity & 0.0167 & 0.0190 \\
\hline
\end{tabular}

Table 7-1. Orbital parameters for the pre-industrial and LGM experiment (after Hewitt and Mitchell, 1997).

\section{- Orography:}

To be as consistent as possible with the shape of the orography in the preindustrial setup (section 5.1), the same dataset (US Navy 10" dataset) was used to 

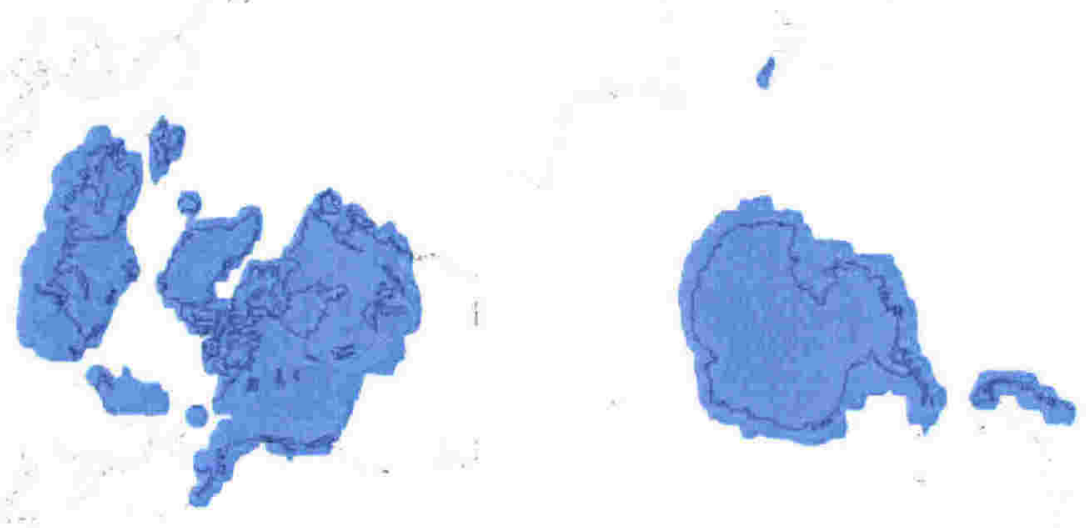

Fig. 7-1. The LGM ice sheets according to Peltier (1994), on HadAM3H grid.

create HadAM3H's LGM orography. The sea level was about 120m lower during the LGM than during pre-industrial times.

Since the US Navy 10" dataset describes only the orography above mean sea level, another dataset that included bathymetry had to be added to it in order to determine the surface of the extra land during the LGM. The ETOPO5 bathymetry data (NOAA, 1988) was interpolated onto the US Navy 10" grid and blended with the US Navy 10" dataset. The sea level of this dataset was lowered by $120 \mathrm{~m}$ by adding $120 \mathrm{~m}$ to all points. Furthermore, the LGM icecaps were inserted into this dataset following Peltier's ice topography (Peltier, 1994. See Fig. 7-1). Peltier's ice data and orography were interpolated onto the higher resolution US Navy/ETOPO5 dataset. The following criterion for the inclusion of the ice sheets was used: If according to Peltier a grid point was covered by ice, Peltier's estimated height of the orography was used if that height was higher than the equivalent height in the US Navy/ETOPO5 dataset. If this was not the case, the height in the US Navy/ETOPO5 dataset was used. The resulting orography dataset was used to create the HadAM3H LGM's orography by interpolating the dataset onto the HadAM3H grid (from $2160 \times 1080$ grid points to $192 \times 145$ grid points). The threshold for setting a grid point to sea or land was set to 0.5 , i.e. if $50 \%$ or more of the points within the averaging area in the US navy/ETOPO5 dataset was land, the HadAM3H grid point was set to land. The 


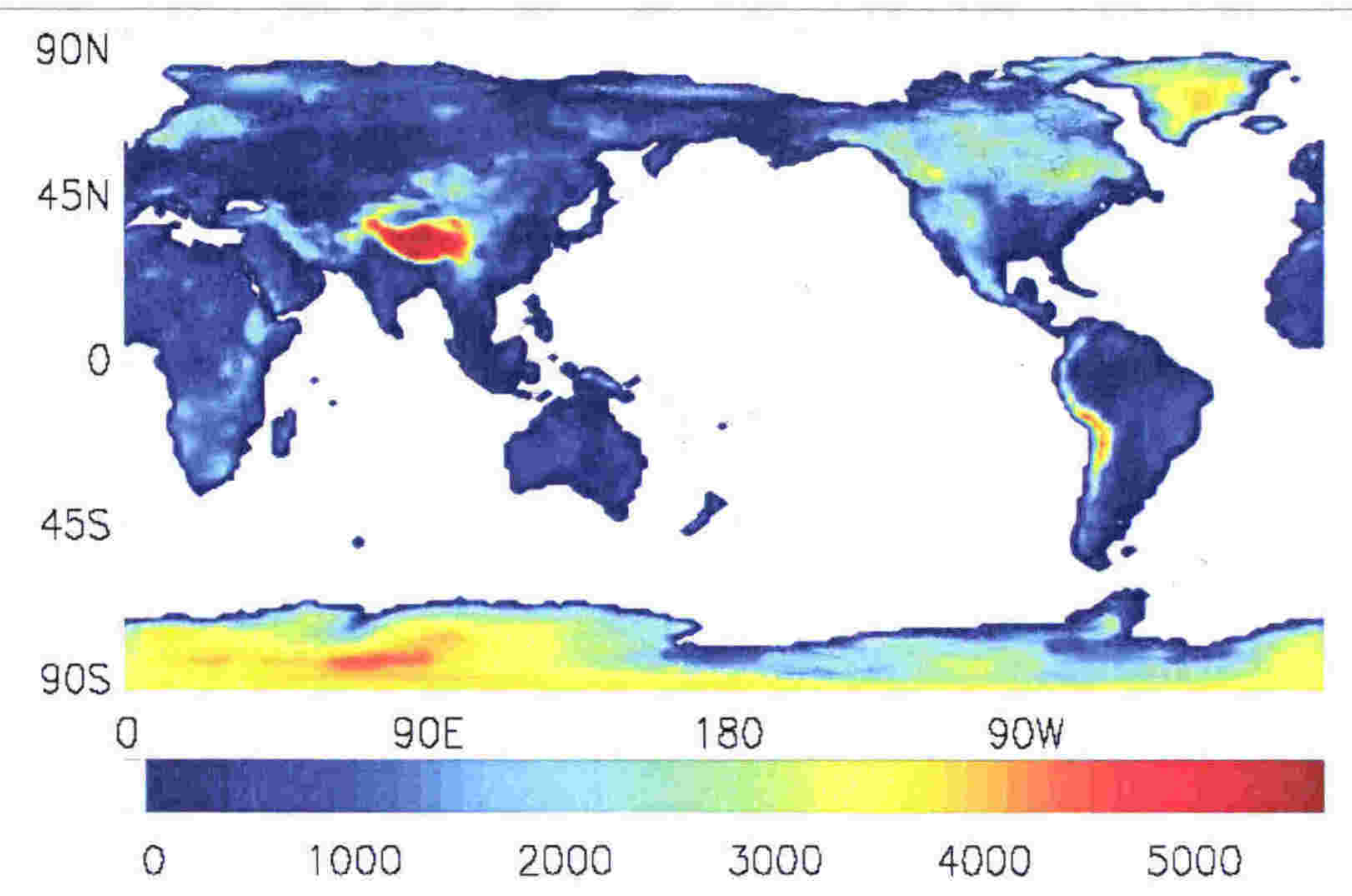

Fig. 7-2. The global orography (in $\mathrm{m}$ ) in the LGM simulation.

LGM orography is pictured in Fig. 7-2.

The landmask associated with the LGM orography differs greatly in several places from the landmask in the pre-industrial simulation. Some of these differences are the closure of the Bering Strait, linking Asia to North America; the closure of the Torres Strait, linking Australia to Papua New Guinea; and the South- East Asian islands forming one landmass. And of importance in this study is that the main islands of New Zealand were connected and formed one landmass.

\section{- SST and sea-ice:}

Just as in the pre-industrial simulation, SSTs and the sea-ice fields were computed by HadCM3, although this time when it was running under LGM conditions. That particular experiment was initialized from present day conditions since there is no global data set of glacial initial conditions available. Several steps were used to accelerate the cooling of the ocean to bring it towards LGM temperatures ${ }^{1}$, and the coupled model ran for 500 modelling years. After

\footnotetext{
${ }^{1}$ Values for the SSTs during the LGM are constrained by proxy data.
} 
that length of modelling time, it had reached a quasi-stable state (details of this experiment and the techniques to accelerate the cooling of the ocean are described in Hewitt, 2000).

Hewitt (2000) discusses how sea-ice in the Northern Hemisphere during the LGM shows a dramatic expansion during the winter months, with the Norwegian Sea, the North Atlantic south of Iceland to $60^{\circ} \mathrm{N}$, the Labrador Sea south to the coast of Newfoundland, the Bering Sea, the Sea of Okhotsk and the Sea of Japan being covered in ice. Most of the glacial sea-ice along the coast of the Fennoscandian Ice Sheet, the interior of the Labrador Sea, and in the Northern Hemisphere Pacific melts during the summer (Fig. 7-3).

Sea-ice in the Southern Hemisphere also shows a dramatic expansion during the winter months, but not as large as indicated by CLIMAP. HadCM3's LGM sea-ice expanded equatorward up to $55^{\circ} \mathrm{S} / 60^{\circ} \mathrm{S}$, where as CLIMAP's sea-ice expanded to $50^{\circ} \mathrm{S}$ (mainly in the Eastern Hemisphere). Areas of major sea-ice expansion during the winter months in HadCM3's simulation are the West Antarctic ice sheet centred on the Ross Sea and the Weddell Sea, and a protrusion of the East Antarctic ice sheet at about $90^{\circ} \mathrm{E}$. These three main areas of wintertime sea-ice expansion show a wave number 3 pattern around Antarctica, with the crests corresponding roughly with the central longitudes of each of the ocean basins. The sea-ice extension is just as large during summer, but the thickness reduces markedly. This would indicate that sea-ice was mainly in the form of icebergs. That icebergs have drifted more equatorward during the LGM has been documented for instance in the New Zealand region (Carter et al., 2002).

The SSTs are in general cooler during the LGM (Fig. 7-4) than during the pre-industrial. A detailed description of these SSTs is given by Hewitt (2000). A most remarkable feature in these SSTs is that there were warmer SSTs in a part of the North Atlantic (Hewitt, 2000: Hewitt et al., 2001: Hewitt et al., 2002), which is at odds with the general theory that cooling in the North Atlantic is linked to the initiation of the ice age (see chapter 3 ). However, apart from the Atlantic, the global distribution of SSTs is very similar to the pre-industrial distribution of SSTs, indicating that the surface circulation patterns were very 


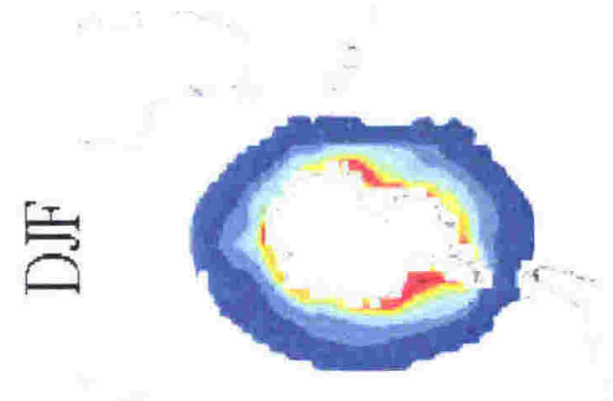

A

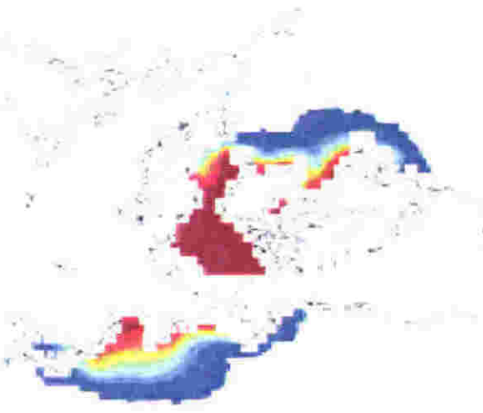

B
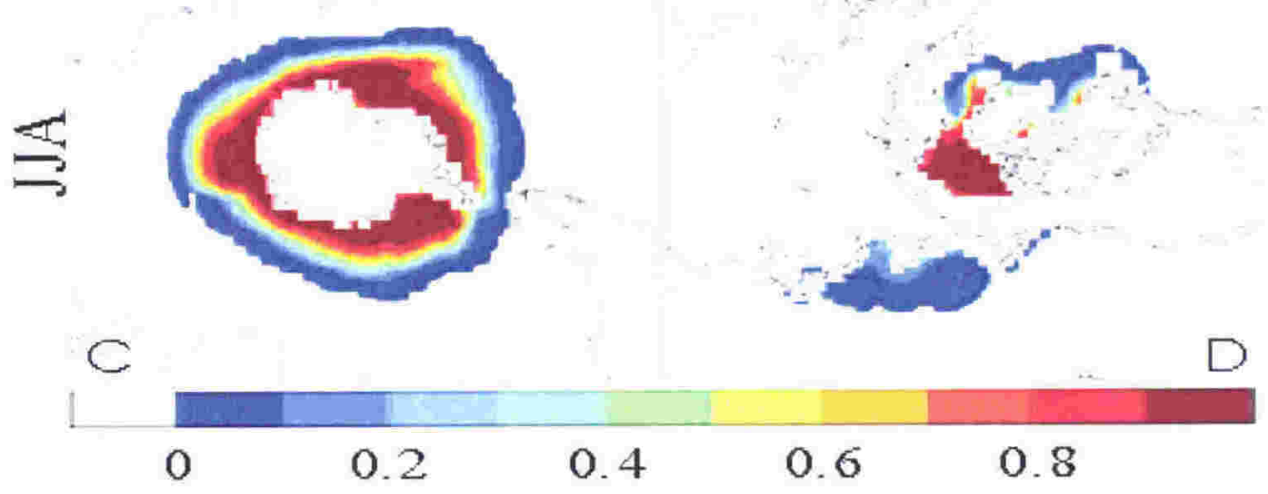

JJA - DJF

DJF - JJA

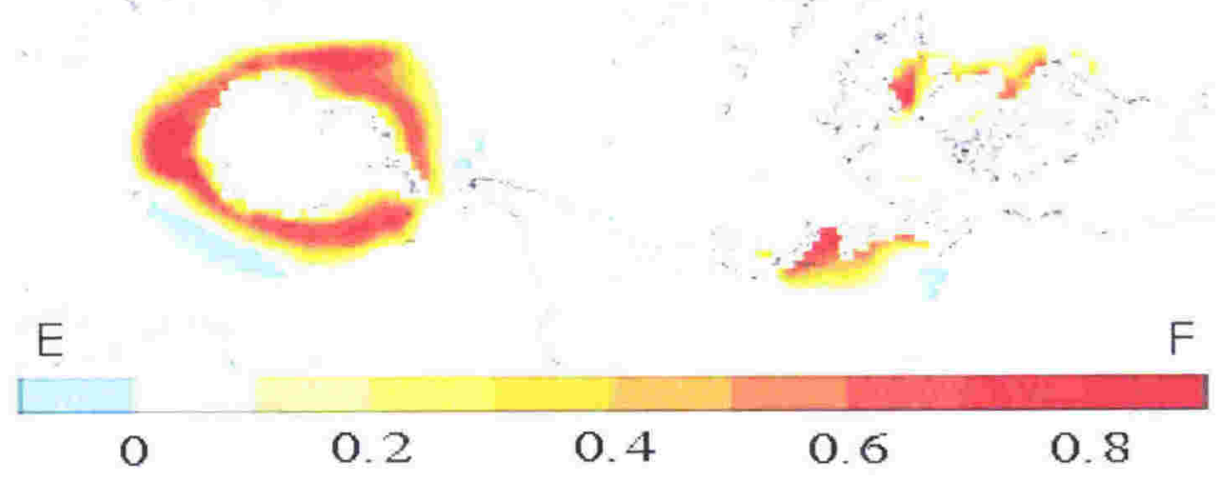

Fig. 7-3. Sea-ice during the LGM. Figure A, C, E are polar projections of the Southern Hemisphere, B, D, F for the Northern Hemisphere. A and B are DJF, C and D are JJA, and $\mathrm{E}$ and $\mathrm{F}$ are the differences in the seasons for each hemisphere. The fields are the seasonal averages. Note that E is JJA-DJF and F is DJF-JJA and that the colourbars are different. Both units refer to the extent of sea-ice cover within the grid box: 0 is no sea-ice, 1 is fully covered by sea-ice.

similar. The main spatial differences in SSTs are related to the occurrence of the ice sheets during the LGM. A more detailed discussion on SSTs is presented in section 7.2.1. 

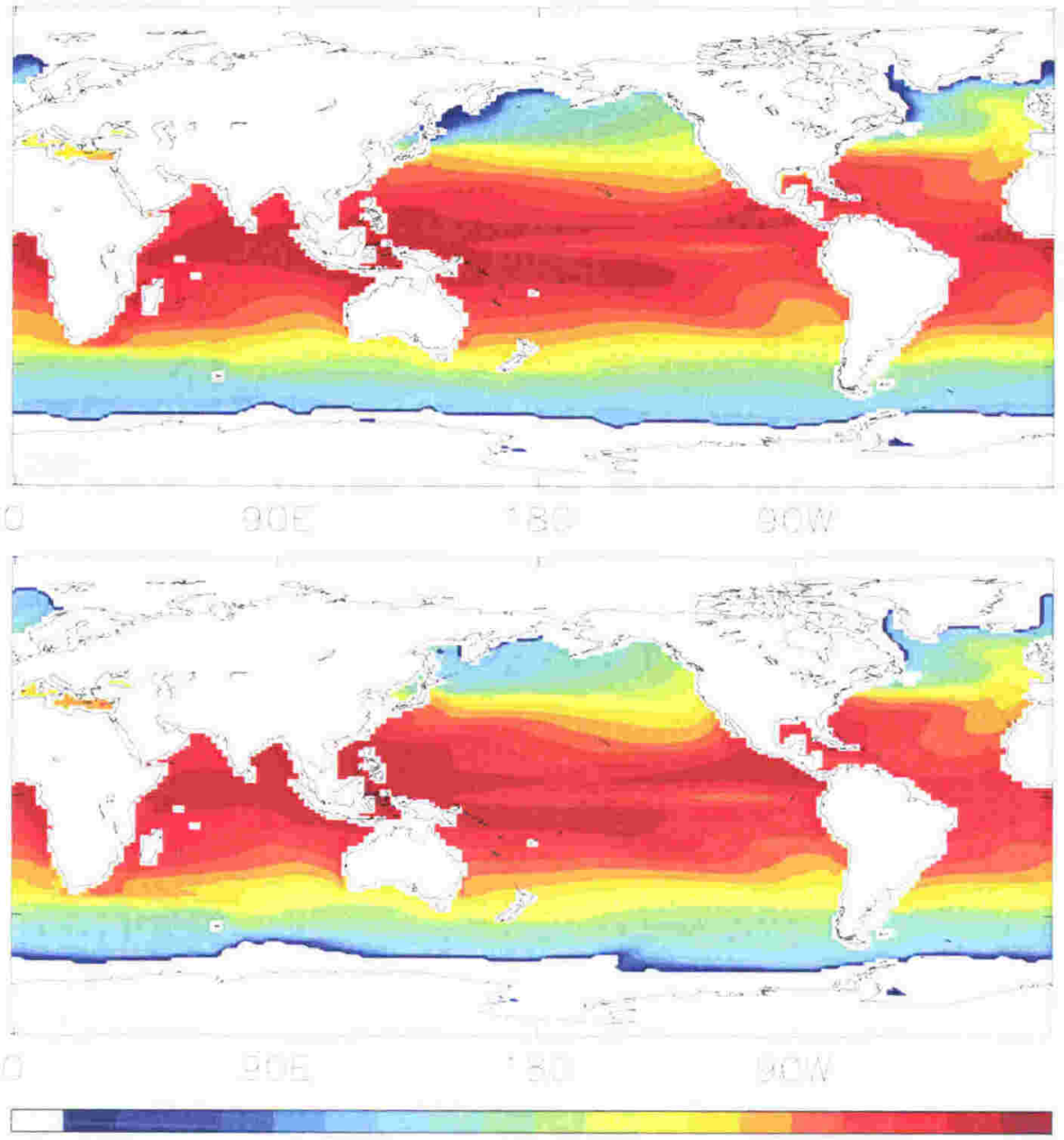

Fig. 7-4. Seasonal averaged SSTs (in K) for DJF and JJA during the LGM. Areas marked in grey are covered for $50 \%$ or more by sea-ice.

\section{- Vegetation and soil:}

The different vegetation distribution during the LGM most likely affected the climate regionally (e.g. Crowley and Baum, 1997). Although quite a lot is known about the vegetation during the LGM, there is no global vegetation dataset available for the LGM that can easily be implemented in the UM. For that reason, UM simulations of the LGM have often used the vegetation distribution of the present day, and have been given either latitude-dependent default values, or have used a simple interpolation technique to attribute vegetation values to new landpoints. The vegetation in this LGM simulation was set up in a similar 
way. New landpoints polewards of $55^{\circ}$ were set to land ice and new landpoints between latitudes $55^{\circ}$ and $25^{\circ}$ were set to "temperate rough grazing". Since new landpoints in the tropics are mainly found in South-East Asia, those new landpoints that are north of the equator were set to tropical rainforest, and those south of the equator were set to tropical savannah (Hope et al., 2004) ${ }^{1}$. The soil properties were set according the same criteria. Overriding these criteria is the position of the ice sheets. All landpoints that were covered by the ice sheets were set to the appropriate value indicating that they were covered in ice. This resulted in unrealistic situations in some places with lush vegetation bordering a kilometre high ice sheet.

\section{- Snow:}

Nothing is known about the snow distribution during the LGM. As a default, the snow amount was set at $50 \mathrm{~m}$ over the permanent ice sheets in the initial conditions in HadCM3. The surface albedo and the height of the ice sheets are fixed, but the snow depth varies in time. Hewitt (2000) discussed the effect of the snow cover on the ice sheet mass balance. Since there is no better snow data available for the LGM simulation in this study, the snow amount, as calculated in the HadCM3 LGM simulation, was set in the initial conditions.

\section{- Chemistry:}

The concentration of atmospheric $\mathrm{CO}_{2}$ was reduced to $200 \mathrm{ppm}$, and concentrations of $\mathrm{CH}_{4}$ and $\mathrm{N}_{2} \mathrm{O}$ were kept at the same levels as in the preindustrial simulation. As in the pre-industrial simulation, the sulphur cycle was excluded. Since there is no data on the ozone distribution and concentration of ozone during the LGM, it was assumed that they were the same as during the pre-industrial. It is understood that the higher levels of aerosols and dust particles during the LGM could have had a measurable affect on the climate of the LGM (e.g. Harvey, 1988), but no attempt has been made to incorporate the glacial

\footnotetext{
${ }^{1}$ This paper describes the vegetation distribution of the whole Austral-Asian region during the LGM. But since the model's global vegetation distribution was not altered for the LGM simulation, only the paper's suggestions of the vegetation cover over the new landpoints were put in the model.
} 
aerosol distribution. There is simply not sufficient data available on the amount and characteristics of the aerosols during the LGM to correctly implement them in the model.

\subsection{Results}

The model's reconstruction of the climate during the LGM is discussed by analysing several atmospheric and oceanic fields. The simulated climate of the LGM is often compared with the pre-industrial climate (chapter 5). Apart from non-linear responses, systematic biases in the model can be filtered out by comparing the LGM output with the pre-industrial output. The LGM simulation is in general not investigated by comparing the climatic findings with geological proxy data. Although, if the simulated climate supports the reconstructions of the LGM based on geological proxy data, it would raise our confidence in our findings.

\subsubsection{Sea Surface Temperature and Sea Ice}

Fig. 7-5 shows the difference in sea-ice cover between the LGM and the preindustrial simulation. For the Northern Hemisphere winter, the main increase in sea-ice takes place in the Norwegian Sea, along the Fennoscandian ice sheet, and the sea of Okhotsk. There are no large differences in sea-ice extent between the pre-industrial simulation and the LGM during the boreal summer months. Seaice in the Southern Hemisphere shows a similar trend. During the austral winter, the sea-ice in the LGM expands much more equatorward then during the preindustrial, especially along the West Antarctic ice sheet. Although the sea-ice extent in the LGM is much more equatorward than during the pre-industrial in summer as well, the difference in the amount of sea-ice is less than during the winter. Since these areas correspond to a low amount of sea-ice in the preindustrial as well, it indicates that icebergs were more common during summer months in the LGM. 


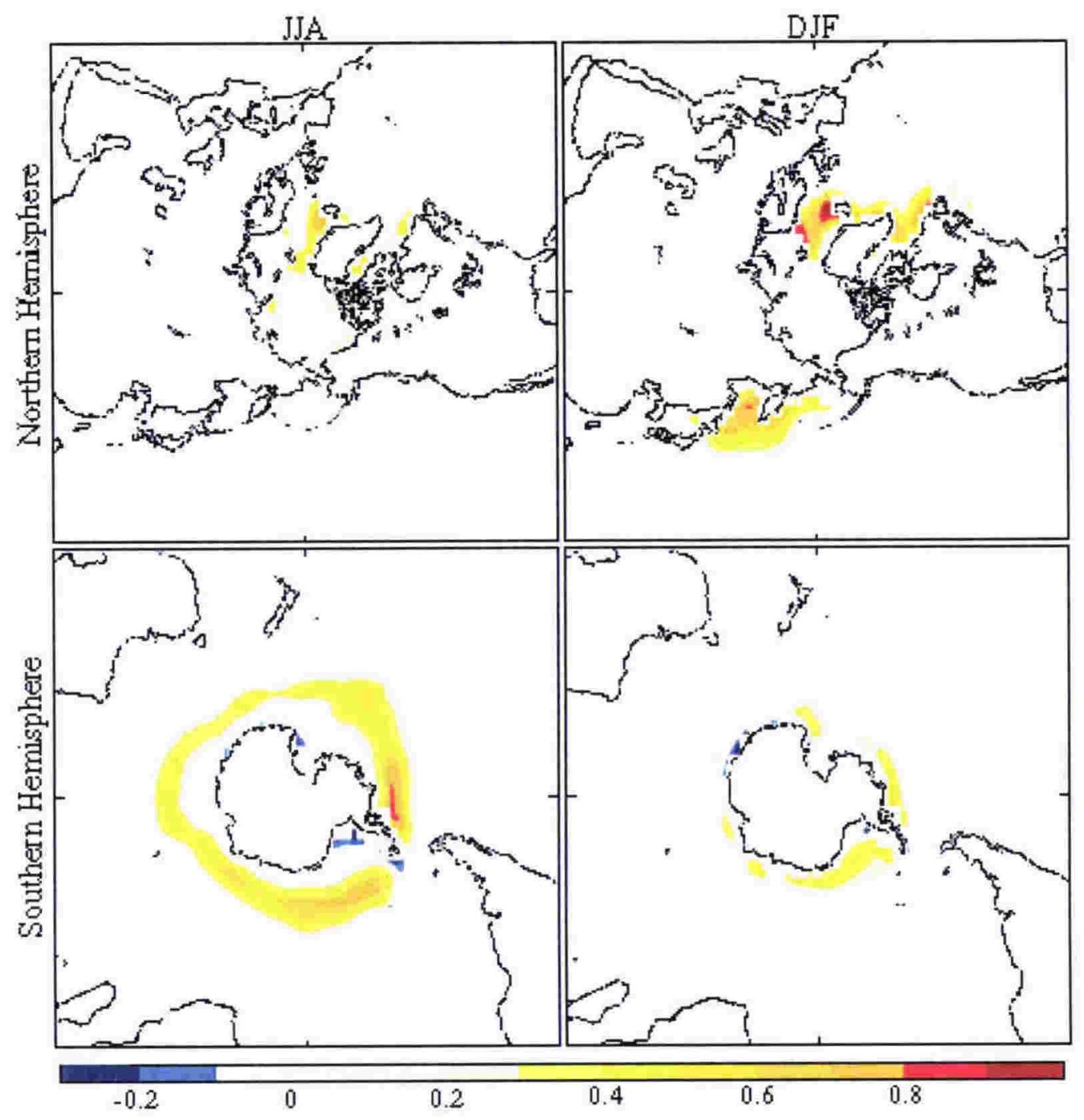

Fig. 7-5. The difference in sea-ice extent between the LGM and the pre-industrial, LGM $_{\text {sea-ice- }}$ pre-industrial $l_{\text {sea-ice }}$ for the Northern (top) and Southern Hemisphere (bottom) for JJA (left) and DJF (right). Units refer to the extent of sea-ice cover within the grid box: 0 is no sea-ice, \pm 1 is fully covered by sea-ice.

Analysis of the SSTs was done by Hewitt (2000), Hewitt et al. (2001), and Hewitt et al. (2002). Only a short summary of their findings is given here.

Fig. 7-6 shows the difference in SST between the pre-industrial and the LGM simulation. As mentioned before, the most striking change in the LGM SSTs, as computed by HadCM3, takes place in the North Atlantic. The meridional overturning cell in the North Atlantic weakens at high latitudes and shifts 

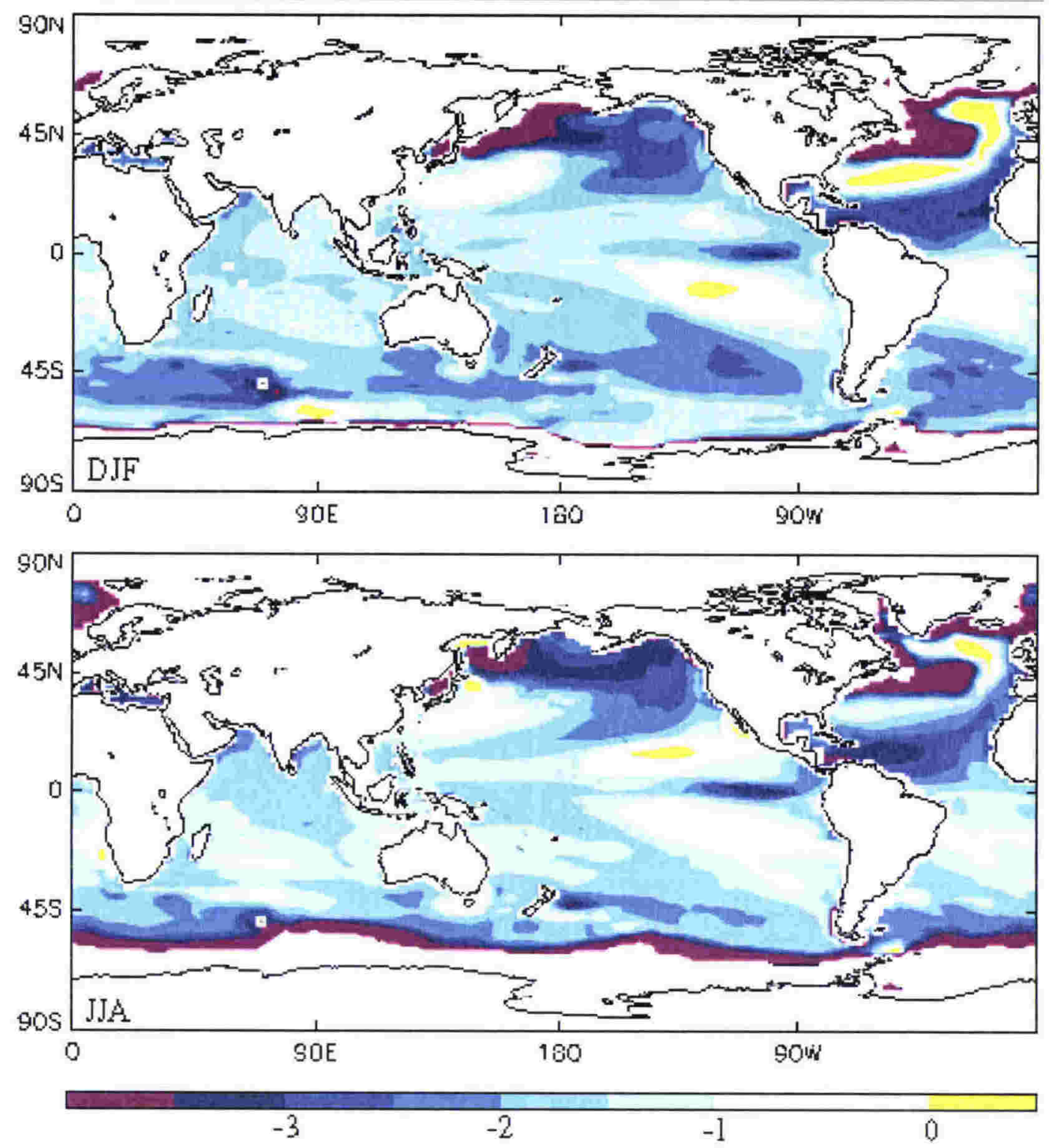

Fig. 7-6. The difference between the LGM and pre-industrial SSTs (in ${ }^{\circ} \mathrm{C}$ ), $\mathrm{LGM}_{\mathrm{SST}}$ - preindustrial $_{\mathrm{SST}}$. Areas marked in grey are covered for $50 \%$ or more in sea-ice in the LGM dataset.

southwards. This is largely the result of the expansion of the Arctic sea-ice (Hewitt, 2000).

The effect of the expansion of the sea-ice is that the Gulfstream is pushed southwards, and is replaced by the cold Labrador Current. The more southerly flow of the Gulfstream in the reconstruction of the LGM results in warmer SSTS in the midlatitudes of the Atlantic and the northerly intrusion of the Labrador Current gives the strong cooling north of these latitudes (Hewitt et al., 2002). At the same time, HadCM3 produces an increase in the strength of the maximum 
overturning, resulting in a more vigorous NATC. The stronger NATC transports more heat northwards along the coast of Western Europe, resulting in the 'warming' in the Labrador Sea. As discussed in chapter 3, a change in the NATC is one of the leading contenders to initiate and/or support a glacial climate. Several possible scenarios for a change in the NATC were discussed and a southwards shift and a reduced NATC were likely options. HadCM3's reconstruction of the LGM seems to agree with a southward movement of the NATC, but disagrees with a weakening of the overturning circulation. However, Kitoh et al. (2001) also found a stronger meridional overturning in the North Atlantic during the LGM in their coupled model.

Hewitt et al. (2002) described some of their findings as model-dependent, since they showed up the simulation with in a coupled model, but not in their simulation with a slab model for the ocean. The coupled model transported heat by the oceans, which a slab model could not. The different ways heat was transported in the models resulted in different responses to the strong atmospheric cooling. The effect of heat transport by the oceans was also an issue when looking at the overall cooling of the Northern and Southern Hemisphere. Hewitt et al. (2002) described that the coupled model transported more heat northwards than a slab model, effectively reducing the cooling of the Northern Hemisphere. Consequently, since more heat was transported northwards, the Southern Hemisphere cooled more in the coupled model.

HadCM3's LGM reconstruction showed an amount of cooling of up to $4^{\circ} \mathrm{C}$ in the Eastern Pacific equatorial waters compared to the pre-industrial simulation. This was described by Hewitt et al. (2002) as the result of the shoaling of the thermocline between $110^{\circ} \mathrm{W}$ and $130^{\circ} \mathrm{W}$ and intensified upwelling in the Eastern Pacific which in turn was the result of a stronger easterly component of the trade winds. The Western Pacific showed a cooling of up to $2^{\circ} \mathrm{C}$. The amount of cooling of the Pacific SSTs in the simulation was therefore larger than described by CLIMAP $(1976 ; 1981)$ but not as large as some other studies have indicated (e.g. Bush and Philander, 1998). Crowley (2000) argued that the amount of cooling in the tropical Pacific SSTs was probably somewhere in between. Furthermore, CLIMAP suggested that the temperature gradient between the 


\begin{tabular}{|r|r|r|r|r|}
\hline & DJF & MAM & JJA & SON \\
\hline \hline NCEP & 5.09 & 2.81 & 1.84 & 3.93 \\
\hline \hline HadAM3H $_{\text {pre-industrial }}$ & 5.74 & 2.56 & 2.58 & 5.01 \\
\hline \hline${\text { HadAM } 3 \mathrm{H}_{\text {LGM }}}^{\prime}$ & 4.59 & 1.34 & 0.78 & 5.49 \\
\hline
\end{tabular}

Table 7-2. Southern Oscillation Index computed from seasonally averaged, nonnormalised, mean sea level pressure difference between Tahiti and Darwin. For comparison, the SOI for NCEP and HadAM3 $\mathrm{H}_{\text {pre-industrial }}$ from Table 5-1 are presented again.

Western and Eastern Pacific was reduced, resulting in a reduction of the trade winds. However, HadCM3's simulation showed an increase in the gradient, very similar as was found by Weaver et al. (1998) and Liu et al. (2000). An increase in the zonal temperature gradient across the tropical Pacific would result in stronger trade winds, which would in turn lead to a stronger Hadley circulation. The ocean in HadCM3 showed an increase in the thermocline slope (Hewitt, 2000), which is unlike CLIMAP which indicated a decrease in the slope of the thermocline. A steepening of the thermocline during the LGM was also found in other studies (e.g. Bush and Philander, 1998: Andreasen et al., 2001).

The increase of the temperature gradient across the tropical Pacific could suggest that the climate characteristics of the LGM were more similar to those of the present day in a La Niña phase. However the SOI shows quite the opposite with the climate of the LGM showing a strong El Niño phase during MAM and JJA, and fairly neutral ENSO conditions during SON and DJF (Table 7-2). Yin and Battisti (2001) found that in understanding past climates, it is more important to correctly model the tropical SST patterns than the absolute temperature difference between the past and present climate. Fig. 7-7 shows the standard deviation in the mean annual SSTs during the LGM. When comparing this figure to the standard deviation plots of the mean annual SSTs in the NCEP climatology and in the pre-industrial simulation (Fig. 5-3), it is clear that the modelled LGM shows stronger interannual variability than those periods. An enhanced ENSO signal was also found in a LGM modelling study done by An et al. (2004).

One can pose the question whether climates with a stronger temperature 


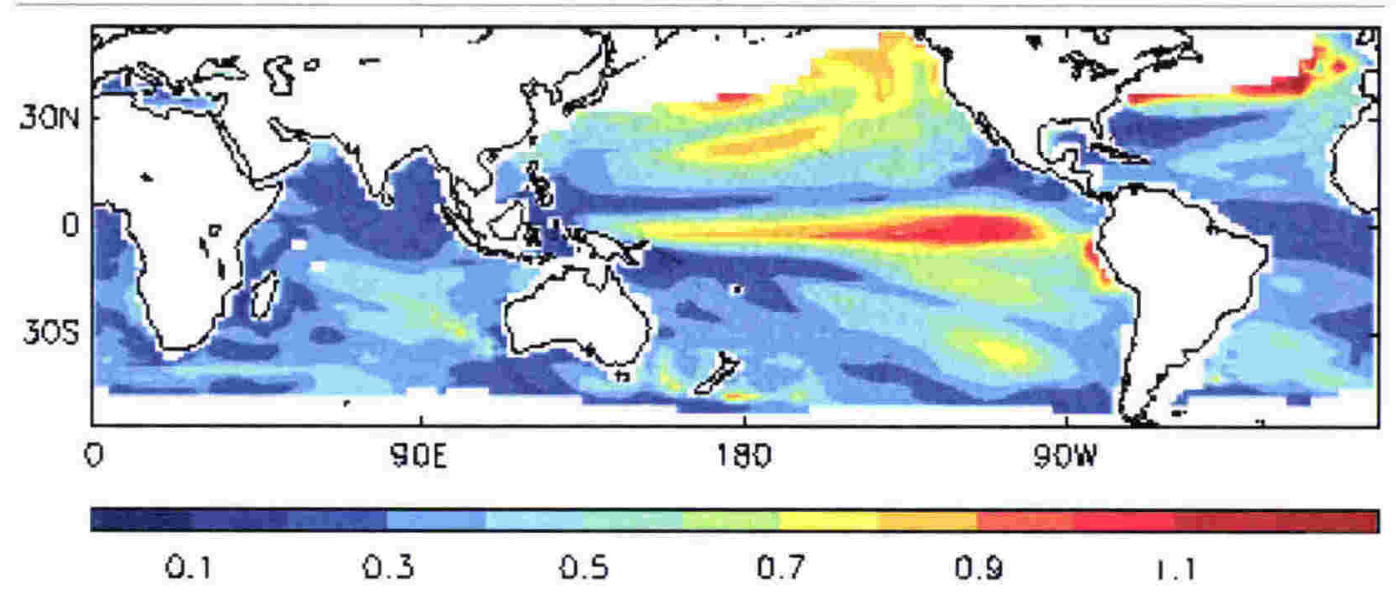

Fig. 7-7. Standard deviation of the mean annual SSTs (in ${ }^{\circ} \mathrm{C}$ ) in the LGM. The additional landmasses of the LGM and the ocean areas that at any time are covered in sea-ice are coloured white.

gradient across the tropical Pacific, but with the mean climatology similar to the present day, experience stronger ENSOs in general. A larger zonal temperature gradient across the Pacific requires a stronger driving force. A weakening of this force can result in a relaxation of the temperature gradient that is larger than in a temperature gradient under ENSO neutral condition in the present day. This would give rise to a stronger ENSO. Before drawing any conclusions on such a possibility in this simulation, it should be investigated whether the enhanced variability in the Pacific SSTs is inherent to the model when run under for LGM conditions. This thought has not been followed up in this thesis, but maybe this hypothesis could partly explain some geological proxy data indicating a La Niñastyle climate, where as other proxy data indicate an El Niño-style climate during the LGM. Since the variability in Pacific SSTs is introduced into HadAM3H through the use of HadCM3's SSTs, it is important to keep in mind the model's dependency on these boundary conditions when comparing the LGM and preindustrial experiments. Note that similar to the pre-industrial simulation, the largest variability of the tropical SSTs during the LGM can be found at $100^{\circ} \mathrm{W}$ $140^{\circ} \mathrm{W}$, where as the present day climate (NCEP climatology) has its largest variability in the Eastern Pacific (see Fig. 5-3). Section 7.2 .8 will analyse in more detail the consequences of the difference in SST variance in the tropical Pacific between the pre-industrial and LGM data. 
Also outside the scope of this thesis but worthwhile to investigate, are the tropical SST patterns during the LGM as modelled by various GCMs. Clement et al. (2001) suggested that colder climates, and then in particular the cooler period of the Younger Dryas, could partially have been initiated by a persistent La Niñalike SST change. However, if the differential cooling between the Western and Eastern Pacific during the LGM as modelled by HadCM3 is reconstructed by other models as well, it would seem that a possible La Niña-style climate during the LGM follows from the LGM conditions and that a La Niña-like SST distribution was not the trigger of the LGM.

The large amount of cooling in the North Pacific is not investigated in this study. Hewitt (2000) noted that most of the Northern Pacific is devoid of glacial climate data, and presents only a few comparisons with geological data. The interannual variability in the Northern Pacific could be the result of a combination of the melting of sea-ice together with a variation in the position of the storm track, and therefore the position of preferred maximum cyclonicity. Although no high frequency data was available, section 7.2 .4 will raise the latter again in the context of interannual variability.

There are no particular dramatic features in the modelled SSTs in the Southern Hemisphere during the LGM. The amount of cooling is consistent with the findings of PMIP using computed SSTs (e.g. Dong and Valdes, 1998: World Climate Research Programme-111, 2000) and are therefore quite a lot warmer than the SSTs as proposed by CLIMAP, especially during the austral winter months. Compared to the pre-industrial simulation, the largest amount of cooling takes place around the edge of the equatorward extended area of sea-ice, as one would expect. Of interest in this study is the maximum in cooling to the east of New Zealand, and the occurrence of high variability in the Tasman Sea and over the Chatham Rise. The subtropical Pacific gyre changed as the result of the strengthened westerly flow, the closure of the Bering Strait and the near closure of the Indonesian throughflow. The change in the subtropical gyre affected the East Australian Current and the Tasman Current. Chapter 8 will discuss in more detail the possible implications of this change to the climate of New Zealand during the LGM. 


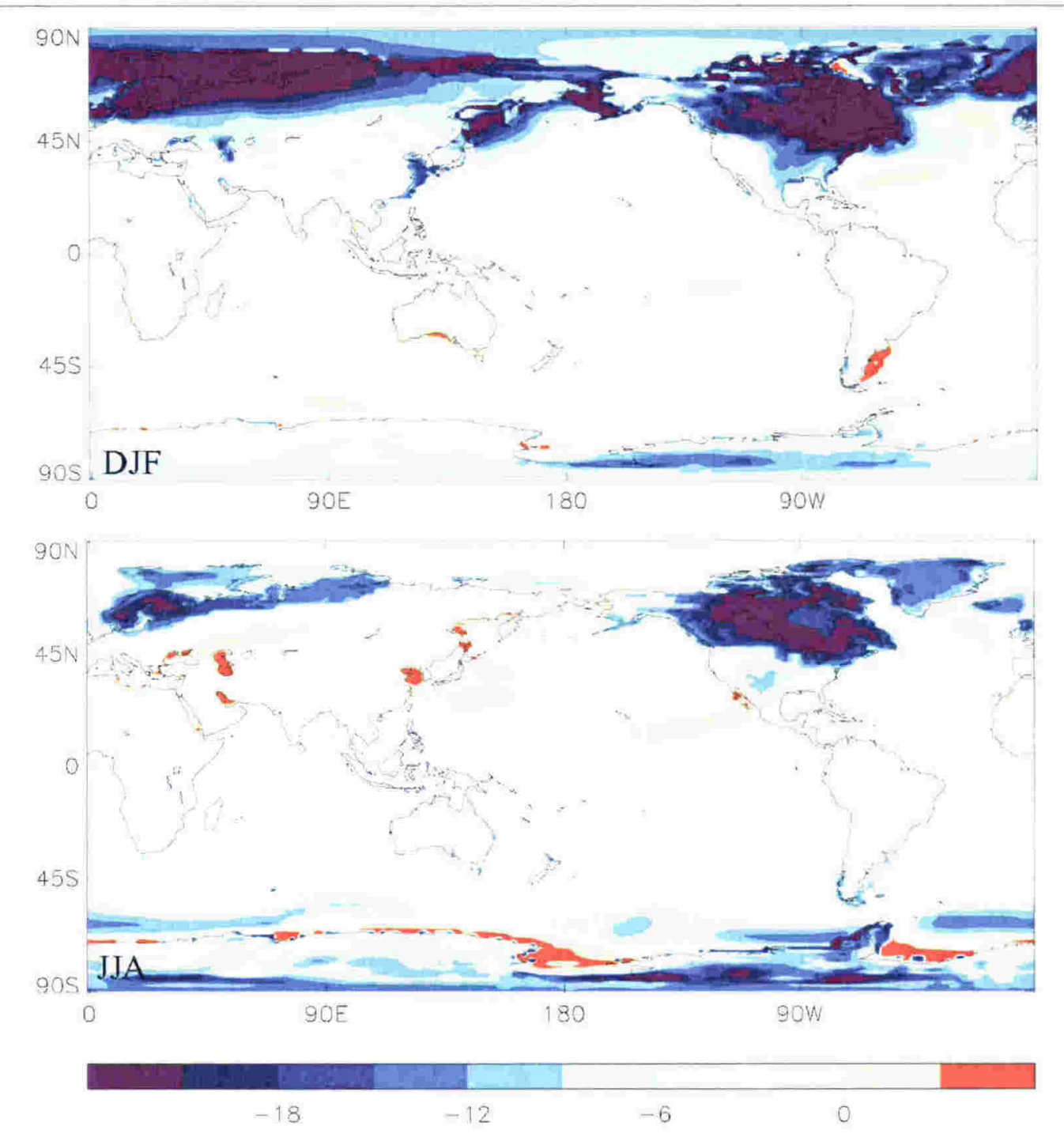

Fig. 7-8. The difference in surface temperature (in ${ }^{\circ} \mathrm{C}$ ) between the LGM and the preindustrial simulation for DJF and JJA.

\subsubsection{Temperature}

The differences between the LGM and pre-industrial surface temperature for DJF and JJA are presented in Fig. 7-8. The LGM climate is colder than the preindustrial climate nearly everywhere. The coastal areas that indicate warming are the result of those points being classified differently for the LGM and preindustrial simulation. Those grid points were ocean points in the pre-industrial simulation and land points during the LGM and so effectively surface temperature is compared against SST. The largest cooling takes place at the high 



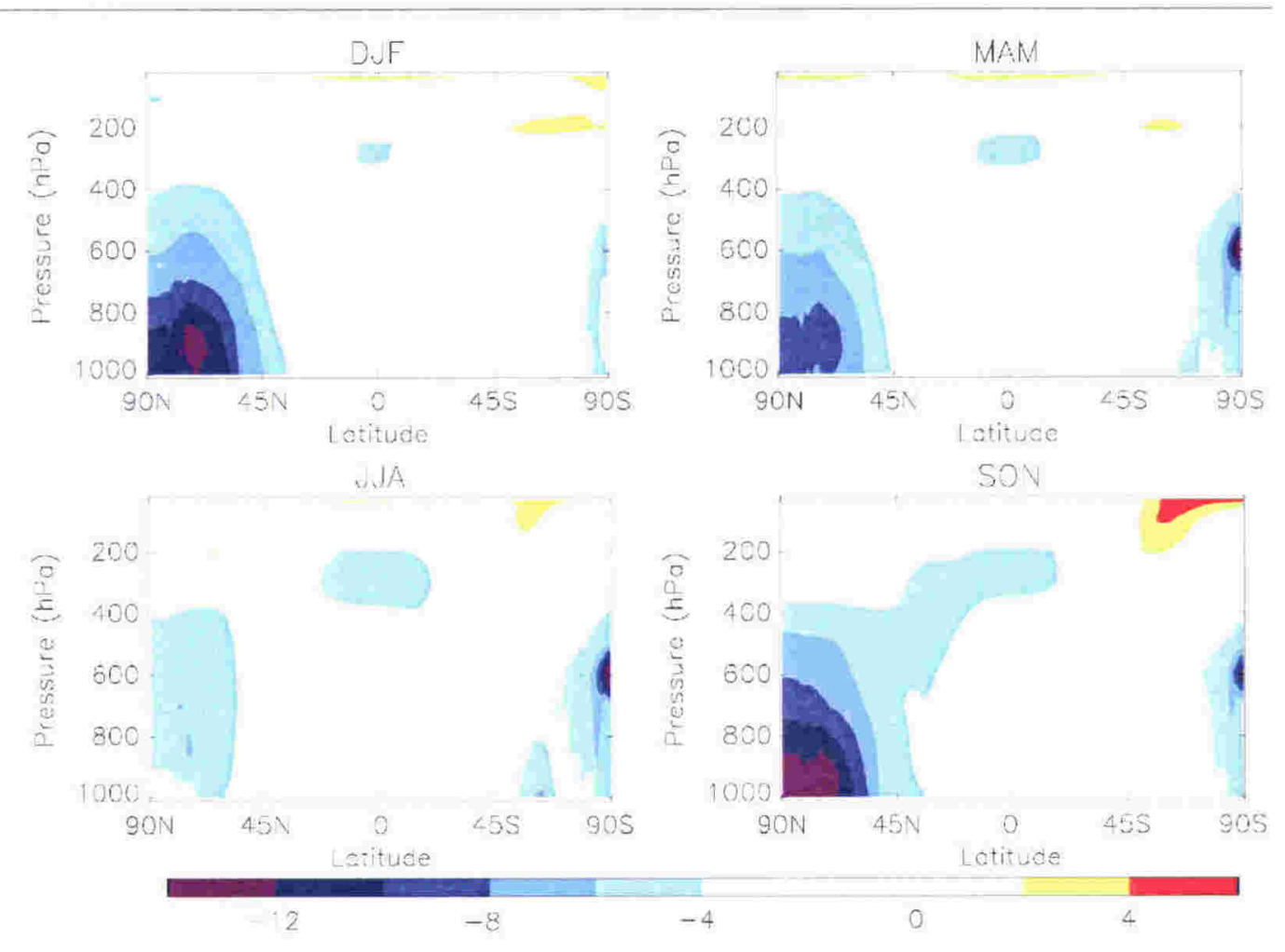

Fig. 7-9. Vertical cross sections of the mean zonal temperature difference $\left(\right.$ in ${ }^{\circ} \mathrm{C}$ ) between the LGM and the pre-industrial simulation.

bottom of the stratosphere of the LGM simulation. When comparing the LGM atmosphere with the pre-industrial atmosphere up to $300 \mathrm{hPa}$, the seasonal amount of cooling was between $3.4^{\circ} \mathrm{C}-4.0^{\circ} \mathrm{C}$ (Table 7-4). Rind et al. (2001) found that the lower stratosphere was warmer during the LGM than in today's climate which they found was mainly the result of the lower concentration of $\mathrm{CO}_{2}$ in the stratosphere during the LGM. The lower concentration of $\mathrm{CO}_{2}$ explains the higher temperatures at high altitudes in Fig. 7-9.

Areas that experience a maximum cooling in the surface temperature also experience strong cooling in the vertical with the large continental Northern Hemisphere ice sheets in particular having a large impact on the amount of cooling. The substantial increase in the meridional temperature gradient in the Northern Hemisphere during the LGM had major consequences for its mean zonal circulation. An increase in meridional temperature gradient is associated with an increase in the strength of the mean westerly flow. The cooling at high latitudes in the Southern Hemisphere is mainly restricted to Antarctica. The 


\begin{tabular}{||c|c|c|}
\hline & Full atmosphere & Atmosphere up to 300hPa \\
\hline DJF & -2.3 & -3.5 \\
\hline \hline MAM & -2.1 & -3.5 \\
\hline \hline JJA & -2.1 & -3.4 \\
\hline \hline SON & -2.5 & -4.0 \\
\hline \hline Annual & -2.3 & -3.6 \\
\hline
\end{tabular}

Table 7-4. The difference in the vertically averaged temperature (in ${ }^{\circ} \mathrm{C}$ ) between the LGM and pre-industrial for the full atmosphere and for the atmosphere up to $300 \mathrm{hPa}$.

meridional temperature gradient in the Southern Hemisphere also increases, but the increase is of a much smaller magnitude than in the Northern Hemisphere.

Of interest are the "corridors" of minimum cooling that can be seen in Fig. 7-9. These areas show up clearly particularly during MAM in the Northern Hemisphere and during SON in the Southern Hemisphere. These "corridors" correspond with areas of maximum meridional heat flux, where heat is transported upward and poleward by the planetary waves. It would seem that it this transport of heat along these "corridors" that slightly offsets the general cooling of the atmosphere in those regions. A different perspective on how the vertical temperature differs during the LGM from the pre-industrial simulation is presented in Fig. 7-10. This figure shows the vertical temperature gradient, or lapse rate, for different zonal bands for the pre-industrial period and the LGM for the seasons of DJF and JJA. The data for these graphs are seasonally averaged temperatures for each zonal band and for each layer in the vertical. Similar as in figure Fig. 7-9, the tropospheric temperatures are lower during the LGM and the largest differences with the pre-industrial simulation are found nearer to the surface and further away from the tropics.

The Northern Hemisphere polar region $(90-75 \mathrm{~N}$ in Fig. $7-10)$ shows the inversion layer at the surface during its winter months, indicating a stable climate at the surface. The effect of the thick continental ice sheets on the Northern Hemisphere higher latitudes (75-60N) during the LGM is to extend the height of this inversion layer, and reduce the vertical temperature gradient directly above it. There is a decrease in the lapse rate with height at the tropopause at the higher 

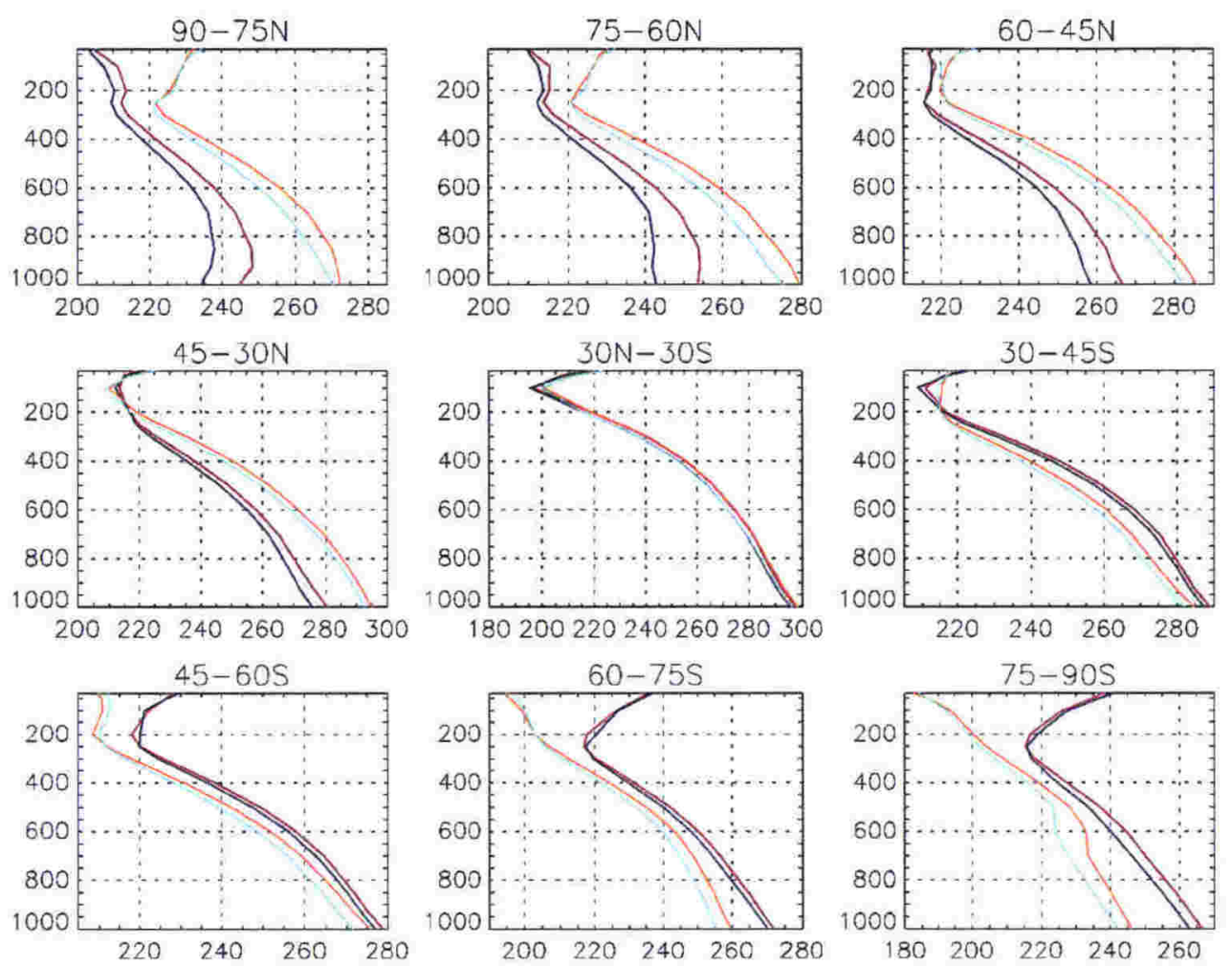

Fig. 7-10. Lapse rate for 9 different zonal bands for the pre-industrial and LGM simulation for DJF and JJA (DJF pre-industral $=$ dark red, $D J F_{L G M}=$ dark blue, JJA $A_{\text {pre-industrial }}=$ orange, $\mathrm{JJA}_{\mathrm{LGM}}=\mathrm{cyan}$. The horizontal scales vary and are chosen so that the differences between the two simulations and the seasonal variation in that particular zonal band are easy to analyse.

latitudes during DJF and there is a reversal of the vertical temperature gradient during JJA resulting in a warming of the lower stratosphere. The height of the tropopause migrates upward when moving from pole to equator (roughly $300 \mathrm{hPa}$ at the pole, $250 \mathrm{hPa}$ at $75-60 \mathrm{~N}, 200 \mathrm{hPa}$ at $60-45 \mathrm{~N}$ and $100 \mathrm{hPa}$ at $45-30 \mathrm{~N})$. As indicated earlier (Fig. 7-9), the tropopause is slightly lower during the LGM than during the pre-industrial period, especially during JJA. This can be seen in Fig. $7-10$ as the points where the LGM's lapse rate crosses the pre-industrial lapse rate.

The LGM and pre-industrial vertical temperature profile for the Southern Hemisphere show the same trends as in the Northern Hemisphere, albeit that the differences between the two simulations is less pronounced and there is the obvious reversal of the seasonal trends. The larger cooling during JJA and stronger seasonality in temperature at high altitudes at the Southern 
Hemisphere's high latitudes are the result of its weaker planetary waves. The planetary waves in the Southern Hemisphere transport less heat upwards and polewards than their counterparts in the Northern Hemisphere. This effectively makes it hard to determine whether the lapse rate had changed at the tropopause at the high latitudes during JJA. On the other hand, the lowering of the tropopause during the LGM, especially during DJF, is easier to recognise, since the vertical temperature profile of the LGM clearly crosses the vertical temperature profile of the pre-industrial simulation. From Fig. 7-9 it was already clear that the lowering of the tropopause was more obvious in the high latitudes of the Southern Hemisphere.

Of note is what happens at the Southern Hemisphere polar latitudes (i.e. Antarctica). The lapse rate shown is an average over a zonal band, and it does not distinguish between areas of different elevations. At certain altitudes, the lapse rate could very well show an inversion similar to the Northern Hemisphere polar region. However, this was not investigated in this study. The lapse rate is isothermal at about $700 \mathrm{hPa}$ during JJA in the pre-industrial simulation and this altitude corresponds with the mean height of the Antarctic continent. The lapse rate below $700 \mathrm{hPa}$ is therefore of no interest. When comparing the pre-industrial lapse rate with the LGM lapse rate during JJA in this region, it is clear that due to the colder surface temperatures during the LGM, the isothermal part of the lapse rate (and maybe even an inversion) has shifted upward (at $500-600 \mathrm{hPa}$ ). This increase in height of a relatively warmer layer will affect the circulation at those heights differently during the LGM than during the pre-industrial.

\subsubsection{Precipitation}

Due to the colder climate, the saturation water vapour pressure is lower and one would expect the global precipitation to be reduced during the LGM (Table 7-5). A reduction in precipitation occurs over both land and ocean, but there is no change in seasonality. The largest reduction in precipitation takes place in the tropics and in the Northern Hemisphere, and is fairly similar for each season. 


\begin{tabular}{|c|c|c|c|c|c|c|c|c|c|c|c|c|}
\hline & 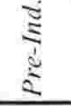 & 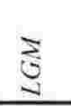 & $\begin{array}{l}\stackrel{\Xi}{\Xi} \\
\vdots\end{array}$ & ड़ & \begin{tabular}{l}
$\vec{\Xi}$ \\
\multirow{2}{1}{} \\
$\Sigma$
\end{tabular} & $\stackrel{\Xi}{G}$ & 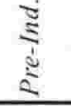 & ज্ & $\begin{array}{l}\stackrel{\Xi}{\Xi} \\
\vdots \\
\Sigma\end{array}$ & ड̇ & 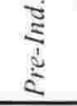 & $\underset{-1}{3}$ \\
\hline DJF & 3.01 & 2.78 & 1.81 & 1.63 & 3.49 & 3.37 & 5.07 & 4.68 & 2.65 & 2.11 & 3.38 & 3.44 \\
\hline MAM & 3.06 & 2.80 & 1.88 & 1.70 & 3.52 & 3.36 & 5.08 & 4.64 & 2.63 & 2.34 & 3.48 & 3.25 \\
\hline JJA & 3.13 & 2.84 & 2.05 & 1.73 & 3.56 & 3.42 & 4.96 & 4.50 & 3.55 & 3.17 & 2.71 & 2.52 \\
\hline SON & 3.03 & 2.76 & 1.82 & 1.62 & 3.51 & 3.34 & 5.08 & 4.78 & 3.53 & 2.93 & 2.54 & 2.59 \\
\hline ANNUAL & 3.06 & 2.80 & 1.89 & 1.67 & 3.52 & 3.37 & 5.05 & 4.65 & 3.09 & 2.64 & 3.03 & 2.95 \\
\hline
\end{tabular}

Table 7-5. Precipitation in $\mathrm{mm}$ /day for the pre-industrial and LGM simulation. The precipitation during the LGM is less for each season and for all areas, except for the Southern Hemisphere (SH) during DJF and SON.
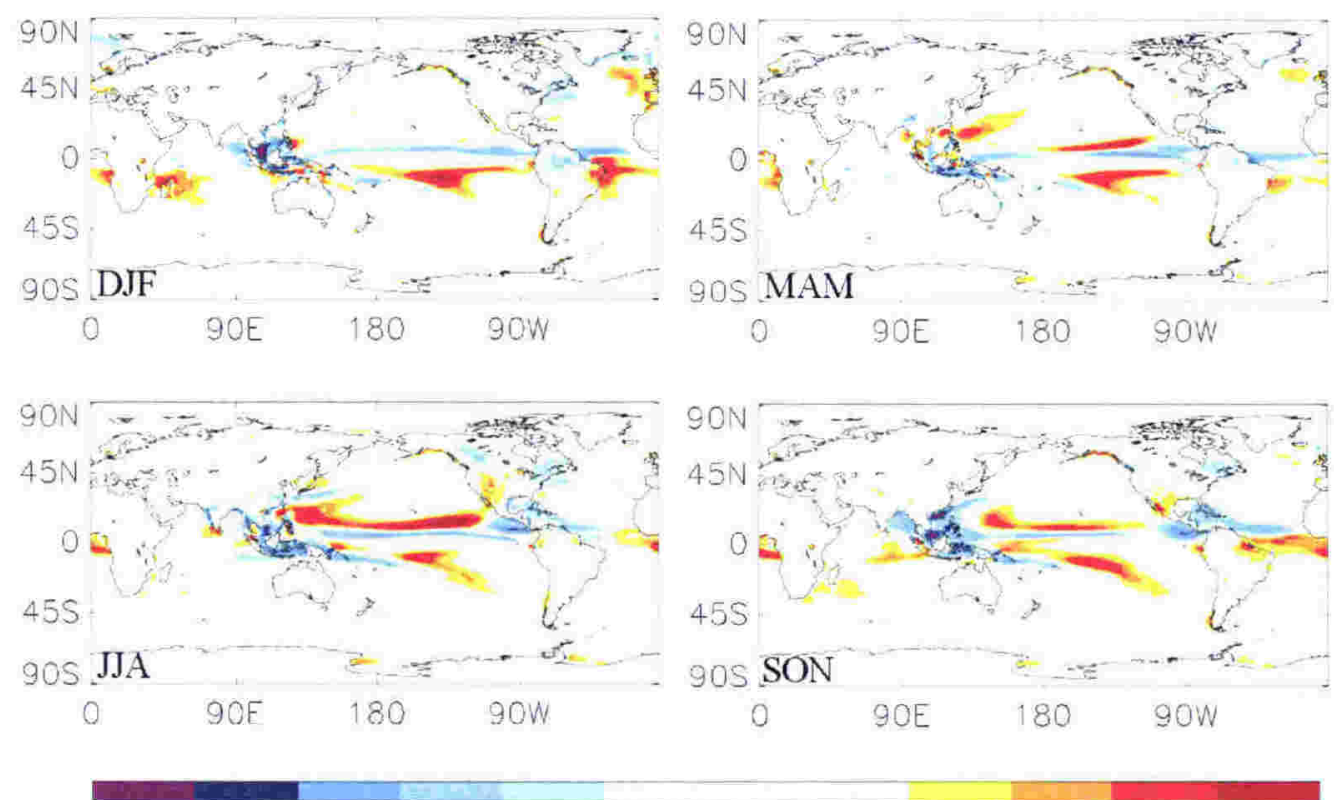

$-12$

$-6$

$-2$

2

6

Fig. 7-11. Difference in seasonal precipitation between the LGM and the pre-industrial simulation (LGM - pre-industrial) in $\mathrm{mm} /$ day.

One intriguing point is that the precipitation in the Southern Hemisphere during SON and DJF has increased slightly.

Fig. 7-11 shows the difference between the LGM and pre-industrial precipitation for each season. The most obvious changes take place in the tropics. There is a major reduction in precipitation over South East Asia during each season. There is less precipitation along the ITCZ, but there is an increase in precipitation just north of the ITCZ, particularly during JJA and SON. This 
would imply that there is not so much a reduction of precipitation along the equatorial Pacific during the LGM, but more a northward shift of the ITCZ. The question arises whether such a pattern is linked to the different distribution of the Western Pacific SSTs in the LGM simulation. That was not investigated in this study. All seasons seem to show an eastward shift of the SPCZ. The eastward shift of the SPCZ during the LGM is consistent with other indicators that the simulated climate of the LGM had a strong presence of El Niño (see Fig. 7-7 and Table 7-2). However, a northward shift of the ITCZ is not consistent with an El Niño phase, and this change might be more related to the initial LGM conditions. This point was not examined any further in this study.

The decrease in precipitation over the Northern Hemisphere takes place mainly over land, and in particular over those areas that are covered by the ice sheets. The Southern Hemisphere experiences mainly a reduction in precipitation south of $50^{\circ} \mathrm{S}$ over the oceans and the Antarctic continent, but shows a slight increase in precipitation in the subtropics and low midlatitudes. Unlike as in the Northern Hemisphere, there are strong seasonal variations, especially of the east coast of both the African and South American continent.

\subsubsection{Mean Sea Level Pressure}

The mean sea level pressure for DJF and JJA during the LGM are presented in Fig. 7-12 (top two plots). When comparing mean sea level pressure between two datasets at different mean sea level heights, it is essential to extrapolate their data onto the same height in order to obtain their absolute differences correctly. In this case, there are three different aspects in finding the common height for the pre-industrial and LGM simulation. During the LGM, the sea level was $120 \mathrm{~m}$ lower than in the pre-industrial simulation, there was more land, and the orography was substantially higher over several regions as the result of the existence of the continental icesheets. There are mathematical techniques that can adjust correctly the LGM mean sea level pressure to the orographic height in the pre-industrial simulation (e.g. a technique that converts pressure levels to sigma levels is presented in Walsh et al., 2000). However, since the uncertainties 


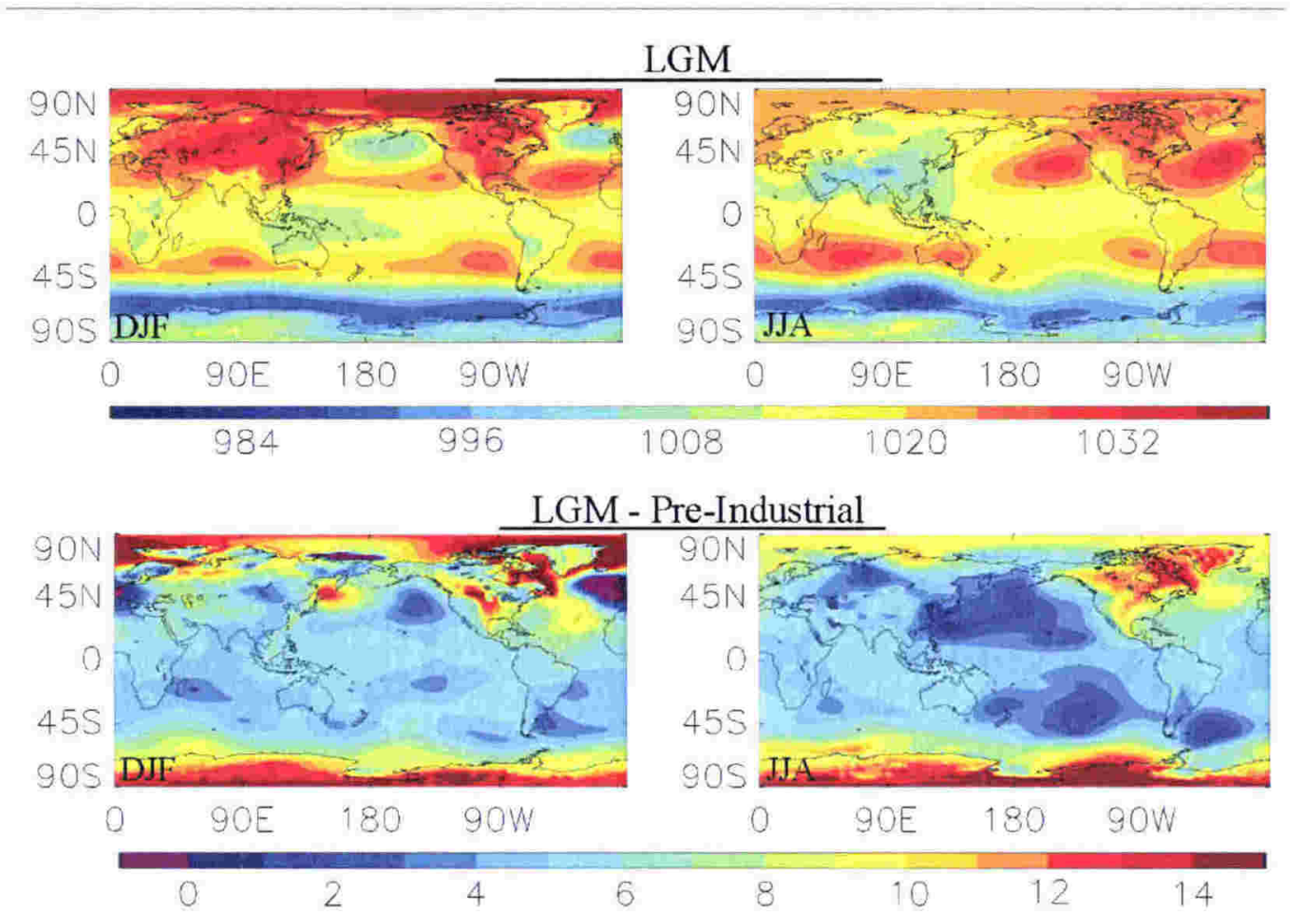

Fig. 7-12. Mean sea level pressure in hPa for the LGM (top) and the difference with the preindustrial mean sea level pressure (MSLP LGM $_{\text {-MSLP }}$ pre-ndustrial, bottom) for DJF (left) and JJA (right).

in the height of the continental icesheets in particular, but also in the height of the sea level during the LGM, could outweigh the differences in the verticallyadjusted mean sea level pressures, it was decided that an absolute comparison of mean sea level pressures between the two simulations was not relevant. However, differences in the spatial patterns of mean sea level pressure (bottom two plots in Fig. 7-12) are less affected by the differences in orography and can indicate differences in wind regimes.

During DJF, the mean sea level pressure difference between the Azores high and the low over the North Atlantic has increased. The net effect of this change would have been an enhancement of the westerly flow over the North Atlantic. In the Pacific, the Aleutian low was relatively deeper, which resulted in a strengthening of the south-westerlies in the region. These features of the mean sea level pressure in the Northern Hemisphere during the LGM were also determined by Dong and Valdes (1998) in their simulation with computed SSTs. 
For the Southern Hemisphere, there is a tendency to an increase in the zonal mean sea level pressure difference between continents and oceans. This is primarily the result of the continents cooling off more than the surrounding oceans The increase in the zonal mean sea level pressure difference between Australia and the Western Pacific is relevant to the climate of New Zealand. The direct consequence of the steeper zonal gradient in mean sea level pressure is that the New Zealand region during the LGM was under the influence of a more dominant south-westerly wind regime, particularly during JJA. This point will return in the chapter to come.

Although the orography of Antarctica in the LGM simulation is the same as in the pre-industrial simulation, there is an apparent differential increase in mean sea level pressure over the continent between the Western and Eastern Hemisphere. This can not simply be explained by the difference in mean sea level heights between the two simulations, and could possibly indicate different changes in wind regimes around the continent. The seasonal variability of mean sea level pressure around Antarctica is only weakly/moderately correlated with the seasonal and spatial increase of sea-ice extent $(0.29,0.39,0.25$ and 0.17 for DJF, MAM, JJA and SON).

Kageyama et al. (1999) investigated the Northern Hemisphere storm tracks during the LGM by looking at the standard deviation of the high frequency (daily) variability of mean sea level pressure. Daily data was not saved in the pre-industrial and LGM simulations, so no comparisons of their stormtracks could be made in this study. The shortest period saved was monthly data, which was the average of 6-hourly data. Interannual variability is discussed in section 7.2.8 and only some differences in winter interannual patterns in mean sea level pressure are presented here.

The standard deviation of the mean sea level pressure for the Northern Hemisphere and Southern Hemisphere winter seasons, as well as their difference with the pre-industrial simulation, are given in Fig. 7-13. Most dramatic changes occur in the Northern Hemisphere. The largest winter (DJF) interannual variability in the Northern Pacific has shifted westward and northwards. There is a strong northward shift of the position of the maximum in winter interannual 

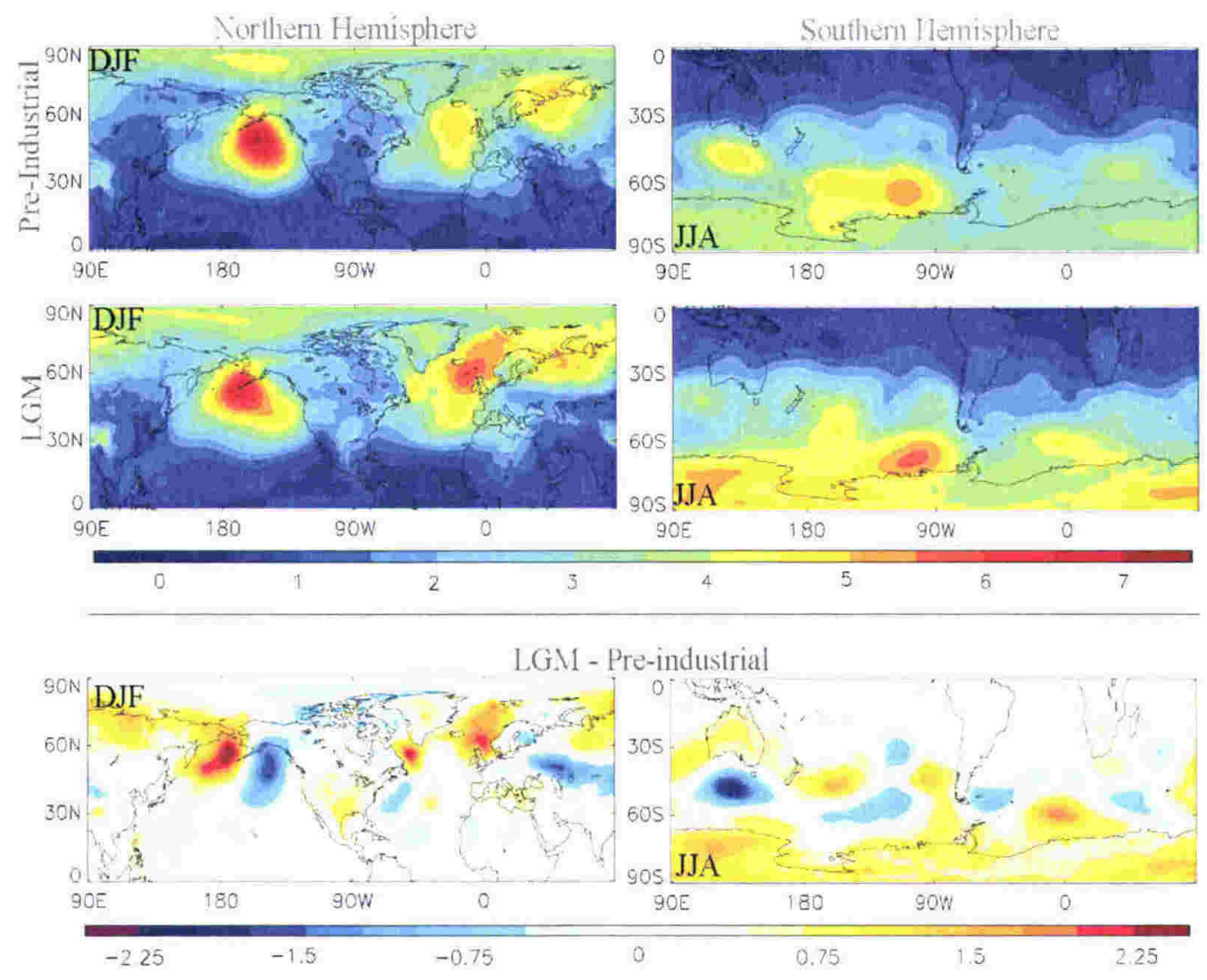

Fig. 7-13. Interannual variability of mean sea level pressure for the Northern (left) and Southern Hemisphere (right) during their winter season for the pre-industrial (top) and LGM simulation (middle) and the differences (bottom) in their patterns (in $\mathrm{hPa}$ ). Note the different scales.

variability in the North Atlantic. Although the amount of variability in the North Pacific remained the same, there is a remarkable increase in the amount of variability in the North Atlantic. This is consistent with the stronger variability in SSTs in this region (refer to Fig. 7-7). It was determined in section 7.2.1 that HadCM3 had computed a strong ENSO signal in the tropical Pacific SSTs. The associated variability would be the dominant variability on the interannual time scale.

The Southern Hemisphere experiences less winter (JJA) interannual variability in mean sea level pressure than the Northern Hemisphere. The position of the maximum in winter interannual variability in the Southern Hemisphere is found in the Eastern Pacific, roughly between $55^{\circ} \mathrm{S}-70^{\circ} \mathrm{S}$ and $90^{\circ} \mathrm{W}-180^{\circ} \mathrm{W}$, from the Ross Sea to the Bellingshausen Sea. The amplitude and 


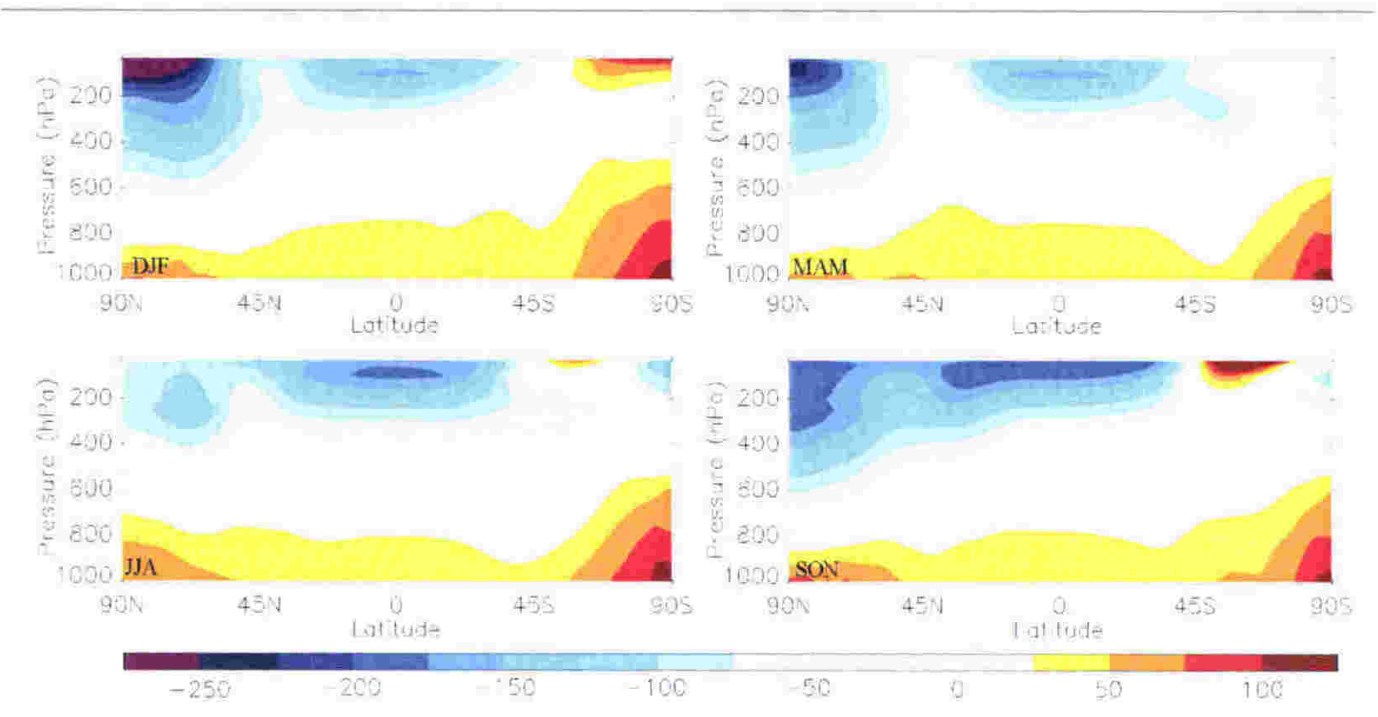

Fig. 7-14. Zonal mean of the differences in geopotential height (in $\mathrm{hPa}$ ) between the LGM and pre-industrial simulation for the four seasons.

position of the maximum does not differ much between the pre-industrial and LGM simulation. The largest differences are found in the midlatitudes. In the zonal mean there is a slight reduction in the winter interannual variability, but the New Zealand region shows an increase during summer and winter, and a reduction during the transitional seasons. Interannual variability in the Southern Hemisphere is further discussed in section 7.2.8.

\subsubsection{Geopotential Height}

The meridional cross sections of the differences in geopotential height between the LGM and pre-industrial simulation are presented for each season in Fig. 7-14. The troposphere is more compact due to the cooler temperatures of the atmosphere in general (Fig. 7-9), and the surface pressure is higher during the LGM $^{1}$. The largest lowering of the geopotential height takes place over the high latitudes in the Northern Hemisphere, where there is a maximum in the amount of cooling as the result of the extended continental ice sheets. The difference in

\footnotetext{
${ }^{1}$ It is important to understand, that due to the lowering of the sea level by $120 \mathrm{~m}$, the surface pressure over sea during the LGM is automatically higher by the equivalent of $120 \mathrm{~m}$ of air, that is, about $14 \mathrm{hPa}$, if all dynamical effects are ignored.
} 
geopotential height between the LGM and the pre-industrial is tilted downwards from the Southern to the Northern Hemisphere as a result of the differential cooling between the Northern and Southern hemisphere. This is in particular the case during DJF and SON. The resulting effect is that the mean zonal westerly flow at high altitudes in the Northern Hemisphere has strengthened more than in the Southern Hemisphere.

The geopotential height at $100 \mathrm{hPa}$ and above is higher at $50^{\circ} \mathrm{S}-70^{\circ} \mathrm{S}$ during most seasons in the LGM than in the pre-industrial simulation. This is consistent with the fact that this area experiences an increase in temperature during the LGM (Fig. 7-9). As indicated earlier, this could be caused both by the lower amount of atmospheric $\mathrm{CO}_{2}$ in the LGM stratosphere as well as a possible deeper penetration of the planetary waves in the stratosphere. The surface maximum in geopotential height difference over Antarctica will result in a decrease in westerly winds at high southern latitudes.

The differences in the $500 \mathrm{hPa}$ height field between the LGM and the preindustrial are quite different for the Northern and Southern Hemisphere (Fig. $7-15)$. The Northern Hemisphere shows a lowering of the $500 \mathrm{hPa}$ height over the pole during the LGM, which is the direct consequence of the much colder surface conditions at the pole. This feature is apparent the whole year round and can be over $150 \mathrm{~m}$ deep, but its position and depth does vary with the seasons. A lower $500 \mathrm{hPa}$ height over the pole increases the meridional gradient, induces cyclonic movement, and therefore strengthens the westerly flow. The zonal winds in the mid and high latitudes during the LGM were therefore stronger than during the pre-industrial. Only over the North Atlantic at $30^{\circ} \mathrm{N}-40^{\circ} \mathrm{N}$ is there a distinctive positive difference between the LGM and pre-industrial $500 \mathrm{hPa}$ height field, which is related to the increased mean sea level pressure in the Atlantic (see Fig. 7-12). The impact of this "high" is to displace the Atlantic storm track northwards.

The characteristics of the geopotential height in the Southern Hemisphere are quite the opposite from the Northern Hemisphere's. There is a positive difference in the $500 \mathrm{hPa}$ height between the LGM and the pre-industrial over Antarctica during all seasons. However, its position and maximum varies through the 

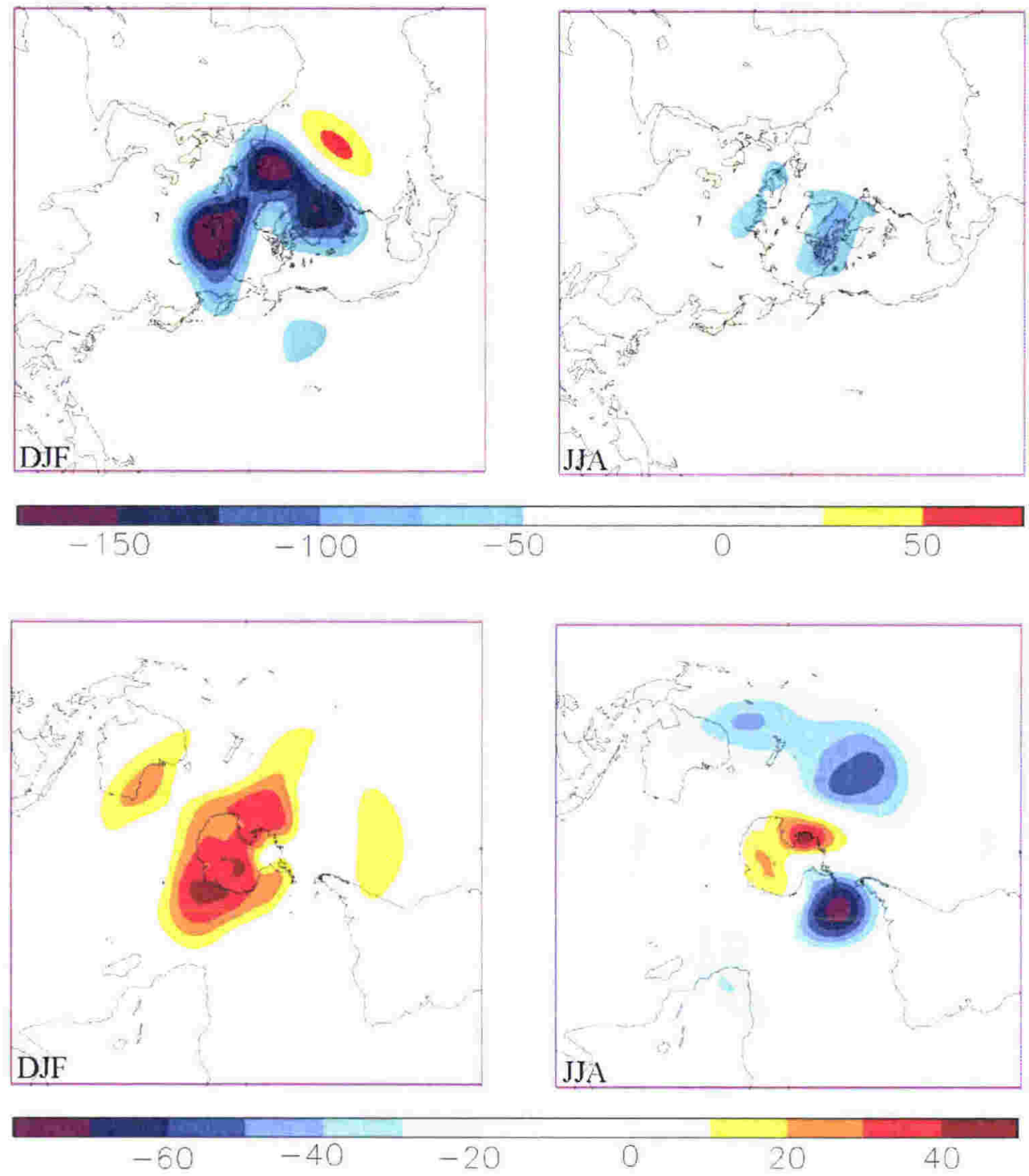

$-40$

$-20$

20

40

Fig. 7-15. Difference in $500 \mathrm{hPa}$ geopotential height (in $\mathrm{m}$ ) between the LGM and preindustrial simulation for the Northern (top) and Southern (bottom) Hemispheres for DJF (left) and JJA (right). Note the different scales.

seasons. The stronger anticyclonic circulation over Antarctica would result in weaker westerly winds at high latitudes. The $500 \mathrm{hPa}$ geopotential height during the LGM is lower over the New Zealand region except during DJF. The position and strength of the associated anticyclonic circulation varies through the seasons. The implied enhanced easterlies from the lower $500 \mathrm{hPa}$ height would result in a decrease of the strength of the westerlies in the New Zealand region. Chapter 8 will discuss in detail the surface wind regimes in New Zealand.

The $500 \mathrm{hPa}$ geopotential height is also analysed for changes in the planetary 

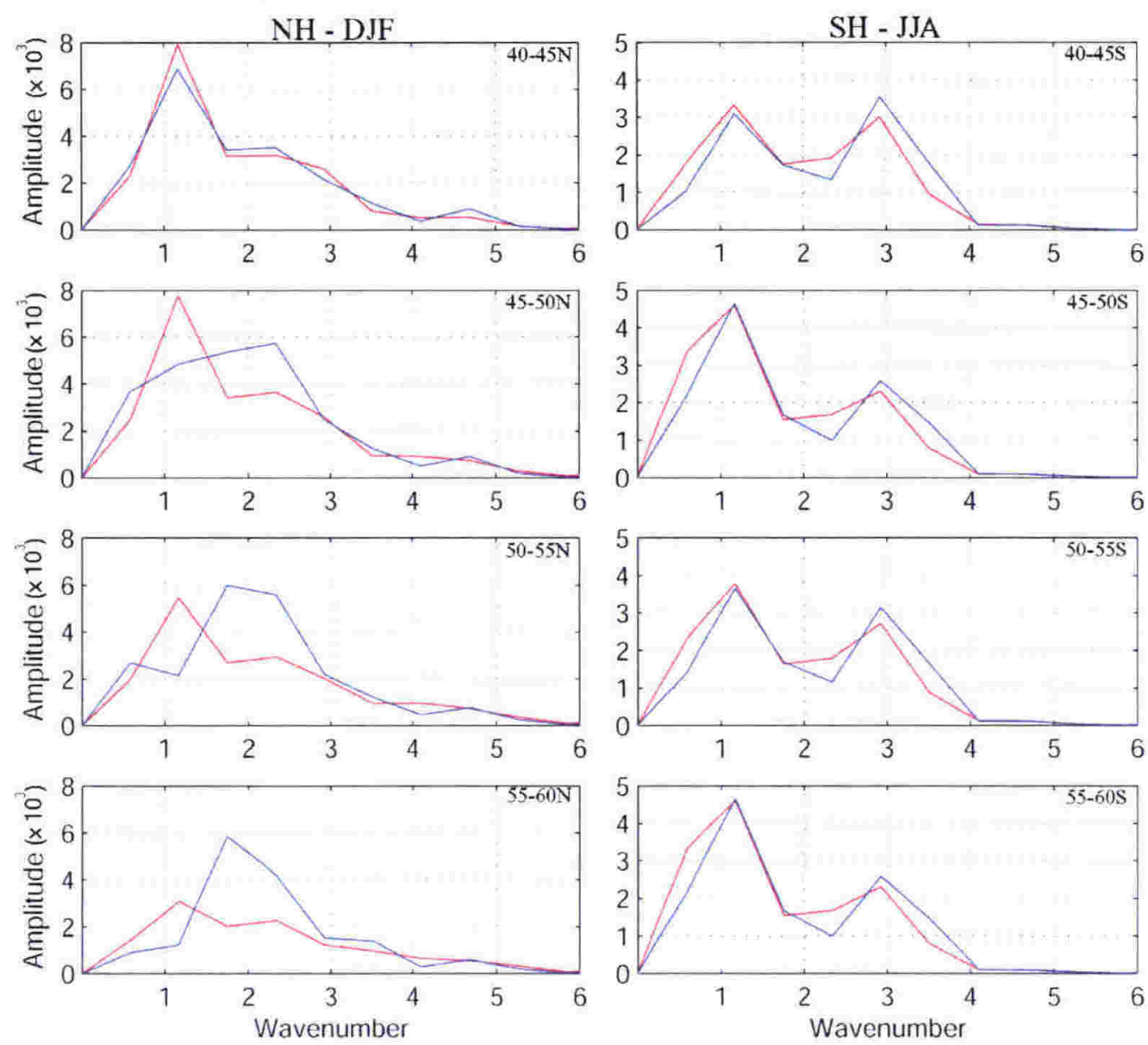

Fig. 7-16. The average power spectra of the mean $500 \mathrm{hPa}$ geopotential height for the preindustrial (red) and LGM (blue) simulations for the Northern (left) and Southern (right) Hemisphere winter seasons for 4 different zonal bands. In total 21 seasons were used for each spectrum. Note the different vertical scales for the $\mathrm{NH}$ and $\mathrm{SH}$.

waves. The average $500 \mathrm{hPa}$ geopotential height is determined for the Northern and Southern Hemisphere for four different zonal bands, $40^{\circ}-45^{\circ}, 45^{\circ}-50^{\circ}, 50^{\circ}$ $55^{\circ}$ and $55^{\circ}-60^{\circ}$. The mean value is subtracted from their zonal average, and the power spectrum is determined for the season of winter of each year. The average power spectra are presented in Fig. 7-16 where the amplitude of the power spectrum is plotted against the dominant wave numbers of the mean zonal circulation. The pre-industrial data is presented in red, the LGM in blue. The column on the left is DJF for the Northern Hemisphere, and the column on the right is JJA for the Southern Hemisphere. Each zonal band is indicated in the top right hand corner. 
The power spectra of the planetary waves in the Northern Hemisphere are analysed first. The $40^{\circ} \mathrm{N}-45^{\circ} \mathrm{N}$ spectrum shows the dominance of wave number 1 in the mean zonal circulation in both the pre-industrial and LGM simulation. There is really no difference between the two patterns. However, there are some considerable differences between the two simulations when moving poleward. The LGM shows a shift in the dominant wave number from wave number 1 to wave numbers 2 and 3 . Analysis of the spatial patterns of the differences in the mean $500 \mathrm{hPa}$ geopotential height shows that the largest changes occur at $30^{\circ} \mathrm{W}$ $100^{\circ} \mathrm{W}$ and $120^{\circ} \mathrm{W}-160^{\circ} \mathrm{W}$. The mean zonal circulation follows a more northern direction at those longitudes as the result of the enhanced temperature and pressure gradient along the eastern site of the Eurasia and American continent (see Fig. 7-8 and Fig. 7-12). This "deflection" in the mean wave number 1 circulation results in an enhancement of wave numbers 2 and 3 . The reduction in dominance of the wave number 1 pattern would also have affected the energy balance of the Northern Hemisphere. The consequences of the change in the Northern Hemisphere planetary waves and the resulting change in their poleward energy transport will be discussed briefly in section 7.2.7.

The Southern Hemisphere shows a different response. The dominant wave numbers for all zonal bands for both the pre-industrial and LGM simulation are wave number 1 and 3 . The power spectra for the two simulations are fairly similar for each latitudinal band. This indicates that the mean zonal circulation of the Southern Hemisphere of the LGM did not differ much from during the preindustrial period. Such a result was also found by Dong and Valdes (1998). The differences in the zonal bands are small. Wave number 1 is dominant over wave number 3 for $45^{\circ} \mathrm{S}-50^{\circ} \mathrm{S}$ and $55^{\circ} \mathrm{S}-60^{\circ} \mathrm{S}$, where as the amplitude in the spectral bands of wave number 1 and 3 are the same for $40^{\circ} \mathrm{S}-45^{\circ} \mathrm{S}$ and $50^{\circ} \mathrm{S}-55^{\circ} \mathrm{S}$. The standard deviation of the amplitude of the dominant wave numbers is smaller than in the Northern Hemisphere. This implies that the dominant wave numbers are much more sharply defined than in the Northern Hemisphere, especially during the LGM. Hence, their peaks are more prominent in their power spectra. The amplitude of wave number 3 in the power spectrum during the other seasons 
range from $15-20 \%$ for DJF and $25-50 \%$ for the transitional seasons compared to its equivalent amplitude during JJA.

Of note is the prominent appearance of wave number 3 which is usually weakly developed in the Southern Hemisphere. But as indicated in chapter 5, HadAM3H overestimates the amplitude of the wave number 3 circulation. Fig. 7-16 shows that the amplitude of the power spectrum of the wave number 3 circulation in the Southern and Northern Hemisphere during their winter months are comparable. There is an indication that the power spectrum has shifted slightly towards the higher wave numbers, with the wave number 3 pattern being slightly stronger during the LGM. It will be shown later that the very small change in the planetary waves can still have a measurable impact on regional climates. A stronger wave number 3 circulation in the Southern Hemisphere is in generally associated with an equatorward expansion of the polar trough and a weakening of the SAO (e.g. Meeh1, 1991: Chen and Yen, 1997: Burnett and McNicoll, 2000) and plays a positive role in the occurrence of blocking in the Southern Hemisphere (e.g. Trenberth and Mo, 1985). A shift to shorter wavelengths means a reduction in meridional heat transport (e.g. Cehelsky and Tung, 1991: Welch and Tung, 1998a: Welch and Tung, 1998b: Solomon and Stone, 2001a). This is further analysed in section 7.2.7.

\subsubsection{Zonal winds}

Compared to the pre-industrial simulation, the North Atlantic jetstream during the LGM shifted northwards and inereased slightly in strength (Fig. 7-17). The Pacific jetstream on the other hand does not indicate a positional shift and decreases slightly in strength during the LGM. These findings are similar to Dong and Valdes (1998) in their simulation with computed SSTs. Other studies found a southward shifted Pacific jet (e.g. Valdes and Hall, 1994: Kitoh et al., 2001), which was the result of a southward shift of the maximum in the meridional temperature gradient. However, the strongest increase in meridional temperature gradient in this study is found along the boundaries of the 

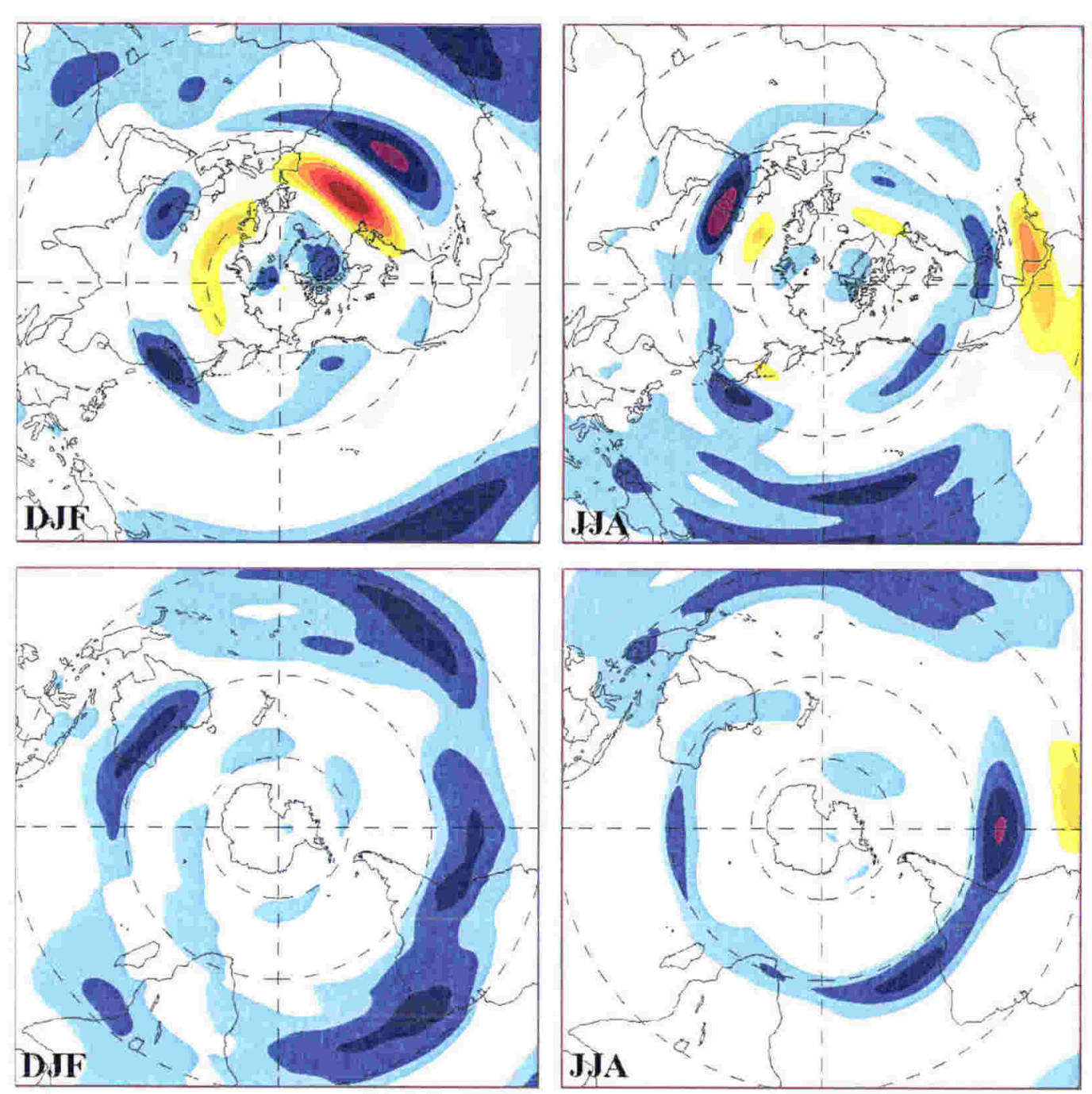

$$
\begin{array}{lllllll}
-8 & -4 & 0 & 4 & 8 & 12 & 16
\end{array}
$$

Fig. 7-17. The difference in the strength of the mean seasonal $250 \mathrm{hPa}$ winds, LGM - preindustrial ( $\mathrm{m} / \mathrm{s}$ ) for the Northern (top) and Southern Hemisphere (bottom) for DJF (left) and JJA (right).

continental ice sheets (compare Fig. 7-1 and Fig. 7-8). This resulted in a strong westerly flow from over the Eurasia continent into the North Pacific.

Kageyama et al. (1999) reported that an eastward extension for both Northern Hemisphere jet streams during the LGM is a common feature for several models. Just as with their discussion on the shift of the storm tracks, this was consistent with the location of the maximum temperature gradients shifting from the landsea edge onto the ice-sea edge. The shift of the jet streams in this simulation is 


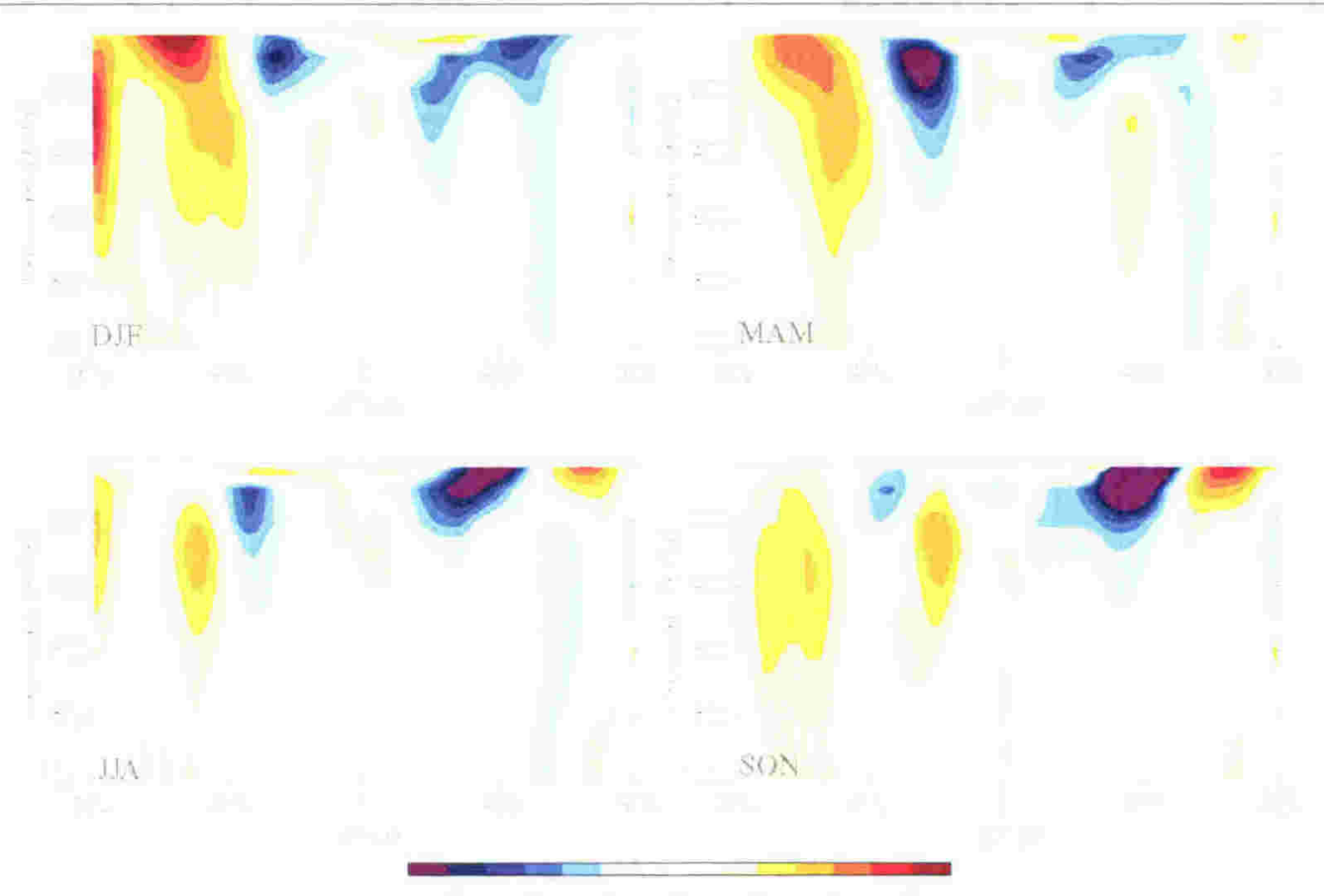

Fig. 7-18. Meridional cross sections of the differences in the average zonal wind $(\mathrm{m} / \mathrm{s})$ between the LGM and the pre-industrial simulation for the four seasons.

also consistent with the shift of the location of the maximum temperature gradients. The more northward directed jet streams are a direct result of the enhanced wave number 2 and 3 pattern in the planetary waves during the LGM as discussed in section 7.2.5.

The meridional cross section of the difference in the mean zonal winds between the LGM and the pre-industrial simulation shows clearly the weakening and the poleward shift of the Northern Hemisphere jet streams (Fig. 7-18). The maximum at about $30^{\circ} \mathrm{N}$ during SON is the result of an early onset of the Atlantic jet stream over North America. The resulting equatorward shift of the onset of the Atlantic jetstream shows up as a local maximum at $30^{\circ} \mathrm{N}$ in the meridional cross section. The weaker extratropical winds in the lower stratosphere are consistent with the findings of Rind et al. (2001). The westerly winds north of $45^{\circ} \mathrm{N}$ strengthen throughout the whole troposphere, where as they hardly change south of $45^{\circ} \mathrm{N}$.

The $250 \mathrm{hPa}$ zonal winds are in general weaker in the Southern Hemisphere, but there are considerable seasonal and spatial variations. The subtropical jet has 
moved slightly poleward at $80^{\circ} \mathrm{E}-170^{\circ} \mathrm{E}$ during DJF, thereby weakening over Australia but strengthening towards $40^{\circ} \mathrm{S}$. The westerly flow downstream of Australia shows a similar pattern. The subpolar jet is weaker and shifts slightly poleward following the stronger gradients in temperature. These gradients had increased as a result of the relatively higher temperatures of the lower stratosphere. This shift of the subpolar jet was also noticed by Rind et al. (2001) who found that this was the result of an increased wave forcing into the lower stratosphere.

Fig. 7-18 shows that the zonal wind speed in the Southern Hemisphere during the LGM was reduced throughout the entire troposphere roughly between $45^{\circ} \mathrm{S}$ $65^{\circ} \mathrm{S}$, and increased slightly at $35^{\circ} \mathrm{S}-45^{\circ} \mathrm{S}$, particularly during MAM. This pattern resembles an equatorward shift of the strongest winds. Such a shift is consistent with the findings of CLIMAP (1976: 1981). However, the response during SON is slightly different. Since the pre-industrial simulation showed an anomalous trend in the SAO during SON, it would seem that this response is more indicative of the anomalous westerly flow in the pre-industrial during this season than that it implies an anomalous response to the LGM conditions. The weaker westerlies are consistent with the difference in geopotential heights (Fig. 7-14).

Fig. 7-19 presents the difference in the strength of the $1000 \mathrm{hPa}$ zonal winds between the pre-industrial and LGM simulation during the four seasons. Data in the tropical and Antarctic regions have been omitted in order to focus on the mid and high latitudes. Apart from SON, it is clear that the westerly winds have increased roughly between $30^{\circ} \mathrm{S}-50^{\circ} \mathrm{S}$, and have decreased poleward of this band. The strongest westerly winds have shifted poleward over the Australian region. Such a shift was also suggested by Wyrwoll et al. (2000) and Hope and Simmonds (2003). Although Fig. 7-19 shows there are local maxima in the zonal wind over Australia, especially in southern Australia during DJF, the strength of the total wind $\left(\left[u^{2}+v^{2}\right]^{1 / 2}\right)$ was less during the LGM. This implies that the meridional wind over Australia, south of roughly $25^{\circ} \mathrm{S}$, was much weaker during the LGM. The reduction of intrusive, moist southerlies in southern Australia could explain why paleoclimatic evidence suggest a drier climate in this region during the LGM (e.g. Colhoun et al., 1996: Hunt and Barrows, 1999). 


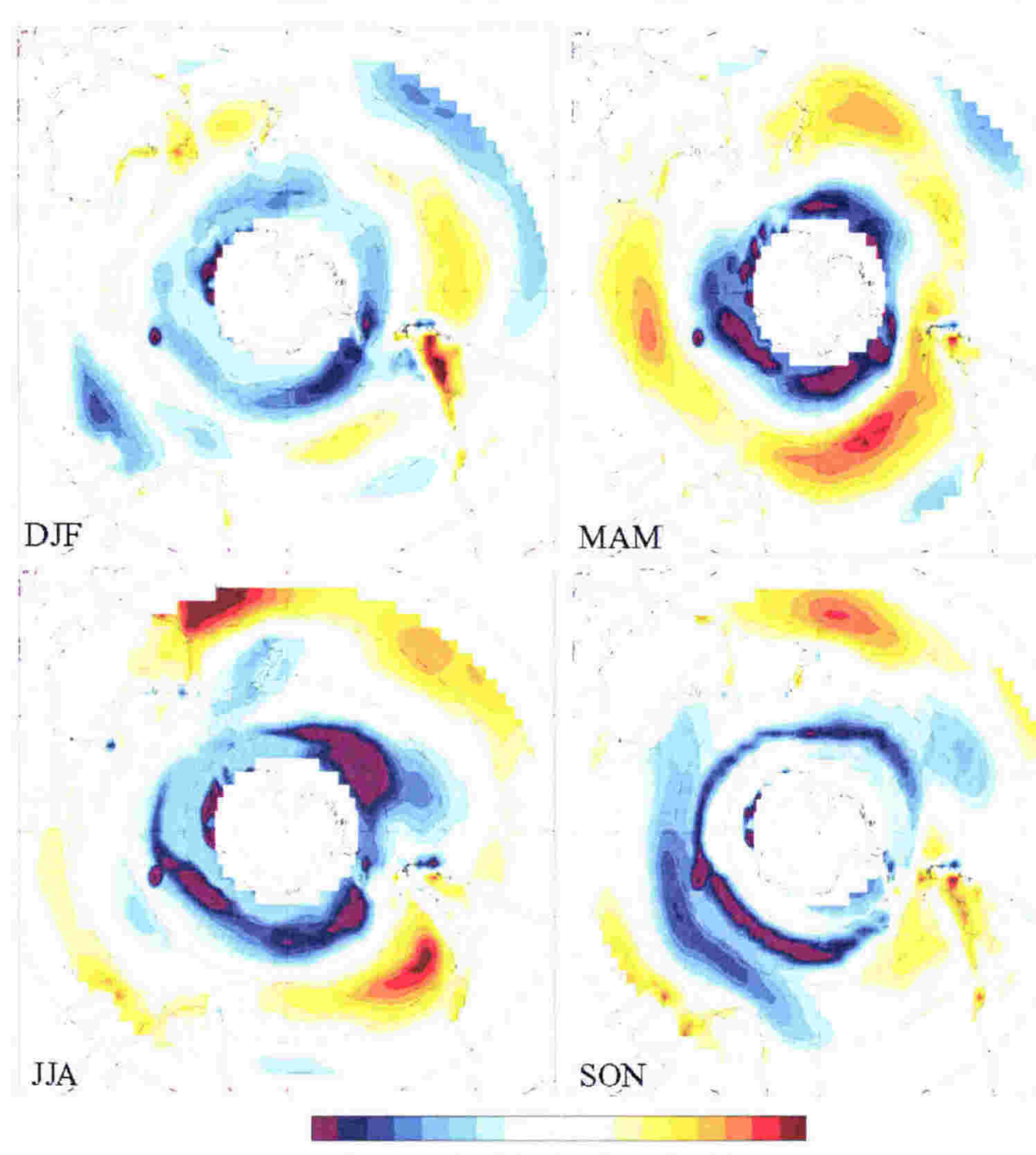

Fig. 7-19. The difference in $1000 \mathrm{hPa}$ zonal winds $(\mathrm{m} / \mathrm{s})$ between the LGM and pre-industrial simulation for the four seasons.

There is an increase in the zonal wind downstream of South America the whole year round. The southern part of South America is covered in glaciers during the LGM, resulting in an enhanced zonal temperature gradient. There is also a steeper temperature gradient between Australia and the South-West Pacific during JJA, which also results in stronger winds coming off the continent. Of particular interest is the weakening in the westerly winds over New Zealand during JJA. During the present day, New Zealand's climate during JJA often experiences "blocking highs". This would suggest that blocking highs over New Zealand were also a feature of the LGM climate. 

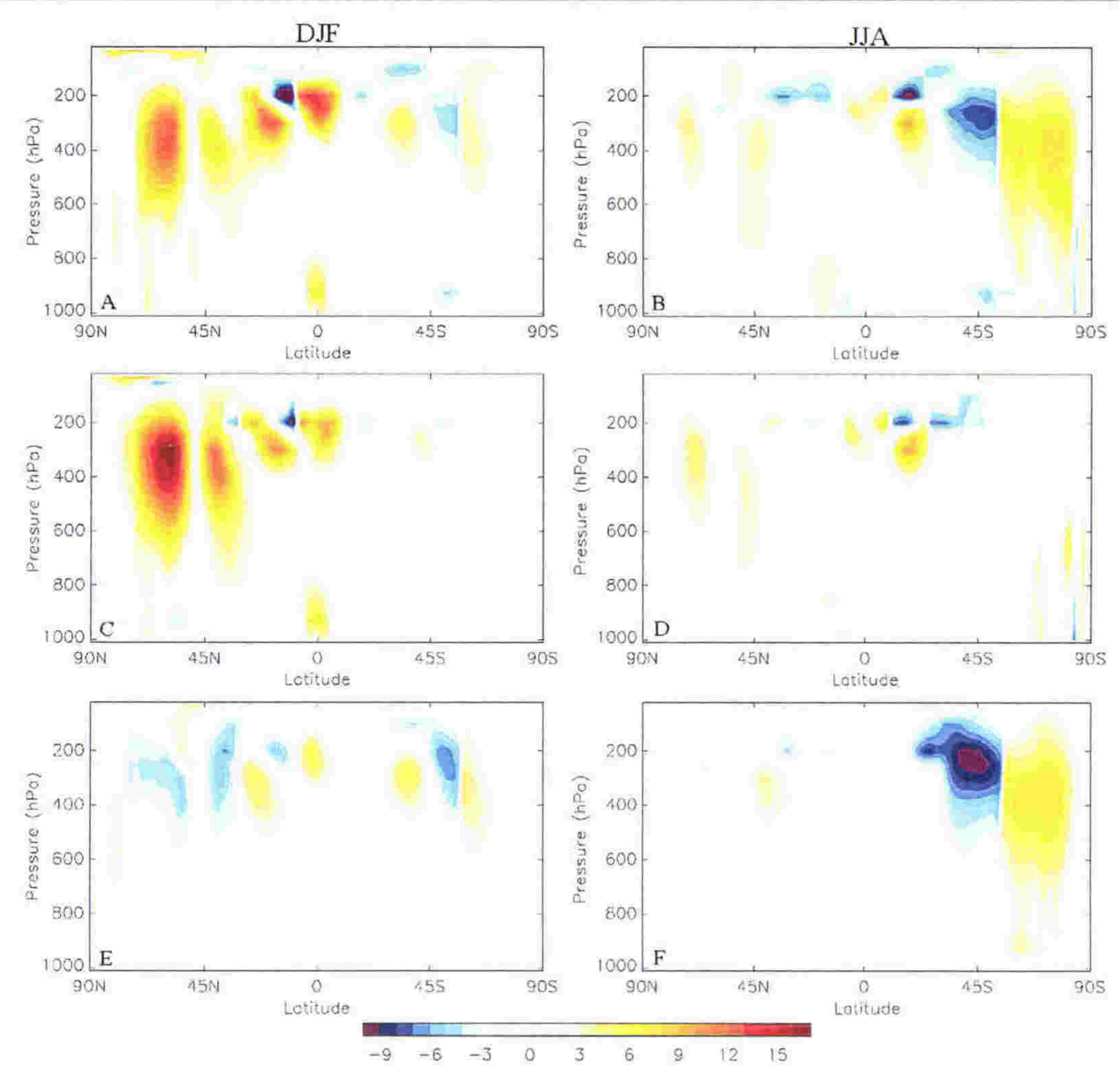

Fig. 7-20. The difference between the absolute values of the LGM and pre-industrial average zonal meridional momentum flux (A and B), its contribution from the mean meridional circulation (C and D) and eddies ( $E$ and F), for DJF (left) and JJA (right). Units are in $\mathrm{m}^{2} / \mathrm{s}^{2}$.

\subsubsection{Meridional Fluxes}

Given the differences in the mean zonal circulation between the LGM and the pre-industrial simulation, it is expected that the meridional transport during the LGM was different too. Fig. 7-20 presents the difference in the absolute values of the total meridional momentum transport and in its two components, the mean meridional circulation and the eddy fluxes, for DJF and JJA .

\footnotetext{
${ }^{1}$ Positive directions are eastward and northward. Although the direction of the fluxes is very important, in order to emphasize the change in their amplitudes, the absolute values of the fluxes are compared. Changes in locations of the fluxes are relatively minor and are taken into account as much as possible in the discussion.
} 
The total meridional momentum flux has changed in both hemispheres, which can be understood by analysing its two components. During the boreal winter months, the contribution of the mean meridional circulation to the total meridional momentum transport in the Northern Hemisphere has increased for both the poleward directed transport in the midlatitudes (Ferrel Cell) and the equatorward directed transport in the higher latitudes (Polar Cell). The maximum difference between the two simulations was at $300 \mathrm{hPa}$ and therefore below the jetstreams. This might be an indication of the colder atmosphere during the LGM being more compact. The eddy momentum fluxes in the Northern Hemisphere show a different pattern, in that they seem to shift slightly equatorward. For the Northern Hemisphere in general, the contribution of the mean meridional circulation to the meridional momentum circulation has increased and the contribution of the eddies has decreased during the LGM.

The Southern Hemisphere also experiences the largest changes in the meridional momentum flux during its winter season. But unlike as in the Northern Hemisphere, it are the eddy momentum fluxes that have changed the most. The poleward eddy meridional momentum flux in the midlatitudes is much weaker and the equatorward eddy meridional momentum flux in the higher latitudes is much stronger during the LGM than in the pre-industrial. Fig. 7-21 shows the differences in the eddy meridional momentum flux into its different components. The differences are between their absolute values ${ }^{1}$.

From section 7.2.5 it was concluded that the most prominent changes in the stationary waves and high altitude wind speeds occurred during the winter in the northern high latitudes. As a result of the enhancement of the wave-like structure of the mean zonal circulation in the Northern Hemisphere, there is both a stronger poleward stationary eddy component in midlatitudes (maximum in difference at $40^{\circ} \mathrm{N}$ ) and an equatorward stationary eddy component at high latitudes (maximum in difference at $60^{\circ} \mathrm{N}$ ). The weak maximum in the stationary eddy component in the Southern Hemisphere shows only a slight descent in

\footnotetext{
' Note: the stationary and transient eddies in the LGM simulation are similar to the pre-industrial simulation and the NCEP climatology presented in Fig. 5-16 and Fig. 5-17, and the positive difference at $60^{\circ} \mathrm{N}$ between the stationary eddies reflects a stronger equatorward component during the LGM.
} 


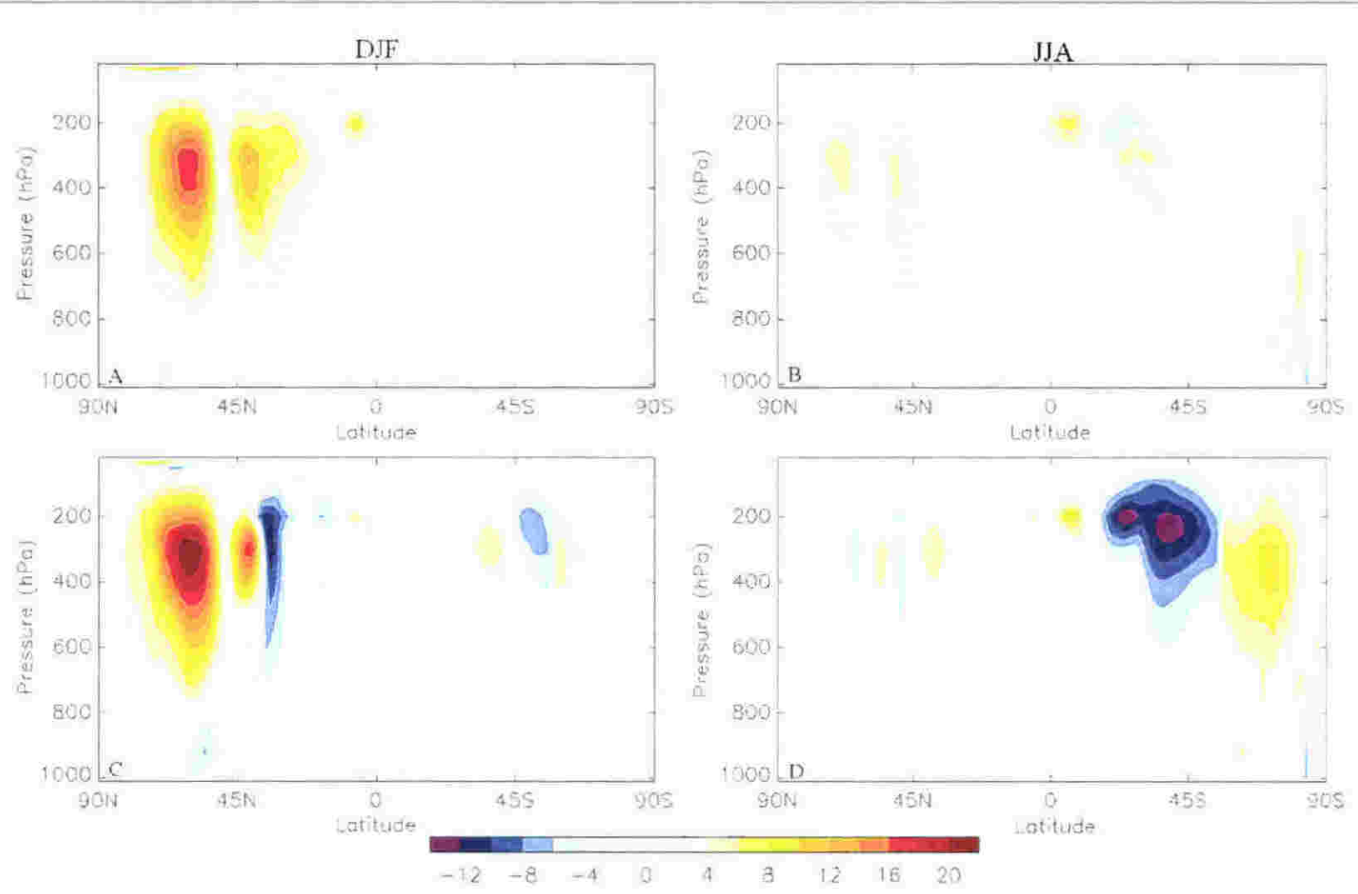

Fig. 7-21. The difference between the absolute values of the LGM and pre-industrial stationary eddy momentum flux (A and B) and the transient eddy momentum flux (C and D) for DJF (left) and JJA (right). Units are in $\mathrm{m}^{2} / \mathrm{s}^{2}$.

altitude between $20^{\circ} \mathrm{S}-40^{\circ} \mathrm{S}$. The transient component in the Northern Hemisphere experiences an increase at high latitudes. It follows the more northward directed and strengthened Atlantic jet, and consequently becomes weaker in the midlatitudes. The transient component in the Southern Hemisphere is weaker in the midlatitudes, which was caused by a reduction in the strength of both the zonal and meridional wind. The transient component increases in the high latitudes, which is the result of an increase in the strength of the equatorward meridional wind.

The difference between the LGM and pre-industrial zonal mean meridional heat transport is dominated by the increase in the Hadley circulation during the LGM, particularly during DJF (Fig. 7-22'). This is a direct consequence of the difference in their SST distribution in the tropical Pacific as was already noted in section 7.2.1. There is clearly also a weak reduction of meridional heat transport

\footnotetext{
${ }^{1}$ The purpose of Fig. 7-22 is to present clearly that the largest changes take place in the tropics and that these are primarily due to changes in the meridional circulation. Hence the rough scale for the figure.
} 


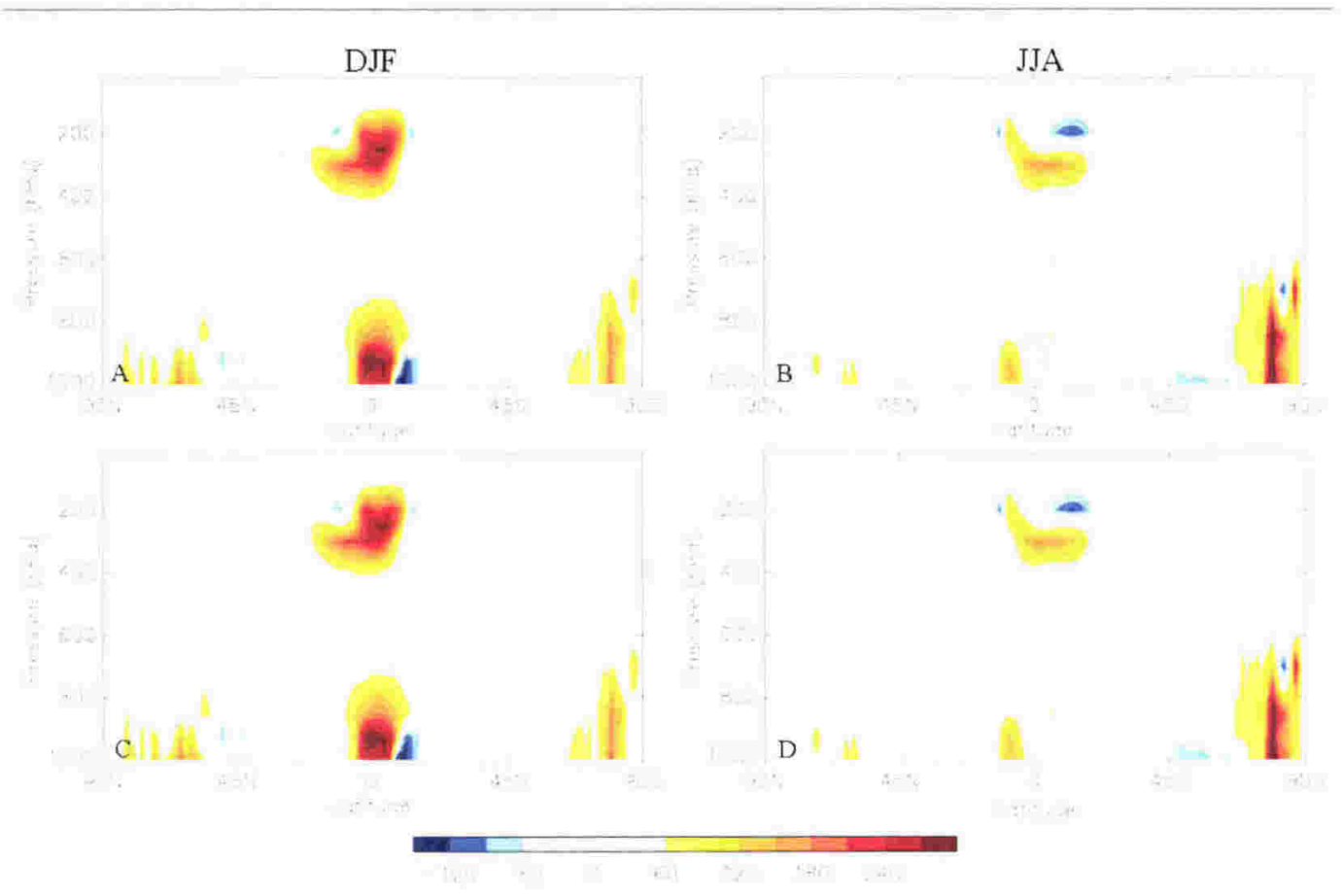

Fig. 7-22. The difference between the absolute values of the LGM and pre-industrial average zonal meridional heat transport ( $\mathrm{A}$ and $\mathrm{B}$ ) and the contribution from the mean meridional circulation (C and D) for DJF (left) and JJA (right). Units are in $\mathrm{mK} / \mathrm{s}$.

by the mean meridional circulation in the lower atmosphere at mid and high latitudes in the Southern Hemisphere and a strong increase of meridional heat transport by the polar cell over Antarctica. Removing the contribution of the mean meridional circulation to the zonal mean meridional heat transport shows the changes in the eddy components, which are prominent in the midlatitudes (Fig. 7-23). The largest changes appear in the Northern Hemisphere, where the meridional heat transport during the LGM is altered as the result of the changes in orography, ice sheets and planetary waves. As the result of the latter, the stationary eddy meridional heat flux increases dramatically. The changes in the transient eddy meridional heat flux show a difficult pattern, but indicate an increase in the poleward heat flux at midlatitudes, and a decrease in the equatorward flux at high latitudes.

The eddy meridional heat flux in the Southern Hemisphere increases in the midlatitudes and is weaker in the high latitudes. This is the result of two processes. There is both an equatorward shift of the maximum in the transient 


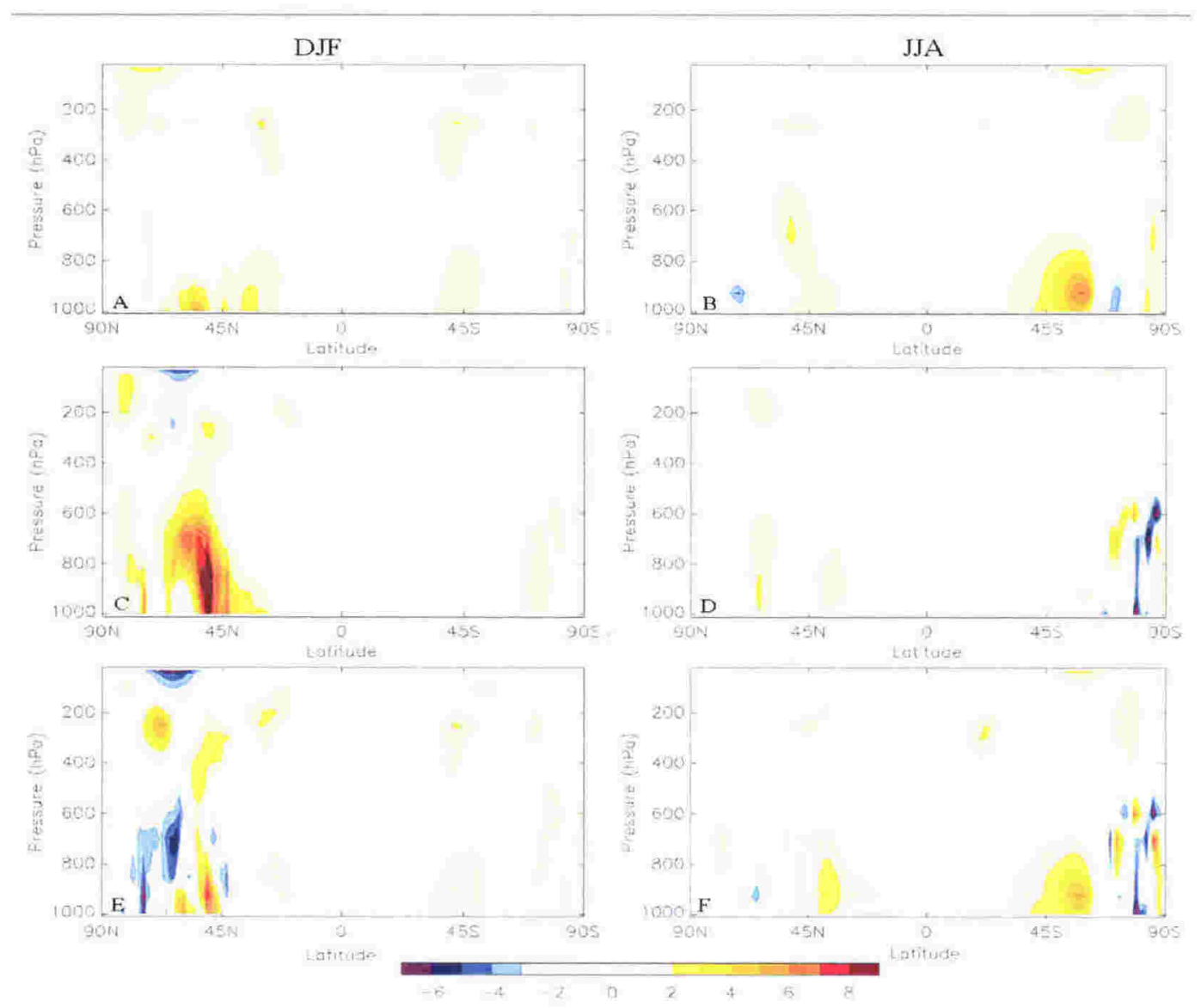

Fig. 7-23. The difference between the absolute values of the LGM and pre-industrial eddy heat flux $(\mathrm{A}$ and $\mathrm{B})$, the stationary $(\mathrm{C}$ and $\mathrm{D})$ and the transient eddy meridional heat flux (E and $\mathrm{F}$ ) for DJF (left) and JJA (right). Units are in $\mathrm{m} . \mathrm{K} / \mathrm{s}$.

eddy meridional heat flux, as well as a general increase in the transient eddy meridional heat flux. Hall et al. (1996b) found the transient meridional heat flux increased by up to $60 \%$. The stationary eddy meridional heat flux hardly changed during the LGM, which is further an indication that the planetary waves had not changed much during the LGM. A dramatic shift in the wavelength of the planetary waves to shorter or longer wavelengths would have altered the amount of energy they could have transported. There is an increase of the stationary eddy meridional heat flux further out from Antarctica as a result of the expansion of the Antarctic ice. This implies an equatorward extension of the extent of the colder katabatic winds coming off the continent. At the same time, there is therefore a reduction in the stationary eddy meridional heat flux over that area that had the strongest katabatic winds in the pre-industrial simulation. 
The difference between the LGM and pre-industrial zonal mean meridional heat transport is given by $\Delta(\overline{\mathrm{v}} \cdot \overline{\mathrm{T}})=(\overline{\mathrm{v}} \cdot \overline{\mathrm{T}})_{\text {LGM }}-(\overline{\mathrm{v}} \cdot \overline{\mathrm{T}})_{\text {Pre-ind. }}$ where $\overline{\mathrm{v}}$ is the mean meridional wind and $\overline{\mathrm{T}}$ is the mean temperature. Table 7-6 shows the correlation of the differences between the LGM and pre-industrial zonal mean meridional heat transport with their differences in meridional wind and temperature at $925 \mathrm{hPa}$ for the zonal band of $35^{\circ} \mathrm{S}-60^{\circ} \mathrm{S}$ for the four seasons. The negative values in the column on the right indicate that the temperature difference between the LGM and pre-industrial simulation acts to enhance meridional heat transport. This is completely in accordance with the fact that the meridional temperature gradient is steeper during the LGM than in the pre-industrial. The very high positive correlation between the differences in mean meridional heat transport and the differences in the meridional wind implies that the reduced zonal mean meridional heat transport in the Southern Hemisphere midlatitudes is caused largely by changes in meridional wind. The net meridional wind at $925 \mathrm{hPa}$ in the midlatitudes is directed polewards ${ }^{1}$. Reduction of the mean meridional heat transport as the result of a reduced poleward directed meridional wind, is the result of less northerlies/more southerlies, and/or the result of weaker northerlies/stronger southerlies. If there was no change in the frequency pattern of the standing waves, a change in the number of northerlies versus southerlies would result in a change in the shape of the planetary waves: the orientation of

\begin{tabular}{||c|c|c|}
\hline & Corr. $\Delta(\overline{\mathrm{v}} \cdot \overline{\mathrm{T}})-\Delta(\overline{\mathrm{v}})$ & Corr. $\Delta(\overline{\mathrm{v}} \cdot \overline{\mathrm{T}})-\Delta(\overline{\mathrm{T}})$ \\
\hline \hline DJF & 0.999 & -0.231 \\
\hline \hline MAM & 0.997 & -0.196 \\
\hline \hline JJA & 0.997 & -0.157 \\
\hline \hline SON & 0.998 & -0.219 \\
\hline
\end{tabular}

Table 7-6. Cross correlation of the difference in mean meridional heat transport with the difference in meridional wind and with the difference in temperature. Difference is LGM pre-industrial, level is at $925 \mathrm{hPa}$, and correlation is for data in the zonal band $35^{\circ} \mathrm{S}-60^{\circ} \mathrm{S}$.

\footnotetext{
' On the Southern Hemisphere, the poleward direction is negative.
} 
the trough becomes less steep. The net effect is that the wave becomes more sinusoidal.

Fig. 7-24 and Table 7-7 give an analysis of the mean seasonal meridional wind between $35^{\circ} \mathrm{S}-60^{\circ} \mathrm{S}$ for the pre-industrial and LGM simulation. Negative values mean northerly winds. The meridional winds in the simulation are stronger during JJA than during DJF. The general shape of the graphs in Fig. 7-24 are quite similar for the pre-industrial and LGM simulations, but the different mean seasonal values for the two simulations indicate the different meridional wind regimes. During all seasons, the average meridional wind is less negative in the LGM than in the pre-industrial. During DJF this is the result of a large increase in the number of southerlies (from $18 \%$ to $25 \%$ of all meridional winds), although the average strength of the southerlies actually decreases (from $1.00 \mathrm{~m} / \mathrm{s}$ to $0.87 \mathrm{~m} / \mathrm{s}$ ). During MAM there is only a small increase in the number of southerlies (from $34 \%$ to $36 \%$ ), but there is quite a shift to stronger southerlies (from $0.86 \mathrm{~m} / \mathrm{s}$ to $1.08 \mathrm{~m} / \mathrm{s}$ ). The most remarkable distribution is during JJA where there is no difference between the simulations in the numbers of northerlies and southerlies. However, there is a shift towards stronger southerlies during the LGM. This results in the largest difference in the mean seasonal meridional wind between the pre-industrial and LGM. One of the consequences of these stronger winds is that the transient eddy meridional heat transport in JJA was much larger during the LGM (Fig. 7-23F). The less negative average value during SON can be explained mainly by a shift from medium towards weak northerly winds.

From the analysis above it is clear that there was not just one factor affecting the differences in meridional wind between $35^{\circ} \mathrm{S}-60^{\circ} \mathrm{S}$. A combination of changes in the strengths and occurrence of the northerly and southerly winds led to a reduced zonal mean meridional heat transport. An increase in the number of southerlies and in the strength of the southerlies would have had a larger effect on the meridional heat transport than only a weakening of the strong northerly winds, since the southerlies would have been able to bring much colder air up north during the LGM than during the pre-industrial. 

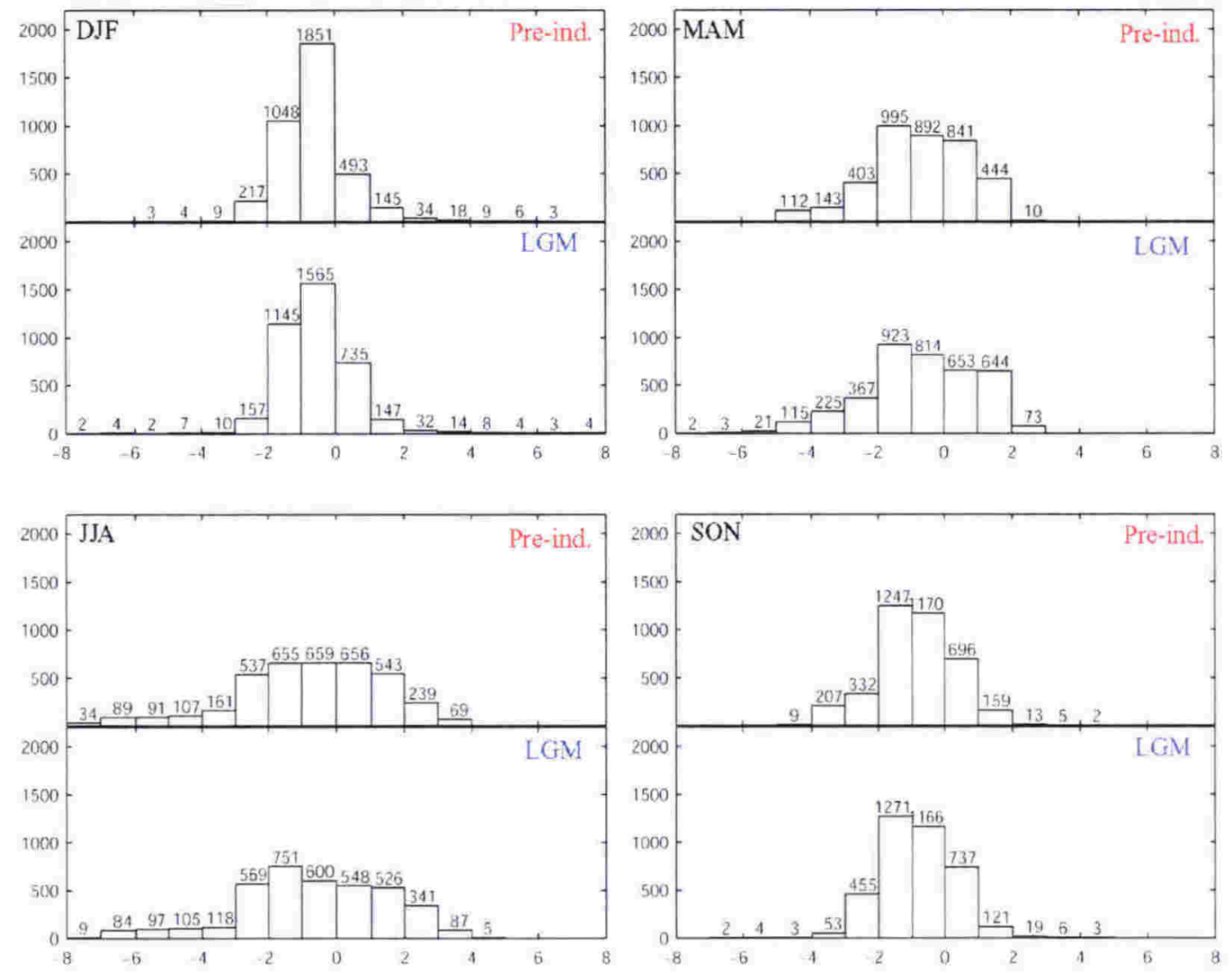

Fig. 7-24. The strength of the mean seasonal meridional wind $(\mathrm{m} / \mathrm{s})$ versus the number of grid points for $35^{\circ} \mathrm{S}-60^{\circ} \mathrm{S}$. The pre-industrial and LGM distributions are paired for each season to facilitate easy comparison. Southerly winds are positive.

\begin{tabular}{|c|c|c|c|c|c|c|c|c|}
\hline & \multicolumn{2}{|c|}{ DJF } & \multicolumn{2}{|c|}{ MAM } & \multicolumn{2}{|c|}{ JJA } & \multicolumn{2}{|c|}{ SON } \\
\hline & 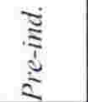 & डे & 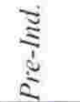 & ड્ & $\stackrel{\Xi}{\stackrel{\Sigma}{*}}$ & है & $\cong$ & ड્ \\
\hline \begin{tabular}{|l} 
Northerlies (negative) \\
\end{tabular} & 3132 & 2893 & 2545 & 2470 & 2333 & 2333 & 2965 & 2954 \\
\hline Average strength (neg) & -0.99 & -1.04 & -1.55 & -1.69 & -2.16 & -2.09 & -1.37 & -1.31 \\
\hline Southerlies (positive) & 708 & 947 & 1295 & 1370 & 1507 & 1507 & 875 & 886 \\
\hline Average strength (pos) & 1.00 & 0.87 & 0.86 & 1.08 & 1.31 & 1.49 & 0.74 & 0.71 \\
\hline Average & -0.63 & -0.56 & -0.73 & -0.70 & -0.79 & -0.68 & -0.88 & -0.83 \\
\hline
\end{tabular}

Table 7-7. Data for this table comes from Fig. 7-24. The number of grid points between $35^{\circ} \mathrm{S}-$ $60^{\circ} \mathrm{S}$ which have a poleward (negative direction) and equatorward (positive direction) directed meridional wind, their average strength (average over all grid points, in $\mathrm{m} / \mathrm{s}$ ), and the average meridional wind for the four seasons $(\mathrm{m} / \mathrm{s})$ for the pre-industrial and LGM simulation.

Although this analysis was for the mean meridional wind at $925 \mathrm{hPa}$, similar findings were found at higher altitudes as well. Another conclusion that can be 
drawn is that a change in the contribution of northerlies and southerlies would have affected the shape of the planetary waves. The heat-transporting polewardtilting phase of the planetary wave would have been reduced at the expense of an increase in the equatorward tilting phase of the wave. This would have resulted in a more sinusoidal wave pattern. An increase in the strength of northerlies and southerlies resulted in an increase of the amplitude of the wave. This is in accordance with the analysis done in section 7.2.5 where it was found that the wave number 3 pattern was slightly enhanced during the LGM.

An increase in the transient eddy meridional heat transport is associated with an increase in baroclinicity. However, there is no clear increase in precipitation at those latitudes (refer Fig. 7-11) during the LGM. The effect of an increase in baroclinicity on the precipitation was countered by the lower specific humidity of the LGM atmosphere. The Southern Hemisphere experienced an equatorward shift in the position of the maximum eddy meridional heat fluxes. This was primarily a response to the equatorward expansion of the sea-ice at the pole. Since the pole was much colder during the LGM, katabatic winds coming of the pole were bringing much colder air equatorward.

\subsubsection{Modes of Variability}

\section{Semi-Annual Oscillation ( $S A O)$}

The SAO signal presented in Fig. 7-25 has been computed as described in chapter 5. What immediately stand out is that the SAO of the LGM is more similar to the SAO of the NCEP climatology than SAO of the pre-industrial simulation. As mentioned in chapter 5 , the pre-industrial simulation failed to model the second peak in the SAO which was the result of an erroneous annual cycle of SSTs at $50^{\circ} \mathrm{S}$. Given the model's experimental design, it is not possible to determine whether the similarity with the SAO of the NCEP climatology was the result of more realistic SSTs in the LGM simulation, or whether the SAO was actually stronger during glacial times. A particularly important point that comes out of the SAO analysis is that there could be an opposite response during SON when comparing midlatitude circulation features of the LGM with the pre- 


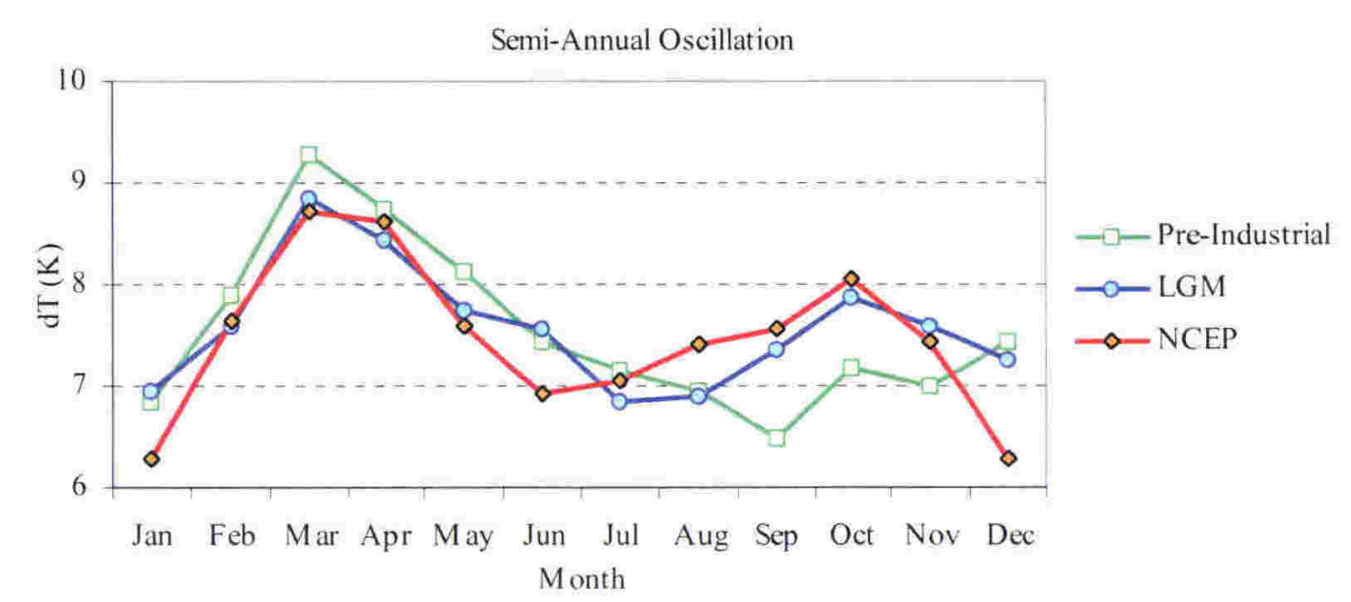

Fig. 7-25. The SAO for the NCEP climatology and the pre-industrial and LGM simulation.

industrial simulation or with the NCEP climatology. The season of spring was often discussed separately, as it did show at times a different response than the other seasons (sections 7.2.6, 7.2.7).

Since the strength of the SAO is indicative of the level of baroclinicity, the cyclonic activity in the LGM during SON was enhanced and closer to the pole during SON than in the pre-industrial simulation. These conclusions are consistent with the increased meridional temperature gradient and meridional heat transport during that time of the year. However, a cautionary note needs to be placed here. Although the meridional temperature gradient determines the seasonal evolution of the SAO in baroclinicity, it is the static stability that determines the amplitudes of its maxima (Walland and Simmonds, 1999). The colder climate of the LGM has a higher static stability than the warmer climate of the pre-industrial. A higher static stability would result in a weaker baroclinic response to an increase in temperature gradient. For that reason the difference in baroclinicity between the two simulations during the first half of the year would be slightly larger than that the difference between their SAOs shows, with the cyclonic activity be reduced during the LGM. By comparing the SAO of the LGM against the SAO of the NCEP climatology, it can be deduced that the LGM has stronger baroclinicity during DJF and weaker baroclinicity during JJA and SON. The onset of the second peak in the SAO of the LGM lags a month behind 
the onset of the second peak in the SAO of the NCEP climatology, but the main difference between the two signals is in their amplitudes. The smaller amplitude of the SAO of the LGM is consistent with an enhanced wave number 3 circulation (section 7.2.5) and an equatorward shift of the subpolar trough (section 7.2.6).

An interesting observation can be made when comparing these findings with those of Menendez et al. (1999). These authors looked at features of the general circulation of the Southern Hemisphere under a "no-ice" scenario. Such a scenario is exactly the opposite to the increase in sea-ice extent that happened during the LGM. The authors do find a reverse response in the general circulation, with weaker mean zonal winds and a decrease in baroclinicity. However, they also find a weaker SAO, which does not follow the other changes. If both our findings regarding the SAO are correct, then the SAO clearly does not show a linear relationship with the extent of sea-ice around Antarctica.

\section{Intraseasonal and Interannual Variations}

The analysis of the intraseasonal and interannual variations is performed as in chapter 5. The EOF analysis is presented in Fig. 7-26. The first three EOFs are very similar to the EOFs for the NCEP climatology and the pre-industrial simulation (Fig. 5-23). The circulation associated with the HLM is again slightly stronger over the South West Pacific and over the Indian Ocean than in the NCEP climatology. The spatial extent of the maximum over the South West Pacific is reduced and slightly enlarged over the Indian Ocean compared to the pre-industrial simulation. The HLM during the LGM accounts for the interannual variation slightly more than that it did in the pre-industrial simulation $(31.2 \%$ versus $29.3 \%$ ). Analysis of the time series shows that the HLM during the LGM does not show the two distinct peaks associated with the vacillation of the negative and positive phase, but shows a maximum in the distribution on the positive site similar as in the pre-industrial simulation (Fig. 7-27). The distribution is slightly more towards the positive during the LGM than in the preindustrial simulation. This would imply that the HLM during the LGM caused 


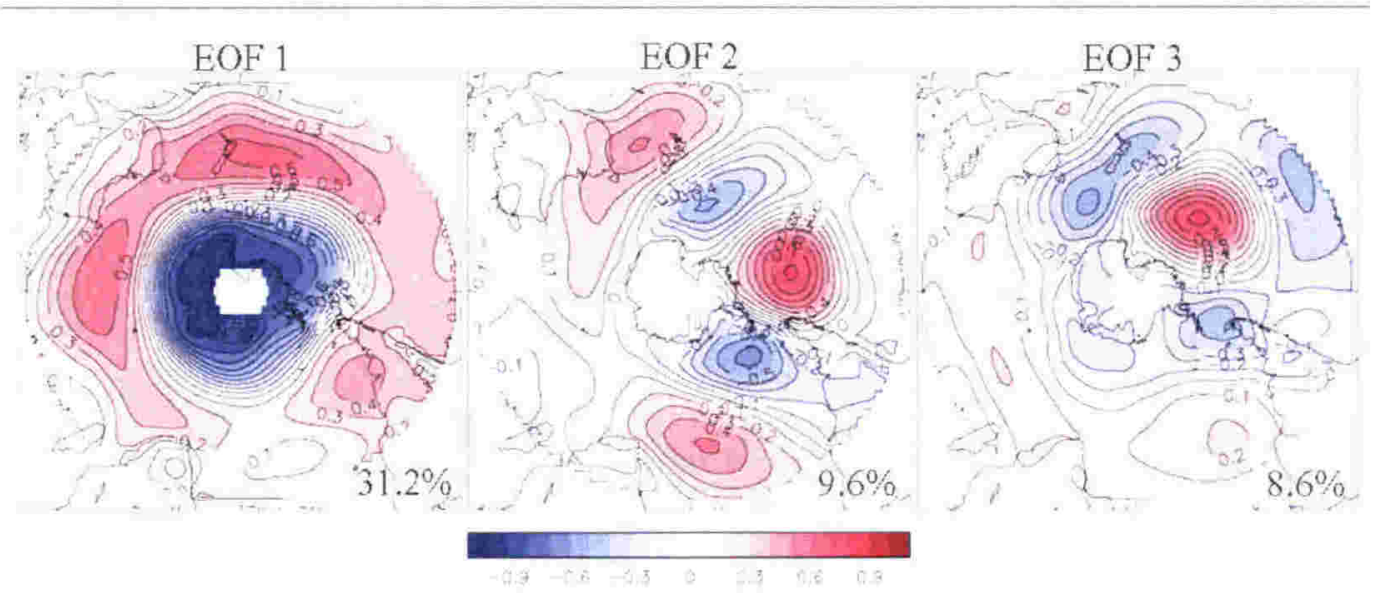

Fig. 7-26. The three leading EOFs of the mean sea level pressure of the LGM simulation.

slightly stronger westerly winds at high latitudes ${ }^{1}$. However, this might also be the effect of a slightly reduced westerly wind in the mean zonal circulation at those latitudes.

Chapter 5 showed that although there was only a weak correlation between the Pacific SST patterns with the timeseries of EOF2 and EOF3 in the preindustrial simulation, the second and third EOF were degenerate, and the variability associated with their patterns strongly resembled the EOF patterns of the NCEP data. The second and third EOF were consequently referred to as ENSO modes. Correlating the timeseries of EOF2 and EOF3 with the SSTs in the LGM simulation does not show the classical ENSO patterns either (Fig. 7-28), although the largest correlations are found in the tropical Pacific. The latter was expected since the interannual variability in the tropical Pacific was larger in the LGM simulation than in the pre-industrial simulation (compare Fig. 7-7 with Fig. 5-3). As in the pre-industrial simulation, the shape of the ENSO correlation pattern differs from the pattern in the present day (Fig. 5-24). The correlation pattern for the third EOF in particular is different, since it seems to indicate that the variability is linked to SST variability in the Western and Central Pacific, rather than in the Eastern Pacific. Whether such a pattern shows up in other fields in the equatorial Tropics and what their impact is on the climate

\footnotetext{
I This should not be confused with the reduction in strength of the mean westerly flow at high latitudes. The two simulations represent two different climate states. In its mean state, the climate of the LGM has a stronger positive HLM phase than the pre-industrial in its mean state.
} 

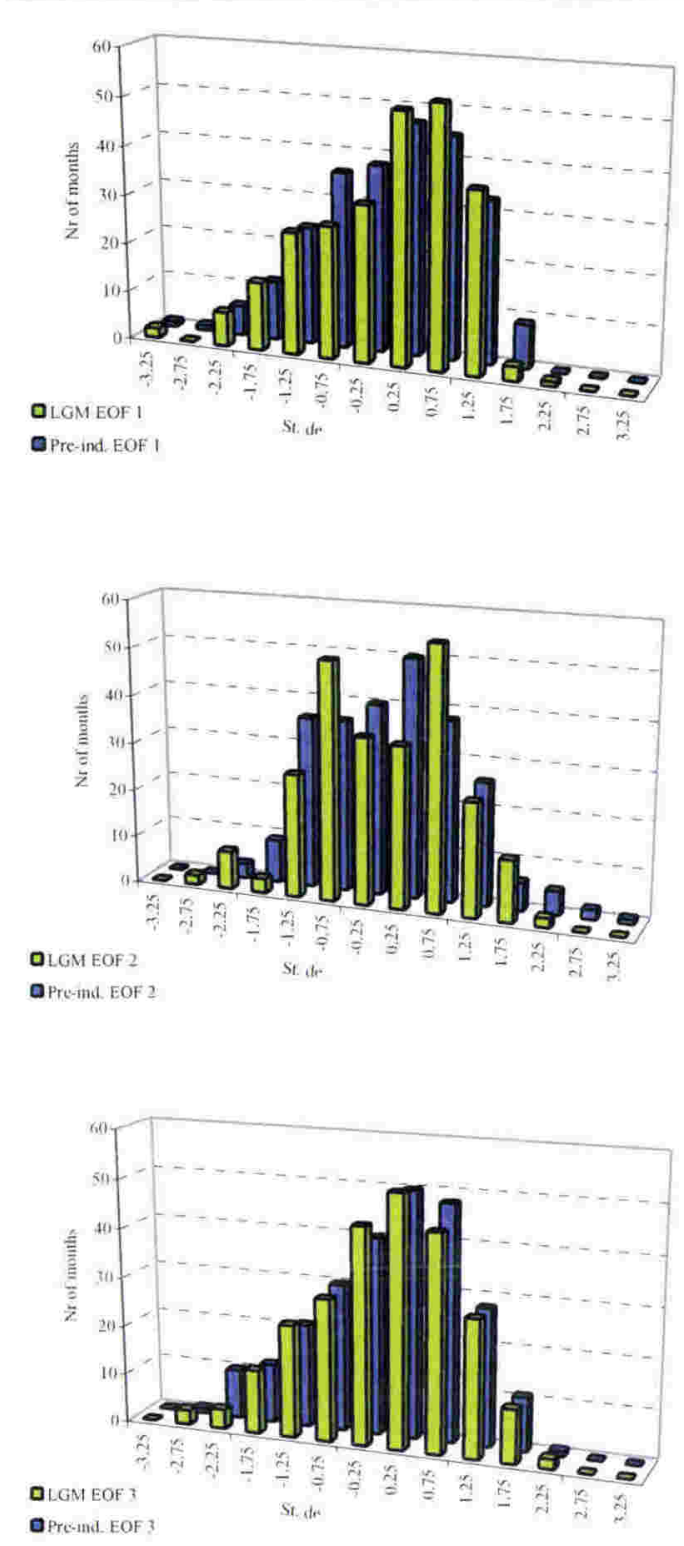

Fig. 7-27. Distribution of the EOF coefficients for the first three EOFs. X-axis is standard deviation and $y$-axis is number of months. The pre-industrial data are given for comparison (see Fig. 5-26).

was not investigated. Using the criterion of North et al. (1982), the second and third eigenvalues overlap slightly, implying some mixing between their patterns. Linear combinations of the second and third eigenvalues could therefore be configured to more closely resemble the characteristic ENSO patterns, hence the second and third EOFs of the LGM are referred to as ENSO modes.

Of interest in this study is the different correlation in the New Zealand region. Fig. 7-28 implies that an El Niño seems to result in higher temperatures in the New Zealand region, opposite to what happens during the present day. Although the correlation patterns are strictly speaking not exactly ENSO patterns, the EOF patterns explaining this variability are referred to as ENSO modes. It is obvious that care should be taken when interpreting these patterns.

The second EOF shows the characteristic "wavetrain" pattern. The start of this pattern is now more comparable to the NCEP EOF 3 pattern (Fig. 5-23), since it starts off from the east coast of Australia, rather than from the south of Australia as in the preindustrial simulation. The gradient in the South West Pacific, and especially over the New Zealand region, is reduced. This pattern enhances the south- 


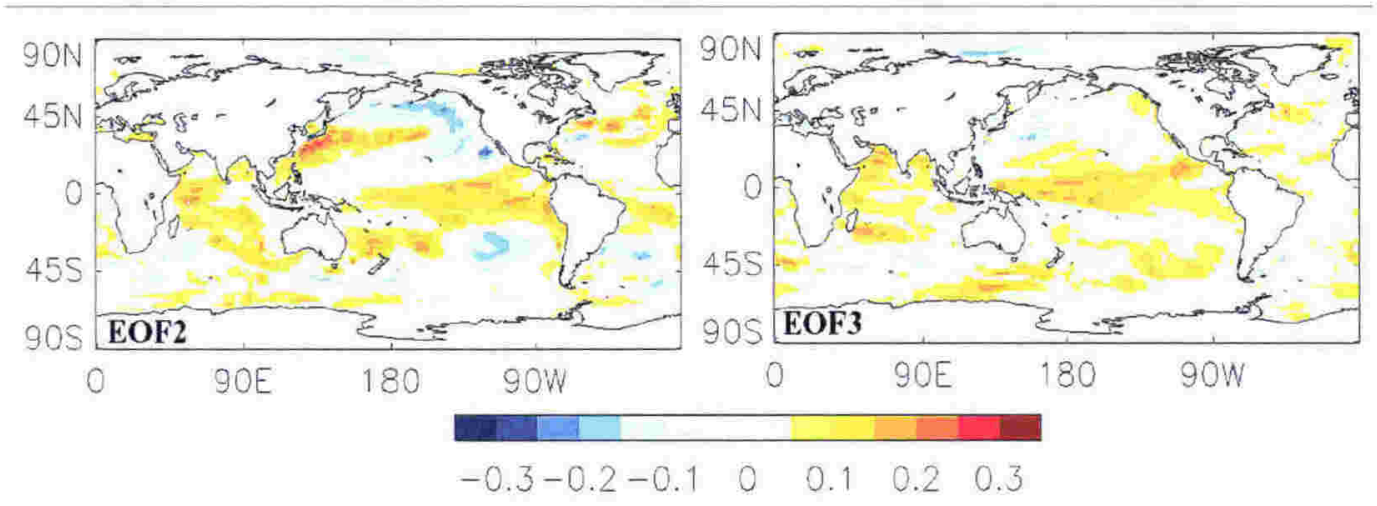

Fig. 7-28. Correlation patterns of the timeseries of EOF2 and EOF3 with SSTs for the LGM simulation.

westerly/north-easterly flow over New Zealand instead of the southerly/northerly flow as it did in the pre-industrial simulation. The wavetrain has a stronger continuation and can easily be identified going into the South Atlantic all the way to Southern Africa. Analysis of the time series indicates symmetry in the occurrence of an El Niño and a La Niña event, with peaks in the standard deviation at both 1 and -1 (Fig. 7-27). Whether that is an artefact of the model, or whether that is a real feature of the climate of the LGM has not been investigated further.

The dipole pattern of the third EOF shows a remarkable change. The characteristics of the dipole, a negative and a positive anomaly over the low and high latitudes of the Pacific respectively, are still there. But the flanks of the low over Antarctica have moved equatorward, and are now shouldering the positive anomalies over the high latitudes. This results in the third EOF also showing a "wavetrain" pattern, albeit about $90^{\circ}$ out of phase with EOF 2. Rather that New Zealand lies between two maxima, as it does in the pattern of the EOF2, New Zealand lies directly under a maximum. Although this pattern only explains $8.6 \%$ of the interannual variability, New Zealand would experience a tendency to lower mean sea level pressures, possibly followed by an increase in wind and baroclinicity, during El Niño years. During La Niña years the opposite would happen and more anticyclones would move over the country. The question can be raised whether the cooler summer temperatures during the LGM result in a present day winter-wavetrain-like intraseasonal variability? The distribution of 
the time signal is very similar to the distribution of the third EOF of the preindustrial simulation (Fig. 7-27). With the interannual variability associated with ENSO during the LGM being of a similar magnitude as in the pre-industrial simulation, it would seem that ENSO should have been felt in New Zealand during the LGM. Although it is difficult to obtain an interannual time resolution in sedimentary samples, Pepper et al. (2004) found the characteristic ENSO period in the spectral analysis of laminae thicknesses. The higher order EOFs (46) range from $4.4 \%$ to $6.3 \%$ and were not analysed.

\subsection{Summary}

This chapter described the climate of the LGM as modelled by HadAM3H. Several features of the global climate and the climate of the Northern Hemisphere were discussed, but the main emphasis was on the climate of the Southern Hemisphere. SSTs and sea-ice had been computed by HadCM3 running under the same LGM conditions as in this simulation. HadAM3H's simulation of the LGM is strongly influenced by these fields. As had been indicated in chapter 5 , the UM has some difficulties in simulating SSTs and sea-ice correctly in the Southern Hemisphere. The modelled LGM climatology has been compared primarily against the modelled pre-industrial climatology with occasional references to geological proxy data. Since the behaviour of HadAM3H was investigated earlier (primarily in chapter 5 but also in e.g. Pope and Stratton, 2002: Hudson and Jones, 2002a), it was possible to separate climatic features of the LGM from model biases, at least in a linear sense.

The main features influencing the climate of the LGM were the large continental ice sheets, cooler SSTs, an increase in the extent of sea-ice, and a lower concentration of atmospheric $\mathrm{CO}_{2}$. The largest amount of cooling in SSTs takes place at high latitudes, particularly in the Northern Hemisphere. The North Atlantic is an exception, where the SSTs are slightly warmer during the LGM. This was caused by an increase of the overturning of the NATC, which in turn brought warm water up north along the coast of Western Europe (Hewitt, 2000: Hewitt et al., 2001: Hewitt et al., 2002). Except for this warming in the North 
Atlantic, the lower SSTs correspond more to the computed SSTs determined by PMIP than the SSTs proposed by CLIMAP. This was expected since HadCM3 had been set up according to the PMIP specified boundary conditions. In the annual mean, the global surface temperature during the LGM was approximately $3.8^{\circ} \mathrm{C}$ cooler than during the present day (pre-industrial). There were considerable geographical and seasonal differences, with the largest cooling to take place at the high latitudes, particularly in the Northern Hemisphere, and during the winter months. The temperature over continents was more reduced than over oceans. As a result of the increase in the zonal difference in temperature between continent and ocean, the zonal mean sea level pressure difference between Australia and the Western Pacific increased This in turn affected the winds downstream of Australia, which probably had an impact on New Zealand's climate. The lower surface temperatures resulted in a slightly lower lapse rate at high latitudes.

Equatorial Pacific SSTs were colder by about $2^{\circ} \mathrm{C}$ in the west and $4{ }^{\circ} \mathrm{C}$ in the east, which fits in the range of $0^{\circ} \mathrm{C}-6^{\circ} \mathrm{C}$ indicated by proxy data and climate simulations. This implies an increased temperature gradient across the Pacific, which resulted in stronger tradewinds and a stronger Hadley circulation. This is opposite to CLIMAP's findings. CLIMAP had a reduced zonal temperature gradient across the Equatorial Pacific, resulting in weaker trade winds. Interannual variability of the Pacific SSTs is larger than during the pre-industrial simulation and is in magnitude more comparable to the variability found in the NCEP climatology. Just as in the pre-industrial simulation, the largest variability occurs more in the El Niño 3.4 area, rather than in the El Niño 1+2 area, as it is in the present day. However, Wittenberg (2002) found that the largest variability in SST shifts westward under a climatic regime with stronger trade winds. The fact that the largest variability in SST during the LGM takes place in the El Niño 3.4 area might therefore be a correct representation. The interannual variability in the Pacific SSTs indicates therefore that ENSO was also a common phenomenon during the LGM, although some of its characteristics might have been different. This could not be investigated in any detail, since HadCM3 has difficulties in simulating the tropical Pacific SSTs correctly (Gordon et al., 2000). 
The amount of precipitation was reduced during the LGM. The largest reductions took place over South East Asia. This might be related to the large reduction in sea surface in this region during the LGM. There are indications that the SPCZ had shifted eastwards, which could be an indication that the climate of the LGM had characteristics common with an El Niño phase. The precipitation is reduced virtually everywhere south of $45^{\circ} \mathrm{S}$. The largest reductions over land took place over the continental ice sheets.

The climate of the Northern Hemisphere was strongly influenced by the large continental ice sheets. The strong cooling in the northern latitudes resulted in a steepening of the meridional temperature gradient. The North Atlantic jetstream shifted northwards, following the steepest meridional temperature gradient shift to the continental ice-sea boundary, and strengthened slightly. The spectrum of the planetary waves shifted from a dominant wave number 1 pattern towards a wave number 2 pattern in the middle to high latitudes. This change in the planetary waves would have affected the climate of the underlying atmosphere. The westerly winds increased onto Europe and North America since the Azores high was relatively higher and the Aleutian low relatively deeper. The warmer SSTs in the North Atlantic would have affected Europe's climate.

The equatorward expansion of the sea-ice in the Southern Hemisphere during the LGM was not as large as according to CLIMAP. The SSTs at the high latitudes were therefore warmer than suggested by CLIMAP. The meridional temperature gradient in the Southern Hemisphere was therefore less steep than as postulated by CLIMAP. The gradient in the Southern Hemisphere was also less steep than in the Northern Hemisphere.

The polar trough shifted equatorward, following the extended edge of the seaice. This conforms to the findings of CLIMAP. The reduced temperature gradient between $50^{\circ} \mathrm{S}-65^{\circ} \mathrm{S}$ results in weaker westerly winds at those latitudes. The equatorward shift of the polar trough shifted the strongest winds equatorward too, and there was a slight increase in the strength of the mean zonal westerlies at $30^{\circ} \mathrm{S}-45^{\circ} \mathrm{S}$. The area of baroclinicity moved northwards with the equatorward shift of the polar trough. There was a decrease in cyclonic activity south of $60^{\circ} \mathrm{S}$, 
and an increase at $30^{\circ} \mathrm{S}-60^{\circ} \mathrm{S}$ during $\mathrm{JJA}$. The increase in baroclinicity in the midlatitudes did not automatically increase the precipitation, since the colder atmosphere of the LGM had a higher static stability and lower saturation vapour pressure. The shift of the zone of baroclinicity can be deduced from the change in eddy meridional heat transport, which increased at $30^{\circ} \mathrm{S}-60^{\circ} \mathrm{S}$ and decreased south of $60^{\circ} \mathrm{S}$. This pattern is similar through the rest of the year, albeit weaker and the transition zone more equatorward. The zonal mean meridional heat transport decreased though, which was the result of a decrease of the contribution of the mean meridional circulation. The meridional heat transport by the stationary eddies hardly changed. This supported the findings of the analysis of the power spectrum of the planetary waves of the Southern Hemisphere in that their frequency pattern was similar as during the pre-industrial. However, this analysis did show that the amplitude of the wave number 3 circulation was slightly larger. A cautionary note needs to be placed here in reference to the model's capability in modelling the planetary wave structure correctly. Since it is known from chapter 5 that the model overestimates the wave pattern of the mean zonal circulation, it is difficult to separate a climatic feature associated with the LGM from the model's bias. But based on the fact that the effects of an enhancement of the planetary waves during the LGM is consistent with the change found in other climatic variables, it was concluded that the slightly larger amplitude of the wave number 3 pattern was a real feature of the climate of the LGM. Since the stationary eddies were similar as during the pre-industrial, the increase in eddy meridional heat transport was taken up by the transient eddies. This would imply an increase in baroclinicity during the LGM.

Analysis on how the zonal mean meridional heat transport was reduced at the Southern Hemisphere midlatitudes shows that the change in the meridional wind is very highly correlated to the change in zonal mean meridional heat transport. It was therefore the change in the meridional wind and not the change in meridional temperature gradient that determined the reduction in the zonal mean meridional heat transport. Analysis of the meridional wind at $35^{\circ} \mathrm{S}-60^{\circ} \mathrm{S}$ for the four seasons showed that a reduction in the number of northerlies and a weakening of the northerlies, as well as an increase in the number of southerlies 
and an increase in the strength of the southerlies, all played their part. An increase in the number of southerlies and an increase in the strength of the southerlies can have a larger impact on the meridional heat transport than the other configurations of change in the meridional wind. The southerlies were able to get much colder air from the higher latitudes during the LGM. The shift in the balance between the number of northerlies versus the number of southerlies implies that the shape of the planetary waves was slightly more sinusoidal. This could explain the different changes in regional climates compared to the changes in the zonal mean. New Zealand's climate might therefore show different changes during the LGM than other landmasses at the same latitude. The state of New Zealand's climate would have been strongly by the changes in the climate of Australia. A colder Australia, with higher mean sea level pressure, has resulted in a poleward movement of the strongest westerly winds, opposite to the meridional shift in the westerly winds in the zonal average. This change would have led to an enhanced southerly flow over New Zealand.

The SAO was weaker during the LGM than during the pre-industrial. It was not possible to directly link the weakening of the SAO to the overall climate of the LGM, since it could simply have been the result of a better representation of the SSTs in the LGM simulation. But since a weakening of the SAO is consistent with an enhancement of the wave number 3 circulation and an equatorward shift of the polar trough, it can be concluded that the SAO was weaker during the LGM.

Interannual variability on the mean flow in the Southern Hemisphere was for a large part again determined by the HLM and ENSO. EOF analysis showed that the HLM ( $1^{\text {st }}$ EOF) accounted for a slightly larger portion of the variability during the LGM than during the pre-industrial (31.2\% vs. 29.3\%). Similar as in the pre-industrial simulation, it showed a preference towards the positive phase. Also as in the pre-industrial simulation, the second and third EOF did not explain exactly the classical ENSO SST patterns in the tropical Pacific, but were referred to as ENSO modes nevertheless. The ENSO signal represented by the wavetrain pattern in the EOF analysis $\left(2^{\text {nd }} E O F, 9.6 \%\right)$ was very similar to the one determined for the NCEP reanalysis. Where as this pattern enhanced a 
southerly/northerly flow over New Zealand during an El Niño/La Niña in the present day, it would stimulate a more south-westerly/north-easterly flow during the LGM. The wavetrain had a very strong continuation and could be identified going all the way across the South Atlantic. The ENSO signal represented by the dipole pattern over the Southern Pacific $\left(3^{\text {rd }}\right.$ EOF, 8.6\%) had changed too. The dipole pattern was still present although its extent was smaller geographically. The high latitude maxima became part of a wavetrain-like feature which followed the wavetrain pattern of the second EOF, but was $90^{\circ}$ out of phase with it. This pattern would have emphasized the different climatic states of El Niño and La Niña over New Zealand. 


\section{LGM simulation:}

\section{New Zealand}

\section{Introduction}

This chapter will discuss the LGM simulation of the regional model NZ_40km. This chapter is arranged the same way as chapter 6 . In the presentation of the data and the following discussion, often only those areas that can be compared against the data from the pre-industrial simulation are presented. That means that the climate of the new landmasses during the LGM is not discussed. However, at times they are mentioned in order to explain a climatic feature of the "mainland" of New Zealand. The LGM climate is mainly compared against the simulated pre-industrial climate, but there are various references to geological proxy data, which would support or contradict the findings. No absolute comparisons are made with the geological proxy data, since the range of proxy findings is too large to describe a unique climate. Therefore, this simulation supplements the existing dataset and ideas about the state of New Zealand's climate during the LGM, rather than it excludes or emphasizes a particular point of view. On the other hand, irreconcilable differences between the two approaches should lead to further investigations. As was mentioned in chapter 6 , the strength of a RCM lies in its capability to model internal variability at high spatial resolution. The internal variability of the RCM under LGM conditions is however impossible to verify with geological proxy 
data. The temporal and spatial resolutions of the geological proxy data are too coarse. Therefore only the mean climatic states of the pre-industrial and LGM climate are compared.

\subsection{Setup}

The RCM used for the simulation of the climate during the LGM is the same as for the pre-industrial simulation, but with the inclusion of the LGM specifications described in section 7.1. However, some settings in this simulation are quite different and need to be determined first. These fields and parameters are discussed below.

\section{- Orography:}

New Zealand's orography during the LGM was for the first part constructed as described in section 7.1. The regional domain was extracted from the global domain. This gave a good land mask for New Zealand during the LGM, but certainly not the right orography. New Zealand's Alps were covered in large glaciers during the LGM. It is not suitable to model these glaciers with Peltier's ice sheets (Peltier, 1994) since the resolution of this dataset is too coarse for the regional model. There are a number of studies that have described the possible extent and height of the New Zealand glaciers during the LGM, but there is no database available that allow for easy inclusion into the UM. For that reason, the extent and height of the glaciers were included under the "best estimate" assumption. The height of the glaciers were defined for the following areas: Fiordland, 1700m; Southern Lakes, 2500m; Mt Cook, 3000m; Rakaia, 2500m; Arthur's Pass, 2000m; Nelson Lakes, Inland Kaikouras up to Marlborough, $1800 \mathrm{~m}$ (Personal communication with J. Shulmeister). Although there are signs that permanent glaciers might have existed in the North Island as well, since their extent would probably not have been very large compared to the resolution of this model, there were no glaciers put on top of the orography of the North Island. In an effort to make the transition between areas of different glacier heights smoother, an arbitrary tapering was applied. Although this might be a 


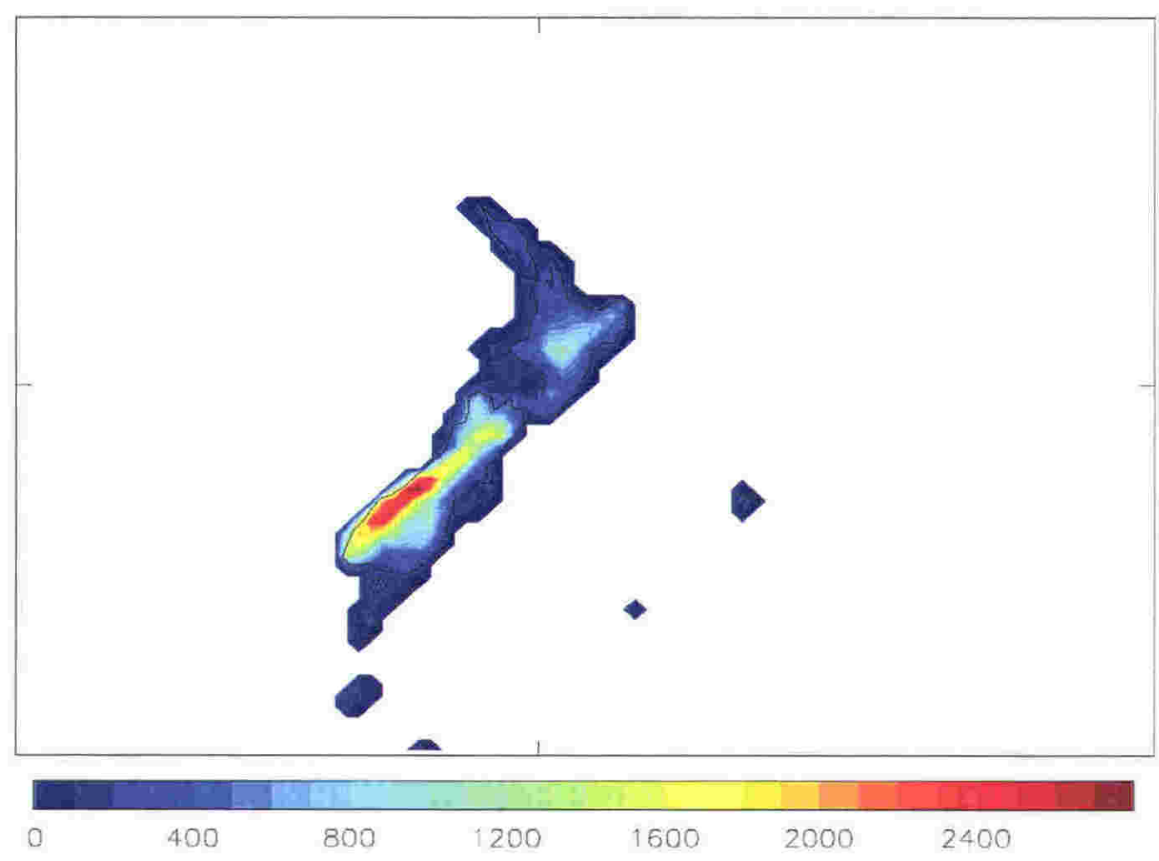

Fig. 8-1. New Zealand's modelled orography for the LGM simulation in its rotated position. Height is in meters.

very rough estimation of the orography of the LGM, since there is no way of knowing what the orography during the LGM was exactly, it was deemed to be appropriate for this model's resolution. As was noted in chapter 6, the modelled orography has a very strong impact on the regional climate, and it is therefore possible that a different estimation of the height of New Zealand's orography during the LGM (more/less glaciers) could produce a different climatic response. However, no sensitivity experiments have been carried out yet to investigate this.

New Zealand's landmass was much larger during the LGM as a result of the lower sea level. Cook Strait and Foveaux Strait disappear, and the three main islands of New Zealand become one large landmass (Fig. 8-1). The Chatham Islands become a bigger island too. The small islands in the far south, the Auckland Islands and Campbell Island, are now large enough to show up in this resolution. The Bounty Islands are to the southeast of New Zealand. The landmass of the LGM undoubtedly had different drainage basins, but similar to the pre-industrial simulation, no river catchments were configured for this simulation. New Zealand's total landmass during the LGM is covered by 288 grid points in the model. 

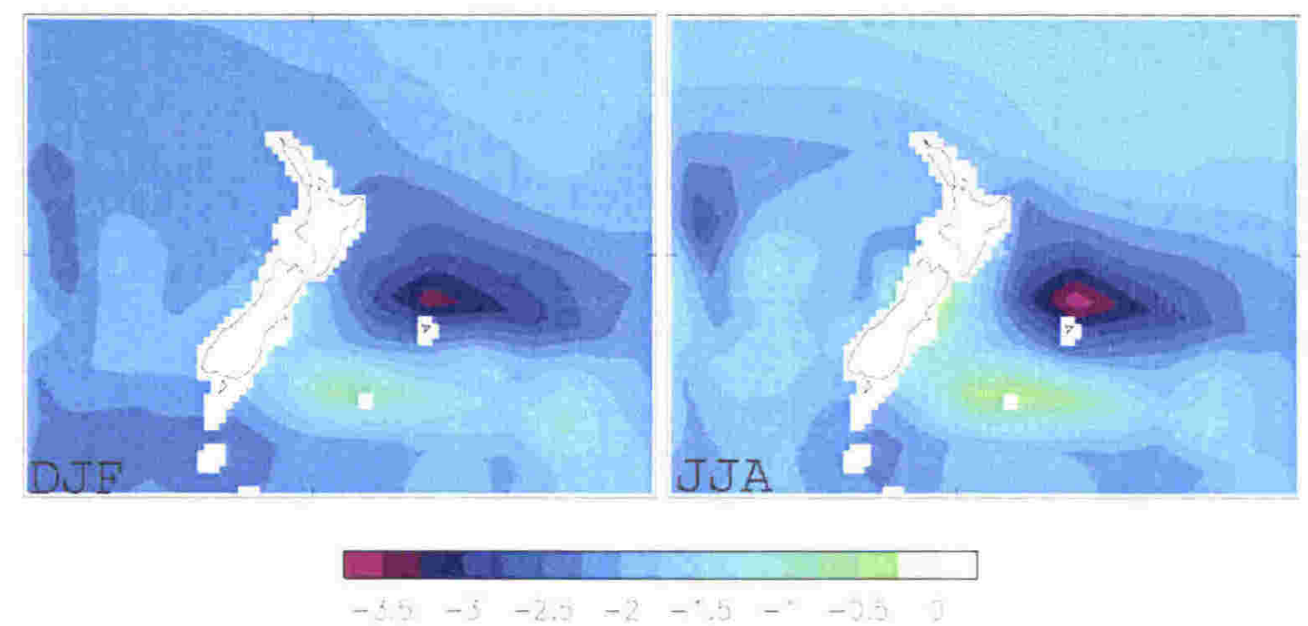

Fig. 8-2. Difference in SST $\left({ }^{\circ} \mathrm{C}\right)$ between the LGM and pre-industrial simulation in NZ_40km for DJF (left) and JJA (right). SSTs are from HadCM3 (Hewitt, 2000).

\section{- SST and sea-ice:}

The SSTs and sea-ice were interpolated from the LGM GCM fields and were updated every month. Although the sea-ice expanded equatorward considerably during the LGM (see Fig. 7-3), and it is known from geological proxy data that icebergs did make it all the way to the Chatham Rise at times (Carter et al., 2002), the RCM does not have any incursions of sea-ice in its domain during the modelled period.

Fig. 8-2 presents the differences in seasonal averages in SST between the LGM and pre-industrial simulation for DJF and JJA. The largest amount of cooling takes place to the east of New Zealand and north of the Chatham Rise. The origin of these waters is to the east of Australia and they were transported by the Tasman Current, the East Auckland Current and the East Coast Current (see Fig. 3-5). The SSTs of these currents are relatively warm. The large amount of cooling to the east of New Zealand indicates therefore that these currents were weaker during the LGM. The weaker currents transported less heat to the east of New Zealand and allowed the cold Southland Current to extend its influence farther northwards, over the Chatham Rise (Nelson et al., 1993: Nelson et al., 2000). At the same time, the smallest reduction in SST takes place south of the Chatham Rise. This region experiences intrusions of cold water from the Antarctic Circumpolar Current (ACC) in the present day, so the small reduction 
in SST is probably more related to the surfacing (lower sea level) of a branch of the ACC than to any possible change in the direction of the ACC. However, Barrows et al. (2000) find that the maximum reduction in SST takes place to the southeast of New Zealand and can be up to $6^{\circ} \mathrm{C}-10^{\circ} \mathrm{C}$. They ascribed that to the equatorward shift of the ACC. Similar findings and suggestions came from other studies (e.g. Nelson et al., 1993: Royal Society of New Zealand, 1994: Nelson et al., 2000). HadCM3 does not simulate an equatorward shift of the ACC in the New Zealand region, which explains why the SSTs do not show such a strong cooling to the southeast of New Zealand. As discussed in chapter 4, HadCM3's main errors in the Southern Hemisphere SSTs are probably associated with the incorrect positioning of the ACC. It is therefore not possible to use this simulation's lack of a shift of the ACC as an argument against the colder temperatures found by other studies. CLIMAP (1976: 1981) gives cooler SSTs during JJA of $2^{\circ} \mathrm{C}-3^{\circ} \mathrm{C}$ to the west and $1^{\circ} \mathrm{C}-2^{\circ} \mathrm{C}$ to the east of New Zealand, and a cooling of only $0^{\circ} \mathrm{C}-2^{\circ} \mathrm{C}$ in the west, and a slight warming to the east during DJF. CLIMAP's findings are at odds with the findings of the studies mentioned earlier. Analysis of simulations using different SSTs will differ considerably.

\section{- Tasman Sea:}

One of the discussion points about the climate of New Zealand during the LGM is whether there were any major changes in the Tasman Sea. Although this study deals with the modelling of the atmosphere, it is worth commenting on how HadCM3 modelled the Tasman Sea during the LGM. It needs to be kept in mind that the resolution of HadCM3 was too low to be able to solve the small-scale features of the very complex currents that are present in the New Zealand area. The correctness of the modelled Tasman Sea is not in question, but its large-scale features are mentioned here since the SSTs and their distribution in the Tasman Sea affect the climate of New Zealand. Furthermore, the strength of the currents in the Tasman Sea during the LGM will give an indication of the state of some atmospheric variables as well.

A close up look at the differences between the pre-industrial and LGM SSTs 

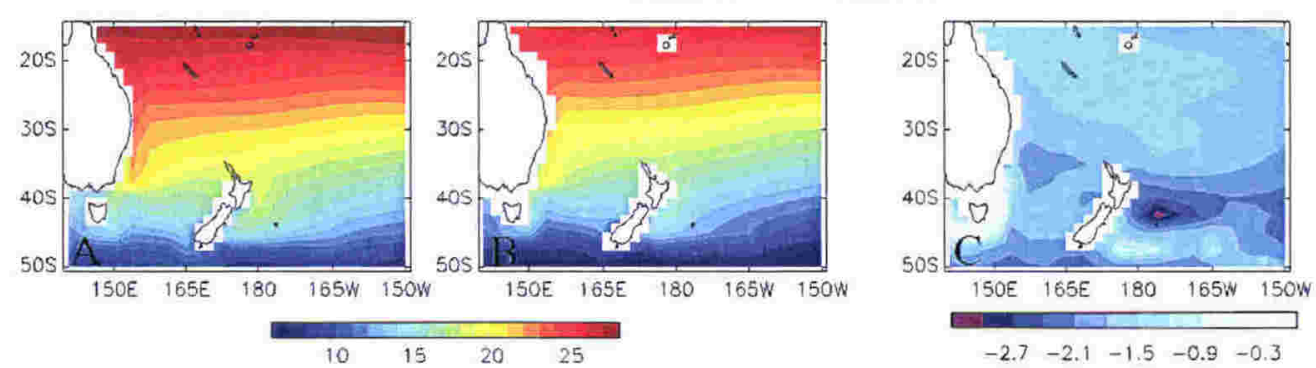

Fig. 8-3. Annual mean SSTs (in ${ }^{\circ} \mathrm{C}$ ) for the South West Pacific for the pre-industrial (A) and the LGM simulation (B). The differences, $\mathrm{SST}_{\mathrm{LGM}}-\mathrm{SST}_{\text {pre-industrial, }}$ are presented in figure $\mathrm{C}$. SSTs were computed by HadCM3 (Hewitt, 2000).

in the Tasman Sea and around New Zealand show some interesting features (Fig. 8-3). The warm East Australian Current (EAC) seems to penetrate less far south during the LGM than during the pre-industrial. But the waters forming the EAC are colder during the LGM. Therefore, it can not be determined convincingly from the SST patterns whether the EAC was weaker during the LGM or whether the cooler SSTs along the east coast of Australia followed the general reduction in SST during the LGM.

The Tasman Current flows from Australia to New Zealand at about $35^{\circ} \mathrm{S}$, and turns around the north of New Zealand (East Auckland Current, EAUC, see Fig. 3-5) to the east coast of New Zealand (East Coast Current, ECC). Although the resolution of $\mathrm{HadCM} 3$ is too coarse to discern this feature in detail, the transport of warm water to the east of New Zealand can be seen by the kink in the isotherms in the SSTs in the pre-industrial simulation (Fig. 8-3A). This feature is virtually missing in the SSTs of the LGM, which is an indication that the strength of the Tasman Current during the LGM was weaker.

The Tasman Sea is clearly colder during the LGM. However, it can not be concluded that that is the result of a possible shift northwards of the colder subAntarctic waters associated with the ACC as was discussed in section 3.3.2. There is not enough detail to draw that conclusion. The colder SSTs in the Tasman Sea can be the result of the cooler SSTs globally.

More information is gained by analysing the stream functions of the two simulations (Fig. 8-4). The data for both pictures in Fig. 8-4 is normalised against the pre-industrial value for Australia. Although the value of the stream function 


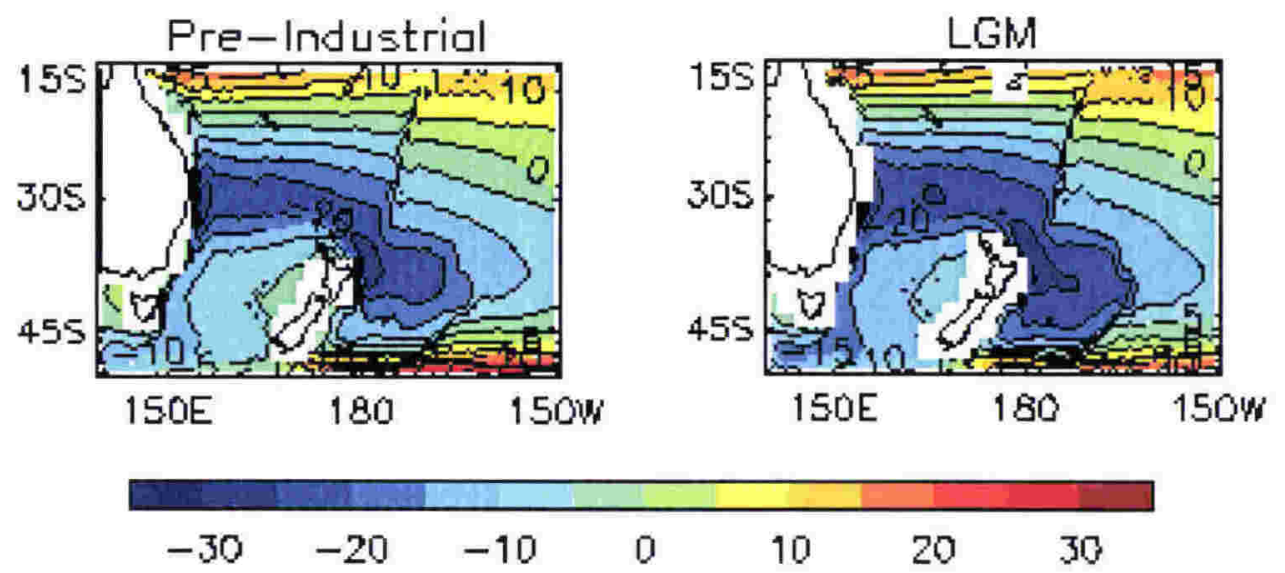

Fig. 8-4. Stream function (in Sv) for the South Western Pacific for the pre-industrial and LGM simulation as modelled by HadCM3 (Hewitt, 2000).

over Australia during the LGM was slightly higher than in the pre-industrial simulation, the data is presented this way in order to improve the visualization of the differences between the two simulations. The downside of this presentation is that absolute comparisons can not be made, and only trends in the data can be investigated. Fig. 8-4 shows that HadCM3 does a reasonably good job in reproducing the main features of the waters around New Zealand. Both the preindustrial and LGM simulation show clearly the Tasman Front, the Deep Western Boundary Current (DWBC), and the East Australian Current (EAC). The Antarctic Circumpolar Current (ACC) just comes in at the bottom of the pictures.

The net southward flow in the Tasman Sea in the pre-industrial simulation is about $4.5 \mathrm{~Sv}$ and nearly $6.9 \mathrm{~Sv}$ in the LGM simulation. This means that HadCM3 transports more water southwards through the Tasman Sea in the LGM simulation than in the pre-industrial simulation. This is mainly caused by an increase in the flow of the branch of the EAC that turns southwards, towards Tasmania (about 2.3Sv). At the same time, HadCM3 does not simulate a decrease in the flow of water that moves northward along the New Zealand coast. In fact, HadCM3 indicates that there might even have been a slight increase in this flow. An increase in the northward flow along the New Zealand west coast would imply a northward shift of the Subtropical Front (STF). Geological proxy data indicates that such a shift might have happened (e.g. Barrows et al., 2000: 
Carter, 2001). There is only the slightest hint of a reduction of the strength of the EAC turning into the Tasman Front. The spacing of the streamlines at the Tasman Front is slightly larger during the LGM. However, this could very well be an effect of the presentation of Fig. 8-4, since any difference between the fronts in the two simulations is smaller than the coarse resolution of the figure.

An strengthening of the southwards branch of the EAC during the LGM was also found by Hirst and Godfrey (1993). They ascribed the cause of the stronger current to the near closure of the Indonesian through flow. The Indonesian through flow is nearly closed in HadCM3 as well, but the stream function for the Indonesian through flow actually indicates that there was a slight increase in the through flow during the LGM. An increase in both the Indonesian through flow and the southwards turning branch of the EAC is consistent with the closure of the Baring Strait and with a possible increase in the subtropical gyre. The strengthened flow through the Tasman Sea during the LGM can be an indicator of a stronger westerly wind during the LGM. Although such a statement is difficult to support with the limited amount of data, it does rhyme with the findings in section 7.2.6 where it was determined that the westerly winds were slightly stronger in the zonal mean during the LGM.

\section{- Vegetation and soil:}

Although quite a lot is known about the vegetation in New Zealand during the LGM, there is no database available which format would allow easy implementation into the UM. For that reason the vegetation and soil characteristics in New Zealand during the LGM were also set up under the "best estimate" assumption. A new dataset that described New Zealand's vegetation and soil characteristics during the LGM was created. Only a very few vegetation and soil characteristics were included and these had to be compatible with the UM codes and descriptions for those parameters. The database was created on the same resolution as the US Navy 10" dataset in order to be consistent with the creation of the new orography. Fig. 8-5 shows the RCM vegetation and soil for New Zealand during the LGM. As mentioned in section 3.3.2, most of New Zealand's vegetation during the LGM was grassland and shrubs. The criterion for 

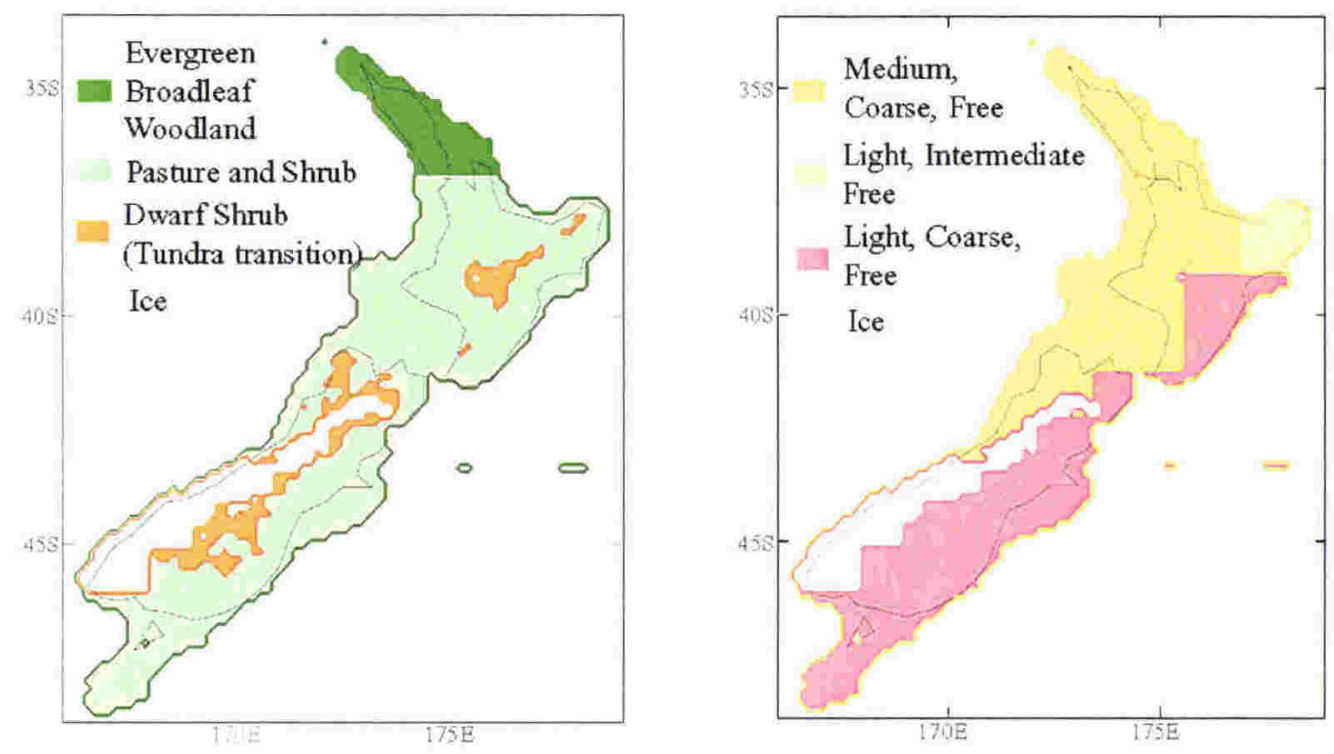

Fig. 8-5. The vegetation and soil data used to create the vegetation and soil parameters for the LGM simulation. The soil characteristics refer to colour, texture and drainage respectively.

the vegetation was that all vegetation above $800 \mathrm{~m}$ was set to dwarf shrub (tundra transition and high altitude wasteland), except for those grid points that were covered in ice (glaciers). Although it is not clear whether the Volcanic Plateau was covered in permanent ice, the grid point associated with Mt Ruapehu was set to ice as well. The vegetation over the rest of the country was set to pasture and shrub, except for the area north of Auckland, where the vegetation was set to evergreen broadleaf woodland. Soils are characterized by three properties, colour, texture and drainage. The east of New Zealand was given light colour, coarse texture and free drainage, the Raukumara Peninsula a light colour, intermediate texture and free drainage, and the rest of the country a medium colour, coarse texture and free drainage, except for those areas covered by glaciers (including Mt Ruapehu) which were set to ice. These datasets were created in consultation with J. Shulmeister. They are very simple, but are reasonably consistent with the general findings of New Zealand's vegetation and soil properties during the LGM.

- Snow:

The extent and the height of the snow pack during the LGM are not known. The 
global simulation had initially a $50 \mathrm{~m}$ high snow pack on top of the ice sheets (Hewitt, 2000) and the simulation lasted long enough for the snow pack to become an integral part of the hydrological cycle. Since this simulation is only over a few years, it was not deemed appropriate to artificially add a thick snow pack onto the regional domain, since the period might be too short for the snow pack to establish equilibrium. Snow cover and soil moisture were not set in the simulation. Snow cover at the end of the simulation is therefore solely the result of the physics and dynamics of the model.

\section{- Chemistry:}

Ozone values were interpolated from the GCM values, and the tropospheric chemistry was set to similar levels as in the GCM. Aerosols were not included.

\subsection{Results}

The regional model ran nested within the GCM for 21 model years. Only the last 16 years of the simulation have been used in the analysis of the RCM data, Salinger and Mullan (1999) describe how New Zealand's climate has varied between 1930-1994. They found that regional temperature and precipitation in New Zealand respond significantly to circulation changes in the southwest Pacific. Since the climatic variations between the LGM and the pre-industrial simulation are more dramatic, it is to be expected that the regional climates in New Zealand will have different responses in temperature and precipitation. The results of the simulation are presented as in chapter 6 .

\subsubsection{Temperature}

Fig. 8-6 shows the mean annual surface temperature during the LGM. The warmest areas are in the Far North, the Coromandel, and the east of the North Island around the Hawke's Bay and Gisborne area. The large extended landmass over the western continental shelf has a similar mean annual surface temperature 


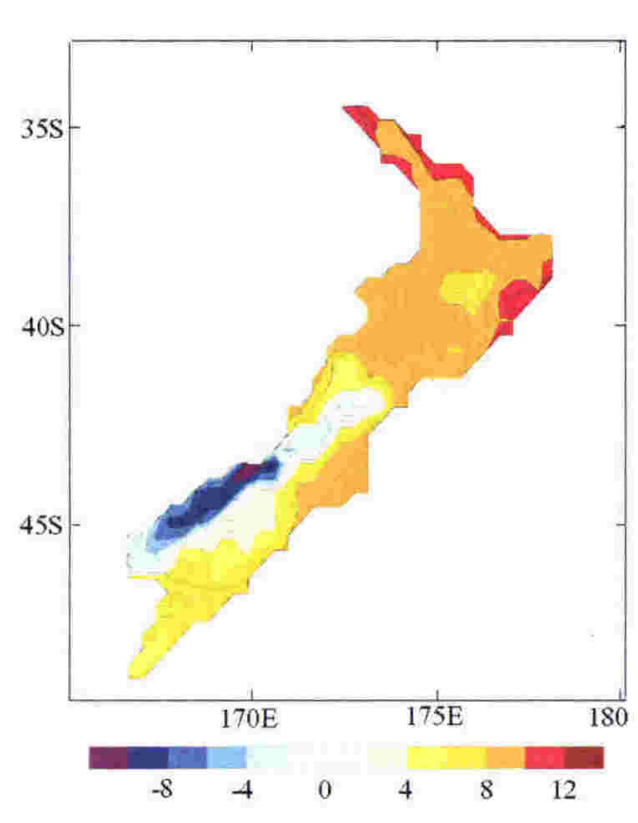

Fig. 8-6. The annual mean surface temperature (in ${ }^{\circ} \mathrm{C}$ ) during the LGM. as in most of the North Island and does not show much geographical variation. In the North Island, only the Volcanic Plateau and one grid point associated with the Northern Tararuas show the cooler mean annual temperatures which are associated with high orography.

The mean annual surface temperature in the Southern Alps stands out again, but it is much lower than in the pre-industrial simulation. Although the surface temperature during the LGM was lower, the large difference between

the LGM and pre-industrial surface temperature in the Southern Alps (compare Fig. 8-6 with Fig. 6-6) are for a large part due to the differences in their modelled heights of orography. The cool area of the Otago district and the warmer region of Canterbury in the present day's climate are present during the LGM as well. The large extended land mass in the south of the country has similar mean annual surface temperatures as the Otago and Southland region.

The main conclusion that can be drawn from the annual mean surface temperatures during the LGM in New Zealand is that temperature regimes that are present in today's climate are also present in the climate of the LGM. Table 8-1 shows the average annual surface temperature for the six regions as well as for New Zealand during the LGM. For comparison, the pre-industrial values of Table 6-1 are presented here again. What immediately stands out is the very large amount of cooling in the West and South South Island (WSSI) area. This large cooling is mainly due to the inclusion of the glaciers in the LGM simulation. Although in the present climate the West Coast and Southland vary coherently and have therefore been grouped together in the same region (WSSI), this would not necessarily have been the case during the LGM. Besides a different global 


\begin{tabular}{|c|c|c|}
\hline Region & LGM & pre-industrial \\
\hline North North Island & 10.97 & 14.00 \\
\hline South-West North Island & 8.74 & 11.48 \\
\hline East North Island & 10.11 & 12.81 \\
\hline North South Island & 6.87 & 9.80 \\
\hline West and South South Island (WSSI) & -1.14 & 6.35 \\
\hline East South Island & 6.05 & 9.99 \\
\hline New Zealand & 5.32 & 9.95 \\
\hline
\end{tabular}

Table 8-1. The annual mean surface temperature for each region of Fig. 6-4 and for the whole of New Zealand for the LGM and the pre-industrial simulation. The LGM values are determined for exactly the same points as for the pre-industrial, that is, the extra land points during the LGM are not included in the calculation.

climatology, the enhanced orography during the LGM could have turned the southern Otago and Southland area into a region responding more like the East Coast; a region "sheltered" by the mountains. When splitting up WSSI into a "West Coast - Fiordland" and a "Southern Otago-Southland" region, the amount of cooling over the former is in the order of $8^{\circ} \mathrm{C}-10^{\circ} \mathrm{C}$, where as in the latter is in the order of $2^{\circ} \mathrm{C}-4^{\circ} \mathrm{C}$. Therefore, the very large amount of cooling in WSSI takes place primarily in the mountains.

The amount of cooling in the North Island is between $2.7^{\circ} \mathrm{C}-3.0^{\circ} \mathrm{C}$, and is quite uniform. The North of the South Island experiences a similar amount of cooling, but the East of the South Island is colder by nearly $4^{\circ} \mathrm{C}$. Several studies have indicated that the SSTs were considerably lower southeast of New Zealand during the LGM. These cold SSTs could have caused much cooler temperatures in the east and southeast of New Zealand. However, the computed SSTs did not reproduce this large cooling (for references and SSTs, see section 8.1, SST and sea-ice). Therefore, the strong cooling in the east of New Zealand in this simulation has to have a different cause. It will be shown later that the reduced westerly wind and an increase in southerlies in this region are contributing factors to the larger amount of cooling in this region. The amount of cooling over the whole of New Zealand of about $4.6^{\circ} \mathrm{C}$ sits exactly in the range of $4^{\circ} \mathrm{C}-5^{\circ} \mathrm{C}$ as was suggested by several other studies (e.g. Markgraf et al., 1992: McGlone et 
al., 1993: Chinn, 1996). However, the average amount of cooling is more in the order of $2.5^{\circ} \mathrm{C}-4^{\circ} \mathrm{C}$ when the very strong cooling over the $\mathrm{Alps}$ is not taken into account. Such a relatively small reduction in the annual mean temperature would still allow for forests to exist in most of New Zealand, which disagrees with geological proxy data that most of New Zealand vegetation during the LGM were grasses (e.g. McGlone et al., 1993). Other factors limiting forest growth should have taken place as well in order to account for this discrepancy. It will be suggested later that the increase in seasonality and the increased incursions of very cold polar air might have contributed to limiting forest growth, and that in the annual mean, the amount of cooling might not have been as large as determined by proxy data. That would imply that the simulated cooling of $2.5^{\circ} \mathrm{C}$ $4^{\circ} \mathrm{C}$ could be a reasonable approximation.

Fig. 8-7 shows the difference in surface temperature between the LGM and the pre-industrial simulation for each season. Only values over the land mass in the pre-industrial simulation have been presented since the variations over sea are primarily related to the differences in SSTs. The strongest cooling is over the Southern Alps, where both the glacier coverage as well as the much higher orography of the LGM results in much lower surface temperatures. The difference in orography height between the LGM and the pre-industrial simulation can be up to $1000 \mathrm{~m}$ for some grid points. If the LGM lapse rate was similar or steeper than today's lapse rate (e.g. $6^{\circ}-8^{\circ} \mathrm{C} / \mathrm{km}$, see Fig. 7-9) then the impact of the glaciers on the amount of cooling ranges from $8^{\circ} \mathrm{C}$ to $10^{\circ} \mathrm{C}$ (It's impact on the amount of cooling as the result of its direct contact with the atmosphere can only be estimated).

There are two regions that need a more detailed analysis. Of note are the differences in the seasonal surface temperatures in the Otago area. Otago can be classified as having a climate similar to a continental climate, since it experiences a strong seasonality, particularly in temperature. Some areas in Otago are not much cooler during DJF, but experience much colder temperatures during winter. This results in an increase in seasonality. This indicates a quite severe climatic regime in Otago, which would have had a significant impact on the existence of certain types of vegetation and the characteristics of soils. 

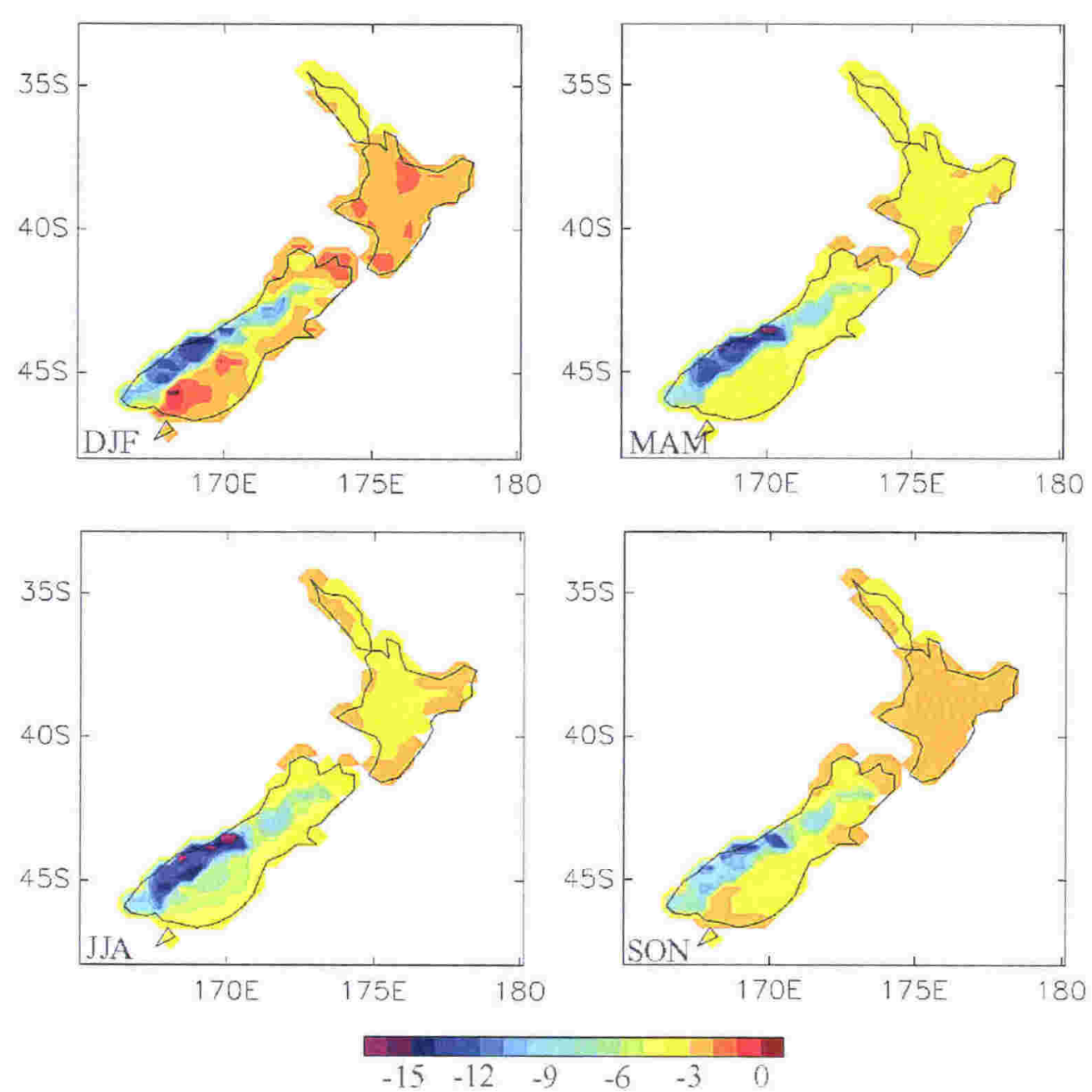

Fig. 8-7. The difference in surface temperature $\left({ }^{\circ} \mathrm{C}\right)$ between the LGM and the preindustrial simulation for each season. Only the difference over the pre-industrial land mass is presented.

The second area that warrants a closer look is the area north of Auckland. Parts of this region show consistently a larger amount of cooling than most of the rest of the North Island. Northland is the area that is most influenced by the warm northerly winds. Although a reduction in northerlies could possibly explain the slightly stronger cooling in this area, it can not explain the sharp change in the amount of cooling over Auckland. The most probable cause is the impact of the different type of vegetation over Northland on the surface temperature. The modelled vegetation has a sharp transition from grass and pasture to forest north of $37^{\circ} \mathrm{S}$ (see Fig. 8-5). 

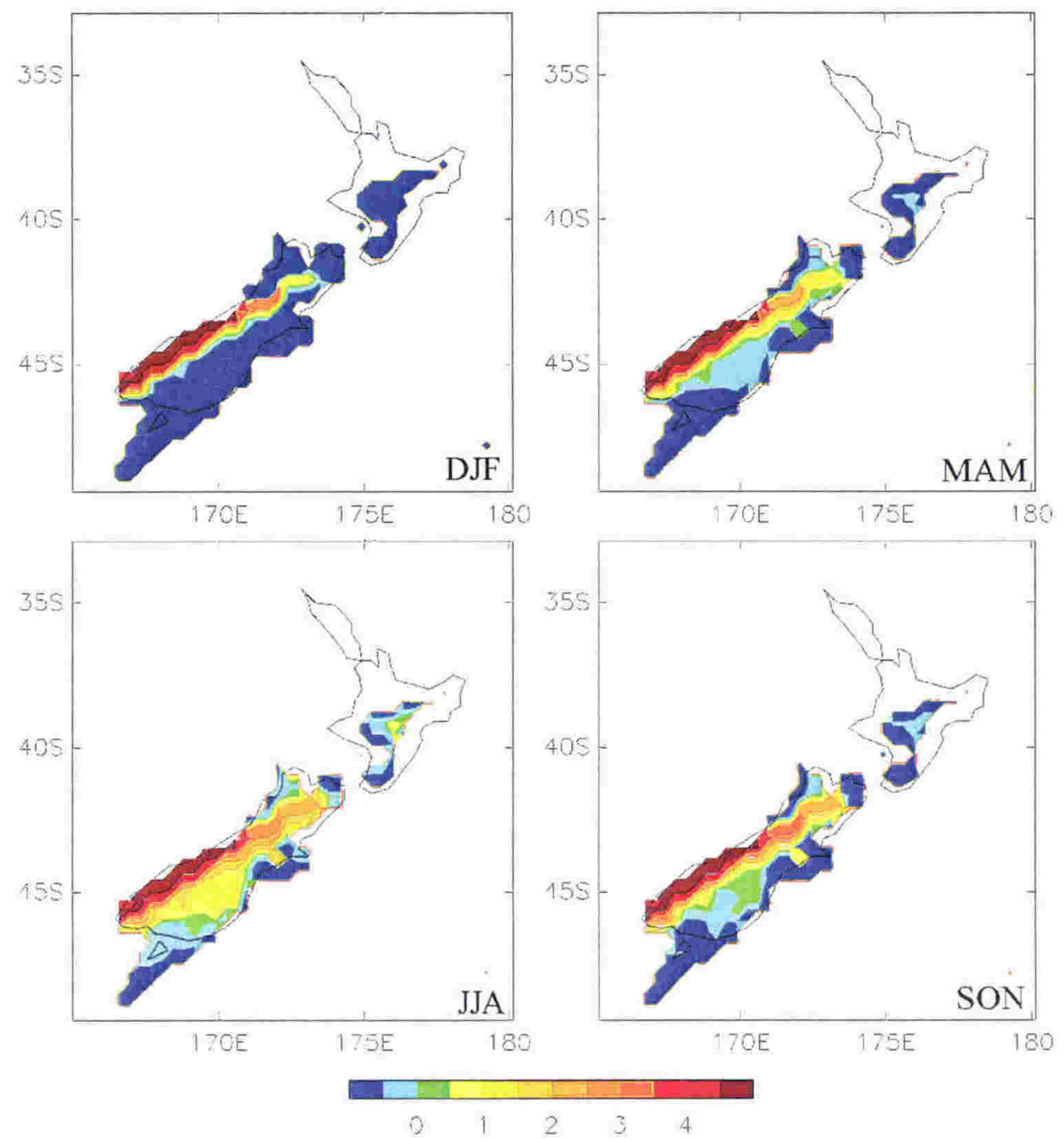

Fig. 8-8. The seasonal snow cover of the greater landmass of New Zealand during the LGM. Snow cover is in $\log 10 \mathrm{~mm}$.

One of the main impacts of the cooler temperatures of the LGM was on the occurrence of snow cover. Precipitation is discussed later in chapter 8.2.2, but Fig. 8-8 shows clearly that snow cover during the LGM was fairly common for most of the South Island during JJA. The snow cover is presented for the greater landmass of New Zealand during the LGM. The data is presented as the log 10 of the amount of snow in $\mathrm{mm}$. The blue colours therefore indicate a snow cover of less than $1 \mathrm{~mm}$ thick. Snow on the ground enhances the regional cooling, since it increases the surface albedo.

The region's mean seasonal surface temperatures are given in Table 8-2. The 


\begin{tabular}{|l|r|r|r|r|r|r|r|r||}
\hline \hline Region & \multicolumn{3}{|c|}{ LGM } & \multicolumn{3}{|c|}{ pre-industrial } \\
\hline & DJF & MAM & JJA & SON & DJF & MAM & JJA & SON \\
\hline \hline NNI & 15.88 & 10.86 & 6.49 & 10.65 & 18.54 & 14.39 & 9.67 & 13.40 \\
\hline \hline SWNI & 13.96 & 8.49 & 4.04 & 8.48 & 16.06 & 11.75 & 7.13 & 10.99 \\
\hline \hline ENI & 16.11 & 9.60 & 4.69 & 10.04 & 18.29 & 12.79 & 7.66 & 12.49 \\
\hline \hline NSI & 12.26 & 6.77 & 1.77 & 6.68 & 14.50 & 9.93 & 5.25 & 9.52 \\
\hline \hline WSSI & 3.99 & -0.86 & -6.80 & -0.89 & 10.74 & 6.82 & 2.15 & 5.70 \\
\hline \hline ESI & 12.63 & 5.72 & -0.55 & 6.39 & 15.60 & 9.88 & 4.38 & 10.09 \\
\hline \hline NZ & 10.74 & 5.26 & -0.06 & 5.34 & 14.71 & 10.22 & 5.38 & 9.51 \\
\hline \hline
\end{tabular}

Table 8-2. Mean seasonal surface temperatures (in ${ }^{\circ} \mathrm{C}$ ) for the LGM and pre-industrial simulation by region.

cooling in the North Island is quite uniform, and the largest amount of cooling takes place during autumn and winter. The seasonality in the amount of cooling in NSI is very similar to that experienced in the North Island. The temperatures are lower by $6.5^{\circ} \mathrm{C}-9.0^{\circ} \mathrm{C}$ in WSSI. The large amount of cooling in the annual mean in ESI was for a large part due to the strong cooling during MAM and JJA.

The very uniform cooling over the regions with low orography, especially over the North Island, gives further support to the suggestion that simple interpolation of the GCM surface temperatures could give adequate values for the surface temperatures of the regional domain. It seems that the impact of the orography during the LGM on the general cooler surface temperatures is similar to the impact of the pre-industrial orography on the pre-industrial temperatures. This is further supported by the cross sections in Fig. 8-9.

The feature that stands out the most in Fig. 8-9 is that the LGM temperature follows the pre-industrial temperature virtually exactly, albeit consistently cooler when compared with comparable points, land versus. land and sea versus. sea. This means that the regional variation during the LGM was not too different to the regional variation in the pre-industrial simulation. As mentioned before, the largest amount of cooling takes place over the Southern Alps and is clearly related to the different modelled heights of the orography. Another aspect of the elevated orography is that there is enhanced lee-side warming. 


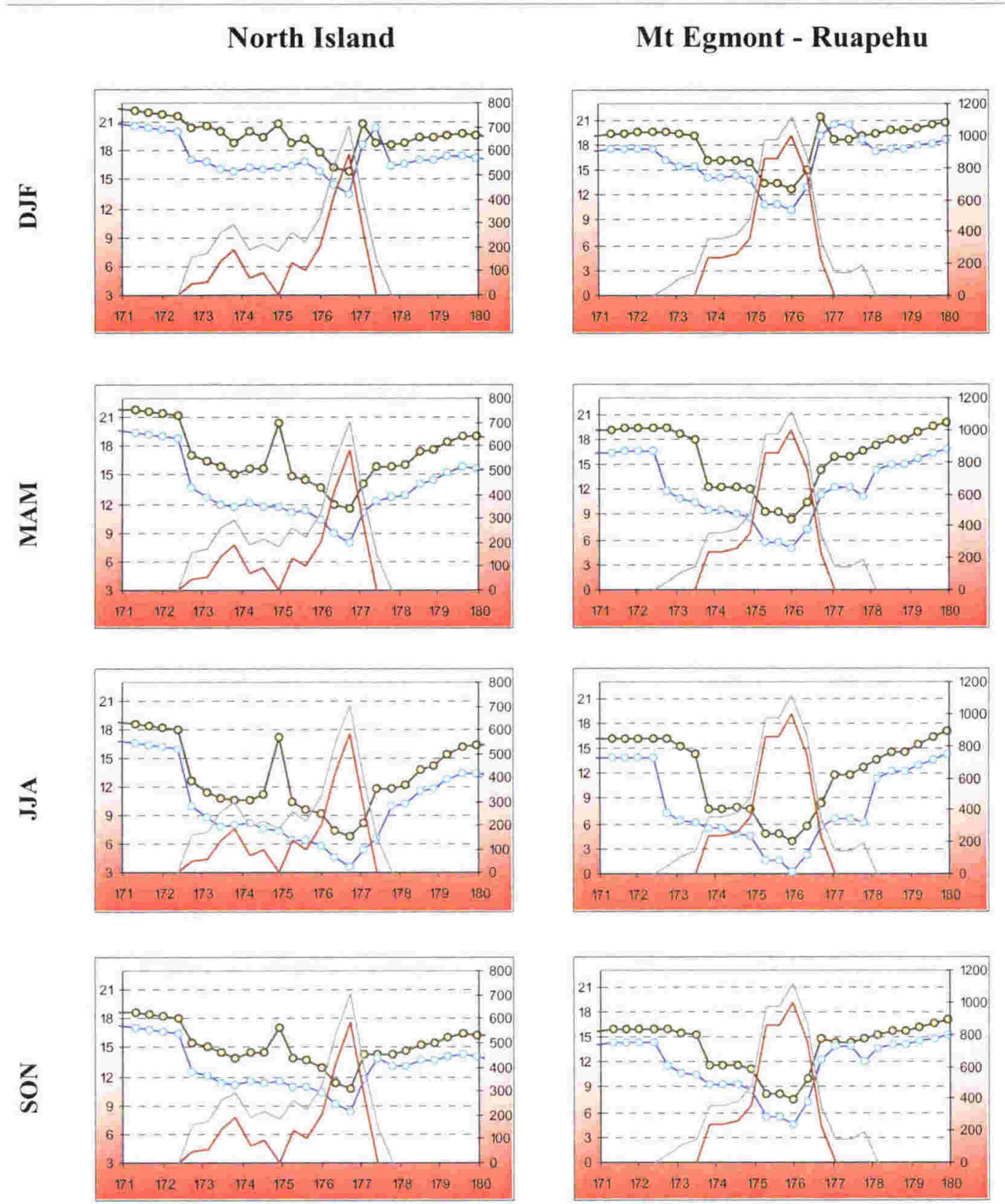

Fig. 8-9. Temperature profiles of the cross sections of Fig. 6-8. X-axis: longitude, Y-axis: left Temperature $\left({ }^{\circ} \mathrm{C}\right)$, right - Height $(\mathrm{m})$. Y-axis scale is different for each cross section. Legend: (irey - NZ LGM orography, Brown - NZ pre-industrial orography, Dark blue with - NZ LGM temperature, Olive green with - NZ pre-industrial temperature.

In the previous section it was discussed that the Tasman Current was weaker during the LGM, which resulted in colder SSTs east of the North Island (see Fig. 8-2). The cross section in Fig. 8-9 shows a larger reduction in surface temperature out at sea, to the east of the country, in the northern transects than in the southern transects. As mentioned earlier, geological proxy data indicate that 


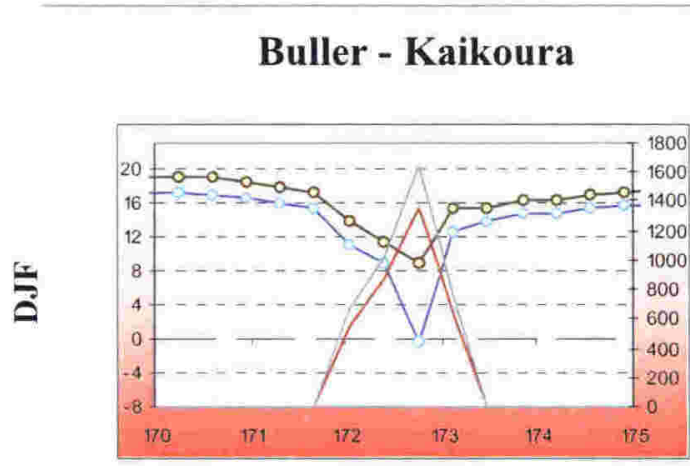

\section{Mt Cook - Banks Peninsula}
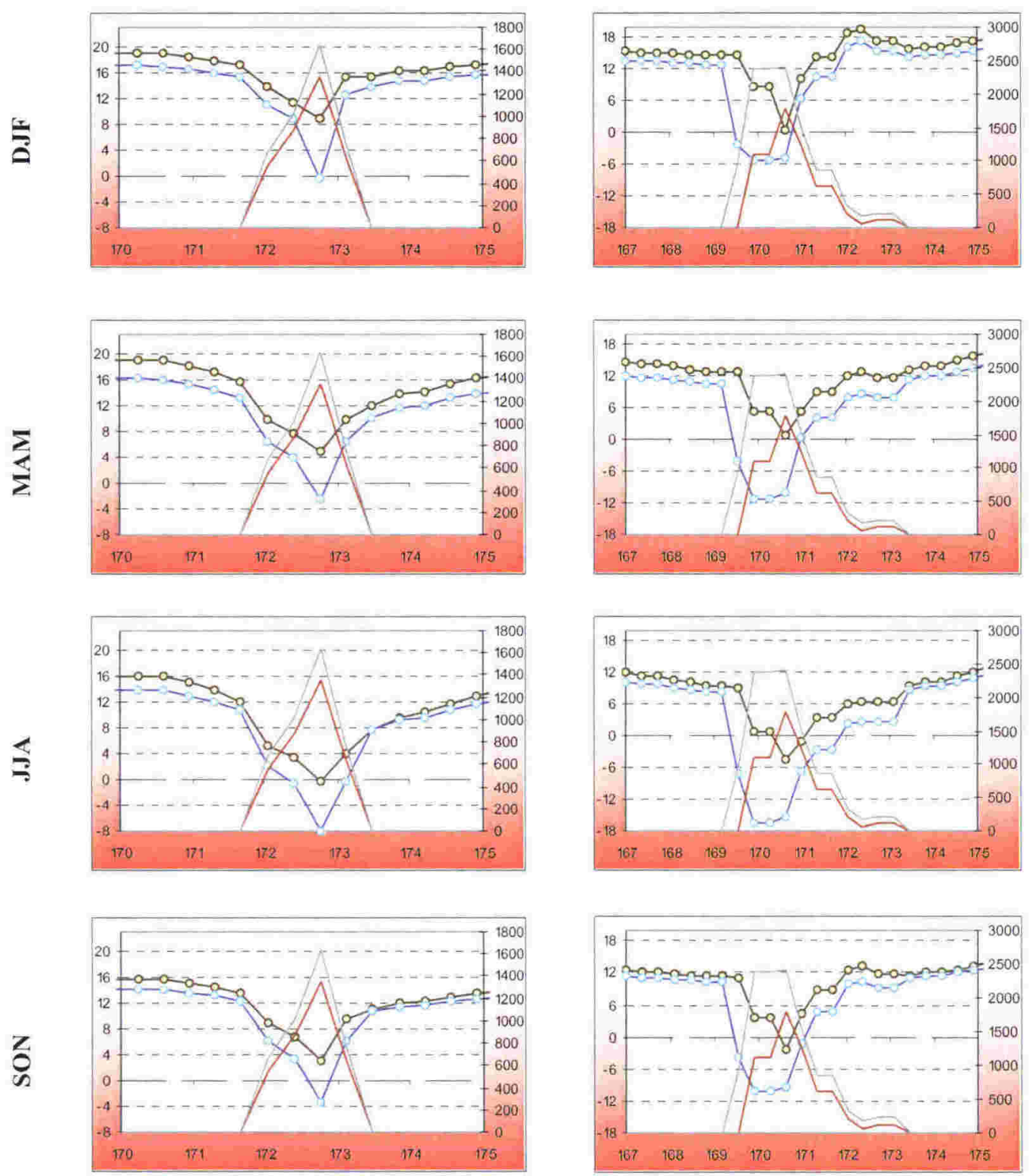

Fig. 8-9. continuation.

there was very strong cooling to the southeast of New Zealand during the LGM, with a possible increase in upwelling of cold water along the east coast of the South Island. If such a scenario had taken place, it undoubtedly would have affected the coastal temperatures in the southeast of the South Island.

The North Island transects show the new landmass in the east. These new land points have higher temperatures than the western areas in these transects, which indicates the lee-side warming that takes place in the east. The Volcanic 

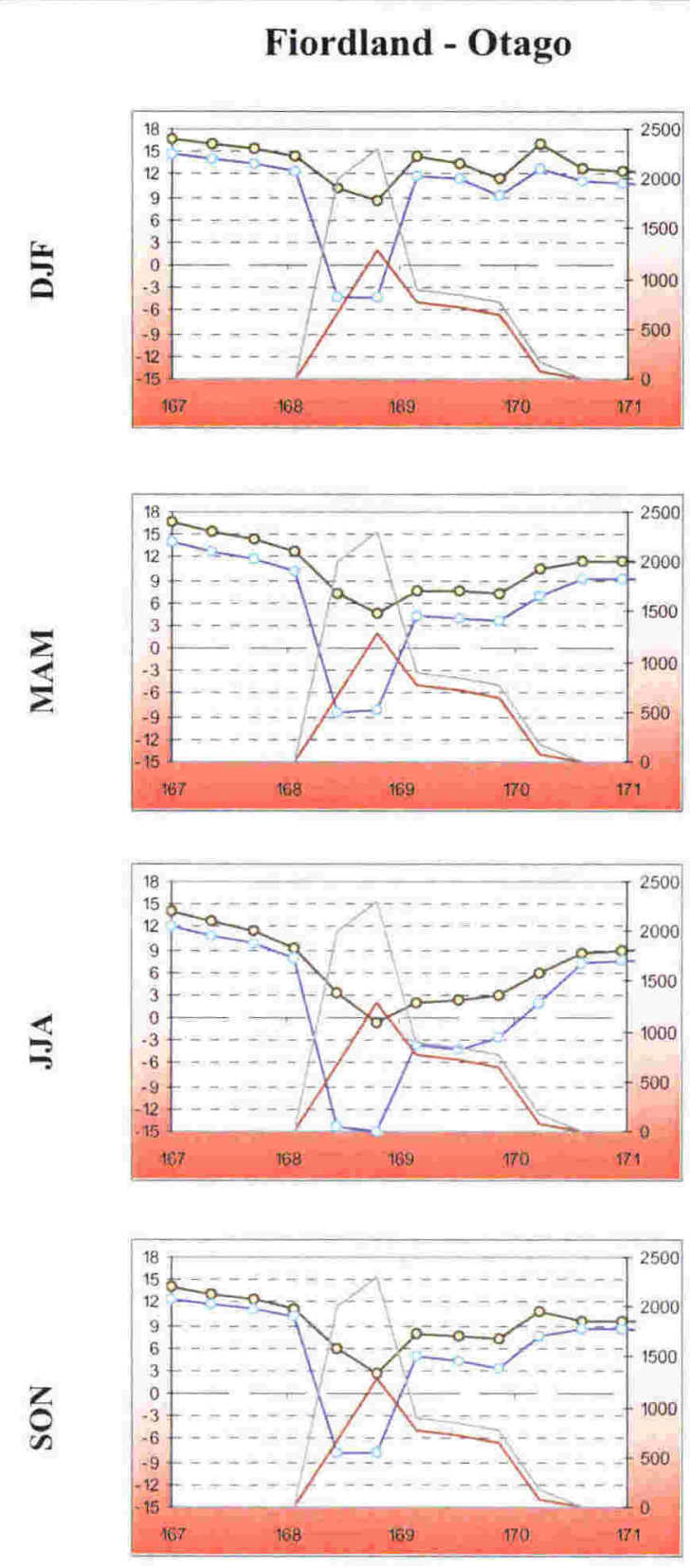

Fig. 8-9. continuation.
Plateau's surface temperatures indicate that it was probably too warm for permanent glaciers to be present on the Plateau, although glaciations could have occurred at smaller scales. These profiles justify the decision not to include glaciers on top of the Volcanic Plateau in the set up of the LGM simulation'.

All South Island transects show that the mountain areas are permanently below freezing level, indicating the existence of the glaciers. The large glaciers did not sit only on top of the Alps, but extended all the way down to sea level on the west coast, where they could still be over a kilometre thick. This effectively shifted the bulk of the modelled orography westward. The result is that the maximum difference in surface

temperature between the two simulations is not over the highest peaks, but at the points of largest difference in modelled orography. This is particularly the case for the West Coast. The change in orography makes it therefore very difficult to compare surface temperature, but also other variables, in this area.

\footnotetext{
${ }^{1}$ Although the model does not support the possibility of permanent glaciers on the Volcanic Plateau, since the real orography is substantially higher than the modelled orography, this statement does not exclude the possibility that glaciers were indeed present on top of the Volcanic Plateau during the LGM.
} 


\subsubsection{Precipitation}

The average annual amount of precipitation in New Zealand during the LGM is shown in Fig. 8-10. Unlike as in Fig. 6-10, the $\log 10$ of the precipitation is presented since no direct comparison needs to be made. The average annual amount of precipitation for each region is given in Table 8-3. The precipitation pattern during the LGM is similar to the pattern of the pre-industrial. The highest amount of rainfall is found in the west, along the Southern Alps, and the driest areas are in the east. The North Island has its highest amount of precipitation over the Volcanic Plateau and has local maxima corresponding to the Tararuas, Mt Egmont and the Ureweras. The amount of precipitation during the LGM is in general less than during the pre-industrial. But there are some remarkable regional variations. There seems to be a westward shift of the main precipitation band along the West Coast, and precipitation in the east of the South Island shows no reduction in precipitation. These patterns are investigated further.

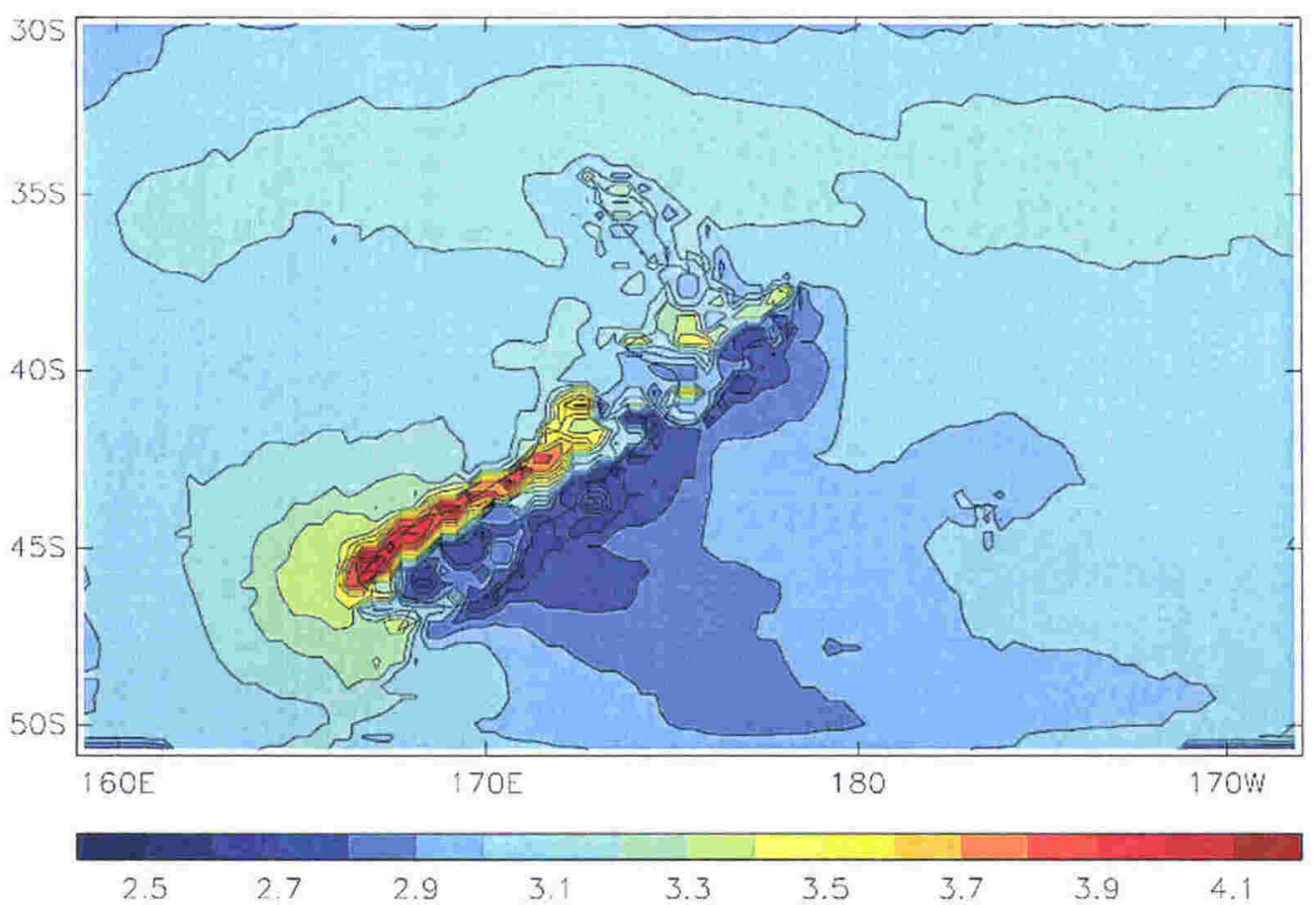

Fig. 8-10. The average annual amount of precipitation $(\log 10(\mathrm{~mm}))$ during the LGM in the regional model. 


\begin{tabular}{|l|c|c|}
\hline \multicolumn{1}{|c|}{ Region } & LGM & Pre-ind. \\
\hline \hline North North Island & 1405.0 & 1556.3 \\
\hline \hline South-West North Island & 1678.8 & 1924.0 \\
\hline \hline East North Island & 1079.7 & 1201.5 \\
\hline \hline North South Island & 2302.5 & 2680.8 \\
\hline \hline West and South South Island & 3638.6 & 3864.7 \\
\hline \hline East South Island & 866.4 & 844.9 \\
\hline \hline New Zealand & 2182.3 & 2363.0 \\
\hline
\end{tabular}

Table 8-3. The average annual amount of precipitation (in $\mathrm{mm}$ ) for each region of

Fig. 6-4 and for the whole of New Zealand.

Fig. 8-11 presents the precipitation differences for each season between the LGM and the pre-industrial simulation (LGM-Pre-industrial). The amount of precipitation in both simulations is presented in Table 8-4. Although the average amount of precipitation over New Zealand is less during the LGM, there is a striking pattern of an increase in precipitation to the west of Westland and Fiordland. At the same time there is also a large reduction in precipitation over the Southern Alps. The cause for these large changes is related to the modelled orography of the LGM. As was clearly presented in the temperature cross sections, the bulk of the modelled LGM orography in the South Island has shifted westward (see Fig. 8-9). The resulting effect is that in the predominantly westerly winds, the air mass has to ascend earlier, more to the west, and higher to cross the divide than during the pre-industrial simulation Precipitation will therefore occur more to the west as well. The resulting "rain shadow" also shifts westward and covers those areas that receive the highest amount of precipitation during the pre-industrial simulation. That explains the large negative difference between both simulations over the Alps. Following the analysis on the West and South South Island region done in section 8.2.1, the West Coast-Fiordland region experiences a reduction in the annual precipitation of $150 \mathrm{~mm}$ (but with a slight increase during DJF), and the Southern Otago - Southland region experiences a reduction of $300 \mathrm{~mm}$. The latter is a reduction of $30 \%$ on its pre-industrial total $(946 \mathrm{~mm})$. Together with the increase in seasonality in temperature, this region would have experienced very harsh conditions during the LGM. A westward 


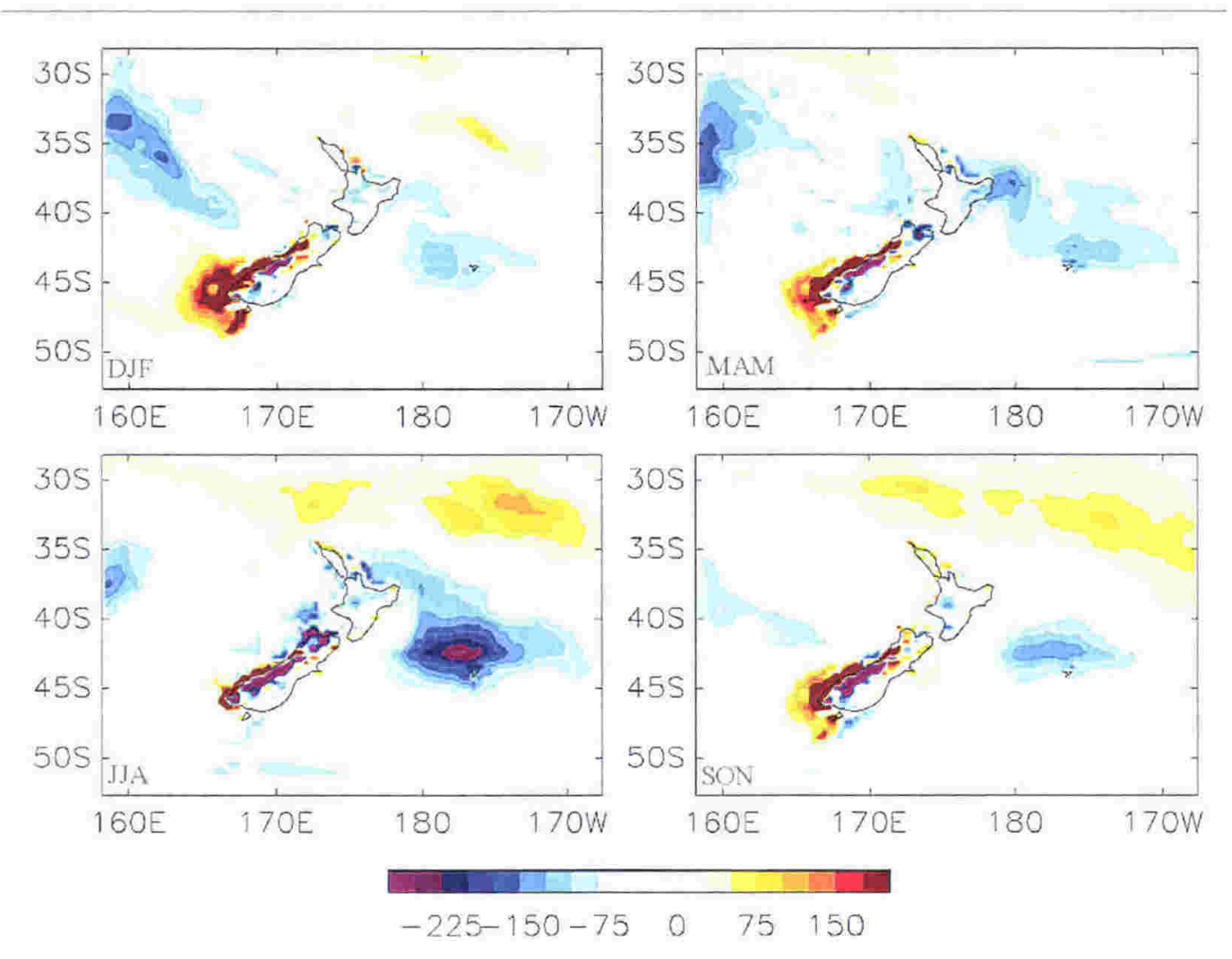

Fig. 8-11. The difference in average seasonal precipitation (in $\mathrm{mm}$ ) between the LGM and preindustrial simulation for each season.

shift of the main precipitation band in the South Island during the LGM confirms geological evidence that expansion of the glaciers to the east of the main divide was limited during the LGM. This topic is further discussed in a paper yet to be completed.

The average annual precipitation during the LGM was reduced over New Zealand except in the east of the South Island. The latter is in contrast with some proxy data which indicate that Canterbury was also drier during the LGM (e.g. Moar, 1980). Although as discussed in section 6.3.2, the model seems to underestimate the precipitation in the east of New Zealand. The apparent lack of a reduction in the precipitation in the east of the South Island does not therefore automatically imply that there was not a real reduction in precipitation during the LGM. Table 8-4 shows that there was virtually no change in the amount of precipitation in the east of the North Island during JJA and SON. The Southern Hemisphere mean zonal circulation in the pre-industrial simulation had its largest 


\begin{tabular}{||r|r|r|r|r|r|r|r|r||}
\hline \multicolumn{1}{|c|}{ Region } & \multicolumn{4}{|c|}{ LGM } & \multicolumn{4}{|c|}{ pre-industrial } \\
\hline \hline & DJF & MAM & JJA & SON & DJF & MAM & JJA & SON \\
\hline \hline NNI & 365.4 & 316.3 & 403.0 & 320.2 & 429.2 & 350.6 & 451.0 & 325.4 \\
\hline \hline SWNI & 424.2 & 356.9 & 426.3 & 471.3 & 490.1 & 395.6 & 489.5 & 548.8 \\
\hline \hline ENI & 247.7 & 219.7 & 388.3 & 223.9 & 292.4 & 299.6 & 383.5 & 226.1 \\
\hline \hline NSI & 552.0 & 497.2 & 585.8 & 667.5 & 604.3 & 611.2 & 777.8 & 687.5 \\
\hline \hline WSSI & 938.9 & 827.5 & 709.9 & 1162.2 & 914.1 & 871.1 & 855.6 & 1223.8 \\
\hline \hline ESI & 217.4 & 186.1 & 247.4 & 215.5 & 213.3 & 188.4 & 262.1 & 181.0 \\
\hline \hline NZ & 554.8 & 486.2 & 504.5 & 636.8 & 575.0 & 531.4 & 591.8 & 664.7 \\
\hline \hline
\end{tabular}

Table 8-4. Average seasonal precipitation ( $\mathrm{mm})$ for the LGM and pre-industrial simulation by region.

biases during SON. It had a weaker SAO, which would have resulted in, among other changes, enhanced precipitation (section 5.2.3). For a region not to show a reduction in precipitation during $\mathrm{SON}$ is therefore quite indicative of precipitation being not less than during the pre-industrial. Areas that experience a similar amount of precipitation as in the pre-industrial during SON are not only in the east, but also in the north of both islands ${ }^{1}$.Precipitation increases in WSSI during DJF.

In the analysis of the characteristics of the Tasman Sea during the LGM, it was concluded that the Tasman Front had weakened, resulting in cooler SSTs along the north and east of the North Island. The large cooling out to the east of New Zealand, particularly during JJA, explains the large local reduction in precipitation. The colder waters north of the Chatham Rise during the LGM would have inhibited convection.

Fig. 8-12 presents the precipitation profiles of the cross sections for both the LGM and pre-industrial data. Of note is how the precipitation patterns of the LGM follow the ones from the pre-industrial simulation very consistently in the cross sections of the North Island. Although the orography was slightly higher,

\footnotetext{
1 The NNI region experiences enhanced precipitation in its most northern region, which is the area that was covered in forests. The question therefore arises what the impact of the vegetation was on the occurrence of precipitation. This has yet to be investigated.
} 


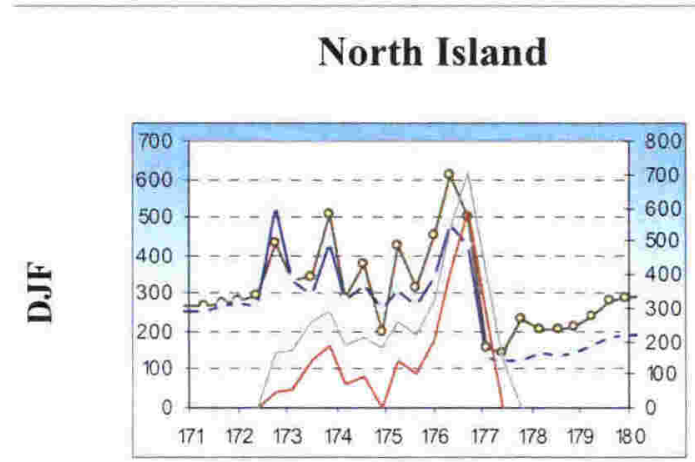

\section{Mt Egmont - Ruapehu}
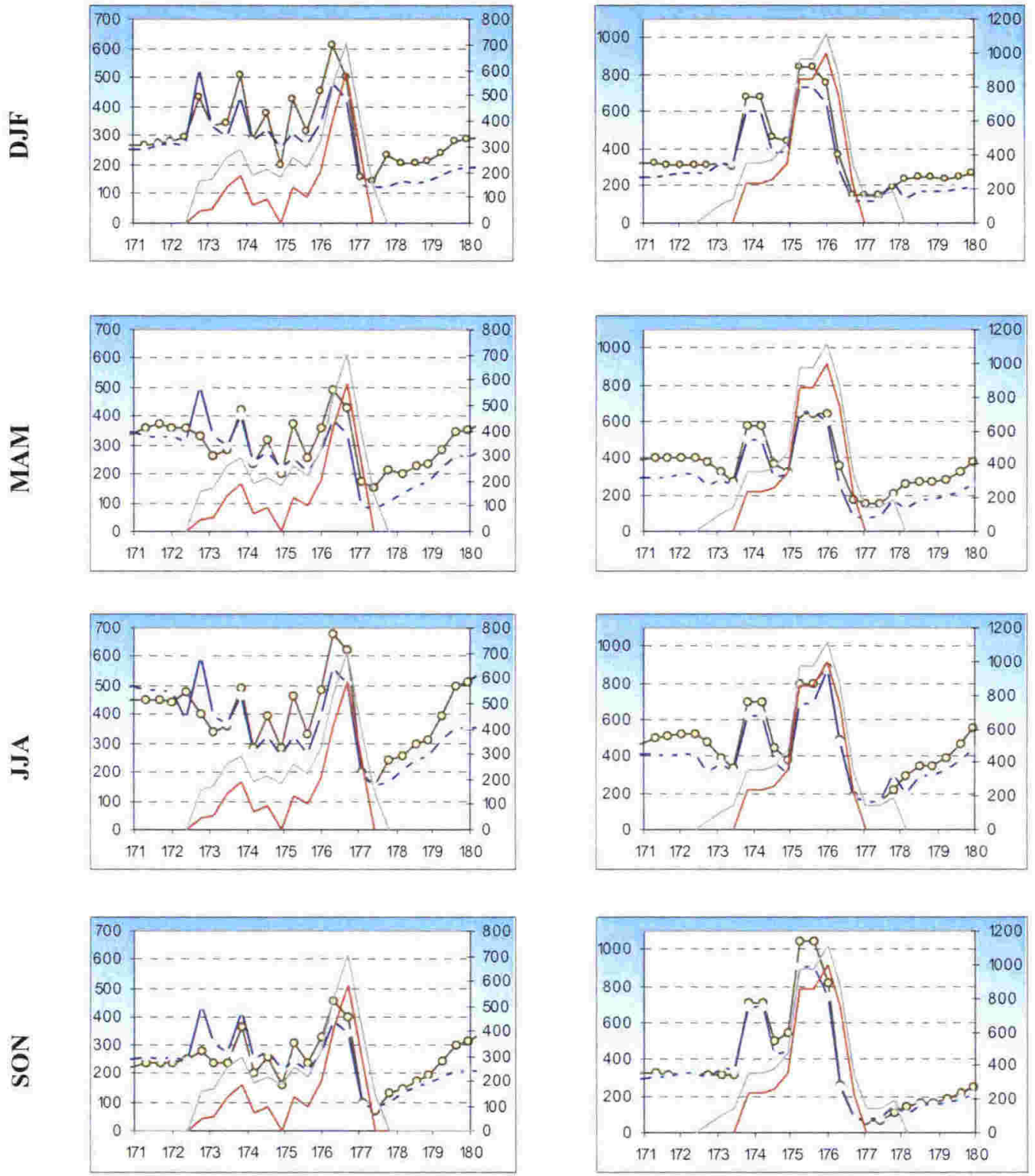

Fig, 8-12. Precipitation profiles of the cross sections in Fig. 6-8. X-axis: longitude, Y-axis: left Precipitation ( $\mathrm{mm})$, right - Height $(\mathrm{m})$. Both y-axis scales change for each cross section. Legend: Grey - NZ LGM orography, Brown - NZ pre-industrial orography, Dark blue with - NZ LGM precipitation, Olive green with - NZ pre-industrial precipitation.

this did not change the modelled precipitation pattern. The only change occurs in the magnitude of the precipitation. The steep increase in precipitation in the Far North is associated with the relatively large increase in the orography at the coastal point. 


\section{Buller - Kaikoura}
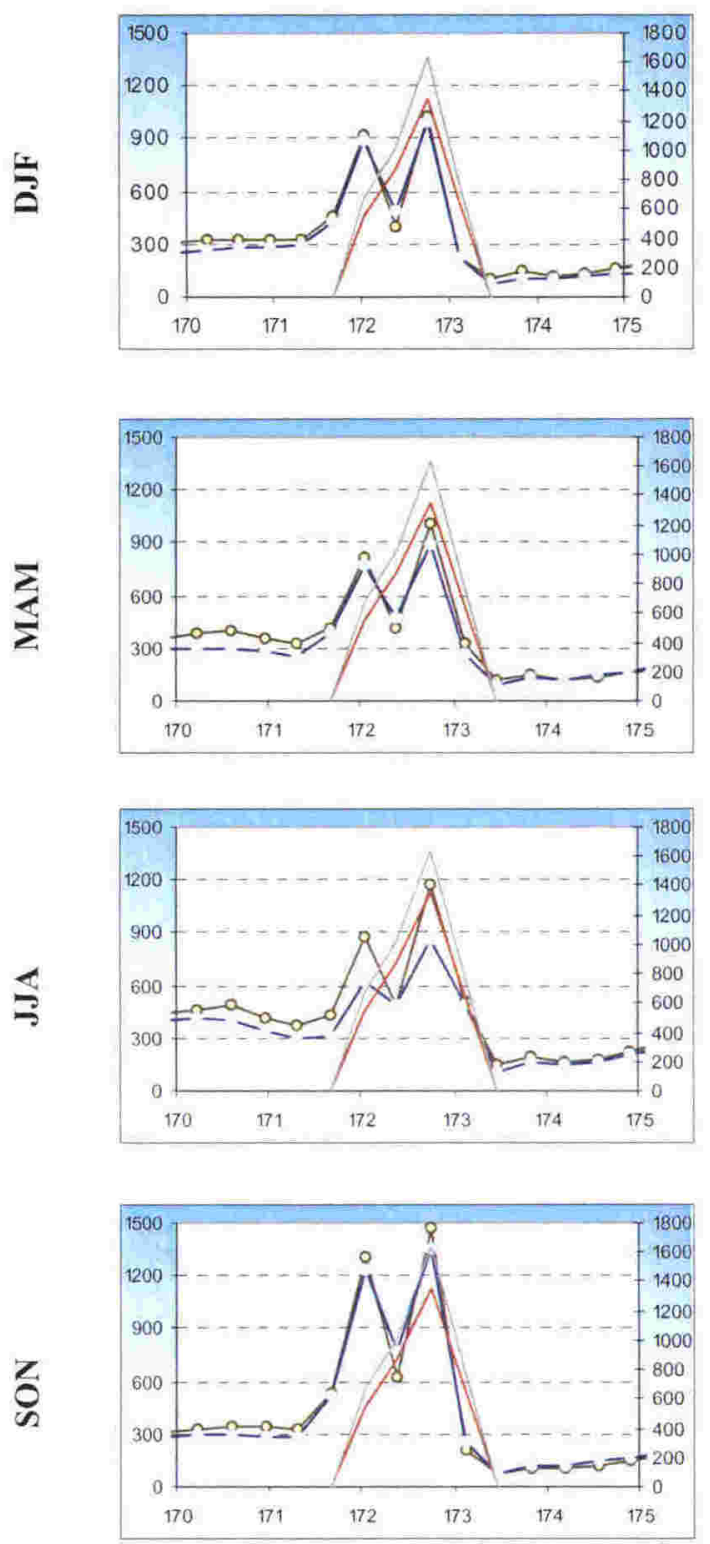

Mt Cook - Banks Peninsula
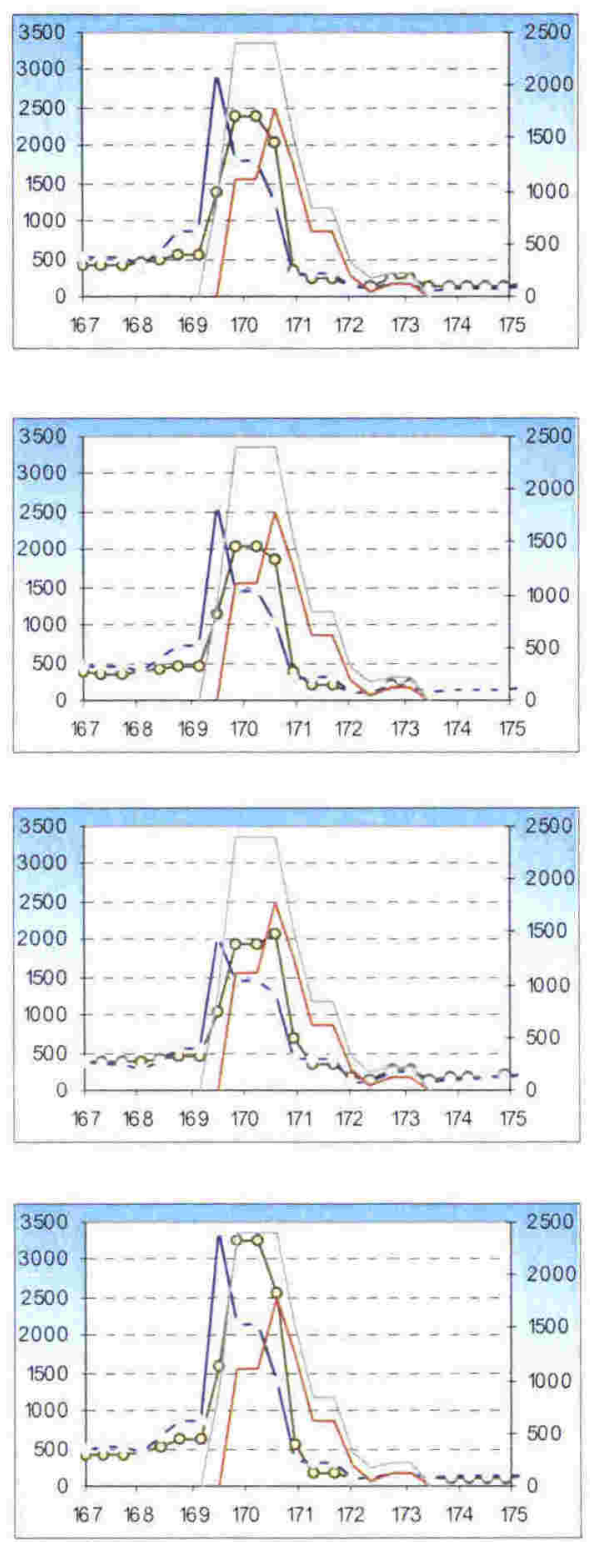

Fig. 8-12. continuation.

As mentioned earlier, the precipitation is generally reduced in the north of the North Island, and this shows up particularly in the areas of high orography, over the Ureweras, Mt Egmont and the Volcanic Plateau. The first grid point of the North Island cross section shows enhanced precipitation during the LGM. This is most likely related to the steep increase in orography, where an atmospheric parcel now has to rise from sea level to over $150 \mathrm{~m}$, instead of only to $30 \mathrm{~m}$. From 


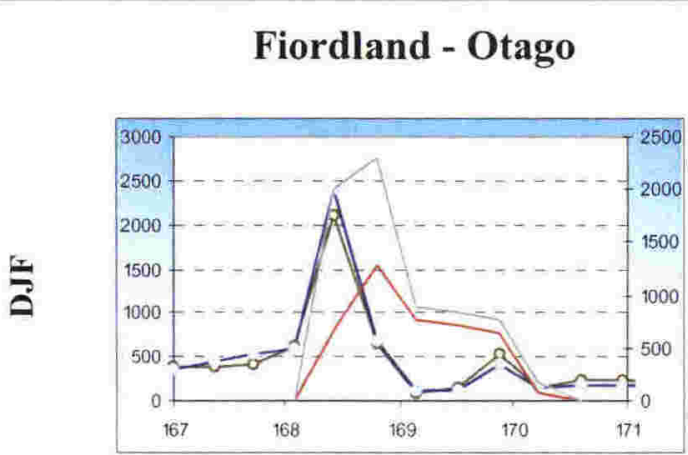

\section{Southern Fiordland - Southland}
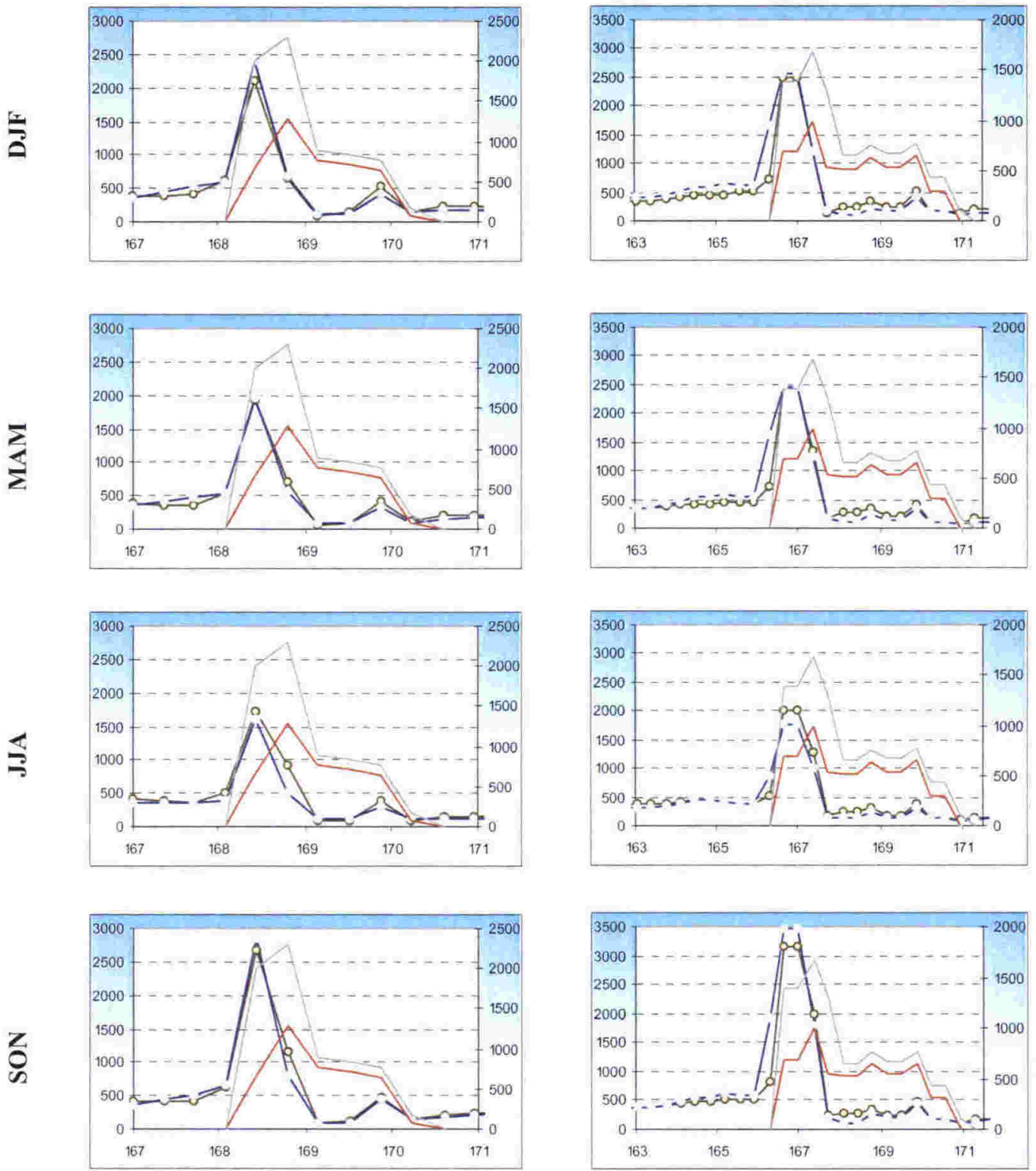

Fig. 8-12. continuation.

the seasonal and regional analysis it was determined that the amount of precipitation during SON was similar to the amount of precipitation in the preindustrial simulation. The North Island cross section shows that precipitation had increased in Northland during SON.

The much gentler rise of orography at the western end of the Mt Egmont Ruapehu cross section did not cause the precipitation to show a sharp peak at the first land point, as it did for the North Island cross section. The precipitation 


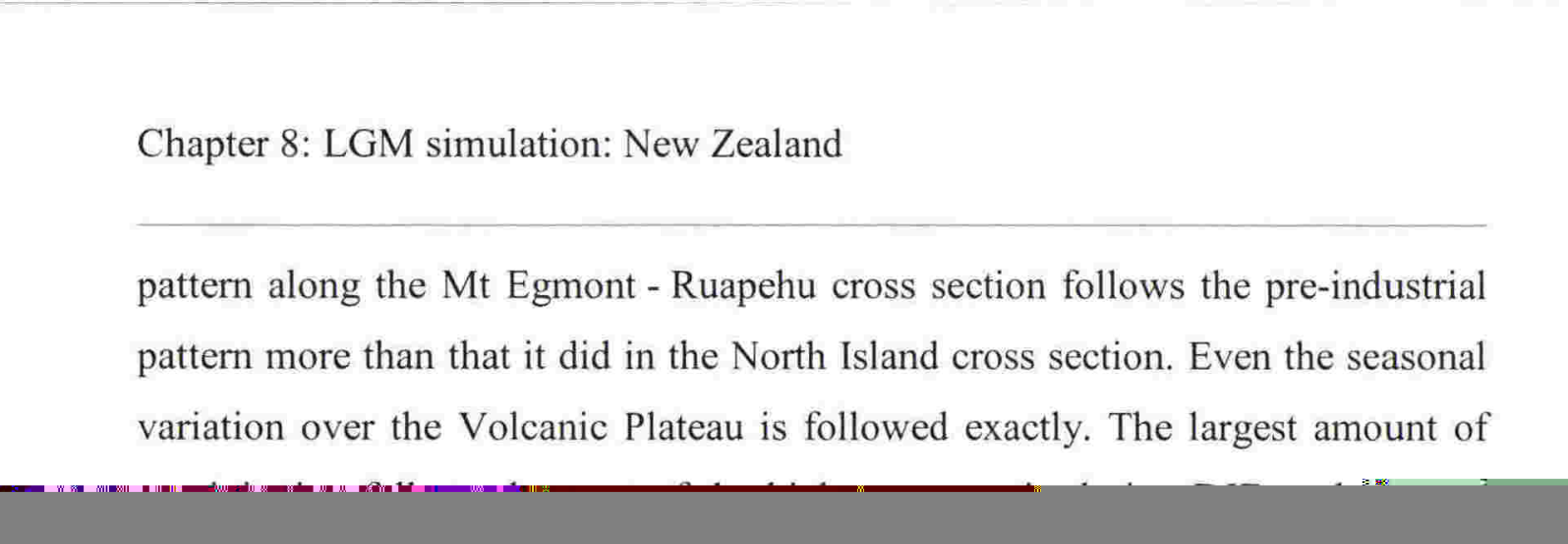


north or south, it would have covered those points. However, such a cross section was not chosen, since any meridional shift in the location of the cross section would cover a smaller range in orography and fewer extremes in data, and would not have added any more information to this analysis. But to support the findings of Fig. 8-11 a zonal cross section, Southern Fiordland-Southland (see Fig. $8-12$ ), is added. This cross section shows clearly the westward shift of the main precipitation band. What is also apparent is that the westward shift is not only restricted to the next grid point to the west, but that it extends well out to sea. In contrast with the Mt Cook - Banks Peninsula cross section, both southern cross sections indicate that the precipitation inland and to the east during the LGM was nearly the same as during the pre-industrial. Only the Mt Aspiring region had slightly reduced precipitation, primarily during JJA and SON.

\subsubsection{Mean Sea Level Pressure \& Winds}

In the previous two sections it was determined that there were considerable regional and seasonal variations in the differences between the pre-industrial and LGM simulations. An obvious cause for these variations could be found in the different wind regimes of the simulations. Fig. 8-13 shows the annual mean sea level pressure in the regional domain. When comparing Fig. 8-13 with Fig. 6-13 it is quite clear that the modelled LGM climate experienced a more dominant south-westerly flow over New Zealand. This is further supported by the data in Table 8-5. Except for the southwest of the North Island, and to a lesser degree for the east of the North Island, the northerly component of the wind has been reduced. Following the discussion in chapter 7 , the reasons for this reduction could be the occurrence of stronger and/or more southerlies, together with weaker and/or less northerlies. The increase in the southerly component of the mean annual meridional wind is completely in accordance with the suggestion made in chapter 7. There it was suggested that the change in the winds in the New Zealand region were influenced by the changes in the planetary waves and the strong cooling over Australia. A colder Australia during the LGM would increase the mean sea level pressure of the overlying air, shifting its strongest 


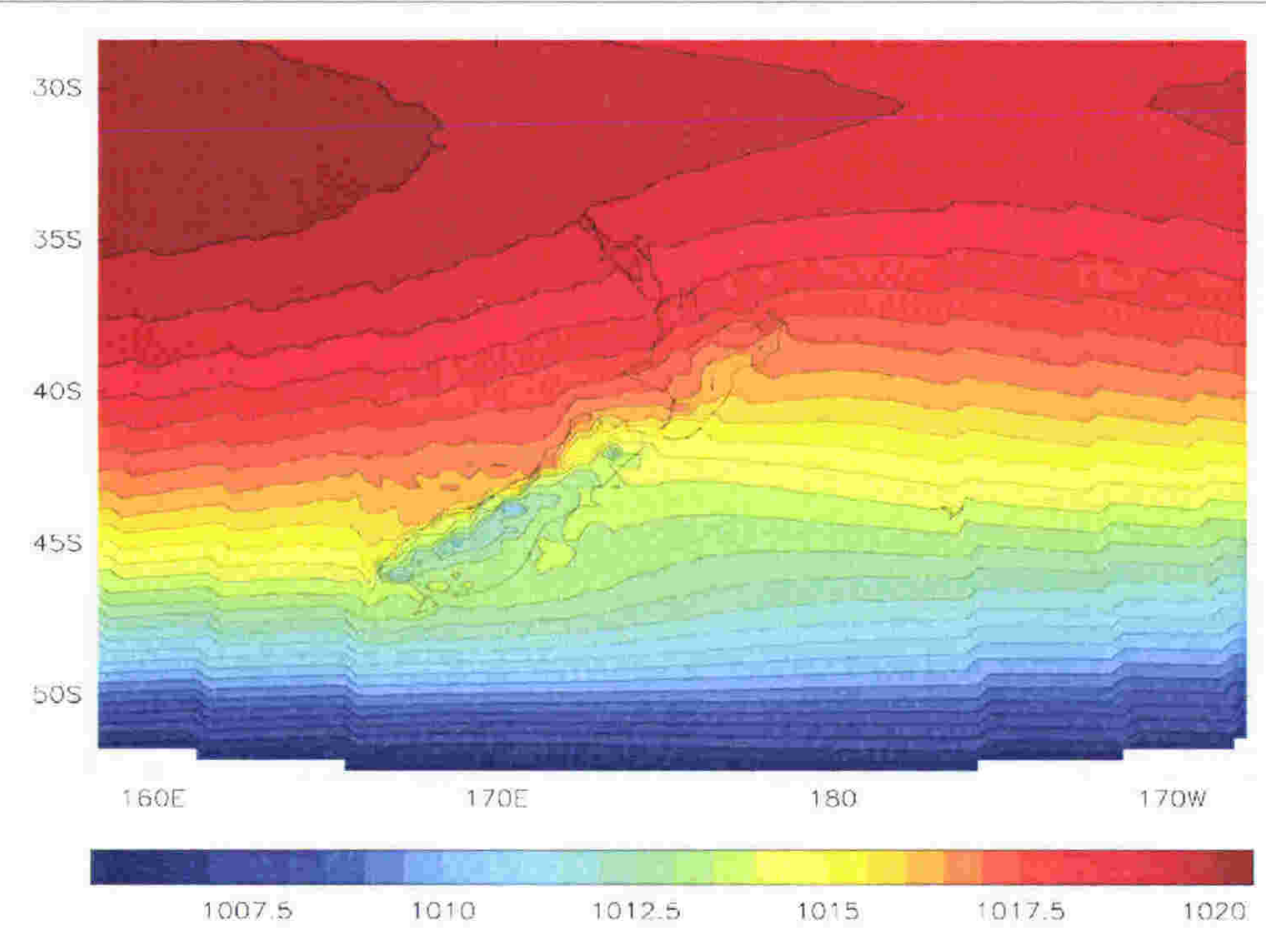

Fig. 8-13. Annual mean sea level pressure (hPa) during the LGM in the RCM.

surface winds polewards. This shift is opposite to the equatorward shift in the zonal mean (Fig. 7-19). A strong equatorward shift of the zonal flow downwind of Australia is required to even out the poleward shift of the westerly winds in the Australian region. This results in a strengthening of the southerly flow over New Zealand.

The stronger westerly flow is also apparent in Table 8-5 which shows an increase in the zonal wind in several regions. However, there are two regions that have a noticeable reduction in the mean zonal wind: WSSI and ESI. The reduction in the zonal wind in WSSI takes place mainly inland. The winds in the Southern Otago - Southland area the pre-industrial simulation were in the annual mean nearly twice as strong as in the West Coast-Fiordland area. The former region has a weakening of the zonal wind by about $7 \%$, where as the zonal wind in the latter region is reduced by $25 \%$. The result is that the mean annual zonal wind in the Southern Otago - Southland area is only about $50 \%$ stronger than in the West Coast - Fiordland area during the LGM. Since the combined regions of WSSI and ESI cover a large part of New Zealand, the average zonal wind over the whole country increases only slightly. 


\begin{tabular}{|c|c|c|c|c|}
\hline & \multicolumn{2}{|c|}{ LGM } & \multicolumn{2}{|c|}{ pre-industrial } \\
\hline & $\mathrm{u}$ wind & $\mathrm{v}$ wind & $\mathrm{u}$ wind & $\mathrm{v}$ wind \\
\hline NNI & 1.841 & 0.543 & 1.483 & 0.356 \\
\hline SWNI & 2.192 & -0.713 & 1.794 & -0.693 \\
\hline ENI & 3.027 & -0.657 & 2.553 & -0.647 \\
\hline NSI & 1.918 & 0.074 & 1.713 & -0.054 \\
\hline WSSI & 1.616 & -0.594 & 1.946 & -0.908 \\
\hline ESI & 1.837 & -0.642 & 2.217 & -0.890 \\
\hline $\mathrm{NZ}$ & 1.923 & -0.329 & 1.913 & -0.521 \\
\hline
\end{tabular}

Table 8-5. The mean annual zonal (u) and meridional wind (v) in $\mathrm{m} / \mathrm{s}$ for each region. For easy comparison, the pre-industrial values are presented again. Westerly and southerly winds are positive.

The seasonal analysis of the zonal and meridional winds over New Zealand, presented in Table 8-6 and Table 8-7, show some more intriguing detail. The westerly wind is weaker the whole year round in the regions WSSI and ESI. It would therefore seem that the two main islands of New Zealand experienced different changes in the zonal wind. Furthermore, there is also an enhanced seasonality with the westerly winds being stronger the whole year round, except during JJA, when each region experiences a weaker westerly wind. A reduction in the strength of the westerly wind over New Zealand during JJA had already shown up in the analysis of the GCM during the LGM (section 7.2.6, Fig. 7-19). A weakening of the westerly wind during JJA is also a common feature in the pre-industrial simulation, and in the present day climate is related to blocking. Trenberth and Mo (1985) linked the occurrence of blocking over the New Zealand region during the winter strongly to the existence of a wave number 3 pattern. It was found in chapter 7 that the wave number 3 pattern during the LGM was slightly enhanced, which would have therefore potentially increased the occurrence of blocking during the LGM. The weaker westerly wind during JJA over the whole of New Zealand is therefore an indication that the occurrence of blocking might have been more common during the winter in the LGM.

The meridional wind analysis shows an increase of the southerly wind in NNI except during JJA. SWNI and ENI have a stronger northerly wind component 


\begin{tabular}{||l|r|r|r|r|r|r|r|r||}
\hline \multicolumn{1}{|c|}{$\mathrm{u}$} & \multicolumn{4}{c|}{ LGM } & \multicolumn{4}{c||}{ pre-industrial } \\
\hline \hline Region & DJF & MAM & JJA & SON & DJF & MAM & JJA & SON \\
\hline \hline NNI & 1.917 & 1.680 & 0.156 & 3.612 & 1.364 & 0.382 & 0.626 & 3.559 \\
\hline \hline SWNI & 2.686 & 2.138 & 0.217 & 3.728 & 2.121 & 1.073 & 0.510 & 3.472 \\
\hline \hline ENI & 3.169 & 3.012 & 1.558 & 4.369 & 2.520 & 1.946 & 1.724 & 4.023 \\
\hline \hline NSI & 2.414 & 1.892 & 0.289 & 3.077 & 1.983 & 1.235 & 0.565 & 3.070 \\
\hline \hline WSSI & 1.703 & 1.751 & 0.708 & 2.303 & 2.126 & 1.790 & 1.011 & 2.855 \\
\hline \hline ESI & 1.473 & 1.878 & 1.714 & 2.284 & 1.902 & 1.985 & 1.897 & 3.083 \\
\hline \hline NZ & 2.030 & 1.934 & 0.735 & 2.994 & 1.964 & 1.419 & 1.036 & 3.232 \\
\hline \hline
\end{tabular}

Table 8-6. The mean seasonal zonal wind (in $\mathrm{m} / \mathrm{s}$ ) for each region for the LGM and the preindustrial simulation.

\begin{tabular}{||l|r|r|r|r|r|r|r|r||}
\hline \multicolumn{1}{|c|}{ V } & \multicolumn{4}{c|}{ LGM } & \multicolumn{4}{c||}{ pre-industrial } \\
\hline \hline Region & DJF & MAM & JJA & SON & DJF & MAM & JJA & SON \\
\hline \hline NNI & 0.253 & 0.877 & 0.443 & 0.600 & 0.098 & 0.725 & 0.542 & 0.060 \\
\hline \hline SWNI & -1.141 & -0.474 & -0.239 & -0.998 & -0.846 & -0.391 & -0.307 & -1.226 \\
\hline \hline ENI & -1.165 & -0.408 & -0.083 & -0.971 & -0.948 & -0.320 & -0.170 & -1.149 \\
\hline \hline NSI & -0.264 & 0.247 & 0.434 & -0.122 & -0.244 & 0.183 & 0.318 & -0.472 \\
\hline \hline WSSI & -0.622 & -0.466 & -0.643 & -0.643 & -0.786 & -0.808 & -0.790 & -1.248 \\
\hline \hline ESI & -0.764 & -0.448 & -0.610 & -0.745 & -0.810 & -0.769 & -0.693 & -1.287 \\
\hline \hline NZ & -0.544 & -0.122 & -0.218 & -0.431 & -0.583 & -0.307 & -0.289 & -0.906 \\
\hline \hline
\end{tabular}

Table 8-7. The mean seasonal meridional wind (in $\mathrm{m} / \mathrm{s}$ ) for each region for the LGM and the pre-industrial simulation.

during the first part of the year, and a weaker northerly wind component during the second half of the year. NSI has a weaker northerly wind component, except during DJF, and the northerly winds are weaker the whole year round in WSSI and ESI. Histogram analysis shows whether the change in the mean meridional wind is due to a decrease/increase in the northerly/southerly winds or whether it is due to a change in their strengths (Fig. 8-14).

The change in the meridional wind over New Zealand is due to both a shift to more southerlies as well as to stronger southerlies. The exception is JJA when there is not a shift to more southerlies but only a shift to stronger southerlies. This change in the meridional wind is similar to the change in the zonal mean 

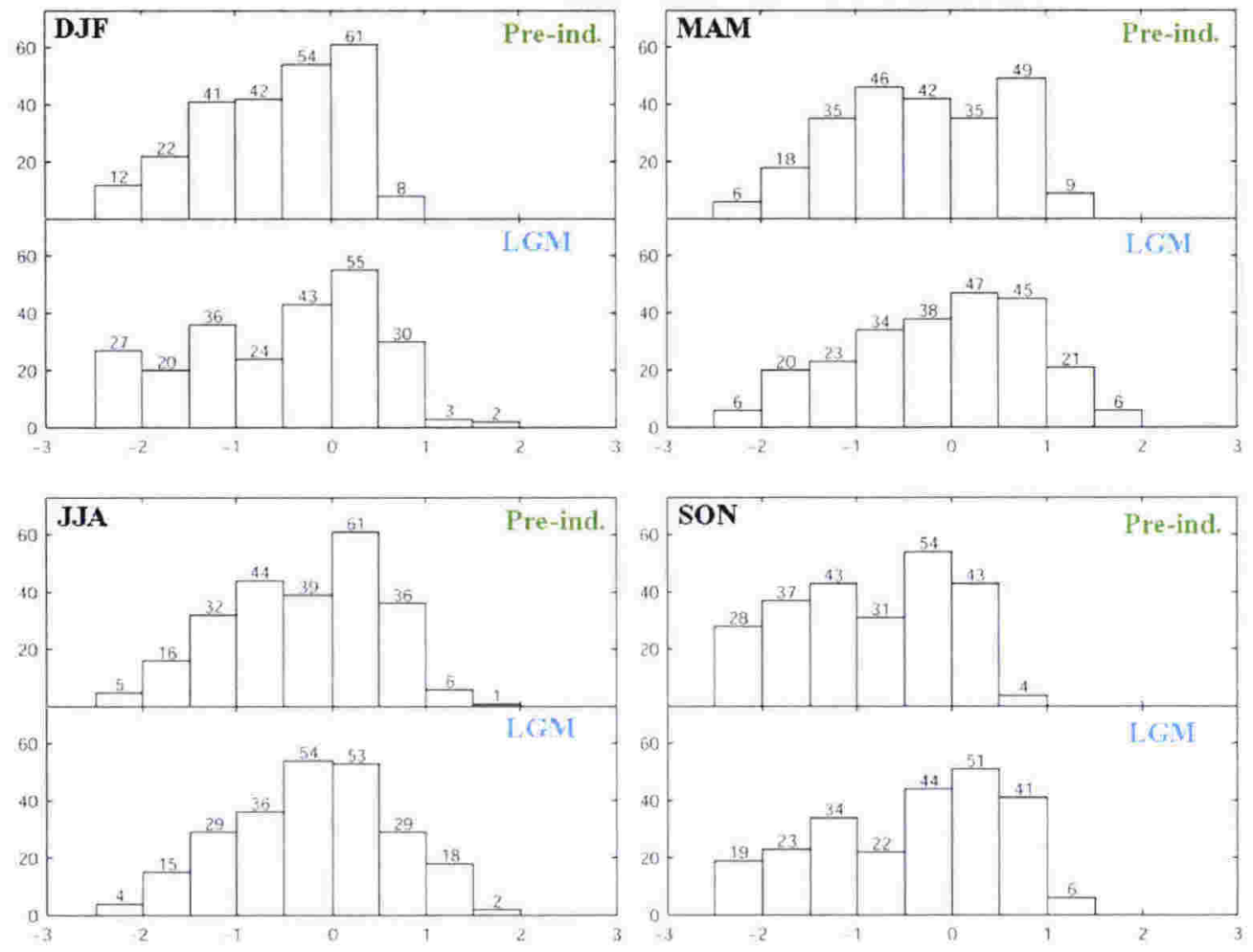

Fig. 8-14. The strength of the meridional wind $(\mathrm{m} / \mathrm{s})$ versus the number of grid points for the New Zealand pre-industrial land mass. The pre-industrial and LGM distributions are paired for each season to facilitate easy comparison. Northerlies are negative, southerlies are positive.

(Fig. 7-24). There are also regional differences within New Zealand. The NNI region, which already experienced a predominantly southerly wind, shows a shift to stronger southerlies. The slight positive increase in the mean meridional wind in SWNI and ENI during the first half of the year is due to a slight increase in the number of grid points showing a mean northerly direction. These regions experience a weakening of the northerlies during the second half of the year, but in general do not show major shifts in the distribution of northerlies versus southerlies at any time of the year. The strongest decrease/increase in the strength of the northerly/southerly winds takes place over WSSI and ESI during the transitional seasons of MAM and SON.

An analysis was done on the change in the meridional wind in the Southern Hemisphere between $35^{\circ} \mathrm{S}-60^{\circ} \mathrm{S}$ in section 7.2.7. It was found that within this zonal band there was a shift towards less northerlies/more southerlies. As 


\begin{tabular}{|l|r|r|r|r|r|}
\hline \multicolumn{2}{|c|}{} & DJF & MAM & JJA & SON \\
\hline \hline \multirow{2}{*}{ GCM } & Pre-ind. & 18.4 & 33.7 & 39.2 & 22.8 \\
\cline { 2 - 6 } & LGM & 24.7 & 35.7 & 39.2 & 23.1 \\
\hline \hline \multirow{2}{*}{ RCM } & Pre-ind. & 28.8 & 38.8 & 43.3 & 19.6 \\
\cline { 2 - 6 } & LGM & 37.5 & 49.6 & 42.5 & 40.8 \\
\hline
\end{tabular}

Table 8-8. The number of grid points (in percentage of the total number of grid points) having a meridional component that is pre-dominantly southerly for the GCM $\left(35^{\circ} \mathrm{S}-60^{\circ} \mathrm{S}\right)$ and RCM for the pre-industrial and LGM simulations.

indicated earlier, such a shift was probably prominent over the New Zealand region. That means that percentage-wise there must have been be a larger shift towards a stronger southerly flow over the New Zealand region than in the zonal mean. Table 8-8 shows the number of grid points that have a meridional wind component that is predominantly southerly for the GCM $\left(35^{\circ} \mathrm{S}-60^{\circ} \mathrm{S}\right)$ and RCM for the pre-industrial and LGM simulation. From this table it is clear that the increase in the southerly component of the meridional wind in the RCM is larger than in the zonal mean for the Southern Hemisphere, supporting the statement made earlier.

Have the different wind patterns in the regions during the LGM affected temperature and precipitation in the regions? From Table 8-2 it was determined that the largest cooling occurred over the South Island. The large amount of cooling in WSSI was for a substantial part ascribed to the differences between the modelled orography in the pre-industrial and LGM simulations, and the inclusion of the glaciers in the LGM simulation. But the large amount of cooling in ESI can not be explained in a similar way. The ESI region also went against the trend of having reduced precipitation during the LGM. There was only a reduction in precipitation during JJA, but virtually none during summer and winter, and even an increase in precipitation during SON.

Correlation maps of temperature versus mean sea level pressure and precipitation versus mean sea level pressure show quite different patterns for the LGM parameters than for the pre-industrial parameters. The correlations are understandably very low in the annual mean $( \pm 0.2)$, and not much can be 
concluded from this, but there are remarkable variations in the seasonal correlation patterns.

\subsubsection{Correlation MSLP-Temperature}

In this section, the seasonal correlation maps of the regional mean seasonal temperatures against the mean sea level pressure at each grid point are presented. The descriptions only deal with the general correlations patterns. Comments on whether a particular wind direction has a stronger/weaker influence on temperature in a particular region, does not imply that that particular wind direction is the most important controlling factor on temperature. Comparisons are primarily made with the equivalent correlation patterns of the pre-industrial simulation (section 6.3.3.1).

\section{- DJF}

(Fig. 8-15) Temperature is stronger positively correlated to MSLP during DJF than in the pre-industrial simulation. The correlation maximum for each region shifts more onto the country. This means that the location of highs and lows over the country dominate the temperature response more strongly than during the pre-industrial simulation. Clear skies will radiate out and receive relatively more heat than during the pre-industrial. Renwick (1998) describes how the occurrence of "blocking highs" during DJF increases in the South West Pacific during El Niño years. It was established from the HadCM3 SST data that ENSO had a significant presence during the LGM (chapter 7). Although New Zealand's climate during the LGM was most likely affected by ENSO, no further investigation was done on the extent of the effect of ENSO on the shift in the correlation patterns ${ }^{1}$. The westerly winds have a stronger cooling effect over all regions during the LGM, except for ENI where there is not much of a difference. What stands out during DJF is the inverse correlation of temperature with the

\footnotetext{
' Since the SSTs in the tropical Pacific as reconstructed by HadCM3 are open to discussion, changes in correlation patterns can be due to incorrectly simulating the SSTs rather than that those changes represent a feature of the climate of the LGM.
} 


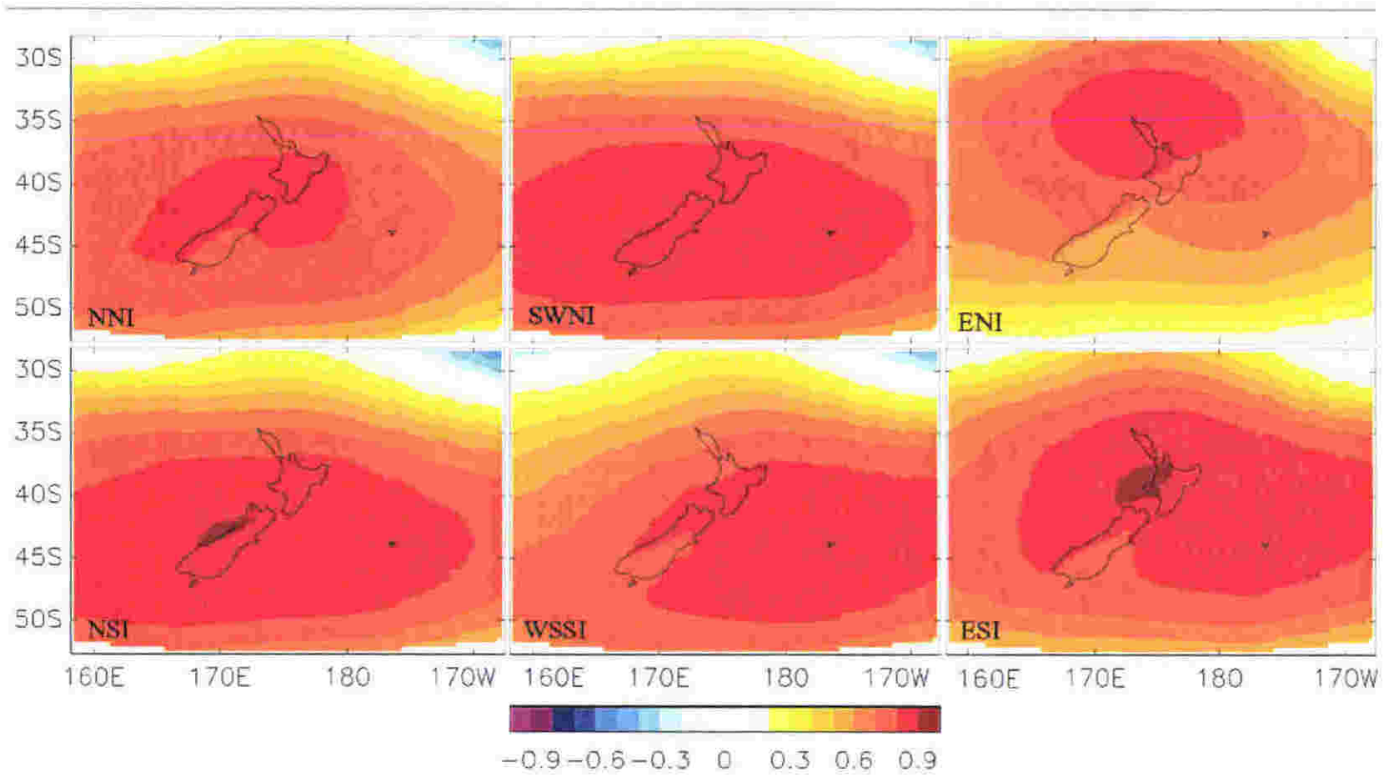

Fig. 8-15. Correlation maps of mean sea level pressure versus temperature for the six different regions during DJF (RCM).

zonal wind over the Alps. Westerly winds give cooling to the whole country, but result in a slight warming over the Alps (see Fig. 8-16). A possible explanation is that since the westerlies have lost their moisture farther to the west (see Fig. $8-12$ ), the dry winds warm up the areas directly downstream of the glaciers. This is in accord with the suggestion made earlier that the precipitation band in the

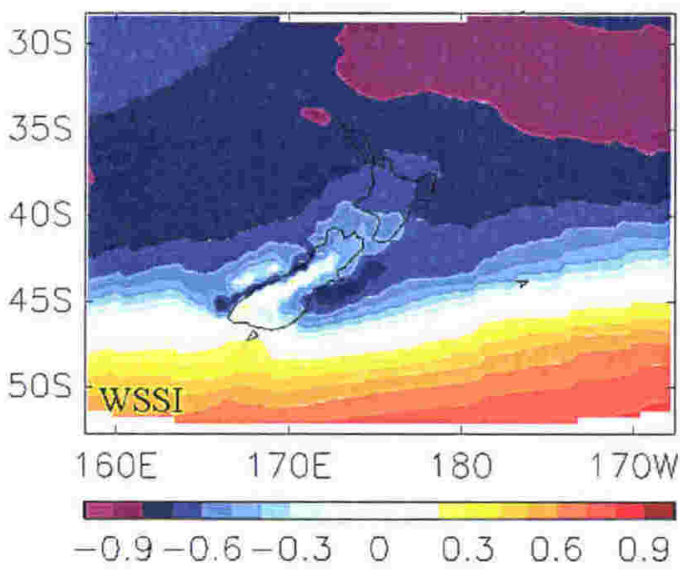

Fig. 8-16. Correlation map of the zonal wind (at $1000 \mathrm{hPa}$ ) versus temperature for WSSI during DJF. Westerly wind is positive.
West Coast shifted westward during the LGM.

It is not possible to separate any possible impacts that a change in the wind regimes might have had on the temperature from the overall cooling in each region. The temperature in WSSI is dominated by the cooling influence of the glaciers and although the cooling in ESI is slightly larger than in the other regions (see Table 8-2), this can not conclusively be ascribed to 


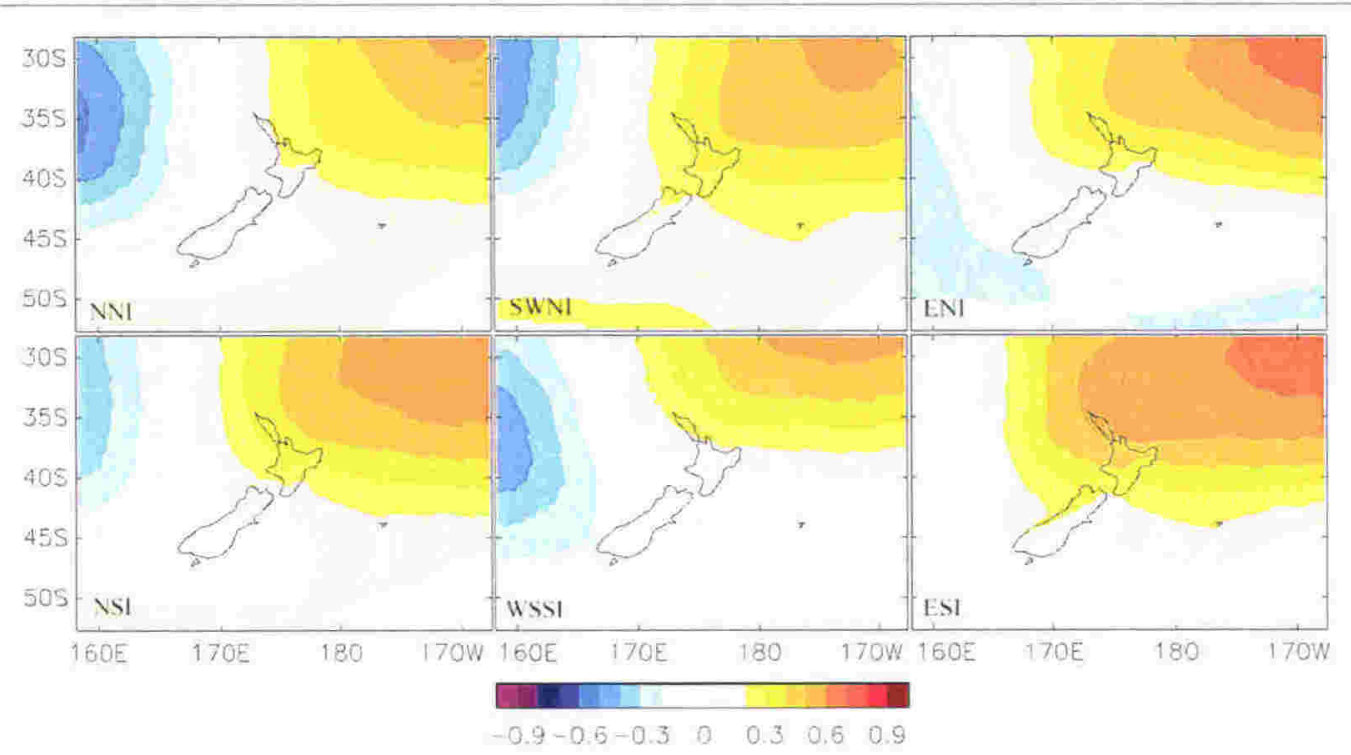

Fig. 8-17. Correlation maps of mean sea level pressure versus temperature for the six different regions during MAM (RCM).

an increase in southerly winds. Other factors (e.g. enhanced outgoing longwave radiation, decrease in westerly wind) will also have played their part.

\section{$-M A M$}

(Fig. 8-17) The correlation patterns for MAM stand out in that they are very similar for each region. They all show the dominance of the meridional wind on

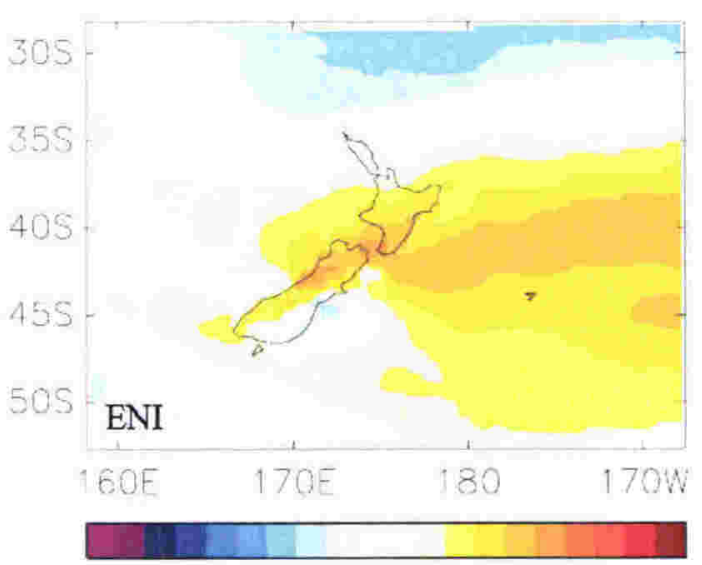

$\begin{array}{llllll}-0.9-0.6-0.3 & 0 & 0.3 & 0.6 & 0.9\end{array}$

Fig. 8-18. Correlation map of the zonal wind (at $1000 \mathrm{hPa}$ ) versus temperature for ENI during MAM. Westerly wind is positive. the temperature response, with correlations up to 0.9 . The advection of warm/cold air has a more prominent influence on the temperature response of each region than in the pre-industrial simulation. The westerly wind has a cooling effect on the far north and south, and results in warming in ENI (Fig. 8-18). The strengthened westerly flow over ENI would have enhanced the föhn effect, thereby 


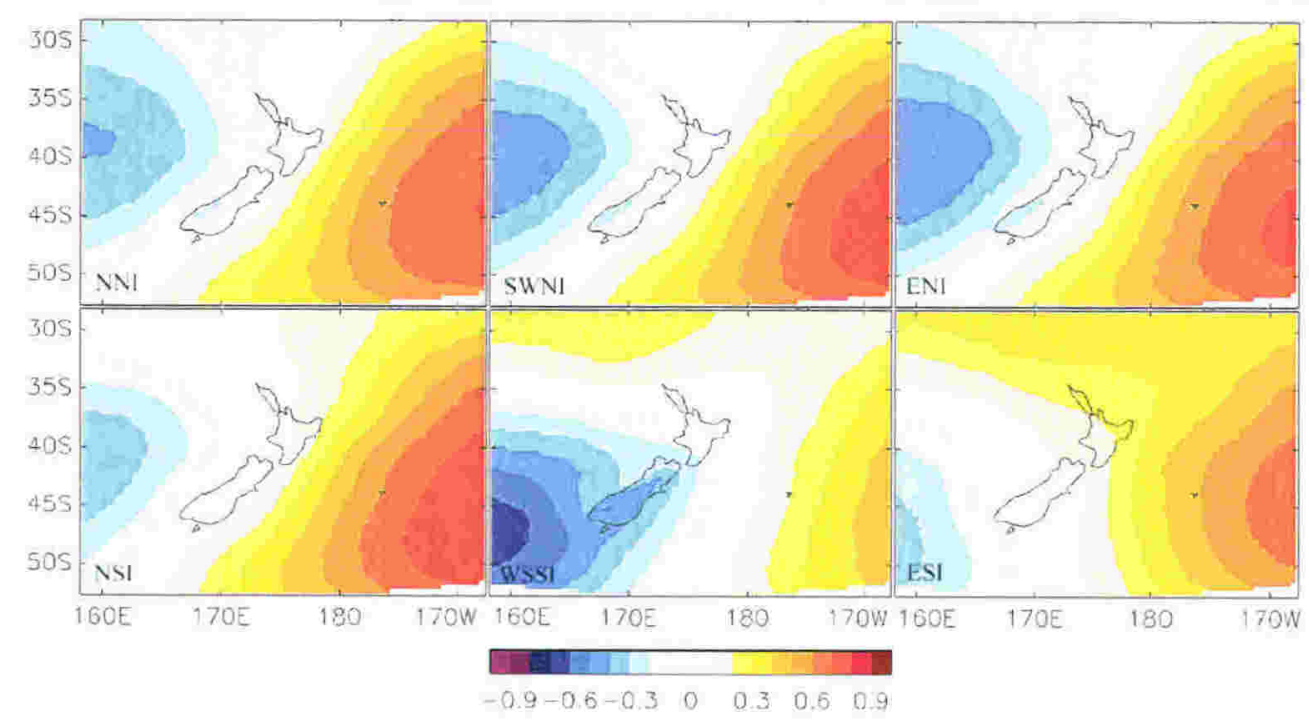

Fig. 8-19. Correlation maps of mean sea level pressure versus temperature for the six different regions during JJA (RCM).

offsetting the general cooling in the region. ENI has the lowest amount of cooling of all regions (Table 8-2).

With temperatures rather uniformly cooler over New Zealand (with the exception of WSSI), the larger amount of cooling in ESI stands out. As mentioned earlier, the southerly component of the wind in this region increases strongly, particularly during the transitional seasons. The increase in the southerlies will therefore have enhanced the amount of cooling in this region.

\section{- JJA}

(Fig. 8-19) The correlation patterns of JJA present stronger positive correlations in the east and weaker negative correlations in the west compared to the correlation patterns for the pre-industrial simulation (see Fig. 6-20). However, the correlation patterns between temperature and the winds are very similar to the equivalent correlation patterns for the pre-industrial simulation (not shown). The meridional wind has the strongest influence, and then in particular the southerly wind which can bring up very cold air from down south. It is in the finer details of the westerly wind where the main differences are, and then in particular in the south. The westerly wind increases its cooling effect slightly on the North Island regions. There is a slight zonal change in the correlation pattern of the 


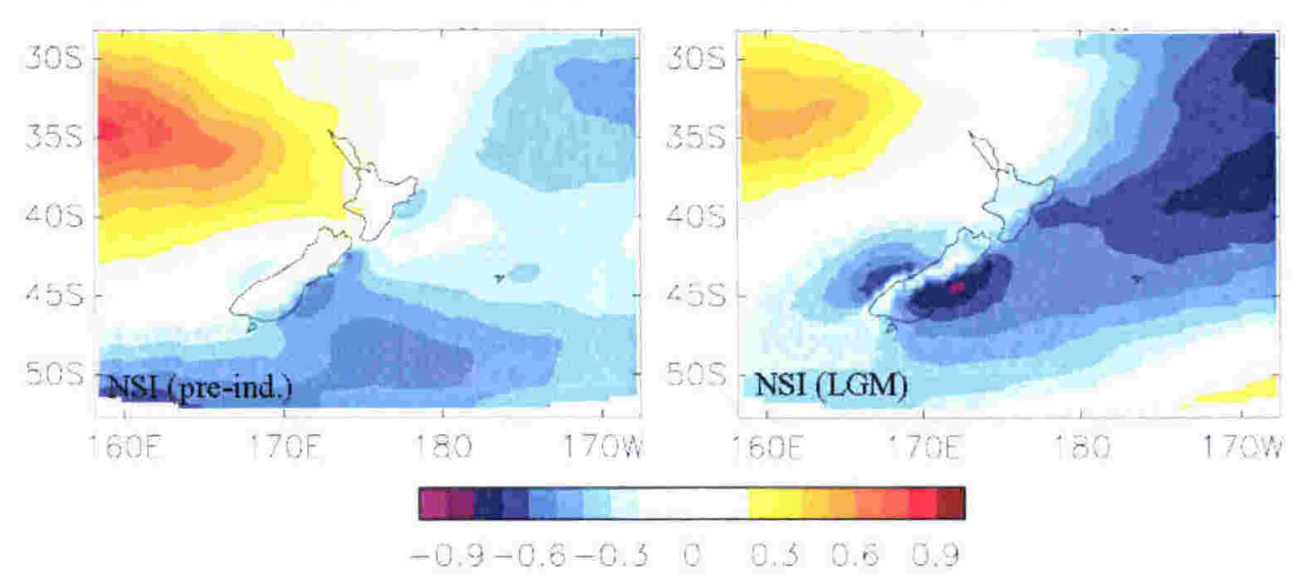

Fig. 8-20. Correlation maps of the zonal wind (at $1000 \mathrm{hPa}$ ) versus temperature for NSI for the pre-industrial (left) and LGM simulation (right) during JJA. Westerly wind is positive.

temperature with the zonal wind in NSI. Although the pattern is the same as for the pre-industrial, the strongest correlations have shifted to an easterly wind to the east of the county (Fig. 8-20). The temperature in NSI is strongly correlated to an easterly wind in the east. This is different than for the pre-industrial simulation, where the correlation was negative (Fig. 8-20). The correlation pattern in WSSI is complicated again but is similar to the pattern during DJF. The westerly wind is negatively correlated to temperature in the west but causes warming over the Alps.

Excluding WSSI from the analysis, the amount of cooling is again quite uniform over the country, except again in ESI. The stronger cooling in this region can this time not directly be linked to a larger increase in the southerly winds, since most regions experience a similar increase in the southerly component. However, ESI would have been more prone to the severity of the cold blast coming from the south, and that could have enhanced its cooling. With its average seasonal temperature below zero (Table 8-2), the region is covered by snow (Fig. 8-8) which increases its surface albedo. Furthermore, with blocking probably more prominent during the LGM, especially during JJA, the amount of radiative cooling would have increased, which would have lead to further cooling. The latter possibility is also supported by the fact that all regions show weaker westerly winds during LGM (Table 8-6). And thirdly, the temperature in 


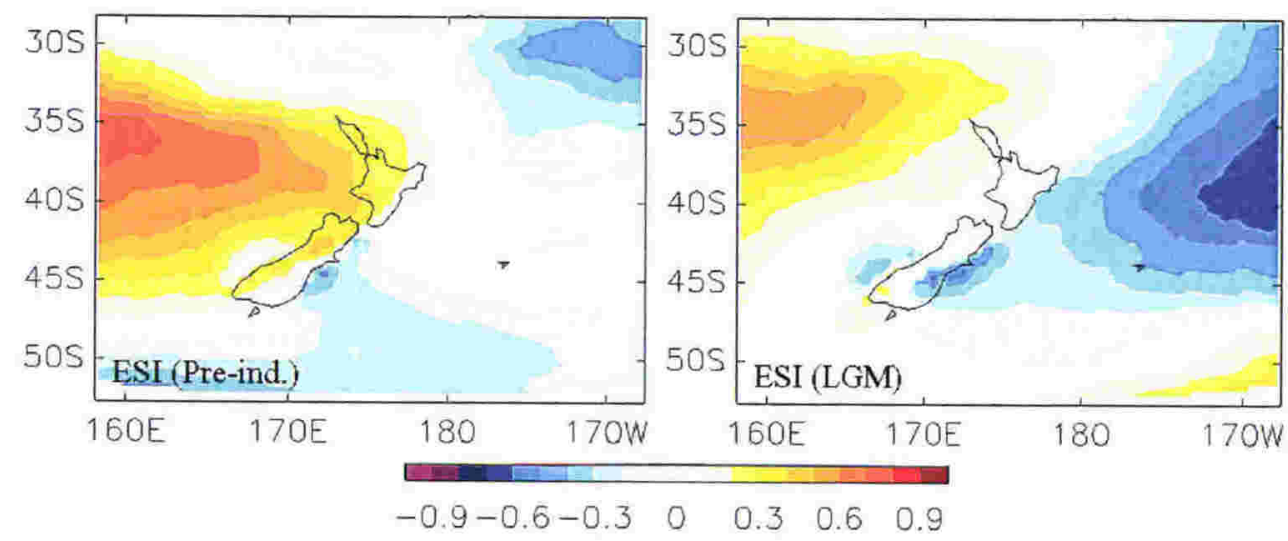

Fig. 8-21. Correlation maps of the zonal wind (at $1000 \mathrm{hPa}$ ) versus temperature for ESI for the pre-industrial (left) and LGM simulation (right) during JJA. Westerly wind is positive.

Otago in particular, and to a lesser extent the coastal areas, are affected by the very cold katabatic winds coming of the mountains (Fig. 8-21).

- SON

(Fig. 8-22) SON has always been the season that has stood out. The mean zonal circulation had its largest anomalous pattern during this season in the preindustrial simulation. Comparisons between the LGM and the pre-industrial simulation for this season have already shown up some different conclusions than during other seasons (e.g. sections 7.2.6, 7.2.7). The correlation maps are dominated by positive correlations just as for the pre-industrial simulation, but they are all slightly weaker. This mainly affects the meridional wind, which has lower correlations with temperature, particularly for the northern regions. The westerly wind still cools NNI, SWNI and NSI, but enhances its impact of warming in ENI and to a lesser extent in ESI. The correlation pattern of WSSI shows the double pattern again of cooling to the west and warming over the Alps under a westerly wind regime, although the correlation is now much weaker $( \pm 0.3)$.

As mentioned under MAM, the largest difference in the amount of cooling between ESI and the northern regions appears during the transitional seasons. ESI experiences stronger cooling than the northern regions when it also has a large increase in southerly winds (Table 8-7). It is therefore suggestive that the 


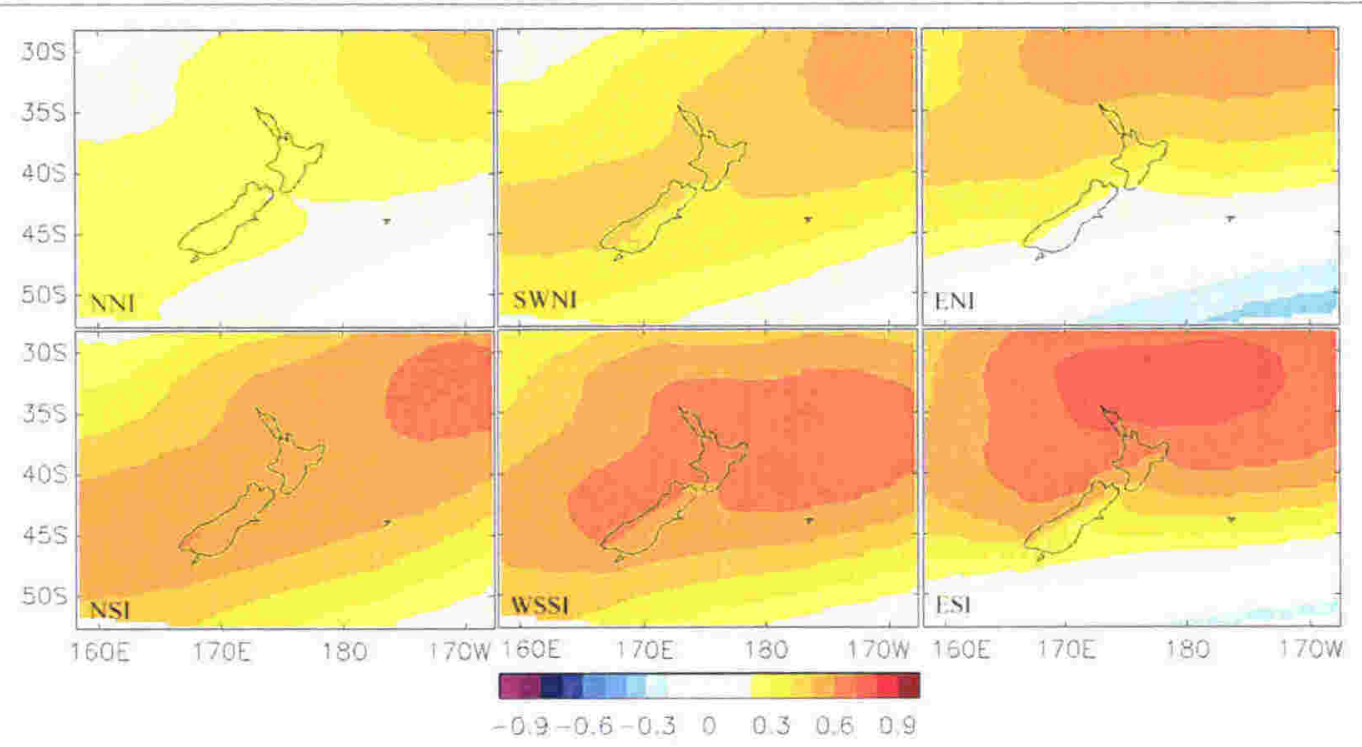

Fig. 8-22. Correlation maps of mean sea level pressure versus temperature for the six different regions during SON (RCM).

increase in the southerly wind component had a measurable effect on the cooling of the regional temperature in ESI. The question that needs to be answered then is why NNI does not experience an increase in cooling even though it experiences a similar increase in the southerly wind. There are three possible explanations. It might be that, similar during JJA, the southerly winds in ESI are coming from far more south and are much colder than the southerlies in NNI. Secondly, there is a strong reduction in the westerly wind in this region, reducing the effect of lee-side warming. And thirdly, large parts of NNI are covered in forest. This kind of vegetation would be able to "regulate" its temperature more than the open grasslands of the south, reducing the effect of the colder winds.

\subsubsection{Correlation MSLP-Precipitation}

In this section, the seasonal correlation maps of the regional mean seasonal precipitation against the mean sea level pressure at each grid point are presented. No distinction has been made on whether a specific wind direction has become more/less important in general in the occurrence of precipitation, or whether it is the result of a change in other factors controlling precipitation. The statements made here only refer to the dominant wind direction, not to the dominant 


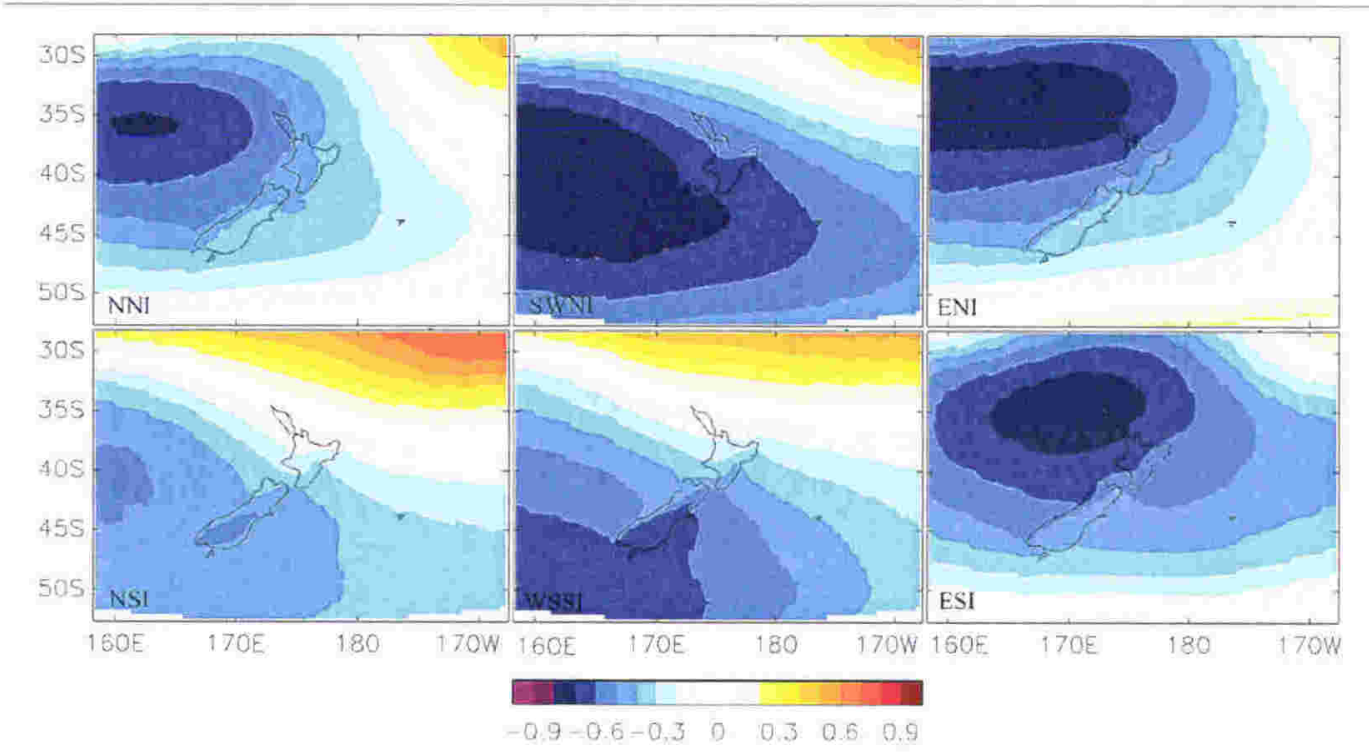

Fig. 8-23. Correlation maps of mean sea level pressure versus precipitation for the six different regions during DJF (RCM).

precipitation process. Comparisons are primarily made with the equivalent correlation patterns of the pre-industrial simulation (section 6.3.3.2).

\section{- DJF}

(Fig. 8-23) All correlation maps for DJF are dominated by a large negative correlation to the west of the country. This indicates that the relative importance of the meridional wind on precipitation is stronger for most regions during the LGM than during the pre-industrial. There are a few changes in the correlation patterns with the zonal wind.

During the pre-industrial, precipitation in NNI was weakly positively correlated to an easterly wind, but during the LGM the positive correlation is with a westerly wind. The mean seasonal precipitation in NNI during DJF was less during the LGM (Table 8-4). The correlation maps show that the occurrence of precipitation in NNI was strongly dependent on moisture coming from the north (Fig. 8-24). However an increase in southerlies over the region (Table 8-7) implies that there were more zones of high/low pressure to the west/east of New Zealand. Besides as the result of the cooler temperatures, the reduction in precipitation in NNI during DJF could therefore also be related to a reduction in the number of tropical lows coming south to New Zealand. SWNI has a stronger 


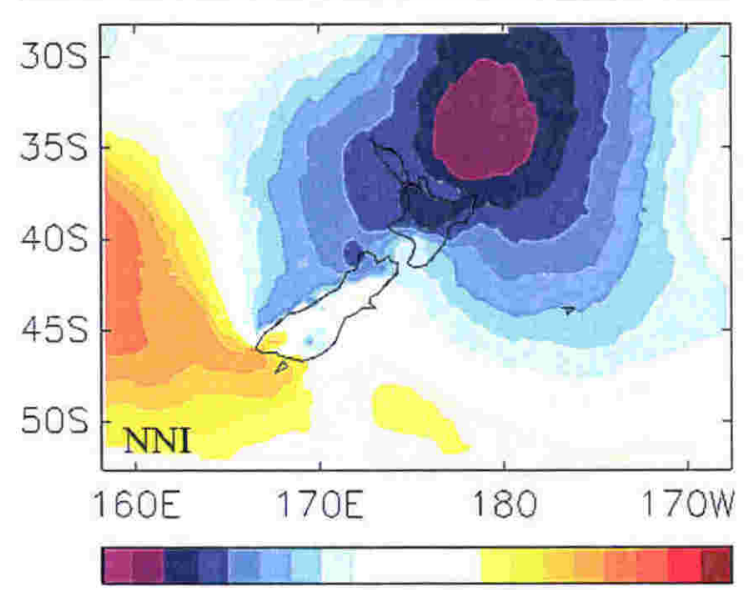

$\begin{array}{llllll}-0.9 & -0.6-0.3 & 0 & 0.3 & 0.6 & 0.9\end{array}$

Fig. 8-24. Correlation map of the meridional wind (at $1000 \mathrm{hPa}$ ) versus precipitation for WSSI during DJF, Northerly wind is negative. positive correlation of precipitation with the westerly wind, and ENI has a stronger correlation with an easterly wind to the south of the country. Since the latter region experiences an increase in westerly winds (Table 8-6), its dependence on moisture coming from the north increases. The reduction in easterly winds is also partly responsible for the overall reduction in precipitation in this region (Table $8-4)$.

It is particularly the correlation with the zonal (westerly) rather than with the meridional (northerly) wind component that increases for NSI. This region experiences a strong increase in westerly wind (Table 8-6), where as the increase in its northerly component is only minor (Table 8-7). However, the overall result was a reduction in precipitation here as well (Table 8-4). Both the two southern regions have difficult patterns. Against the trend set by the northern regions, WSSI and ESI both show an increase in precipitation (Table 8-4), a reduction in the westerly wind (Table 8-6) and a reduction in the northerly wind (Table 8-7). The correlation patterns for WSSI for the pre-industrial and LGM simulation are very similar, and indicate therefore that changes in precipitation are not related to changes in advection. The increase in precipitation is probably related to the lowering of the condensation level as a result of the cold temperatures of the glaciers and the steep increase in orography. Both effects result in an increase in the geographical extent of the area which temperatures are below the condensation/freezing level. This would allow a relatively larger amount of moisture to precipitate. The effect of the increase in orography on precipitation can also be seen in the correlation of precipitation with the westerly wind (Fig. $8-25)$. The westerly wind is negatively correlated with precipitation just offshore of WSSI and positively correlated over the Alps. As the moisture-bearing 


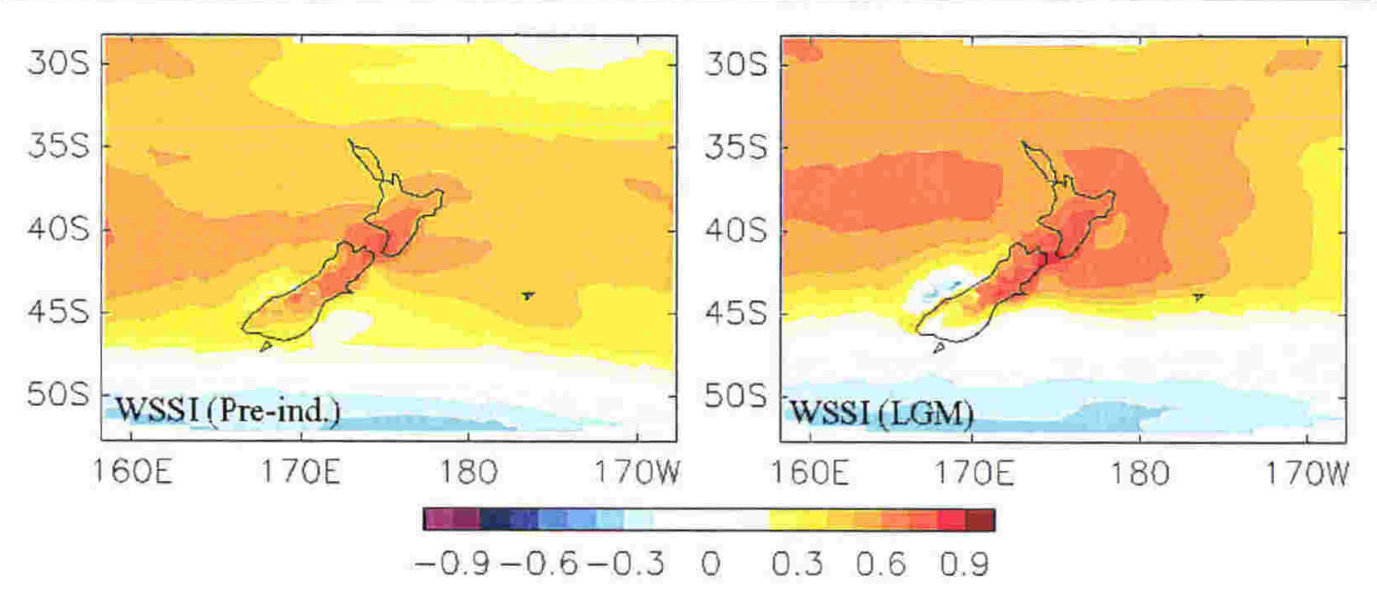

Fig. 8-25. Correlation maps of the zonal wind (at $1000 \mathrm{hPa}$ ) versus precipitation for WSSI for the pre-industrial (left) and LGM simulation (right) during DJF. Westerly wind is positive.

westerly wind approaches the land, it experiences a strong backflow when it tries to cross the mountains. In the seasonal mean, this results in a weak easterly flow (at $1000 \mathrm{hPa}$ ). The airmass needs a major uplift in order to cross the mountains, and precipitation is strongly enhanced. This was clearly pictured in Fig. 8-11 (DJF). There is also a weak negative correlation with precipitation over southern Southland. This region is now well inland in the enlarged landmass of New Zealand during the LGM and sits in the rain shadow of the high orography of Fiordland.

Precipitation in ESI is very strongly correlated with a low in mean sea level pressure just to the northwest of the North Island. This implies that precipitation in ESI is very strongly correlated to a northerly flow to the east of the North Island and a southerly flow to the south. With the strength of the westerly winds in ESI being negatively correlated to the occurrence of precipitation (Mullan et al., 2001), the strong reduction in the westerly wind in this region would therefore enhance the occurrence of precipitation as well. It would make it easier for easterlies/southerlies to penetrate deeper inland. The increase in the number of southerlies in ESI is therefore at least partly the cause of the apparent increase in precipitation. As mentioned earlier, it should be noted that the model seems to underestimate the precipitation in the east in the first place (section 6.3.2). A small increase in precipitation during the LGM does therefore not directly imply that there was a real increase in precipitation in the east of the South Island. For 


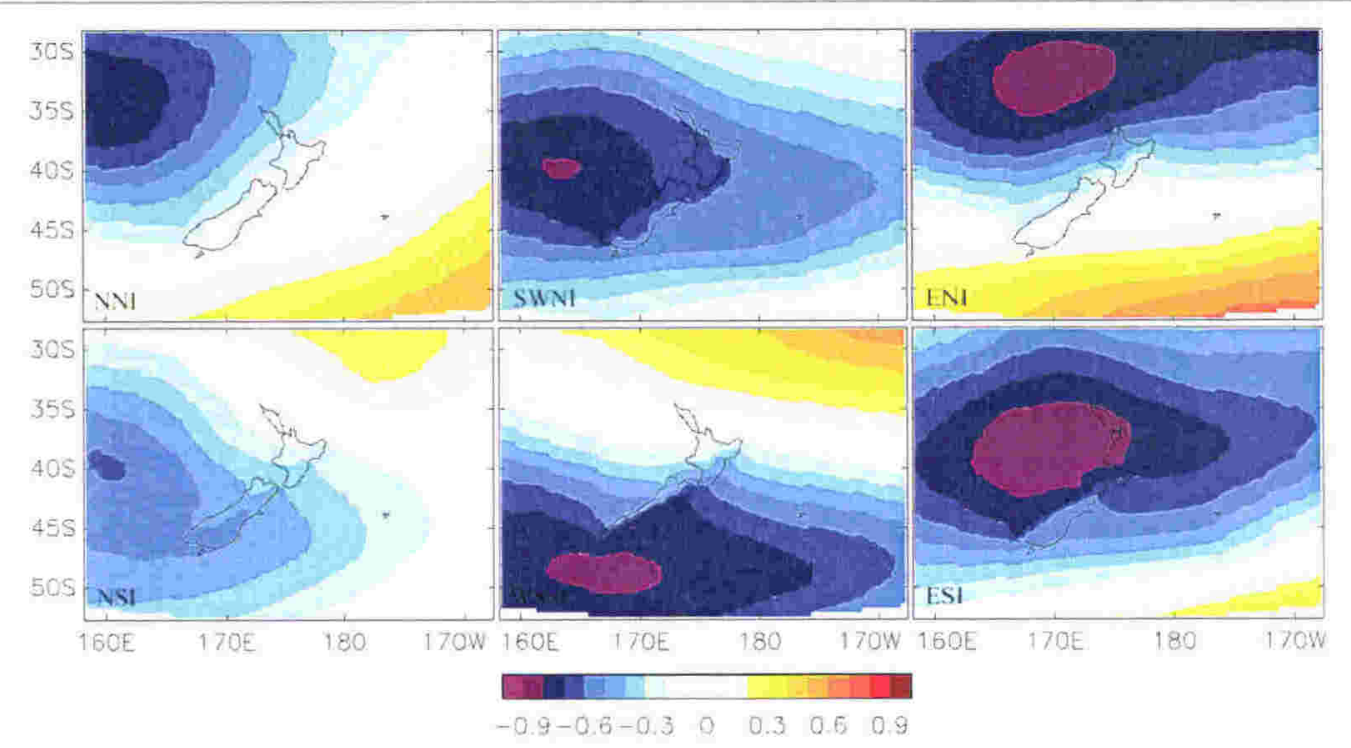

Fig. 8-26. Correlation maps of mean sea level pressure versus precipitation for the six different regions during MAM (RCM).

each region, precipitation is strongly negatively correlated with temperature, except for the mountain ranges in WSSI. Warm air can contain more moisture, so ascent of a warm moist parcel will result in an increase in precipitation.

\section{$-M A M$}

(Fig. 8-26) Differences between the pre-industrial and LGM correlation patterns for the North Island regions are relatively small. Precipitation in NNI has a weaker correlation with a low to the northwest. The maximum negative correlation in SWNI shifts slightly westward. The main implication of this shift is that the positive correlation of precipitation with the westerly wind shifts slightly southwards. Precipitation in ENI is weaker correlated to a minimum to the northwest of the regional domain in its correlation pattern with the meridional wind (Fig. 8-27). This implies that ENI gets relatively less precipitation from airmasses that originally came from the north and have curled onto the North Island, than that it did in the pre-industrial simulation. The latter pattern fits in nicely with the fact that the region experiences an increase in westerly winds (Table 8-6), thereby reducing the occurrence of moist easterly onshore flows. All northern areas experience a reduction in precipitation during MAM, but the reduction in ENI is larger $(>25 \%)$ than for the other North Island regions $(10 \%)$. 


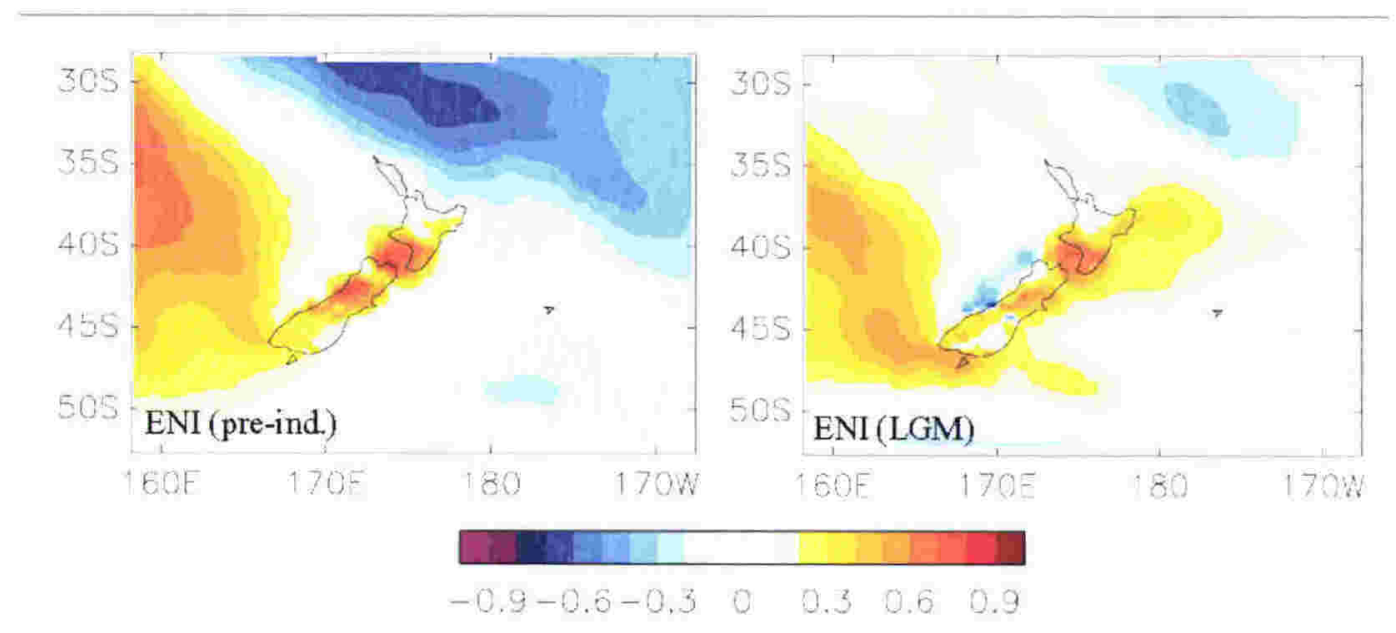

Fig. 8-27. Correlation maps of the meridional wind (at $1000 \mathrm{hPa}$ ) versus precipitation for ENI for the pre-industrial (left) and LGM simulation (right) during MAM. Northerly wind is negative.

Since the strengthened westerly wind also had an impact on the temperature regime in ENI, it is fair to say that the westerly wind exerted its influence on the climate of ENI during MAM. Since the model underestimates precipitation in this region during MAM quite clearly (Fig. 6-11), such a strong simulated reduction in precipitation would indicate that ENI was indeed quite drier during MAM in the LGM.

The correlation pattern for NSI has changed the most. It shows that in the zonal direction the region's precipitation is now correlated with a westerly wind, rather than with an easterly wind as it was in the pre-industrial simulation (Fig. 8-28). This is quite intriguing. In the pre-industrial simulation, NSI gets its moisture both from tropical lows descending on New Zealand (hence the northeasterly wind component) and from frontal depressions that are embedded in the mean westerly circulation. The enhanced westerly flow during the LGM could make it harder for tropical lows to descend on New Zealand and therefore prevent moisture transport to the southern regions.

The increase and eastward shift of the maximum negative correlation for ESI results in changes in the precipitation-wind direction correlation patterns. The correlation with a westerly wind to the north and a southerly wind to the south of New Zealand shifts southwards by about $5^{\circ}$, and the correlations with the meridional winds become weaker. ESI experiences a strong increase in the 


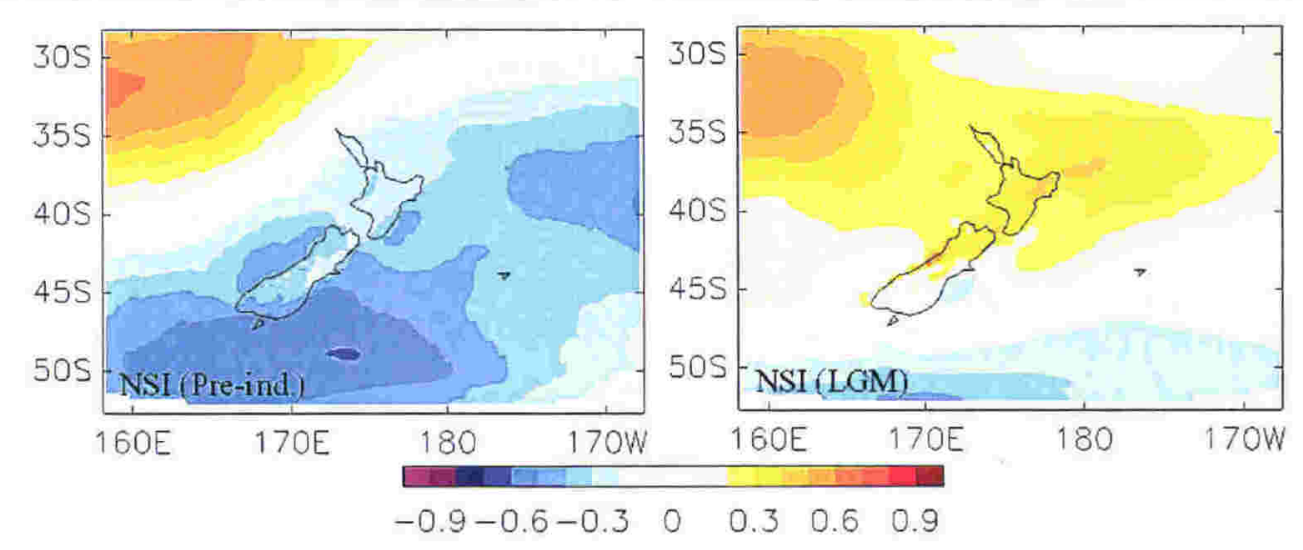

Fig. 8-28. Correlation maps of the zonal wind (at $1000 \mathrm{hPa}$ ) versus precipitation for NSI for the pre-industrial (left) and LGM simulation (right) during MAM. Westerly wind is positive.

southerly wind with virtually no change in precipitation. It would seem that the increase of incursions of southerly storms bringing precipitation in the region offsets the general reduction in precipitation as the result of the colder temperatures.

\section{- JJA}

(Fig. 8-29) The strong reduction of the westerly wind in NNI (see Table 8-6) shifts the correlation pattern slightly eastward but otherwise does not change much. Precipitation in this region is still highly correlated to north-easterly winds. The reduction in precipitation, about $10 \%$, is similar to the reduction during DJF and MAM (Table 8-4). No discernable influence of a different wind regime can be detected in the precipitation pattern during JJA. The opposite is true for SWNI. Precipitation in SWNI is correlated to northerlies to the northeast of the country rather than to the northwest of the country (Fig. 8-30). At the same time, the correlation pattern with westerly winds is much weaker, which could be an indication of the effect of a weaker westerly wind during this period (Table 8-7). In general, precipitation in this region comes now more from a northerly direction. The pattern for ENI is similar to the pre-industrial pattern, but the maxima are stronger. This indicates the stronger correlation of precipitation in this region to a north-easterly flow. This area stands out from all other areas 


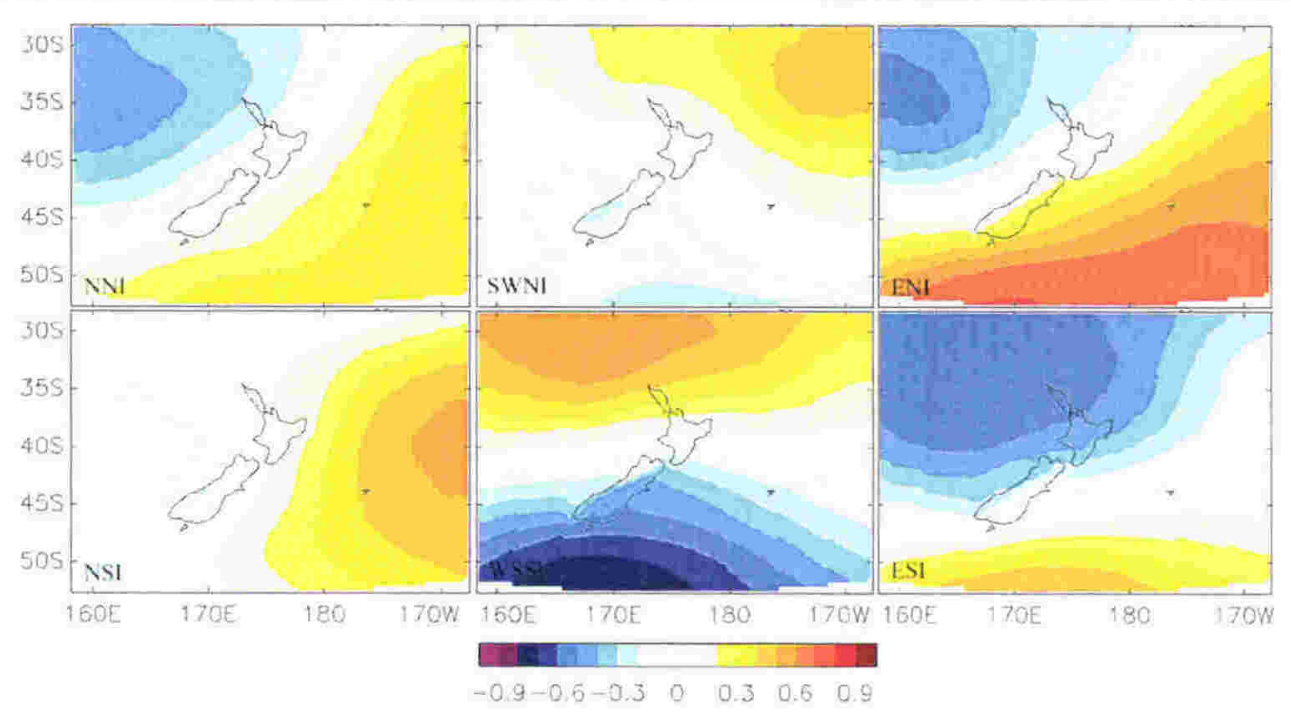

Fig. 8-29. Correlation maps of mean sea level pressure versus precipitation for the six different regions during JJA (RCM).

since it is the only region that experiences an increase in precipitation during JJA (Table 8-4) ${ }^{1}$. Without any significant change in the correlation patterns, precipitation seems to be enhanced under current climate wind regimes. In the present day, JJA experiences an increase in the occurrence of blocking highs to the east of New Zealand, and it has been indicated before that blocking was at least an as common feature during the LGM as it is now. Blocking highs to the east of New Zealand would induce weaker westerly winds over the country (a common feature for all regions during JJA, see Table 8-6) and give enhanced north-easterly flows onto ENI. The correlation pattern of ENI could therefore be an indication of the occurrence of blocking highs in the New Zealand region during JJA.

The different correlation pattern for NSI indicates that precipitation in this area is during the LGM more correlated to an easterly wind, rather than to a westerly wind (Fig. 8-31), but it is still strongly correlated to northerly winds. Such a pattern is more similar to that for ENI, in that the occurrences of blocking highs are influential on directing moisture to this area. Although the configuration of the correlation pattern for WSSI does not change much, the

\footnotetext{
1 This does not automatically mean that there was a real increase in precipitation, since the model underestimates precipitation in this region during JJA (Fig. 6-11).
} 


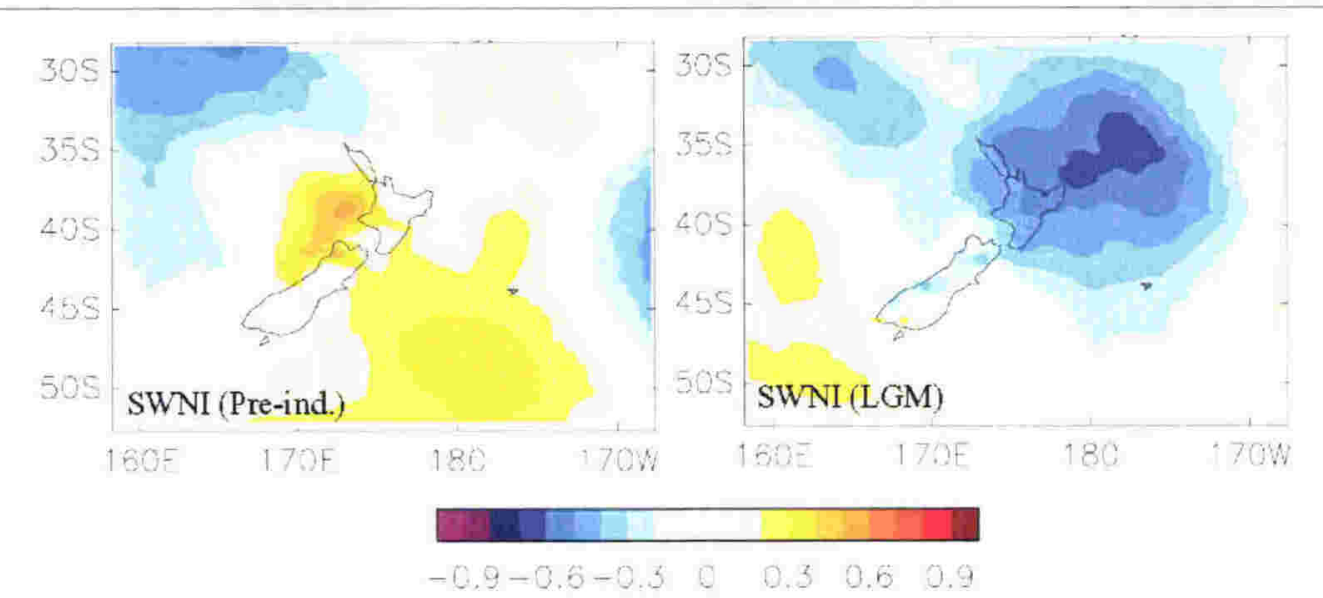

Fig. 8-30. Correlation maps of the meridional wind (at $1000 \mathrm{hPa}$ ) versus precipitation for SWNI for the pre-industrial (left) and LGM simulation (right) during JJA. Northerly wind is negative.

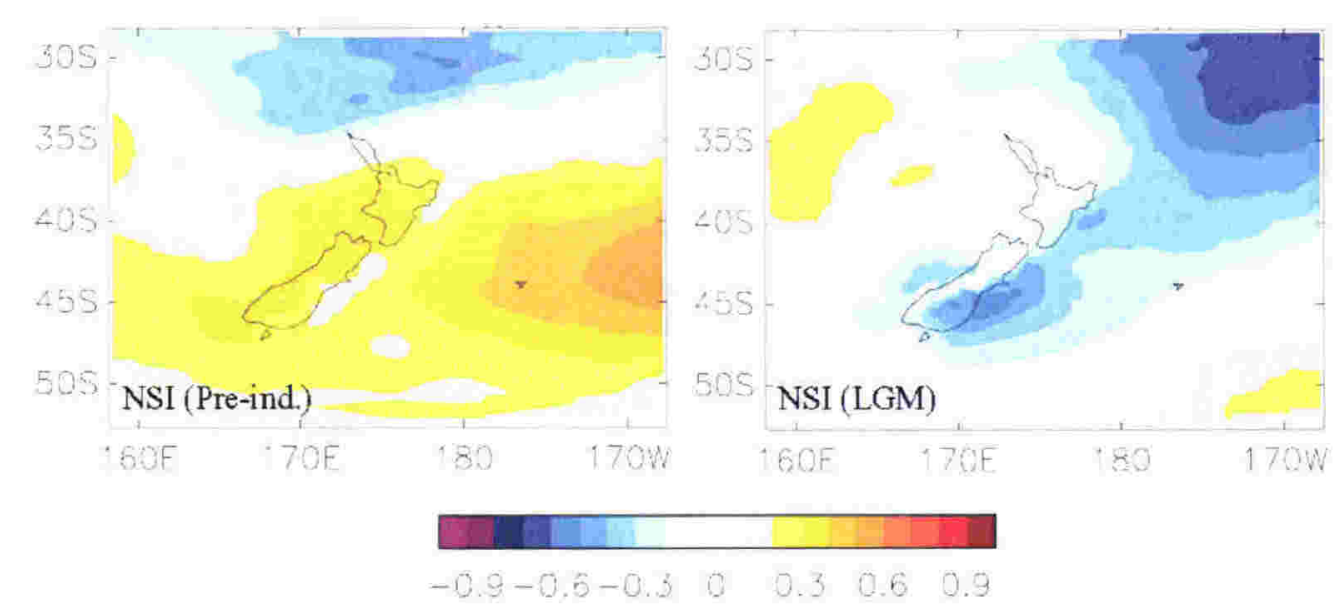

Fig. 8-31. Correlation maps of the zonal wind (at 1000hPa) versus precipitation for NSI for the pre-industrial (left) and LGM simulation (right) during JJA. Westerly wind is positive.

reduction in the correlation maxima makes precipitation in this region only weakly correlated to a northerly wind to the northwest of the regional domain.

Northerly winds will cause dry conditions downwind of the mountains in inland Otago and Southland. The correlation pattern for ESI does not change dramatically although the correlation of precipitation with a northerly wind to the east of the country decreases slightly and shifts slightly more to the north. Precipitation in ESI is slightly reduced. But since the mean seasonal temperature 


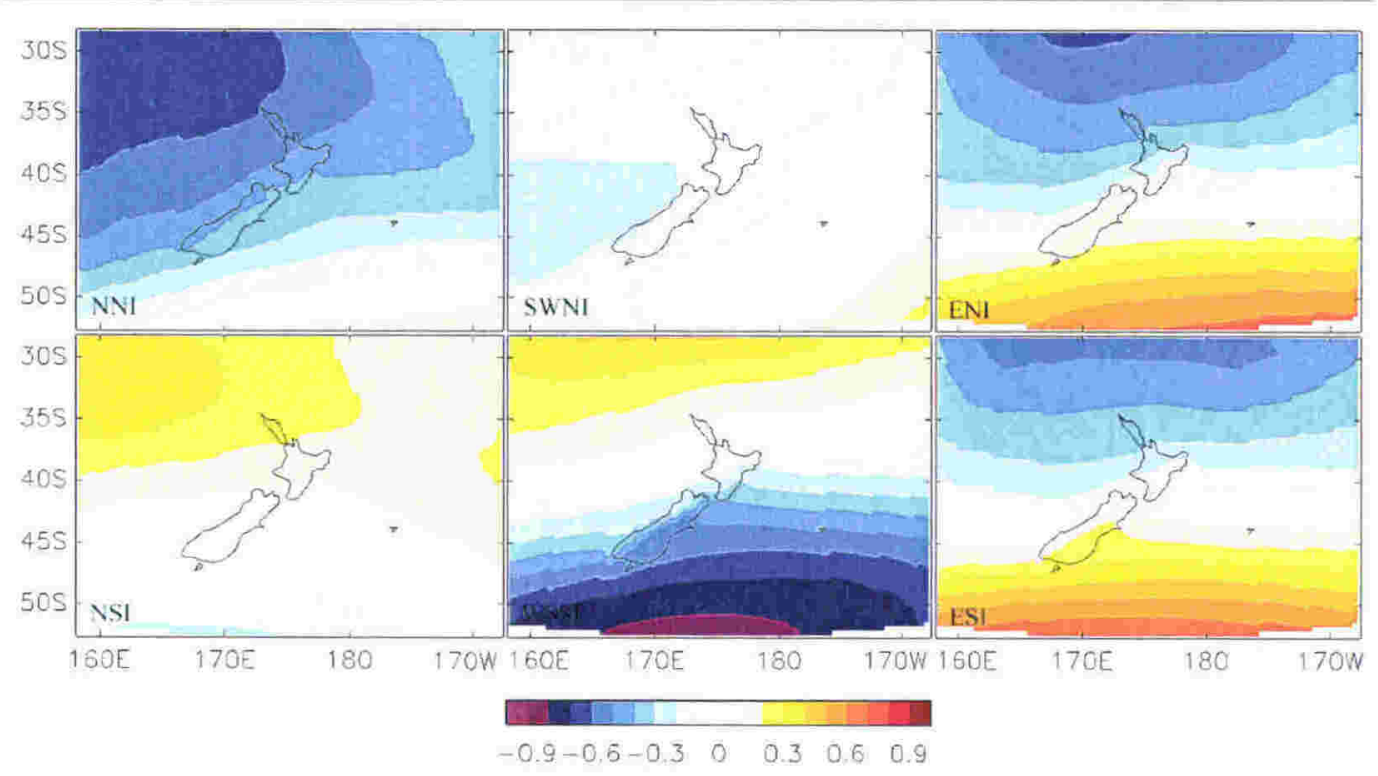

Fig. 8-32. Correlation maps of mean sea level pressure versus precipitation for the six different regions during $\mathrm{SON}(\mathrm{RCM})$.

is below freezing in ESI (Table 8-2), this region, together with WSSI, would get its precipitation mainly in the form of snow. Most of the South Island was blanketed by snow during JJA (Fig. 8-8), which in turn increased the surface albedo, causing further cooling.

$-S O N$

(Fig. 8-32) The correlation patterns during SON are in general weaker than during the pre-industrial (Fig. 6-36). Although the southerly wind in NNI increases ten-fold during SON (Table 8-7), it is the westerly wind that is still very strongly correlated to the occurrence of precipitation in this region. The amount of precipitation hardly changed in NNI (Table 8-4). The increase in general baroclinicity would have countered the reduction in precipitation as the result of the cooler conditions. In chapter 7 it was already indicated that baroclinicity in the LGM during SON was enhanced compared to the preindustrial simulation.

Precipitation in SWNI is only weakly correlated to any wind direction. The correlation pattern still indicates the influence of the moisture-bearing westerly wind coming onto the country, and precipitation in SWNI correlates with a 
northerly wind in the east and a southerly wind to the west of the country. The pattern for ENI does not change much, and precipitation is even more strongly correlated to the easterly wind than in the pre-industrial simulation. This follows from the fact that ENI is under the influence of very strong westerly winds (Table 8-6). Such a regime usually brings dry conditions to this region. Although the westerly wind dominates in this region, the amount of precipitation in ENI is similar as during the pre-industrial (Table 8-4). This is due to the incursions of southerly storms onto the whole south and east of the country. All southern regions and ENI show a strong increase in the southerly wind component (Table 8-7). These regions would have experienced more extreme conditions during the LGM than during the present day, with them often having been under the influence of strong, warm, dry westerly winds, alternated with stronger and colder southerly winds. To explain the lack of proxy data indicating the existence of forests in New Zealand during the LGM, it was assumed that a general cooling of about $4.5^{\circ} \mathrm{C}-5^{\circ} \mathrm{C}$ was required. However, the harshness and the severity of the climate, together with the enhanced seasonality, might very well have been the limiting factor on forest growth, and not the absolute cooling. Such a scenario would imply that the average cooling could have been less than the suggested amount and be more in line with the cooling of $2.5^{\circ} \mathrm{C}-4^{\circ} \mathrm{C}$ found here (section 8.2.1).

The correlation pattern for NSI is quite different again. Precipitation is slightly stronger correlated to the westerly wind, but shows virtually no correlation with the meridional wind. As mentioned before in the discussion of ENI, this region also experienced only a minor reduction in precipitation, a strong westerly wind and an increased southerly wind component. It would therefore also have experienced an increase in its variability, both in temperature as well as in precipitation. Precipitation in WSSI and ESI are strongly positively correlated to westerly and easterly winds respectively. Similar as during MAM, WSSI and ESI experience a very strong increase in the southerly winds. For WSSI it is the westerly wind that goes south around the South Island and then turns north that brings the moisture, mainly to Otago, and then in particular to its coastal region. But with the westerly wind weaker in inland Otago, moist air can 
travel inland easier as well. The strong reduction in westerly wind in ESI enhances the correlation of precipitation with a southerly wind.

\subsection{Summary}

The climate of New Zealand during the Last Glacial Maximum was investigated using an RCM, which was embedded within a GCM. The setup for the glacial conditions was similar as in the GCM and was discussed in chapter 7. Some fields were remarkably different and were determined separately. Most noticeable was the different orography of New Zealand during the LGM. New Zealand's modelled orography in the LGM simulation was higher than in the preindustrial simulation, partly as the result of the lower sea level but mainly as the result of the extensive glaciers covering the land. New Zealand's modelled LGM orography was higher by $1 \mathrm{~km}$ compared to the orography in the pre-industrial simulation in some areas. As was determined in chapter 6, the modelled orography plays a major part on the outcome of the climate simulation. Incorrect representation of the orography could therefore give anomalous responses. Since it is impossible to know what the orography exactly was during the LGM, it should be kept in mind that the simulated climate might have been slightly different with a different modelled orography. However, it is believed that if the orography was modelled slightly different, along established thoughts about what the LGM orography might have been, it would not have affected the general results of this study. The orography modulates the regional responses, but a small change in its modelled height does not reverse its effect. From chapter 6 it was already determined that the RCM was not capable of reconstructing the exact values for climatic parameters like temperature and precipitation, but that it does a good job in reconstructing regional aspects and seasonal trends. These two variables were examined again in the analysis of New Zealand's climate during the LGM, together with the possible effects of the changes in wind regimes for the six regions defined in chapter 6 on these variables. 
Cross sections of temperature showed how the temperature followed the preindustrial values very well, albeit that they were consistently colder. Only where there was a change in the orography did the trends in the cross sections differ. There were much colder temperatures in the mountains during the LGM. This was partly because of the inclusion of the glaciers, but also for a large part as the result of the higher orography. The extended landmass to the east of the North Island showed similar temperatures during DJF and SON as the current east coast. However, this area experienced much colder temperatures than the current coastal areas during MAM and SON.

From proxy data it was determined that the average annual temperature in New Zealand during the LGM might have been lower by $4.5^{\circ} \mathrm{C}-5^{\circ} \mathrm{C}$. The mean annual cooling found here $\left(4.6^{\circ} \mathrm{C}\right)$ sits exactly within this range, but this value was controlled by the very strong cooling that took place over the Alps. It was impossible to compare the LGM and pre-industrial temperatures in the Alps against each other, because their equivalent grid points were at different heights. Adjusting the average annual cooling for the large effect of the enhanced orography of the Alps gave an estimated cooling of about $8^{\circ} \mathrm{C}-10^{\circ} \mathrm{C}$ over WSSI and a calculated cooling of $2.5^{\circ} \mathrm{C}-4^{\circ} \mathrm{C}$ for the rest of New Zealand. The strongest seasonal cooling took place during MAM and JJA, followed by SON. Since it was known that the modelled westerly wind was too strong, particularly during $\mathrm{SON}$, it was difficult to indicate whether the modelled amount of cooling was too low or too high. But in general, the seasonal temperature differences increased during the LGM. Apart from WSSI, the largest cooling consistently took place in ESI. ESI experienced a strong increase in the number of southerlies, particularly during MAM and SON. Southerly winds were able to bring up much colder polar air and moved right up the east coast. Of a lesser impact on the temperature was the snow cover. The South Island experienced snow fall during the whole year, although only during JJA was there a substantial snow pack on the ground. Snow on the ground increased the albedo of the surface, reflecting incoming solar radiation, and would have enhanced the amount of cooling of the land. 
Precipitation patterns were also heavily influenced by the increase in height of the orography. The Southern Alps acted already as a barrier to the prevailing westerly winds in the pre-industrial simulation, but that became even more the case during the LGM. Although the colder atmosphere of the LGM was less capable of containing moisture, there was an increase in precipitation just to the west of the Alps, particularly during DJF. The westward shift of the precipitation band along the West Coast of the South Island and Fiordland was the result of the extension of the glaciers all the way out to the sea. The modelled orography was not only higher than in the pre-industrial simulation, but its bulk had also shifted westwards. The westerly wind, bringing moist air to the land, had to ascend earlier, more to the west. Convection, cloud formation and eventually precipitation, would therefore have occurred more to the west as well. The effect of the westward migration of the main precipitation band was a reduction in precipitation over the Alps. Just as with the analysis of temperature, precipitation patterns in WSSI were difficult to compare between the two simulations, since most data was calculated at different grid point heights. Similar to the preindustrial simulation, the precipitation patterns followed the orography quite well. Overall, the mean precipitation was reduced in New Zealand, varying between $10 \%-20 \%$ less than in the pre-industrial simulation, except for some notable exceptions. There was virtually no reduction in precipitation in ESI and during DJF and SON there was even a slight increase in precipitation. This was ascribed to a reduction of the strength in the westerly wind, and to the increase of strong incursions of southerly winds. The latter could also have been influential in keeping the precipitation levels virtually the same in NSI and ENI during SON. However, there was a strong reduction in precipitation over and directly east of the Alps, especially in inland Otago and Southland. It was noted however that since the model underestimates precipitation in the east of the country, an apparent increase, or lack of reduction, in precipitation in the east, does not automatically imply that precipitation in those regions was the same as during the present day. It could therefore be possible that those regions experienced a modest decrease in precipitation during the LGM. 
Similar as in the pre-industrial simulation, temperature and precipitation followed the orography quite well. It could therefore be suggested that the general trend in these variables could probably also have been obtained by simply interpolating the orographically corrected GCM values. However, the geological proxy data set does not show the same resolution in space and in time as CLIDB. Any variation between the GCM interpolated values and the CLIDB data could be an indication of a regional climate effect. Such a pattern would never show up if downscaling was applied to the LGM simulation, since the error in the geological data could be much larger than the error in the interpolated value of the GCM. Hence this was a case for using a RCM. This becomes even clearer when investigating the different regional wind regimes of the different climates on the temperature and precipitation patterns.

A change in the wind patterns played a role in the analysis of both temperature and precipitation. From the analysis of the mean zonal circulation in the Southern Hemisphere it was concluded that there was a change in the modelled wind regimes during the LGM, with a stronger westerly wind and more incursions of southerlies in the latitudes covering New Zealand. It was determined that the New Zealand region experienced more and stronger incursions of southerly winds than in the zonal mean. This followed from the fact that there was an enhanced wave number 3 pattern in the mean zonal circulation with a stronger northward flow to the east of Australia.

The reduction of northerlies, and therefore the increase in southerlies, in NNI could be an indication of fewer tropical lows being able to descend down on New Zealand. This would have reduced the advection of warm moist air to the region. But the increase in the occurrence and strength of the southerly winds did not happen all over New Zealand, nor did it happen during every season. Both SWNI and ENI experienced an increase in northerlies during DJF and MAM. The largest increase in southerlies happened in WSSI and ESI, particularly during MAM and SON, and resulted in the largest cooling in New Zealand. As the result of the increase in the geographical extent of the land with temperatures below the 
condensation level, the lowering of the condensation level, and the massive increase in the modelled orography, precipitation in WSSI only decreased by about $5 \%$ during MAM and SON, and increased slightly during DJF (2\%). The larger reduction in precipitation during JJA (17\%) is similar as in all other regions. But since precipitation in ESI already depended strongly on a southerly component in the wind direction in the pre-industrial simulation, the general reduction in moisture available for precipitation during the LGM is countered in ESI by the increase of incursions of southerly winds. The resulting effect was that there was virtually no change in precipitation in ESI. The effect of the increase in southerlies travelled up the country, and affected NSI and ENI during JJA and SON as well.

Although in the mean zonal circulation the westerly wind increased slightly in the midlatitudes, it showed a variable pattern over New Zealand. The westerly wind increased substantially over the North Island and over NSI, especially during DJF (increased by $20 \%-45 \%$ ) and MAM (increased by $50 \%-400 \% !^{1}$ ). The increase in the strength of the westerly wind during SON in these areas was relatively small $(1 \%-8 \%)$ when compared to the pre-industrial simulation. This was more the result of comparing the wind with an anomalously strong westerly wind in the pre-industrial simulation than that the westerly wind during SON was relatively weak. The westerly wind was still the strongest during SON. The stronger westerly wind could have acted as a barrier to tropical lows coming down on New Zealand, enhancing the drier conditions in the northern regions. Nevertheless, those northern regions still depended strongly on precipitation coming from the north, which was indicated by the higher correlation (increase of dependency) of precipitation with the meridional (northerly) wind during DJF and MAM. The stronger westerly wind over ENI would have prevented moisture-bearing easterlies to come ashore, thereby enhancing the dry conditions

\footnotetext{
${ }^{1}$ This is a comparison of regional averages. Grid points with an easterly wind (negative direction) would cancel out the westerly wind (positive direction) of another grid point. During the LGM, more grid points showed a predominant (and stronger) westerly flow, thereby strongly enhancing the differences in the zonal wind direction between the two simulations. Hence the extreme value of $400 \%$.
} 
in the east. ENI showed a stronger correlation between precipitation and an easterly flow than in the pre-industrial simulation. The stronger westerly winds increased their cooling effect for NSI and the North Island regions during DJF, and enhanced warming in ENI during MAM. The temperature response during DJF depended on the location of a high over the country more strongly than during the pre-industrial. It was the advection of weather systems that affected the temperature response during the other seasons. A stronger westerly wind would support the geological evidence of enhanced upwelling in the east (e.g. Nelson et al., 2000).

The two southern regions were remarkably different in that they experienced a weaker westerly wind (up to 5\%-25\% weaker) the whole year round, although, as with temperature and precipitation, it was difficult to analyse the data for WSSI, since comparisons were made between points at different heights. The weaker westerly wind affected both the precipitation and temperature characteristics of the two regions. A weaker westerly wind on the lee-side of the mountain range resulted in an increase in precipitation, since moist easterlies were able to come onshore more easily. As the result of the westward shift of the main precipitation band, the westerly was quite dry when it crossed the mountains. The dry westerly wind in turn warmed up the Alps and Fiordland in particular, although the correlation between westerly wind and temperature was only up to +0.3 . The exception was during MAM, when the westerly wind did not have a warming influence over the mountains. But this was still in sharp contrast with the negative correlation (cooling) during the pre-industrial. Therefore, besides that the precipitation band shifted westward during the LGM, the area that was affected by a dry warm föhn wind moved westward as well. This had consequences especially for northern Southland/Central Otago during DJF where the cooling was reduced as a result of the weaker westerly wind. It is therefore possible that these regions did not experience any cooling during the LGM, and might even have experienced slightly warmer temperatures than during the present day. ESI experienced the strongest westerly winds during the transition seasons of MAM and SON, when these winds had a warming effect on 
the region. During summer and winter, the westerly wind was weaker, allowing easterlies to come ashore more easily, and thereby cooling ESI. This is similar to the pre-industrial simulation, except that the westerly winds had a warming effect on ESI during JJA as well.

JJA was often left out in the previous discussion. This was because JJA showed some different characteristics. All regions had a weaker westerly wind during JJA. Blocking highs are a common feature during JJA in the present day. It was already suggested in chapter 7 that the occurrence of blocking highs could have increased over the New Zealand region, particularly during JJA, as the result of the enhanced wave number 3 pattern of the mean zonal circulation. The reduction in the strength of the westerly wind in all regions during JJA follows therefore the suggestion of an increase in blocking during the LGM very well. An increase in blocking during JJA would have enhanced the cooling as well, particularly over the South Island, since clear skies would have enhanced the amount of outgoing longwave radiation. At the same time, the enhanced incoming radiation would have had less effect since most of the South Island was covered in snow. There was virtually no change in the number of gridpoints experiencing a mean southerly flow during JJA, but there was a shift to stronger southerlies. Stronger southerlies would have been able to drag the very cold polar air all the way into New Zealand. The southerly component increased for nearly all regions, indicating that the cold blasts were moving all the way up the country.

To what extent the stronger ENSO signal during the LGM had affected New Zealand's climate has not been investigated. HadCM3 does not reconstruct the variability in the tropical SSTs correctly and it is therefore difficult to link a change in ENSO patterns to any climatic features of the LGM. The very short period of only 16 years of analysis did show that there was not a dominant El Niño or La Niña signal (chapter 7). If El Niño was dominant during the whole period, than it can be expected that New Zealand had experienced a more south(westerly) flow, explaining some of the increases in the southerlies 
described here. With the variability as the result of ENSO explaining a similar amount of interannual variability during the LGM and during the pre-industrial, it is therefore clear that the modelled changes in New Zealand's climate of the LGM are primarily related to the glacial conditions and not to ENSO patterns.

New Zealand's climate during the LGM was undeniably harsh. The North was cooler, drier and windier, and the South was cold and bleak. Such a climate regime would have affected the existence of certain vegetation types. Geological proxy data indicates a cooling of about $4.5^{\circ} \mathrm{C}-5^{\circ} \mathrm{C}$. Together with a drier climate, such a cold climate would severely limit the existence of forests. However, the cooling found here was less. There should therefore be other factors limiting vegetation growth. It is proposed here that it was the increase in the extremes of the climate that limited the growth of certain vegetation types. With precipitation in the south similar to the present day, the existence of forests in the south could be affected mainly by the colder temperatures and the strong incursions of bleak southerly winds, where as the limitation to forest growth in the north could be mainly related to the drier conditions and stronger westerly winds. It is not known to the author whether it would be possible to find such a distinction in the geological data.

The main anomaly between this simulation and geological proxy data was in the position of the ocean currents around New Zealand. Suggestions that the Tasman Front had weakened and had shifted northwards were to a certain extent simulated in the GCM, and therefore showed up in the SSTs in the RCM. But there was no equatorward shift of the ACC into New Zealand's regional domain. Such a strong northward shift of the ACC would have cooled particularly the SSTs in the south and east of the South Island (a cooling of $6^{\circ} \mathrm{C}-10^{\circ} \mathrm{C}$ was determined by Barrows et al., 2000), and would have affected their local climates. 


\section{Summary and Conclusions}

\subsection{Summary}

In order to improve our capability in making future climate predictions, it is necessary to understand climates from the past. Past climates can inform us about the intricacies of global circulations and regional climate patterns under different forcings. Climate modellers and geologists work together in their efforts in reconstructing and understanding those climates. The latter finds a multitude in proxy data, which helps draw a hypothesis about past circulations, where as the former alters boundary conditions and model settings in order to simulate the climate from the past. Combining both approaches, climate models should be able to reproduce the climate features implied by the geological proxy data, and geologists should be able to either find proof supporting, or contradicting, model findings or indicate shortcomings in the model. The aim of this study was to investigate the climate of New Zealand during the Last Glacial Maximum. The approach to this was through climate modelling. The models used were versions of the UKMO Unified Model. Output from a GCM detailed the general circulation and emphasis was placed on the circulation in the Southern Hemisphere midlatitudes. Regional detail was obtained by embedding a RCM within the GCM.

In order to be able to separate data representing a "real" climate pattern from the biases and errors in the model's output, it is imperative to investigate the performance of the model first. For that reason, the GCM and RCM were first set up under pre-industrial conditions and their output was compared against present 
day climatologies; the NCEP 1971-2000 climatology and the NIWA CLIDB. It is assumed that the climate of the pre-industrial era was not that much different from today's climate, and that such a comparison is therefore valid. Simulating the pre-industrial climate rather than the present day climate simplifies the model considerably. Simulating the pre-industrial climate eliminates the need to include the increasing concentrations of greenhouse gases in the atmosphere we experience today. This means that the tropospheric chemistry can be kept constant in the model. The particular versions of the UM used were the HadAM3H and HadRM3H (NZ_40km) configurations. HadAM3H is a global atmospheric model with a resolution twice that of the well-established and documented model HadAM3 (e.g. Pope et al., 2000), with 192 points in longitude, 145 points in latitude and 19 levels in the vertical. Initial conditions and monthly updated SSTs and sea-ice fields were provided by its coupled GCM version, HadCM3 (Gordon et al., 2000). NZ_40km is an RCM, which physics are similar to that of $\operatorname{HadAM} 3 \mathrm{H}$ with only a few changes in some parameterization schemes. Its horizontal resolution of the New Zealand domain was $0.36^{\circ}$, or about $40 \mathrm{~km}$, and there were $95 \times 68$ points. The initial and boundary conditions were supplied by $\mathrm{HadAM} 3 \mathrm{H}$.

The biases in HadAM3H were similar to the ones in HadAM3. Relevant to this study is that the surface westerly winds in midlatitudes were too strong, the distribution of HadCM3's computed SSTs in the Pacific was not completely correct, and there were cooler SSTs in the North Pacific and warmer SSTs in the Southern Hemisphere high latitudes. It was found further that HadAM $3 \mathrm{H}$ overestimated the amplitude, and therefore the strength, of the planetary waves in the Southern Hemisphere, which in turn affected meridional transport.

The climate of the LGM was reconstructed using Peltier's (1994) ice sheets and computed SSTs and sea-ice fields from a HadCM3 LGM simulation (Hewitt, 2000). The latter was set up according to the glacial boundary conditions specified by PMIP (World Climate Research Programme-111, 2000), and the SSTs were therefore more similar to the values as determined by PMIP than those by CLIMAP (1976: 1981). $\mathrm{CO}_{2}$ levels were set to $200 \mathrm{ppm}$ and orbital 
parameters were based on the calculations of Berger (1978). Although emphasis was placed on the general circulation in the Southern Hemisphere, some aspects of the Northern Hemisphere circulation as well as some climatic patterns in the tropical Pacific were discussed. The modelled mean global temperature during the LGM was $3.8^{\circ} \mathrm{C}$ colder than in a pre-industrial simulation, but there were considerable geographical and seasonal differences. In the annual mean the cooling in the Northern Hemisphere was $5.2^{\circ} \mathrm{C}$, where as for the Southern Hemisphere the cooling was only $2.3^{\circ} \mathrm{C}$. Precipitation was reduced everywhere and during all seasons, except for the Southern Hemisphere during DJF and SON. The latter was probably related to precipitation patterns in the ITCZ and SPCZ, while precipitation was reduced everywhere south of about $45^{\circ} \mathrm{S}$. The largest reduction in precipitation took place over South East Asia, although one has to keep in mind that there was a large reduction in sea surface in South East Asia during the LGM.

\subsection{Conclusions}

\subsubsection{Global climate during the LGM}

Features of the Northern Hemisphere circulation during the LGM have been investigated in several other studies, and have been used to compare this simulation's output against (e.g. Manabe and Broccoli, 1985: Rind, 1987: Hall et al., 1996a: Dong and Valdes, 1998). It was found that particularly the enlarged continental ice sheets had a large impact on the general circulation in the Northern Hemisphere. The meridional temperature gradient increased as a result of the strong polar cooling. This caused a strengthening of the westerly winds. The North Atlantic jetstream shifted northwards and increased slightly in strength as a result of a warming in the North Atlantic (e.g. Hewitt, 2000: Hewitt et al., 2001). The Pacific jetstream did not shift southwards as was indicated by several other studies (e.g. Valdes and Hall, 1994: Kitoh et al., 2001) and decreased in strength. The latter findings were therefore similar to Dong and Valdes (1998) for their simulation with computed SSTs. In general, the strongest 
westerly winds were found along the boundaries of the continental ice sheets, where the largest gradients in meridional temperature resided. This resulted in a shift in the planetary waves from a dominant wave number 1 pattern towards a wave number 2 pattern. This shift in turn affected the underlying regional climates.

The increase in the meridional temperature gradient, as a result of the very strong cooling at the poles, was slightly muted by a cooling in the tropics. The climate of the tropics can drive some climate patterns in the midlatitudes through teleconnections, and for that reason some attention was paid to what happened in the tropical Pacific during the LGM. Computed SSTs were $2^{\circ} \mathrm{C}$ cooler in the Western Pacific, and $4^{\circ} \mathrm{C}$ cooler in the Eastern Pacific. This amount of cooling was slightly larger than found by CLIMAP. But these values were within the range found by many other modelling studies and by those estimated from several geological proxy data. It suggested also that there was in increase in the east-west gradient, again opposite to CLIMAP's findings, but similar to some other modelling studies (e.g. Weaver et al., 1998: Liu et al., 2000: Andreasen et al., 2001). The increase in the east-west gradient intensified the Hadley circulation, and suggested that the climate of the LGM was more towards a La Niña-style climate. At the same time, there was strong interannual variation in the Pacific's tropical SSTs in the Niño 3-4 area. This is different than in the present day, since the maximum in interannual variability in the NCEP climatology takes place in the Niño 1-2 region. The fact that the maximum in interannual variability in the simulation was not in the Niño 1-2 area seemed to be more an artefact of the model, since it also appeared in the Niño 3-4 area in the pre-industrial simulation. However, the increased interannual variability in the tropical Pacific SSTs seemed to be a real feature of the LGM climate. It indicates that there was a strong ENSO signal during the LGM, although its characteristics might have been a bit different.

The changes in the mean zonal circulation in the Southern Hemisphere were not as profound as in the Northern Hemisphere. This was mainly because the Southern Hemisphere did not have large continental ice sheets to influence the 
general circulation. The computed sea-ice expanded equatorward up to $55^{\circ} \mathrm{S} / 60^{\circ} \mathrm{S}$. This was not as vast as suggested by CLIMAP which suggested that the sea-ice expanded to $50^{\circ} \mathrm{S}$. The expansion of the sea-ice clearly affected the general circulation. It is therefore very important to get the expansion, and therefore the dynamics, of the sea-ice correct when simulating the climate of the LGM in the Southern Hemisphere.

The most indicative difference between the climate of the LGM and the present day (pre-industrial) in the Southern Hemisphere was the difference in their wind regimes. It was the change in the mean westerly circulation, and its related change in other climate parameters, that characterized the different state of the Southern Hemisphere's climate during the LGM. At the same time, regional climates in the Southern Hemisphere were particularly affected by the changes in the meridional winds. It was expected that the westerly winds were stronger during the LGM as a result of the increase in the meridional temperature gradient, but this strengthening of the westerly wind did not happen throughout the entire atmosphere. Compared to the pre-industrial simulation, the zonal mean of the geopotential height showed a steeper dipping slope from Antarctica to the Arctic than in the pre-industrial simulation. This indicated a force countering the increase in the westerly wind in the Southern Hemisphere as a result of an increase in the meridional temperature gradient. The subpolar trough shifted slightly equatorward, following the expansion of the sea-ice, and therefore shifted the strongest westerly winds equatorward as well. In the zonal mean, the westerly wind decreased throughout the entire troposphere roughly between $45^{\circ} \mathrm{S}-65^{\circ} \mathrm{S}$, with a slight, but consistent, increase at $35^{\circ} \mathrm{S}-45^{\circ} \mathrm{S}$. The strengthening and equatorward shift of the westerly wind is consistent with the findings of CLIMAP. However, there were some intriguing regional variations. Consistent with the findings of Wyrwoll et al. (2000) and Hope and Simmonds (2003), the strongest westerly winds for the Australian region shifted poleward, particularly during JJA. This was the result of an increase in mean sea level pressure over Australia. This shift was opposite to the shift in the zonal mean. Other regions required therefore a strong equatorward shift to counter the poleward shift of the 
westerly winds to the south of Australia. In the zonal mean, the winds curved northwards downstream of Australia, thereby increasing the southerly component of the meridional flow over the New Zealand region.

The zonal mean meridional winds between $35^{\circ} \mathrm{S}-60^{\circ} \mathrm{S}$ underwent a change in both the distribution of northerlies versus southerlies, as well as in their strengths. Although the mean meridional wind was always a northerly, there was an increase in southerly winds during all seasons except during JJA. During the winter months there was mainly a shift to stronger northerlies and southerlies. The increase in southerly winds had a strong impact on the regional climate of New Zealand.

Analysis of the $500 \mathrm{hPa}$ geopotential height showed that the planetary wave structure had a slight enhancement of the wave number 3 pattern. A cautionary note was placed on this finding, since it is known that the UM exaggerates the wave number 3 circulation in the Southern Hemisphere, and the question can therefore be raised whether the enhanced wave number 3 circulation was a real feature of the circulation during the LGM or that it was an artefact of the model. However, it was found that a slight enhancement of the wave number 3 circulation pattern was consistent with other climate patterns, and was therefore seen as a true feature of the circulation during the LGM. The amplitude of this pattern would have slightly increased during JJA as the result of the increase in the strength of both the northerlies and southerlies. The planetary waves would have shown a slightly more sinusoidal pattern during the other seasons as a result of the change in the distribution of the northerlies and southerlies. Such a change is completely in accordance with the fact that the zonal mean meridional heat transport was reduced during the LGM since it results in a reduction of the heat transporting poleward-tilting phase. Although the zonal mean meridional heat transport was reduced in the Southern Hemisphere midlatitudes, there was a small increase in the transient eddy component. An increase in this term is associated with an increase in cyclonic activity. However, the increase in baroclinicity did not lead to an increase in precipitation since the colder climate of the LGM had reduced moisture content and a larger static stability. A stronger 
wave number 3 circulation in the Southern Hemisphere may have played a positive role in the occurrence of blocking in the Southern Hemisphere (e.g. Trenberth and Mo, 1985). It was suggested that blocking over New Zealand could have been enhanced during the LGM, particularly during JJA.

The main modes of circulation variability on monthly and longer timescales changed slightly during the LGM. All the changes in the circulation in the Southern Hemisphere which were mentioned above (an equatorward shift of the polar trough and the westerly winds, and an enhanced wave number 3 pattern in the mean zonal circulation) are consistent with a weaker SAO (e.g. Meehl, 1991: Chen and Yen, 1997: Burnett and McNicoll, 2000). The pattern of the SAO remained the same however, with the peaks appearing during March and October. EOF analysis showed that interannual variability was again dominated by the HLM and ENSO. The HLM accounted for a slightly larger portion of the variability during the LGM than during the pre-industrial $(31.2 \%$ vs. $29.3 \%)$, and showed a preference towards the positive phase. Since HadCM3 reconstructed the variability in the tropical SSTs inadequately, the simulated ENSO signals were not identical to the classical ENSO patterns. However, variability that was linked to Pacific SST patterns have been referred to as ENSO patterns throughout this thesis. The EOFs associated with ENSO variability explain a similar amount of the total variability as for the pre-industrial simulation $(9.6 \%$ versus $9.7 \%$ and 8.6\% versus $9.6 \%$ ), even though the ENSO signal was stronger in the tropics during the LGM. The $2^{\text {nd }}$ EOF showed a strong wavetrain pattern going from East Australia all the way into the South Atlantic, very similar to the wavetrain pattern found in the analysis of the NCEP data. Such a pattern enhances southwesterly flows over the New Zealand region during El Niño years. The $3^{\text {rd }}$ EOF showed a different pattern and was more similar to a wavetrain pattern too. It also went roughly from East Australia to the South Atlantic, but was weaker and was $90^{\circ}$ out of phase with the wavetrain pattern associated with the $2^{\text {nd }} E O F$. This pattern enhanced the occurrence of low-pressure zones moving right over New Zealand during El Niño years. Depending on the correctness of HadCM3's tropical SSTs, it could be suggested that ENSO potentially would have had a 
stronger signal in New Zealand during the LGM than in the present day, since the different influences of El Niño and La Niña on New Zealand's climate would have been enhanced. The south-westerly flow associated with the $2^{\text {nd }}$ EOF would draw up much colder air than during the present day, where as the north-easterly flow during La Niña years would only have been slightly cooler than during the present day. A similar comparison goes for the variability associated with the $3^{\text {rd }}$ EOF, when during El Niño years there was an increase in cool westerlies, with only a relatively small decrease in precipitation, where as during La Niña years there were more high-pressure zones covering New Zealand, leading to drier and less cool conditions. Both patterns therefore lead to an enhancement of the different signatures of El Niño and La Niña in New Zealand.

\subsubsection{New Zealand's climate during the LGM}

New Zealand's regional climate during the LGM was mainly described through temperature, precipitation and wind patterns. When comparing the modelled LGM climate with the pre-industrial simulation, it had to be kept in mind that as the result of the enhanced orography during the LGM, data for the two simulations was determined at different heights. Cross sections of temperature and precipitation showed that these variables followed the orography very consistently. The difference in orography could therefore not explain the variations in the differences in these climate parameters. The main conclusions about New Zealand's climate during the LGM were:

\section{- Temperature:}

The modelled annual average cooling over New Zealand was $4.6^{\circ} \mathrm{C}$, which sits in well within the estimation of $4.5^{\circ} \mathrm{C}-5^{\circ} \mathrm{C}$ based on geological proxy data (e.g. McGlone et al., 1993). However the amount of cooling in the simulation was strongly influenced by the enormous increase in the modelled orography of the Southern Alps. The main mountain ranges in the South Island were much higher during the LGM as the result of the inclusion of massive glaciers, which in the West Coast and Fiordland extended all the way to the Tasman Sea. Omitting the 
effect of the different orography led to a cooling of $8^{\circ} \mathrm{C}-10^{\circ} \mathrm{C}$ in WSSI and $2.5^{\circ} \mathrm{C}$ $4^{\circ} \mathrm{C}$ for the rest of New Zealand. The cooling in the North Island was less $\left(2.5^{\circ} \mathrm{C}\right.$ $\left.3.3^{\circ} \mathrm{C}\right)$ than in the east of the South Island $\left(3^{\circ} \mathrm{C}-5^{\circ} \mathrm{C}\right)$. The seasonal variations in temperature increased since the cooling was larger during the winter than during the summer.

\section{- Precipitation:}

The precipitation was reduced almost everywhere, varying between 10\%-20\% less than in the pre-industrial simulation. The exceptions were in the east of the South Island the whole year round, in ENI during JJA and SON and in NNI during SON, where there was no reduction in precipitation. Since the model underestimates precipitation in the east, the apparent lack of a reduction in precipitation in the eastern regions does therefore not automatically imply that precipitation in the east was similar to the present day. The precipitation pattern over the Alps had altered considerably as the result of the existence of the massive glaciers. The precipitation band had shifted westward, thereby reducing the amount of precipitation over the Alps and to the region immediately on the lee-side of the mountain range, in particular inland Otago and Southland. These areas experienced the largest reduction in precipitation.

\section{- Winds:}

Together with the changes in the winds in the general circulation of the Southern Hemisphere, the change in the wind regimes in New Zealand during the LGM were the most profound findings in this study. Cooler temperatures and a reduction in precipitation were expected, but the change in the winds and their change in their correlation patterns with temperature and precipitation in New Zealand were indicative of the different climate of the LGM in New Zealand. There were also remarkable differences between the North Island and most of the South Island. The westerly wind was considerably stronger over the North Island and the north of the South Island, but was weaker over the rest of the South Island. The exception was during JJA when the westerly wind was weaker everywhere. This may have been associated with enhanced blocking during this 
season. The stronger westerly wind could have acted as a barrier to tropical lows coming down towards New Zealand, thereby enhancing the drier conditions in the northern regions. The stronger westerly wind would definitely have prevented moist easterlies coming ashore in ENI, thereby enhancing the drier conditions in the east. The stronger westerly winds had a strong cooling effect for the North Island, particularly during the summer months.

The westerly winds were $5 \%-25 \%$ weaker over most of the South Island. The weaker westerly wind in the east of the South Island allowed moist easterlies to come ashore more easily. The resulting relative increase in precipitation in this area countered the general reduction in precipitation as a result of the drier LGM conditions in general. The westerly wind had a warming effect on the land during MAM and SON, and had a cooling effect during the summer and winter. The reduction in westerly wind in WSSI was more difficult to establish, since as the result of the massive increase in the orography, there was often a net easterly directly to the west of the glaciers. After having lost its moisture more to the west, the westerly wind was dry over the Alps and had therefore a warming effect on the land. It was possible that some areas in inland Otago and Southland were not cooler during the summer months during the LGM as a result of this föhn wind.

The change in the meridional wind over New Zealand was felt strongly over most of the South Island. The increase in southerlies had a strong cooling effect on the South Island and would have contributed to the enhanced cooling that took place there. The southerlies were able to drag much colder air than in the present day from the polar region onto New Zealand. The strongest increase in southerly winds took place during MAM and SON, which coincided with the strongest cooling in the South. With precipitation in the east linked to a southerly component in the wind, an increase in southerlies therefore also countered (at least partially) the general reduction in precipitation. The opposite was true for the northern region, where a decrease in northerlies could be associated with a decrease in the numbers of (tropical) lows coming down and therefore reducing the advection of moist air to the region. 
Proxy data have indicated that New Zealand during the LGM was in general colder by $4.5^{\circ} \mathrm{C}-5^{\circ} \mathrm{C}$, drier and probably windier. Most of the results here agree to a certain point with those findings. However, this study was able to give a more detailed regional and seasonal description and those findings were re-examined. The estimated cooling of $4.5^{\circ} \mathrm{C}-5^{\circ} \mathrm{C}$ was mainly based on proxy data indicating the existence, or the lack of certain vegetation types, and to a lesser degree, to the lowering of snowlines. The cooling of $2.5^{\circ} \mathrm{C}-4^{\circ} \mathrm{C}$ found in this study would therefore seem to be too low to explain those findings. However, it was suggested here that it was not the absolute cooling but the increase in extremes that determined the existence of vegetation types in New Zealand. The LGM climate in New Zealand was much harsher, with an increase in seasonality in temperature and incursions of strong southerly, polar winds. Those conditions, together with a reduction in precipitation, would have affected plant growth, hence the vegetation changed to those plant types that could cope with the harsher conditions. It was suggested that in some isolated and/or sheltered areas forests could still have existed. If that were the case, it might very well be possible that the South and North Island have had different limiting conditions for those areas. The east of the South Island did not experience a real reduction in precipitation, but had increased snowfall, and a weaker westerly wind. Wind and precipitation would therefore probably not have been the limiting factor for forests growth in the east of the South Island. The cold temperatures, and particularly the ones associated with the polar blasts, would have been the main limiting factor for forests to grow in the South Island. It can therefore be suggested that those areas that were sheltered from direct exposure to the cold southerlies might still have had conditions that suited forests. The opposite is true for the North Island. The cooling was much more reduced, but there were drier and windier conditions, especially in the east. For forests to be able to exist, they should have been protected from the strongest wind, and should have been in soils that retain their moisture well. Such a situation might have happened in NNI, since proxy data indicates that the north of the North Island was still covered in forests. Although the modelled westerly wind during the LGM in NNI increased, it was not stronger than what the rest of the North Island experiences 
today. Nor was the reduction in precipitation in NNI such that it was drier than conditions experienced in the east of the North Island during the present day. Since these areas are covered by forest in the present day, it is therefore plausible that forests were most likely common in NNI during the LGM. The suggestions regarding the different limited factors for vegetation growth for both islands are mere speculations, but it would be interesting to find out whether these can be backed up by geological/biological evidence.

Although this study did not involve any ocean modelling, some of the ocean data around New Zealand was analysed. The SSTs in the Tasman Sea were about $2^{\circ} \mathrm{C}-3^{\circ} \mathrm{C}$ cooler. It was not possible to determine whether this amount of cooling was solely the result of the general cooler conditions during the LGM, or whether a change in the currents had affected the cooling as well. Geological proxy data does suggest that the currents around New Zealand during the LGM might have been different. Although the resolution of the coupled model was too low to discern the small-scale currents around New Zealand, there were indications in the SSTs and streamline patterns that the Tasman Front was slightly weaker during the LGM. A reduction in the Tasman Current gave rise to colder SSTs to the waters to the north and the east of the North Island. These findings are consistent with geological proxy data (e.g. Barrows et al., 2000). However, geological data also indicate that there were much lower SSTs to the southeast of New Zealand, over the Campbell Plateau (e.g. Nelson et al., 2000: Carter et al., 2002), which were possibly associated with a northward shift of the ACC. Such a shift was not observed in this simulation however. The fact that the model did not show an equatorward shift of the $\mathrm{ACC}$ does not mean that such a shift did not happen at the LGM. It is known that the location of the ACC is not well modelled in HadCM3 (Gordon et al., 2000).

\subsection{Discussion}

At the start of this thesis it was mentioned that geologists and climate modellers have come together in studying past climates. One can supply physical 
constraints on some climatic parameters, the other can develop possible scenarios for climates of the past based on mathematical equations. When these separate studies support each other findings, we feel that we have improved our understanding on a particular aspect of our climate. But often it happens that the studies contradict each other. Although this study investigated the LGM through climate modelling, by no means is it implied that all the findings here are the correct ones. Particularly where the climate simulation contradicts the proxy record, modellers should question their model. For instance, adjusting parameterizations might then lead to improvements in the simulation. On the other hand, due to its spatial and temporal limitation of information that can be gained from geological proxy data, geologists should set out to obtain more and better proxy data in order to improve the description of the climatic parameter.

In this study several simplifications were made and known errors were acknowledged and were taken into account when drawing conclusions. The most obvious anomalies were that the modelled westerly wind was too strong and the amplitude of the wave number 3 pattern of the mean zonal circulation in the Southern Hemisphere was too large. Although the atmosphere is a strong nonlinear system, it has been assumed that the different aspects of the climate during the LGM found in this study were the result of running the model under the general glacial conditions and not as the anomalous result of one or more of the parameterizations, or in the settings of the model, to a set of different initial and boundary conditions. Improvements therefore could then be found in improving the parameterizations or initial settings. The GCM configuration used in this study, HadAM3H, was an improvement on HadAM3 particularly because it was capable of simulating the climate in the Northern Hemisphere more realistically. Since the model originates from the UK, it is understandable that in the update of the model emphasis was placed on improving the simulation of the Northern Hemisphere circulation. However, such a model does not have to result in a better simulation of the climate of the Southern Hemisphere. To improve the simulation of the climate in the Southern Hemisphere, a model configuration should be set up that in particular deals with the main features of the Southern Hemisphere circulation. If such improvements were made, a better simulation of 
Southern Hemisphere climates during other times, such as the LGM, would be likely too. In this particular case, the configuration should improve the wave number 3 pattern in the general circulation (together with its related exchanges in the meridional direction), the strength of the westerly wind, the ocean currents in general, but the position of the ACC in particular, and the extent of sea-ice. All these changes should lead to improved reconstructions of regional models in the Southern Hemisphere as well. For the New Zealand region during the LGM, it can be very crucial to include a possible northward shift of the ACC, as indicated by proxy data. Such a change in the position of the ACC will definitely affect the temperatures of the coastal areas, and will probably affect also more inland regions. Alternatively, this study of the climate of New Zealand during the LGM could be done again by using SSTs computed from proxy data. Such a study could then investigate the effect of much colder SSTs to the east and south of New Zealand on the climate of New Zealand.

Undoubtedly, improvements in the regional climate model could be made (e.g. resolution, representation of orography, and its effect on precipitation, baroclinicity, lee wave effects, etc., vegetation and soil characteristics, etc.), but it is felt that the greatest importance should be placed on getting the initial and boundary conditions correctly. The largest anomalies were during SON and they were related to the anomalous general circulation during that season.

Several simplifications were made in the GCM configuration. Due to the lack of an LGM global vegetation database that can easily be implemented into the UM, the vegetation during the LGM was prescribed very similar as for the preindustrial simulation. However, from geological proxy data we know that the vegetation during the LGM was different. The question that can be raised is how much the simulated climate of the LGM would have been different if the correct vegetation and soils had been included. Or in other words, what is the sensitivity of the model to the different kind of vegetation. The main impact of including the correct vegetation in the simulation of the LGM would be the vegetation's effect on the exchanges of gases (respiration) and the hydrological cycle. But since the 
former is kept constant in the simulation of the LGM, and the latter was weaker during the LGM, it could be suggested that the impact would have been limited.

Aerosols in the atmosphere have a cooling effect on the mean global temperature. Geological proxy data have indicated that the amount of aerosols in the atmosphere during the LGM was much higher than during the present day. A drier, windier climate, and an increase in land area, all contributed to this increase. Using an EBM, Harvey (1988) found that the effect of the increased concentration of the aerosols on the climate of the LGM could be a cooling of $2^{\circ} \mathrm{C}-3^{\circ} \mathrm{C}$. Although the UM includes a simple scheme to incorporate the effect of aerosols on radiation (Cusack et al., 1998), it is not known what the distribution, nature and radiative properties were of the aerosols during the LGM. For that reason they were not included in this simulation.

The temperature response of the high latitudes in the Southern Hemisphere impacts on the SAO, the strength of the westerly wind and the position of the polar trough. One of the main controls on the temperature of the Southern Hemisphere's high latitudes is the extent of sea-ice. It was found that the extent of sea-ice was also linked to cyclonicity in the high latitudes (e.g. Cavalieri and Parkinson, 1981: Simmonds and Wu, 1993). It is therefore crucial for the simulation of a Southern Hemisphere climate to correctly incorporate the seasonal distribution and extent of sea-ice. It can be expected that a full dynamical sea-ice model could lead to an improved simulation of the climate of the Southern Hemisphere.

It is felt that the greatest improvement in the regional climate model could be made in the representation of the mountains. New Zealand's regional climates have always been affected by the regional influences of the local orography, especially in the south of the country. Including orography correctly is therefore very important. However, with the climate recordings in the mountains taken at different heights than the equivalent height of the gridpoints in the model, plus the large, very localised (high resolution), effect of mountain slopes and valleys on the local climate, it will always be impossible to directly compare modelled and observed data. Generalisation (smoothing) and interpolation of data will 
always be necessary when one wants to model the climatic effects of steep mountains like those in New Zealand. Parameterizations for cloud formation, convection, precipitation and others probably would need to be specifically determined for a land mass like New Zealand.

Although this study used only mean monthly and mean seasonal data as output, more information could be obtained if daily minimum and maximum data were available too. In this simulation, vegetation and soil properties were simplified, and no drainage system was included. If these features had been included in the simulation, they certainly would have affected the climatic daily extremes. Since these were not included, analysis of daily extremes was less relevant here, but should be important in accessing the internal variability of the regional model. 


\title{
Abbreviations and Acronyms
}

\author{
AABW \\ Antarctic Bottom Water \\ ACC \\ Antarctic Circumpolar Current \\ $\mathrm{AD}$ \\ Anno Domino \\ AGCM \\ Atmospheric General Circulation Model \\ $\mathrm{AAO}$ \\ Antarctic Oscillation \\ BMRC \\ Bureau of Meteorological Research Centre \\ BP \\ Before Present \\ $\mathrm{CCN}$ \\ Cloud Condensation Nuclei \\ CGCM \\ Coupled General Circulation Model \\ CFL \\ Courant-Friedrich-Levy \\ CLIDB \\ Climate Database \\ CLIMAP \\ Climate: Long-Range Investigation, Mapping and \\ Prediction \\ DC \\ D'Urville Current \\ DJF \\ December-January-February \\ DWBC \\ Deep Western Boundary Current \\ EAC \\ East Australian Current \\ EAUC \\ East Auckland Current \\ EBM \\ Energy Balance Model \\ ECC \\ East Cape Current \\ ECMWF \\ European Centre for Medium-Range Weather Forecasting \\ EMIC \\ Earth System Models of Intermediate Complexity \\ ENI \\ East North Island \\ EOF \\ Empirical Orthogonal Functions
}




\begin{tabular}{|c|c|}
\hline ESI & East South Island \\
\hline ENIAC & Electrical Numerical Integrator And Calculator \\
\hline ENSO & El Niño - Southern Oscillation \\
\hline ESM & Earth System Model \\
\hline GCM & General Circulation Model \\
\hline HadAM3 & Hadley Centre Atmospheric Model version 3 \\
\hline HadAM3H & $\begin{array}{l}\text { Hadley Centre Atmospheric Model version } 3 \text { High } \\
\text { resolution }\end{array}$ \\
\hline HadCM3 & Hadley Centre Coupled Model version 3 \\
\hline HadOM3 & Hadley Centre Ocean Model version 3 \\
\hline HadRM3H & Hadley Centre Regional Model version 3 High resolution \\
\hline HLM & High Latitude Mode \\
\hline IAS & $\begin{array}{l}\text { Institute for Advanced Study (Meteorology project in } \\
\text { Princeton) }\end{array}$ \\
\hline IPCC & Intergovernmental Panel on Climate Change \\
\hline ITCZ & Intertropical Convergence Zone \\
\hline JJA & June-July-August \\
\hline $\mathrm{km}$ & kilometre \\
\hline Ky & thousand years \\
\hline LBC & Lateral Boundary Conditions \\
\hline LGM & Last Glacial Maximum \\
\hline MAM & March-April-May \\
\hline $\mathrm{hPa}$ & hecto Pascal \\
\hline MOSES & Met Office Surface Exchange Scheme \\
\hline MSLP & Mean Sea Level Pressure \\
\hline My & Million years \\
\hline NADW & North Atlantic Deep Water \\
\hline NATC & North Atlantic Thermohaline Circulation \\
\hline NCEP & National Centers for Environmental Prediction \\
\hline NH & Northern Hemisphere \\
\hline NNI & North North Island \\
\hline NSI & North South Island \\
\hline
\end{tabular}


NIWA

OAGCM

OGCM

PD

PMIP

$\mathrm{ppb}$

ppm

$\mathrm{RCM}$

REOF

SAF

SAM

SAO

$\mathrm{SC}$

SH

SOI

SON

SPCZ

SPJ

SST

STF

STJ

SWNI

TF

THC

UGAMP

UKMO

UM

WAUC

WC

WHS

WSSI
National Institute of Water and Atmospheric Research

Ocean-Atmosphere General Circulation Model

Ocean General Circulation Model

Present Day

Paleoclimate Modeling Intercomparison Project

parts per billion

parts per million

Regional Climate Model

Rotated Empirical Orthogonal Functions

SubAntarctic Front

Southern Annual Mode

Semi Annual Oscillation

Southland Current

Southern Hemisphere

Southern Oscillation Index

September-October-November

South Pacific Convergence Zone

Sub Polar Jet

Sea Surface Temperature

Sub Tropical Front

Sub Tropical Jet

South West North Island

Tasman Front

Thermohaline Circulation

United Kingdom Global Atmospheric Modelling Programme

United Kingdom Meteorological Office

Unified Model

West Auckland Current

Westland Current

Wilson and Henderson-Sellers

West and South South Island 


\section{List of figures}

Fig. 2-1. Schematic diagram representing the Earth's annual global mean

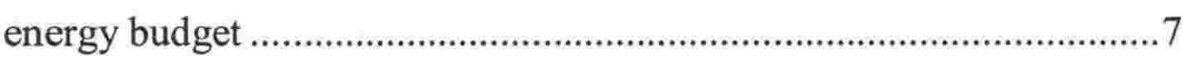

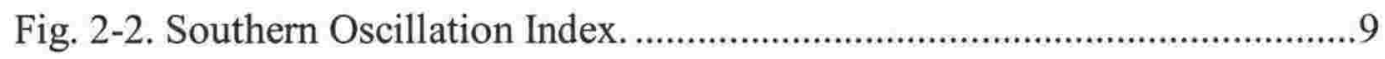

Fig. 2-3. Reconstruction of the wandering of the South Pole during the

Paleozoic. .12

Fig. 2-4. The changes in mean global temperature during the Quatenary. .15

Fig. 2-5. Schematic diagram illustrating the three main orbital changes.
A) Obliquity. 16
B) Eccentricity. 16
C) Precession.

Fig. 2-6. Graphical representation of the orbital variations in time and their power spectra.

Fig. 2-7. Heinrich events.

A) Icebergs break off of the ice sheet, carrying debris with it, which is subsequently deposit in the deep North Atlantic. 20

B) A very schematic time series indicating the cyclicity of Heinrich and Dansgaard-Oeschger cycles. 20

Fig. 2-8. Temperature fluctuations in the last 400.000 years. 26

Fig. 3-1. Zonal mean sea surface temperature anomalies between the LGM and PD simulations for DJF and JJA. .40

Fig. 3-2. The meridional temperature difference between latitudes $50^{\circ} \mathrm{S}$ and $65^{\circ} \mathrm{S}$ for climatological $500 \mathrm{hPa}$ temperature. 
Fig. 3-3. Timescales associated with modes identified by EOF analysis.

Fig. 3-4. The four leading rotated EOFs of 30-day low-pass filtered $500 \mathrm{hPa}$ geopotential heights, shown as covariance maps. 46

Fig. 3-5. Currents around New Zealand. 50

Fig. 4-1. Zero dimensional climate model with ice albedo feedback. 61

Fig. 4-2. Temperature versus the sine of latitude. 63

Fig. 4-3. Vertical distributions of the radiative heat balance components for the thermal equilibrium of a clear atmosphere. 64

Fig. 4-4. The Sequence of the models. 75

Fig. 4-5. New Zealand in the South-West Pacific and the Regional Domain $\mathrm{NZ} 440 \mathrm{~km}$. 85

Fig. 5-1. The global orography in the pre-industrial simulation. 90

Fig. 5-2. $\mathrm{SST}_{\mathrm{HadCM} 3}-\mathrm{SST}_{\mathrm{NCEP}}$ for the seasonal averaged JJA and DJF

Fig. 5-3. Standard deviation of the mean annual SST for the NCEP

reanalysis and $\mathrm{HadAM} 3 \mathrm{H}$. 95

Fig. 5-4. Polar plots of the Northern and Southern Hemisphere of seaice $_{\text {HadAM } 3 \mathrm{H}}$ - sea-ice NCEP, seasonally averaged for JJA and DJF. .96

Fig. 5-5. The difference in surface temperature between the pre-industrial HadAM3H and NCEP re-analysis for the seasonal averaged JJA and DJF.

Fig. 5-6. Zonally averaged temperature difference between HadAM3H and NCEP climatology.

Fig. 5-7. Average daily total precipitation for the four seasons as modelled by $\mathrm{HadAM} 3 \mathrm{H}$.

Fig. 5-8. Precipitation and LW TOA.

A) Difference in annual averaged precipitation between the preindustrial HadAM3H and NCEP climatology.

B) Difference in annual averaged outgoing LW radiation (TOA) between the pre-industrial HadAM3H and the NCEP climatology 100

Fig. 5-9. Mean Sea Level Pressure for JJA and DJF from HadAM3H and the NCEP re-analysis, and the difference between them. 
Fig. 5-10. The difference in $500 \mathrm{hPa}$ geopotential height between HadAM3H and the NCEP climatology for JJA and DJF. 104

Fig. 5-11. Polar projections of the seasonal differences in $500 \mathrm{hPa}$ geopotential heights between HadAM3H and the NCEP climatology.

Fig. 5-12. 200hPa wind speed for HadAM3H, the NCEP climatology, and the difference between them.

Fig. 5-13. A schematic diagram illustrating the $200 \mathrm{hPa}$ anomalies in the Southern Hemisphere. 108

Fig. 5-14. 200hPa mean zonal wind for HadAM3H, NCEP and $|\mathrm{HadAM} 3 \mathrm{H}|-|\mathrm{NCEP}|$ for the zonal band $20^{\circ} \mathrm{S}-20^{\circ} \mathrm{N}$ for JJA and DJF.

Fig. 5-15. Covariance of $u$ and $v$ of NCEP and HadAM3H for DJF and JJA.

Fig. 5-16. Stationary eddy momentum flux for NCEP and HadAM3H for DJF and JJA

Fig. 5-17. Transient eddy momentum flux for NCEP and HadAM3H for DJF and JJA

Fig. 5-18. Covariance of $\mathrm{v}$ and T of NCEP and HadAM3H for DJF and JJA.

Fig. 5-19. Stationary eddy heat flux for NCEP and HadAM3H for DJF and JJA.

Fig. 5-20. Transient eddy heat flux for NCEP and HadAM3H for DJF and JJA.

Fig. 5-21. Meridional temperature differences between latitude $50^{\circ} \mathrm{S}$ and $65^{\circ} \mathrm{S}$ for climatological $500 \mathrm{hPa}$ temperatures for $\mathrm{HadAM} 3 \mathrm{H}$ and NCEP

Fig. 5-22. Zonal mean 500hPa temperature for HadAM3H and NCEP for $50^{\circ} \mathrm{S}$ and $65^{\circ} \mathrm{S}$.

Fig. 5-23. Intraseasonal variation of the Southern Hemisphere $\left(20^{\circ} \mathrm{S}-80^{\circ} \mathrm{S}\right)$ circulation as presented by EOF analysis. 
Fig. 5-24. Correlation patterns of the timeseries of EOF2 and EOF3

(NCEP) with SST.

Fig. 5-25. Correlation patterns of the timeseries of EOF2 and EOF3

$(\operatorname{HadAM} 3 \mathrm{H})$ with SSTs.

Fig. 5-26. Distribution of the EOF coefficients for the first three EOFs for

NCEP and HadAM3H.

Fig. 6-1. CLIDB temperature and rainfall.

New Zealand Average Daily Temperature

New Zealand Mean Annual Rainfall.

Fig. 6-2. The orography and the domain of the regional model NZ_40km in

its rotated position

Fig. 6-3. Average SSTs for DJF and JJA for NZ_40km.

Fig. 6-4. The modelled DJF mean sea level pressure in the domain of the simulation.

$\mathrm{A}+\mathrm{B})$ Rotated back to its original position

$\mathrm{C}+\mathrm{D})$ Zoomed in on the land areas

Fig. 6-5. The grid points and the different regional areas of the New

Zealand landmass.

Fig. 6-6. The mean annual surface temperatures $\left({ }^{\circ} \mathrm{C}\right)$ in the RCM and

CLIDB.

Fig. 6-7. The difference in mean seasonal surface temperature between

NZ_40m and CLIDB.

Fig. 6-8. The cross sections for the temperature and precipitation profiles.

Fig. 6-9. Temperature cross sections, pre-industrial.

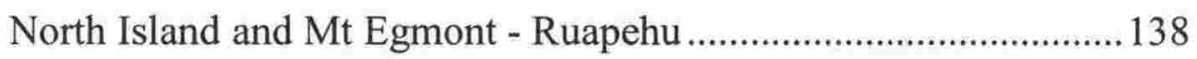

Buller Kaikoura and Mt Cook - Banks Peninsula ............................ 139

Fiordland - Otago ....................................................................... 140

Fig. 6-10. The mean annual precipitation for the RCM and CLIDB................ 142

Fig. 6-11. The difference in mean seasonal precipitation between the

NZ_40km and CLIDB.

Fig. 6-12. Precipitation cross sections, pre-industrial.

North Island and Mt Egmont - Ruapehu 146 
Buller - Kaikoura and Mt Cook - Banks Peninsula.

Fiordland - Otago

Fig. 6-13. Annual mean sea level pressure for NCEP and the RCM.

Fig. 6-14. Correlation maps of mean sea level pressure (NCEP) versus observed temperature (CLIDB) for the six different regions during DJF.

Fig. 6-15. Correlation maps of mean sea level pressure versus temperature for the six different regions during DJF.

Fig. 6-16. Correlation maps of mean sea level pressure (NCEP) versus temperature (CLIDB) for the six different regions during MAM....156

Fig. 6-17. Correlation maps of mean sea level pressure versus temperature for the six different regions during MAM.

Fig. 6-18. Correlation maps of the zonal wind (at $1000 \mathrm{hPa}$ ) versus temperature for ENI and ESI during MAM.

Fig. 6-19. Correlation maps of mean sea level pressure (NCEP) versus temperature (CLIDB) for the six different regions during JJA

Fig. 6-20. Correlation maps of mean sea level pressure versus temperature for the six different regions during JJA.

Fig. 6-21. Correlation map of the meridional wind (at $1000 \mathrm{hPa}$ ) versus temperature for SWNI during JJA

Fig. 6-22. Correlation maps of the zonal wind (at $1000 \mathrm{hPa}$ ) versus temperature for ESI during MAM and for ESI during JJA.

Fig. 6-23. Correlation maps of mean sea level pressure (NCEP) versus temperature (CLIDB) for the six different regions during SON

Fig. 6-24. Correlation maps of mean sea level pressure versus temperature for the six different regions during SON

Fig. 6-25. The correlation map of the zonal wind (at $1000 \mathrm{hPa}$ ) versus temperature and the correlation map of the meridional wind (at $1000 \mathrm{hPa}$ ) versus temperature for NNI during SON.

Fig. 6-26. Correlation maps of mean sea level pressure (NCEP) versus precipitation (CLIDB) for the six different regions during DJF. 
Fig. 6-27. Correlation maps of mean sea level pressure versus precipitation for the six different regions during DJF.

Fig. 6-28. The correlation map of the zonal wind (at $1000 \mathrm{hPa}$ ) versus precipitation and the correlation map of the meridional wind (at $1000 \mathrm{hPa}$ ) versus precipitation for NNI during DJF. 165

Fig. 6-29. Correlation maps of mean sea level pressure (NCEP) versus precipitation (CLIDB) for the six different regions during MAM. 166

Fig. 6-30. Correlation maps of mean sea level pressure versus precipitation for the six different regions during MAM.

Fig. 6-31. The correlation map of the zonal wind (at $1000 \mathrm{hPa}$ ) versus precipitation and the correlation map of the meridional wind (at $1000 \mathrm{hPa}$ ) versus precipitation for SWNI during MAM

Fig. 6-32. Correlation maps of mean sea level pressure (NCEP) versus precipitation (CLIDB) for the six different regions during JJA.......169

Fig. 6-33. Correlation maps of mean sea level pressure versus precipitation for the six different regions during JJA

Fig. 6-34. Correlation maps of the meridional wind (at $1000 \mathrm{hPa}$ ) versus precipitation for NNI and SWNI during JJA.

Fig. 6-35. Correlation maps of mean sea level pressure (NCEP) versus precipitation (CLIDB) for the six different regions during SON..... 172

Fig. 6-36. Correlation maps of mean sea level pressure versus precipitation for the six different regions during SON.

Fig. 6-37. Correlation maps of the zonal wind (at $1000 \mathrm{hPa}$ ) versus precipitation for NSI during MAM and for NSI during SON.

Fig. 7-1. The LGM ice sheets according to Peltier on HadAM3H grid 180

Fig. 7-2. The global orography in the LGM simulation. 181

Fig. 7-3. Sea-ice during the LGM.

Fig. 7-4. Seasonal averaged SSTs for DJF and JJA (LGM). 184

Fig. 7-5. The difference in sea-ice extent between the LGM and the preindustrial for the Northern and Southern Hemisphere for JJA and DJF. 
Fig. 7-6. The difference between the LGM and pre-industrial SSTs. 188

Fig. 7-7. Standard deviation of the mean annual SSTs in the LGM.

Fig. 7-8. The difference in surface temperature between the LGM and the pre-industrial simulation for DJF and JJA.

Fig. 7-9. Vertical cross sections of the mean zonal temperature difference between the LGM and the pre-industrial simulation.

Fig. 7-10. Lapse rate for 9 different zonal bands for the pre-industrial and LGM simulation for DJF and JJA.

Fig. 7-11. Difference in seasonal precipitation between the LGM and the pre-industrial simulation.

Fig. 7-12. Mean sea level pressure in $\mathrm{hPa}$ for the LGM and the difference with the mean sea level pressure of the pre-industrial for DJF and JJA.

Fig. 7-13. Low frequency variability of mean sea level pressure for the Northern and Southern Hemisphere during their winter season for the pre-industrial and LGM simulation and the differences in their patterns.

Fig. 7-14. Zonal mean of the differences in geopotential height between the LGM and pre-industrial simulation for the four seasons. .204

Fig. 7-15. Difference in 500hPa geopotential height between the LGM and pre-industrial simulation for the Northern and Southern Hemispheres for DJF and JJA. .206

Fig. 7-16. The average power spectra of the mean $500 \mathrm{hPa}$ geopotential height for the pre-industrial and LGM simulations for the Northern and Southern Hemisphere winter seasons for 4 different zonal bands.

Fig. 7-17. The difference in the strength of the mean seasonal $250 \mathrm{hPa}$ winds, LGM - pre-industrial, for the Northern and Southern Hemisphere for DJF and JJA

Fig. 7-18. Meridional cross sections of the differences in the average zonal wind between the LGM and the pre-industrial simulation for the four seasons. 
Fig. $7-19$. The difference in $1000 \mathrm{hPa}$ zonal winds between the LGM and pre-industrial simulation for the four seasons.

Fig. 7-20. The difference between the absolute values of the LGM and preindustrial average zonal meridional momentum flux, its contribution from the mean meridional circulation and eddies, for DJF and JJA.

Fig. 7-21. The difference between the absolute values of the LGM and preindustrial stationary eddy momentum flux and the transient eddy momentum flux for DJF and JJA

Fig. 7-22. The difference between the absolute values of the LGM and preindustrial average zonal meridional heat transport and the contribution from the mean meridional circulation for DJF and JJA

Fig. 7-23. The difference between the absolute values of the LGM and preindustrial eddy heat flux (A and B), the stationary and the transient eddy meridional heat flux for DJF and JJA

Fig. 7-24. The strength of the mean seasonal meridional wind $(\mathrm{m} / \mathrm{s})$ versus the number of grid points for $35^{\circ} \mathrm{S}-60^{\circ} \mathrm{S}$

Fig. 7-25. The SAO for the NCEP climatology and the pre-industrial and

LGM simulation

Fig. 7-26. The three leading EOFs of the mean sea level pressure of the

LGM simulation.

Fig. 7-27. Distribution of the EOF coefficients for the first three EOFs (LGM).

Fig. 7-28. Correlation patterns of the timeseries of EOF2 and EOF3 with SSTs for the LGM simulation.

Fig. 8-1. New Zealand's modelled orography for the LGM simulation in its rotated position

Fig. 8-2. Difference in SST between the LGM and pre-industrial simulation in NZ_40km for DJF and JJA

Fig. 8-3. Annual mean SSTs for the South West Pacific for the preindustrial and the LGM simulation. 
Fig. 8-4. Stream function for the South Western Pacific for the preindustrial and LGM simulation as modelled by HadCM3.

Fig. 8-5. The vegetation and soil data used to create the vegetation and soil parameters for the LGM simulation.

Fig. 8-6. The annual mean surface temperature during the LGM.

Fig. 8-7. The difference in surface temperature between the LGM and the pre-industrial simulation for each season (NZ regional domain).....247

Fig. 8-8. The seasonal snow cover of the greater landmass of New Zealand during the LGM.

Fig. 8-9. Temperature cross sections, LGM:

North Island and Mt Egmont - Ruapehu ........................................250

Buller - Kaikoura and Mt Cook - Banks Peninsula.........................251

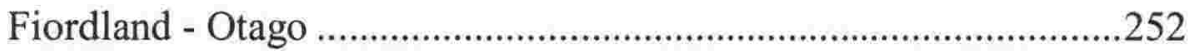

Fig. 8-10. The total annual amount of precipitation during the LGM in the regional model.

Fig. 8-11. The difference in average seasonal precipitation between the

LGM and pre-industrial simulation for each season.

Fig. 8-12. Precipitation cross sections, LGM.

North Island and Mt Egmont - Ruapehu 257

Buller - Kaikoura and Mt Cook - Banks Peninsula...........................258

Fiordland - Otago and Southern Fiordland - Southland ...................259

Fig. 8-13. Annual mean sea level pressure during the LGM in the RCM. ........262

Fig. 8-14. The strength of the meridional wind versus the number of grid points for the New Zealand pre-industrial land mass.

Fig. 8-15. Correlation maps of mean sea level pressure versus temperature for the six different regions during DJF (RCM).

Fig. 8-16. Correlation map of the zonal wind (at $1000 \mathrm{hPa}$ ) versus temperature for WSSI during DJF. 268

Fig. 8-17. Correlation maps of mean sea level pressure versus temperature for the six different regions during MAM (RCM). 269

Fig. 8-18. Correlation map of the zonal wind (at $1000 \mathrm{hPa}$ ) versus temperature for ENI during MAM. 
Fig. 8-19. Correlation maps of mean sea level pressure versus temperature for the six different regions during JJA (RCM).

Fig. 8-20. Correlation maps of the zonal wind (at 1000hPa) versus temperature for NSI for the pre-industrial and LGM simulation during JJA

Fig. 8-21. Correlation maps of the zonal wind (at $1000 \mathrm{hPa}$ ) versus temperature for ESI for the pre-industrial and LGM simulation during JJA

Fig. 8-22. Correlation maps of mean sea level pressure versus temperature for the six different regions during SON (RCM).

Fig. 8-23. Correlation maps of mean sea level pressure versus precipitation for the six different regions during DJF (RCM).

Fig. 8-24. Correlation map of the meridional wind (at $1000 \mathrm{hPa}$ ) versus precipitation for WSSI during DJF.

Fig. 8-25. Correlation maps of the zonal wind (at $1000 \mathrm{hPa}$ ) versus precipitation for WSSI for the pre-industrial and LGM simulation during DJF. 276

Fig. 8-26. Correlation maps of mean sea level pressure versus precipitation for the six different regions during MAM (RCM).

Fig. 8-27. Correlation maps of the meridional wind (at $1000 \mathrm{hPa}$ ) versus precipitation for ENI for the pre-industrial and LGM simulation during MAM.

Fig. 8-28. Correlation maps of the zonal wind (at $1000 \mathrm{hPa}$ ) versus precipitation for NSI for the pre-industrial and LGM simulation during MAM.

Fig. 8-29. Correlation maps of mean sea level pressure versus precipitation for the six different regions during JJA (RCM).

Fig. 8-30. Correlation maps of the meridional wind (at $1000 \mathrm{hPa}$ ) versus precipitation for SWNI for the pre-industrial and LGM simulation during JJA. 
List of figures

Fig. 8-31. Correlation maps of the zonal wind (at $1000 \mathrm{hPa}$ ) versus precipitation for NSI for the pre-industrial and LGM simulation

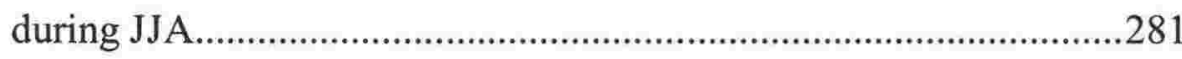

Fig. 8-32. Correlation maps of mean sea level pressure versus precipitation for the six different regions during SON (RCM). .282 


\section{List of tables}

Table 2-1. The Geological Time Scale. ............................................................... 10

Table 2-2. Principal sources of proxy data for paleoclimate reconstructions. .....23

Table 2-3. Characteristics of natural archives.......................................................24

Table 4-1. Coordinates of the regional domain NZ_40km..............................85

Table 5-1. Southern Oscillation Index for the four seasons for HadAM3H

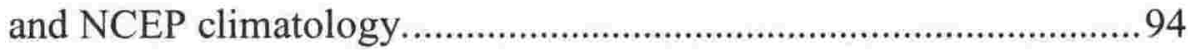

Table 6-1. The mean annual surface temperature for each region in New Zealand and for the whole of New Zealand as determined from CLIDB and NZ_40km.

Table 6-2. Mean seasonal surface temperatures of CLIDB and NZ_40km by region.

Table 6-3. The mean annual precipitation for each region of New Zealand and for the whole of New Zealand as determined from CLIDB and NZ_40km.

Table 6-4. The mean seasonal precipitation of CLIDB and NZ_40km by region.

Table 7-1. Orbital parameters for the pre-industrial and LGM experiment....... 179

Table 7-2. Southern Oscillation Index (LGM).

Table 7-3. The difference in average seasonal surface temperature between the LGM and the pre-industrial simulation.

Table 7-4. The difference in the vertically averaged temperature between the LGM and pre-industrial for the full atmosphere and for the atmosphere up to $300 \mathrm{hPa}$.

Table 7-5. Precipitation for the pre-industrial and LGM simulation. 199 
Table 7-6. Cross correlation of the difference in mean meridional heat transport with the difference in meridional wind and with the difference in temperature

Table 7-7. Statistics of the mean seasonal meridional wind.

Table 8-1. The annual mean surface temperature for each region in New Zealand and for the whole of New Zealand for the LGM and the pre-industrial simulation

Table 8-2. Mean seasonal surface temperatures for the LGM and preindustrial simulation by region.

Table 8-3. The average annual amount of precipitation for each region in New Zealand and for the whole of New Zealand (LGM).

Table 8-4. Average seasonal precipitation ( $\mathrm{mm}$ ) for the LGM and preindustrial simulation by region.

Table 8-5. The mean annual zonal and meridional wind for each region.

Table 8-6. The mean seasonal zonal wind for each region for the LGM and the pre-industrial simulation

Table 8-7. The mean seasonal meridional wind for each region for the

LGM and the pre-industrial simulation. .264

Table 8-8. The number of grid points having a meridional component that is pre-dominantly southerly for the GCM $\left(35^{\circ} \mathrm{S}-60^{\circ} \mathrm{S}\right)$ and RCM for the pre-industrial and LGM simulations. .266 


\section{References}

Alley, R. B., P. U. Clark, L. D. Keigwin, and R. S. Webb, 1999: Making sense of Millenial-scale climate change. Mechanisms of Global Climate Change at Millenium Time Scale., P. U. Clark, Webb, R.S., Keigwin, L.D., Ed.

An, S.-I., A. Timmermann, L. Bejarno, F.-F. Jin, F. Justino, Z. Liu, and A. W. Tudhope, 2004: Modeling evidence for enhanced El Niño-Southern Oscillation amplitude during the Last Glacial Maximum. Paleoceanography, 19 (PA4009), $1-11$.

Andreasen, D. H., A. C. Ravelo, and A. J. Broccoli, 2001: Remote forcing at the Last Glacial Maximum in the Tropical Pacific Ocean. Journal of Geophysical Research, 106 (C1), 879-897.

Augustin, L., C. Barbante, P. R. F. Barnes, J.-M. Barnola, M. Bigler, E. Castellano, O. Cattani, J. Chappellaz, D. Dahl-Jensen, B. Delmonte, G. Dreyfus, G. Durand, S. Falourd, H. Fischer, J. Flückiger, M. Hansson, P. Huybrechts, G. Jugie, S. J. Johnsen, J. Jouzel, P. Kaufmann, J. Kipfstuhl, F. Lambert, V. Y. Lipenkov, G. C. Littot, A. Longinelli, R. Lorrain, V. Maggi, V. MassonDelmotte, H. Miller, R. Mulvaney, J. Oerlemans, H. Oerter, G. Orombelli, F. Parrenin, D. A. Peel, J. R. Petit, D. Raynaud, C. Ritz, U. Ruth, J. Schwander, U. Siegenthaler, R. Souchez, B. Stauffer, J. P. Steffensen, B. Stenni, T. F. Stocker, I. E. Tabacco, R. Udisti, R. S. W. van de Wal, M. van den Broeke, J. Weiss, F. Wilhelms, J. G. Winther, E. W. Wolff, and M. Zucchelli, 2004: Eight glacial cycles from an Antarctic ice core. Nature, 429, 623-628.

Bard, E., 2002: Climate shock: Abrupt changes over millenium time scales. Physics Today, 55 (12), 32-38.

Barnola, J.-M., D. Raynaud, Y. S. Korotkevich, and C. Lorius, 1987: Vostok ice core provides 160,000 -year record of atmospheric $\mathrm{CO}_{2}$. Nature, 329, 408-414.

Barron, E. J. and W. M. Washington, 1984: The role of geographic variables in explaining paleoclimates: Results from Cretaceous climate model sensitivity studies. Journal of Geophysical Research, 89 (D1), 1267-1279. 
Barrows, T. T., S. Juggins, P. De Deckker, J. Thiede, and J. I. Martinez, 2000: Sea-surface temperatures of the Southwest Pacific ocean during the Last Glacial Maximum. Paleoceanography, 15 (1), 95-109.

Bennett, K. D., S. G. Haberle, and S. H. Lumley, 2000: The Last GlacialHolocene transition in Southern Chile. Science, 290, 325-328.

Berger, A. and M. F. Loutre, 1994: Precession, eccentricity, obliquity, insolation and paleoclimates. Long-Term Climatic Variations: data and modeling, J.-C. Duplessy and M.-T. Spyridakis, Eds., Springer-Verlag, 107-151.

Berger, A., M. F. Loutre, and V. Dehant, 1989: Pre-Quatenary Milankovitch frequencies. Nature, 342, 133.

Berger, A. L., 1978: Long term variations of daily insolation and Quaternary climatic changes. Journal of the Atmospheric Sciences, 35, 2362-2367.

Bintanja, R. and J. Oerlemans, 1996: The effect of reduced ocean overturning on the climate of the last glacial maximum. Climate Dynamics, 12, 523-533.

Bjornsson, H. and L. A. Mysak, 2001: Present day and Last Glacial Maximum ocean thermohaline circulation in a zonally averaged coupled ocean-sea-iceatmosphere model. Journal of Climate, 14, 1422-1439.

Bond, G. C. and R. Lotti, 1995: Iceberg discharges into the North Atlantic on millenial time scales during the Last Glaciation. Science, 267, 1005-1010.

Bradley, R. S., 1999: Paleoclimatology: Reconstructing climates of the Quatenary. 2 ed. Vol. 64, International Geophysics Series, Academic Press, 613 pp.

Broccoli, A. J., 2000: Tropical cooling at the Last Glacial Maximum: An atmosphere-mixed layer ocean model simulation. Journal of Climate, 13, 951976.

Broccoli, A. J. and S. Manabe, 1987: The influence of continental ice, atmospheric $\mathrm{CO}_{2}$, and land albedo on the climate of the last glacial maximum. Climate Dynamics (1), 87-99.

Broecker, W. S., 1994: Massive iceberg discharges as triggers for global climate change. Nature, 372, 421-424.

Brown, J. and I. Simmonds, 2004: Sensitivity of the $\delta^{18} \mathrm{O}$-temperature relationship to the distribution of continents. Geophysical Research Letters, 31 (doi:10.1029/2004GL019870), L09208.

Budd, W. F., B. Coutts, and R. Warner, 1998: Modelling the Antarctic and Northern Hemisphere ice-sheet changes with global climate through the glacial cycle. Annals of Glaciology, 27, 153-160. 
Burckle, L. H. and R. Mortlock, 1998: Sea-ice extent in the Southern Ocean during the Last Glacial Maximum: Another approach to the problem. Annals of Glaciology, 27, 302-304.

Burnett, A. W. and A. R. McNicoll, 2000: Interannual variations in the Southern Hemisphere winter circumpolar vortex: Relationships with the semiannual oscillation. Journal of Climate, 13, 991-999.

Bush, A. B. G. and S. G. H. Philander, 1998: The Role of Ocean-Atmosphere Interactions in Tropical Cooling During the Last Glacial Maximum. Science, 279, 1341-1344.

Carter, L., 2001: Currents of change: The ocean flow in a changing world. Water and Atmosphere, 9 (4), 15-17.

Carter, L., H. L. Neil, and L. Northcote, 2002: Late Quatenary ice-rafting events in the SW Pacific Ocean, off eastern New Zealand. Marine Geology, 191, 19-35.

Cavalieri, D. J. and C. L. Parkinson, 1981: Large-scale variations in observed Antarctic sea ice extent and associated atmospheric circulation. Monthly Weather Review, 109, 2323-2336.

Cehelsky, P. and K.-K. Tung, 1991: Nonlinear baroclinic adjustment. Journal of the Atmospheric Sciences, 48 (17), 1930-1947.

Charney, J. G., 1947: The dynamics of long waves in a baroclinic westerly current. Journal of Meteorology, 4 (5), 135-162.

Charney, J. G., 1950: Progress in dynamic meteorology. Bulletin of the American Meteorological Society, 31 (7), 231-236.

Chen, T.-C. and M.-C. Yen, 1997: Interdecadal variation of the Southern Hemisphere circulation. Journal of Climate, 10, 805-812.

Chinn, T. J., 1996: The Southern Hemisphere glacial record - Antarctica and New Zealand. Papers and Proceedings of the Royal Society of Tasmania, 130 ((2)), 17-24.

Claussen, M., L. A. Mysak, A. J. Weaver, M. Crucifix, T. Fichefet, M. F. Loutre, S. L. Weber, J. Alcamo, V. A. Alexeev, A. Berger, R. Calov, A. Ganopolski, H. Goosse, G. Lohmann, F. Lunkeit, I. I. Mokhov, V. Petoukhov, P. Stone, and Z. Wang, 2002: Earth system models of intermediate complexity: Closing the gap in the spectrum of climate systems models. Climate Dynamics, 18, 579-586.

Clement, A. C., M. A. Cane, and R. Seager, 2001: An orbitally driven tropical source for abrupt climate change. Journal of Climate, 14 (No. 11), 2369-2375.

CLIMAP, 1976: The surface of the ice-age Earth. Science, 191 (4232), 11311137. 
CLIMAP, 1981: Seasonal reconstructions of the Earth's surface at the Last Glacial Maximum. Geological Society of America map chart series, MC-36.

Colhoun, E. A., D. Hannan, and K. Kiernan, 1996: Late Wisconsin glaciation of Tasmania. Papers and Proceedings of the Royal Society of Tasmania, 130 ((2)), 33-45.

Cox, P. M., R. A. Betts, C. B. Bunton, R. L. H. Essery, P. R. Rowntree, and J. Smith, 1999: The impact of new land surface physics on the GCM simulation of climate and climate sensitivity. Climate Dynamics, 15, 183-203.

Cressman, G. P., 1972: Dynamic weather prediction. Meteorological Challenges: A History, D. P. McIntyre, Ed., Information Canada, 179-207.

Crosta, X., J. J. Pichon, and L. H. Burckle, 1998a: Application of modern analog technique to marine Antarctic diatoms: Reconstruction of maximum sea-ice extent at the Last Glacial Maximum. Paleoceanography, 13 (No. 3), 284-297.

Crosta, X., J. J. Pichon, and L. H. Burckle, 1998b: Reappraisal of Antarctic seasonal sea-ice at the Last Glacial Maximum. Geophysical Research Letters, 25 (14), 2703-2706.

Crowley, T. J., 2000: CLIMAP SST's revisited. Climate Dynamics, 16, 241-255.

Crowley, T. J. and G. R. North, 1991: Paleoclimatology. Vol. 18, Oxford Monographs on Geology and Geophysics, Oxford University Press, 339 pp.

Crowley, T. J. and S. K. Baum, 1997: Effect of vegetation on an ice-age climate model simulation. Journal of Geophysical Research, 102 (D14), 16,463-16,480.

Crowley, T. J., J. G. Mengel, and D. A. Short, 1987: Gondwanaland's seasonal cycle. Nature, 329, 803-807.

Crowley, T. J., S. K. Baum, and K. Y. Kim, 1993: General circulation model sensitivity experiments with pole-centered supercontinents. Journal of Geophysical Research, 98 (D5), 8793-8800.

Cullen, M. J. P., 1993: The unified forecast/climate model. The Meteorological Magazine, 122 (No. 1449), 81-94.

Cullen, M. J. P. and T. Davies, 1991: A conservative split-explicit integration scheme with fourth-order horizontal advection. Quarterly Journal of the Royal Meteorological Society, 117, 993-1002.

Cusack, S. and J. M. Edwards, 1999: Investigating k distribution methods for parameterizing gaseous absorption in the Hadley Centre climate model. Journal of Geophysical Research, 104 (D2), 2051-2057. 
Cusack, S., J. M. Edwards, and R. Kershaw, 1999b: Estimating the subgrid variance of saturation, and its parameterization for use in a GCM cloud scheme. Quarterly Journal of the Royal Meteorological Society, 125, 3057-3076.

Cusack, S., A. Slingo, J. M. Edwards, and M. Wild, 1998: The radiative impact of a simple aerosol climatology on the Hadley Centre atmospheric GCM. Quarterly Journal of the Royal Meteorological Society, 124, 2517-2526.

Davies, L. A. and A. R. Brown, 2001: Assessment of which scales of orography can be credibly resolved in a numerical model. Quarterly Journal of the Royal Meteorological Society, 127, 1225-1237.

Delaygue, G., V. Masson, J. Jouzel, and R. D. Koster, 2000: The origin of Antarctic precipitation: A modelling approach. Tellus, 52B, 19-36.

Delaygue, G., J. Jouzel, V. Masson, R. D. Koster, and E. Bard, 2000: Validity of the isotopic thermometer in central Antarctica: Limited impact of glacial precipitation seasonality and moisture origin. Geophysical Research Letters, 27 (17), 2677-2680.

Delecluse, P., 1994: Modelling the ocean circulation. Long-Term Climatic Variations: data and modeling, J.-C. Duplessy and M.-T. Spyridakis, Eds., Springer-Verlag, 73-106.

Dokken, T. M. and E. Jansen, 1999: Rapid changes in the mechanism of ocean convection during the last glacial period. Nature, 401, 458-461.

Dong, B. and P. J. Valdes, 1998: Simulations of the Last Glacial Maximum climates using a general circulation model: Prescribed versus computed sea surface temperatures. Climate Dynamics, 14, 571-591.

Dong, B. and P. J. Valdes, 2000: Climates at the Last Glacial Maximum: Influence of model horizontal resolution. Journal of Climate, 13, 1554-1573.

Drost, F., A. B. Mullan, B. Bhaskaran, and J. A. Renwick, 2005: Report on the effect of the size of the regional domain in New Zealand's regional climate model on the modelled climatology. Internal Report, 32 pages pp. National Institute of Water and Atmospheric Research, Wellington.

Duplessy, J.-C. and N. J. Shackleton, 1985: Response of global deep-water circulation to Earth's climatic change 135,000-107,000 years ago. Nature, 316, 500-507.

Eady, E. T., 1949: Long waves and cyclone waves. Tellus, 1 (3), 33-52.

Eddy, J. A. and R. S. Bradley, 1991: Changes in time in the temperature of the Earth. EarthQuest, 5 (1). 
Edwards, J. M., 1996: Efficient calculation of infrared fluxes and cooling rates using the two-stream equations. Journal of the Atmospheric Sciences, 53 (No. 13), 1921-1932.

Edwards, J. M. and A. Slingo, 1996: Studies with a flexible new radiation code. I: Choosing a configuration for a large-scale model. Quarterly Journal of the Royal Meteorological Society, 122, 689-719.

Farrell, B. F., 1990: Equable climate dynamics. Journal of the Atmospheric Sciences, 47 (24), 2986-2995.

Fenessy, M. J. and J. Shukla, 2000: Seasonal prediction over North America with a regional model nested in a global model. Journal of Climate, 13, 2605-2627.

Fichefet, T., S. Hovine, and J.-C. Duplessy, 1994: A model study of the Atlantic thermohaline circulation during the last glacial maximum. Nature, 372, 252-255.

Fleming, J. R., 1996: Historical essays on meteorology 1919-1995. BraunBrumfeld, $617 \mathrm{pp}$.

Frakes, L. A., J. E. Francis, and J. I. Syktus, 1994: Climate modes of the Phanerozoic. Cambridge University Press, 274 pp.

Frei, C., J. H. Christensen, M. Déqué, M. Jacob, R. G. Jones, and P. L. Vidale, 2003: Daily precipitation statistics in regional climate models: Evaluation and intercomparison for the European Alps. Journal of Geophysical Research, 108 (D3), 4124-4142.

Ganopolski, A. and S. Rahmstorf, 2001: Rapid changes of glacial climate simulated in a coupled climate model. Nature, 409, 153-158.

Ganopolski, A., S. Rahmstorf, V. Petoukhov, and M. Claussen, 1998: Simulation of modern and glacial climates with a coupled global model of intermediate complexity. Nature (391), 351-356.

Garcia, R. G., 1991: Parameterization of planetary wave breaking in the Middle Atmosphere. Journal of the Atmospheric Sciences, 48 (11), 1405-1419.

Genthon, C., J.-M. Barnola, D. Raynaud, C. Lorius, J. Jouzel, N. I. Barkov, Y. S. Korotkevich, and V. M. Kotlyakov, 1987: Vostok ice core: Climatic response to $\mathrm{CO}_{2}$ and orbital forcing changes over the last climatic cycle. Nature, 329, 414418.

Gerard, J. C. and V. Dols, 1990: The warm cretaceous climate: Role of the longterm carbon cycle. Geophysical Research Letters, 17 (10), 1561-1564.

Ghil, M. and K. C. Mo, 1991: Intraseasonal oscillations in the global atmosphere. Part II: Southern Hemisphere. Journal of the Atmospheric Sciences, 48 (5), 780 790. 
Giorgi, F., 1990: Simulation of regional climate using a limited area model nested in a general circulation model. Journal of Climate, 3, 941-963.

Giorgi, F. and M. R. Marinucci, 1996: An investigation of the sensitivity of simulated precipitation to model resolution and its implications for climate studies. Monthly Weather Review, 124, 148-166.

Gordon, C., C. Cooper, C. A. Senior, H. Banks, J. M. Gregory, T. C. Johns, J. F. B. Mitchell, and R. A. Wood, 2000: The simulation of SST, sea ice extents and ocean heat transports in a version of the Hadley Centre coupled model without flux adjustments. Climate Dynamics, 16, 147-168.

Gregory, D., 1995: A consistent treatment of the evaporation of rain and snow for use in large-scale models. Monthly Weather Review, 123, 2716-2732.

Gregory, D. and P. R. Rowntree, 1990: A mass flux convection scheme with representation of cloud ensemble characteristics and stability-dependent closure. Monthly Weather Review, 118, 1483-1506.

Gregory, D. and S. Allen, 1991: The effect of convective scale downdraughts upon NWP and climate simulations. Ninth Conference Numerical Weather Prediction, Denver, Colorado, 122-123.

Gregory, D., R. Kershaw, and P. M. Inness, 1997: Parameterization of momentum transport by convection. II: Tests in single-column and general circulation models. Quarterly Journal of the Royal Meteorological Society, 123, 1153-1183.

Gregory, J., 1999: Representation of the radiative effect of convective anvils. Technical Note 7, 20 pp. Hadley Centre, Bracknell.

Gregory, J. M. and D. Morris, 1996: The sensitivity of climate simulations to the specification of mixed phase clouds. Climate Dynamics, 12, 641-651.

Hall, N. M. J., B. Dong, and P. J. Valdes, 1996a: Atmospheric equilibrium, instability and energy transport at the last glacial maximum. Climate Dynamics, 12, 497-511.

Hall, N. M. J., P. J. Valdes, and B. Dong, 1996b: The maintenance of the last great ice sheets: A UGAMP GCM study. Journal of Climate, 9, 1004-1019.

Hartmann, D. L., 1994: Global physical climatology. Vol. 56, International Geophysics Series, Academic Press, 411 pp.

Hartmann, D. L., 1995: A PV view of zonal flow vacillation. Journal of the Atmospheric Sciences, 52 (14), 2561-2576.

Harvey, L. D. D., 1988: Climatic impact of ice-age aerosols. Nature, 334, 333335. 
Harvey, L. D. D., 1989: An energy balance climate model study of radiative forcing and temperature response at $18 \mathrm{ka}$. Journal of Geophysical Research, 94 (D10), 12873-12884.

Hayashi, Y. and D. G. Golder, 1987: Effects of wave-wave and wave-mean flow interactions on the growth and maintenance of transient planetary waves in the presence of a mean thermal restoration force. Journal of the Atmospheric Sciences, 44 (22), 3392-3401.

Hays, J. D., J. Imbrie, and N. J. Shackleton, 1976: Variations in the Earth's orbit: Pacemaker of the Ice Ages. Science, 194 (4270), 1121-1132.

Heinrich, H., 1988: Origin and consequences of cyclic ice rafting in the Northeast Atlantic Ocean during the past 130,000 years. Quatenary Research, 29, 142-152.

Held, I. M., 1978: The vertical scale of an unstable baroclinic wave and its importance for eddy heat flux parameterizations. Journal of the Atmospheric Sciences, 35, 572-576.

Held, I. M. and P. J. Phillips, 1992: Sensitivity of the eddy momentum flux to meridional resolution in atmospheric GCMs. Journal of Climate, 6, 499-507.

Hewitt, C. D., 2000: A study of the mechanisms of climate change at the Last Glacial Maximum, Department of Meteorology, The University of Reading, 178.

Hewitt, C. D. and J. F. B. Mitchell, 1997: Radiative forcing and response of a GCM to ice age boundary conditions: Cloud feedback and climate sensitivity. Climate Dynamics, 13, 821-834.

Hewitt, C. D., A. J. Broccoli, J. F. B. Mitchell, and R. J. Stouffer, 2001: A coupled model study of the last glacial maximum: Was part of the North Atlantic relatively warm? Geophysical Research Letters, 28 (8), 1571-1574.

Hewitt, C. D., R. J. Stouffer, A. J. Broccoli, J. F. B. Mitchell, and P. J. Valdes, 2002: The effect of ocean dynamics in a coupled GCM simulation of the Last Glacial Maximum. Climate Dynamics, 20, 203-218.

Hirst, A. C. and J. S. Godfrey, 1993: The role of Indonesian throughflow in a global ocean GCM. Journal of Physical Oceanography, 23 (6), 1057-1086.

Hope, G., A. P. Kershaw, S. van der Kaars, S. Xiangjun, P.-M. Liew, L. E. Heusser, H. Takahara, M. S. McGlone, N. Miyoshi, and P. T. Moss, 2004: History of vegetation and habitat change in the Austral-Asian region. Quaternary International, 118-119, 103-126.

Hope, P. and I. Simmonds, 2003: Mechanisms driving meridional shifts in the Southern Hemisphere westerlies in extreme climates. Seventh International Conference on Southern Hemisphere Meteorology and Oceanography, Wellington, AMS, 256-257. 
Hovine, S. and T. Fichefet, 1994: A model study of the glacial oceanic circulation. Long-Term Climatic Variations: data and modeling, J.-C. Duplessy and M.-T. Spyridakis, Eds., Springer-Verlag, 481-489.

Hudson, D. A. and R. G. Jones, 2002a: Simulations of present-day and future climate over southern Africa using HadAM3H. Hadley Centre technical note 38, 36 pp. Hadley Centre, Meteorological Office, Bracknell.

Hudson, D. A. and R. G. Jones, 2002b: Regional climate model simulations of present-day and future climates of southern Africa. Hadley Centre technical note 39, 41 pp. Hadley Centre, Meteorological Office, Bracknell.

Hunt, G. and T. Barrows, 1999: CLIMANZ: Preliminary palaeoclimatic maps. Quaternary Australasia, 17 (2), 16-30.

Hurrell, J. W. and H. van Loon, 1994: A modulation of the atmospheric annual cycle in the Southern Hemisphere. Tellus, 46A, 325-338.

Hurrell, J. W., H. van Loon, and D. J. Shea, 1998: The mean state of the Troposphere. Meteorology of the Southern Hemisphere, D. J. Karoly and D. G. Vincent, Eds., American Meteorological Society, 1-46.

Huybrechts, P., 1990: A 3-D model for the Antarctic ice sheet: A sensitivity study on the glacial-interglacial contrast. Climate Dynamics, 5, 79-92.

Imbrie, J., 1994: Measuring the gain of the climate system's response to Milankovitch forcing in the precession and obliquity bands. Long-Term Climatic Variations: data and modeling, J.-C. Duplessy and M.-T. Spyridakis, Eds., Springer-Verlag, 403-410.

Imbrie, J., J. Hays, D. Martinson, D. McIntyre, A. Mix, A. Horley, J. Pisias, W. Prell, and N. Shackleton, 1984: The orbital theory of Pleistocene climate: Support from a revised chronology of the marine delta ${ }^{18} \mathrm{O}$ record. Milankovitch and Climate, A. Berger, J. Imbrie, J. Hays, G. Kukla, and B. Saltzman, Eds., Reidel, 269-306.

IPCC, 2001: Climate change 2001: Synthesis report. A contribution of working groups I, II and III to the third assessment report of the Intergovernmental Panel on Climate Change. Report 052101507 3, 398 pp. Cambridge University Press, Cambridge.

James, I. N., 1988: On the forcing of planetary-scale Rossby waves by Antarctica. Quarterly Journal of the Royal Meteorological Society, 114, 619637.

Jones, D. A. and I. Simmonds, 1993: A climatology of Southern Hemisphere extratropical cyclones. Climate Dynamics, 9, 131-145.

Joseph, D., 1980: Navy 10" global elevation values. National Center for Atmospheric Research notes on the FNWC terrain data set, NCAR, $3 \mathrm{pp}$. 
Jouzel, J., R. B. Alley, K. M. Cuffey, W. Dansgaard, P. Grootes, G. Hoffmann, S. J. Johnsen, D. Koster, D. Peel, C. A. Shuman, M. Stievenard, M. Stuiver, and J. White, 1997: Validity of the temperature reconstruction from water isotopes in ice cores. Journal of Geophysical Research, 102 (C12), 26,471-26,487.

Kageyama, M. and P. J. Valdes, 2000: Synoptic-scale perturbations in AGCM simulations of the present and Last Glacial Maximum climates. Climate Dynamics, 16, 517-533.

Kageyama, M., P. J. Valdes, G. Ramstein, C. D. Hewitt, and U. Wyputta, 1999: Northern Hemisphere storm tracks in Present Day and Last Glacial Maximum climate simulations: A comparison of the European PMIP models. Journal of Climate., 12, 742-760.

Kalnay, E., M. Kanamitsu, R. Kistler, W. Collins, D. Deaven, L. Gandin, M. Iredell, S. Saha, G. White, J. Woollen, Y. Zhu, M. Chelliah, W. Ebisuzaki, W. Higgins, J. Janowiak, K. C. Mo, C. Ropelewski, J. Wang, A. Leetmaa, R. Reynolds, R. Jenne, and D. Joseph, 1996: The NCEP/NCAR 40-year reanalysis project. Bulletin of the American Meteorological Society, 77 (No. 3), 437-471.

Kantha, L. H. and C. A. Clayson, 2000: Small Scale Processes in Geophysical Fluid Flows. first ed. Vol. 67, International Geophysics Series, Academic Press, $888 \mathrm{pp}$.

Karoly, D. J., 1983: Standing wave climatologies for the Southern Hemisphere. First International Conference for the Southern Hemisphere, 100-103.

Karoly, D. J., 1989: Southern Hemisphere circulation features associated with El Niño - Southern Oscillation events. Journal of Climate, 2, 1239-1252.

Karoly, D. J., 1990: The role of transient eddies in low-frequency zonal variations of the Southern Hemisphere circulation. Tellus, 42A (1), 41-50.

Karoly, D. J., D. G. Vincent, and D. J. Shea, 1998: General circulation. Meteorology of the Southern Hemisphere, D. J. Karoly and D. G. Vincent, Eds., American Meteorological Society, 47-86.

Katzfey, J. J., 1995: Simulation of extreme New Zealand precipitation events. Part I: Sensitivity to orography and resolution. Monthly Weather Review, 123, 737-754.

Kida, H., T. Koide, H. Sasaki, and M. Chiba, 1991: A new approach for coupling a limited area model to a GCM for regional climate simulations. Journal of Meteorological Society of Japan, 69 (6), 723-728.

Kidson, J. W., 1988a: Indices of the Southern Hemisphere zonal wind. Journal of Climate, 1, 183-194.

Kidson, J. W., 1988b: Interannual variations in the Southern Hemisphere circulation. Journal of Climate, 1, 1177-1198. 
Kidson, J. W., 1991: Intraseasonal variations in the Southern Hemisphere circulation. Journal of Climate, 4, 939-953.

Kidson, J. W., 1999: Principal modes of Southern Hemisphere low-frequency variability obtained from NCEP-NCAR reanalyses. Journal of Climate, 12, 2808-2830.

Kidson, J. W., M. J. Revell, B. Bhaskaran, A. B. Mullan, and J. A. Renwick, 2002: Convection patterns in the Tropical Pacific and their influence on the atmospheric circulation at higher latitudes. Journal of Climate, 15, 137-159.

Kiehl, J. T. and K. E. Trenberth, 1997: Earth's annual global mean energy budget. Bulletin of the American Meteorological Society, 78 (No. 2), 197-208.

Kistler, R., E. Kalnay, W. Collins, S. Saha, G. White, J. Woollen, M. Chelliah, W. Ebisuzaki, M. Kanamitsu, V. Kousky, H. van den Dool, R. L. Jenne, and M. Fiorino, 2001: The NCEP-NCAR 50-year reanalysis: Monthly means CD-ROM and documentation. Bulletin of the American Meteorological Society, 82 (No. 2), 247-267.

Kitoh, A., S. Murakami, and H. Koide, 2001: A simulation of the Last Glacial Maximum with a coupled atmosphere-ocean GCM. Geophysical Research Letters, 28 (11), 2221-2224.

Koutavas, A., J. Lynch-Stieglitz, T. M. Marchitto Jr., and J. P. Sachs, 2002: El Niño-like pattern in ice age Tropical Pacific sea surface temperature. Nature, 297, 226-230.

Krinner, G. and C. Genthon, 1998: GCM simulations of the Last Glacial Maximum surface climate of Greenland and Antarctica. Climate Dynamics, 14, 741-758.

Krinner, G., D. Raynaud, C. Doutriaux, and H. Dang, 2000: Simulations of the Last Glacial Maximum ice sheet surface climate: Implications for the interpretation of ice core air content. Journal of Geophysical Research, 105 (D2), 2059-2070.

Kumar, A., F. Yang, L. Goddard, and S. Schubert, 2004: Differing trends in the tropical surface temperatures and precipitation over land and oceans. Journal of Climate, 17, 653-664.

Kushner, P. J., I. M. Held, and T. L. Delworth, 2001: Southern Hemisphere atmospheric circulation response to global warming. Journal of Climate, 14, 2238-2249.

Lautenschlager, M., U. Mikolajewicz, E. Maier-Reimer, and C. Heinze, 1992: Application of ocean models for the interpretation of atmospheric general circulation model experiments on the climate of the Last Glacial Maximum. Paleoceanography, 7 (NO. 6), 769-782. 
Levis, S., A. F. Foley, and D. Pollard, 1999: $\mathrm{CO}_{2}$, climate, and vegetation feedbacks at the Last Glacial Maximum. Journal of Geophysical Research, 104 (D24), 31,191-31,198.

Lewis, J. M., 1998: Clarifying the dynamics of the general circulation: Phillips's 1956 experiment. Bulletin of the American Meteorological Society, 79 (1), 39-60.

Li, D. and K. P. Shine, 1995: A 4-dimensional ozone climatology for UGAMP models. UGAMP Internal Report No. 35, 44 pages pp. UK Universities Global Atmospheric Modeling Programme, .

Limpasuvan, V. and D. L. Hartmann, 1999: Eddies and the annular modes of climate variability. Geophysical Research Letters, 26 (20), 3133-3136.

Lin, R.-Q., R.-X. Huang, and J. R. Apel, 1992: A study of the astronomical theory of ice ages in a two-dimensional nonlinear climate model. Journal of Geophysical Research, 97 (D9), 10029-10036.

Liu, Z., S. Shin, P. Behling, W. Prell, M. Trend-Staid, S. P. Harrison, and J. E. Kutzbach, 2000: Dynamical and observational constraints on Tropical Pacific sea surface temperatures at the last glacial maximum. Geophysical Research Letters, 27 (1), 105-108.

Lunt, D. J. and P. J. Valdes, 2001: Dust transport to Dome C, Antaretica, at the Last Glacial Maximum and present day. Geophysical Research Letters, 28 (2), 295-298.

Madden, R. A. and P. R. Julian, 1971: Detection of a 40-50 day oscillation in the zonal wind in the Tropical Pacific. Journal of the Atmospheric Sciences, 28 (5), 702-708.

Manabe, S. and R. F. Strickler, 1964: Thermal equilibrium of the atmosphere with a convective adjustment. Journal of the Atmospheric Sciences, 21, 361-385.

Manabe, S. and A. J. Broccoli, 1985: A comparison of climate model sensitivity with data from the Last Glacial Maximum. Journal of the Atmospheric Sciences, 42 (23), 2643-2651.

Manabe, S. and R. J. Stouffer, 1999: The role of thermohaline circulation in climate. Tellus, 51 A-B (1), 91-109.

Markgraf, V., 1991: Younger Dryas in southern South America. Boreas, 20, 6369.

Markgraf, V., J. R. Dodson, A. P. Kershaw, M. S. McGlone, and N. Nicholls, 1992: Evolution of late Pleistocene and Holocene climates in the circum-South Pacific land areas. Climate Dynamics, 6, 193-211. 
Marotzke, J., 1989: Instabilities and multiple steady states of the Thermohaline Circulation. Oceanic Circulation Models: Combining Data and Dynamics, D. L. T. Anderson and J. Willebrand, Eds., Kluwer Academic Publishers, 605.

McGlone, M. S., 1995: Lateglacial landscape and vegetation change and the Younger Dryas climatic oscillation in New Zealand. Quatenary Science Reviews, 14, 867-881.

McGlone, M. S., M. J. Salinger, and N. T. Moar, 1993: Paleovegetation studies of New Zealand's climate since the Last Glacial Maximum. Global Climates since the LGM, 294-316.

McGregor, J. L. and K. Walsh, 1994: Climate change simulations of Tasmanian precipitation using multiple nesting. Journal of Geophysical Research, 99 (D10), 20889-20905.

Meehl, G. A., 1991: A reexamination of the mechanism of the semiannual oscillation in the Southern Hemisphere. Journal of Climate, 4, 911-926.

Meehl, G. A., J. W. Hurrell, and H. van Loon, 1998: A modulation of the mechanism of the semiannual oscillation in the Southern Hemisphere. Tellus, 50A, 442-450.

Menendez, C. G., V. Serafini, and H. Le Treut, 1999: The storm tracks and the energy cycle of the Southern Hemisphere: Sensitivity to sea-ice boundary conditions. Annales Geophysicae, 17, 1478-1492.

Mitchell, J. F. B., 1993: Modelling of palaeoclimates: Examples from the recent past. Climate Research Technical note 37, 21 pp. Hadley Centre Meteorological Office, London Rd., Bracknell, UK.

Mo, K. C., 2000: Relationships between low-frequency variability in the Southern Hemisphere and sea surface temperature anomalies. Journal of Climate, 13, 3599-3610.

Moar, N. T., 1980: Late Otiran and Early Aranuian grassland in central South Island. New Zealand Journal of Ecology, 3, 4-12.

Moberg, A. and P. D. Jones, 2004: Regional climate model simulations of daily maximum and minimum near-surface temperatures across Europe compared with observed station data 1961-1990. Climate Dynamics, 23 (7-8), 695-715.

Morse, D. L., E. D. Waddington, and E. J. Steig, 1998: Ice age storm trajectories inferred from radar stratigraphy at Taylor Dome, Antarctica. Geophysical Research Letters, 25 (17), 3383-3386.

Moura, A. D. and P. H. Stone, 1976: The effects of spherical geometry on baroclinic Instability. Journal of the Atmospheric Sciences, 33, 602-616. 
Mullan, A. B., 1998: Southern Hemisphere sea-surface temperatures and their contempory and lag association with New Zealand temperature and precipitation. International Journal of Climatology, 18, 817-840.

Mullan, A. B. and B. J. McAvaney, 1995: Validation of high latitude tropospheric circulation in the Southern Hemisphere. First International AMIP Scientific Conference, 205-210.

Mullan, A. B., D. S. Wratt, and J. A. Renwick, 2001: Transient model scenarios of climate changes for New Zealand. Weather and Climate, 21, 3-34.

Nebeker, F., 1995: Calculating the weather. Vol. 60, International Geophysics Series, Academic Press, 255 pp.

Nelson, C. S., P. J. Cooke, C. H. Hendy, and A. M. Cuthbertson, 1993: Oceanographic and climatic changes over the past 160.000 years at deep sea drilling project site 594 off southeastern New Zealand, Southwest Pacific Ocean. Paleoceanography, 8 (4), 435-458.

Nelson, C. S., I. L. Hendy, H. L. Neil, C. H. Hendy, and P. P. E. Weaver, 2000: Last Glacial jetting of cold waters through the Subtropical Convergence Zone in the Southwest Pacific off eastern New Zealand, and some geological implications. Palaeogeography, Palaeoclimatology, Palaeoecology, 156, $103-$ 121.

NOAA, cited 2003: National Geophysical Data Center: Data announcement 88MGG-02: Digital relief of the surface of the Earth. [Available online from http://www.ngdc.noaa.gov/mgg/global/etopo5.HTML.]

Noone, D. and I. Simmonds, 2002: Associations between $\delta^{18} \mathrm{O}$ of water and climate parameters in a simulation of atmospheric circulation for 1979-95. Journal of Climate, 15, 3150-3169.

North, G. R., R. F. Cahalan, and J. A. Coakley Jr, 1981: Energy balance climate models. Reviews of Geophysics and Space Physics, 19 (1), 91-121.

North, G. R., J. G. Mengel, and D. A. Short, 1983: Simple energy balance model resolving the seasons and the continents: Application to the astronomical theory of the Ice Ages. Journal of Geophysical Research, 88 (C11), 6576-6586.

North, G. R., T. L. Bell, R. F. Cahalan, and F. J. Moeng, 1982: Sampling errors in the estimation of emperical orthogonal functions. Monthly Weather Review, 110, 699-706.

Paillard, D., 1998: The timing of Pleistocene glaciations from a simple multiplestate climate model. Nature, 391, 378-381.

Palmer, T. N., G. J. Shutts, and R. Swinbank, 1986: Alleviation of a systematic westerly bias in general circulation and numerical weather prediction models 
through an orographic gravity wave drag parameterization. Quarterly Journal of the Royal Meteorological Society, 112 (474), 1001-1039.

Peltier, W. R., 1994: Ice Age paleotopography. Science, 265, 195-201.

Penney, A. C., 2001: Climate Database (CLIDB) user's manual. NIWA Technical Report. National Institute of Water and Atmospheric Research, Wellington.

Pepper, A. C., J. Shulmeister, and D. C. Nobes, 2004: Possible ENSO signals prior to the Last Glacial Maximum, during the last deglaciation and the early Holocene, from New Zealand. Geophysical Research Letters, 31, L15206.

Petit, J. R., J. Jouzel, D. Raynaud, N. I. Barkov, J.-M. Barnola, I. Basile, M. Benders, J. Chapellaz, M. Davis, G. Delaygue, M. Delmotte, V. M. Kotlyakov, M. Legrand, V. Y. Lipenkov, C. Lorius, L. Pepin, C. Ritz, E. Saltzman, and M. Stievenard, 1999: Climate and atmospheric history of the past 420,000 years from the Vostok ice core, Antarctica. Nature, 399, 429-436.

Phillips, N. A., 1954: Energy transformations and meridional circulations associated with simple baroclinic waves in a two-level, quasi-geostrophic model. Tellus, 6, 273-286.

Pope, V. D. and R. A. Stratton, 2002: The processes governing horizontal resolution sensitivity in a climate model. Climate Dynamics, 19, 211-236.

Pope, V. D., M. L. Gallani, P. R. Rowntree, and R. A. Stratton, 2000: The impact of new physical parameterizations in the Hadley Centre climate model: HadAM3. Climate Dynamics, 16 (2-3), 123-146.

Press, F. and R. Siever, 1982: Earth. third ed. W.H. Freeman and Company, 613 pp.

Quintanar, A. I. and C. R. Mechoso, 1995: Quasi-stationary waves in the Southern Hemisphere. Part II: Generation mechanisms. Journal of Climate, 8, 2673-2690.

Renwick, J. A., 1998: ENSO-related variability in the frequency of South Pacific blocking. Monthly Weather Review, 126, 3117-3123.

Renwick, J. A., 2002: Southern Hemisphere circulation and relations with sea ice and sea surface temperature. Journal of Climate, 15, 3058-3068.

Renwick, J. A., 2005: Persistent positive anomalies in the Southern Hemisphere circulation. Monthly Weather Review, 133, 977-988.

Renwick, J. A. and M. J. Revell, 1999: Blocking over the South Pacific and Rossby wave propagation. Monthly Weather Review, 127, 2233-2247. 
Renwick, J. A., J. J. Katzfey, K. C. Nguyen, and J. L. McGregor, 1998: Regional model simulation of New Zealand climate. Journal of Geophysical Research, 103 (D6), 5973-5982.

Ridout, J. A., B. Chertouk, and R. Gelaro, 1994: Response of a general circulation model to a change in cloud solar forcing: Model feedbacks and comparison with satellite data. Journal of Geophysical Research, 99 (D9), 18555-18576.

Rind, D., 1987: Components of Ice Age circulation. Journal of Geophysical Research, 92 (D4), 4241-4281.

Rind, D., 1988: Dependence of warm and cold climate depiction on climate model resolution. Journal of Climate, 1, 965-997.

Rind, D., 1998: Latitudinal temperature gradients and climate change. Journal of Geophysical Research, 123 (D6), 5943-5971.

Rind, D., M. Chandler, P. Lonergan, and J. Lerner, 2001: Climate change and the middle atmosphere 5. Paleostratosphere in cold and warm climates. Journal of Geophysical Research, 106 (D17), 20,195-20,212.

Rossby, C.-G. and V. P. Starr, 1949: Interpretations of the angular-momentum principle as applied to the general circulation of the atmosphere. Journal of Meteorology, 6, 288.

Royal Society of New Zealand, 1994: Paleoclimates and climate modelling. National Science Strategy Committee for Climate Change Workshop, Science House, Royal Society of New Zealand, Wellington, SIR Publishing, 48.

Sadourny, R., 1994: Global atmospheric modelling. Long-Term Climatic Variations: data and modeling, J.-C. Duplessy and M.-T. Spyridakis, Eds., Springer-Verlag, 43-71.

Salinger, M. J., 1980a: New Zealand climate: I. Precipitation patterns. Monthly Weather Review, 108, 1892-1904.

Salinger, M. J., 1980b: New Zealand climate: II. Temperature patterns. Monthly Weather Review, 108, 1905-1912.

Salinger, M. J. and A. B. Mullan, 1999: New Zealand climate: Temperature and precipitation variations and their links with atmospheric circulation. International Journal of Climatology, 19 (10), 1049-1071.

Salinger, M. J., J. A. Renwick, and A. B. Mullan, 2001: Interdecadal Pacific Oscillation and South Pacific climate. International Journal of Climatology, 21, 1705-1721.

Saltzman, B., 1990: Three basic problems of paleoclimatic modeling: A personal perspective and review. Climate Dynamics, 5, 67-78. 
Seidov, D. and B. J. Haupt, 1997: Global ocean thermohaline conveyor at present and in the late Quaternary. Geophysical Research Letters, 24 (22), 2817-2820.

Shackleton, N. J., J. Backman, H. Zimmerman, D. V. Kent, M. A. Hall, D. G. Roberts, D. Schnitker, J. G. Baldauf, A. Desprairies, R. Homrighausen, P. Huddlestun, J. B. Keene, A. J. Kaltenback, K. A. O. Krumsiek, A. C. Morton, J. W. Murray, and J. Westberg-Smith, 1984: Oxygen isotope calibration of the onset of ice-rafting and history of glaciation in the North Atlantic region. Nature, 307, 620-623.

Shulmeister, J., I. Goodwin, J. A. Renwick, K. Harle, L. Armand, M. S. McGlone, E. Cook, J. R. Dodson, P. P. Hesse, P. Mayewski, and M. Curran, 2004: The Southern Hemisphere westerlies in the Australasian sector over the last glacial cycle: A synthesis. Quatenary International, 118-119, 23-53.

Simmonds, I., 1996: Climatic role of Southern Hemisphere extratropical cyclones and their relationship with sea ice. Papers and Proceedings of the Royal Society of Tasmania, 130 (2), 95-100.

Simmonds, I., 1998: The role of models in understanding climate change. Palaeoclimates, 3 ((1-3)), 7-23.

Simmonds, I. and X. Wu, 1993: Cyclone behaviour response to changes in winter Southern Hemisphere sea-ice concentration. Quarterly Journal of the Royal Meteorological Society, 119, 1121-1148.

Simmonds, I. and D. A. Jones, 1998: The mean structure and temporal variability of the semiannual oscillation in the southern extratropics. International Journal of Climatology, 18, 473-504.

Simmonds, I. and K. Keay, 2000a: Mean Southern Hemisphere extratropical cyclone behaviour in the 40-year NCEP-NCAR reanalysis. Journal of Climate, 13, 873-885.

Simmonds, I. and K. Keay, 2000b: Variability of Southern Hemisphere extratropical cyclone behaviour, 1958-97. Journal of Climate, 13, 550-561.

Simmons, A. J. and D. M. Burridge, 1981: An energy and angular-momentum conserving vertical finite-difference scheme and hybrid vertical coordinates. Monthly Weather Review, 109, 758-766.

Sinclair, M. R., 1995: A climatology of cyclogenesis for the Southern Hemisphere. Monthly Weather Review, 123 (6), 1601-1619.

Sinclair, M. R. and M. J. Revell, 2000: Classification and composite diagnosis of extratropical cyclogenesis events in the Southern Pacific. Monthly Weather Review, 128 (4), 1089-1105.

Slingo, A., 1989: A GCM parameterization for the shortwave radiative properties of water clouds. Journal of the Atmospheric Sciences, 46 (no. 10), 1419-1427. 
Smagorinsky, J., 1963: General circulation experiments with the Primitive Equations. Monthly Weather Review, 91 (3), 99-164.

Smagorinsky, J., 1983: The beginnings of numerical weather prediction and general circulation modelling: Early recollections. Advances in Geophysics, 25, $3-37$.

Smith, R. N. B., 1990: A scheme for predicting layer clouds and their water content in a general circulation model. Quarterly Journal of the Royal Meteorological Society, 116, 435-460.

Solomon, A. and P. H. Stone, 2001a: Equilibration in an Eddy Resolving Model with Simplified Physics. Journal of the Atmospheric Sciences, 58, 561-574.

Stammerjohn, S. E., M. R. Drinkwater, R. C. Smith, and X. Liu, 2003: Iceatmosphere interactions during sea-ice advance and retreat in the western Antarctic Peninsula region. Journal of Geophysical Research, 108 (C10), 27 (1$15)$.

Starr, V. P., 1948: An essay on the general circulation of the Earth's atmosphere. Journal of Meteorology, 5 (No. 2), 39-43.

Stephens, G. L. and P. J. Webster, 1981: Clouds and climate: Sensitivity of simple systems. Journal of the Atmospheric Sciences, 38, 235-247.

Stone, P. H., 1978: Baroclinic instability. Journal of the Atmospheric Sciences, 35, 561-571.

Taljaard, J. J., 1967: Development, distribution and movement of cyclones and anticyclones in the Southern Hemisphere during the IGY. Journal of Applied Meteorology, 6 (NO. 6), 973-987.

Tarasov, L. and W. R. Peltier, 1997: A high-resolution model of the 100ka iceage cycle. Annals of Glaciology, 25, 58-64.

Taylor, K. E., 1994: Climate models for the study of paleoclimates. Long-Term Climatic Variations: data and modeling, J.-C. Duplessy and M.-T. Spyridakis, Eds., Springer-Verlag, 21-41.

Thompson, P. D., 1990: Charney and the revival of numerical weather prediction. The Atmosphere - A Challenge: The Science of Jule Gregory Charney, R. S. Lindzen, E. N. Lorenz, and G. W. Platzmann, Eds., American Meteorological Society, 93-119.

Toracinta, E. R., R. J. Oglesby, and D. H. Bromwich, 2004: Atmospheric response to modified CLIMAP ocean boundary conditions during the Last Glacial Maximum. Journal of Climate, 17, 504-522. 
Trenberth, K. E., 1976: Fluctuations and trends in indices of the Southern Hemisphere circulation. Quarterly Journal of the Royal Meteorological Society, 102 (431), 65-75.

Trenberth, K. E., 1980: Planetary waves at $500 \mathrm{mb}$ in the Southern Hemisphere. Monthly Weather Review, 108, 1378-1389.

Trenberth, K. E., 1986: The signature of a blocking episode on the general circulation in the Southern Hemisphere. Journal of Atmospheric Sciences, 43 (19), 2061-2069.

Trenberth, K. E., 1991: Storm tracks in the Southern Hemisphere. Journal of the Atmospheric Sciences, 48 (19), 2159-2178.

Trenberth, K. E., Ed., 1992: Climate system modeling. Press Syndicate of the University of Cambridge, $788 \mathrm{pp}$.

Trenberth, K. E. and K. C. Mo, 1985: Blocking in the Southern Hemisphere. Monthly Weather Review, 113, 3-21.

Valdes, P. J. and N. M. J. Hall, 1994: Mid-latitude depressions during the Last Ice-Age. Long-Term Climatic Variations: data and modelling, J.-C. Duplessy and M.-T. Spyridakis, Eds., Springer Verlag, 511-531.

van Loon, H., 1956: Blocking action in the Southern Hemisphere. Notos, 5, 171177.

van Loon, H., 1967: The half-yearly oscillations in middle and high southern latitudes and the coreless winter. Journal of the Atmospheric Sciences, 24, 472 486.

van Loon, H., 1979: The association between latitudinal temperature gradient and eddy transport. Part I: Transport of sensible heat in winter. Monthly Weather Review, 107, 525-534.

van Loon, H. and R. L. Jenne, 1972: The zonal harmonic standing waves in the Southern Hemisphere. Journal of Geophysical Research, 77 (6), 992-1003.

van Loon, H. and J. W. Kidson, 1993: The association between latitudinal temperature gradient and eddy transport Part III: The Southern Hemisphere. Australian Meteorological Magazine, 42, 31-37.

van Loon, H., J. W. Kidson, and A. B. Mullan, 1993: Decadal variation of the annual cycle in the Australian dataset. Journal of Climate, 6, 1227-1231.

Walland, D. J. and I. Simmonds, 1999: Baroclinicity, meridional temperature gradients, and the southern semiannual oscillation. Journal of Climate, 12, 33763382. 
Walsh, K. J. E., I. Simmonds, and M. Collier, 2000: Sigma-coordinate calculation of topographically forced baroclinicity around Antarctica. Dynamics of Atmospheres and Oceans, 33, 1-29.

Weaver, A. J., M. Eby, A. F. Fanning, and E. C. Wiebe, 1998: Simulated influence of carbon dioxide, orbital forcing and ice sheets in the climate of the Last Glacial Maximum. Nature, 394, 847-853.

Webb, R. S., D. H. Rind, S. J. Lehman, R. J. Healy, and D. Sigman, 1997: Influence of ocean heat transport on the climate of the Last Glacial Maximum. Nature, 385, 695-699.

Welch, W. T. and K. Tung, 1998a: Non-linear baroclinic adjustment and wavenumber selection in a simple case. Journal of the Atmospheric Sciences, $\mathbf{5 5}$ (8), 1285-1302.

Welch, W. T. and K. Tung, 1998b: On the equilibrium spectrum of transient waves in the atmosphere. Journal of the Atmospheric Sciences, 55, 2833-2851.

Williams, K. D., D. H. Hassell, R. G. Jones, and J. M. Murphy, 2001: A comparison of the climate sensitivity of HadSM3 and HadSM3H, 16 pp. Hadley Centre

Wilson, M. F. and A. Henderson-Sellers, 1985: A global archive of land cover and soils data for use in general circulation climate models. Journal of Climatology, 5 (2), 119-143.

Wittenberg, A. T., 2002: ENSO response to altered climates, Atmospheric and Oceanic Sciences, Princeton University, 311.

World Climate Research Programme-111, 2000: Proceedings of the third PMIP workshop. Paleoclimate Modelling Intercomparison Project (PMIP), La Huardiere, Canada, 271.

Wyrwoll, K.-H., B. Dong, and P. J. Valdes, 2000: On the position of the Southern Hemisphere westerlies at the Last Glacial Maximum: An outline of AGCM simulation results and evaluation of their implications. Quaternary Science Reviews, 19, 881-898.

Yin, J. H. and D. S. Battisti, 2001: The importance of Tropical sea surface temperature patterns in simulations of Last Glacial Maximum climate. Journal of Climate, 14, 565-581. 